\title{
Motion Compensation and Robotic Control of Maritime Cranes
}

by

\section{Ryan A. McKenzie}

A thesis submitted to the Faculty of Graduate and Postdoctoral Affairs in partial fulfillment of the requirements for the degree of

\section{Master of Applied Science in Mechanical Engineering}

Ottawa-Carleton Institute for Mechanical and Aerospace Engineering Department of Mechanical and Aerospace Engineering Carleton University Ottawa, Ontario

(C) 2020

Ryan A. McKenzie 


\section{Abstract}

Maritime operations occur in a rapidly changing and extremely dangerous environment. To improve handling of cargo while at sea, this thesis develops a method for combining active-heave compensation and anti-pendulum control for a combined world-frame compensation system. State estimation algorithms are applied using low-cost inertial sensors attached to the deck of the ship and to the body of the load. The control system is validated with physical experiments on a test-scale motion

platform, as well as hardware-in-the-loop test-scale simulations. The results show potential for $49.2-99.5 \%$ reduction in settling time, $41.1-98.4 \%$ reduction in distance travelled, and 34.6-84.0\% reduction in root-mean-squared error for energy dissipation tests; as well as potential improvements in set-point tracking performance compared to uncompensated cases when base excitation is applied. 
To my wife, my parents, and all of my family;

I could not have done this without you. 


\section{Acknowledgments}

The author acknowledges the feedback and support of Dr. Rishad Irani of the MultiDomain Laboratory at Carleton University, as well as the editorial contributions of family and friends throughout the revision process.

The author acknowledges the in-kind contribution of DSA (Dynamic Systems Analysis Ltd) for the use of their ShipMo3D software.

The author acknowledges the support of the Natural Sciences and Engineering Research Council of Canada (NSERC). Cette recherche a été financée par le Conseil de recherches en sciences naturelles et en génie du Canada (CRSNG). 


\section{Table of Contents}

Abstract $\quad$ i

Acknowledgments $\quad$ iii

Table of Contents $\quad$ iv

List of Tables $\quad$ ix

List of Figures $\quad$ xi

List of Abbreviations and Symbols $\quad$ xix

1 Introduction 1

1.1 Contributions ....................... 8

1.2 Organization . . . . . . . . . . . . . . . . 9

2 Literature Review $\quad 10$

2.1 Motion Compensation . . . . . . . . . . . . . . 10

2.1.1 Heave Compensation . . . . . . . . . . . . . . . . . . 11

2.1.2 Anti-Pendulum Control . . . . . . . . . . . . . . . 15

2.1.3 Combined Heave Compensation and Anti-Pendulum Control . 20

2.1.4 Applications in the Current Research . . . . . . . . . . . . . 21 
2.2 Robotic Control and Modelling . . . . . . . . . . . . . . 23

2.2.1 Robotic Control and Manual Operation . . . . . . . . . . . 23

2.2.2 Robotic Modelling . . . . . . . . . . . . . . . . . . 24

2.2.3 Applications in the Current Research . . . . . . . . . . . . . . 28

2.3 Conclusion/Summary . . . . . . . . . . . . . . . . . 29

3 Design of Experiment and Simulation 30

3.1 System Overview . . . . . . . . . . . . . . . . . . . . . 31

3.2 Input Commands and Control System Implementation . . . . . . . . 34

3.3 Ship Motion Profiles . . . . . . . . . . . . . . . . . . . 38

3.4 Experimental Apparatus . . . . . . . . . . . . . . . 45

3.4.1 Multibody System 1: Motion Platform . . . . . . . . . . 46

3.4.2 Multibody System 2: Knuckle Boom Crane . . . . . . . . . . 46

3.4.3 Electric Actuators . . . . . . . . . . . . . . . . . 52

3.4.4 Actuator Sensors . . . . . . . . . . . . . . . 53

3.4.5 IMU Sensor . . . . . . . . . . . . . . . . . . . . 55

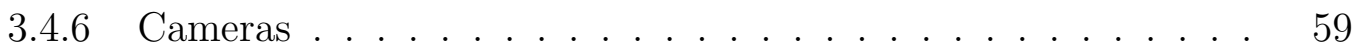

3.5 Test-Scale Simulation . . . . . . . . . . . . . . . . . . 61

3.5.1 Multibody System 1: Motion Platform . . . . . . . . . . . 65

3.5.2 Multibody System 2: Knuckle Boom Crane . . . . . . . . . 65

3.5.3 Electric Actuators . . . . . . . . . . . . . . 65

3.5.4 Sensors . . . . . . . . . . . . . . . . . . 68

3.6 Procedure for Testing and Analysis . . . . . . . . . . . . 70

3.7 Conclusion/Summary . . . . . . . . . . . . . . . . 71

4 Derivation of System Models $\quad 72$

4.1 Kinematics of the Motion Platform . . . . . . . . . . . . . 73 
4.1.1 Simscape-Based Inverse Kinematics . . . . . . . . . . . . . 76

4.1.2 Simscape-Based Forward Kinematics . . . . . . . . . . . . . . 78

4.2 Kinematics of the Knuckle Boom Crane . . . . . . . . . . . . . . . . . 82

4.2.1 Reference Frames and Joint Variables . . . . . . . . . . . . . . 85

4.2.2 Base Actuator Mechanism . . . . . . . . . . . . . . . . . 89

4.2.3 Boom Actuator Mechanism . . . . . . . . . . . . . . . . . 94

4.2.4 Jib Actuator Mechanism . . . . . . . . . . . . . . . 96

4.2.5 Forward and Inverse Kinematics of the Full Knuckle Boom Crane 98

4.2.6 Summary of Kinematic Models . . . . . . . . . . . . . . . . . 104

4.2.7 Confirmation of Kinematic Models . . . . . . . . . . . . . . . 107

4.3 Multibody Dynamics of the Pendulum Load . . . . . . . . . . . . . 109

4.3.1 Derivation with Lagrangian Mechanics . . . . . . . . . . . . . 109

4.3.2 Confirmation of Dynamic Models . . . . . . . . . . . . . . . 121

4.4 Conclusion/Summary . . . . . . . . . . . . . . . . . 127

5 State Estimation and Sensor Fusion $\quad 128$

5.1 Estimating Ship Motion . . . . . . . . . . . . . . 129

5.1.1 Orientation Algorithm: Complementary Filter . . . . . . . . . 132

5.1.2 Estimating Yaw, Pitch, and Roll . . . . . . . . . . . . . . . . 141

5.1.3 Position Algorithm: Extended Kalman Filter . . . . . . . . . . 144

5.2 Estimating Load Motion . . . . . . . . . . . . . . . . 149

5.2.1 Estimating Pendulum Angles and Position . . . . . . . . . . . 151

5.3 Removal of Estimation Biases and Filtering . . . . . . . . . . 155

5.4 Conclusion/Summary . . . . . . . . . . . . . . . 156

6 Motion Compensation and Control 157

6.1 Independent Joint Control . . . . . . . . . . . . . . . . . . . . 158 
6.2 LQR-Based Motion Compensation . . . . . . . . . . . . . . . . 164

6.3 Conclusion/Summary . . . . . . . . . . . . . . . . . . . 172

$\begin{array}{lll}7 & \text { Results and Discussion } & 173\end{array}$

7.1 Summary of Results . . . . . . . . . . . . . . . . . . 176

7.2 Energy Dissipation . . . . . . . . . . . . . . . . . . 181

7.2 .1 Off-Axis Motion . . . . . . . . . . . . . . . . 190

7.2.2 Additional Performance Metrics . . . . . . . . . . . . . . . . . 192

7.2.3 Natural Damping of the Pendulum . . . . . . . . . . . . . . 197

7.2.4 Preliminary Simulation Results . . . . . . . . . . . . . 201

7.2.5 Energy Dissipation Results . . . . . . . . . . . . . 207

7.3 Fixed Set-Point Tracking . . . . . . . . . . . . . . . . . 231

7.3.1 Ship Motion Results . . . . . . . . . . . . . . 235

7.3.2 Natural Frequency Excitation Results . . . . . . . . . . . . . . 240

7.4 Variable Set-Point Tracking . . . . . . . . . . . . . . . . . 242

7.5 Conclusion/Summary . . . . . . . . . . . . . . . . 247

8 Conclusions and Recommendations $\quad 249$

8.1 Objectives . . . . . . . . . . . . . . . . . . 249

8.2 Contributions . . . . . . . . . . . . . . . 250

8.3 Future Work . . . . . . . . . . . . . . . . . . . . 251

A Ship Motion Profiles $\quad 263$

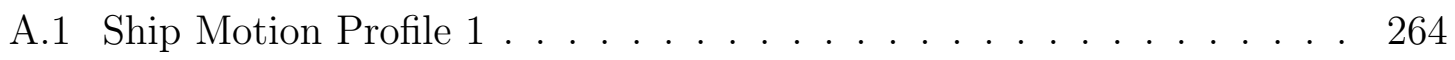

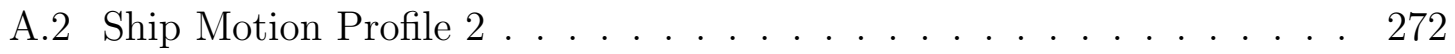

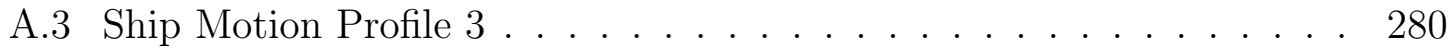

$\begin{array}{ll}\text { B Mechanical Drawings } & 288\end{array}$ 
C Mathematical Preliminaries

C.1 Rotation Sequences . . . . . . . . . . . . . . . . . . 356

C.2 Rotation Matrices. . . . . . . . . . . . . . . . . . . . 359

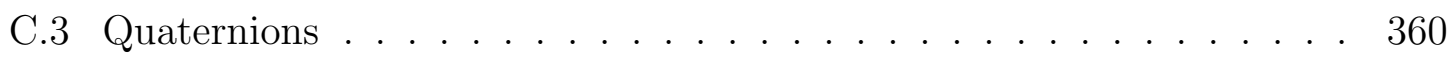

C.4 Transformation Sequences . . . . . . . . . . . . . . . 363

C.5 Homogeneous Transformation Matrices . . . . . . . . . . 366

C.6 Dual Quaternions . . . . . . . . . . . . . . . . . . 367

D Expanded Derivation of System Models 370

D.1 Confirmation of Kinematic Models . . . . . . . . . . . . 370

D.2 Matrix Form of Equations of Motion . . . . . . . . . . 382

D.3 Confirmation of Dynamic Models . . . . . . . . . . . . 386 


\section{List of Tables}

3.1 Data sent between the HIL/OITL simulation and the myRIO. . . . . 37

3.2 Crane actuator lower and upper deadbands. . . . . . . . . . . . . . 52

4.1 Node and edge descriptions for the motion platform. . . . . . . . 73

4.2 Summary of inverse kinematic model differences for Motion Profile $1 . \quad 80$

4.3 Summary of inverse kinematic model differences for various set-points. 81

4.4 Node and edge descriptions for the knuckle boom crane. . . . . . . . 84

4.5 Summary of kinematic model differences for the knuckle boom crane. 108

5.1 Summary of complementary filter parameters for the ship estimation. 140

5.2 Summary of complementary filter parameters for the load estimation. $\quad 150$

6.1 Independent joint control parameters. . . . . . . . . . . . . . . . . 162

7.1 Summary of mean energy dissipation results. . . . . . . . . . . . . 180

7.2 Summary of mean fixed set-point tracking results. . . . . . . . . . . . 180

7.3 Summary of variable set-point tracking results. . . . . . . . . . . . . . 180

7.4 Energy dissipation experiments/simulations (001-040). . . . . . . . 182

7.5 Energy dissipation experiments/simulations (041-080). . . . . . . 183

7.6 Fixed set-point tracking experiments/simulations (081-096). . . . . . 232

7.7 Variable set-point tracking experiments/simulations (097-104) . . . . 242 
C.1 Quaternion operations $[1] . \ldots \ldots$. . . . . . . . . . 361

C.2 Dual quaternion operations $[1,2] \ldots \ldots$. . . . . . . . . . 368 


\section{List of Figures}

1.1 Example of a knuckle boom crane adapted from [3] . . . . . . . . . . 2

1.2 Convention for coordinate frames and ship degrees of freedom $[4,5]$. . 3

1.3 The effect of heave compensation over a period of time. . . . . . . . 5

1.4 The energy dissipation effect of anti-pendulum control. . . . . . . . 6

3.1 System overview. . . . . . . . . . . . . . . . . . . . . . 31

3.2 Control system overview. . . . . . . . . . . . . . . . . . . . . . 32

3.3 Overview of the physical system and/or simulation. . . . . . . . . . 33

3.4 Operator Task 2: Variable set-point tracking. . . . . . . . . . . . 36

3.5 Convention for coordinate frames and ship degrees of freedom (repeated for convenience). . . . . . . . . . . . . . . 38

3.6 Motion platform with linear actuator labels. . . . . . . . . . . . 39

3.7 Motion platform coordinate frame conventions. . . . . . . . . . . . . . 42

3.8 Ship Motion Profile 1: Full-scale and test-scale displacements [6]. . . 43

3.9 Ship Motion Profile 1: LA1 set-points, feedback, and filtered feedback. 44

3.10 Test-scale experimental apparatus. . . . . . . . . . . . . 45

3.11 Test-scale knuckle boom crane. . . . . . . . . . . . . . . . . . 47

3.12 Crane tip workspace. . . . . . . . . . . . . . . . . . 48

3.13 Cable path with the jib retracted. . . . . . . . . . . . . 49

3.14 Cable path with the jib extended. . . . . . . . . . . . . . 49 
3.15 Extended cable length as a function of the jib actuator displacement. $\quad 50$

3.16 Crane potentiometers and encoder noise data. . . . . . . . . . 54

3.17 IMU reference frame. . . . . . . . . . . . . . . . . 56

3.18 IMU1 noise data. . . . . . . . . . . . . . . . . . . . . . . . . . 57

3.19 IMU2 noise data. . . . . . . . . . . . . . . . . . . . . . 58

3.20 Example of stereo vision with GoPro cameras. Key tracking points are indicated by dashed lines and red/black markers. . . . . . . . . . . . 60

3.21 Simscape model visualisation. . . . . . . . . . . . . . . . . . 63

3.22 Overview of the Simscape model block diagram. . . . . . . . . . . . 64

3.23 Simscape IMU model. . . . . . . . . . . . . . . . . . . . . . 69

4.1 Diagram of the motion platform. . . . . . . . . . . . . . . 74

4.2 Kinematic structure of the motion platform. . . . . . . . . . . . 74

4.3 Visualisation of the Simscape model used for the inverse kinematics of the motion platform. . . . . . . . . . . . . . 77

4.4 Block diagram of the Simscape model used for the inverse kinematics of the motion platform. . . . . . . . . . . . . . . . 79

4.5 Diagram of the knuckle boom crane. . . . . . . . . . . . . . 83

4.6 Kinematic structure of the knuckle boom crane. . . . . . . . . . . . . 84

4.7 Diagram of the knuckle boom crane. . . . . . . . . . . . . . 85

4.8 Base actuator mechanism. . . . . . . . . . . . . . . . . . . . . 90

4.9 Boom actuator mechanism. . . . . . . . . . . . . . . 95

$4.10 \mathrm{Jib}$ actuator mechanism. . . . . . . . . . . . . . . . . . . . . 97

4.11 Knuckle boom crane forward and inverse kinematic problems. . . . . 99

4.12 Model evaluation points in the crane's workspace. . . . . . . . . . . . 108

4.13 Diagram of the variable length spherical pendulum. . . . . . . . . . 109 
4.14 Simscape model of the variable length spherical pendulum used to confirm the dynamic analysis. . . . . . . . . . . . . . . . . . . . 121

4.15 Differences in the free response of each dynamic model caused by varying initial angles. . . . . . . . . . . . . . . . . . 123

4.16 Differences in the free response of each dynamic model for various initial angles and lengths. . . . . . . . . . . . . . . . . 125

5.1 Diagram of the ship motion estimation problem. . . . . . . . . . 129

5.2 Diagram of the load motion estimation problem. . . . . . . . . . . . 149

5.3 Diagram of the variable length pendulum with twist. . . . . . . . . 151

6.1 Block diagram of the PID algorithm. . . . . . . . . . . . . . . . 162

6.2 Block diagram of the LQR-based algorithm. . . . . . . . . . . . . . 170

6.3 Block diagram of the LQR-based algorithm as equivalent PD controllers.171

7.1 World position tracking data for energy dissipation tests. . . . . . . . 185

7.2 Pendulum response with exponential fitting for Test 020 Repetition 001 (uncompensated/free response of a $700 \mathrm{~mm}$ pendulum). . . . . . 188

7.3 Pendulum response with exponential fitting for Test 060 Repetition 001 (compensated response of a $700 \mathrm{~mm}$ pendulum) . . . . . . . . . . 189

7.4 Ellipsoid fitting result for Test 020 Repetition 001 (uncompensated/free response of a $700 \mathrm{~mm}$ pendulum). . . . . . . . . . . . . . . . . 195

7.5 Ellipsoid fitting result for Test 060 Repetition 001 (compensated response of a $700 \mathrm{~mm}$ pendulum). . . . . . . . . . . . . . . 195

7.6 Decay constants found from fitting exponential decay functions to the angular response of each uncompensated test case (Test 001-040). The mean value is indicated by a dashed line. . . . . . . . . . . . . . 200 
7.7 Pendulum response with exponential fitting for Simulation 020-1 (uncompensated/free response of a $700 \mathrm{~mm}$ pendulum) . . . . . . . . . . 203

7.8 Pendulum response with exponential fitting for Simulation 060-1 (compensated response of a $700 \mathrm{~mm}$ pendulum with truth data feedback). 204

7.9 Pendulum response with exponential fitting for Simulation 060-2 (compensated response of a $700 \mathrm{~mm}$ pendulum with simulated sensor feedback). . . . . . . . . . . . . . . . . . 205

7.10 The crane tip velocity and pendulum angle for truth and simulated sensor simulations, Simulation 060-1 and Simulation 060-2, respectively. 206

7.11 Significant peak angles in the positive $\theta_{x}$ direction for all energy dissipation tests (Test 001-080) and simulations (Simulation 001-1/2-0801/2). The experimental results are summarized by the mean of all repetitions with error bars to indicate the standard errors. The results are grouped by the mean initial angle, the negative of which is indicated by a dashed line. . . . . . . . . . . . . . . . . . . . . . . 212

7.12 Significant peak angles in the negative $\theta_{y}$ direction for all energy dissipation tests (Test 001-080) and simulations (Simulation 001-1/2-0801/2). The experimental results are summarized by the mean of all repetitions with error bars to indicate the standard errors. The results are grouped by the mean initial angle, the negative of which is indicated by a dashed line. . . . . . . . . . . . . . . . . 213 
7.13 Maximum height relative to the set-point as an indication of the potential energy introduced to the system for all energy dissipation tests (Test 001-080) and simulations (Simulation 001-1/2-080-1/2). The experimental results are summarized by the mean of all repetitions with error bars to indicate the standard errors, and the results are grouped by the mean initial angle. . . . . . . . . . . . . . . . .

7.14 Load position estimate for the first 10 seconds of the compensated sensor-based simulation, Simulation 060-2. The large errors in the zdirection over the first 2 seconds of the initialization period result in an abrupt change in pendulum length/height in some cases. . . . . . 218

7.15 Load position estimate for the first 10 seconds of the uncompensated sensor-based simulation, Simulation 020-2. The estimation is shown to converge to the actual value with a phase lag within this time, which suggests a minimum initialization period for the estimation algorithm.

7.16 Time constants for the decay of peak $\theta_{x}$ angles for all energy dissipation tests (Test 001-080) and simulations (Simulation 001-1/2-080-1/2). The experimental results are summarized by the mean of all repetitions with error bars to indicate the standard errors, and the results are grouped by the mean initial angle. . . . . . . . . . . . . . . . . . . 223

7.17 Time constants for the decay of peak $\theta_{y}$ angles for all energy dissipation tests (Test 001-080) and simulations (Simulation 001-1/2-080-1/2). The experimental results are summarized by the mean of all repetitions with error bars to indicate the standard errors, and the results are grouped by the mean initial angle. . . . . . . . . . . . . . . . 
7.18 Time constants for the decay of peak heights for all energy dissipation tests (Test 001-080) and simulations (Simulation 001-1/2-080-1/2). The experimental results are summarized by the mean of all repetitions with error bars to indicate the standard errors, and the results are grouped by the mean initial angle. . . . . . . . . . . . . .

7.19 Distance travelled above a noise threshold during the test period for all energy dissipation tests (Test 001-080) and simulations (Simulation 001-1/2-080-1/2). The experimental results are summarized by the mean of all repetitions with error bars to indicate the standard errors, and the results are grouped by the mean initial angle. . . . . . . . . . 227

7.20 Root-mean-squared error (RMSE) of the distance between the load position and the set-point during the test period for all energy dissipation tests (Test 001-080) and simulations (Simulation 001-1/2-0801/2). The experimental results are summarized by the mean of all repetitions with error bars to indicate the standard errors, and the results are grouped by the mean initial angle. . . . . . . . . . . . . . 230

7.21 World position tracking data for fixed set-point tests. . . . . . . . . . 234

7.22 Ellipsoid volume, distance travelled, and set-point tracking RMSE for fixed set-point tracking tests and simulations with base excitation derived from scaled ship motion (Test 081-083 and 089-091; Simulation 081-1/2-083-1/2 and 089-1/2-091-1/2). The experimental results are summarized by the mean of all repetitions with error bars to indicate the standard errors, and the results are grouped by the corresponding ship motion profile. . . . . . . . . . . . . . . . . 239 
7.23 Ellipsoid volume, distance travelled, and set-point tracking RMSE for fixed set-point tracking tests and simulations with sinusoidal base excitation derived from the natural frequency of the pendulum $w_{n}=\sqrt{g / l_{p}}$ (Test 084-088 and 092-096; Simulation 084-1/2-088-1/2 and 092-1/2096-1/2). The experimental results are summarized by the mean of all repetitions with error bars to indicate the standard errors, and the results are grouped by the corresponding motion profile. . . . . . . . 241

7.24 World position tracking data for variable set-point tests. . . . . . . . 244

7.25 Relative ellipsoid volume, distance travelled, and set-point tracking RMSE for variable set-point tracking tests and simulations with base excitation derived from scaled ship motion (Test 097-104; Simulation 097-1/2-104-1/2). The experimental results are summarized by the mean of all repetitions with error bars to indicate the standard errors, and the results are grouped by the corresponding ship motion profile. 246

A.1 Ship Motion Profile 1: Full-scale and test-scale displacements [6]. . . 264 A.2 Ship Motion Profile 1: Linear actuator set-points. . . . . . . . . . . 265

A.3 Ship Motion Profile 1: LA1 set-points, feedback, and filtered feedback. 266

A.4 Ship Motion Profile 1: LA2 set-points, feedback, and filtered feedback. 267

A.5 Ship Motion Profile 1: LA3 set-points, feedback, and filtered feedback. 268

A.6 Ship Motion Profile 1: LA4 set-points, feedback, and filtered feedback. 269

A.7 Ship Motion Profile 1: LA5 set-points, feedback, and filtered feedback. 270

A.8 Ship Motion Profile 1: Test-scale ship motion resulting from the filtered linear actuator feedback. . . . . . . . . . . . . . . . 271

A.9 Ship Motion Profile 2: Full-scale and test-scale displacements. . . . . 272 A.10 Ship Motion Profile 2: Linear actuator set-points. . . . . . . . . . 273 
A.11 Ship Motion Profile 2: LA1 set-points, feedback, and filtered feedback. 274

A.12 Ship Motion Profile 2: LA2 set-points, feedback, and filtered feedback. 275

A.13 Ship Motion Profile 2: LA3 set-points, feedback, and filtered feedback. 276

A.14 Ship Motion Profile 2: LA4 set-points, feedback, and filtered feedback. 277

A.15 Ship Motion Profile 2: LA5 set-points, feedback, and filtered feedback. 278

A.16 Ship Motion Profile 2: Test-scale ship motion resulting from the filtered

linear actuator feedback. . . . . . . . . . . . . . . . . . 279

A.17 Ship Motion Profile 3: Full-scale and test-scale displacements. . . . . 280

A.18 Ship Motion Profile 3: Linear actuator set-points. . . . . . . . . . . . 281

A.19 Ship Motion Profile 3: LA1 set-points, feedback, and filtered feedback. 282

A.20 Ship Motion Profile 3: LA2 set-points, feedback, and filtered feedback. 283

A.21 Ship Motion Profile 3: LA3 set-points, feedback, and filtered feedback. 284

A.22 Ship Motion Profile 3: LA4 set-points, feedback, and filtered feedback. 285

A.23 Ship Motion Profile 3: LA5 set-points, feedback, and filtered feedback. 286

A.24 Ship Motion Profile 3: Test-scale ship motion resulting from the filtered

linear actuator feedback. . . . . . . . . . . . . . . . . . . 287

C.1 Yaw-pitch-roll rotation sequence. . . . . . . . . . . . . . . . 357

C.2 Surge-sway-heave-yaw-pitch-roll transformation sequence. . . . . . . . 364

D.1 Simscape model of the knuckle boom crane used to confirm the kinematic analysis. . . . . . . . . . . . . . . . . . . . . 371

D.2 Simscape block diagram of the knuckle boom crane used to confirm the kinematic analysis. . . . . . . . . . . . . . . . . . . . . . . 372

D.3 Simscape block diagram for the base actuator mechanism. . . . . . . 373 


\title{
List of Abbreviations and Symbols
}

\author{
List of Abbreviations \\ 3D 3-Dimensional \\ AHC Active Heave Compensation \\ APC Anti-Pendulum Control \\ CAD Computer-Aided Design \\ CF Complementary Filter \\ DAE Differential Algebraic Equation \\ DC Direct Current \\ D-H Denavit-Hartenberg \\ DOF Degree(s) of Freedom \\ EKF Extended Kalman Filter \\ FFT Fast Fourier Transform \\ FPGA Field Programmable Gate Array \\ FPS Frames-Per-Second
}




\begin{tabular}{ll} 
FSMC & Fuzzy Sliding Mode Control \\
FOV & Field of View \\
HIL & Hardware-in-the-Loop \\
IMU & Inertial Measurement Unit \\
LA & Linear Actuator \\
LED & Light-Emitting Diode \\
LERP & Linear Interpolation \\
LQR & Linear Quadratic Regulator \\
MPC & Model Predictive Control \\
NI & National Instruments \\
ODE & Ordinary Differential Equation \\
RMSE & Root-Mean-Squared Error \\
OITL & Operator-in-the-Loop \\
PD & Proportional-Derivative \\
PHC & Passive Heave Compensation \\
PWD & Proportional-Integral-Derivative \\
\hline
\end{tabular}


RPR

RRR

SLERP

SMC

SPA

SVD

TCP

ZV

ZVD

ZVDD

\section{List of Symbols}

$A_{i}$

$\{\tilde{A}, \tilde{B}, \tilde{C}, \tilde{D}\}$

$S_{\vec{a}}$

${ }^{S} \vec{a}_{S / W}$

$B_{i}$
Revolute-Prismatic-Revolute Configuration of a Robotic Manipulator

Revolute-Revolute-Revolute Configuration of a Robotic Manipulator

Spherical Linear Interpolation

Sliding Mode Control

Signal Prediction Algorithm

Singular Value Decomposition

Transmission Control Protocol (internet protocol)

Zero Vibration

Zero Vibration Derivative

Zero Vibration Derivative Derivative

Amplitude of the $i^{\text {th }}$ mode or sine wave used to represent periodic motion

Set of linearised system matrices

Acceleration vector with components expressed in frame $S$

Acceleration vector for general/relative motion of frame $S$ with respect to frame $W$ with components expressed in frame $W$

Body frame of the $i^{\text {th }}$ rigid body or rigid body group 


$\begin{array}{ll}B(\xi, \dot{\xi}) & \text { Coriolis/Centrifugal matrix defined in terms of the } \xi \text { general- } \\ & \text { ized coordinates and } \dot{\xi} \text { velocities } \\ \vec{b}_{i} & \text { Offset vector for linear actuator base attachment points } \\ c_{(i, j)} & \text { Constants that define rigid offsets } \\ c_{x}, c_{y} & \text { Compact representations of } \cos \left(\theta_{x}\right) \text { and } \cos \left(\theta_{y}\right) \\ c_{\theta_{x}}, c_{\theta_{y}} & \text { Damping constants for the pendulum due to aerodynamic drag } \\ d & \text { Displacement }\end{array}$

$d_{j a}^{*} \quad$ Displacement associated with $j^{\text {th }}$ joint along the local $a$ axis

$d_{j a, 0}^{*} \quad$ Initial displacement associated with $j^{\text {th }}$ joint along the local $a$ axis

$E_{K} \quad$ Kinetic energy

$E_{P} \quad$ Potential energy

e Error-state vector

$e_{a} \quad$ Acceleration magnitude error for the complementary filter

$e_{j} \quad$ The $j^{\text {th }}$ edge of a graph diagram

$e(t), e_{k} \quad$ Error signals (continuous, discrete)

$F_{\xi} \quad$ Force associated with coordinate $\xi$

$f(x, \tilde{u}) \quad$ General form of a nonlinear system of equations

$f_{i} \quad$ Frequency $(\mathrm{Hz})$ of the $i^{t h}$ mode or sine wave used to represent periodic motion 


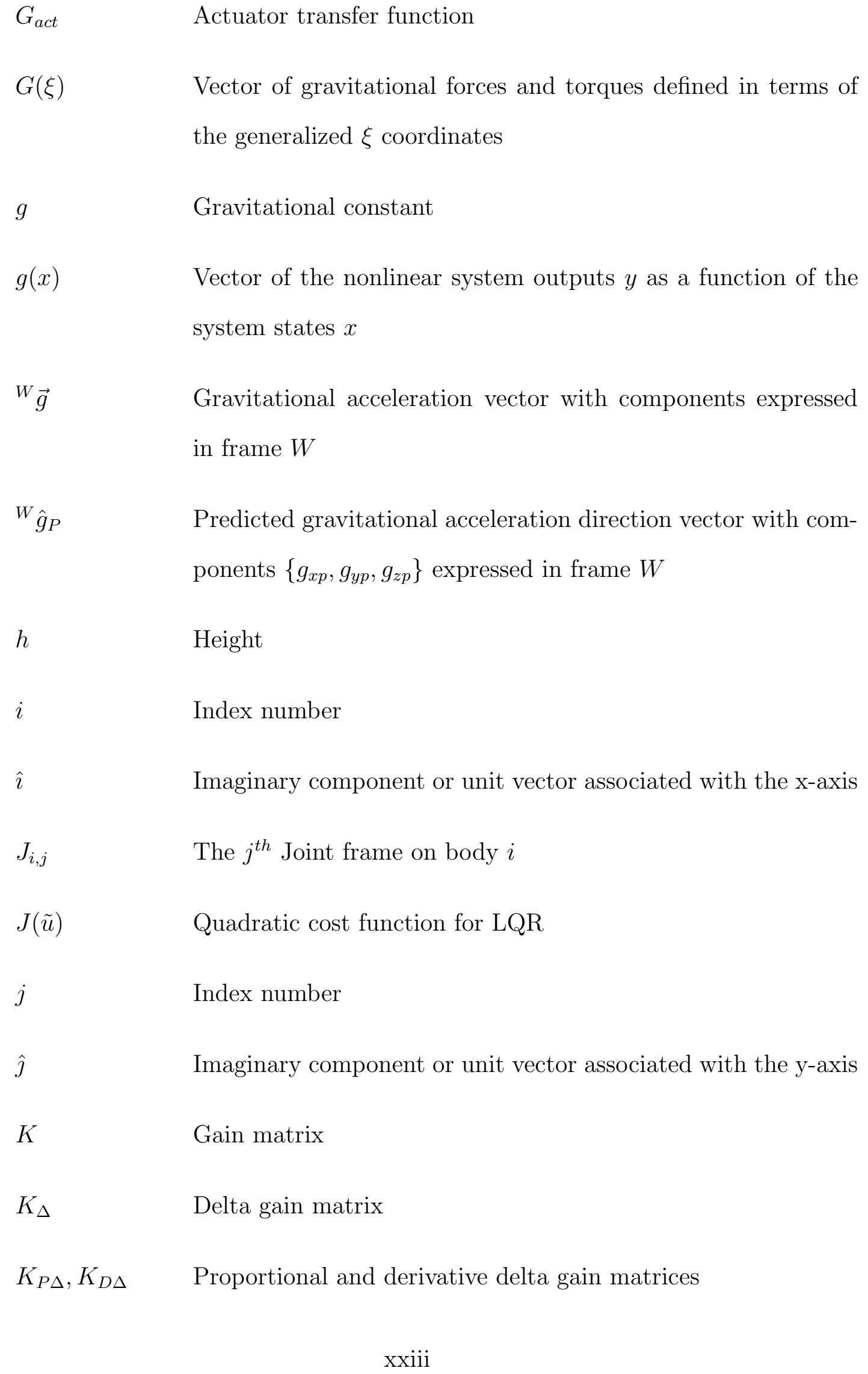




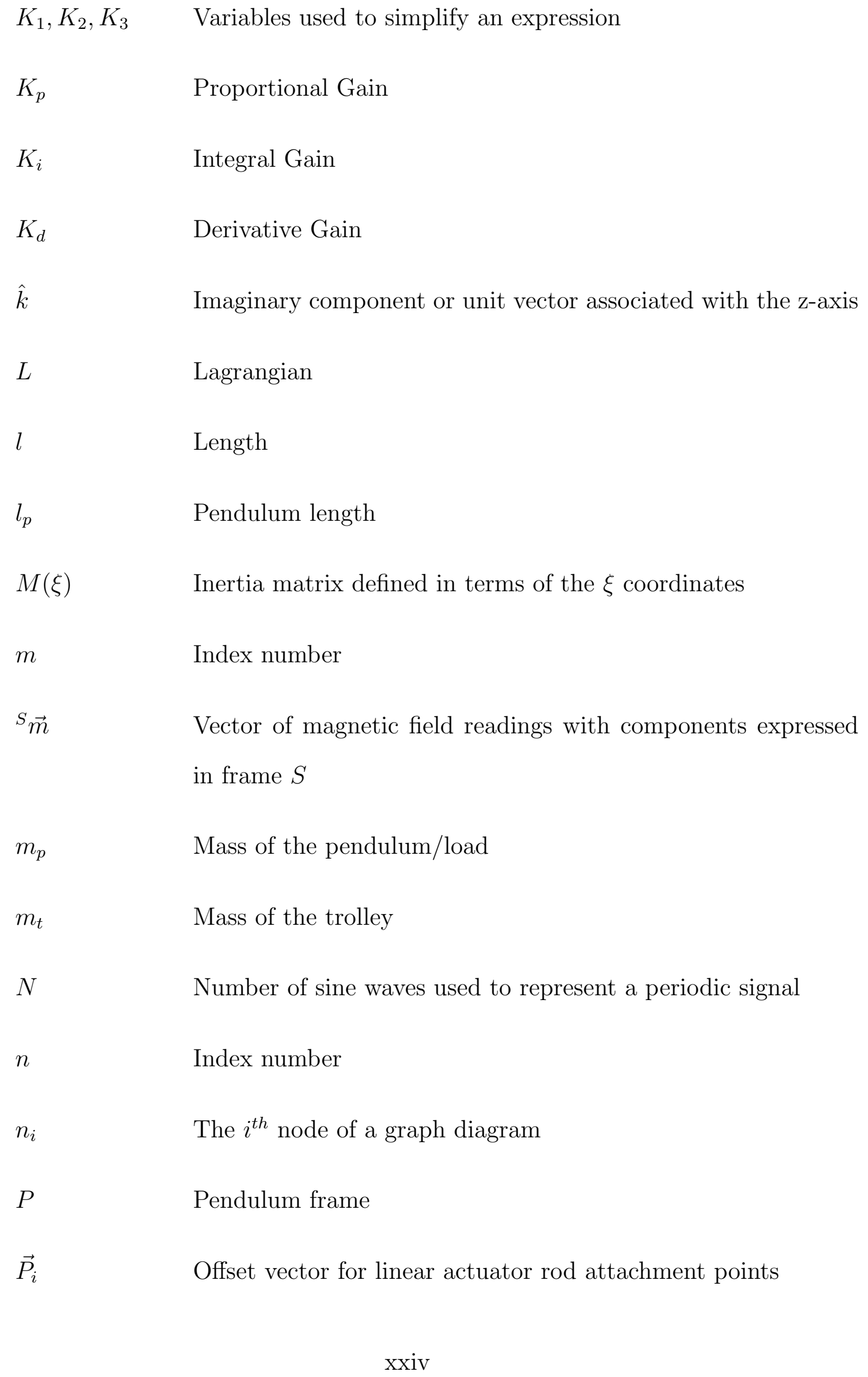


$\{Q, R, N\} \quad$ Quadratic cost function matrices for LQR

$\mathrm{Q}_{I} \quad$ Identity dual quaternion

$\mathbf{Q}_{j} \quad$ Dual quaternion associated with the $j^{\text {th }}$ joint

${ }^{A} \hat{\mathbf{Q}}_{B} \quad$ Dual quaternion transformation representing the pose of frame $B$ with respect to frame $A$

${ }^{A} \hat{\mathbf{Q}}_{D, B} \quad$ The dual/translational component of a dual quaternion transformation representing the pose of frame $B$ with respect to frame $A$

${ }^{A} \hat{\mathbf{Q}}_{R, B} \quad$ The real/rotation component of a dual quaternion transformation representing the pose of frame $B$ with respect to frame A

qI Identity quaternion

${ }^{W} \mathbf{q}_{S} \quad$ Quaternion transformation representing the orientation of frame $S$ with respect to frame $W$ with components $q_{i}$

$\Delta \mathbf{q}_{a} \quad$ Delta quaternion that represents a difference in orientation

${\widehat{\Delta \mathbf{q}_{a}}} \quad$ Interpolation-based delta quaternion that represents a difference in orientation subject to linear or spherical linear interpolation (LERP or SLERP).

$r(t), r_{k} \quad$ Controller reference signal (continuous, discrete)

${ }^{W} \vec{r}_{S / B} \quad$ Vector representing a rigid arm or offset of frame $S$ with respect to frame $B$ with components expressed in frame $W$ 


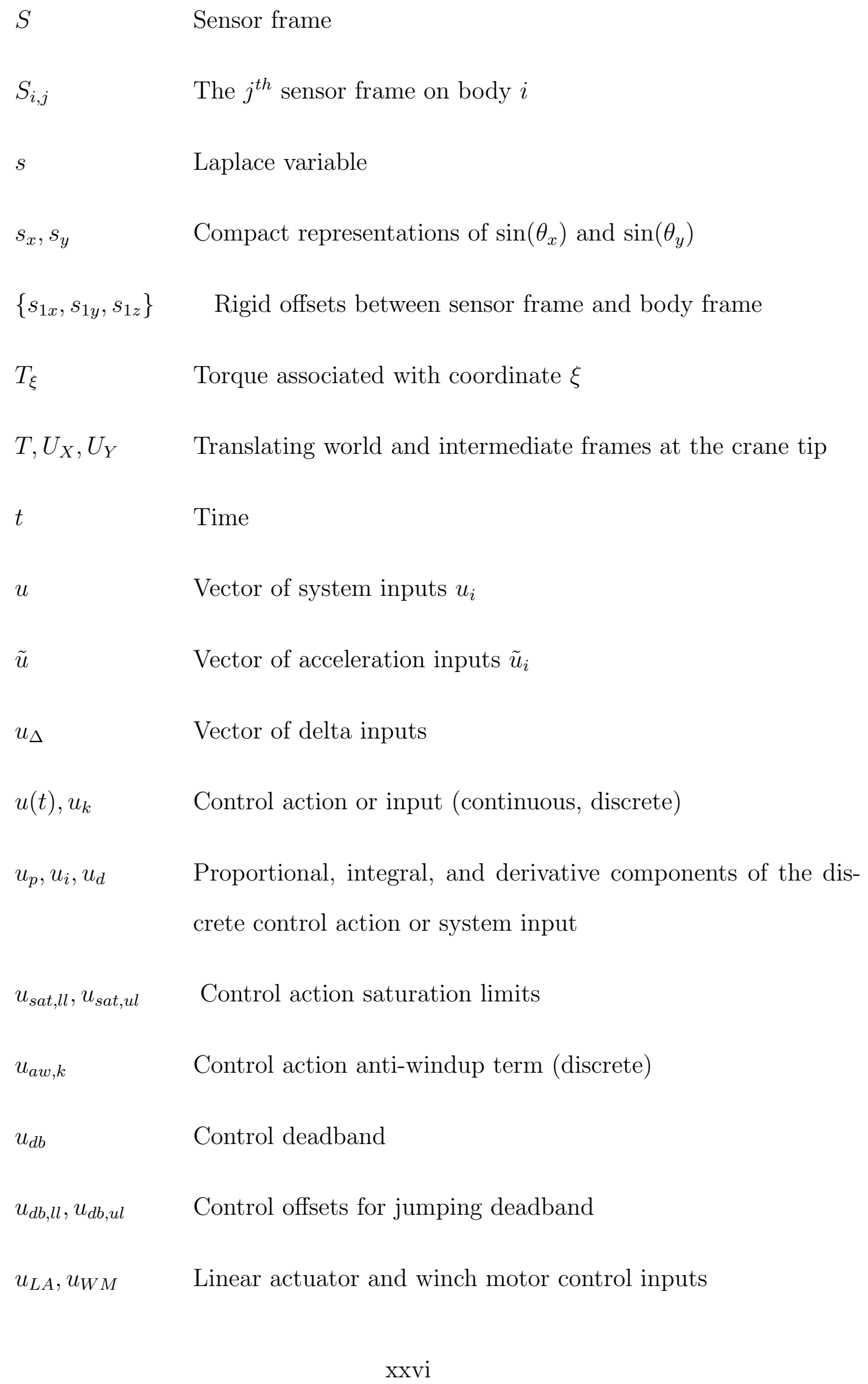

Sensor frame

$S_{i, j}$

$s$

$s_{x}, s_{y}$

$\left\{s_{1 x}, s_{1 y}, s_{1 z}\right\} \quad$ Rigid offsets between sensor frame and body frame

$T_{\xi}$

$T, U_{X}, U_{Y}$

$t$

$u$

$\tilde{u}$

$u_{\Delta}$

$u(t), u_{k}$

$u_{p}, u_{i}, u_{d}$

$u_{\text {sat }, l l}, u_{\text {sat }, u l}$

$u_{a w, k}$

$u_{d b}$

$u_{d b, l l}, u_{d b, u l}$

$u_{L A}, u_{W M}$

The $j^{\text {th }}$ sensor frame on body $i$

Laplace variable

Compact representations of $\sin \left(\theta_{x}\right)$ and $\sin \left(\theta_{y}\right)$

Torque associated with coordinate $\xi$

Translating world and intermediate frames at the crane tip

Time

Vector of system inputs $u_{i}$

Vector of acceleration inputs $\tilde{u}_{i}$

Vector of delta inputs

Control action or input (continuous, discrete)

Proportional, integral, and derivative components of the dis-

crete control action or system input

Control action saturation limits

Control action anti-windup term (discrete)

Control deadband

Control offsets for jumping deadband

Linear actuator and winch motor control inputs 
$v_{p x}, v_{p y}$

$v(t)$

W

$w_{i}, w_{n}$

$x$

$x, y, z$

$x_{t}, y_{t}, z_{t}$

$x_{p}, y_{p}, z_{p}$

$y$

$y(t), y_{k}$

$\alpha_{a}, \alpha_{m}$

$\bar{\alpha}_{a}, \bar{\alpha}_{m}$

$\beta$

$\beta_{a, l l}, \beta_{a, u l}$
Velocity

Components of the world-frame velocities of the pendulum load directed along its local $\mathrm{x}$ - and $\mathrm{y}$-axes

Time-varying offset term for a periodic signal

World frame

Natural frequency or Eigenfrequency ( $\mathrm{rad} / \mathrm{s}$ ) of the $i^{\text {th }}$ mode or sine wave used to represent periodic motion

Vector of system states $x_{i}$

Cartesian coordinates or axes

Cartesian coordinates for the crane tip

Cartesian coordinates for the pendulum load

Vector of system outputs $y_{i}$

System output signal (continuous, discrete)

Complementary filter gains/weights for interpolation

Complementary filter constant gains for adaptive interpolation

Complementary filter parameter for selecting the method of interpolation

Complementary filter parameters used to determine the adaptive gain from the acceleration magnitude error 
$\beta_{m}$

$\Delta$

$\epsilon$

$\zeta$

${ }^{W} \hat{\eta}_{P},{ }^{W} \hat{\eta}_{P}$

$\theta$

$\theta_{x}, \theta_{y}, \theta_{z}$

$\theta_{j a}^{*}$

$\theta_{j a, 0}^{*}$

$\kappa, \kappa_{\theta_{x}}, \kappa_{\theta_{y}}$

$\Lambda(\xi, u)$

$\Lambda_{\xi}$

$\Lambda_{u}$

$\lambda$
Complementary filter parameter for interpolation based on the magnetic field readings

Used to indicate change in a variable or quantity

Dual number

Generalized coordinate

Predicted and actual magnetic field direction vectors with components defined by $\left\{\eta_{x}, \eta_{y}, \eta_{z}\right\}$

Angular displacement

Pendulum angles

Angular displacement associated with the $j^{\text {th }}$ joint about the local $a$ axis

Initial angular displacement associated with the $j^{\text {th }}$ joint about the local $a$ axis

Damping constants for pendulum damping

Constraint matrix defined in terms of coordinates $\xi$ and inputs $u$

Partial derivative of the constraint matrix with respect to coordinates $\xi$

Partial derivative of the constraint matrix with respect to inputs $u$

Vector of $\lambda_{i}$ Lagrange multipliers 
$\lambda_{\theta_{x}}, \lambda_{\theta_{y}}$

$\mu$
Decay constants for pendulum damping

Mean

Vector of generalized coordinates with components $\xi_{i}$

Mathematical constant $(\pi \approx 3.14)$

Standard deviation

Vector of generalized forces/torques with components $\tau_{i}$

Time constants for pendulum damping

Discrete state transition matrix

Phase of the $i^{\text {th }}$ mode or sine wave used to represent periodic motion

Matrix of terms in the constrained nonlinear state equations related to the Coriolis/centrifugal and gravitational components of the equations of motion

Matrix of terms in the constrained nonlinear state equations related to the constrained acceleration inputs

Subtended angle used for spherical linear interpolation of quaternions

Vector of angular rates with components expressed in frame $S$ 


\section{Chapter 1}

\section{Introduction}

The \$36-billion maritime industry is an integral part of the Canadian economy [7]. Maritime activities can include transportation, offshore construction, scientific research, search and rescue operations, and national defence. Despite many advancements in the industry, worker safety is an ongoing concern [8]. Harsh maritime conditions lead to a high risk of workplace-related injury, as well as damage to equipment and/or goods. Research has shown that maritime cranes are involved in up to $12.1 \%$ of offshore injuries [9], and human error is cited as the root cause for $66-89 \%$ of such accidents $[10,11]$. These cranes operate in harsh conditions and suffer from control challenges such as disturbances from waves and wind. These operating conditions result in undesired motion that may lead to damage, injury, or death. The concerns are magnified by human error from an unintuitive joint control interface and a reliance on operator reactive control. The work carried out in this thesis seeks to reduce the undesired motion of the load during maritime crane operations by decoupling the motion of the load from that of the ship. Furthermore, the work seeks to reduce the occurrence of human error by developing robotic control of maritime cranes with an intuitive world-frame control structure. 
Maritime cranes are available in many different types and configurations with a range of sizes depending on the application. A common type of crane used in the maritime industry is the knuckle boom crane. An example of a knuckle boom crane is shown in Figure 1.1 with labels indicating the main components. The knuckle boom crane provides three degrees of freedom to position the crane tip, including relative rotation of the tower, the boom, and the jib. The load acts as a variable length pendulum with two angular degrees of freedom and a single linear degree of freedom. Although the pendulum length is controlled by extending or retracting cable, the angular degrees of freedom cannot be directly controlled and the crane is considered an underactuated system. The potential for a third underactuated rotation about the cable axis, the potential for double-pendulum effects at the crane hook, and the flexibility of the cable are not considered for the majority of the current work. The scope of the current research is limited to a knuckle boom crane due to its availability and usefulness in a large range of maritime operations. It is anticipated that the methods and concepts presented herein may be modified and extended to other crane types in future work.

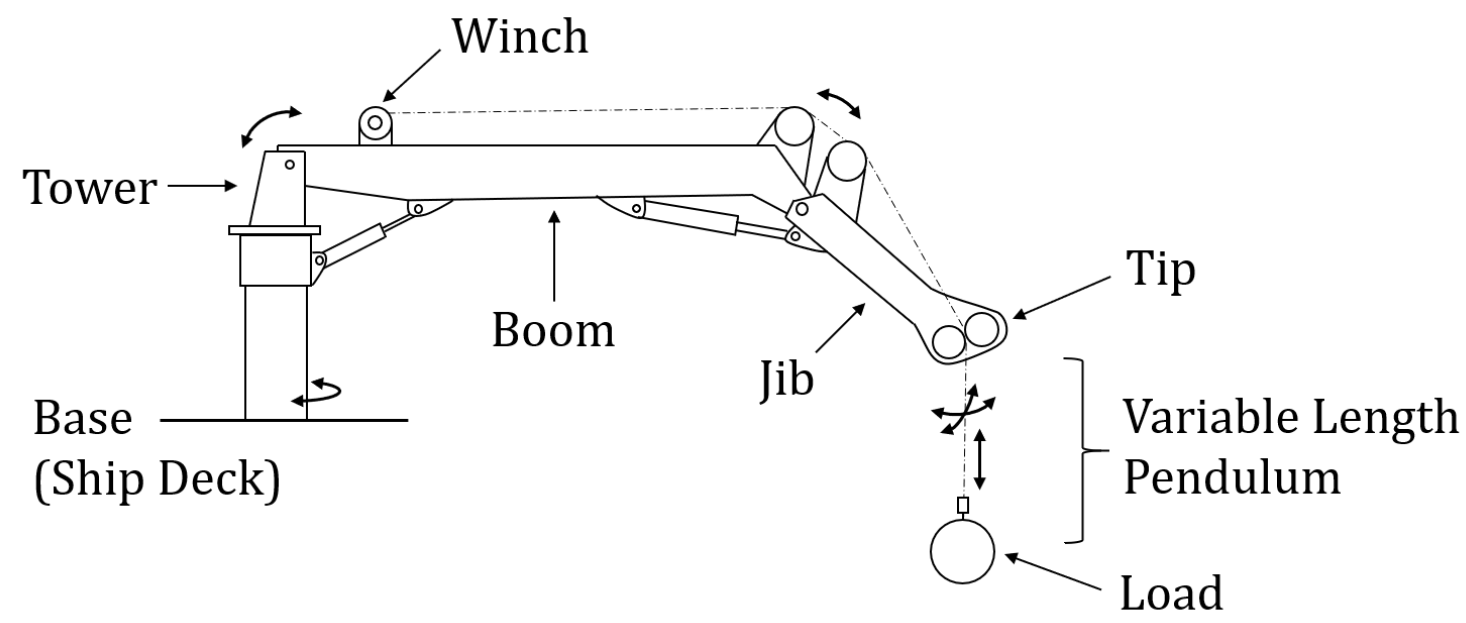

Figure 1.1: Example of a knuckle boom crane adapted from [3]. 
As previously noted, the operating environment for maritime cranes may involve disturbances from waves and wind. These disturbances cause the ship to move with six degrees of freedom, which are defined as surge, sway, heave, roll, pitch, and yaw $[4,5]$. The world and translating world frames are shown in Figure 1.2 with arrows to indicate the positive directions. In the current research, the world frame is defined as a standard right-handed reference frame with a North-East-Down configuration. For simplicity, the ship is assumed to have a Northern heading, and the ship motion is defined in a translating world frame that moves with the nominal velocity of the ship. The ship motion excites the base of the crane and leads to both linear and rotational movement of the suspended load. This undesired and often unanticipated motion may result in dangerous collisions, cable breakage due to increased tension and/or resonance, passenger discomfort in launch and recovery operations, and many other undesirable situations.

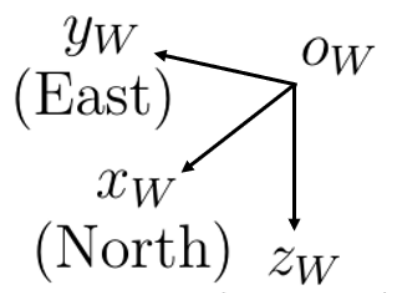

(Down)

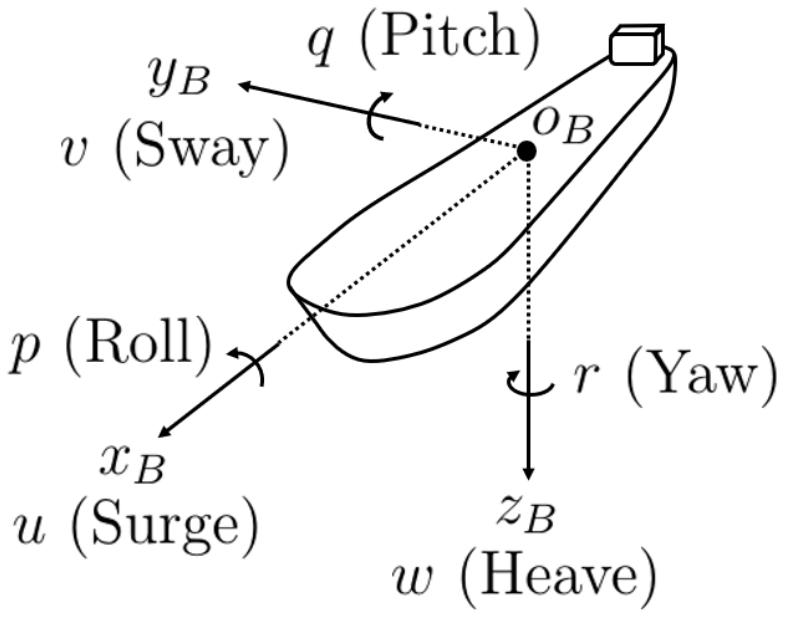

Figure 1.2: Convention for coordinate frames and ship degrees of freedom $[4,5]$. 
In some cases, it may be possible to wait for lower sea states and thereby avoid extreme situations. However, delaying an operation is not always possible, practical, or economically viable. Therefore, this research will focus on applying the existing crane and actuators with a modified control system to compensate for the ship motion. The compensation system will be used to decouple the load motion from that of the ship, and will allow the operator to focus on performing the required task with fewer distractions.

The primary research will combine two existing methods to reduce undesired motion, namely heave compensation and anti-pendulum control. The former is used to decouple the vertical heave motion of the load from that of the vessel, whereas the latter is used to reduce the pendulation of the load. A typical heave compensation technique is illustrated in Figure 1.3 with time progressing from left to right. The periodic heave motion of the crane tip is indicated by the blue line, the desired load positions are shown with dashed outlines and may be achieved by varying the cable length, and the large black circles indicate how the load acts with a fixed cable length. Similarly, an anti-pendulum control technique is illustrated in Figure 1.4. The uncompensated motion is shown on the left with the load swinging about the crane tip as a result of a disturbance. If the crane tip is moved such that kinetic energy is dissipated from the load, the swinging can be reduced and/or eliminated as shown on the right. In literature and industry, each method has proven to be successful [12-16]; however, few articles exist that combine these methods [17] and world-frame sensing and positioning of the load is not explicitly considered. To address this gap in knowledge, the primary research will build on the heave compensation work of [13] and the anti-pendulum work of $[14,17]$ to develop a combined compensation system that attempts to maintain a desired world-frame position with realistic sensor feedback. 
The first objective is to develop a method for combining active-heave compensation and anti-pendulum control for a combined world-frame compensation system.

The world-frame compensation system will attempt to maintain a desired position of the load relative to the world frame by manipulating the existing crane actuators. This form of compensation is directly applicable to a variety of at-sea operations, which may include offshore construction or ship-to-platform transfer. In this thesis work, a proportional controller with deadbands will be used in combination with an inverse kinematic model to track world-frame set-points while issuing commands to reduce undesired heave and pendulation.

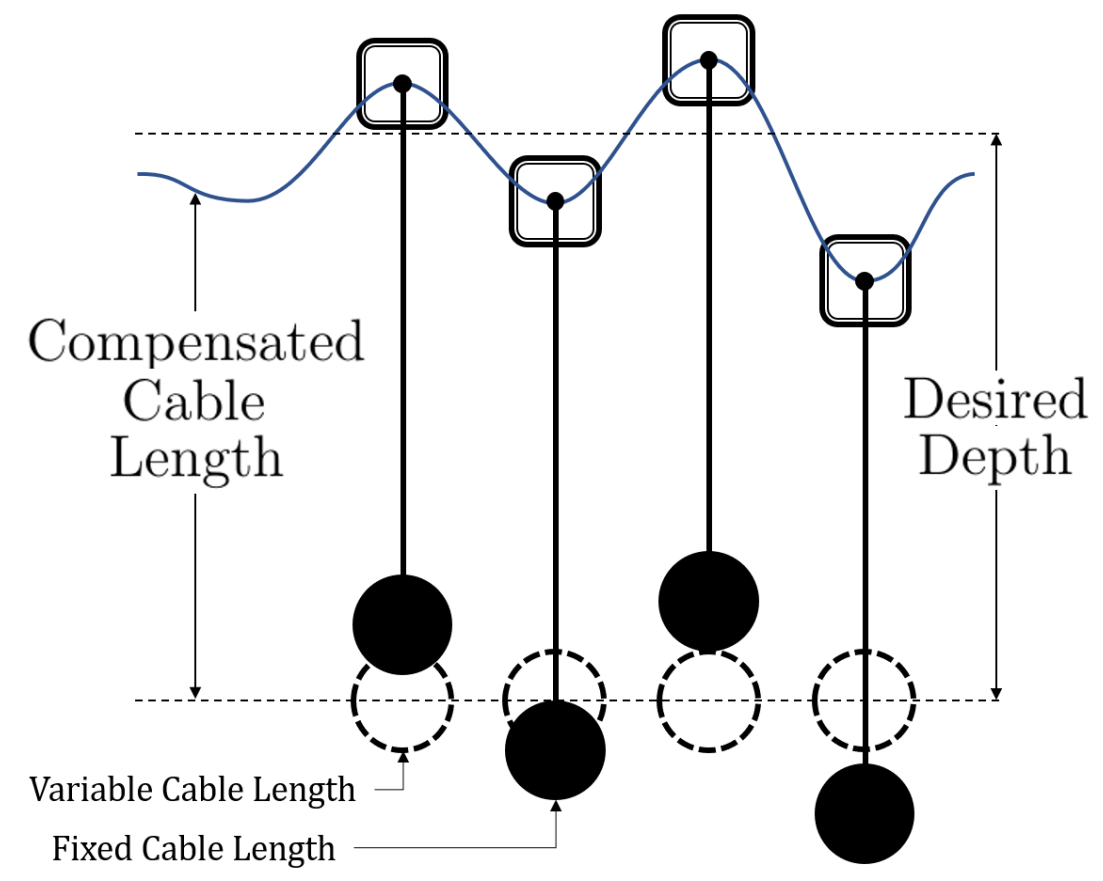

Figure 1.3: The effect of heave compensation over a period of time. 
It is noted that the performance of a world-frame compensation system depends entirely on the ability of the system to estimate key states, including the pose of the ship and the load. Therefore, in the current thesis work, a quaternion-based sensor fusion algorithm will be applied to estimate the angular motion of both the load and the ship. The signal-to-noise ratio of the test-scale accelerations are not appropriate for linear motion estimates of the ship; however, an algorithm from previous work will be included for completeness. For the proposed solution, wireless inertial measurement units (IMUs) will be placed on the load and ship to provide feedback for performing the pose estimations. The IMU-based solution is low-cost and would require very little modification of existing industrial systems. Inertial measurements are not subject to the limitations that result from visual obstructions such as sea spray, inclement weather, or crashing waves; and IMU-based technology is widely used within the industry.

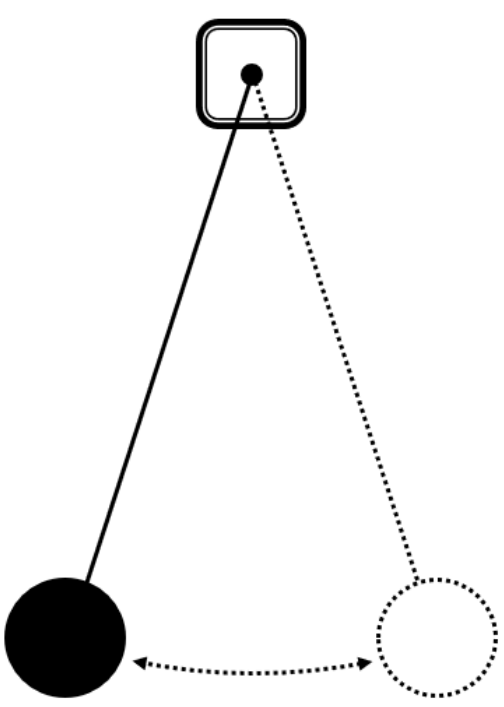

(a) Uncompensated Motion

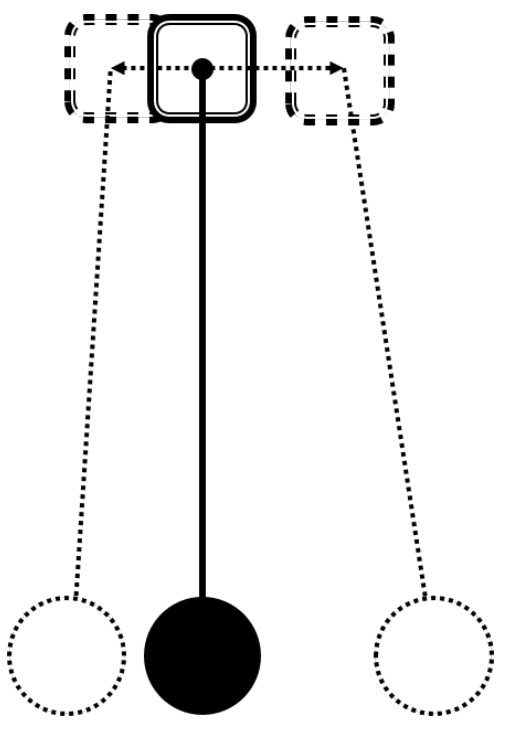

(b) Compensated Motion

Figure 1.4: The energy dissipation effect of anti-pendulum control. 
The secondary research will reduce the potential for human error by developing world-frame control. In current systems, the operator manually controls a series of rotational joints to indirectly control the load [17]. To achieve a linear reach motion, the operator must typically rotate two or more joints simultaneously. In the field of robotics, this control method is referred to as joint-space control [18]. Joint-space control is challenging and may result in many deviations from the optimal trajectory due to human error $[17,19]$. In addition, the operator must account for a delay between the crane and load motion due to the inertia of the load. To address these challenges, an operator will issue commands to control the linear motion of the load relative to the world frame, and a controller will solve inverse kinematic equations to convert these linear commands to joint commands. Although inverse kinematic control is common in the field of robotics [18], it has yet to be widely applied to industrial maritime cranes due to the complex motion, disturbances, inertial effects, and variable cable length. A world-frame control scheme could allow for improved ship-to-ship and/or ship-to-platform transfers in future work, and it is well-suited for the eventual automation of such tasks.

The second objective is to integrate the robotic control technique with the combined compensation system for hardware-in-the-loop testing. The work seeks to evaluate the effectiveness of the control and compensation techniques through test-scale experiments and simulations.

To achieve the above goals, a systematic and concurrent approach will be applied. A robotic model will be created in MATLAB and Simulink to simulate the crane, sensors, actuators, winch, and pendulum load. The operator controls will be added to allow for simulation with operator-in-the-loop testing in future work, and an experimental apparatus will be developed by modifying an existing instrumented testbed for use in the flume tank at Carleton University [20]. System identification techniques 
will be applied to estimate transfer functions and deadbands for the real-world actuators, as well as to quantify the natural damping of the pendulum and sensor noise. Existing ship motion data from [21] and [6] will be applied without compensation to study the no-control case. Finally, the combined compensation system will be implemented with a sensor fusion algorithm to estimate the ship and load motion. The performance of the compensation system relative to the uncompensated case will be evaluated through a variety of disturbance rejection, fixed set-point tracking, and variable set-point tracking experiments and simulations.

\subsection{Contributions}

The primary contributions of the research discussed in this thesis are:

- Further development of a test-scale experimental apparatus for evaluation of motion compensation systems.

- Development of parametric hardware-in-the-loop simulations to facilitate future development and testing. Although operator studies and human factors analysis are beyond the scope of the current work, the simulations allow for operatorin-the-loop testing in future work.

- Development of a combined compensation system with potential for extended application to a variety of crane types in future work.

- Application of a robotic control method to reduce the potential for operator error during compensated crane operations, as well as to facilitate task automation in future work.

- Application of sensor fusion techniques to estimate both the ship and load motion through low-cost inertial sensor feedback. 
- Test-scale experimental validation and simulations of the compensation and control techniques for a variety of test conditions.

\subsection{Organization}

To meet the key objectives outlined above and to effectively present the research, this thesis is organized into a series of eight chapters with several appendices. Following this introductory chapter, a literature review is included in Chapter 2. The design of the experimental apparatus, the input motion profiles, and the simulation parameters are described in Chapter 3. In Chapter 4, the relevant system models are developed for simulation and control. The methods used to estimate the motion of the ship, load, and crane using the available and/or simulated sensor feedback are developed in Chapter 5. The motion compensation algorithm is developed in Chapter 6. The details of the experimental and simulated results are provided in Chapter 7. Finally, the conclusions and recommendations for future work are summarized in Chapter 8. 


\section{Chapter 2}

\section{Literature Review}

This chapter reviews the limitations, benefits, drawbacks, and applicability of the literature as it relates to the objectives of the current research. In Section 2.1, motion compensation is discussed for both heave compensation and anti-pendulum control of maritime cranes as it relates to the first objective of the current thesis work. In Section 2.2, robotic modelling and control methods are discussed as they are related to both the first and second objectives.

\subsection{Motion Compensation}

Motion compensation is an ongoing concern in the maritime industry. The two main types of compensation are heave compensation and anti-pendulum control. For a more extensive review of vertical heave compensation, the reader is referred to the work of Woodacre et al. [22]. Similarly, for a more extensive review of anti-pendulum control, the reader is referred to the work of Ramli et al. [12] and Abdel-Rahman et al. [23]. The term anti-pendulum control, as opposed to anti-sway control, is used in this thesis work to avoid confusion with the swaying motion of the ship. The anti- 
pendulum control method is intended to reduce pendulation caused by the operator commands and/or ship motion in any direction and, therefore, is considered a more precise term for maritime crane applications.

Within the current section, heave compensation is discussed in Subsection 2.1.1, anti-pendulum control is discussed in Subsection 2.1.2, and compensation techniques that combine heave and anti-pendulum control are presented in Subsection 2.1.3. Finally, applications of the motion compensation literature in the context of the current thesis work are described in Subsection 2.1.4.

\subsubsection{Heave Compensation}

Heave compensation is used to decouple the vertical motion of the load from that of the ship. There are several established methods, which may be classified as either active heave compensation (AHC) or passive heave compensation (PHC). An active system applies closed-loop control and requires an energy input to perform the task, whereas a passive system acts as an open-loop vibration isolator [22]. Although it is sometimes less expensive to implement a passive system, Hatleskog and Dunnigan suggest that PHC is limited to approximately $80 \%$ reduction in heave motion [24]. In contrast, AHC systems may approach 84\%-99\% reduction in heave motion [22]; however, it is noted that most systems are evaluated in simplified simulations with limited experimental validation. In the current research, AHC will be applied due to its higher potential for decoupling the load and ship motion. The active system may also make use of existing equipment with very few modifications.

In its simplest form, the goal of an AHC system is to reduce the load velocity to zero by controlling the relative motion between the load and vessel. Assuming only 
vertical motion, the heave velocity of the load may be represented as

$$
v_{\text {load }}=v_{\text {vessel }}+v_{\text {load } / \text { vessel }},
$$

where the relative velocity of the load with respect to the vessel may be controlled via existing crane actuators (ex. winch or hydraulic cylinders). In this ideal case, the heave motion is reduced by setting the relative velocity to cancel the heave velocity of the vessel such that

$$
v_{\text {load } / \text { vessel }}=-v_{\text {vessel }}
$$

and the resultant velocity of the load from Equation 2.1 is held at zero. Equivalently, the effect of this velocity cancellation may be considered as simply maintaining the vertical world-frame position of the load by rejecting the base excitation of the vessel.

The vessel motion is typically sensed through an inertial measurement unit (IMU), which provides linear accelerations and angular rates to describe the ship motion [22]. Although many researchers only consider the heave motion of the vessel, it is important to consider all degrees of freedom to fully decouple the vertical motion $[25,26]$. When the vessel rolls or pitches, the crane will also rotate about the axis of rotation, and, as a result, the vertical position of the tip will change. When combined with the heave motion of the vessel, this added vertical displacement may significantly affect the response of the system. The placement of the crane with respect to the axis of rotation determines the magnitude of the added displacement, so this additional displacement will be accounted for in the current research.

It is also noted that, for both experiments and simulations, realistic ship motion and IMU sensor data should be used to determine the system response. This data is not commonly available for real-world systems and may be difficult to generate/acquire, so the majority of researchers apply simplistic sine waves to verify control 
techniques and cite this concern for future work. The real-world scenarios are much more complex, and an investigation of the response to these conditions is necessary to fully evaluate a compensation system. In the current research, motion data from the DSTO-TR-0093 report and ShipMo3D simulations will be applied to allow for consistency/benchmarking with previous work $[6,13,21,27]$.

As previously mentioned, the closed-loop AHC method requires a controller and one or more actuators to operate. A common technique is to use the winch to extend or retract cable as the ship moves up or down, respectively. This winch-based technique has been applied by many researchers with various controllers, and it is currently used in the maritime industry. The winch-based technique was also examined by Woodacre et al. in a review of heave compensation systems and related work $[13,22,28]$. The authors reviewed many techniques for heave compensation, and analysed the effectiveness of various controllers. The authors concluded that predictive control techniques could account for system lag, and thereby improve tracking performance.

Woodacre et al. confirmed that a model predictive controller with integral action and a preceding signal prediction algorithm (MPC+PI+SPA) could improve performance of an AHC system [13,28]. The AHC task was accomplished through the use of a hydraulic winch, and the system was evaluated through simulations and experiments for various operating conditions. The MPC+PI+SPA was compared to a standard PID controller, and the results showed improved robustness and decreased tracking error for the majority of test cases. Realistic motion data was applied for a single axis compensation, the work focused on set-point tracking for towing applications, and the work did not consider pendulum dynamics that would be present in a crane system.

An alternative to the winch-based AHC algorithm was recently presented by Chu 
et al. [17]. The technique was based on inverse kinematics, and the authors used the linear actuators of a knuckle boom crane to maintain a constant vertical position of the crane tip. The boom and jib hydraulic cylinders were actuated to perform the compensation task rather than relying on the winch to vary the cable length. Although the approach was effective for small heave motion, it was not as effective for high sea states due to physical limitations of the crane's range of motion. The system also experienced lag when hydraulic models were included, which further decreased performance.

In the current research, the inverse kinematics and the winch-based approaches will be partially combined. In particular, the crane actuators will be used to maintain a constant tip position, and the winch will be used to adjust the cable length to account for deviations between the desired tip position and the sensed position. This combination of inverse kinematics and winch control may improve set-point tracking for the load position if the tip approaches the boundary of its workspace, or if the winch response is significantly faster than that of the crane actuators. Although maintaining the tip position is not always possible or practical for large heave motions, it may have several benefits over automatically varying the cable length for specific industrial applications. In future work, it may be beneficial to implement the systems in parallel and allow the operator to switch the heave compensation method based on the operational goals.

In addition to heave compensation, anti-pendulum control is required to fully decouple the motion of the load from that of the ship. 


\subsubsection{Anti-Pendulum Control}

Anti-pendulum control (APC) is applied to reduce the pendulum motion of the load. In land-based systems, the pendulum motion is produced predominantly through operator control actions with some potential for wind-induced motion. Therefore, feed-forward techniques are quite commonly applied for land-based cranes with some feedback techniques used to remove external excitation. However, for maritime crane applications, the external excitation is much larger due to a fully mobile base that is affected by both wind and waves. Longer cable lengths may also cause the pendulum to experience resonance if the natural frequency is excited by the low-frequency ship motion, and the operator control actions combined with potentially high inertia loads may induce significant pendulum motion. Both feed-forward and feedback anti-pendulum control methods have been applied in the literature.

Feed-forward APC is an open-loop method that may reduce operator-induced excitation of the load during crane operations. The feed-forward method does not require additional sensors for determining the load position, so it is unable to reduce motion caused by external excitation without the addition of feedback control. Ramli et al. [12] reviewed several existing feed-forward methods, which include input shaping, filtering, and command smoothing. The input shaping method was identified as the most popular and was more often applied for APC. The input shaping method applies knowledge of the natural frequencies and damping ratios of a system to adjust a command signal with impulses. This method has been applied to nonlinear systems; however, it is sensitive to parameter uncertainties and typically requires an initial angle of zero to avoid increased oscillation. Input shaping has also been combined with various feedback controllers to reduce the effects of external disturbances [12]. Ramli

et al. suggest that adaptive input shaping could account for parameter uncertainties through online estimation of natural frequencies and damping ratios [12]. 
Syvertsen explored several input shaping techniques for offshore cranes, including zero vibration $(\mathrm{ZV})$, zero vibration derivative (ZVD), and zero vibration derivative derivative (ZVDD) shapers [29]. Syvertsen showed that the ZV shaper resulted in vibrations of $20 \mathrm{~cm}$, the ZVD shaper resulted in vibrations of $5 \mathrm{~cm}$, and the ZVDD shaper resulted in vibrations of $2-3 \mathrm{~cm}$; whereas the unshaped commands resulted in $100 \mathrm{~cm}$ vibrations. It is noted that the ZVDD shaper was the most robust to modelling errors based on a sensitivity analysis. However, the increased robustness corresponded to an increased delay in operator commands. The input shapers were evaluated in simulation for a simplistic 45 degree rotation of a single joint without fully considering realistic operator commands, external excitation, or non-zero initial angles. The shaper parameters were fixed during the simulation as well, which may reduce effectiveness of the less robust methods as natural frequencies and damping ratios frequently change during crane operations. It is also noted that Martin and Irani [30] have shown that input shapers are effective; however, due to significant time lags, they are better suited for automated tasks.

In future work, the combination of feed-forward and feedback techniques may prove necessary when investigating human factors. However, an in-depth analysis of human factors is beyond the scope of the current thesis work. Due to the aforementioned limitations of feed-forward methods, the current research will focus predominantly on a feedback compensation system to actively reduce all forms of excitation.

Feedback APC is a closed-loop method that may reduce pendulum motion caused by external excitation and/or operator actions during crane operations. The feedback method requires additional sensors to determine the motion of the load, and a controller is applied to reduce the pendulum motion based on sensor feedback.

van Albada et al. recently suggested an APC algorithm based on energy dissipation [14]. The method ensures that negative work is performed to remove kinetic 
energy from the load. The presented approaches include continuously moving the crane tip in the direction of the load, and/or retracting or extending cable as the load moves away from or towards the crane tip, respectively. Although the crane tip motion accounts for the majority of the energy dissipation effect, it was found that the combination of the two methods was able to reduce motion $10 \%$ faster than using the crane tip alone [14]. The authors suggest that the crane tip and winch should be actuated at a predefined maximum acceleration and maximum velocity, and that extrapolation should be performed to anticipate and prepare for directional changes. In this way, the direction of acceleration may be reversed ahead of time to ensure that the tip and winch are consistently moving in the correct direction. Although it is noted that the method works for three-dimensional motion, results were only presented for the planar case with pendulum motion caused by a simplistic sine wave excitation, an initial angle, or control actions to move between two points. The method was also applied in later work by Chu et al. [17], and, for the planar case, the crane tip commands may be summarized as

$$
v_{s p, t i p}=\left\{\begin{array}{ll}
v_{\text {max }, \text { tip }} & x_{\text {load }}>x_{\text {tip }} \\
-v_{\text {max }, \text { tip }} & x_{\text {load }}<x_{\text {tip }}
\end{array},\right.
$$

where $v_{s p, t i p}$ is the tip velocity set-point, $v_{\max , t i p}$ is the pre-defined maximum velocity, $x_{t i p}$ represents the crane tip location, and $x_{\text {load }}$ represents the load position. 
Similarly, the winch commands may be summarized as

$$
v_{\text {sp }, \text { winch }}= \begin{cases}-v_{\text {max winch }} & v_{\text {load }}>v_{\text {tip }} \text { and } x_{\text {load }}>x_{\text {tip }} \\ -v_{\text {max,winch }} & v_{\text {load }}<v_{\text {tip }} \text { and } x_{\text {load }}<x_{\text {tip }} \\ v_{\text {max }, \text { winch }} & v_{\text {load }}>v_{\text {tip }} \text { and } x_{\text {load }}<x_{\text {tip }} \\ v_{\text {max }, \text { winch }} & v_{\text {load }}<v_{\text {tip }} \text { and } x_{\text {load }}>x_{\text {tip }}\end{cases}
$$

where $v_{s p \text { winch }}$ is the winch/cable velocity set-point, $v_{\text {max winch }}$ is the pre-defined maximum velocity set-point for the winch, $x_{t i p}$ and $x_{\text {load }}$ are as defined above, and $v_{\text {tip }}$ and $v_{\text {load }}$ are the tip and load velocities, respectively. In this case, the positive winch/cable velocity corresponds to extending cable such that the cable is extended as the load moves towards the tip and retracted as the load moves away from the tip as described above. The anticipation of changes in direction slightly increases the complexity; however, the control algorithm is a bang-bang controller. The authors also suggest a general energy dissipation method that involves integrating the equations of motion to choose a case that minimizes the kinetic energy.

It is also noted that typical winch-based AHC algorithms may issue commands that are opposite to those dictated by the APC algorithm above. In such cases, the AHC algorithm may perform positive work on the system and thereby increase the energy associated with the pendulum motion. Although the majority of the energy dissipation effort is typically realised through the crane tip for APC, variations in cable length caused by AHC may reduce the effectiveness of the system. This concern further motivates the use of crane tip motion for AHC to avoid significant interaction between the two compensation efforts.

Ramli et al. [12] identify sliding mode control (SMC) as a potential solution that is very robust and less sensitive to model uncertainties and external disturbances. 
Suthakorn and Parker applied SMC for APC of a maritime crane [15]. The authors considered the pitch and yaw motions of the ship, which they modelled as simple sine waves. The SMC method was shown to effectively reduce the pendulum motion caused by these disturbances.

Ngo et al. also applied a variation of SMC to an offshore container crane, which they defined as fuzzy sliding mode control (FSMC) [31]. In this algorithm, the control gain in SMC was adjusted with fuzzy tuning, and a saturation function was applied to reduce the chattering that is often observed in SMC. The rolling motion of a mobile harbour was considered as a simple sine wave, and several simulations and experiments were performed to validate the proposed controller. The authors showed that the FSMC algorithm was slightly more effective than SMC at reducing pendulum motion. A prediction algorithm was also applied to compensate for the rolling motion of the vessel, which further improved performance. Chattering was shown to be reduced, and robustness was shown by varying cable length to $\pm 28 \%$ of a nominal value. However, the cases considered by Ngo et al. were simplified and cannot be considered entirely conclusive. Further investigation is required to determine the benefits of FSMC over SMC, as the authors only considered a planar case with simple rolling motion. For a complete APC system, the motion of a variable length spherical pendulum should be considered with excitation of all degrees of freedom of the vessel. Investigation of operator interactions and interactions with AHC systems would also be beneficial in evaluating the control strategy. To further improve robustness of the system, it may be possible to add an adaptation mechanism that accounts for large variations in cable length. The addition of adaptive characteristics to SMC was also suggested by Ramli et al. [12] as a means of improving robustness.

Woodacre [13] and Ramli et al. [12] also described MPC as having many potential benefits, including robustness, the ability to deal with constraints, and closed-loop 
stability. However, Ramli et al. suggest that it is more sensitive to modelling errors than SMC. Woodacre showed that the first-order model of the winch could be misidentified with up to a decade deviation in the corner frequency before degradation of the response was observed.

Kimiaghalam et al. applied MPC combined with a feed-forward controller to reduce load oscillations for a maritime crane [32]. The feed-forward controller was used to provide the compensation for the vessel motion, and the MPC was intended to correct any deficiencies of the feed-forward control. The authors showed that the control algorithm was able to effectively maintain a position for rolling motion, which was simulated as a simple sine wave. However, the full motion of the vessel was not considered, the crane was a specific type that allowed the load to be suspended from two points on the boom, and the ability of the system to facilitate transfer between two points was not fully explored. Beyond the work of Martin and Irani [30], few other articles exist that apply MPC to maritime cranes and/or address these gaps in knowledge.

In the following subsection, the combination of anti-pendulum control and active heave compensation is briefly discussed.

\subsubsection{Combined Heave Compensation and Anti-Pendulum Control}

In currently available literature, there is little research that combines AHC and APC. A simple combination was applied by $\mathrm{Chu}$ et al. [17,33] using inverse kinematics to move the crane tip and extend or retract cable from the winch. However, the focus of the work was on bond graph modelling of the crane system, so few details were provided regarding the AHC and APC systems. The authors applied inverse 
kinematics and PID controllers, and the base excitations were not fully considered. The only results provided were for a simplistic case with an initial angle and heave motion based on a simple sine wave. Although the results were positive for the cases shown, the potential interaction of the systems was not explored, operator commands were not considered, and the remaining five degrees of freedom of the vessel motion were not included. The majority of the literature develops AHC and APC in isolation, which may cause issues when integrating the two systems.

In the next subsection, the applications of the motion compensation literature to the current work are summarized.

\subsubsection{Applications in the Current Research}

The work discussed in the above subsections will be extrapolated to the current research in several ways, including:

1. An energy dissipation technique that is similar to the work of van Albada et al. [14] and Chu et al. [33] will be developed in combination with an inverse kinematic model to allow for world-frame compensation based on inertial sensor feedback.

2. Multiple degrees of freedom of ship motion will be considered, and realistic motion profiles will be applied from existing work and commercially available simulation software.

3. A test-scale apparatus will be developed, and physical experiments will be performed to evaluate the real-world performance of the compensation system and provide a proof-of-concept/benchmark for future research efforts.

4. Hardware-in-the-loop (HIL) test-scale simulations will be performed, which will decrease development time, allow for real-time performance evaluation for a 
variety of simulated conditions, and ensure consistency between the control system applied in the simulations and experiments. Woodacre [13] noted several discrepencies between the simulation and experimental results, which were attributed to different implementations of the controller in LabVIEW for experiments and in MATLAB/Simulink for simulation. The current work seeks to avoid these discrepancies through HIL simulation.

To track a world-frame set-point and perform both AHC and APC, key system states must be estimated with relative accuracy. Although the actuator positions may be detected through simplistic sensors, the pose (position and orientation) of the ship and load form a more complex estimation problem. Many authors consider the use of low-cost inertial measurement units for pose estimation problems throughout the aerospace and maritime industries [34-38], and this technology is widely accepted within the maritime industry [39]. In the current work, an orientation estimation based on a complementary filter (CF) described by Valenti et al. [34] will be applied to determine the ship orientation and the pose of the load. The algorithm developed by Valenti et al. was intended to reduce the impact of magnetic field fluctuations on the roll and pitch angles, and it showed improved convergence and performance characteristics when compared to other filtering algorithms in previous work [34]. For completeness, a linear estimation algorithm will also be briefly discussed based on the work of Küchler et al. [35]. It is anticipated that the algorithm, which was developed for estimating heave motion from accelerometer data, may be extended to estimate periodic surge, sway, and heave motion for a complete linear motion estimate.

To effectively apply the information gained through estimation techniques to the world-frame compensation problem and to perform the necessary experiments and simulations, robotic modelling and control techniques must also be applied. Robotic modelling and control is briefly discussed in the following section. 


\section{$2.2 \quad$ Robotic Control and Modelling}

Robotic control and modelling techniques are required to resolve world-frame motion into the correct joint motions for the purpose of compensation and set-point tracking, as well as to perform simulations. In Subsection 2.2.1, robotic control and manual techniques and considerations for operator-based crane control are briefly discussed. Robotic modelling techniques are discussed in Subsection 2.2.2, and applications to the current research are summarized in Subsection 2.2.3.

\subsubsection{Robotic Control and Manual Operation}

In the maritime industry, cranes are typically controlled in the joint-space. That is, the operator issues manual commands for each joint individually in an effort to influence the motion of the load. This control method has been criticized in literature by several authors, including Chu et al. [17] and Sanfilippo et al. [40]. For knuckle boom cranes, or cranes that involve rotational degrees of freedom, several authors suggest a control approach based on inverse kinematics. In this case, the operator issues linear commands in a Cartesian frame and a controller automatically converts to the necessary joint commands. This approach was applied by Chu et al. to control the tip position of a knuckle boom crane, and the authors suggested that the result was easier control [17]. However, the topic of operator control and/or human factors was not explored in detail, and few articles exist that focus on this important issue. Based on the assumptions and suggestions of the existing literature, it is hypothesized that the inverse kinematic approach may improve controllability by allowing operators to issue commands in a more intuitive Cartesian frame.

Also, as discussed by Ramli et al. [12] and Vaughan et al. [41], the operator acts as a type of competing feedback controller that may interact with the designed control 
system in a variety of ways. Vaughan et al. [41] noted that the effect of feed-forward control on operator performance had been studied in the literature, but that interaction with feedback control was not well documented. This observation led to a study that compared manual control to proportional-derivative (PD) feedback control and feed-forward input shaping control. The study involved 12 novice operators with the goal of moving a bridge crane through a simple obstacle course. The manual control resulted in significantly higher deviation between operators, more undesired motion, and longer completion times [41]. The experiments were performed on a bridge crane, which would allow the operators to control the system in a Cartesian frame without the base-excitations that would be present in a maritime system. Despite the seemingly intuitive nature of such a system relative to maritime cranes, the operatorinduced excitation alone caused significant issues. Based on the observations and the added difficulty of maritime crane control, it is hypothesized that semi-automatic control methods may produce even more dramatic improvements compared to manual commands when maritime conditions are considered.

In the following subsection, robotic modelling is discussed.

\subsubsection{Robotic Modelling}

To achieve the motion compensation objectives of this thesis, a system model must be derived. Given the control challenges and vast number of applications, an extensive amount of crane modelling and control research exists. The dynamic modelling techniques applied in the literature include the Lagrange method, Kane's method, and the bond graph approach. For a more extensive review of crane modelling techniques, the reader is referred to the work of Ramli et al. [12] and Abdel-Rahman et al. [23]. To develop and evaluate the previously discussed control algorithm, several models must be generated for the test-scale knuckle boom crane. The required models include 
a forward and inverse kinematic model of the crane, a multi-body dynamic model of the pendulum load, and multi-domain system models to incorporate the actuator dynamics.

\section{Kinematic Model}

Given a defined geometry of a vessel, the placement of the knuckle boom crane, and the crane dimensions, a kinematic model may be formed. Ship motion data is typically reported in accelerations and/or velocities about the centre of mass, so it will be used to drive the motion of the vessel directly for realistic movement. The motion of the crane and load is assumed to have a negligible effect on the motion of the vessel for the current research. A kinematic model is also required for the crane control algorithm, which involves transformations between coordinate frames and inverse kinematics to convert from world-frame Cartesian to joint commands.

To begin modelling, cranes are typically represented as a series of links and joints. The primitive joints may be revolute (rotational) or prismatic (linear). For the main structure of the knuckle boom crane (Figure 1.1) considered in this research, three revolute joints are used to represent rotation about the base, rotation of the first boom, and rotation of the second boom. The crane tip is represented as a point on the link corresponding to the second boom, and an additional set of three kinematic structures are used to represent the actuator mechanisms.

After identifying all of the links and joints, a set of coordinate frames must be assigned for each joint. A common convention for coordinate frame assignment is the Denavit-Hartenberg (D-H) Convention, which is described in [42] and applied to a similar knuckle boom crane by Chu et al. [17]. The D-H method provides a systematic approach for determining transformations between the frames, and allows these transformations to be represented by only four parameters due to constraints 
applied in the frame assignment process. The D-H Convention was originally developed for use with homogeneous transformation matrices, so some authors question its use for alternative transformation operators [2]. Although homogeneous transformation matrices have been used extensively in the field of robotics, quaternions and dual quaternions are frequently discussed in current robotic research $[1,2,43]$. Quaternion-based methods may be used as a direct substitution for transformation matrices in many cases, and the reader is referred to Appendix $\mathrm{C}$ for a brief review of key topics as they are applied in the current thesis work. For a more in-depth discussion of quaternion properties, the reader is referred to $[1,2,43-46]$.

As quaternions are required for the pose estimation algorithms, they are also used in the current thesis work to represent frame transformations during the derivation of the kinematic models. In particular, dual quaternions are used as a direct alternative to the homogeneous transformation matrices that are commonly applied in the field of robotics. The D-H Convention will not be applied to allow for arbitrary frame assignment without redundant transformations, and the modelling procedure will represent the kinematic structure through transformation loops/sequences.

It is also noted that quaternion-based modelling methods are typically used in aerospace or advanced robotic fields; however, there is little evidence of their widespread use in the maritime industry. Therefore, the current thesis work seeks to bridge this gap in knowledge through the introduction of simplistic quaternion-based methods. It is anticipated that future control strategies may benefit from screw-theoretic modelling, which is a typical application of dual quaternions [1, 43, 47, 48].

Regardless of the chosen modelling technique, the result is a kinematic model that is necessary for many control algorithms. It is noted that the crane tip is commonly treated as the end effector in existing research, including the work of Chu et al. [17]. However, in the current research, the load itself is considered the end effector of the 
under-actuated system and set-points are intended to control its motion relative to the world frame by adjusting tip position and cable length.

In addition to the kinematic models, a multi-body dynamic model of the pendulum load must be developed.

\section{Multibody Dynamic Model}

The majority of researchers apply a lumped-mass approach for multibody dynamic modelling, and Lagrangian methods are typically used to generate multibody dynamic models. The Lagrangian technique is commonly applied in robotics [42], and involves assigning generalized coordinates and forming a Lagrangian function based on the difference between kinetic and potential energies. Derivatives are taken from the Lagrangian to form the equations of motion as

$$
\frac{d}{d t}\left(\frac{\delta L}{\delta \dot{\xi}_{i}}\right)-\frac{\delta L}{\delta \xi_{i}}=\tau_{i},
$$

where $t$ is time, $L$ is the Lagrangian, $\xi_{i}$ is the generalized coordinate and $\tau_{i}$ is the generalized force or torque for the $i^{t h}$ equation of motion. In robotics, the generalized coordinates are typically selected as the joint variables to avoid unnecessary constraints. For the current thesis work, an explicit dynamic model of the main structure of the crane is not explicitly required; however, the cable/pendulum system must be considered. In the current research, a variable length spherical pendulum will be modelled in Simscape with confirmation via the Lagrangian methods. There are many methods of cable modelling depending on the model requirements $[27,49,50]$; however, flexible cable models are considered beyond the scope of the current work. Also, a single-pendulum system with a point mass will be considered for simplicity, which is consistent with the majority of existing literature. 


\section{Multi-Domain System Models}

As was noted by Chu [33], the dynamics of the hydraulic components/actuators are not negligible for simulation and control of an offshore crane. For simple validation purposes, many authors do not consider the full hydraulic system. Researchers have applied various methods for modelling the hydraulic systems, including a system identification and transfer function approach applied by Woodacre [13]. Chu et al. applied a bond graph approach to modelling the full hydraulic system, which was integrated into a real-time simulator for a knuckle boom crane [33,51-53]. The bond graph method is a powerful tool for modelling multi-domain systems. It is based on energy flow, and allows for easy modification of key parameters. In contrast, system identification is based on experimental data, the resulting models may be more realistic, and nonlinear characteristics may be identified. For the test-scale system in the current research, system identification techniques will be used to identify transfer functions for the actuators.

\subsubsection{Applications in the Current Research}

1. A kinematic model will be developed to include the ship, IMU, and crane; and inverse kinematics will be used to control the world-frame position of the crane. The inverse kinematic control is similar to the work of Chu et al.; however, quaternion-based methods will be used throughout the modelling procedure.

2. The Lagrangian method will be applied to generate a simplistic multibody dynamic model of the pendulum load, which will be compared to Simscape Multibody simulation results.

3. System identification techniques will be applied to identify transfer functions and other key parameters for the sensors and actuators. 


\subsection{Conclusion/Summary}

This chapter reviewed the limitations, benefits, drawbacks, and applicability of the literature as it relates to the objectives of the current research. In Section 2.1, motion compensation was discussed for both heave compensation and anti-pendulum control of maritime cranes, which is related to the first objective of the current thesis work. In Section 2.2, robotic modelling and control methods were briefly discussed as they are related to both the first and second objectives.

The next chapter outlines the equipment used in the current thesis work. 


\section{Chapter 3}

\section{Design of Experiment and}

\section{Simulation}

This chapter provides an overview of the experimental apparatus, control architecture, and simulation framework that will be referred to and/or used throughout the thesis. In Section 3.1, a system overview and control architecture is provided. In Section 3.2, the input commands and practical considerations regarding the implementation of the control system on a real-time controller for hardware-in-the-loop (HIL) simulations and experiments are described. In Section 3.3, the operating conditions and ship motion profiles are discussed. The experimental apparatus is described in Section 3.4, and the corresponding simulations are described in Section 3.5. Finally, the procedure for testing and analysis is briefly described in Section 3.6. 


\subsection{System Overview}

The system considered in the current work is summarized in Figure 3.1 and consists of several input motion profiles, an input command, a feedback control system, and the physical system and/or simulation. The input motion profiles are provided as kinematic set-points that correspond to either real or simulated ship motion. The input command is used in this thesis to mimic a crane operator's command. The commands adjust the control set-points based on the system output. A feedback control system combines these input commands with sensor feedback signals to actuate the system and drive it towards a desired state. In a real system, the operator commands are unpredictable and subject to a variety of human errors, so predefined set-point profiles are used in test cases to improve repeatability.

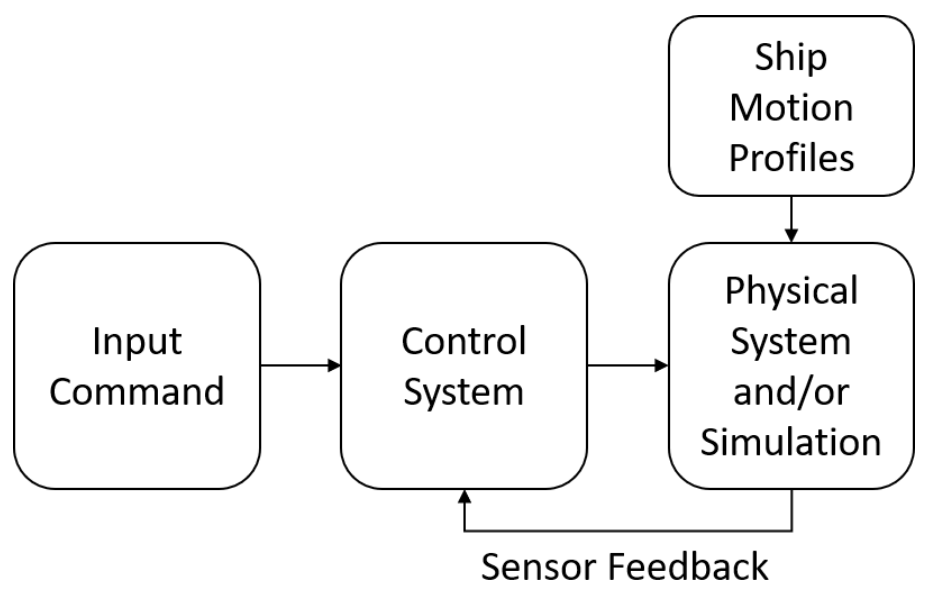

Figure 3.1: System overview.

Figure 3.2 shows an expanded block diagram of the "Control System." The control system receives the input commands, as well as feedback signals from a variety of sensors. The input commands are processed to determine the desired motion, and the sensor feedback is used to estimate the current motion via a state estimation and sensor fusion algorithm. Based on the available information, a control set-point 
is calculated and provided to the combined compensation controller. The controller attempts to track the set-point while minimizing undesired motion by generating kinematic commands corresponding to a desired crane tip motion. The control actions are transformed to joint commands with an inverse kinematic model, and these commands are processed prior to sending the control signals to the physical system and/or simulation.

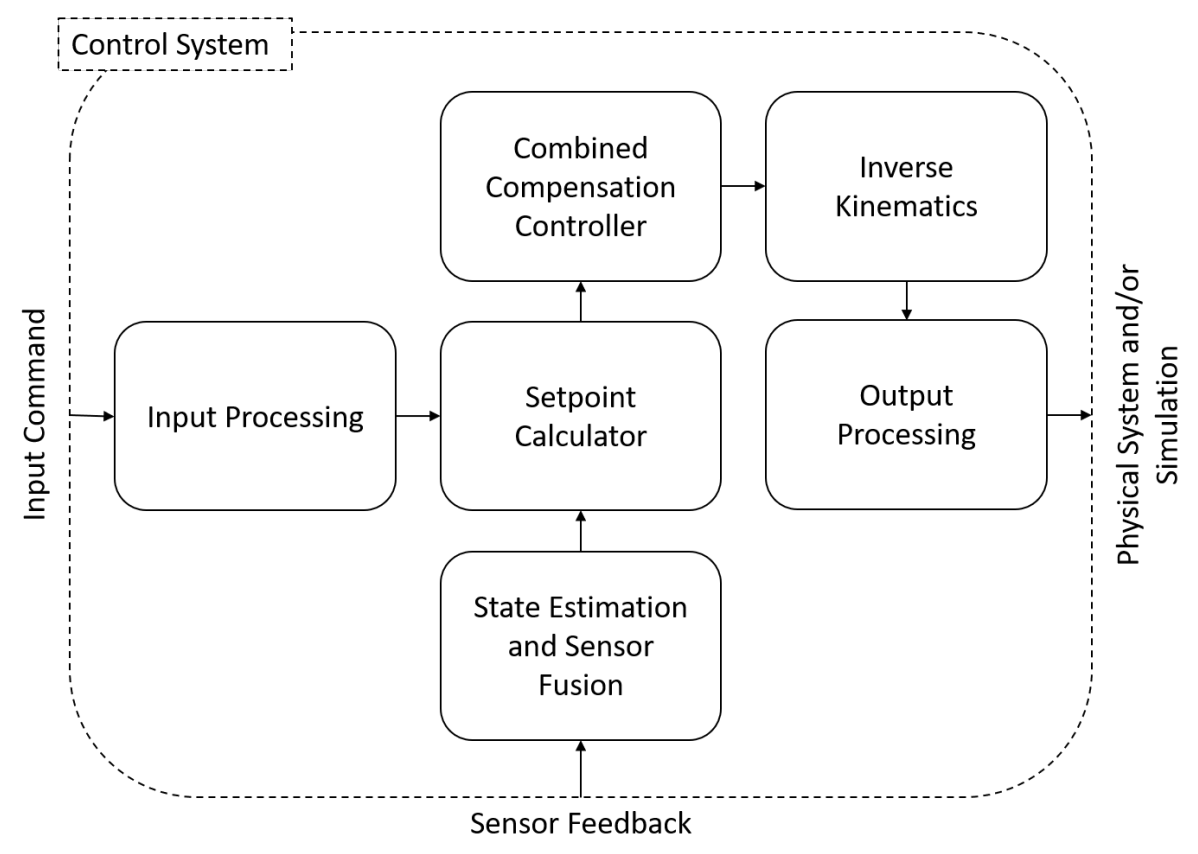

Figure 3.2: Control system overview.

An expanded block diagram of the "Physical System and/or Simulation" subsystem is provided in Figure 3.3. The full-scale movement of the ship is converted to test-scale motion for use with the motion platform during test-scale simulations and experiments. In simulations, the kinematics are specified as smooth displacement, velocity, and acceleration profiles with ideal set-point tracking. In the experiments, the displacement profiles are provided as set-points to actuator control boards. The motion of the platform or ship deck causes base excitation of the knuckle boom crane, which leads to motion of the attached load. The control signals are provided to the 
crane actuators via control boards, and several sensors are used to determine key system states. The details of the physical system are discussed in Section 3.4, and the simulation is discussed in Section 3.5.

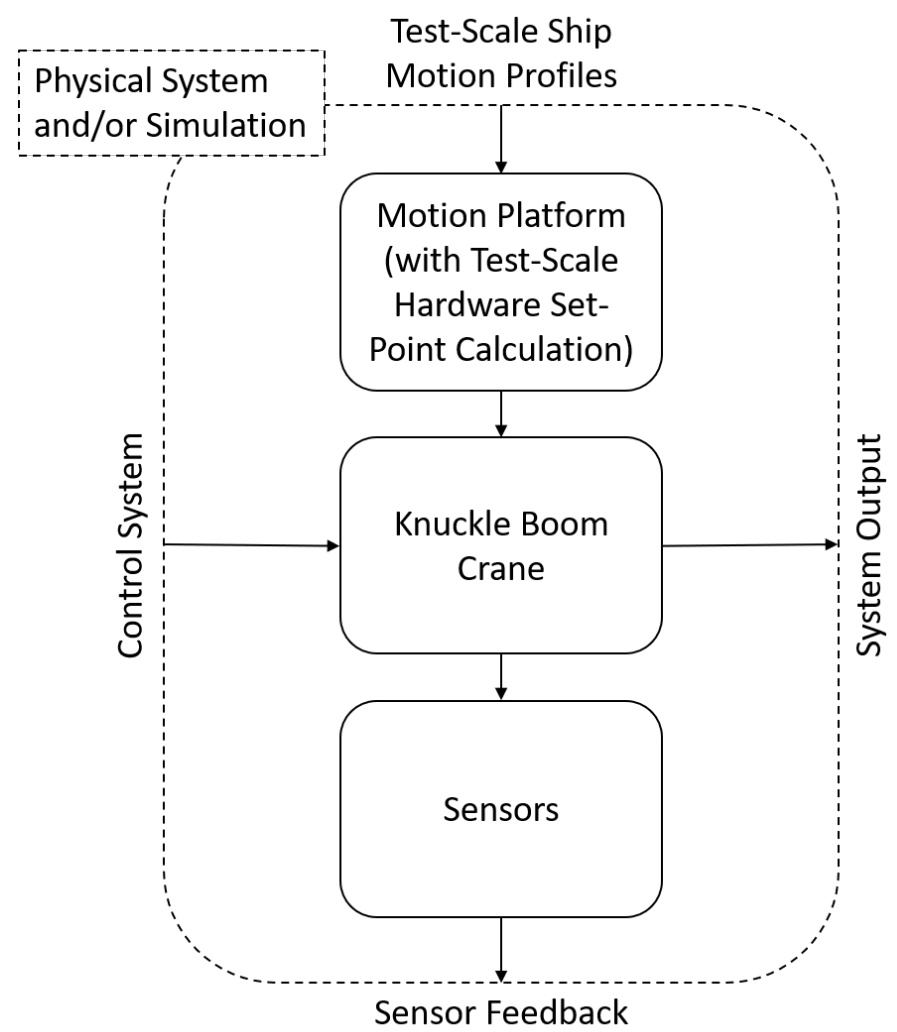

Figure 3.3: Overview of the physical system and/or simulation.

The remaining sections of this chapter provide details regarding the above system components. The input commands and the control system implementation are discussed in the following section. 


\subsection{Input Commands and Control System Imple- mentation}

In this thesis, two 'operator tasks' with predefined set-point profiles are considered. The first task is to maintain the world-frame position of the suspended load. Assuming ideal inverse kinematics and compensation, this task should not require additional input from an operator. In the current work, the fixed set-point will be considered as a desired tip location of $\left\{x_{t d}, y_{t d}, z_{t d}\right\}=\{3,-502,-153\}$ (mm) with a pendulum length

of $700 \mathrm{~mm}$ unless otherwise stated. The tip location corresponds to a physical marker within the experimental apparatus to allow for easier confirmation when setting up for the experiments. For the second task, a 120-second pick-and-place operation is considered. Figure 3.4 illustrates the pick-and-place operation with graphs that represent the desired cable length and world-frame tip x/y/z-positions to define the time-varying set-points for the duration of the test. To perform the operation, the cable length is extended to a 'pick-up' location, the cable is retracted, the load is moved along the body of the ship, the cable is extended to a 'place' location, and the cable is retracted to its original length to complete the operation.

During experiments, the crane and motion platform are controlled using two National Instruments (NI) myRIO controllers, which provide a set of reconfigurable inputs and outputs for digital and analog signals. The motion platform controller outputs set-points to the five linear actuator control boards for position control, and a trigger signal is received from the crane controller to start the motion at the beginning of the test.

The crane controller acquires input data from five joystick axes and a toggle switch on the operator input device, which is bypassed using the aforementioned set-point profiles for repeatability in the current work. The crane controller also acquires data 
from two inertial measurement units (IMUs) on the ship/load, three linear actuator potentiometer feedback signals, and an encoder positioned on the winch shaft. The controller outputs set-points to three linear actuator control boards and the winch motor. The controller also sends the aforementioned trigger signal to the motion platform controller and records all feedback and outputs for analysis.

During physical experiments, the real actuators and sensors are used by the crane controller. However, for performing simulations, a hardware-in-the-loop and operatorin-the-loop (HIL/OITL) approach is taken to decrease the development time and allow for realistic control actions during the simulation. The HIL/OITL simulation is accomplished by redirecting the crane controller outputs to a Simulink model rather than the physical actuators. Similarly, the feedback signals from the Simulink model are sent to the crane controller in place of the physical sensor signals. The Simulink model runs on the host computer and communicates with the myRIO over a virtual network connection by sending and receiving the data described in Table 3.1. The signals are modified to match the resolution and format of the real-world signals to ensure that the control algorithm is consistent for both simulations and experiments.

The ship motion profiles, the corresponding coordinate systems, and the method used to emulate the motion during experiments and simulations are discussed in the following section. 

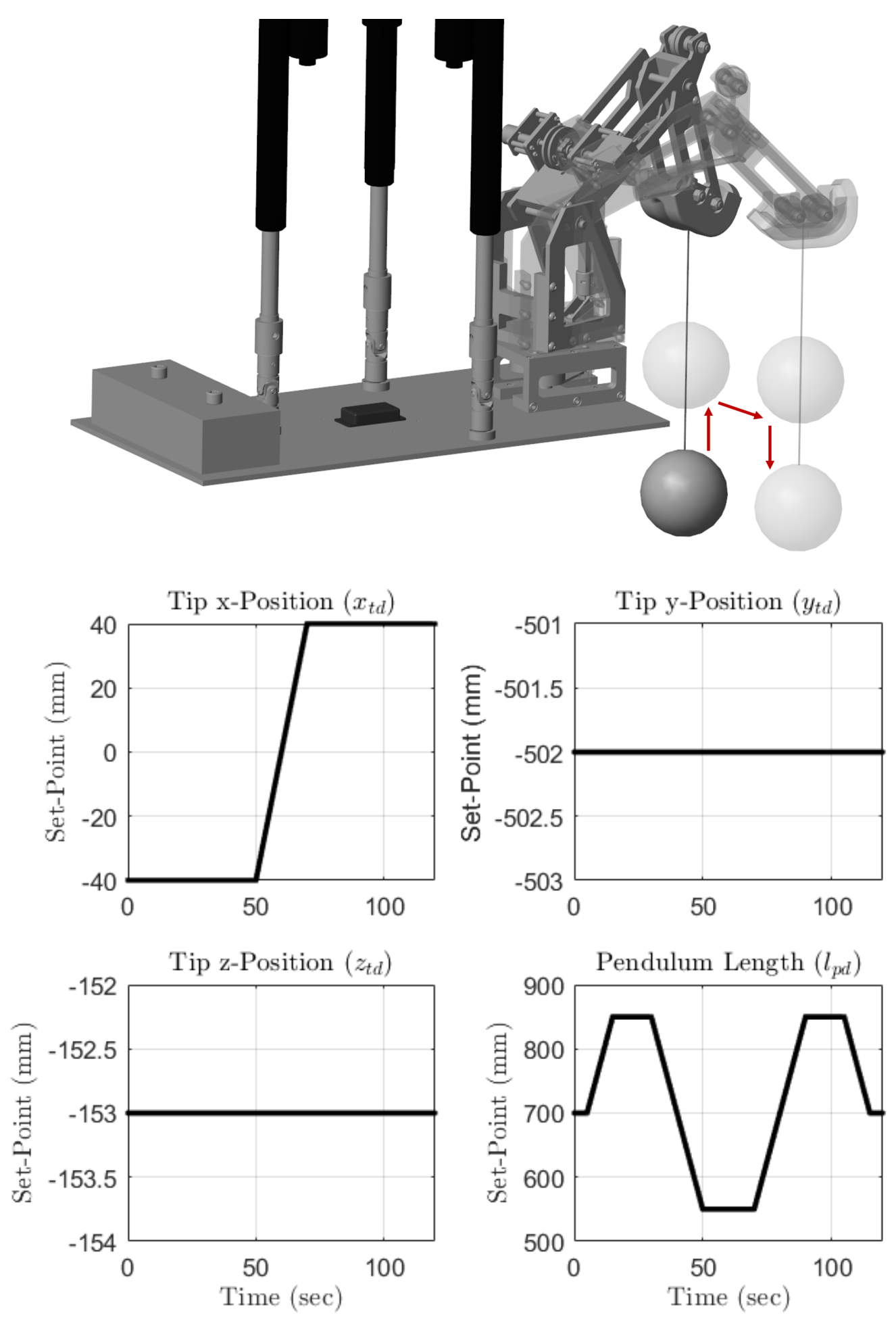

Figure 3.4: Operator Task 2: Variable set-point tracking. 
Table 3.1: Data sent between the HIL/OITL simulation and the myRIO.

\begin{tabular}{|c|c|}
\hline Simulation-to-myRIO & myRIO-to-Simulation \\
\hline Base Actuator Feedback & Base Actuator Control Voltage \\
Boom Actuator Feedback & Boom Actuator Control Voltage \\
Jib Actuator Feedback & Jib Actuator Control Voltage \\
Encoder Feedback & Winch Motor PWM Duty Cycle \\
IMU1 Time & Load X/Y/Z Set-Point \\
IMU1 Gyroscope X & Load X/Y/Z Estimate \\
IMU1 Gyroscope Y & Ship Surge/Sway/Heave Estimate \\
IMU1 Gyroscope Z & Ship Roll/Pitch/Yaw Estimate \\
IMU1 Accelerometer X & \\
IMU1 Accelerometer Y & \\
IMU1 Accelerometer Z & \\
IMU1 Magnetometer X & \\
IMU1 Magnetometer Y & \\
IMU1 Magnetometer Z & \\
IMU2 Time & \\
IMU2 Gyroscope X & \\
IMU2 Gyroscope Y & \\
IMU2 Gyroscope Z & \\
IMU2 Accelerometer X & \\
IMU2 Accelerometer Y & \\
IMU2 Accelerometer Z & \\
IMU2 Magnetometer X & \\
IMU2 Magnetometer Y & \\
IMU2 Magnetometer Z & \\
Tip X/Y/Z Truth Data & \\
Pendulum $\theta_{x} / \theta_{y}$ Truth Data & \\
Ship Surge/Sway/Heave Truth Data & \\
Ship Roll/Pitch/Yaw Truth Data & \\
\hline
\end{tabular}




\subsection{Ship Motion Profiles}

As previously stated, the ship motion is specified as a combination of surge, sway, and heave displacements with roll, pitch, and yaw angles. The ship motion is specified in a translating world frame with positive directions as indicated by Figure 1.2, which is repeated here as Figure 3.5 for convenience. In this thesis work, the ship is assumed to have a Northern heading.

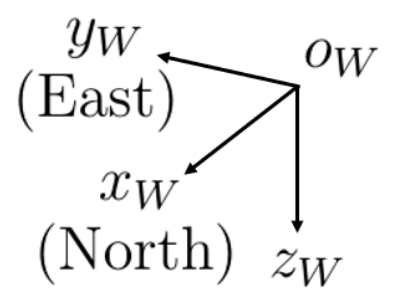

(Down)

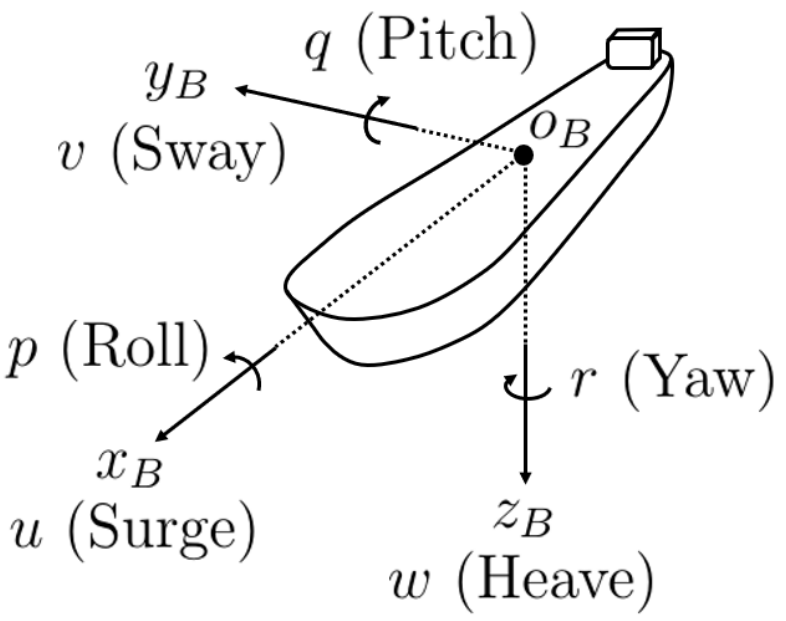

Figure 3.5: Convention for coordinate frames and ship degrees of freedom (repeated for convenience).

For the test-scale experiments and simulations, a five-degree-of-freedom motion platform is used to approximate the scaled ship motion. The motion platform is shown in Figure 3.6 with labels indicating the numbered actuators and key components. The motion platform, which was developed in previous work [20], uses five linear actuators (LA1-LA5) to move a "ship deck" over a body of water. A knuckle boom crane is attached to the ship deck to compensate for the platform's motion. The 
first and second actuators control the surge and sway motion, whereas the remaining three actuators control the heave, roll, and pitch. The motion platform is unable to explicitly perform yaw motion, which is considered negligible and set to zero for the test-scale system.

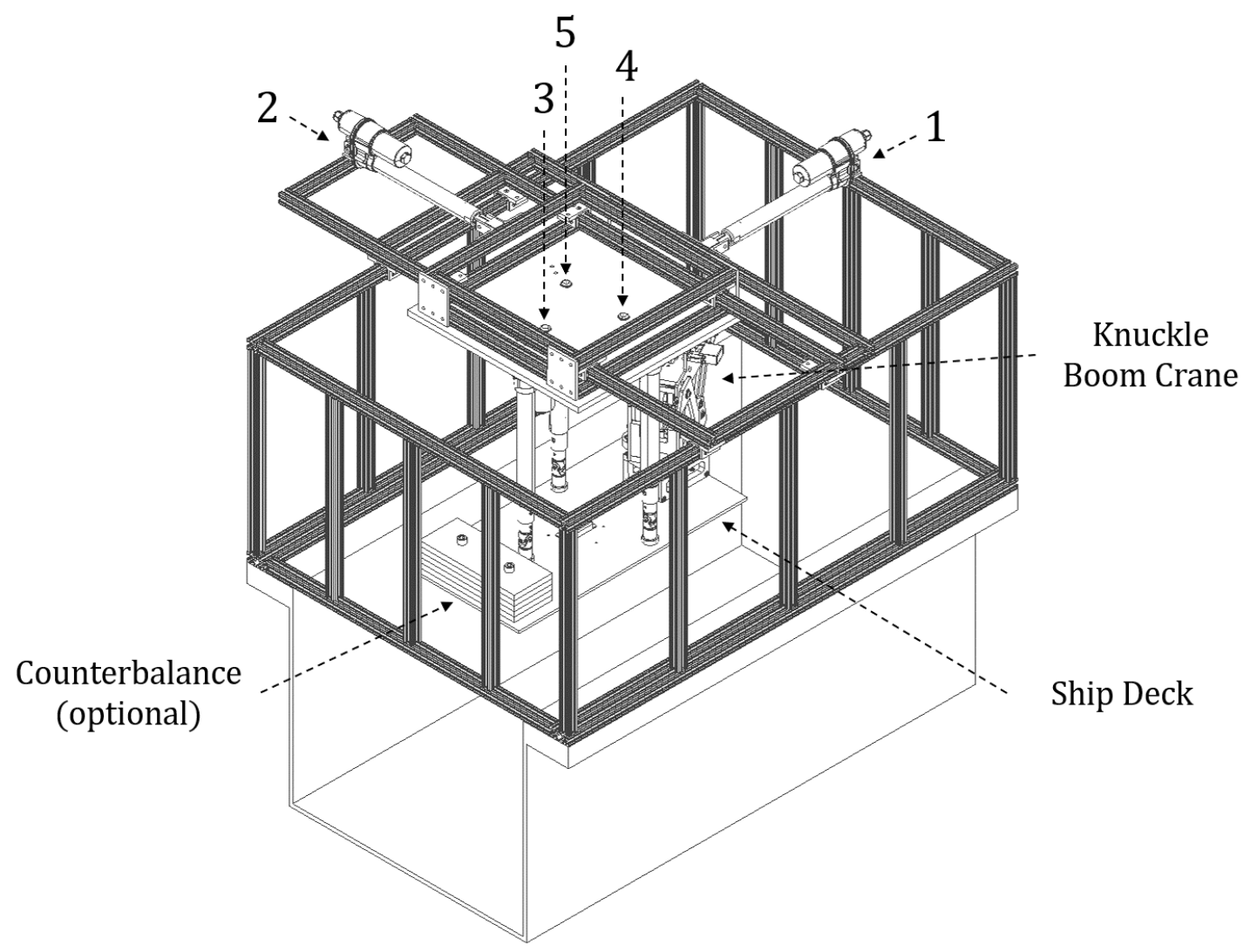

Figure 3.6: Motion platform with linear actuator labels.

To emulate ship motion using the motion platform, the linear displacements are scaled to within the 0-200 $\mathrm{mm}$ range of the actuators by applying the same scale factor to the surge, sway, and heave displacements. The scale factor is chosen to ensure the maximum actuator velocity of $20 \mathrm{~mm} / \mathrm{s}$ is not exceeded. The test-scale ship motion is then converted to linear actuator set-points using an inverse kinematic model, which is discussed in Chapter 4. When converting to actuator set-points, 
two common configurations are considered. The first configuration is with the crane positioned at the stern (back) of the vessel, whereas the second configuration is with the crane positioned at the port (left) side of the vessel. Given that the crane is fixed to the motion platform, emulating the two configurations may be accomplished by resolving the motion from one of two reference frames when calculating the set-points. The reference frames are shown in Figure 3.7a where the crane is located at the stern, and Figure 3.7b where the crane is located at the port side. The $o_{B} x_{B} y_{B} z_{B}$ frame corresponds to the ship frame shown in Figure 3.5. These coordinate frames are used to pre-process the test-scale motion data prior to applying the inverse kinematics. Although the option to switch between the two coordinate frames is implemented in the current work, the crane is assumed to be located at the port side with the configuration shown in Figure 3.7b.

Three motion profiles are considered for the test cases. The first motion profile is adapted from [6], whereas the second and third profiles are generated with ShipMo3D [54]. Each of the profiles correspond to a similarly-sized vessel with an approximate length of $120 \mathrm{~m}$, and the profiles are defined using the reference frame in Figure 3.5. The first motion profile is shown in Figure 3.8 as surge, sway, heave, roll, pitch, and yaw displacements plotted as a function of time. The left axis of each plot indicates the full-scale measurement, whereas the right axis indicates the corresponding test-scale measurement. The test-scale measurements are generated by scaling the full-scale data based on the peak-to-peak value of the dominant displacement and then shifting the resultant data to be centred at zero displacement relative to the nominal position. The angular motion is equivalent except for the yaw motion, which is set to zero due to the physical limitations of the motion platform. The data is shown over a 120 second period, which is the length of time used for all test cases.

Selecting the port-side configuration from Figure 3.7b and using the inverse kine- 
matic model (Chapter 4), the test-scale profiles are converted to position set-points for the motion platform actuators. However, the system is unable to perfectly track the set-points, so the position feedback signals are used to estimate the real motion of each actuator. An example of the process applied for LA1 using Ship Motion Profile 1 is shown in Figure 3.9. The upper plot indicates the set-point, the recorded feedback, and the filtered feedback corresponding to the displacement of the actuator. The filtered feedback is achieved by fitting a smoothing spline to the data in MATLAB, and its first and second derivatives are shown in the lower two plots. The filtered displacement, velocity, and acceleration profiles are used as kinematic set-points to drive the test-scale simulations as described in Section 3.5. By using the filtered feedback instead of the actuator set-points, the motion of the simulated platform will match the motion of the physical platform more closely. The complete set of ship motion profiles and the corresponding actuator motion profiles are provided in Appendix A.

The experimental apparatus, including the motion platform, knuckle boom crane, and associated sensors and actuators, is described in the following section. 


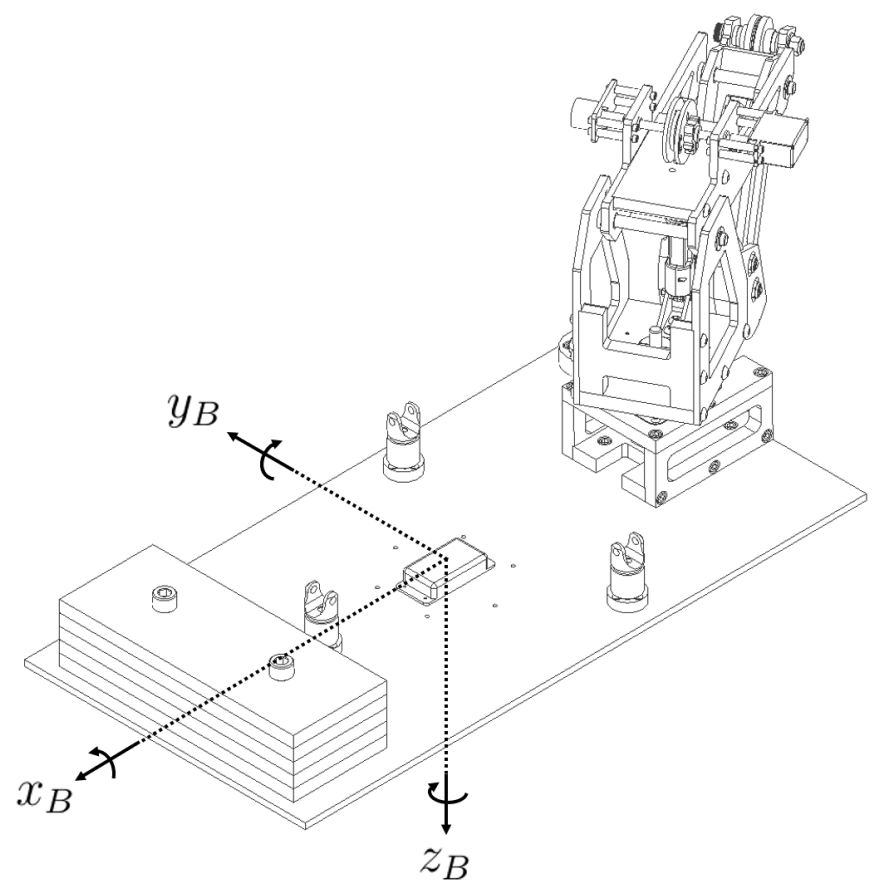

(a) Configuration 1: Crane located at the stern (back) of the vessel.

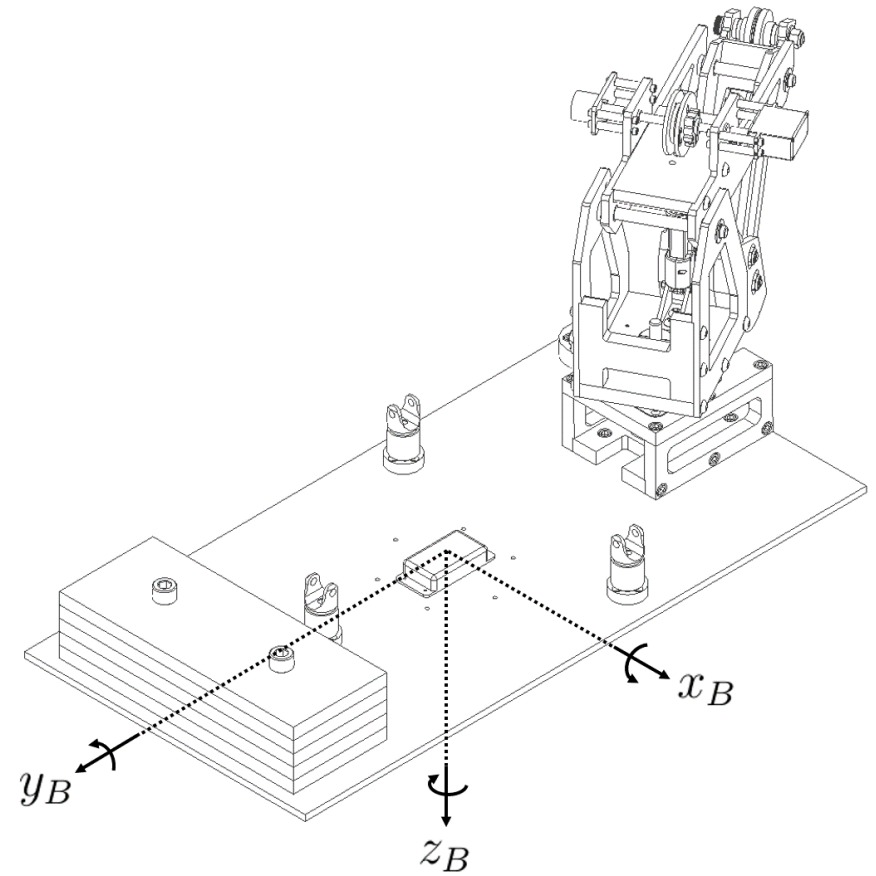

(b) Configuration 2: Crane located at the port (left) side of the vessel.

Figure 3.7: Motion platform coordinate frame conventions. 

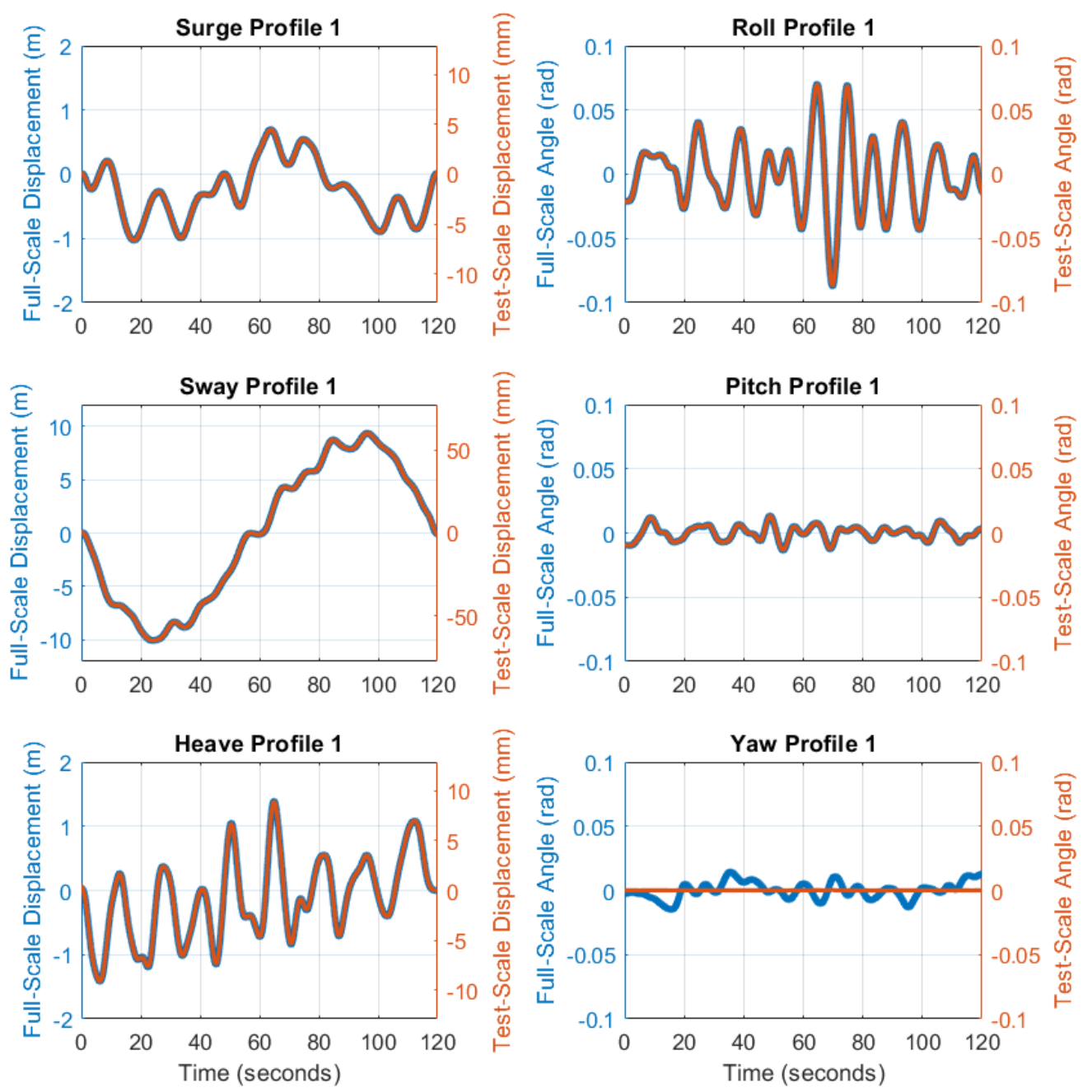

Figure 3.8: Ship Motion Profile 1: Full-scale and test-scale displacements [6]. 

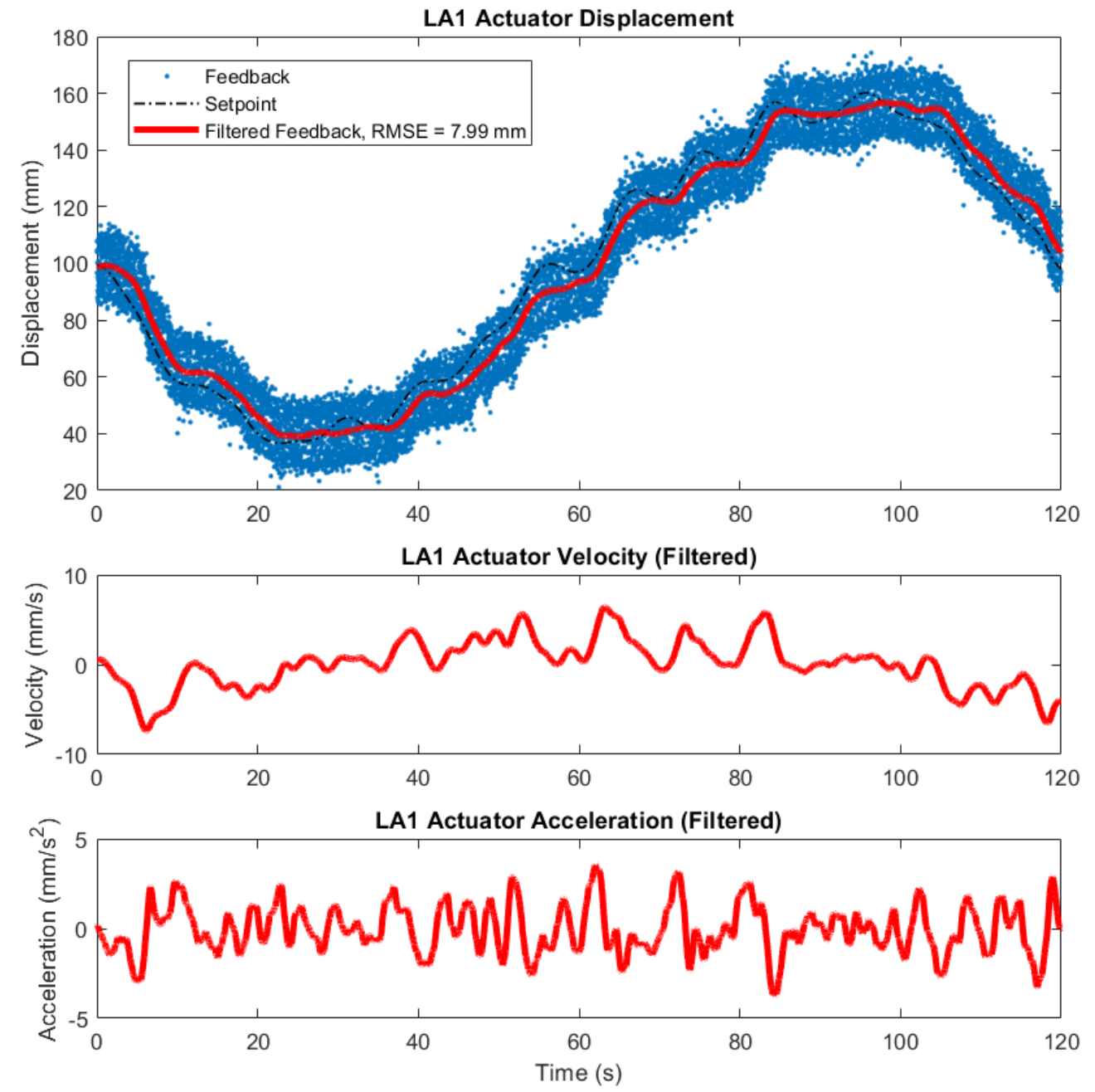

Figure 3.9: Ship Motion Profile 1: LA1 set-points, feedback, and filtered feedback. 


\subsection{Experimental Apparatus}

The experimental apparatus, shown in Figure 3.10, consists of two multibody systems: motion platform and knuckle boom crane. Additionally, there are several electric actuators and sensors in both of these systems, and each system is controlled with a National Instruments myRIO controller. The main components of the apparatus are discussed in the following subsections.

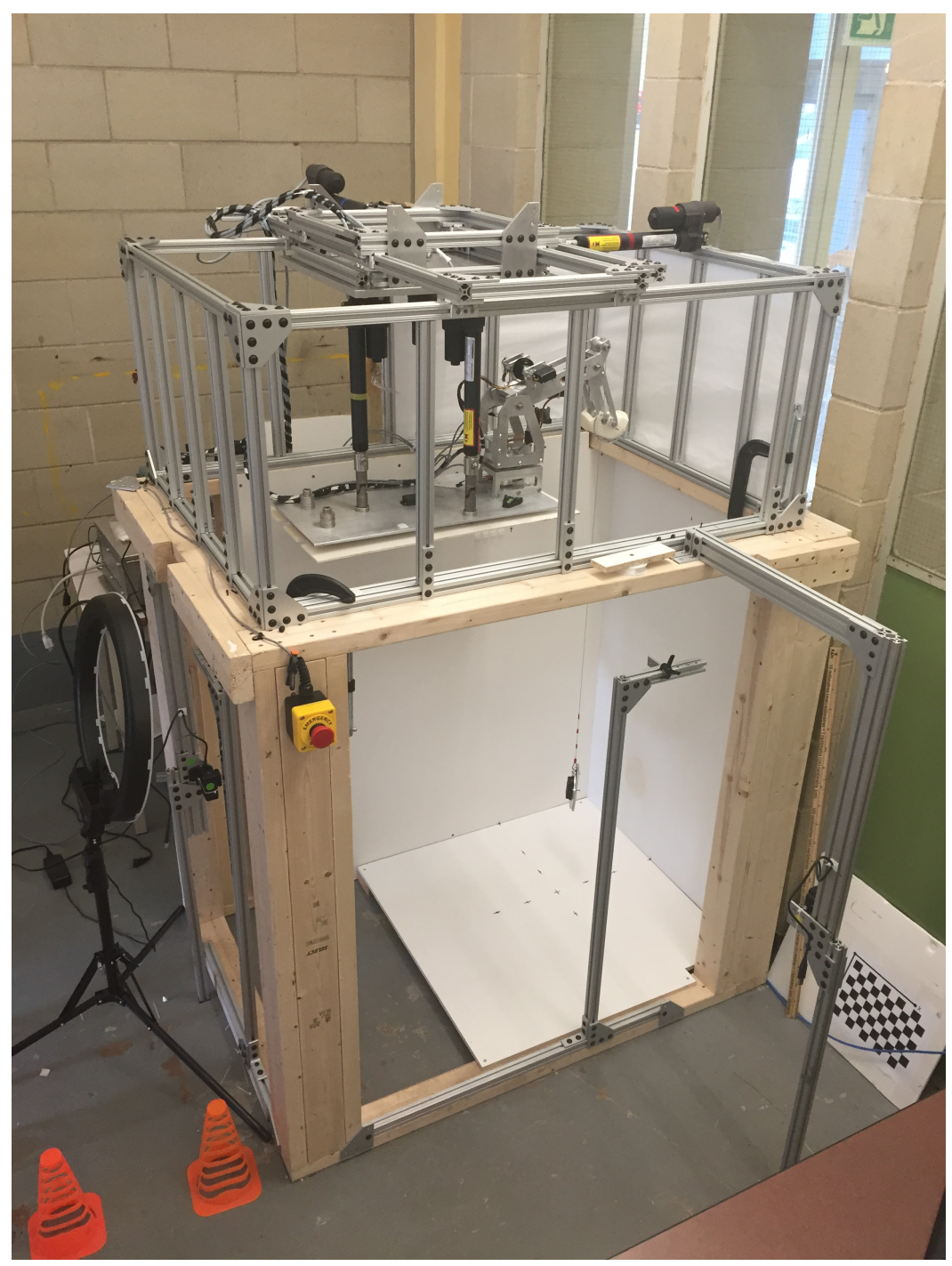

Figure 3.10: Test-scale experimental apparatus. 


\subsubsection{Multibody System 1: Motion Platform}

The motion platform, which was developed in [20], consists of a support structure, a two-degree-of-freedom trolley system, and a three-degree-of-freedom parallel manipulator that supports a platform ("ship deck"). The trolley system allows for surge and sway motion, whereas the parallel manipulator allows for heave, roll and pitch. The dynamics of the motion platform are not explicitly considered for control or simulation. In the current work, the motion platform is used to track kinematic set-points to approximate the motion of a vessel.

\subsubsection{Multibody System 2: Knuckle Boom Crane}

In this thesis work, a test-scale knuckle boom crane was designed, built and tested for use on the motion platform. The details of the knuckle boom crane design are provided as mechanical drawings in Appendix B. An illustration of the knuckle boom crane is provided in Figure 3.11 and highlights the main components, including the base, tower, boom, jib, and load with an associated winch/cable/pulley system. The base is rigidly connected to the motion platform and supports the tower on a single degree-of-freedom turntable to allow for relative rotation about a vertical axis. The tower supports the boom with one degree of freedom about a horizontal axis, and the boom supports the jib with another degree of freedom about a horizontal axis. The crane tip is located at the free end of the jib, and its position is controlled by actuating the three aforementioned degrees of freedom.

The base-to-tip portion of the crane may be considered as a robotic system with three revolute joints (an RRR manipulator). Each joint is controlled with a linear electric actuator for comparison with the industrial case in which hydraulic cylinders are typically used. The actuators add revolute joints at their connection points with 


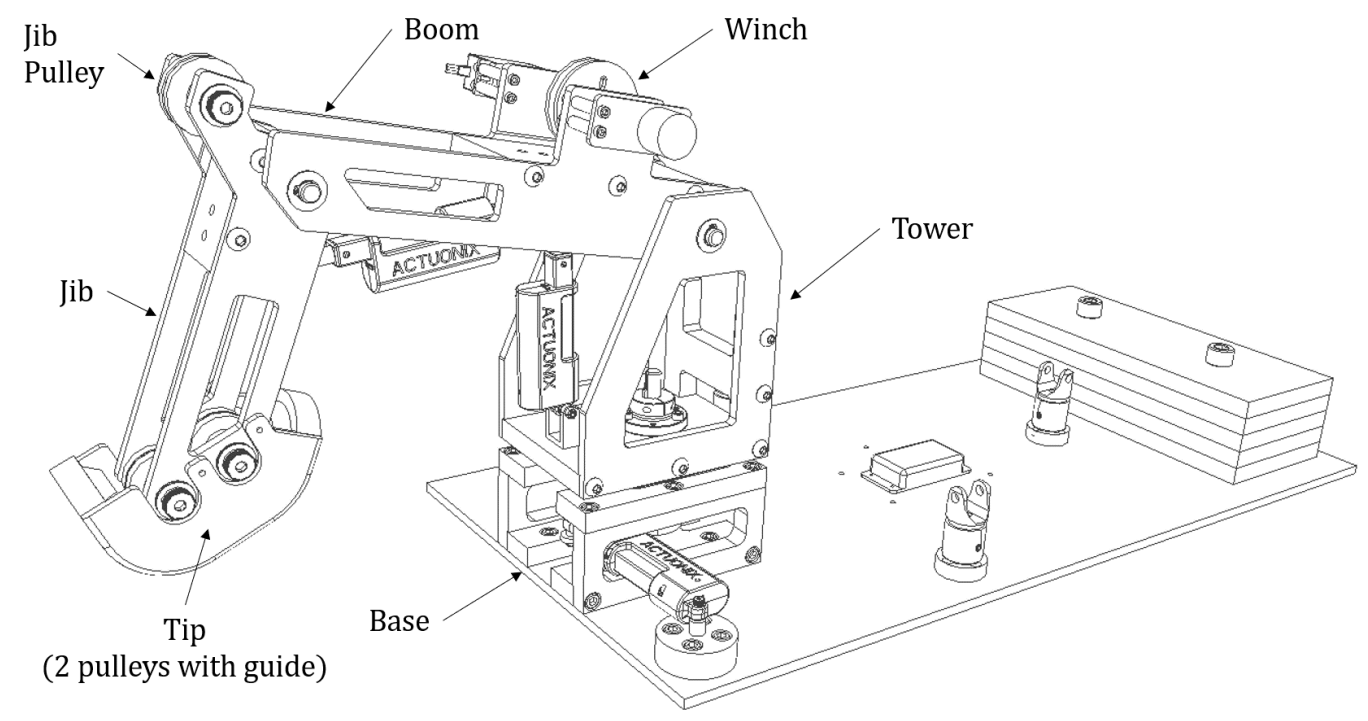

Figure 3.11: Test-scale knuckle boom crane.

an actuated prismatic joint between them to create an RPR configuration in parallel with the main revolute joint. The motion of each joint is limited by the length of the actuators, which may extend from 0-50 mm. Given the constrained motion, Figure 3.12 shows the workspace of the crane tip where each point represents a possible position of the crane tip relative to the base of the crane located at the origin. The points are generated by considering 0-50 $\mathrm{mm}$ displacements of each actuator.

The final component of the knuckle boom crane is the load with its associated winch/cable/pulley system. The winch motor is located near the tower, which is common for industrial cranes. A flexible coated steel cable is coiled on the winch pulley, runs along the boom to a pulley attached to the jib, and extends through the crane tip. The crane tip is located between two pulleys, and a 3D-printed guide is used to direct the cable into the grooves of these pulleys to prevent jamming. The cable extends from the crane tip to the load, which acts as a variable-length pendulum. The pendulum length varies as the winch rotates; however, the pendulum length is also a function of the jib rotation due to the varying wrap angle and position of the 


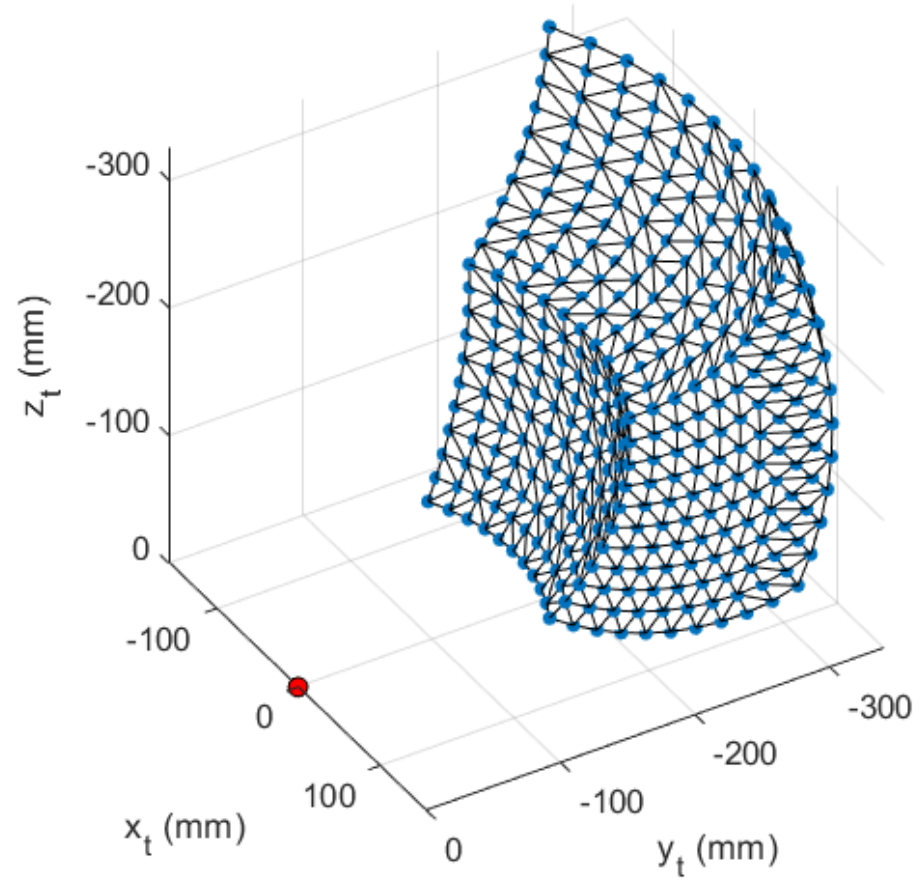

Figure 3.12: Crane tip workspace.

jib pulley. Figure 3.13 and Figure 3.14 show the cable paths for the retracted and extended jib positions, respectively, and the cable path is illustrated by a red line. In Figure 3.13, the distance from the winch to the jib pulley is at a maximum, so more cable is required to span this distance. As the jib extends, the distance from the winch to the jib pulley decreases to its minimum length in Figure 3.14. For a constant winch rotation, the variations in cable length required to span these internal distances directly correspond to variations in the extended cable or pendulum length.

Figure 3.15 plots the variation in the extended cable or pendulum length caused by extending the jib actuator. The points represent measured values, and the dashed line represents a quadratic fit described by the annotation. The measurements are taken from the crane tip to the load and are given relative to the extended cable length when the jib actuator is fully retracted. 


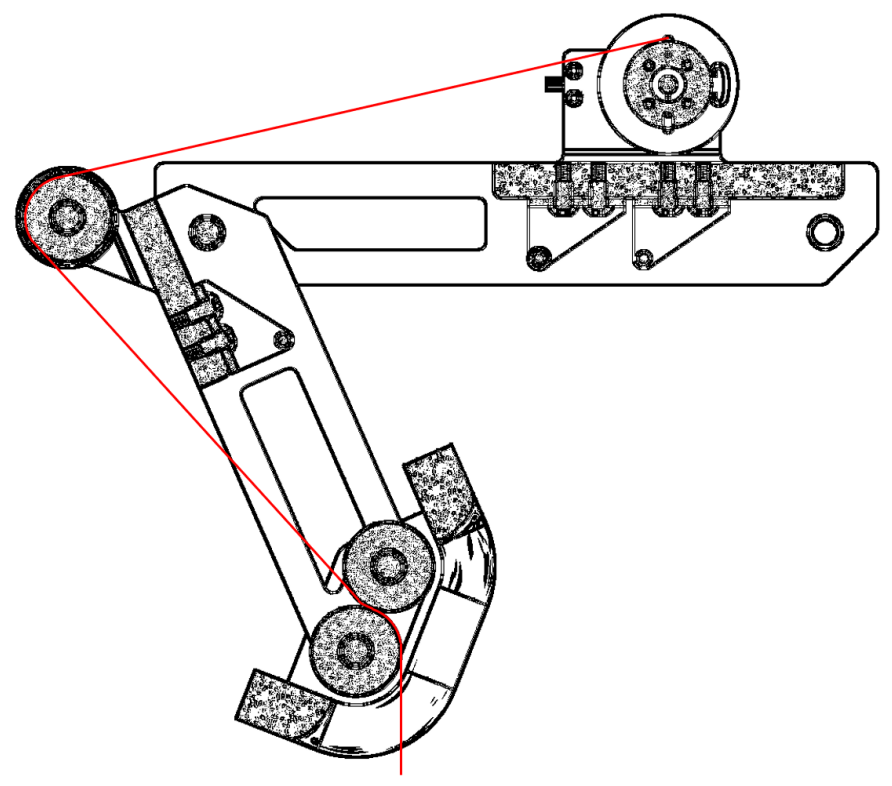

Figure 3.13: Cable path with the jib retracted.

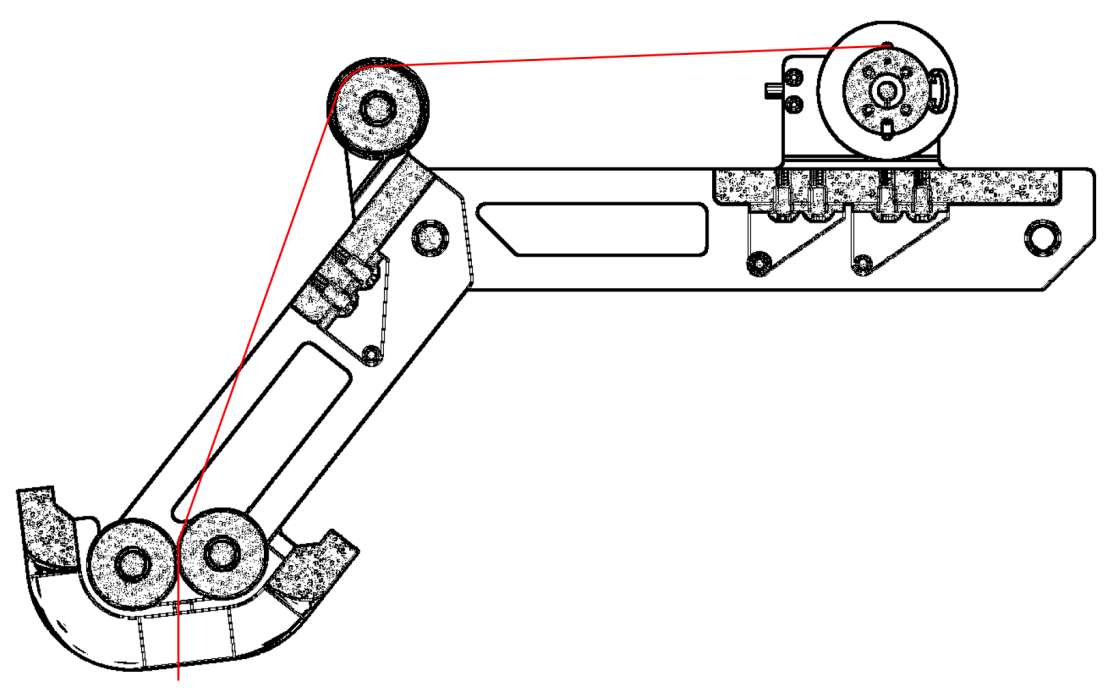

Figure 3.14: Cable path with the jib extended. 


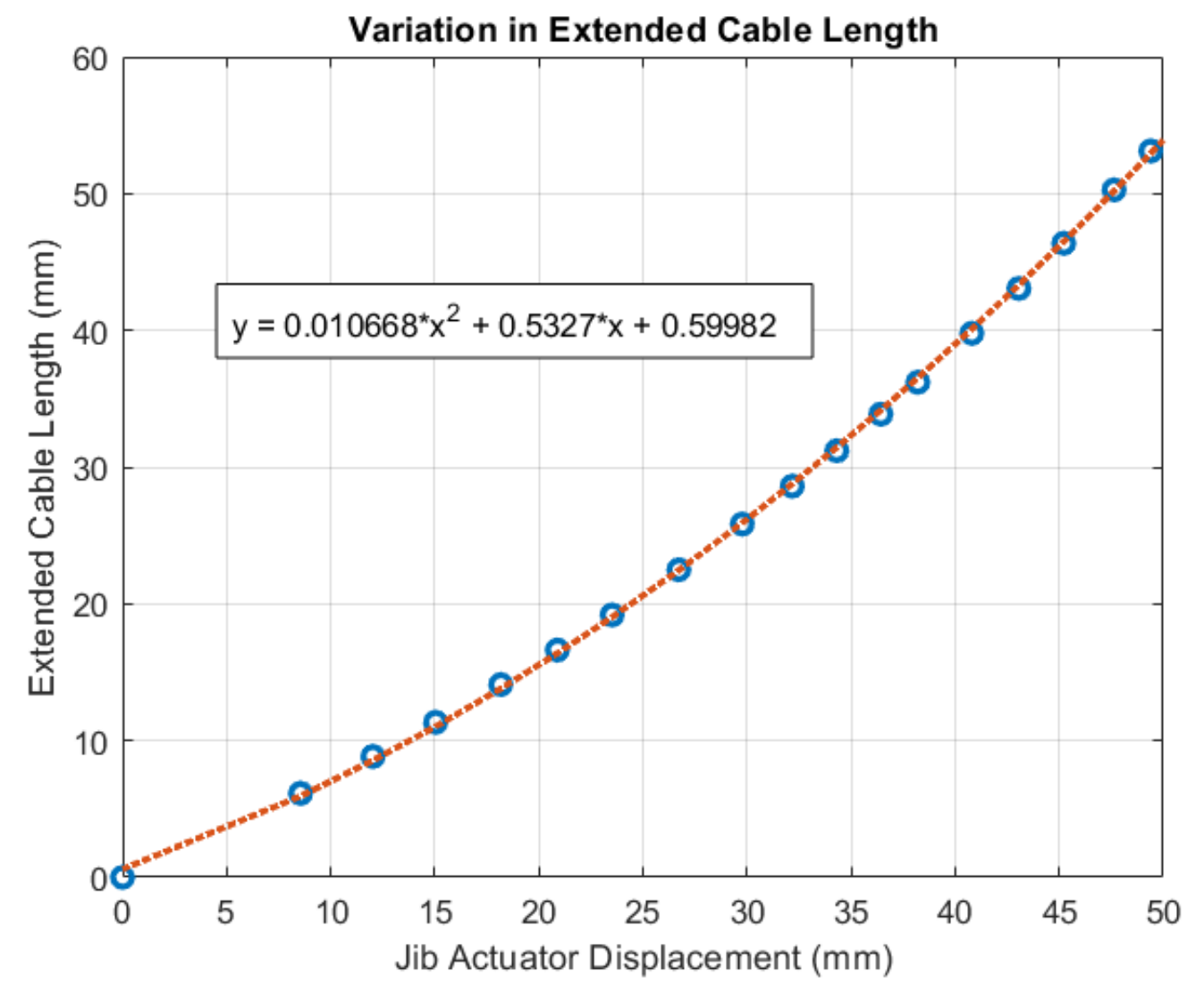

Figure 3.15: Extended cable length as a function of the jib actuator displacement. 
A similar process may be applied to determine the variations in pendulum length that occur as a result of rotating the winch pulley. As the winch rotates, cable is fed through the pulley system and directly increases the extended cable or pendulum length. The variations in cable length are approximately linear with a slope that represents the mean diameter of the wrapped cable. A mean diameter of $41.26 \mathrm{~mm}$ is used, in combination with the relative rotation of the winch, to determine the amount of cable that has been extended as a result of rotating the winch from its initial position.

Considering an initial pendulum length of $325 \mathrm{~mm}$ when the jib is fully retracted and the winch is in its home/initial position, the total pendulum length may be determined from the above considerations as

$$
\begin{aligned}
l_{p} & =l_{p, 0}+\Delta l_{p, w m}+\Delta l_{p, j i b}, \\
l_{p, 0} & =325 \\
\Delta l_{p, w m} & =\left(\frac{\Delta \theta_{w m}}{360}\right) \pi(41.26), \\
\Delta l_{p, j i b} & =(0.010668) d_{j i b}^{2}+(0.5327) d_{j i b}+0.59982,
\end{aligned}
$$

where $l_{p}$ is the extended cable or pendulum length $(\mathrm{mm}), \Delta l_{p, w m}$ is the change in pendulum length $(\mathrm{mm})$ that results from rotating the winch $\Delta \theta_{w m}$ degrees from its initial position, and $\Delta l_{p, j i b}$ is the change in length $(\mathrm{mm})$ that results from extending the jib linear actuator $d_{j i b}$ millimetres from its zero position. Equations $3.1-3.4$ can be used to keep a constant pendulum length as the knuckle boom crane moves to compensate for disturbances via its electric actuators. 


\subsubsection{Electric Actuators}

The test-scale crane uses three linear electric actuators from Actuonix with $50 \mathrm{~mm}$ stroke lengths and 64:1 gear ratios [55]. The linear actuators are controlled with Simple Motor Controllers, which are supplied by Pololu and act as proportional speed controllers [56]. The control actions provided by the myRIO to either the actuator control boards or the simulation models are voltages in the range of 0-3.3 VDC where 0 VDC corresponds to full-speed retraction, 1.65 VDC is the stationary/neutral voltage, and 3.3 VDC is full-speed extension. However, due to the load on each of the actuators, deadbands are observed for each of these input voltages. The deadbands are expressed relative to the stationary/neutral voltage in Table 3.2. For feedback control, the input/control voltages are calculated within a zero-centred shifted/linearised range and then converted to the full range 0-3.3 VDC physical signal by jumping the deadbands specified in Table 3.2 and adding the stationary voltage. A similar process is applied for the winch motor by jumping the deadband specified in Table 3.2 and shifting; however, the winch motor is controlled directly with 5 VDC pulse width modulation (PWM) to set the speed of rotation by varying the duty cycle from $0-1(0-100 \%)$ with a neutral/stationary value of $0.5(50 \%)$.

Table 3.2: Crane actuator lower and upper deadbands.

\begin{tabular}{|l|c|c|c|c|}
\hline & $\begin{array}{c}\text { Base } \\
\text { Actuator }\end{array}$ & $\begin{array}{c}\text { Boom } \\
\text { Actuator }\end{array}$ & $\begin{array}{c}\text { Jib } \\
\text { Actuator }\end{array}$ & $\begin{array}{c}\text { Winch } \\
\text { Motor }\end{array}$ \\
\hline Lower Deadband & $-0.145 \mathrm{~V}$ & $-0.145 \mathrm{~V}$ & $-0.145 \mathrm{~V}$ & -0.0165 \\
Upper Deadband & $0.145 \mathrm{~V}$ & $0.525 \mathrm{~V}$ & $0.175 \mathrm{~V}$ & 0 \\
\hline
\end{tabular}




\subsubsection{Actuator Sensors}

Each of the three linear actuators for the base, boom, and jib of the crane are equipped with potentiometers to measure the actuator displacement as a voltage from 0-5 VDC. By setting the actuator displacements to stationary values, the noise of each feedback signal is quantified by collecting feedback data over a period of time. The corresponding noise data for the base, boom, and jib actuators are represented by histograms in the upper-left, upper-right, and lower-left plots of Figure 3.16, respectively. The bars represent the number of samples taken for each voltage, and the red lines represent probability density functions for approximate normal distributions derived from the data. The mean $\mu$ and standard deviation $\sigma$ for each normal distribution is shown in the title of the corresponding plot for convenience. The winch motor displacement is sensed with a 12-bit encoder, which returns a digital signal to indicate the angle of rotation from 0-360 degrees. For angles exceeding 360 degrees, a softwarebased counter is used to track the number of full rotations and thereby determine the absolute angle of rotation when controlling the winch. The noise in the encoder signal is quantified through the same procedure as for the linear actuator feedback, and the resulting histogram with an approximate normal distribution is shown in the lower-right plot of Figure 3.16. For each feedback signal, the standard deviation from Figure 3.16 is used to apply normally distributed noise and thereby emulate the feedback signals during the simulations. The mean values indicated by the histograms correspond to the actual positions at the time the data was collected and are not applied in simulation. 

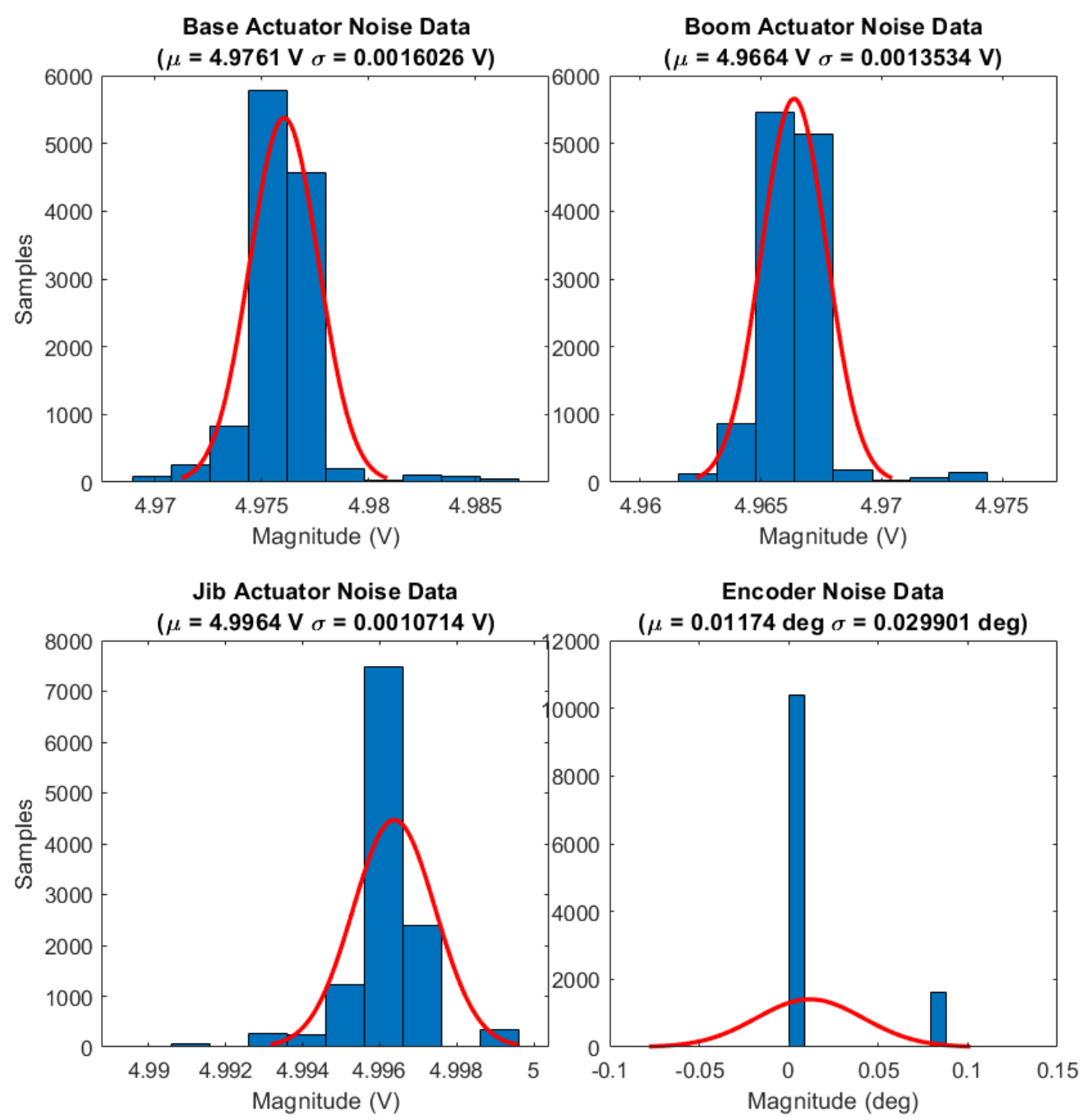

Figure 3.16: Crane potentiometers and encoder noise data. 


\subsubsection{IMU Sensor}

The system also involves the use of two Yost Labs 3-Space Inertial Measurement Units (IMUs) [57] to sense the pose of the ship and load. Both IMUs have a local measurement frame shown in Figure 3.17. The first IMU is attached near the centre of the motion platform, whereas the second IMU is attached to the load. These IMUs are referred to as IMU1 and IMU2, respectively. Each IMU provides nine digital feedback signals, including three angular rates, three linear accelerations, and three magnetic field measurements. To simulate these sensors, the sensor noise is quantified by collecting feedback data for several minutes while the sensors remain stationary. The collected data is described by the histograms in Figure 3.18 for IMU1 and Figure 3.19 for IMU2. Again, a red line is shown to approximate the normal distribution of each signal with the mean and standard deviation shown in the title of the corresponding plot. The tags 'Gyro,' 'Acc,' and 'Mag' are used to denote the gyroscopic sensors, accelerometers, and magnetometers; and a capital 'X,' 'Y,' or ' $\mathrm{Z}$ ' denotes the direction of the measurement within the reference frame shown in Figure 3.17. The sensor biases cannot accurately be quantified by the mean values as the 'true' directions of magnetic north and gravity are not known with sufficient accuracy and the mean values may be partially attributed to the mounting configuration. It is assumed that the effects of sensor biases are effectively removed following an approximation with low-pass filters during an initialization period. The deviations are directly applied as normally distributed noise on each signal for the purpose of simulation. 


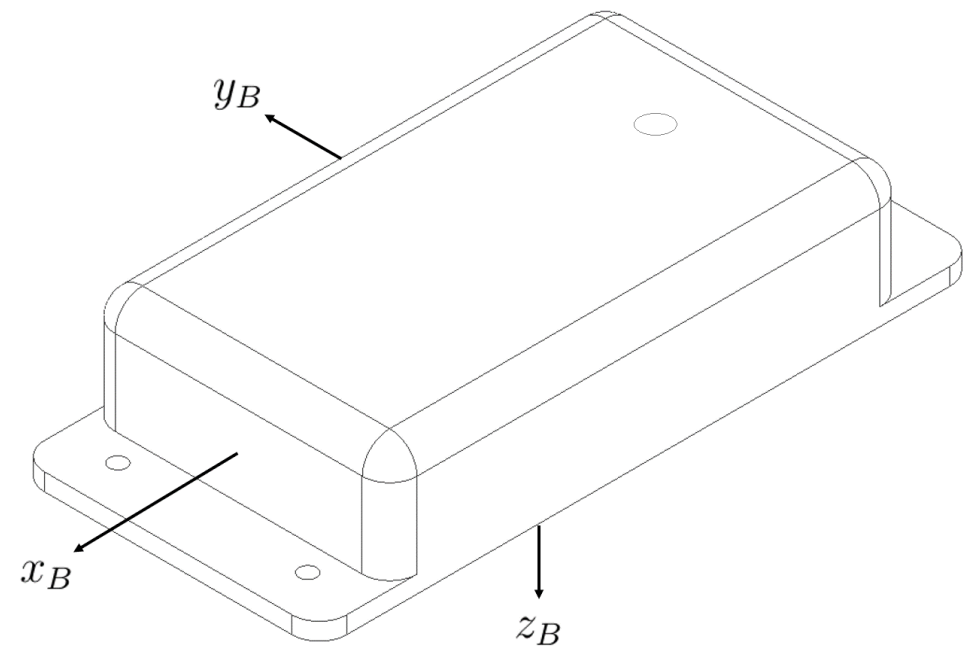

Figure 3.17: IMU reference frame. 

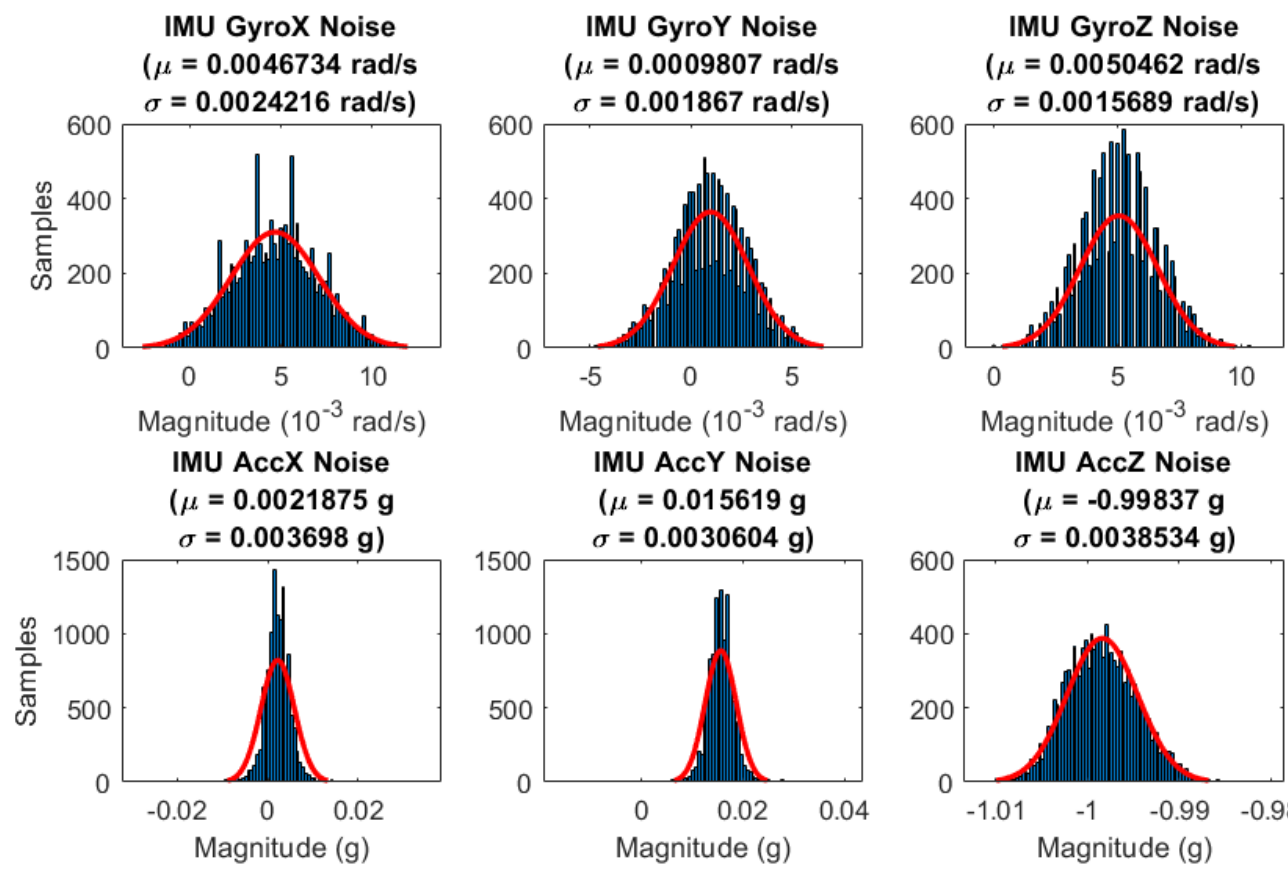

Magnitude $\left(10^{-3} \mathrm{rad} / \mathrm{s}\right)$

IMU AccY Noise

( $\mu=0.015619 \mathrm{~g}$

Magnitude $\left(10^{-3} \mathrm{rad} / \mathrm{s}\right)$

IMU AccZ Noise

$(\mu=-0.99837 \mathrm{~g}$
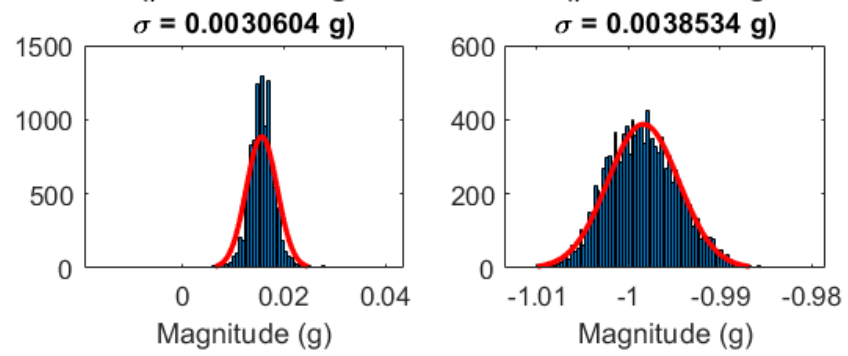

IMU MagX Noise

$(\mu=0.12871 \mathrm{G}$
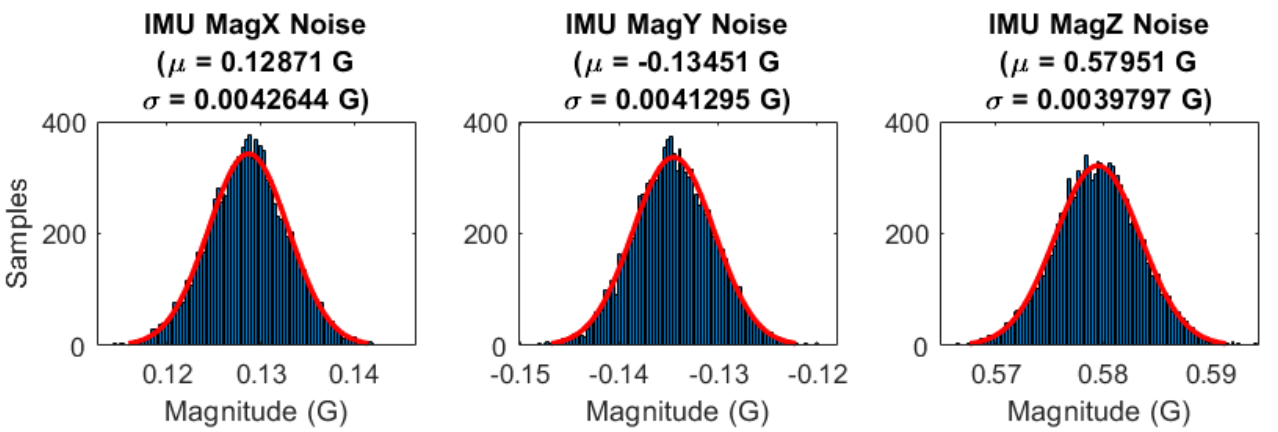

Figure 3.18: IMU1 noise data. 


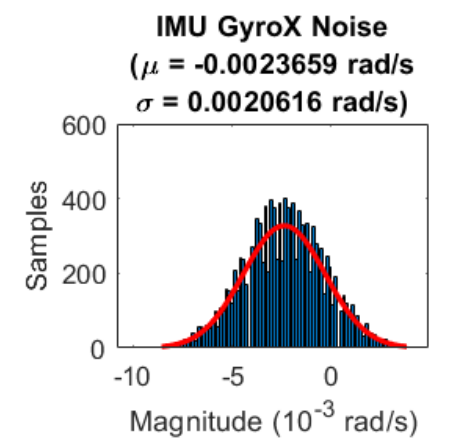

IMU AccX Noise

$(\mu=0.0046198 \mathrm{~g}$

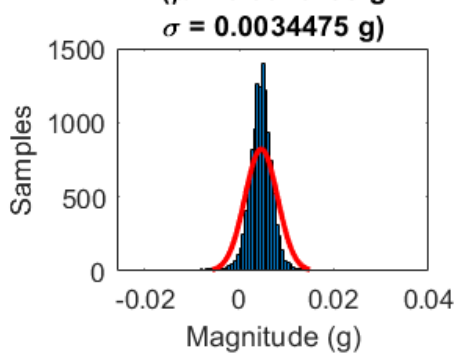

IMU MagX Noise

$(\mu=0.20833 \mathrm{G}$

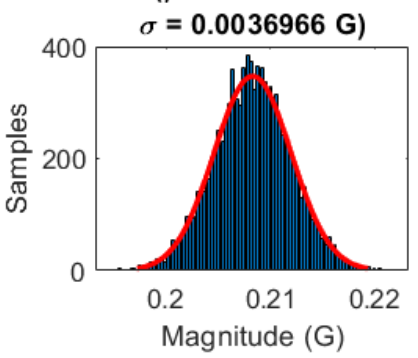

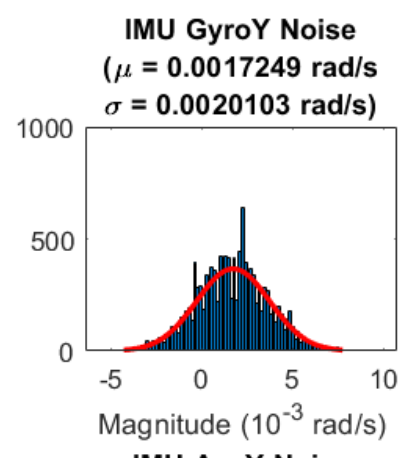

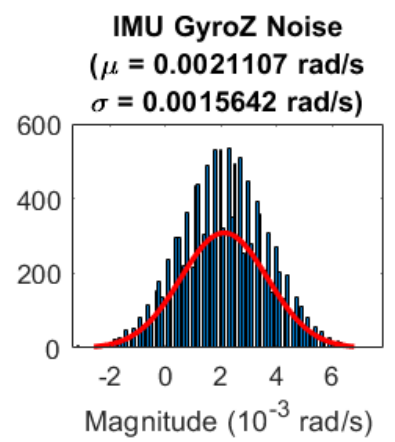

IMU AccY Noise

( $\mu=0.033476 \mathrm{~g}$

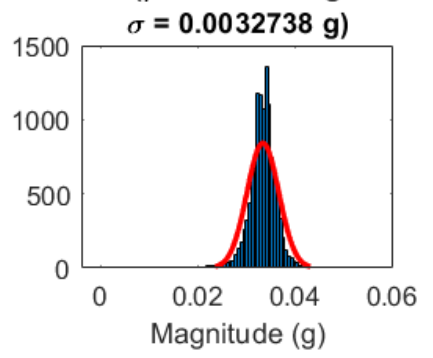

IMU MagY Noise

$(\mu=-0.17674 \mathrm{G}$

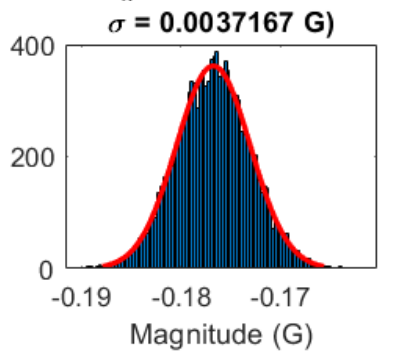

IMU AccZ Noise

$(\mu=-0.9725 \mathrm{~g}$

$\sigma=0.0023695 \mathrm{~g}$ )

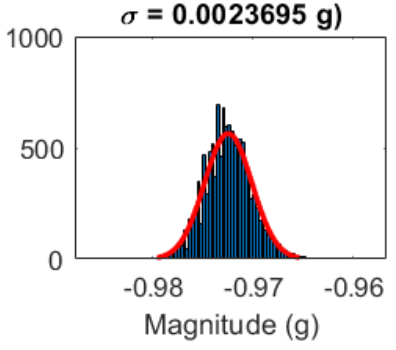

IMU MagZ Noise

$(\mu=0.54822 \mathrm{G}$

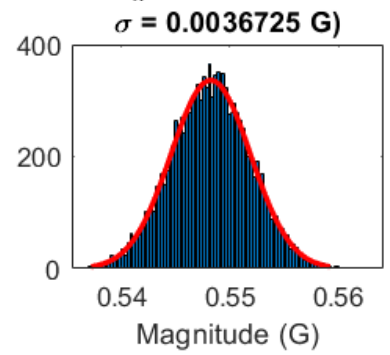

Figure 3.19: IMU2 noise data. 


\subsubsection{Cameras}

The sensors described above are essential for the control algorithms and simulations applied throughout the current work. In addition to these sensors, two GoPro HERO7 Black cameras [58] are used to collect data during the experiments. The cameras are set to 30 frames-per-second (FPS) with 1080p video resolution and a linear field of view (FOV). The cameras are used as a stereo vision system to measure the position of the load relative to the world frame. A checkerboard calibration procedure is applied using the Stereo Camera Calibrator App in MATLAB, and the videos are synchronised with an LED that turns on at the beginning of each experiment. An example of stereo vision data collected during an experiment is shown in Figure 3.20 where the first camera (CAM1) is positioned to look down the surge axis, and the second camera (CAM2) is positioned to look down the sway axis. Tracking software was developed to find pixel locations of key points on the motion platform via the black cross on the bottom of the platform, the floor (world) via the black cross at the bottom of the figure, and the load/cable as indicated by the dashed lines and markers on the cable in the figure.

Now that the physical system has been established along with the corresponding sensors, the following section discusses the test-scale simulations. 

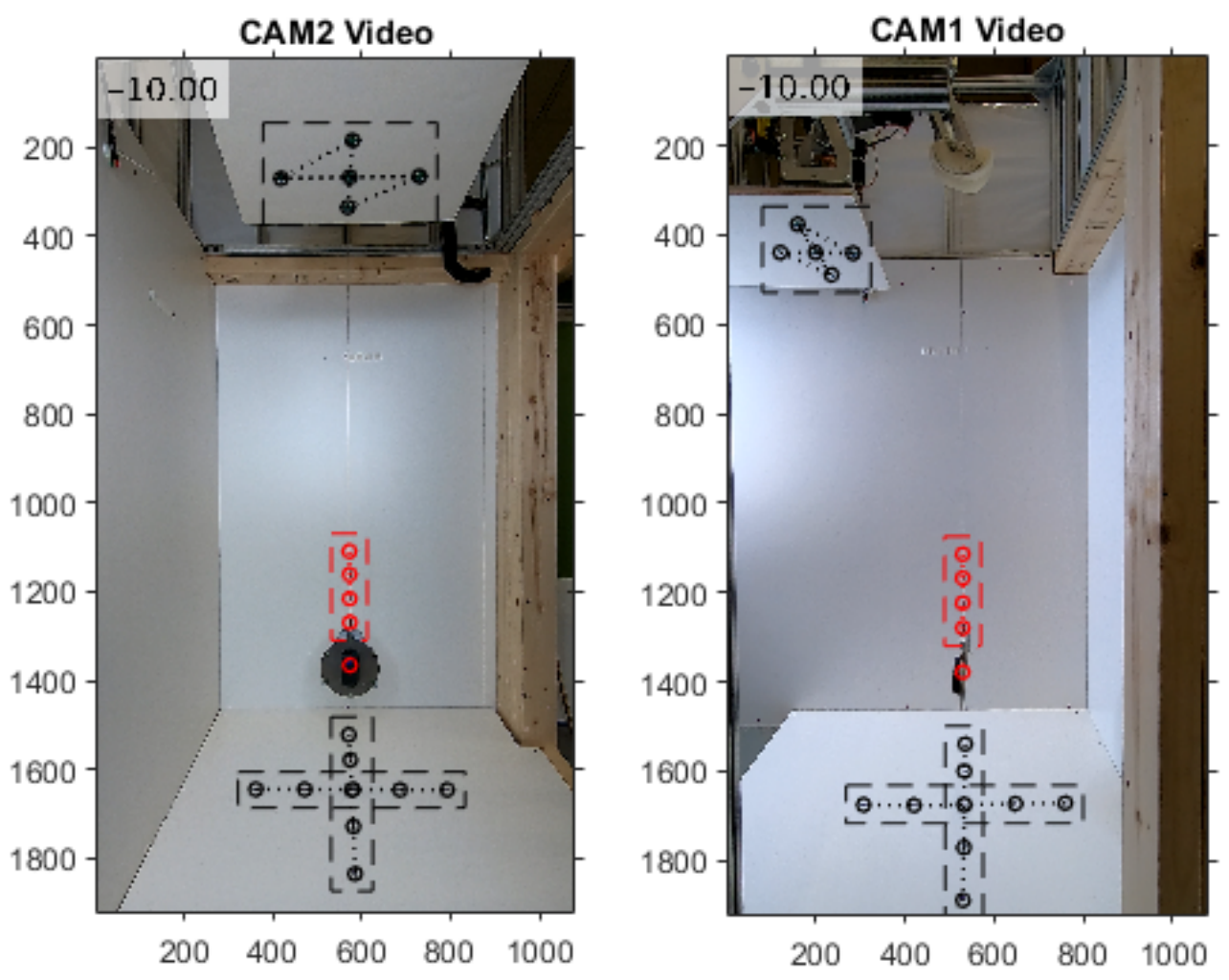

Figure 3.20: Example of stereo vision with GoPro cameras. Key tracking points are indicated by dashed lines and red/black markers. 


\subsection{Test-Scale Simulation}

The test-scale simulation consists of two multibody systems (motion platform and knuckle boom crane), several electric actuators, and sensors. The visualisation of the completed model and its high-level block diagram are provided in Figure 3.21 and Figure 3.22, respectively. In Figure 3.21, the visualisation of the test-scale system shows the motion platform mounted on the flume tank at Carleton University with the crane and load suspended above the water. The functions of the high-level blocks shown in Figure 3.22 are described as

\section{Ship Motion:}

Supplies the test-scale ship motion profiles as kinematic set-points for the motion platform.

\section{Test-Scale Motion Platform:}

Contains the Simscape model of the test-scale motion platform, which is driven through its kinematic set-points.

\section{Parametric System Model:}

Contains the parametrized models of the knuckle boom crane and actuators. The parameters are loaded in MATLAB prior to running the simulation, including frame offsets, initial lengths, link lengths, actuator placement, and IMU locations.

\section{Sensor Models:}

Contains sensor models to emulate the linear actuator feedback, the winch encoder feedback, and the two IMUs. Optional noise is applied to the sensor data, and the signals are converted to the same form as used in the FPGA of the myRIO for the physical measurements. Quantization error 
is also applied to the signals to match the resolution of the physical measurements. The output of the block also includes truth data for the tip location, pendulum angles, and ship motion for use in truth data simulations.

\section{myRIO Controller (HIL/OITL):}

Sends the feedback signals to the myRIO controller via TCP communication over a virtual network. For hardware-in-the-loop (HIL) simulations, the myRIO directly replaces the physical feedback with the simulated feedback in the control software. The myRIO allows for operator interactions via an input device for operator-in-the-loop (OITL) testing in future work. The outputs of the control software include the actuator control actions (voltages and duty cycle), the load set-point, the ship motion estimate, and the load position estimate.

\section{Load Set-Point Viewer:}

Displays the load set-point as a partially transparent green indicator for visualisation.

\section{Test-Scale Estimate Viewer:}

Displays the estimated ship and load positions/orientations with partially transparent red indicators for visualisation.

In the following subsections, the simulated multibody systems, electric actuators, and sensors are briefly discussed. 


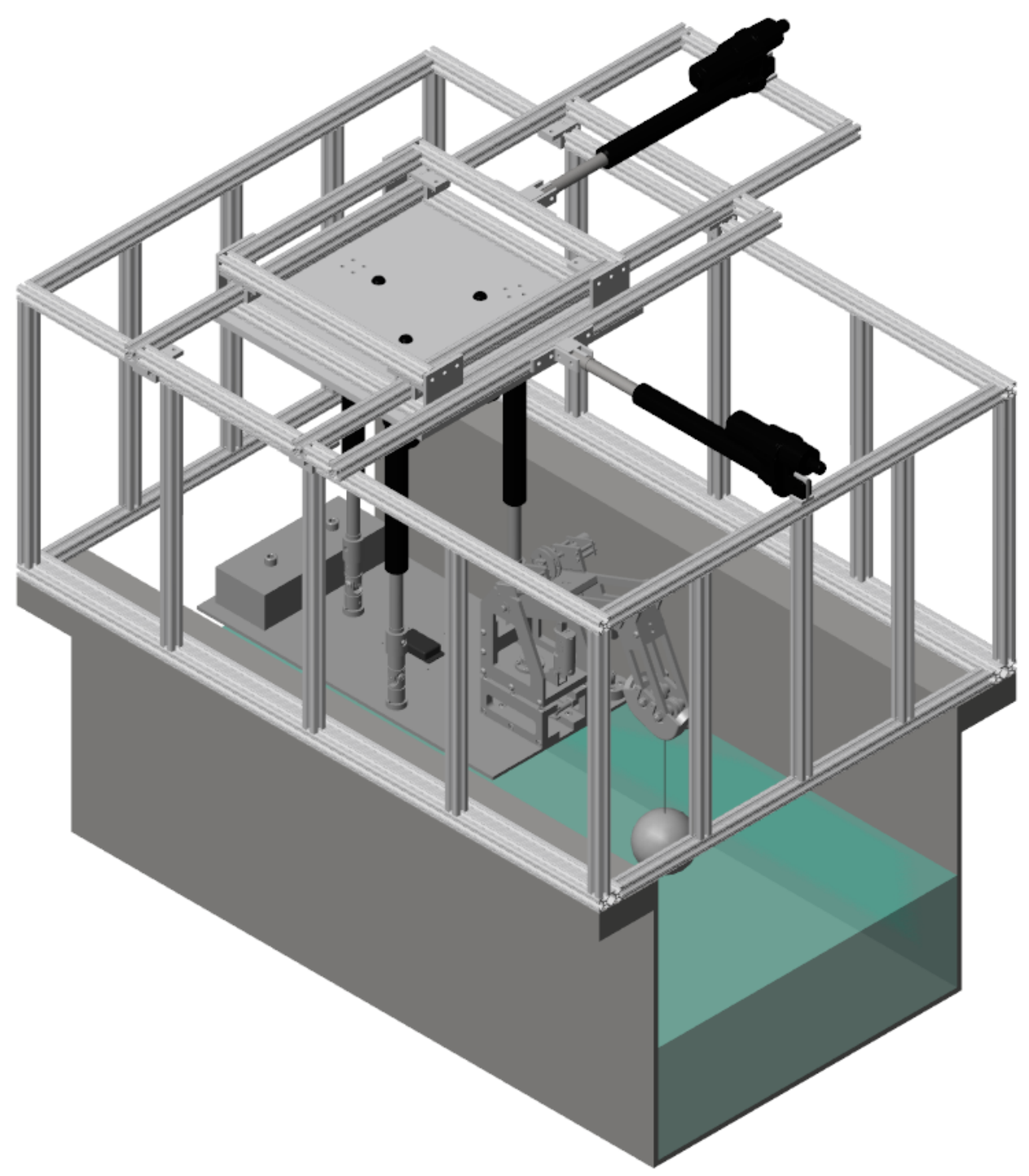

Figure 3.21: Simscape model visualisation. 


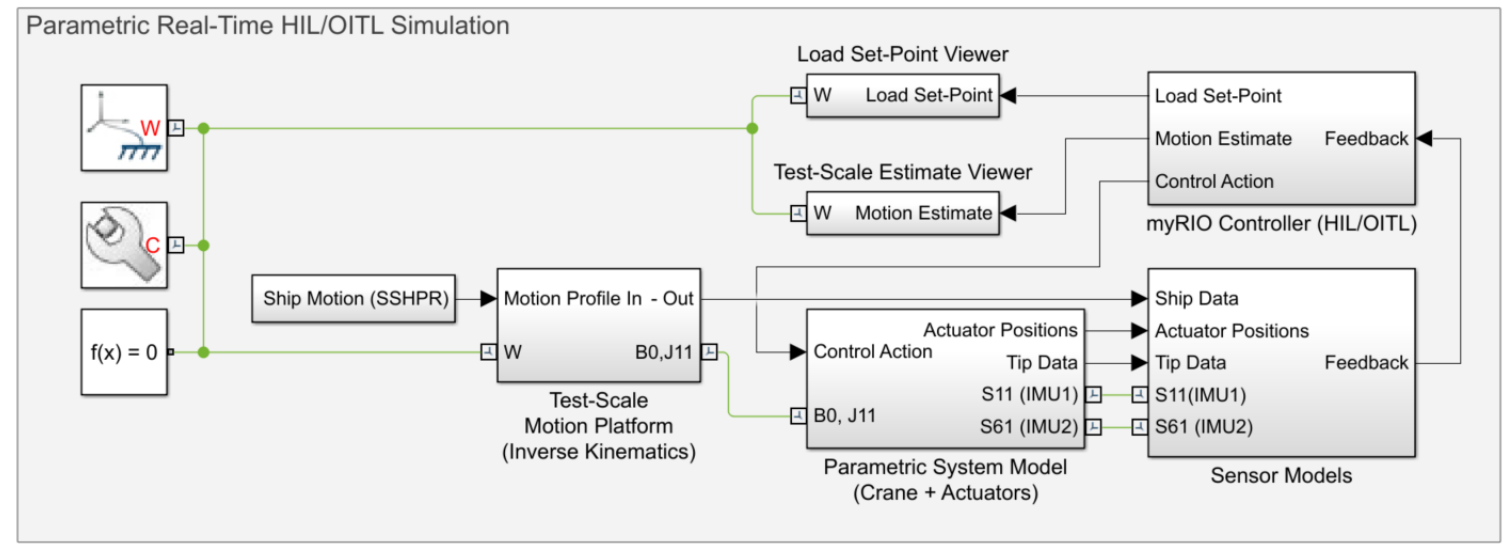

Figure 3.22: Overview of the Simscape model block diagram. 


\subsubsection{Multibody System 1: Motion Platform}

As part of the current thesis work, the motion platform model is assembled in computer-aided design (CAD) software and imported into Simulink as a Simscape Multibody model. In the test-scale simulations, prismatic joints, which correspond to the motion platform actuators, are driven to match the filtered feedback from the experimental apparatus. The motion platform is driven via kinematic inputs to excite the base of the knuckle boom crane.

\subsubsection{Multibody System 2: Knuckle Boom Crane}

The test-scale crane is assembled in computer-aided design (CAD) software based on the specifications in Appendix B. The material properties are included in the model and the inertial properties are derived from the geometry. The CAD model is imported into Simulink as a Simscape Multibody model, and the load is considered a point mass for simplicity. Damping torques, which are a result of aerodynamic drag on the body of the load, are included in the simulation using parameters and equations that will be discussed in Chapter 7. The crane is assembled to allow for force control with inertial effects in future work; however, kinematic control is considered in the current thesis work and the crane joints are driven using transfer functions that represent the actuators' dynamics.

\subsubsection{Electric Actuators}

To model/simulate the linear actuators of the crane, their responses are represented by transfer functions with upper and lower input deadbands, input saturation, and output saturation. The transfer functions are identified with the actuators mounted on the crane to include inertial effects in the identification procedure. The input 
deadbands are listed in Table 3.2 and are a result of the load carried by each actuator, the input saturation represents the range of voltages that may be applied (0-3.3 VDC), and the output saturation represents the range of motion $(0-50 \mathrm{~mm})$. The control actions received from the myRIO correspond to voltages in the range of $0-3.3$ VDC that match those supplied to the physical actuators as described in Section 3.4.3, which include deadband jumps and shifts. These deadband jumps and shifts must be removed from the voltages supplied by the myRIO to linearise them for use with the simulated transfer functions. The transfer functions used to simulate the linear actuators are identified as

$$
\begin{aligned}
G_{B A S E} & =\frac{185.6}{s(s+13.8)} & {\left[\frac{m m}{V}\right], } \\
G_{B O O M} & =\frac{458}{s(s+31.61)} & {\left[\frac{m m}{V}\right], } \\
G_{J I B} & =\frac{640.09}{s(s+48.32)} & {\left[\frac{m m}{V}\right], }
\end{aligned}
$$

where $G_{B A S E}, G_{B O O M}$, and $G_{J I B}$ are the transfer functions associated with the base, boom, and jib linear actuators for shifted linearised input voltages in the range of -1.65 to 1.65 VDC after removal of the deadband jump. The output of each transfer function corresponds to the position of the corresponding actuator. The asymmetrical deadband jumps for reverse and forward motion correspond to the lower and upper deadbands listed in Table 3.2. A similar identification procedure is applied for the winch motor, which yields a transfer function of

$$
G_{W I N C H}=\frac{37928}{s(s+24.43)} \quad\left[\frac{\mathrm{deg}}{\% \text { duty cycle }}\right]
$$

where $G_{W I N C H}$ is the winch motor transfer function with shifted linearised inputs in the range of -0.17 to 0.17 after removal of the deadband jump. The output of the 
transfer function corresponds to the degrees rotation of the winch. The asymmetrical deadband jump for reverse and forward motion correspond to the lower and upper deadbands listed in Table 3.2. The degrees rotation of the winch is used in combination with the jib position to calculate and drive the simulated pendulum length in accordance with Equations 3.1-3.4.

To use the myRIO voltages/inputs with the linear transfer functions in simulation, the following steps are taken:

1. The saturation limits are applied to the input signal, and it is shifted by subtracting the stationary/neutral value of 1.65 VDC for the linear actuators or 0.5 for the winch motor to achieve a zero-centred input.

2. The known deadband jump is removed from the signal by shifting it either up or down for reverse or forward motion based on the identified asymmetrical deadbands listed in Table 3.2 to linearise the transfer function input.

3. The linearised input is applied to the corresponding simulated transfer function.

4. The transfer function output is offset for an optional non-zero starting position.

5. The output is saturated to the $0-50 \mathrm{~mm}$ range for the linear actuators, no saturation is applied to the winch motor to allow for continuous rotation, and the final outputs are used to drive the kinematics of the associated joint in simulation. 


\subsubsection{Sensors}

Within simulation, the actuator feedback signals are determined by sensing the relevant joints, converting the units to match the corresponding hardware signals, and applying sensor noise based on the standard deviations of Figure 3.16. Quantization errors are also applied through data type conversions to match the resolution of the signals in the physical system. The IMU feedback, however, requires several additional steps to calculate. Figure 3.23 shows the Simscape model of an IMU. To emulate an IMU, the angular velocities (wx, wy, wz) and linear accelerations (ax, ay, az) of the IMU's reference frame are sensed in the world frame using a transform sensor. The world-frame gravity constant is added to the corresponding acceleration measurement as a vector with a negative (upwards) z-component to match how gravity is sensed by the physical IMU. The magnetometer field reading is assumed to be a unit vector in the Northern direction for the current work. The angular velocity, linear acceleration, and magnetic field measurement vectors are then transformed from the world frame to the sensor frame using a quaternion transformation that is returned by the transform sensor. Finally, prior to sending the simulated feedback to the myRIO, noise is added to each signal based on the standard deviations of Figure 3.18 for IMU1 and Figure 3.19 for IMU2.

The simulations allow for direct examination of the system response, so cameras are not required to analyse the system. However, to match the experimental conditions, the simulation results are resampled at a frequency of $30 \mathrm{~Hz}$ for consistency with the 30 FPS camera frame rate used during experimental analysis. 


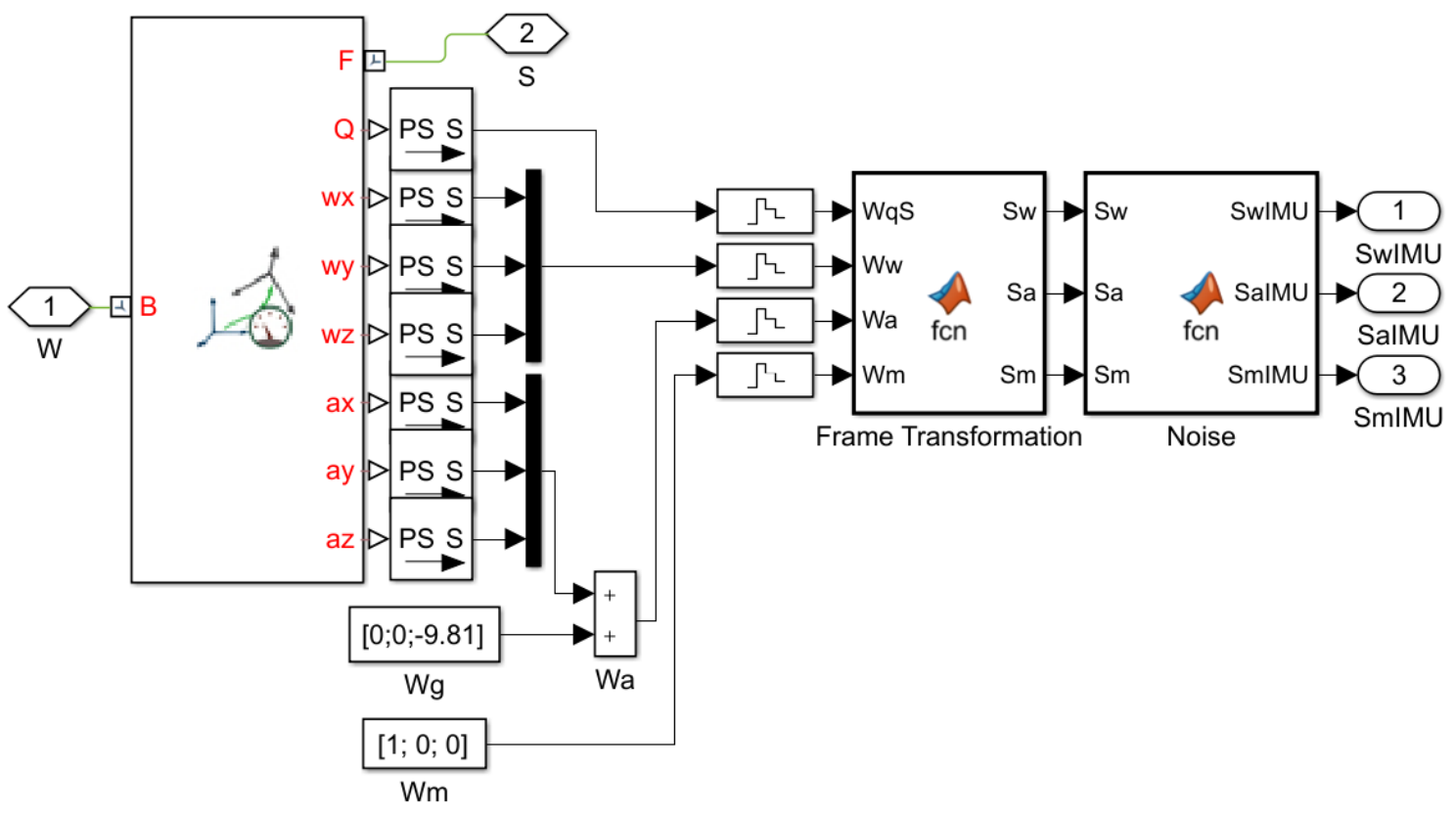

Figure 3.23: Simscape IMU model. 


\subsection{Procedure for Testing and Analysis}

To perform the experiments which will be compared to simulation, the pendulum length is adjusted and confirmed, the cameras are activated, the load is manually pulled to a marker that is positioned for the appropriate starting angle, and the load is released while activating an LED to indicate the start time in each camera. For the compensated tests, the compensation system is activated when the switch is triggered for the LED. The tests are performed for a duration of 120 seconds with an additional 10 seconds of recording before and after the test to allow for initialization of the estimation algorithms, as well as to allow for initialization of the machine vision software that was developed for the analysis. Four red markers with uniform spacing are attached to the cable a set distance above the pendulum load to visually track its motion, and five markers are drawn on the bottom of the deck to allow for vision-based ship motion tracking.

To analyse the test results, the videos from each camera are loaded into MATLAB, the start time is identified in each video via the LED, and the initial marker positions are identified and manually confirmed. The markers are automatically tracked through each frame of the videos to identify their pixel coordinates throughout the duration of the test. Using stereo parameters found through a calibration procedure with the Stereo Camera Calibrator App in MATLAB [59], the pixel coordinates are converted to 'world' coordinates and expressed in the reference frame of the first camera. However, the camera frame provides an unintuitive reference for viewing and analysing the results, so it is desirable to express the coordinates in a frame that is aligned with the previously defined world frame of the compensation system. The world frame of the compensation system represents a 'North East Down' (XYZ) frame while assuming a Northern heading for the ship. For simplicity, the world 
frame is offset to the floor underneath the motion platform and several additional markers are drawn along the $\mathrm{X}$ and $\mathrm{Y}$ axes for the analysis. By detecting the world frame markers on the floor in the camera frame and using a 'model' that contains the actual locations of these marker points expressed in the world frame, the cross covariance matrix is formed and singular value decomposition (SVD) is applied to

find a transformation that maps the world-frame model points to the camera-frame measurement points. The transformation is applied to convert all the camera frame coordinates to the world frame for analysis.

For test cases that involve variable set-points and/or ship motion, the motion is started with the same trigger used to active the LED to synchronise the motion at the start of each test. The detailed results of the analysis, the calculation of performance metrics, and the various test cases will be described in Chapter 7.

\subsection{Conclusion/Summary}

This chapter provided an overview of the system, the operating conditions, ship motion profiles, operator tasks, the experimental apparatus, and the hardware-in-theloop simulations that are referred to and/or used throughout this thesis. The work presented in this chapter is essential for meeting the first and second key objectives of this thesis. 


\section{Chapter 4}

\section{Derivation of System Models}

This chapter describes the kinematic and dynamic models that are applied throughout the current thesis work. In Section 4.1, a previously-developed inverse kinematic model of the motion platform is presented within the context of the current work. The output of the model is compared to that of an existing software package, and the root-mean-squared differences (RMSD) between the two models are discussed. Due to significant differences attributed to simplifying assumptions in the previous work [20], the software-based model is used to convert test-scale ship motion profiles into linear actuator set-points. Next, the forward and inverse kinematic models of the knuckle boom crane are developed and confirmed in Section 4.2. Dual quaternion transformations are applied as they are compact, efficient, and may be considered a generalization of planar complex number analysis to three-dimensional problems. Some mathematical preliminaries regarding dual quaternions are provided in Appendix C. Finally, a dynamic model of the load is developed and confirmed in Section 4.3 as a variable length spherical pendulum suspended from a three-degree-of-freedom trolley that represents the crane tip. 


\subsection{Kinematics of the Motion Platform}

A physical diagram of the motion platform is provided in Figure 4.1 by separating the mechanism into ten groups of bodies that are connected by 11 joints. The corresponding kinematic structure is illustrated using a graph theoretic approach in Figure 4.2 and the graph components are described in Table 4.1. The nodes $n_{i}$ represent the groups of rigidly connected rigid bodies or links, including the five linear actuators LA1-LA5. The solid edges $e_{j}$ represent the simplified physical joints or kinematic pairs connecting these grouped bodies. An additional dashed edge $e_{12}$ is used to represent a virtual joint with a total of six degrees of freedom. This virtual joint is not included when discussing the degrees of freedom of the platform as it does not represent a physical joint or influence the mobility of the platform. However, the virtual joint is useful for defining the forward kinematic problem where the virtual $e_{12}$ joint is driven by the physical $e_{1}-e_{11}$ joints, as well as for defining the inverse kinematic problem where the physical $e_{1}-e_{11}$ joints are driven by the virtual $e_{12}$ joint.

Table 4.1: Node and edge descriptions for the motion platform.

\begin{tabular}{|c|l|c|l|}
\hline$n_{i}$ & Node Description & $e_{j}$ & Edge Description \\
\hline$n_{0}$ & Ground/World, Support, LA1 Base & $e_{1}$ & 1-DOF Prismatic Joint \\
$n_{1}$ & LA1 Rod, Y-Gantry, LA2 Base & $e_{2}$ & 1-DOF Prismatic Joint \\
$n_{2}$ & LA2 Rod, X-Gantry & $e_{3}-e_{5}$ & 1-DOF Revolute Joint \\
$n_{3}-n_{5}$ & LA3-LA5 Base & $e_{6}-e_{8}$ & 2-DOF Cylindrical Joint \\
$n_{6}-n_{8}$ & LA3-LA5 Rod & $e_{9}-e_{11}$ & 2-DOF Universal Joint \\
$n_{9}$ & Platform or Ship Deck & $e_{12}$ & 6-DOF Virtual Joint \\
\hline
\end{tabular}




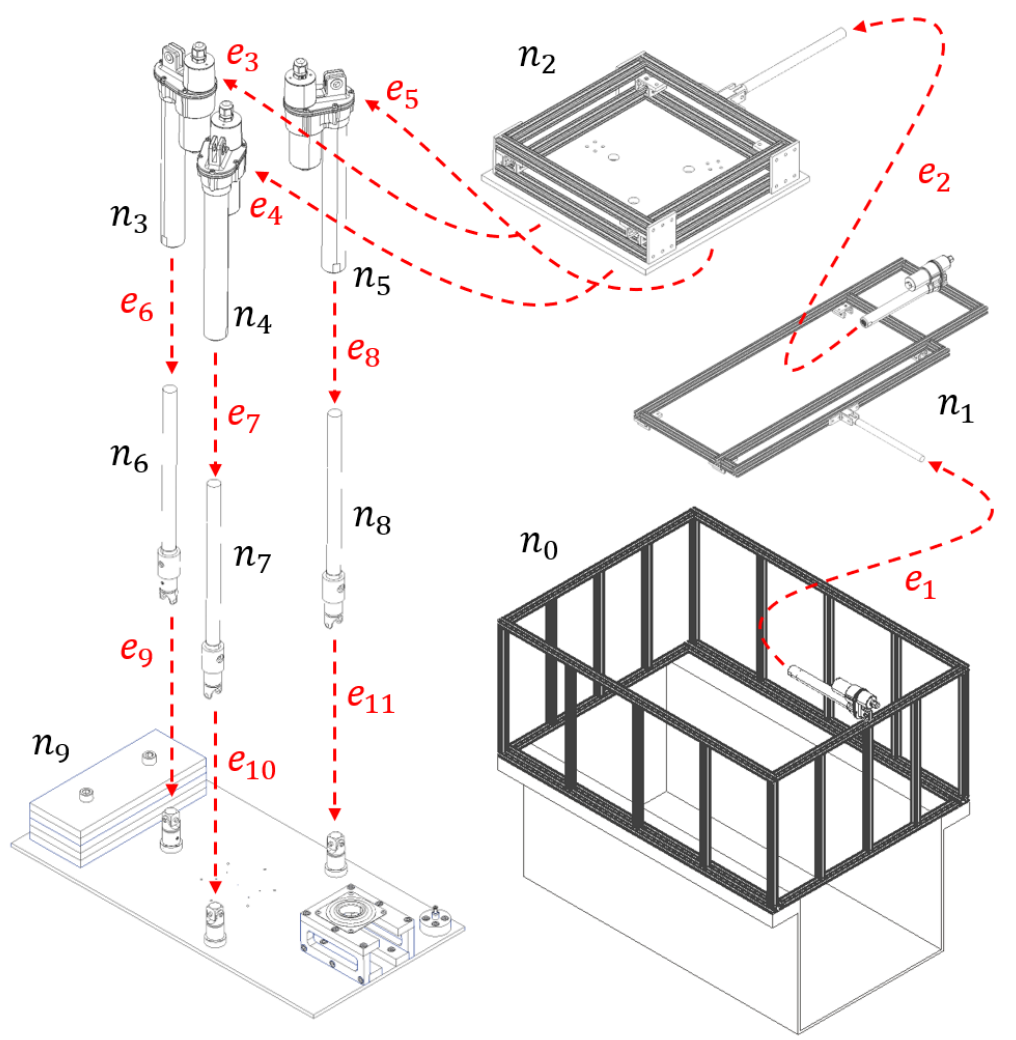

Figure 4.1: Diagram of the motion platform.

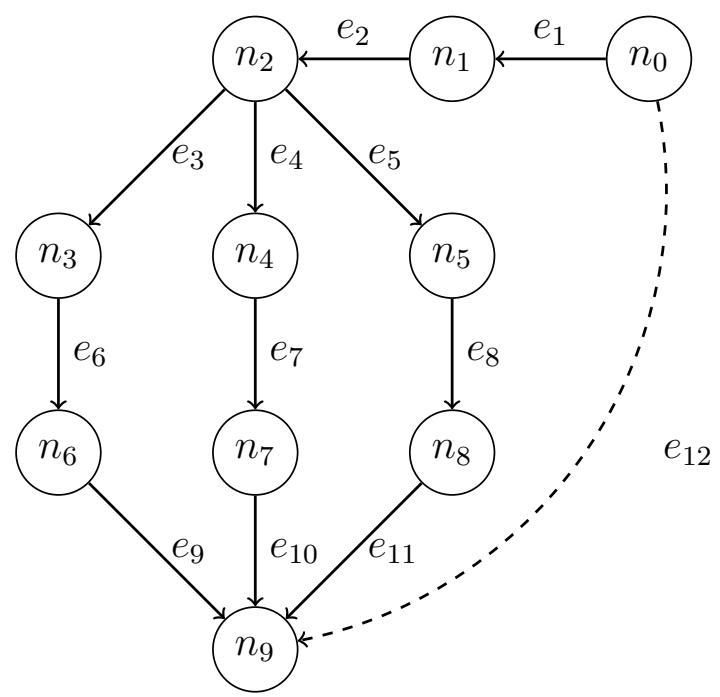

Figure 4.2: Kinematic structure of the motion platform. 
The mobility of the motion platform may be calculated using the standard Kutzbach criterion for spatial mechanisms [60], which yields a mobility of five. The same result is achieved if the virtual joint is included as it has no impact on the system's mobility. From the mobility, it is possible to independently control five degrees of freedom of the motion platform through the five linear actuators. The controlled degrees of freedom are selected as the surge, sway, heave, roll, and pitch motion of the deck. The yaw motion of the deck, a sixth degree of freedom, must either be identically zero or be dependent on the other five degrees of freedom. In previous work [20], the yaw motion was assumed to be negligible and several simplifying assumptions were made to derive an inverse kinematic model. By applying a slight change of notation to match the current work, the model may be stated as

$$
\begin{aligned}
& l_{1}=d_{\text {sway }}, \\
& l_{2}=d_{\text {surge }}, \\
& l_{3}=f_{3}\left(d_{\text {heave }}, \theta_{\text {roll }}, \theta_{\text {pitch }}\right)=\sqrt{\left(P_{3 x}-b_{3 x}\right)^{2}+\left(P_{3 y}-b_{3 y}\right)^{2}+\left(P_{3 z}-b_{3 z}\right)^{2}} \\
& l_{4}=f_{4}\left(d_{\text {heave }}, \theta_{\text {roll }}, \theta_{\text {pitch }}\right)=\sqrt{\left(P_{4 x}-b_{4 x}\right)^{2}+\left(P_{4 y}-b_{4 y}\right)^{2}+\left(P_{4 z}-b_{4 z}\right)^{2}} \\
& l_{5}=f_{5}\left(d_{\text {heave }}, \theta_{\text {roll }}, \theta_{\text {pitch }}\right)=\sqrt{\left(P_{5 x}-b_{5 x}\right)^{2}+\left(P_{5 y}-b_{5 y}\right)^{2}+\left(P_{5 z}-b_{5 z}\right)^{2}}
\end{aligned}
$$

where $l_{i}$ correspond to the extended lengths for the five linear actuators, LA1-LA5, expressed as functions of the deck motion. The vectors $\overrightarrow{b_{i}}=\left[b_{i x}, b_{i y}, b_{i z}\right]^{T}$ define the points at which the LA3-LA5 actuator bases attach to the gantry system, and the vectors $\vec{P}_{i}=\left[P_{i x}, P_{i y}, P_{i z}\right]^{T}$ define the points at which the actuator rods attach to the deck. The $\vec{P}_{i}$ vectors are expressed in the same reference frame at the $\overrightarrow{b_{i}}$ vectors by applying a heave, roll, and pitch transformation matrix. Equating the vector lengths to the actuator lengths in this manner represents simplifying assumptions that the platform does not experience significant yaw motion, and that the actuator bases 
are mounted on three universal joints instead of the three $120^{\circ}$-offset revolute joints for the purpose of this calculation. Previous work also assumed that the surge and sway displacements were the extended lengths for LA1 and LA2. However, it is noted that this assumption introduces error in surge and sway motion when the offsets of the universal joints $\left(e_{9}-e_{11}\right)$ are considered. The maximum error in surge and/or sway motion is reported as $\pm 17.32 \mathrm{~mm}$ in previous work [20]. The provided inverse kinematic model in Equations 4.1-4.5 does not explicitly deal with the errors in linear motion caused by the universal joint offsets. However, the need to correct the surge and sway error during trajectory planning was noted, and correction factors were applied to the extended lengths of LA3-LA5 $\left(l_{3}-l_{5}, e_{6}-e_{8}\right)$ to reduce the heave error introduced by the offsets when defining heave relative to the fully retracted position of the actuators [20].

\subsubsection{Simscape-Based Inverse Kinematics}

In the current work, the inverse kinematics are performed using the kinematic structure of the motion platform in Figure 4.2 and Table 4.1. The analysis is performed in Simulink/Simscape, and the results are compared to Equations 4.1-4.5 to investigate the effects of the simplifying assumptions. The construction of the Simscape Multibody model, which is shown in Figure 4.3 with its corresponding block diagram in Figure 4.4, is labelled to match the graph theoretic representation in Figure 4.2. In Figure 4.3, a simplified triangular model of the platform is constructed using the dimensions reported by McPhee [20] where the vertices of the triangles represent the locations at which LA3-LA5 bases $\left(n_{3}-n_{5}\right)$ connect to the "X-Gantry" (base, $\left.n_{2}\right)$ and "Ship Deck" (platform, $\left.n_{9}\right)$. The offsets caused by the universal joints $\left(e_{9}-e_{11}\right)$ are also included in the model, and the resulting universal joint locations are indicated by blue spheres. Additionally, the side of the platform that supports the crane is 
indicated by a red prism and the world frame is displayed with the platform in its neutral position. In Figure 4.4, the 6-DOF virtual joint $e_{12}$ is constructed in Simscape using a 3-DOF Cartesian joint for surge, sway, and heave; and three revolute joints for roll, pitch, and yaw. The rotation sequence used for the revolute joints is defined as z-y-x from the base (B) to the follower (F). To facilitate comparison of the results, the world frame is defined at the neutral position of the platform where all actuators are extended to $100 \mathrm{~mm}$ and the surge, sway, heave, roll, pitch, and yaw displacements are zero.

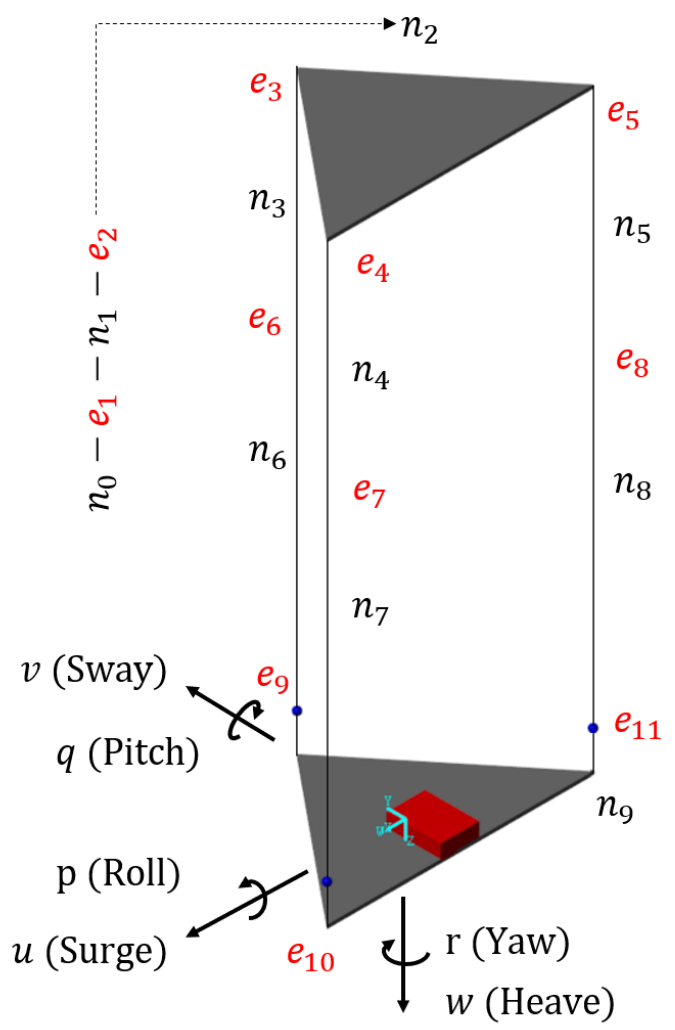

Figure 4.3: Visualisation of the Simscape model used for the inverse kinematics of the motion platform.

The Simscape Multibody model is implemented in parallel with a function block containing the inverse kinematic model to facilitate a direct comparison of the out- 
puts. The first test-scale ship motion profile from Chapter 3 is supplied to both models, and the required displacements of the LA1-LA5 actuators are recorded. In addition to these displacements, the Simscape model calculates the dependent yaw motion that results from setting the controlled degrees of freedom of the platform.

The difference in the required linear actuator displacement of the previously derived model relative to the Simscape model is recorded as root-mean-squared differences (RMSD) for each actuator in the left-hand-side of Table 4.2. For the test-scale ship motion, the maximum RMSD is recorded as $1.512 \mathrm{~mm}$, which may be attributed to the linear motion error introduced by the universal joint offsets. The other recorded differences are under $0.4 \mathrm{~mm}$ and are likely caused by a combination of the simplifying assumptions.

\subsubsection{Simscape-Based Forward Kinematics}

To investigate the effect of the actuator differences on the ship motion, the Simscape model is reconfigured to act as a forward kinematic model and convert the calculated linear actuator set-points back to surge, sway, heave, roll, pitch, and yaw displacements. The root-mean-squared difference of each displacement dataset is calculated relative to the desired ship motion, and the differences are summarized in the right-hand-side of Table 4.2. As the yaw motion is not accounted for in the inverse kinematic model, it is listed as a RMSD relative to the desired zero yaw. 


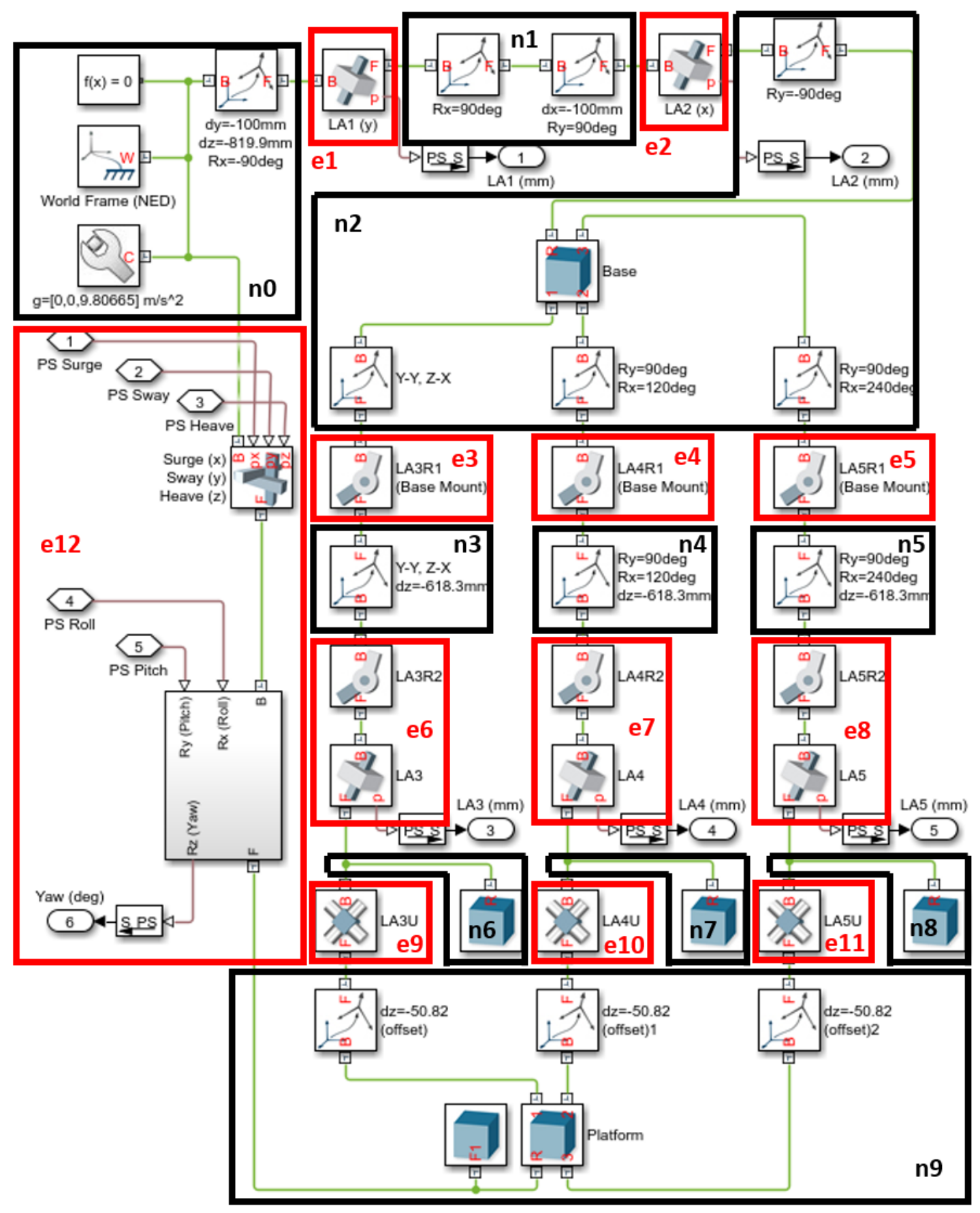

Figure 4.4: Block diagram of the Simscape model used for the inverse kinematics of the motion platform. 
Table 4.2: Summary of inverse kinematic model differences for Motion Profile 1.

\begin{tabular}{|c|c|c|c|}
\hline \multicolumn{2}{|c|}{ Actuator Displacement Differences } & \multicolumn{2}{|c|}{ Ship Motion Differences } \\
\hline Actuator Displacement & RMSD & Degree of Freedom & RMSD \\
\hline LA1 & $1.512 \mathrm{~mm}$ & Sway & $1.484 \mathrm{~mm}$ \\
LA2 & $0.372 \mathrm{~mm}$ & Surge & $0.396 \mathrm{~mm}$ \\
LA3 & $0.150 \mathrm{~mm}$ & Heave & $0.039 \mathrm{~mm}$ \\
LA4 & $0.140 \mathrm{~mm}$ & Roll & $6.8 \mathrm{e}-05 \mathrm{deg}$ \\
LA5 & $0.145 \mathrm{~mm}$ & Pitch & $3.3 \mathrm{e}-04 \mathrm{deg}$ \\
- & - & Yaw & $0.0049 \mathrm{deg}$ \\
\hline
\end{tabular}

For the ship motion applied as part of the current thesis, the largest RMSD recorded in Table 4.2 is $1.484 \mathrm{~mm}$ for sway, which is a direct result of the linear actuator difference observed for LA1. The majority of the difference is attributed to the linear motion caused by the universal joint offsets, and it is considered negligible compared to the set-point tracking error of the actuators.

However, the ship motion applied in this thesis is well within the limits of the motion platform. The platform was designed to operate with up to $20^{\circ}$ roll and pitch [20]. As these design limits are approached, the differences associated with the previously developed inverse kinematic model increase substantially. The differences of the inverse kinematic model are reported for three test cases in Table 4.3. The test cases include $20^{\circ}$ roll, $20^{\circ}$ pitch, and combined $20^{\circ}$ roll $/$ pitch with the other controlled degrees of freedom specified as zero. The reported differences are significant with up to $21.62 \mathrm{~mm}$ difference in linear displacement and $3.21^{\circ}$ difference in angular displacement. The actual yaw from the Simulink/Simscape inverse kinematic model is reported in the bottom row of the table as it represents a previously unmodelled error that is not directly controllable due to a dependence on the other specified degrees of freedom. That is, it is not possible to entirely remove the yaw error without causing error in the other degrees of freedom. 
Table 4.3: Summary of inverse kinematic model differences for various set-points.

\begin{tabular}{|c|c|c|c|}
\hline Displacement & $20^{\circ}$ Roll & $20^{\circ}$ Pitch & $20^{\circ}$ Roll/Pitch \\
\hline LA1 & $-21.62 \mathrm{~mm}$ & $5.99 \mathrm{~mm}$ & $-14.30 \mathrm{~mm}$ \\
LA2 & $0.00 \mathrm{~mm}$ & $17.38 \mathrm{~mm}$ & $7.59 \mathrm{~mm}$ \\
LA3 & $-2.93 \mathrm{~mm}$ & $-3.09 \mathrm{~mm}$ & $-2.83 \mathrm{~mm}$ \\
LA4 & $-2.95 \mathrm{~mm}$ & $-1.88 \mathrm{~mm}$ & $8.43 \mathrm{~mm}$ \\
LA5 & $-2.95 \mathrm{~mm}$ & $-2.82 \mathrm{~mm}$ & $-9.59 \mathrm{~mm}$ \\
\hline Surge & $0.00 \mathrm{~mm}$ & $17.34 \mathrm{~mm}$ & $6.81 \mathrm{~mm}$ \\
Sway & $-21.62 \mathrm{~mm}$ & $6.07 \mathrm{~mm}$ & $-15.14 \mathrm{~mm}$ \\
Heave & $-2.94 \mathrm{~mm}$ & $-2.55 \mathrm{~mm}$ & $-0.08 \mathrm{~mm}$ \\
Roll & $0.01 \mathrm{deg}$ & $-0.21 \mathrm{deg}$ & $-1.10 \mathrm{deg}$ \\
Pitch & $0.00 \mathrm{deg}$ & $-0.17 \mathrm{deg}$ & $-3.21 \mathrm{deg}$ \\
Yaw & $0.00 \mathrm{deg}$ & $0.04 \mathrm{deg}$ & $2.329 \mathrm{deg}$ \\
\hline Yaw (Simscape) & $(0.00 \mathrm{deg})$ & $(0.00 \mathrm{deg})$ & $(2.952 \mathrm{deg})$ \\
\hline
\end{tabular}

It is noted that the above analysis does not consider the universal joint correction factors that were applied to the LA3-LA5 actuators in previous work [20]. Applying the correction yields 12-96\% reduction in the heave, roll, and pitch differences in some cases. However, in other cases, the differences increase by $9.1-4500 \%$ due to a small angle assumption, and the linear surge/sway motions remain uncorrected. Given that the surge/sway motions represent the majority of the reported difference, the benefits of applying the correction are reduced. Due to the improved accuracy of the Simulink/Simscape model for the various test cases, the model is used to generate the linear actuator set-points for the three ship motion profiles discussed in Chapter 3. Using the set-points generated with the Simulink/Simscape model, the motion platform causes the crane to move based on the defined ship motion profile.

In this section, the kinematics of the motion platform were analysed using a Simulink/Simscape model and the results were compared to a previously-developed inverse kinematic model. In the next section, a kinematic model of the test-scale knuckle boom crane is developed for use in the motion compensation and control algorithms of Chapter 6. 


\subsection{Kinematics of the Knuckle Boom Crane}

A physical diagram of the knuckle boom crane is provided in Figure 4.5 by separating the mechanism into six groups of rigid bodies that are connected by 16 joints. The corresponding kinematic structure is illustrated using a graph theoretic approach in Figure 4.6 and the graph components are described in Table 4.4. The nodes $n_{i}$ represent groups of rigidly connected bodies or links, and the solid edges $e_{j}$ represent the simplified physical joints or kinematic pairs connecting these grouped bodies. The $e_{1}$ joint is used to represent the motion of the ship deck, which was discussed in the previous section. It is noted that a 6-DOF joint is used for the ship motion as the crane control algorithm assumes no knowledge of the constraints introduced by the motion platform. The main structure of the crane is indicated by double arrows $e_{2}-e_{7}$ in Figure 4.6, which is considered a serial manipulator with three revolute joints and a variable length pendulum to represent the cable/load. As cable dynamics and doublependulum effects are beyond the scope of the current work, the $e_{7}$ joint is considered to be welded so that there is no relative motion between the cable and the load. The three dashed edges, $e_{8}-e_{10}$ in Figure 4.6, are included to represent compound virtual joints with six degrees of freedom. The virtual joints are not included when discussing the degrees of freedom of the crane as they do not represent physical joints and do not influence the local mobility of the crane. The first virtual joint $e_{8}$ is used for the local forward and inverse kinematic problems regarding the position of the crane tip relative to the base. These two kinematic problems represented by $e_{8}$ are the central focus of this section and will be discussed further below. The second and third virtual joints, $e_{9}$ and $e_{10}$, are used to represent the inverse kinematic problems of determining

joint variables based on the feedback from the two IMUs, which will be discussed in Chapter 5. 
In Figure 4.6, three parallel chains $e_{j}^{\prime} e_{j}^{\prime \prime} e_{j}^{\prime \prime \prime}$ are included to represent the actuation of the main revolute joints $e_{j}$. Each of the main revolute joints is controlled with a linear actuator that adds a revolute joint, the actuator base, a prismatic joint, the actuator rod, and another revolute joint in parallel with the crane's revolute joint. These three kinematic substructures are used to relate the position of the crane's revolute joints to the corresponding actuator positions for the forward and inverse kinematic problems of this section. The substructures are identified as they provide three loops $e_{j} e_{j}^{\prime} e_{j}^{\prime \prime} e_{j}^{\prime \prime \prime}$ for $j=\{2,3,4\}$ that may be solved independently of the loop $e_{2} e_{3} e_{4} e_{8}$ considered for the main serial structure. Each of these kinematic loops will be analysed in the following subsections to relate the crane tip position to the corresponding actuator positions, but first the reference frames and joint variables must be defined.

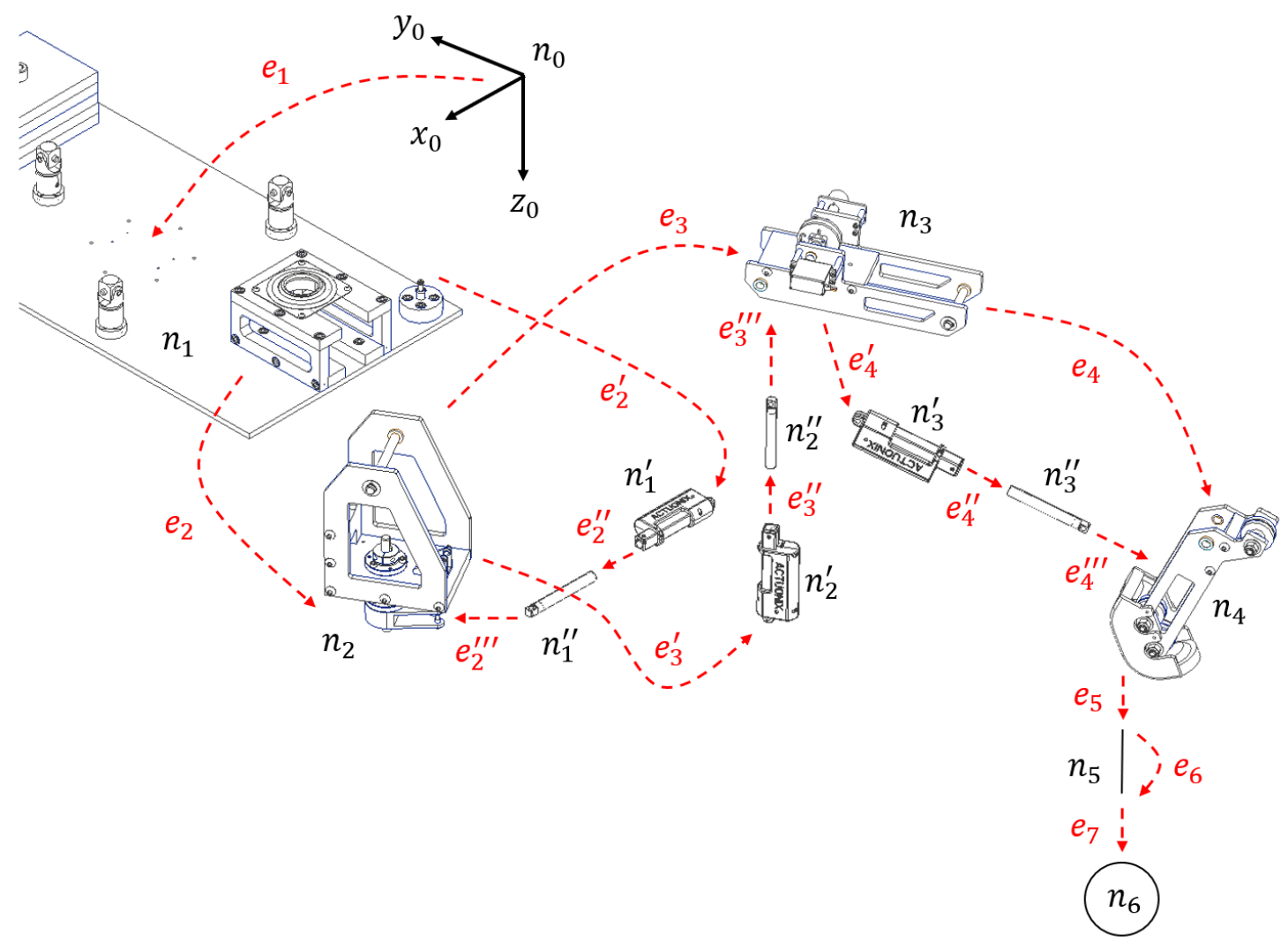

Figure 4.5: Diagram of the knuckle boom crane. 


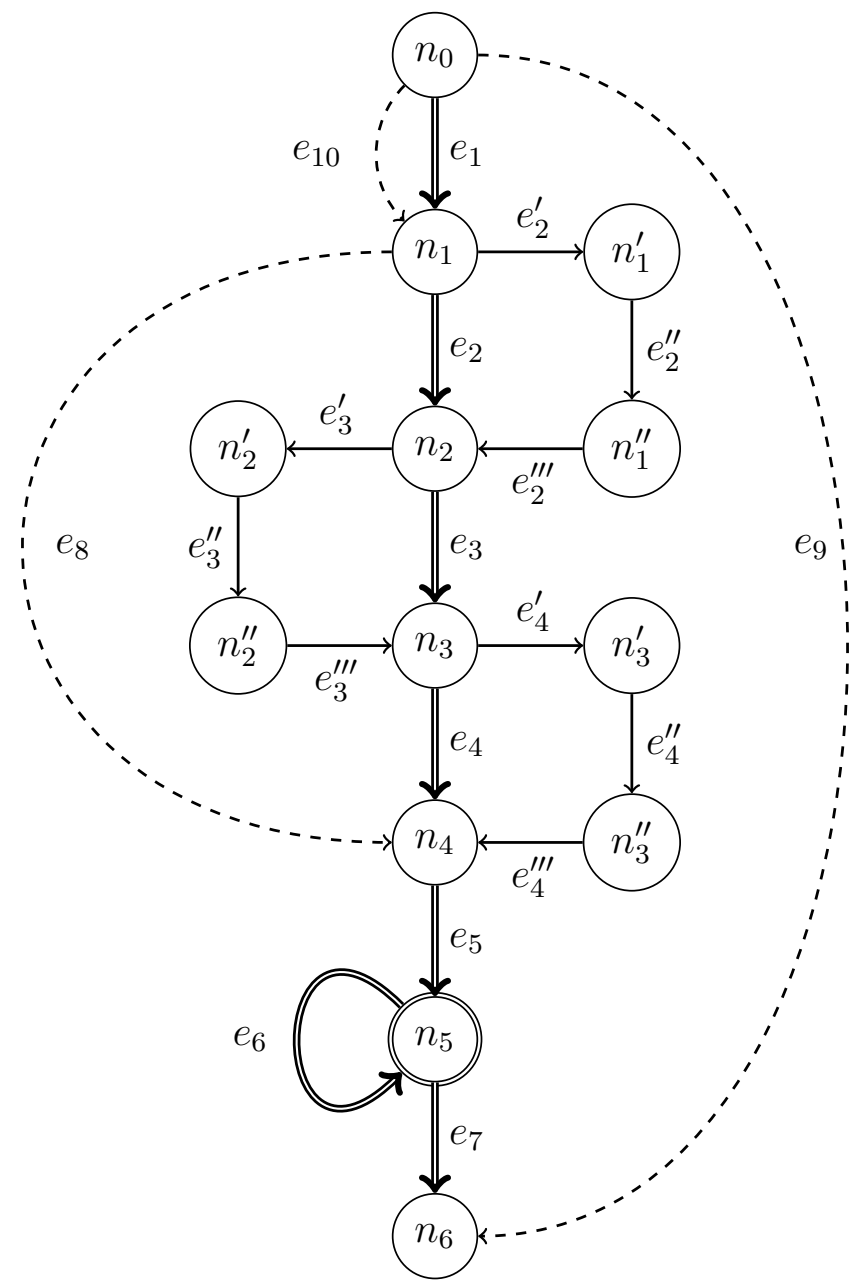

Figure 4.6: Kinematic structure of the knuckle boom crane.

Table 4.4: Node and edge descriptions for the knuckle boom crane.

\begin{tabular}{|c|l|c|l|}
\hline$n_{i}$ & Node Description & $e_{j}$ & Edge Description \\
\hline$n_{0}$ & Ground / World & $e_{1}$ & 6-DOF Joint \\
$n_{1}$ & Platform or Ship Deck, Crane Base & $e_{2}-e_{4}$ & 1-DOF Revolute Joint \\
$n_{2}$ & Crane Tower & $e_{5}$ & 3-DOF Spherical Joint \\
$n_{3}$ & Crane Boom & $e_{6}$ & 1-DOF Prismatic Joint \\
$n_{4}$ & Crane Jib & $e_{7}$ & 0-DOF Welded Joint \\
$n_{5}$ & Variable Length Cable & $e_{8}^{-} e_{10}$ & 6-DOF Virtual Joint \\
$n_{6}$ & Load & - & - \\
\hline$n_{i}^{\prime}$ & Joint Actuator Base & $e_{j}^{\prime}$ & 1-DOF Revolute Joint \\
$n_{i}^{\prime \prime}$ & Joint Actuator Rod & $e_{j}^{\prime \prime}$ & 1-DOF Prismatic Joint \\
- & - & $e_{j}^{\prime \prime \prime}$ & 1-DOF Revolute Joint \\
\hline
\end{tabular}




\subsubsection{Reference Frames and Joint Variables}

A physical representation of the main serial kinematic structure is shown in Figure 4.7 with labels indicating the nodes $n_{0}-n_{6}$ and the edges $e_{1}-e_{7}$ that correspond to rigid bodies and joints, respectively. The $i^{\text {th }}$ node corresponds to the $i^{\text {th }}$ rigid body, which is labelled with a body frame $B_{i}$ at the centre of gravity, a primary joint frame $J_{i, 1}$ at the point of connection to the $(i-1)^{t h}$ body, a secondary joint frame $J_{i, 2}$ at the point of connection to the $(i+1)^{t h}$ body, and a sensor frame $S_{i, s}$ to indicate the frame used by the $s^{\text {th }}$ sensor attached to the body if applicable. In this case, the $S_{1,1}$ and $S_{6,1}$ frames correspond to the IMU1 and IMU2 sensors described in Chapter 3. The world frame $W_{0}$ is also assigned body and joint frames for convenience.

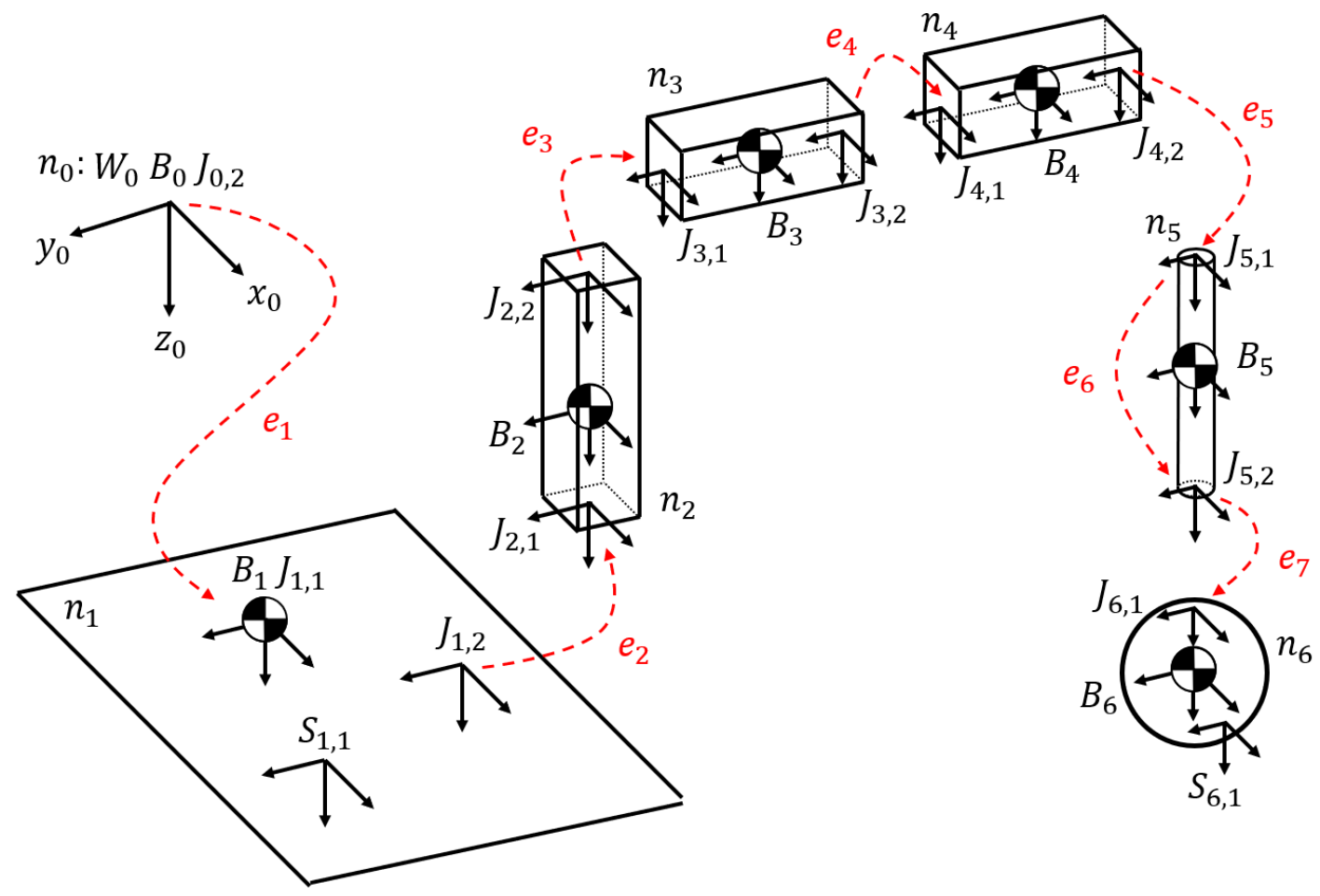

Figure 4.7: Diagram of the knuckle boom crane. 
Considering the physical joints of the crane and applying the Kutzbach criterion for spatial mechanisms [60] to $n_{1}-n_{4}$ yields a mobility of three. These degrees of freedom correspond to the linear displacements of the crane tip, which are controlled by using the linear actuators to vary the revolute joint angles. If the pendulum load is also considered, the mobility equation yields a value of 6 for $n_{1}-n_{6}$ in the simplified model. The pendulum load adds a fourth controlled degree of freedom that corresponds to the cable length, as well as two uncontrolled degrees of freedom corresponding to the pendulum angles. In this chapter, the pendulum load is considered separately from the main serial structure to generalize the resulting control algorithm to other types of cranes. The pendulum load will be analysed during the development of the multibody dynamic model in Section 4.3, as well as within the state estimation algorithm of Chapter 5.

In the main serial kinematic chain, the forward kinematic problem of determining the pose or transformation of any body frame $B_{n}$ relative to any previous body frame $B_{m}$ may be represented as a generalized dual quaternion product,

$$
{ }^{{ }^{B}} \hat{\mathbf{Q}}_{B_{n}}=\prod_{i=m+1}^{n}\left({ }^{B_{i-1}} \hat{\mathbf{Q}}_{J_{i-1,2}}^{J_{i-1,2}} \hat{\mathbf{Q}}_{J_{i, 1}}^{J_{i, 1}} \hat{\mathbf{Q}}_{B_{i}}\right), \quad n>m
$$

where the transformations are written in a compact notation such that ${ }^{A} \hat{\mathbf{Q}}_{C}=$ ${ }^{A} \hat{\mathbf{Q}}_{B}{ }^{B} \hat{\mathbf{Q}}_{C}={ }^{A} \hat{\mathbf{Q}}_{B}^{B} \hat{\mathbf{Q}}_{C}$ represents the pose of frame $C$ relative to frame $A$, and the dual quaternion product $\prod_{i=m+1}^{n}$ involves successive post multiplication of the first term by the following terms to define the transformation sequence. 
The joints $e_{j}=\left\{e_{1}-e_{7}\right\}$ are defined by dual quaternions $\mathbf{Q}_{1}-\mathbf{Q}_{7}$, which are either identity dual quaternions $\mathbf{Q}_{I}$ for welded joints or transformation sequences that are functions of the independent joint variables $d_{j}^{*}$ or $\theta_{j}^{*}$ such that

$$
\begin{aligned}
& { }_{J_{0,2}} \hat{\mathbf{Q}}_{J_{1,1}}=\mathbf{Q}_{1}\left(d_{1 x}^{*}, d_{1 y}^{*}, d_{1 z}^{*}, \theta_{1 z}^{*}, \theta_{1 y}^{*}, \theta_{1 x}^{*}\right) \\
& { }^{J_{1,2}} \hat{\mathbf{Q}}_{J_{2,1}}=\mathbf{Q}_{2}\left(\theta_{2 z}^{*}\right) \\
& { }_{2,2} \hat{\mathbf{Q}}_{J_{3,1}}=\mathbf{Q}_{3}\left(\theta_{3 x}^{*}\right) \\
& J_{3,2} \hat{\mathbf{Q}}_{J_{4,1}}=\mathbf{Q}_{4}\left(\theta_{4 x}^{*}\right) \\
& J_{4,2} \hat{\mathbf{Q}}_{J_{5,1}}=\mathbf{Q}_{5}\left(\theta_{5 x}^{*}, \theta_{5 y}^{*}, \theta_{5 z}^{*}\right) \\
& J_{5,1} \hat{\mathbf{Q}}_{J_{5,2}}=\mathbf{Q}_{6}\left(d_{6 z}^{*}\right) \\
& J_{5,2} \hat{\mathbf{Q}}_{J_{6,1}}=\mathbf{Q}_{7}=\mathbf{Q}_{I}
\end{aligned}
$$

where the rotation angles for the $j^{\text {th }}$ joint are defined as a rotation of $\theta_{j a}^{*}$ about the local $a$ axis, and the linear motion is defined as a displacement of $d_{j a}^{*}$ along the local $a$ axis. For joint transformations with multiple variables, the left-to-right/firstto-last sequence of the joint variables listed in Equations 4.7-4.13 is used to define successive frames in the sequence. The positive directions of the joint variables follow the standard right-hand-rule for the corresponding axis. It is noted that the sixth joint is considered an internal prismatic joint that defines the variable length of the fifth rigid body. 
When considering the main serial portion of the knuckle boom crane, the three revolute joints for the base, boom, and jib are defined as

$$
\begin{aligned}
& \text { Base: }{ }^{J_{1,2}} \hat{\mathbf{Q}}_{J_{2,1}}=\mathbf{Q}_{2}\left(\theta_{2 z}^{*}\right) \\
& =\cos \left(\frac{\theta_{2 z}^{*}}{2}\right)+0 \hat{\imath}+0 \hat{\jmath}+\sin \left(\frac{\theta_{2 z}^{*}}{2}\right) \hat{k}+\frac{\epsilon}{2}(0+0 \hat{\imath}+0 \hat{\jmath}+0 \hat{k}), \\
& \text { Boom: }{ }^{J_{2,2}} \hat{\mathbf{Q}}_{J_{3,1}}=\mathbf{Q}_{3}\left(\theta_{3 x}^{*}\right) \\
& =\cos \left(\frac{\theta_{3 x}^{*}}{2}\right)+\sin \left(\frac{\theta_{3 x}^{*}}{2}\right) \hat{\imath}+0 \hat{\jmath}+0 \hat{k}+\frac{\epsilon}{2}(0+0 \hat{\imath}+0 \hat{\jmath}+0 \hat{k}), \\
& \text { Jib: }{ }^{J_{3,2}} \hat{\mathbf{Q}}_{J_{4,1}}=\mathbf{Q}_{4}\left(\theta_{4 x}^{*}\right) \\
& =\cos \left(\frac{\theta_{4 x}^{*}}{2}\right)+\sin \left(\frac{\theta_{4 x}^{*}}{2}\right) \hat{\imath}+0 \hat{\jmath}+0 \hat{k}+\frac{\epsilon}{2}(0+0 \hat{\imath}+0 \hat{\jmath}+0 \hat{k}),
\end{aligned}
$$

where the joint variables $\left\{\theta_{2 z}^{*}, \theta_{3 x}^{*}, \theta_{4 x}^{*}\right\}$ are controlling the extended lengths $\left\{d_{2 x}, d_{3 z}, d_{4 z}\right\}$ of the corresponding linear actuators. The extended lengths of the actuators are measured relative to the fully retracted lengths $\left\{d_{2 x, 0}, d_{3 z, 0}, d_{4 z, 0}\right\}$.

In the following three subsections, the forward and inverse kinematic models of each parallel actuator mechanism will be developed. The inverse kinematic problem is defined as determining the extended length required to achieve a specified angle for the corresponding revolute joint, whereas the forward kinematic problem is defined as determining the joint angle from the extended length. To analyse the parallel mechanisms for each of the actuators, intermediate frames $L A-L A^{\prime \prime \prime}$ are assigned to the four bodies within the kinematic chain when defining the parallel joint transformations in the following sections. After providing the inverse kinematic models for each of these parallel joint mechanisms, the main serial kinematic structure will be analysed and the models will be confirmed with Simulink/Simscape. 


\subsubsection{Base Actuator Mechanism}

The revolute joint $e_{2}$, which allows the tower to rotate about the base of the crane, is shown in Figure 4.8 within the context of its parallel mechanism as described by the loop $e_{2} e_{2}^{\prime} e_{2}^{\prime \prime} e_{2}^{\prime \prime \prime}$ in Figure 4.6. Additional frames and transformation are superimposed on the diagram for use in the kinematic analysis. The $J_{1,2}$ and $J_{2,2}$ frames were previously defined; however, the $L A-L A^{\prime \prime \prime}$ frames are added to define intermediate transformations for the revolute joint between the deck and the actuator base, the prismatic joint between the actuator base and the actuator rod, and the revolute joint between the actuator rod and the tower. The prismatic joint displacement is the sum of the extended length and the fully retracted length, and rigid offsets are included to define the $x$ - and $y$-distances to $L A$ as $\left\{c_{2 x, 1}, c_{2 y, 1}\right\}$ and to $L A^{\prime \prime \prime}$ as $\left\{0, c_{2 y, 2}\right\}$ with respect to $J_{1,2}$ and $J_{2,1}$, respectively. In the configuration shown, $J_{2,1}$ has rotated about $J_{1,2}$ to a home position that is defined with a fully retracted actuator $d_{2 x}=0$ and a corresponding joint angle of $\theta_{2 z}^{*}=\theta_{2 z 0}$. Using the frames defined in Figure 4.8 as a representation of the parallel kinematic structure illustrated in Figure 4.6 and introducing intermediate joint angles $\theta_{2 z}^{\prime}$ and $\theta_{2 z}^{\prime \prime}$ for the additional revolute joints, the relevant transformations may be defined as

$$
\begin{aligned}
{ }^{J_{1,2}} \hat{\mathbf{Q}}_{L A} & =1+0 \hat{\imath}+0 \hat{\jmath}+0 \hat{k}+\frac{\epsilon}{2}\left(0-c_{2 x, 1} \hat{\imath}-c_{2 y, 1} \hat{\jmath}+0 \hat{k}\right), \\
{ }^{L A} \hat{\mathbf{Q}}_{L A^{\prime}} & =\cos \left(\frac{\theta_{2 z}^{\prime}}{2}\right)+0 \hat{\imath}+0 \hat{\jmath}+\sin \left(\frac{\theta_{2 z}^{\prime}}{2}\right) \hat{k}+\frac{\epsilon}{2}(0+0 \hat{\imath}+0 \hat{\jmath}+0 \hat{k}), \\
{ }^{L A^{\prime}} \hat{\mathbf{Q}}_{L A^{\prime \prime}} & =1+0 \hat{\imath}+0 \hat{\jmath}+0 \hat{k}+\frac{\epsilon}{2}\left(0+\left(d_{2 x}+d_{2 x, 0}\right) \hat{\imath}+0 \hat{\jmath}+0 \hat{k}\right), \\
{ }^{L A^{\prime \prime}} \hat{\mathbf{Q}}_{L A^{\prime \prime \prime}} & =\cos \left(\frac{\theta_{2 z}^{\prime \prime}}{2}\right)+0 \hat{\imath}+0 \hat{\jmath}+\sin \left(\frac{\theta_{2 z}^{\prime \prime}}{2}\right) \hat{k}+\frac{\epsilon}{2}(0+0 \hat{\imath}+0 \hat{\jmath}+0 \hat{k}), \\
{ }^{J_{2,1}} \hat{\mathbf{Q}}_{L A^{\prime \prime \prime}} & =1+0 \hat{\imath}+0 \hat{\jmath}+0 \hat{k}+\frac{\epsilon}{2}\left(0+0 \hat{\imath}-c_{2 y, 2} \hat{\jmath}+0 \hat{k}\right) .
\end{aligned}
$$




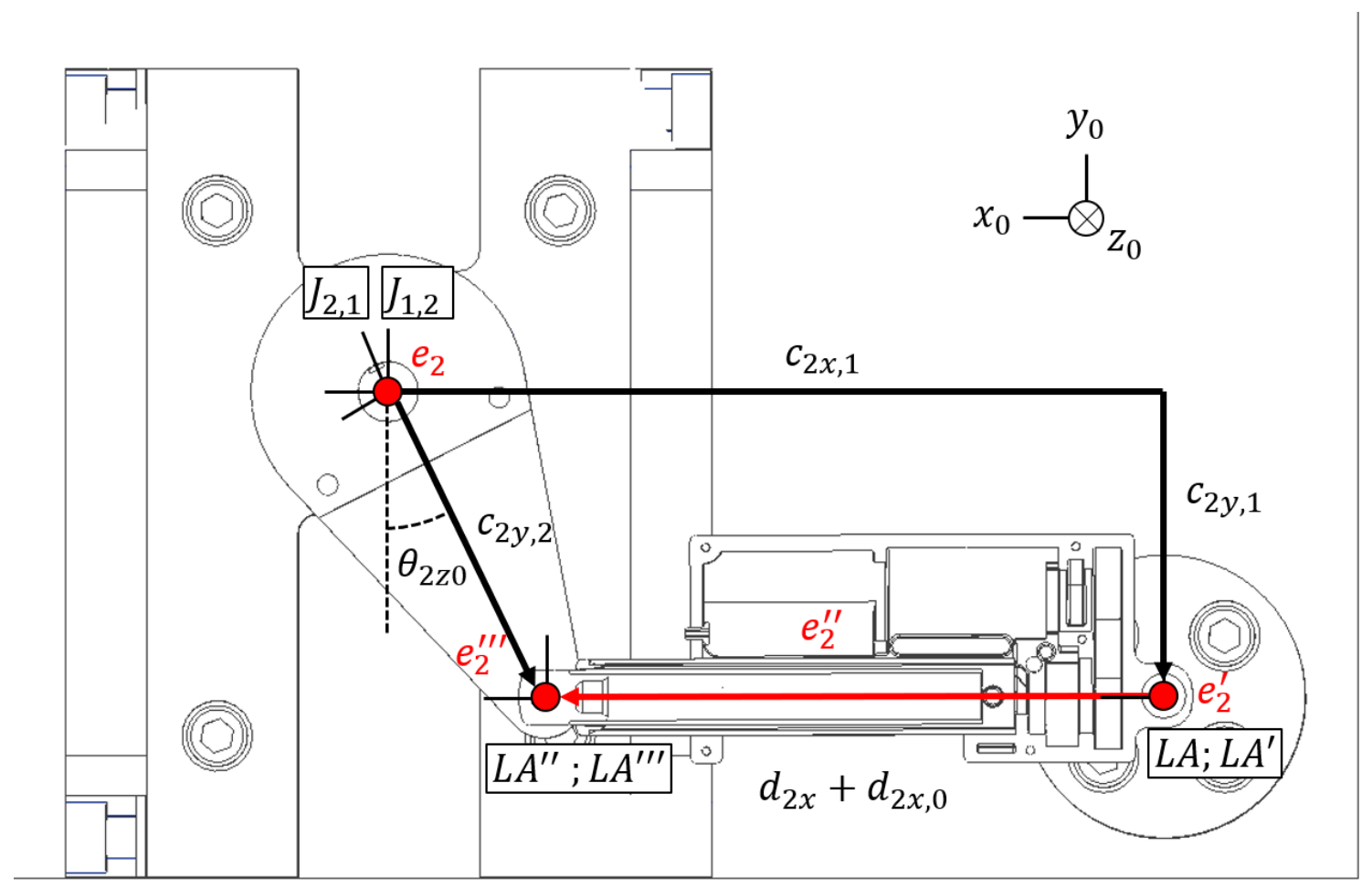

Figure 4.8: Base actuator mechanism.

The above transformations define a kinematic chain that connects the joint frame on the first body $J_{1,2}$ to the joint frame on the second body $J_{2,1}$ through the linear actuator joints $e_{2}^{\prime} e_{2}^{\prime \prime} e_{2}^{\prime \prime \prime}$. The kinematic chain is defined by the dual quaternion product of the above transformations in sequence while noting that the final transformation must be conjugated prior to multiplication. This relationship between the two joint frames was also expressed in Equation 4.14 in terms of the revolute joint variable. By equating the two equivalent expressions for the relationship between the frames, the loop closure is achieved and the forward kinematic and inverse kinematic problems may be solved from the resulting equation. 
Equating the transformation between frames $J_{1,2}$ and $J_{2,1}$ defined by the revolute joint $e_{2}$ to the same transformation defined by the parallel joints $e_{2}^{\prime} e_{2}^{\prime \prime} e_{2}^{\prime \prime \prime}$ yields

$$
J_{1,2} \hat{\mathbf{Q}}_{J_{2,1}}={ }^{J_{1,2}} \hat{\mathbf{Q}}_{L A}^{L A} \hat{\mathbf{Q}}_{L A^{\prime}}^{L A^{\prime}} \hat{\mathbf{Q}}_{L A^{\prime \prime}}^{L A^{\prime \prime}} \hat{\mathbf{Q}}_{L A^{\prime \prime \prime}}^{L A^{\prime \prime \prime}} \hat{\mathbf{Q}}_{J_{2,1}}
$$

where ${ }^{L A^{\prime \prime \prime}} \hat{\mathbf{Q}}_{J_{2,1}}={ }^{J_{2,1}} \hat{\mathbf{Q}}_{L A^{\prime \prime \prime}}^{*}$ is conjugated to reverse the direction of the transformation and complete the kinematic loop. Performing the dual quaternion multiplication on the right-hand-side, equating the components, and selecting the non-trivial/nonzero equations provides

$$
\begin{aligned}
\cos \left(\frac{\theta_{2 z}^{*}}{2}\right)= & \cos \left(\frac{\theta_{2 z}^{\prime}}{2}+\frac{\theta_{2 z}^{\prime \prime}}{2}\right) \\
\sin \left(\frac{\theta_{2 z}^{*}}{2}\right)= & \sin \left(\frac{\theta_{2 z}^{\prime}}{2}+\frac{\theta_{2 z}^{\prime \prime}}{2}\right) \\
0= & \left(d_{2 x}+d_{2 x, 0}\right) \cos \left(\frac{\theta_{2 z}^{\prime}}{2}-\frac{\theta_{2 z}^{\prime \prime}}{2}\right)-c_{2 x, 1} \cos \left(\frac{\theta_{2 z}^{\prime}}{2}+\frac{\theta_{2 z}^{\prime \prime}}{2}\right) \\
& -c_{2 y, 1} \sin \left(\frac{\theta_{2 z}^{\prime}}{2}+\frac{\theta_{2 z}^{\prime \prime}}{2}\right)-c_{2 y, 2} \sin \left(\frac{\theta_{2 z}^{\prime}}{2}+\frac{\theta_{2 z}^{\prime \prime}}{2}\right) \\
0= & c_{2 y, 2} \cos \left(\frac{\theta_{2 z}^{\prime}}{2}+\frac{\theta_{2 z}^{\prime \prime}}{2}\right)-c_{2 y, 1} \cos \left(\frac{\theta_{2 z}^{\prime}}{2}+\frac{\theta_{2 z}^{\prime \prime}}{2}\right) \\
& +c_{2 x, 1} \sin \left(\frac{\theta_{2 z}^{\prime}}{2}+\frac{\theta_{2 z}^{\prime \prime}}{2}\right)+\left(d_{2 x}+d_{2 x, 0}\right) \sin \left(\frac{\theta_{2 z}^{\prime}}{2}-\frac{\theta_{2 z}^{\prime \prime}}{2}\right),
\end{aligned}
$$

where Equations 4.23 and 4.24 originate from the real component of the dual quaternions and show closure of the orientation loop, whereas Equations 4.25 and 4.26 come from the dual components of the dual quaternions and represent closure of the displacement vector loop. Equations 4.23 and 4.24 give the relationship $\theta_{2 z}^{*}=\theta_{2 z}^{\prime}+\theta_{2 z}^{\prime \prime}$, which may be substituted into Equations 4.25 and 4.26. 
Performing the substitution isolating the $\left(d_{2 x}+d_{2 x, 0}\right)$ terms yields

$$
\begin{gathered}
\left(d_{2 x}+d_{2 x, 0}\right) \cos \left(\frac{\theta_{2 z}^{\prime}}{2}-\frac{\theta_{2 z}^{\prime \prime}}{2}\right)=c_{2 y, 2} \sin \left(\frac{\theta_{2 z}^{*}}{2}\right)+c_{2 y, 1} \sin \left(\frac{\theta_{2 z}^{*}}{2}\right)+c_{2 x, 1} \cos \left(\frac{\theta_{2 z}^{*}}{2}\right) \\
-\left(d_{2 x}+d_{2 x, 0}\right) \sin \left(\frac{\theta_{2 z}^{\prime}}{2}-\frac{\theta_{2 z}^{\prime \prime}}{2}\right)=c_{2 y, 2} \cos \left(\frac{\theta_{2 z}^{*}}{2}\right)-c_{2 y, 1} \cos \left(\frac{\theta_{2 z}^{*}}{2}\right)+c_{2 x, 1} \sin \left(\frac{\theta_{2 z}^{*}}{2}\right)
\end{gathered}
$$

which allows the solution to proceed similar to a planar four-bar analysis. Taking the square of both sides of Equations 4.27 and 4.28 and adding the resultant equations yields a relationship where the two intermediate angles $\theta_{2 z}^{\prime}$ and $\theta_{2 z}^{\prime \prime}$ are eliminated and only the constant offsets, the joint variable, and the actuator length remain,

$$
\left(d_{2 x}+d_{2 x, 0}\right)^{2}=c_{2 x, 1}^{2}+c_{2 y, 1}^{2}+c_{2 y, 2}^{2}+2 \sin \left(\theta_{2 z}^{*}\right) c_{2 x, 1} c_{2 y, 2}-2 \cos \left(\theta_{2 z}^{*}\right) c_{2 y, 1} c_{2 y, 2} .
$$

The inverse kinematic problem of determining the actuator extended length $d_{2 x}$ in terms of the joint variable $\theta_{2 z}^{*}$ is solved directly from Equation 4.29 such that

$$
d_{2 x}=\sqrt{c_{2 x, 1}^{2}+c_{2 y, 1}^{2}+c_{2 y, 2}^{2}+2 \sin \left(\theta_{2 z}^{*}\right) c_{2 x, 1} c_{2 y, 2}-2 \cos \left(\theta_{2 z}^{*}\right) c_{2 y, 1} c_{2 y, 2}}-d_{2 x, 0} .
$$

The forward kinematic problem requires the joint variable in terms of the actuator length, which may also be solved from Equation 4.29 by first applying the following half-angle identities,

$$
\begin{aligned}
\sin (\theta) & =\frac{2 \tan \left(\frac{\theta}{2}\right)}{1+\tan ^{2}\left(\frac{\theta}{2}\right)}, \\
\cos (\theta) & =\frac{1-\tan ^{2}\left(\frac{\theta}{2}\right)}{1+\tan ^{2}\left(\frac{\theta}{2}\right)} .
\end{aligned}
$$


Using these half-angle identities, Equation 4.29 takes a quadratic form in terms of $\tan \left(\frac{\theta_{2 z}^{*}}{2}\right)$ and the solution for the joint angle $\theta_{2 z}^{*}$ in terms of the extended actuator length $d_{2 x}$ is found with the quadratic equation as

$$
\begin{aligned}
\theta_{2 z}^{*} & =2 \operatorname{atan}_{2}\left(\frac{-B \pm \sqrt{B^{2}-4 A C}}{2 A}\right) \\
A & =K_{1}+K_{3} \\
B & =2 K_{2} \\
C & =K_{1}-K_{3} \\
K_{1} & =-\left(d_{2 x}+d_{2 x, 0}\right)^{2}+c_{2 x, 1}^{2}+c_{2 y, 1}^{2}+c_{2 y, 2}^{2} \\
K_{2} & =2 c_{2 x, 1} c_{2 y, 2} \\
K_{3} & =2 c_{2 y, 1} c_{2 y, 2},
\end{aligned}
$$

where values $K_{1}, K_{2}, K_{3}, A, B, C$, are defined to simplify the expression and the $\operatorname{atan}_{2}$ is the four-quadrant arctangent function. The constants defined in this analysis may be directly measured from the CAD files of the test-scale knuckle boom crane as $d_{2 x, 0}=97 \mathrm{~mm}, c_{2 x, 1}=122 \mathrm{~mm}, c_{2 y, 1}=47.825 \mathrm{~mm}$, and $c_{2 y, 2}=53.975 \mathrm{~mm}$ where the reported precision is based on the actual dimensions specified within the CAD file. It is noted that substitution of these values into Equation 4.33-4.39 for the fully retracted position $d_{2 x}=0$ results in a home configuration where the base joint angle is $\theta_{2 z}^{*}=\theta_{2 z 0}=-27.6^{\circ}$.

In the following subsection, a similar process is applied to the boom actuator mechanism to solve its forward and inverse kinematic problems. 


\subsubsection{Boom Actuator Mechanism}

The revolute joint $e_{3}$, which defines the rotation of the boom relative to the tower of the crane, is shown in Figure 4.9 within the context of its parallel mechanism as described by the loop $e_{3} e_{3}^{\prime} e_{3}^{\prime \prime} e_{3}^{\prime \prime \prime}$ in Figure 4.6. The frames $L A-L A^{\prime \prime \prime}$ are assigned as with the base actuator mechanism, and rigid offsets define the $y$ - and $z$-distances to $L A$ as $\left\{c_{3 y, 1}, c_{3 z, 1}\right\}$ and to $L A^{\prime \prime \prime}$ as $\left\{c_{3 y, 2}, c_{3 z, 2}\right\}$ with respect to $J_{2,2}$ and $J_{3,1}$, respectively. In the configuration shown, $J_{3,1}$ has rotated about $J_{2,2}$ to a home position that is defined with a fully retracted actuator $d_{3 z}=0$ and a corresponding joint angle of $\theta_{3 x}^{*}=\theta_{3 x 0}$. Using the frames defined in Figure 4.9 as a representation of the parallel kinematic structure illustrated in Figure 4.6, the transformations may be defined as

$$
\begin{aligned}
{ }^{J_{2,2}} \hat{\mathbf{Q}}_{L A} & =1+0 \hat{\imath}+0 \hat{\jmath}+0 \hat{k}+\frac{\epsilon}{2}\left(0+0 \hat{\imath}-c_{3 y, 1} \hat{\jmath}+c_{3 z, 1} \hat{k}\right), \\
{ }^{L A} \hat{\mathbf{Q}}_{L A^{\prime}} & =\cos \left(\frac{\theta_{3 x}^{\prime}}{2}\right)+\sin \left(\frac{\theta_{3 x}^{\prime}}{2}\right) \hat{\imath}+0 \hat{\jmath}+0 \hat{k}+\frac{\epsilon}{2}(0+0 \hat{\imath}+0 \hat{\jmath}+0 \hat{k}), \\
{ }^{L A^{\prime}} \hat{\mathbf{Q}}_{L A^{\prime \prime}} & =1+0 \hat{\imath}+0 \hat{\jmath}+0 \hat{k}+\frac{\epsilon}{2}\left(0+0 \hat{\imath}+0 \hat{\jmath}+\left(d_{3 z}+d_{3 z, 0}\right) \hat{k}\right), \\
{ }^{L A^{\prime \prime}} \hat{\mathbf{Q}}_{L A^{\prime \prime \prime}} & =\cos \left(\frac{\theta_{3 x}^{\prime \prime}}{2}\right)+\sin \left(\frac{\theta_{3 x}^{\prime \prime}}{2}\right) \hat{\imath}+0 \hat{\jmath}+0 \hat{k}+\frac{\epsilon}{2}(0+0 \hat{\imath}+0 \hat{\jmath}+0 \hat{k}), \\
{ }^{J_{3,1}} \hat{\mathbf{Q}}_{L A} & =1+0 \hat{\imath}+0 \hat{\jmath}+0 \hat{k}+\frac{\epsilon}{2}\left(0+0 \hat{\imath}-c_{3 y, 2} \hat{\jmath}+c_{3 z, 2} \hat{k}\right) .
\end{aligned}
$$

The inverse kinematic problem is solved by expressing the extended actuator length $d_{3 z}$ in terms of the joint variable $\theta_{3 x}^{*}$. Forming the transformation loop and applying the method from the previous subsection yields

$$
d_{3 z}=\sqrt{c_{3 y, 1}^{2}+c_{3 y, 2}^{2}+c_{3 z, 1}^{2}+c_{3 z, 2}^{2}+K_{2} \sin \left(\theta_{3 x}^{*}\right)-K_{3} \cos \left(\theta_{3 x}^{*}\right)}-d_{3 z, 0} .
$$




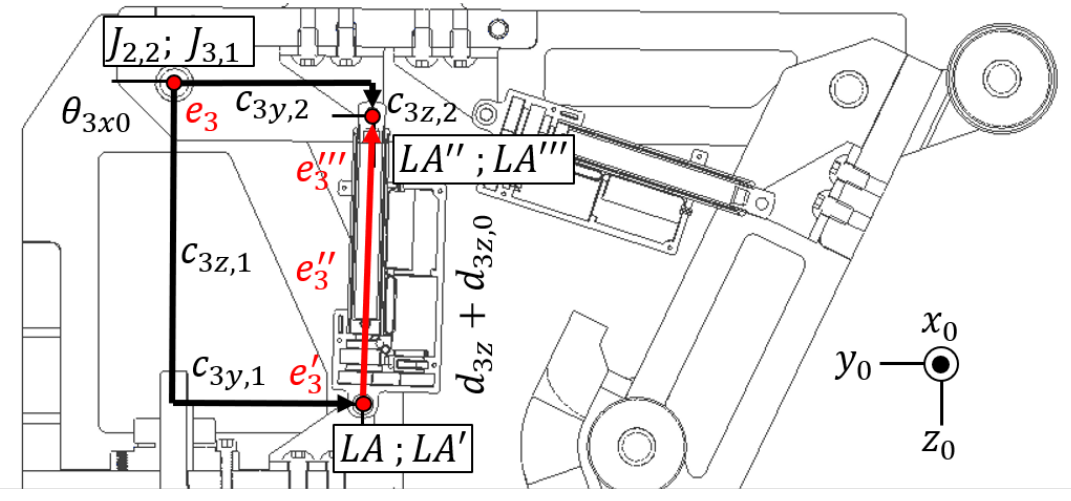

Figure 4.9: Boom actuator mechanism.

The constants $K_{2}$ and $K_{3}$ are defined during the forward kinematic solution, which expresses the joint variable $\theta_{3 x}^{*}$ in terms of the extended actuator length $d_{3 z}$ as

$$
\begin{aligned}
\theta_{3 x}^{*} & =2 \operatorname{atan}_{2}\left(\frac{-B \pm \sqrt{B^{2}-4 A C}}{2 A}\right) \\
A & =K_{1}+K_{3} \\
B & =2 K_{2} \\
C & =K_{1}-K_{3} \\
K_{1} & =-\left(d_{3 z}+d_{3 z, 0}\right)^{2}+c_{3 y, 1}^{2}+c_{3 y, 2}^{2}+c_{3 z, 1}^{2}+c_{3 z, 2}^{2} \\
K_{2} & =2\left(c_{3 y, 2} c_{3 z, 1}-c_{3 y, 1} c_{3 z, 2}\right) \\
K_{3} & =2\left(c_{3 y, 1} c_{3 y, 2}+c_{3 z, 1} c_{3 z, 2}\right) .
\end{aligned}
$$

The constants defined in this subsection are reported with the precision of the CAD file as $d_{3 z, 0}=97 \mathrm{~mm}, c_{3 y, 1}=62.865 \mathrm{~mm}, c_{3 z, 1}=107.315 \mathrm{~mm}, c_{3 y, 2}=66.04 \mathrm{~mm}$, and $c_{3 z, 2}=9.525 \mathrm{~mm}$. It is noted that substitution of these values into Equation 4.46 for a fully retracted position $d_{3 z}=0$ results in a home configuration with a boom joint angle of $\theta_{3 x}^{*}=\theta_{3 x 0}=-0.73^{\circ}$. In the following subsection, this process is applied once more to the jib actuator mechanism. 


\subsubsection{Jib Actuator Mechanism}

The revolute joint $e_{4}$, which defines the rotation of the jib relative to the boom of the crane, is shown in Figure 4.10 within the context of its parallel mechanism as described by the loop $e_{4} e_{4}^{\prime} e_{4}^{\prime \prime} e_{4}^{\prime \prime \prime}$ in Figure 4.6. The frames $L A-L A^{\prime \prime \prime}$ are assigned as with the boom actuator mechanism, and rigid offsets define the $y$ - and $z$-distances to $L A$ as $\left\{c_{4 y, 1}, c_{4 z, 1}\right\}$ and to $L A^{\prime \prime \prime}$ as $\left\{c_{4 y, 2}, c_{4 z, 2}\right\}$ with respect to $J_{3,2}$ and $J_{4,1}$, respectively. In the configuration shown, $J_{4,1}$ has rotated about $J_{3,2}$ to a home position that is defined with a fully retracted actuator $d_{4 z}=0$ and a corresponding joint angle of $\theta_{4 x}^{*}=\theta_{4 x 0}$. Using the frames defined in Figure 4.10 as a representation of the parallel kinematic structure illustrated in Figure 4.6, the transformations may be defined as

$$
\begin{aligned}
{ }^{J, 2} \hat{\mathbf{Q}}_{L A} & =1+0 \hat{\imath}+0 \hat{\jmath}+0 \hat{k}+\frac{\epsilon}{2}\left(0+0 \hat{\imath}+c_{4 y, 1} \hat{\jmath}+c_{4 z, 1} \hat{k}\right), \\
{ }^{L A} \hat{\mathbf{Q}}_{L A^{\prime}} & =\cos \left(\frac{\theta_{4 x}^{\prime}}{2}\right)+\sin \left(\frac{\theta_{4 x}^{\prime}}{2}\right) \hat{\imath}+0 \hat{\jmath}+0 \hat{k}+\frac{\epsilon}{2}(0+0 \hat{\imath}+0 \hat{\jmath}+0 \hat{k}), \\
{ }^{L A^{\prime}} \hat{\mathbf{Q}}_{L A^{\prime \prime}} & =1+0 \hat{\imath}+0 \hat{\jmath}+0 \hat{k}+\frac{\epsilon}{2}\left(0+0 \hat{\imath}+0 \hat{\jmath}+\left(d_{4 z}+d_{4 z, 0}\right) \hat{k}\right), \\
{ }^{L A^{\prime \prime}} \hat{\mathbf{Q}}_{L A^{\prime \prime \prime}} & =\cos \left(\frac{\theta_{4 x}^{\prime \prime}}{2}\right)+\sin \left(\frac{\theta_{4 x}^{\prime \prime}}{2}\right) \hat{\imath}+0 \hat{\jmath}+0 \hat{k}+\frac{\epsilon}{2}(0+0 \hat{\imath}+0 \hat{\jmath}+0 \hat{k}), \\
{ }^{J_{4,1}} \hat{\mathbf{Q}}_{L A} & =1+0 \hat{\imath}+0 \hat{\jmath}+0 \hat{k}+\frac{\epsilon}{2}\left(0+0 \hat{\imath}-c_{4 y, 2} \hat{\jmath}+c_{4 z, 2} \hat{k}\right) .
\end{aligned}
$$

The inverse kinematic problem is solved by expressing the extended actuator length $d_{4 z}$ in terms of the joint variable $\theta_{4 x}^{*}$. Forming the transformation loop and applying the method from the previous subsection yields

$$
d_{4 z}=\sqrt{c_{4 y, 1}^{2}+c_{4 y, 2}^{2}+c_{4 z, 1}^{2}+c_{4 z, 2}^{2}+K_{2} \sin \left(\theta_{4 x}^{*}\right)-K_{3} \cos \left(\theta_{4 x}^{*}\right)}-d_{4 z, 0} .
$$




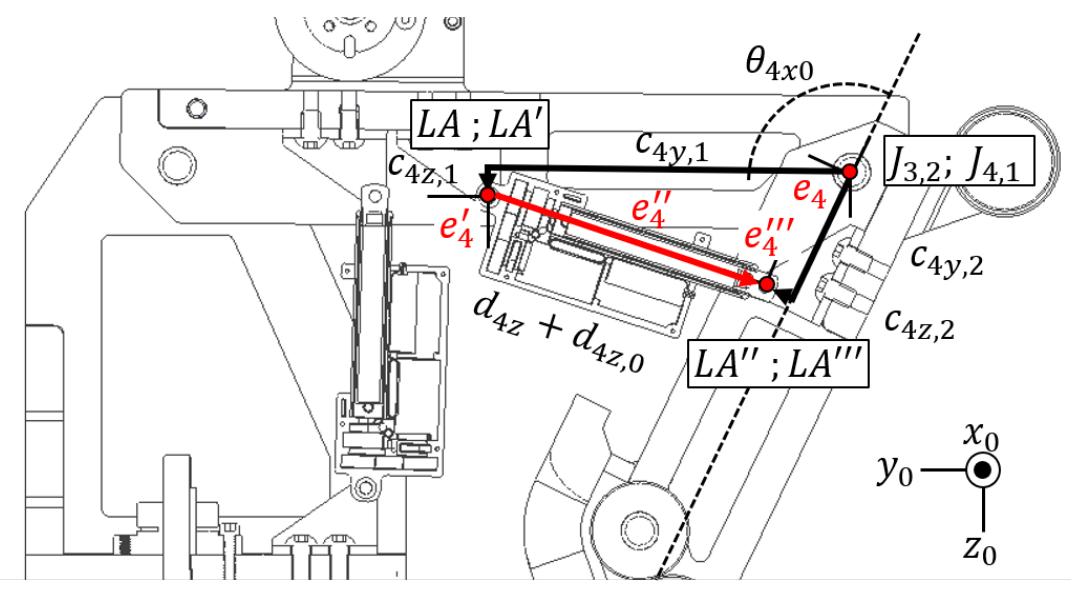

Figure 4.10: Jib actuator mechanism.

The constants $K_{2}$ and $K_{3}$ are defined during the forward kinematic solution, which expresses the joint variable $\theta_{4 x}^{*}$ in terms of the extended actuator length $d_{4 z}$ as

$$
\begin{aligned}
\theta_{4 x}^{*} & =2 \operatorname{atan}_{2}\left(\frac{-B \pm \sqrt{B^{2}-4 A C}}{2 A}\right) \\
A & =K_{1}+K_{3} \\
B & =2 K_{2} \\
C & =K_{1}-K_{3} \\
K_{1} & =-\left(d_{4 z}+d_{4 z, 0}\right)^{2}+c_{4 y, 1}^{2}+c_{4 y, 2}^{2}+c_{4 z, 1}^{2}+c_{4 z, 2}^{2} \\
K_{2} & =2\left(c_{4 y, 2} c_{4 z, 1}+c_{4 y, 1} c_{4 z, 2}\right) \\
K_{3} & =2\left(-c_{4 y, 1} c_{4 y, 2}-+c_{4 z, 1} c_{4 z, 2}\right) .
\end{aligned}
$$

The constants defined in this subsection are reported with the precision of the CAD file as $d_{4 z, 0}=97 \mathrm{~mm}, c_{4 y, 1}=120.8532 \mathrm{~mm}, c_{4 z, 1}=9.525 \mathrm{~mm}, c_{4 y, 2}=46.99 \mathrm{~mm}$, and $c_{4 z, 2}=9.525 \mathrm{~mm}$. It is noted that substitution of these values into Equation 4.59 for a fully retracted position $d_{4 z}=0$ results in a home configuration with a jib joint angle of $\theta_{4 x}^{*}=\theta_{4 x, 0}=-115^{\circ}$. In the following subsection, the forward and inverse kinematics of the full crane are analysed. 


\subsubsection{Forward and Inverse Kinematics of the Full Knuckle Boom Crane}

The forward kinematic problem is defined as finding the crane tip position $\left\{x_{t}, y_{t}, z_{t}\right\}$ based on the joint variables $\left\{\theta_{2 z}^{*}, \theta_{3 x}^{*}, \theta_{4 x}^{*}\right\}$, whereas the inverse kinematic problem is defined as finding the joint variables $\left\{\theta_{2 z}^{*}, \theta_{3 x}^{*}, \theta_{4 x}^{*}\right\}$ based on desired values for the crane tip position $\left\{x_{t}, y_{t}, z_{t}\right\}$.

The pose of any frame on the $i^{\text {th }}$ body is defined relative to the body frame $B_{i}$ indicated in Figure 4.7 using only linear displacements to simplify the resulting transformations. To further simplify the transformations, the exact locations of the body frames are not considered for the current kinematic analysis. Instead of defining the exact locations of the body frames $B_{2}-B_{5}$, the transformations between joint frames are considered as the current kinematic analysis only requires knowledge of the crane tip location at $J_{4,2}$ relative to the motion platform frame at $J_{1,2}$. The forward kinematic problem from Equation 4.6 is reformulated to define the pose of the crane tip frame relative to the ship deck as a dual quaternion transformation ${ }_{1,2}^{\mathbf{Q}_{J_{4,2}}}$ such that

$$
{ }^{J_{1,2}} \hat{\mathbf{Q}}_{J_{4,2}}=\prod_{i=2}^{4}\left({ }^{J_{i-1,2}} \hat{\mathbf{Q}}_{J_{i, 1}}^{J_{i, 1}} \hat{\mathbf{Q}}_{J_{i, 2}}\right)
$$

where ${ }^{J_{i-1,2}} \hat{\mathbf{Q}}_{J_{i, 1}}$ are the previously defined transformations corresponding to each of the revolute joints. The remaining dual quaternions ${ }^{J_{i, 1}} \hat{\mathbf{Q}}_{J_{i, 2}}$ are rigid transforms that define the relative pose of the joint frames on each body as

$$
\begin{aligned}
& { }^{J_{2,1}} \hat{\mathbf{Q}}_{J_{2,2}}=1+0 \hat{\imath}+0 \hat{\jmath}+0 \hat{k}+\frac{\epsilon}{2}\left(0+0 \hat{\imath}+0 \hat{\jmath}-l_{2 z} \hat{k}\right), \\
& { }^{J_{3,1}} \hat{\mathbf{Q}}_{J_{3,2}}=1+0 \hat{\imath}+0 \hat{\jmath}+0 \hat{k}+\frac{\epsilon}{2}\left(0+0 \hat{\imath}-l_{3 y} \hat{\jmath}+0 \hat{k}\right), \\
& { }^{J_{4,1}} \hat{\mathbf{Q}}_{J_{4,2}}=1+0 \hat{\imath}+0 \hat{\jmath}+0 \hat{k}+\frac{\epsilon}{2}\left(0+0 \hat{\imath}-l_{4 y} \hat{\jmath}+0 \hat{k}\right),
\end{aligned}
$$


where the $\pm l_{i a}$ terms define the distance between the joint frames on the $i^{\text {th }}$ body as measured along the local $a$-axis with signs inherited from the direction of the corresponding axis.

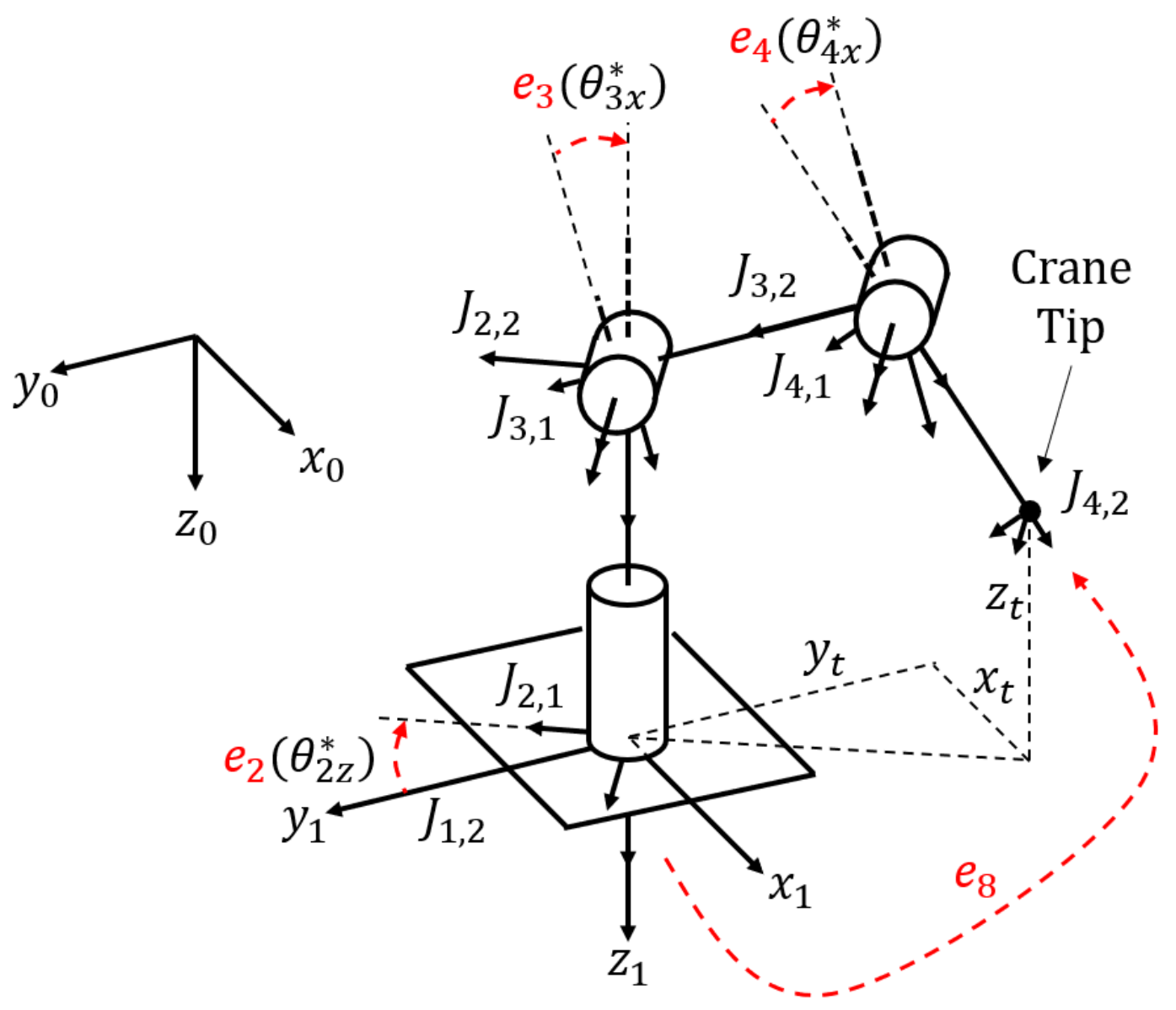

Figure 4.11: Knuckle boom crane forward and inverse kinematic problems.

The 6-DOF virtual joint from the deck to the crane tip that was defined by $e_{8}$ may be used to represent the pose of the tip frame relative to the base frame as a dual quaternion ${ }^{J_{1,2}} \hat{\mathbf{Q}}_{J_{4,2}}$ that is the concatenation of a pure rotation and a pure translation such that

$$
{ }^{J_{1,2}} \hat{\mathbf{Q}}_{J_{4,2}}={ }^{J_{1,2}} \hat{\mathbf{Q}}_{D, J_{4,2}}{ }^{J_{1,2}} \hat{\mathbf{Q}}_{R, J_{4,2}},
$$


where the pure rotation component is a real quaternion that is represented by ${ }^{J_{1,2}} \hat{\mathbf{Q}}_{R, J_{4,2}}$, and the pure translation component is an identity real quaternion combined with a dual quaternion represented by ${ }^{J_{1,2}} \hat{\mathbf{Q}}_{R, J_{4,2}}$. The pure translation component defines a new translating coordinate frame with axes that are aligned with the $J_{1,2}$ frame and an origin that is coincident with the origin of the $J_{4,2}$ frame. To solve the forward and inverse kinematic problems a transformation loop is formed using the above transformation for $e_{8}$ and an equivalent transformation for $e_{2} e_{3} e_{4}$. The equivalent transformation ${ }^{J_{1,2}} \hat{\mathbf{Q}}_{J_{4,2}}$ for $e_{2} e_{3} e_{4}$ is defined by substituting the dual quaternion terms into Equation 4.66 as

$$
\begin{aligned}
J_{1,2} \hat{\mathbf{Q}}_{J_{4,2}}= & \cos \left(\frac{\theta_{2 z}^{*}}{2}\right) \cos \left(\frac{\theta_{3 x}^{*}}{2}+\frac{\theta_{4 x}^{*}}{2}\right)+\hat{\imath} \cos \left(\frac{\theta_{2 z}^{*}}{2}\right) \sin \left(\frac{\theta_{3 x}^{*}}{2}+\frac{\theta_{4 x}^{*}}{2}\right)+ \\
& \hat{\jmath} \sin \left(\frac{\theta_{2 z}^{*}}{2}\right) \sin \left(\frac{\theta_{3 x}^{*}}{2}+\frac{\theta_{4 x}^{*}}{2}\right)+\hat{k} \sin \left(\frac{\theta_{2 z}^{*}}{2}\right) \cos \left(\frac{\theta_{3 x}^{*}}{2}+\frac{\theta_{4 x}^{*}}{2}\right)+ \\
& \frac{\epsilon}{2} \sin \left(\frac{\theta_{2 z}^{*}}{2}\right)\left(l_{2 z} c_{3+4}+l_{3 y} \sin \left(\frac{\theta_{3 x}^{*}}{2}-\frac{\theta_{4 x}^{*}}{2}\right)+l_{4 y} s_{3+4}\right)+ \\
& \frac{\epsilon \hat{\imath}}{2} \sin \left(\frac{\theta_{2 z}^{*}}{2}\right)\left(l_{3 y} \cos \left(\frac{\theta_{3 x}^{*}}{2}-\frac{\theta_{4 x}^{*}}{2}\right)+l_{4 y} c_{3+4}+l_{2 z} s_{3+4}\right)- \\
& \frac{\epsilon \hat{\jmath}}{2} \cos \left(\frac{\theta_{2 z}^{*}}{2}\right)\left(l_{3 y} \cos \left(\frac{\theta_{3 x}^{*}}{2}-\frac{\theta_{4 x}^{*}}{2}\right)+l_{4 y} c_{3+4}+l_{2 z} s_{3+4}\right)- \\
& \frac{\epsilon \hat{k}}{2} \cos \left(\frac{\theta_{2 z}^{*}}{2}\right)\left(l_{2 z} c_{3+4}+l_{3 y} \sin \left(\frac{\theta_{3 x}^{*}}{2}-\frac{\theta_{4 x}^{*}}{2}\right)+l_{4 y} s_{3+4}\right)
\end{aligned}
$$

where $c_{3+4}=\cos \left(\frac{\theta_{3 x}^{*}}{2}+\frac{\theta_{4 x}^{*}}{2}\right)$ and $s_{3+4}=\sin \left(\frac{\theta_{3 x}^{*}}{2}+\frac{\theta_{4 x}^{*}}{2}\right)$ are used to simplify the expression. 
The transformation loop is completed by setting Equation 4.70 equal to Equation 4.71. The transformation loop contains information regarding both the orientation and position of the crane tip frame relative to the base. However, the current kinematic analysis is concerned only with defining the relationship between the position of the crane tip and the joint variables. The position information may be extracted through right multiplying by the conjugate of the real component of the transformation. Performing the multiplication to the transformation loop and substituting the translations $x_{t}, y_{t}, z_{t}$ into the translation quaternion ${ }^{J_{1,2}} \hat{\mathbf{Q}}_{D, J_{4,2}}$ of the virtual 6-DOF joint yields

$$
\begin{aligned}
{ }_{J_{1,2}} \hat{\mathbf{Q}}_{D, J_{4,2}} & =1+0 \hat{\imath}+0 \hat{\jmath}+0 \hat{k}+\frac{\epsilon}{2}\left(+0+x_{t} \hat{\imath}+y_{t} \hat{\jmath}+z_{t} \hat{k}\right) \\
x_{t} & =\sin \left(\theta_{2 z}^{*}\right)\left(l_{4 y} \cos \left(\theta_{3 x}^{*}+\theta_{4 x}^{*}\right)+l_{3 y} \cos \left(\theta_{3 x}^{*}\right)\right) \\
y_{t} & =-\cos \left(\theta_{2 z}^{*}\right)\left(l_{4 y} \cos \left(\theta_{3 x}^{*}+\theta_{4 x}^{*}\right)+l_{3 y} \cos \left(\theta_{3 x}^{*}\right)\right) \\
z_{t} & =-l_{2 z}-l_{4 y} \sin \left(\theta_{3 x}^{*}+\theta_{4 x}^{*}\right)-l_{3 y} \sin \left(\theta_{3 x}^{*}\right),
\end{aligned}
$$

where Equations 4.73-4.75 define the crane tip position as a function of the joint variables and thereby solve the forward kinematic problem.

The forward kinematic solution is also in a convenient form for solving the inverse kinematic problem, which expresses the joint variables in terms of the crane tip position as is necessary for Cartesian control. In the following, a desired position of the crane tip relative to its base frame is selected by substituting $\left\{x_{t}, y_{t}, z_{t}\right\}=$ $\left\{x_{t d}, y_{t d}, z_{t d}\right\}$ into Equations 4.73-4.75. Squaring and adding Equations 4.73 and 4.74 yields eliminates $\theta_{2 z}^{*}$ from the resultant equation as

$$
\sqrt{x_{t d}^{2}+y_{t d}^{2}}=l_{4 y} \cos \left(\theta_{3 x}^{*}+\theta_{4 x}^{*}\right)+l_{3 y} \cos \left(\theta_{3 x}^{*}\right) .
$$


Substituting the result back into Equations 4.73 and 4.74, rearranging to isolate the sine and cosine terms, and then dividing the resulting equations provides an expression for the tangent of $\theta_{2 z}^{*}$ as

$$
\tan \left(\theta_{2 z}^{*}\right)=\frac{\sin \left(\theta_{2 z}^{*}\right)}{\cos \left(\theta_{2 z}^{*}\right)}=\frac{x_{t d}}{-y_{t d}}
$$

such that the base revolute joint variable $\theta_{2 z}^{*}$ may be directly solved by taking the arctangent. The solution for $\theta_{2 z}^{*}$ represents the first inverse kinematic equation of the crane as

$$
\theta_{2 z}^{*}=\operatorname{atan}_{2}\left(\frac{x_{t d}}{-y_{t d}}\right)
$$

where the $\operatorname{atan}_{2}$ function is used to return a quadrant-corrected angle.

Returning to Equations 4.75 and 4.76 , isolating the terms with $\left(\theta_{3 x}^{*}+\theta_{4 x}^{*}\right)$, squaring the equations and adding the result yields an expression solely in terms of constant offsets and the boom revolute joint variable $\theta_{3 x}^{*}$ as

$x_{t d}^{2}+y_{t d}^{2}+2 l_{2 z} z_{t d}+l_{3 y}^{2}+l_{2 z}^{2}+z_{t d}^{2}+2\left(l_{3 y} l_{2 z}+l_{3 y} z_{t d}\right) \sin \left(\theta_{3 x}^{*}\right)-2 l_{3 y} \sqrt{\left(x_{t d}^{2}+y_{t d}^{2}\right)} \cos \left(\theta_{3 x}^{*}\right)=l_{4 y}^{2}$

which may be solved by applying the half angle identities and following the same process used to solve the forward kinematics of the planar four-bar actuator mechanisms such that, 


$$
\begin{aligned}
\theta_{3 x}^{*} & =2 \operatorname{atan}_{2}\left(\frac{-B \pm \sqrt{B^{2}-4 A C}}{2 A}\right) \\
A & =K_{1}+K_{3} \\
B & =2 K_{2} \\
C & =K_{1}-K_{3} \\
K_{1} & =x_{t d}^{2}+y_{t d}^{2}+2 l_{2 z} z_{t d}+l_{3 y}^{2}+l_{2 z}^{2}+z_{t d}^{2}-l_{4 y}^{2} \\
K_{2} & =2\left(l_{3 y} l_{2 z}+l_{3 y} z_{t d}\right) \\
K_{3} & =2 l_{3 y} \sqrt{\left(x_{t d}^{2}+y_{t d}^{2}\right)} .
\end{aligned}
$$

Equations 4.80-4.86 define the second set of inverse kinematic equations for the main serial structure of the crane.

The final revolute joint angle $\theta_{4 x}^{*}$, which represents the rotation of the jib relative to the boom, may be found in terms of $\theta_{3 x}^{*}$ by isolating the $\left(\theta_{3 x}^{*}+\theta_{4 x}^{*}\right)$ terms in Equations 4.75 and 4.76. Division of the resulting equations yields an expression for the tangent of the sum of the angles as

$$
\tan \left(\theta_{3 x}^{*}+\theta_{4 x}^{*}\right)=\frac{l_{4 y} \sin \left(\theta_{3 x}^{*}+\theta_{4 x}^{*}\right)}{l_{4 y} \cos \left(\theta_{3 x}^{*}+\theta_{4 x}^{*}\right)}=\frac{-z_{t d}-l_{2 z}-l_{3 y} \sin \left(\theta_{3 x}^{*}\right)}{\sqrt{x_{t d}^{2}+y_{t d}^{2}}-l_{3 y} \cos \left(\theta_{3 x}^{*}\right)} .
$$

Equation 4.87 provides the third inverse kinematic equation and the solution for the jib revolute joint angle $\theta_{4 x}^{*}$ as

$$
\theta_{4 x}^{*}=\operatorname{atan}_{2}\left(\frac{-z_{t d}-l_{2 z}-l_{3 y} \sin \left(\theta_{3 x}^{*}\right)}{\sqrt{x_{t d}^{2}+y_{t d}^{2}}-l_{3 y} \cos \left(\theta_{3 x}^{*}\right)}\right)-\theta_{3 x}^{*}
$$

The kinematic models are summarized and confirmed in the following subsections. 


\subsubsection{Summary of Kinematic Models}

In summary, several forward kinematic models were derived in this section, including:

- the base actuator model as defined in Equations 4.33-4.39;

- the boom actuator model as defined in Equations 4.46-4.52;

- the jib actuator model as defined in Equations 4.59-4.65; and

- the knuckle boom crane model as defined in Equations 4.73-4.75.

Several inverse kinematic models were also derived in this section, including:

- the base actuator model as defined in Equation 4.30;

- the boom actuator model as defined in Equation 4.45;

- the jib actuator model as defined in Equation 4.58; and

- the knuckle boom crane model as defined in Equations 4.78, 4.80-4.86, and 4.88.

The method applied to derive the models was essentially an extension of the planar vector loop analysis with complex numbers to a three-dimensional loop analysis with dual quaternion transformations. In summary, the procedure applied was to:

1. Separate the mechanism into $n$ groups of rigidly connected bodies or links by disconnecting them at the $J$ joints and labelling them from $n_{0}$ to $n_{n-1}$.

2. Label the $j^{\text {th }}$ joint as $e_{j}$, and assign an aligned pair of reference frames at the connection points of the joint on each of the two joined bodies. 
3. Define joint variables $\theta_{j a}^{*}$ and/or $d_{j a}^{*}$ for each joint to relate the reference frame on the first joined body to the reference frame on the second joined body. The joint variables are labelled with the joint number $j$ and the $a$ axis about/along which they operate. If applicable, define a transformation sequence with intermediate frames for joints with multiple variables.

4. Express the joint transformations as a set of dual quaternions in terms of the joint variables $\theta_{j a}^{*}$ and/or $d_{j a}^{*}$.

5. Express the relationships between joint frames on the same rigid body as a set of dual quaternions in terms of constant offsets $c$ or link lengths $l$.

6. If the kinematic structure contains a loop, define the inverse kinematic problem as specifying the input variable in terms of the output variable, and define the forward kinematic problem as specifying the output variable in terms of the input variable.

7. If the kinematic structure does not contain a loop, assign a virtual joint with up to six degrees of freedom to define a closed loop for the inverse kinematic and/or forward kinematic problem. The virtual joint is selected with enough degrees of freedom to avoid introducing additional constraints or altering the mobility of the system by matching the degrees of freedom of the motion space that the mechanism operates within.

8. Form the dual quaternion loop equations by equating transformation sequences connecting identical base and follower reference frames.

An approach based on transformation loops within kinematic structures was applied throughout the kinematic analysis to form an intuitive representation of joint 
and frame transformations. Dual quaternions were used to represent the transformations as they provide a convenient and compact expression for the pose of one frame relative to another.

In the following subsection, the kinematic analysis of this chapter is concluded and confirmed by comparing the output of each model to the output of similar models developed within existing multibody software. 


\subsubsection{Confirmation of Kinematic Models}

To confirm the forward kinematic models, function blocks containing the mathematical models from the previous subsections are implemented in parallel with a Simscape model that is provided in Appendix D. The actuator lengths are specified with kinematic set-points ranging from $0-50 \mathrm{~mm}$ with $5 \mathrm{~mm}$ increments to generate a 3 dimensional array that is representative of the entire workspace of the crane tip. The corresponding tip locations are shown as blue dots in Figure 4.12 relative to the base frame $J_{1,2}$. For each set-point, the forward kinematic equations are evaluated and recorded. The root-mean-squared-difference (RMSD) for the entire dataset relative to the Simscape model output is recorded in the upper half of Table 4.5. The results indicate a negligible difference that may be attributed to numerical error, which confirms the forward kinematic models from the previous subsections.

To confirm the inverse kinematic models, the set-points are specified as the Cartesian crane tip locations indicated by the blue dots in Figure 4.12. The models are evaluated in parallel, and the RMSD for the inverse kinematic model dataset relative to that of the Simscape model is recorded in Table 4.5. The results indicate a negligible difference that may be attributed to numerical error, which confirms the inverse kinematic models from the previous subsections. In the next section, a dy-

namic model of the pendulum load is developed for use in the control algorithm of Chapter 6. 


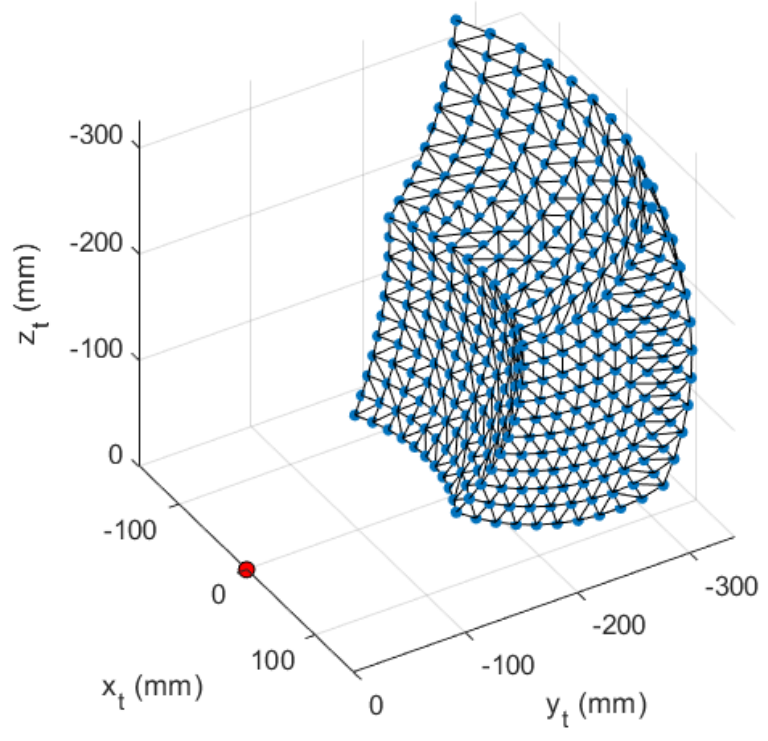

Figure 4.12: Model evaluation points in the crane's workspace.

Table 4.5: Summary of kinematic model differences for the knuckle boom crane.

\begin{tabular}{|c|c|c|}
\hline Model Reference & Variable Estimated & RMSD \\
\hline Equation 4.33-4.39 & $\theta_{2 z}$ & $1.4612 \mathrm{e}-14 \mathrm{deg}$ \\
Equation 4.46-4.52 & $\theta_{3 x}$ & $2.2466 \mathrm{e}-14 \mathrm{deg}$ \\
Equation 4.59-4.65 & $\theta_{4 x}$ & $4.3850 \mathrm{e}-14 \mathrm{deg}$ \\
Equation 4.73 & $x_{t}$ & $1.1206 \mathrm{e}-14 \mathrm{~mm}$ \\
Equation 4.74 & $y_{t}$ & $3.7701 \mathrm{e}-14 \mathrm{~mm}$ \\
Equation 4.75 & $z_{t}$ & $4.0647 \mathrm{e}-14 \mathrm{~mm}$ \\
\hline Equation 4.30 & $d_{2 x}$ & $1.3838 \mathrm{e}-14 \mathrm{~mm}$ \\
Equation 4.45 & $d_{3 z}$ & $3.8886 \mathrm{e}-14 \mathrm{~mm}$ \\
Equation 4.58 & $d_{4 z}$ & $3.4227 \mathrm{e}-14 \mathrm{~mm}$ \\
Equation 4.78 & $\theta_{2 z}$ & $4.3987 \mathrm{e}-15 \mathrm{deg}$ \\
Equation 4.80-4.86 & $\theta_{3 x}$ & $1.8351 \mathrm{e}-14 \mathrm{deg}$ \\
Equation 4.88 & $\theta_{4 x}$ & $3.9581 \mathrm{e}-14 \mathrm{deg}$ \\
\hline
\end{tabular}




\subsection{Multibody Dynamics of the Pendulum Load}

\subsubsection{Derivation with Lagrangian Mechanics}

In this section, a multibody dynamic model of a variable length spherical pendulum with a three-degree-of-freedom trolley is developed with Lagrangian mechanics. The schematic of the system is illustrated in Figure 4.13, and the corresponding dynamic model will be used for the control system in Chapter 6. The mass of the pendulum load is defined as $m_{p}$, the mass of the trolley is defined as $m_{t}$, and the cable mass is considered negligible for the current research. The trolley is used to represent the position of the crane tip, which is given relative to the world frame as $\left\{x_{t}, y_{t}, z_{t}\right\}$. The joint angles are taken relative to the translating coordinate frame and are defined simply as $\theta_{x}$ and $\theta_{y}$. The distance from the crane tip to the centre of mass of the pendulum is defined as $l_{p}$, and the load is considered a point mass for simplicity.

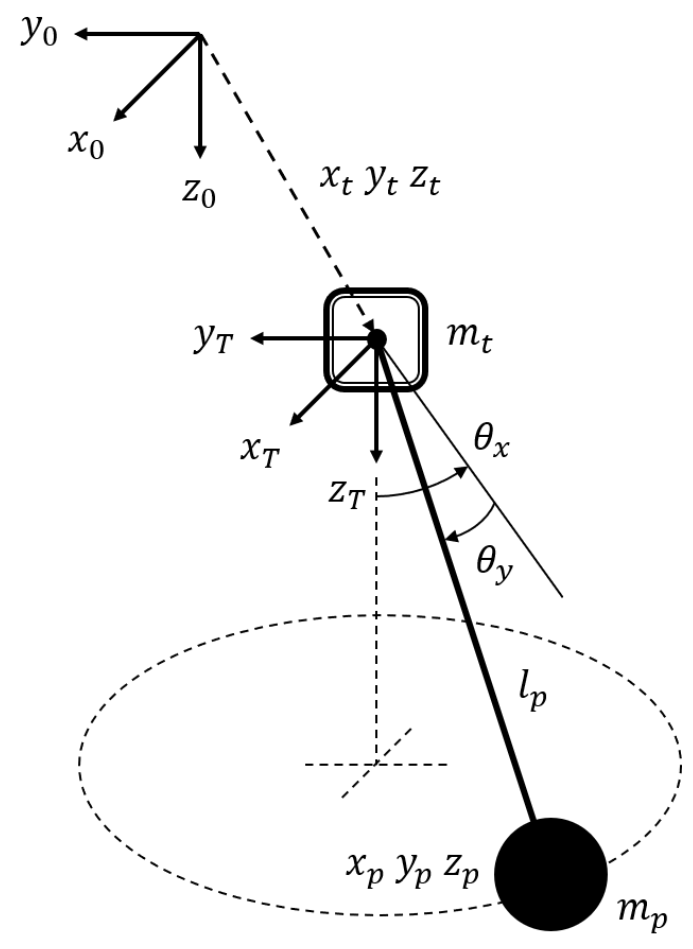

Figure 4.13: Diagram of the variable length spherical pendulum. 
To determine the position of the pendulum mass relative to the world frame, a dual quaternion transformation sequence is defined. The transformation sequence relates the pendulum frame $P$ to the world frame $W_{0}$, and is defined as

$$
{ }^{W_{0}} \hat{\mathbf{Q}}_{P}={ }^{W_{0}} \hat{\mathbf{Q}}_{T}^{T} \hat{\mathbf{Q}}_{U_{X}}^{U_{X}} \hat{\mathbf{Q}}_{U_{Y}}^{U_{Y}} \hat{\mathbf{Q}}_{P}
$$

where the intermediate frames $T, U_{X}$, and $U_{Y}$ are introduced to represent a translating frame attached to the crane tip, a tip frame that has rotated through the $\theta_{x}$ angle, and a frame that has rotated through both the $\theta_{x}$ and $\theta_{y}$ angles of the pendulum, respectively. The transformations between the five frames are defined as

$$
\begin{aligned}
{ }^{W_{0}} \hat{\mathbf{Q}}_{T} & =1+0 \hat{\imath}+0 \hat{\jmath}+0 \hat{k}+\frac{\epsilon}{2}\left(0+x_{t} \hat{\imath}+y_{t} \hat{\jmath}+z_{t} \hat{k}\right), \\
{ }^{T} \hat{\mathbf{Q}}_{U_{X}} & =\cos \left(\frac{\theta_{x}}{2}\right)+\sin \left(\frac{\theta_{x}}{2}\right) \hat{\imath}+0 \hat{\jmath}+0 \hat{k}+\frac{\epsilon}{2}(0+0 \hat{\imath}+0 \hat{\jmath}+0 \hat{k}), \\
{ }^{U_{X}} \hat{\mathbf{Q}}_{U_{Y}} & =\cos \left(\frac{\theta_{y}}{2}\right)+0 \hat{\imath}+\sin \left(\frac{\theta_{y}}{2}\right) \hat{\jmath}+0 \hat{k}+\frac{\epsilon}{2}(0+0 \hat{\imath}+0 \hat{\jmath}+0 \hat{k}), \\
{ }^{U_{Y}} \hat{\mathbf{Q}}_{P} & =1+0 \hat{\imath}+0 \hat{\jmath}+0 \hat{k}+\frac{\epsilon}{2}\left(0+0 \hat{\imath}+0 \hat{\jmath}+l_{p} \hat{k}\right),
\end{aligned}
$$

where the linear and angular displacements correspond to those shown in Figure 4.13. Performing the multiplication and extracting the displacement information yields the position of the pendulum relative to the world frame as

$$
\begin{aligned}
& x_{p}=x_{t}+l_{p} \sin \left(\theta_{y}\right), \\
& y_{p}=y_{t}-l_{p} \cos \left(\theta_{y}\right) \sin \left(\theta_{x}\right), \\
& z_{p}=z_{t}+l_{p} \cos \left(\theta_{y}\right) \cos \left(\theta_{x}\right),
\end{aligned}
$$

which, for the simplistic pendulum-trolley system, may be confirmed through trigonometric projections of the pendulum length. 
To derive a set of dynamic equations that represent the multibody system, the Lagrangian is formed as

$$
L=E_{K}-E_{P}
$$

where $E_{K}$ is the total kinetic energy of the system, and $E_{P}$ is the total potential energy of the system. Assuming a datum at the origin of the world frame, the potential energy may be expressed directly from the $z$-coordinates of the trolley and pendulum. Considering the North-East-Down configuration of the world frame, the potential energy of the system may be expressed as

$$
E_{P}=-m_{t} g z_{t}-m_{p} g z_{p}
$$

where $g$ is the Earth's gravitational constant. The trolley velocities may be directly expressed as $\left\{\dot{x}_{t}, \dot{y}_{t}, \dot{z}_{t}\right\}$, whereas the pendulum velocities may be expressed as $\left\{\dot{x}_{p}, \dot{y}_{p}, \dot{z}_{p}\right\}$ by differentiating Equations $4.94-4.96$ with respect to time. The kinetic energy of the system may be expressed in terms of these velocities as

$$
E_{K}=\frac{1}{2} m_{t}\left({\dot{x_{t}}}^{2}+{\dot{y_{t}}}^{2}+\dot{z}_{t}^{2}\right)+\frac{1}{2} m_{p}\left({\dot{x_{p}}}^{2}+{\dot{y_{p}}}^{2}+\dot{z}_{p}^{2}\right)
$$

which, in combination with the potential energy from Equation 4.98, completes the information required to form the Lagrangian as defined in Equation 4.97. 
The $i^{\text {th }}$ equation of motion is derived from the Lagrangian using vectors of generalized coordinates $\xi$ and forces/torques $\tau$ as

$$
\begin{aligned}
\frac{d}{d t}\left(\frac{\partial L}{\partial \dot{\xi}_{i}}\right) & -\frac{\partial L}{\partial \xi_{i}}=\tau_{i}, \\
{\left[\xi_{1}, \xi_{2}, \xi_{3}, \xi_{4}, \xi_{5}, \xi_{6}\right]^{T} } & =\left[x_{t}, y_{t}, z_{t}, \theta_{x}, \theta_{y}, l_{p}\right]^{T}, \\
{\left[\tau_{1}, \tau_{2}, \tau_{3}, \tau_{4}, \tau_{5}, \tau_{6}\right]^{T} } & =\left[F_{x_{t}}, F_{y_{t}}, F_{z_{t}}, T_{\theta_{x}}, T_{\theta_{y}}, F_{l_{p}}\right]^{T},
\end{aligned}
$$

where $\xi_{i}$ represents the $i^{t h}$ generalized coordinate, and $\tau_{i}$ represents the $i^{t h}$ generalized force/torque applied at the corresponding coordinate. Evaluating Equation 4.100 for $i=\{1,2,3,4,5,6\}$ provides the equations of motion as

$$
\begin{aligned}
& F_{x_{t}}=-m_{p} l_{p} s_{y} \dot{\theta}_{y}{ }^{2}+2 m_{p} \dot{l}_{p} c_{y} \dot{\theta_{y}}+\left(m_{t}+m_{p}\right) \ddot{x_{t}}+m_{p} \ddot{l_{p}} s_{y}+m_{p} l_{p} \ddot{\theta_{y}} c_{y}, \\
& F_{y_{t}}=m_{p} l_{p} c_{y} s_{x} \dot{\theta}_{x}^{2}+2 m_{p} l_{p} c_{x} s_{y} \dot{\theta}_{x} \dot{\theta}_{y}-2 m_{p} \dot{l}_{p} c_{x} c_{y} \dot{\theta}_{x}+m_{p} l_{p} c_{y} s_{x} \dot{\theta}_{y}{ }^{2}+ \\
& 2 m_{p} \dot{l}_{p} s_{x} s_{y} \dot{\theta}_{y}+\left(m_{t}+m_{p}\right) \ddot{y}_{t}-m_{p} \ddot{l}_{p} c_{y} s_{x}+m_{p} l_{p} \ddot{\theta}_{y} s_{x} s_{y}-m_{p} l_{p} \ddot{\theta}_{x} c_{x} c_{y}, \\
& F_{z t}=-m_{p} l_{p} c_{x} c_{y} \dot{\theta}_{x}{ }^{2}+2 m_{p} l_{p} s_{x} s_{y} \dot{\theta_{x}} \dot{\theta_{y}}-2 m_{p} \dot{l}_{p} c_{y} s_{x} \dot{\theta_{x}}-m_{p} l_{p} c_{x} c_{y} \dot{\theta}_{y}{ }^{2}- \\
& 2 m_{p} \dot{l}_{p} c_{x} s_{y} \dot{\theta}_{y}+\left(m_{t}+m_{p}\right)\left(\ddot{z}_{t}-g\right)+m_{p} \ddot{l}_{p} c_{x} c_{y}-m_{p} l_{p} \ddot{\theta}_{x} c_{y} s_{x}-m_{p} l_{p} \ddot{\theta}_{y} c_{x} s_{y}, \\
& T_{\theta_{x}}=m_{p} l_{p} c_{y} g s_{x}-m_{p} l_{p} c_{y} \ddot{y}_{t} c_{x}-m_{p} l_{p} c_{y} \ddot{z}_{t} s_{x}+m_{p} l_{p}^{2} c_{y} \ddot{\theta}_{x} c_{y}+ \\
& 2 m_{p} l_{p} c_{y} \dot{l}_{p} \dot{\theta}_{x} c_{y}-2 m_{p} l_{p}^{2} c_{y} \dot{\theta}_{x} \dot{\theta}_{y} s_{y} \\
& T_{\theta_{y}}=m_{p} l_{p}^{2} c_{y} s_{y} \dot{\theta}_{x}^{2}+m_{p} l_{p}^{2} \ddot{\theta}{ }_{y}+2 m_{p} l_{p} \dot{l}_{p} \dot{\theta}_{y}+m_{p} l_{p} \ddot{x}_{t} c_{y}+m_{p} l_{p} g c_{x} s_{y}- \\
& m_{p} l_{p} \ddot{z}_{t} c_{x} s_{y}+m_{p} l_{p} \ddot{y}_{t} s_{x} s_{y} \\
& F_{l_{p}}=-m_{p} l_{p} \dot{\theta}_{y}^{2}+m_{p} \ddot{l}_{p}+m_{p} \ddot{x}_{t} s_{y}-m_{p} g c_{x} c_{y}+m_{p} \ddot{z}_{t} c_{x} c_{y}- \\
& m_{p} \ddot{y}_{t} c_{y} s_{x}-m_{p} l_{p} \dot{\theta}_{x}^{2} c_{y}^{2},
\end{aligned}
$$

where the terms $c_{x}=\cos \left(\theta_{x}\right), s_{x}=\sin \left(\theta_{x}\right), c_{y}=\cos \left(\theta_{y}\right)$, and $s_{y}=\sin \left(\theta_{y}\right)$ are substituted to simplify the equations. 
Returning to the generalized notation, the equations of motion may be expressed in a standard matrix-vector form as

$$
M(\xi) \ddot{\xi}+B(\xi, \dot{\xi}) \dot{\xi}+G(\xi)=\tau
$$

where $\xi$ and $\tau$ are the previously defined $6 \times 1$ vectors of generalized coordinates and forces, $M(\xi)$ is the $6 \times 6$ positive definite and symmetric inertia matrix, the resultant of $B(\xi, \dot{\xi}) \dot{\xi}$ is a vector of the Coriolis and centrifugal terms which is represented here as the product of a $6 \times 6$ matrix and the generalized velocities, and $G(\xi)$ is a $6 \times 1$ vector of the gravitational forces and torques. The matrix/vector definitions of these terms are provided in Appendix D. It is noted that the matrix defined by $(\dot{M}-2 B)$ is skew symmetric and the system is linear in parameters, which is similar to the work of [61] and may be useful for nonlinear adaptive force control in future work.

In the above system, the inputs are included within the generalized forces $\tau$. However, for the current thesis, the desired inputs are the crane tip motion and the varying pendulum length. To change the form of the inputs, holonomic constraints are applied using Lagrange multipliers. A set of four algebraic constraint equations are added to the matrix-vector form as

$$
\begin{aligned}
M(\xi) \ddot{\xi}+B(\xi, \dot{\xi}) \dot{\xi}+G(\xi) & =\tau-\Lambda_{\xi}^{T} \lambda, \\
\Lambda(\xi, u) & =0,
\end{aligned}
$$

where $\lambda=\left[\lambda_{1}, \lambda_{2}, \lambda_{3}, \lambda_{4}\right]^{T}$ is a $4 \times 1$ vector of Lagrange multipliers, and $\Lambda_{\xi}$ is the partial derivative of the constraint matrix $\Lambda(\xi, u)$ with respect to the coordinates $\xi$. 
The constraint equations are defined within the $4 \times 6$ constraint matrix as

$$
\Lambda(\xi, u)=\left[\begin{array}{cccccc}
x_{t}-u_{x_{t}} & 0 & 0 & 0 & 0 & 0 \\
0 & y_{t}-u_{y_{t}} & 0 & 0 & 0 & 0 \\
0 & 0 & z_{t}-u_{z_{t}} & 0 & 0 & 0 \\
0 & 0 & 0 & 0 & 0 & l_{p}-u_{l_{p}}
\end{array}\right]
$$

where $u=\left[u_{x_{t}}, u_{y_{t}}, u_{z_{t}}, 0,0, u_{l_{p}}\right]^{T}$ is a $6 \times 1$ vector of the time-varying kinematic inputs. The zero elements of the input vector correspond to the unactuated/unconstrained degrees of freedom $\theta_{x}$ and $\theta_{y}$. In the current form, Equations 4.110 and 4.111 represent a set of second-order differential algebraic equations (DAEs). To reduce the system to a set of ordinary differential equations (ODEs), the kinematic constraints are expressed in terms of acceleration by taking time derivatives of the multi-variable function as

$$
\begin{aligned}
& \dot{\Lambda}(\dot{\xi}, \dot{u})=\frac{d}{d t} \Lambda(\xi, u)=\frac{\partial \Lambda}{\partial \xi} \frac{d \xi}{d t}+\frac{\partial \Lambda}{\partial u} \frac{d u}{d t}=\Lambda_{\xi} \dot{\xi}+\Lambda_{u} \dot{u}, \\
& \ddot{\Lambda}(\ddot{\xi}, \ddot{u})=\frac{d}{d t} \dot{\Lambda}(\dot{\xi}, \dot{u})=\frac{\partial \dot{\Lambda}}{\partial \dot{\xi}} \frac{d \dot{\xi}}{d t}+\frac{\partial \dot{\Lambda}}{\partial \dot{u}} \frac{d \dot{u}}{d t}=\Lambda_{\xi} \ddot{\xi}+\Lambda_{u} \ddot{u}
\end{aligned}
$$

where it is noted that $\Lambda_{u}=-\Lambda_{\xi}$ due to the form of the applied constraints. In the current research, no other external force inputs are considered, so Equation 4.110 may be restated with $\tau=0$ and the constraints expressed in terms of acceleration as

$$
\begin{aligned}
M(\xi) \ddot{\xi}+B(\xi, \dot{\xi}) \dot{\xi}+G(\xi)+\Lambda_{\xi}^{T} \lambda & =0, \\
\Lambda_{\xi} \ddot{\xi} & =-\Lambda_{u} \ddot{u},
\end{aligned}
$$


Solving Equation 4.115 for the generalized accelerations $\ddot{\xi}$, substituting the result into Equation 4.116, and solving for the Lagrange multipliers yields

$$
\lambda=\left(\Lambda_{\xi} M^{-1}(\xi) \Lambda_{\xi}^{T}\right)^{-1}\left(\Lambda_{u} \ddot{u}-\Lambda_{\xi} M^{-1}(\xi)(B(\xi, \dot{\xi}) \dot{\xi}+G(\xi))\right)
$$

where the $4 \times 4$ matrix $\Lambda_{\xi} M^{-1} \Lambda_{\xi}^{T}$ is invertible as the defined constraints are independent [62]. In future work, these Lagrange multipliers could be used to approximate and enforce limits on the constraint forces, including the cable tension.

Returning to Equation 4.115 and substituting the expression for the Lagrange multipliers while noting that $\Lambda_{u}=-\Lambda_{\xi}$ from the definition of the constraints yields a set of ordinary differential equations,

$$
M \ddot{\xi}+B \dot{\xi}+G-\Lambda_{\xi}^{T}\left(\Lambda_{\xi} M^{-1} \Lambda_{\xi}^{T}\right)^{-1} \Lambda_{\xi} M^{-1}(B \dot{\xi}+G)=\Lambda_{\xi}^{T}\left(\Lambda_{\xi} M^{-1} \Lambda_{\xi}^{T}\right)^{-1} \Lambda_{\xi} \ddot{u}
$$

where dependence on the generalized variables is omitted to simplify the expression. Solving the set of equations for the generalized acceleration $\ddot{\xi}$ yields

$$
\begin{gathered}
\ddot{\xi}=\Psi_{B G}(\xi, \dot{\xi})+\Psi_{\ddot{u}}(\xi, \dot{\xi}) \ddot{u}, \\
\Psi_{B G}(\xi, \dot{\xi})=\left[\begin{array}{c} 
\\
0 \\
0 \\
0 \\
\frac{-g s_{x}-2 c_{y} i_{p} \dot{\theta}_{x}+2 l_{p} s_{y} \dot{\theta}_{x} \dot{\theta}_{y}}{c_{y} l_{p}} \\
\frac{-c_{y} l_{p} s_{y} \dot{\theta}_{x}^{2}-2 i_{p} \dot{\theta}_{y}-c_{x} s_{y} g}{l_{p}} \\
0
\end{array}\right],
\end{gathered}
$$




$$
\Psi_{\ddot{u}}(\xi, \dot{\xi})=\left[\begin{array}{cccccc}
1 & 0 & 0 & 0 & 0 & 0 \\
0 & 1 & 0 & 0 & 0 & 0 \\
0 & 0 & 1 & 0 & 0 & 0 \\
0 & \frac{c_{x}}{c_{y} l_{p}} & \frac{s_{x}}{c_{y} l_{p}} & 0 & 0 & 0 \\
\frac{-c_{y}}{l_{p}} & \frac{-s_{x} s_{y}}{l_{p}} & \frac{c_{x} s_{y}}{l_{p}} & 0 & 0 & 0 \\
0 & 0 & 0 & 0 & 0 & 1
\end{array}\right],
$$

which represents a system of nonlinear second-order ordinary differential equations.

It is noted that Equations 4.119-4.121 may also be found directly from Equations $4.103-4.108$ by selecting constraint forces as

$$
\begin{aligned}
& F_{c, x_{t}}=-m_{p} l_{p} s_{y} \dot{\theta}_{y}^{2}+2 m_{p} \dot{l}_{p} c_{y} \dot{\theta}_{y}+\left(m_{t}+m_{p}\right) \ddot{u}_{x_{t}}+m_{p} \ddot{l}_{p} s_{y}+m_{p} l_{p} \ddot{\theta}_{y} c_{y}, \\
& F_{c, y_{t}}=m_{p} l_{p} c_{y} s_{x} \dot{\theta}_{x}^{2}+2 m_{p} l_{p} c_{x} s_{y} \dot{\theta}_{x} \dot{\theta}_{y}-2 m_{p} \dot{l}_{p} c_{x} c_{y} \dot{\theta}_{x}+m_{p} l_{p} c_{y} s_{x} \dot{\theta}_{y}^{2}+ \\
& 2 m_{p} \dot{l}_{p} s_{x} s_{y} \dot{\theta}_{y}+\left(m_{t}+m_{p}\right) \ddot{u}_{y_{t}}-m_{p} \ddot{l}_{p} c_{y} s_{x}+m_{p} l_{p} \ddot{\theta}_{y} s_{x} s_{y}-m_{p} l_{p} \ddot{\theta}_{x} c_{x} c_{y}, \\
& F_{c, z_{t}}=-m_{p} l_{p} c_{x} c_{y} \dot{\theta}_{x}^{2}+2 m_{p} l_{p} s_{x} s_{y} \dot{\theta}_{x} \dot{\theta}_{y}-2 m_{p} \dot{l}_{p} c_{y} s_{x} \dot{\theta}_{x}-m_{p} l_{p} c_{x} c_{y} \dot{\theta}_{y}^{2}- \\
& 2 m_{p} \dot{l}_{p} c_{x} s_{y} \dot{\theta}_{y}+\left(m_{t}+m_{p}\right)\left(\ddot{u}_{z_{t}}-g\right)+m_{p} \ddot{l}_{p} c_{x} c_{y}-m_{p} l_{p} \ddot{\theta}_{x} c_{y} s_{x}-m_{p} l_{p} \ddot{\theta}_{y} c_{x} s_{y}, \\
& T_{c, \theta_{x}}=0 \text { (unconstrained) } \\
& T_{c, \theta_{y}}=0 \text { (unconstrained) } \\
& F_{c, l_{p}}=-m_{p} l_{p} \dot{\theta}_{y}^{2}+m_{p} \ddot{u}_{l_{p}}+m_{p} \ddot{x}_{t} s_{y}-m_{p} g c_{x} c_{y}+m_{p} \ddot{z}_{t} c_{x} c_{y}- \\
& m_{p} \ddot{y}_{t} c_{y} s_{x}-m_{p} l_{p} \dot{\theta}_{x}^{2} c_{y}^{2}
\end{aligned}
$$

where the subscript ' $c$ ' is used to indicate constraint forces/torques. Substitution of the constraint forces/torques of Equations 4.122-4.127 as the applied forces/torques in Equations 4.103-4.108 provides 


$$
\begin{aligned}
\ddot{x}_{t} & =\ddot{u}_{x_{t}}, \\
\ddot{y}_{t} & =\ddot{u}_{y_{t}}, \\
\ddot{z}_{t} & =\ddot{u}_{z_{t}}, \\
\ddot{\theta_{x}} & =\left(\frac{-g s_{x}-2 c_{y} \dot{l_{p}} \dot{\theta}_{x}+2 l_{p} s_{y} \dot{\theta_{x}} \dot{\theta_{y}}}{c_{y} l_{p}}\right)+\left(\frac{\ddot{u}_{y_{t}} c_{x}+\ddot{u}_{z_{t}} s_{x}}{c_{y} l_{p}}\right), \\
\ddot{\theta_{y}} & =\left(\frac{-c_{y} l_{p} s_{y} \dot{\theta}_{x}^{2}-2 \dot{l_{p}} \dot{\theta_{y}}-c_{x} s_{y} g}{l_{p}}\right)+\left(\frac{-\ddot{u}_{x_{t}} c_{y}-\ddot{u}_{y_{t}} s_{x} s_{y}+\ddot{u}_{z_{t}} c_{x} s_{y}}{l_{p}}\right), \\
\ddot{l_{p}} & =\ddot{u}_{l_{p}},
\end{aligned}
$$

where Equations 4.128-4.133 are equivalent to the set of Equations 4.119-4.121 found by applying the constraints using Lagrange multipliers.

These equations may be rewritten as a system of nonlinear first-order ordinary differential equations by assigning state variables $x_{1}=\xi$ and $x_{2}=\dot{\xi}$, as well as an input vector of $\tilde{u}=\ddot{u}$. The nonlinear system of equations is given in a general form,

$$
\dot{x}=f(x, \tilde{u})
$$

where the functional dependence of the state velocity vector on the state and input vectors is defined by the expanded form as

$$
\left[\begin{array}{c}
\dot{x_{1}} \\
\dot{x_{2}}
\end{array}\right]=\left[\begin{array}{c}
x_{2} \\
\Psi_{B G}\left(x_{1}, x_{2}\right)
\end{array}\right]+\left[\begin{array}{c}
0 \\
\Psi_{\ddot{u}}\left(x_{1}, x_{2}\right)
\end{array}\right] \tilde{u},
$$

where $\Psi_{B G}$ and $\Psi_{\ddot{u}}$ are the previously defined matrices. Although the state evolution of the system is defined by Equations 4.134 and 4.135, the pendulum position is not explicitly defined by these equations. Instead, the pendulum position is defined as the system output within a set of nonlinear output equations. 
The nonlinear output equations define the pendulum position in the world frame as a function of the system states. The nonlinear output equations are given in a general form as

$$
y=g(x),
$$

where $y$ is the output vector. The functional dependence of the output vector on the state vector is defined by the expanded form as

$$
\left[\begin{array}{c}
x_{p} \\
y_{p} \\
z_{p}
\end{array}\right]=\left[\begin{array}{c}
x_{t}+l_{p} \sin \left(\theta_{y}\right) \\
y_{t}-l_{p} \cos \left(\theta_{y}\right) \sin \left(\theta_{x}\right) \\
z_{t}+l_{p} \cos \left(\theta_{x}\right) \cos \left(\theta_{y}\right)
\end{array}\right],
$$

where Equations 4.94-4.96 are substituted to define the pendulum position.

Although the above system of nonlinear equations is useful for simulation and nonlinear control in future work, the control algorithm described in Chapter 6 requires a linearised state-space model. The nonlinear equations may be approximated by a linear state-space model of the form

$$
\begin{aligned}
& \dot{x}=\tilde{A} x+\tilde{B} \tilde{u}, \\
& y=\tilde{C} x+\tilde{D} \tilde{u},
\end{aligned}
$$

where the system matrices $\{\tilde{A}, \tilde{B}, \tilde{C}, \tilde{D}\}$ are defined by evaluating Jacobian matrices at an operating point [63]. In the current work, the operating point is considered to be an equilibrium point of the form

$$
x_{e}=\left[\begin{array}{llllllllllll}
x_{t}^{*} & y_{t}^{*} & z_{t}^{*} & 0 & 0 & l_{p}^{*} & 0 & 0 & 0 & 0 & 0 & 0
\end{array}\right]^{T},
$$

where $x_{e i}^{*}$ indicates that any value of the state may define an equilibrium point. 
The first system matrix $\tilde{A}$ is defined by evaluating the Jacobian of Equation 4.134 with respect to the state vector as

$$
\tilde{A}=\left(\frac{\partial f}{\partial x}\right)_{x_{e}}=\left[\begin{array}{cccccccccccc}
0 & 0 & 0 & 0 & 0 & 0 & 1 & 0 & 0 & 0 & 0 & 0 \\
0 & 0 & 0 & 0 & 0 & 0 & 0 & 1 & 0 & 0 & 0 & 0 \\
0 & 0 & 0 & 0 & 0 & 0 & 0 & 0 & 1 & 0 & 0 & 0 \\
0 & 0 & 0 & 0 & 0 & 0 & 0 & 0 & 0 & 1 & 0 & 0 \\
0 & 0 & 0 & 0 & 0 & 0 & 0 & 0 & 0 & 0 & 1 & 0 \\
0 & 0 & 0 & 0 & 0 & 0 & 0 & 0 & 0 & 0 & 0 & 1 \\
0 & 0 & 0 & 0 & 0 & 0 & 0 & 0 & 0 & 0 & 0 & 0 \\
0 & 0 & 0 & 0 & 0 & 0 & 0 & 0 & 0 & 0 & 0 & 0 \\
0 & 0 & 0 & 0 & 0 & 0 & 0 & 0 & 0 & 0 & 0 & 0 \\
0 & 0 & 0 & \frac{-g}{l_{p}^{*}} & 0 & 0 & 0 & 0 & 0 & 0 & 0 & 0 \\
0 & 0 & 0 & 0 & \frac{-g}{l_{p}^{*}} & 0 & 0 & 0 & 0 & 0 & 0 & 0 \\
0 & 0 & 0 & 0 & 0 & 0 & 0 & 0 & 0 & 0 & 0 & 0
\end{array}\right]
$$

where dependence on the pendulum length is indicated by elements containing $l_{p}^{*}$. It is noted that the four non-zero eigenvalues of the form $s= \pm w_{n} \hat{\imath}$ correspond to the approximate natural frequencies of the spherical pendulum where $w_{n}=\sqrt{g / l_{p}^{*}}$, which indicates that the frequency response of the linear system may deteriorate as the length changes. Due to the dependence on the pendulum length, the Jacobian must be re-evaluated to linearise the system about a new operating point if significant changes in the length occur. 
The remaining system matrices $\{\tilde{B}, \tilde{C}, \tilde{D}\}$ are defined as

$$
\begin{aligned}
& \tilde{B}=\left(\frac{\partial f}{\partial \tilde{u}}\right)_{x_{e}}=\left[\begin{array}{cccccc}
0 & 0 & 0 & 0 & 0 & 0 \\
0 & 0 & 0 & 0 & 0 & 0 \\
0 & 0 & 0 & 0 & 0 & 0 \\
0 & 0 & 0 & 0 & 0 & 0 \\
0 & 0 & 0 & 0 & 0 & 0 \\
0 & 0 & 0 & 0 & 0 & 0 \\
1 & 0 & 0 & 0 & 0 & 0 \\
0 & 1 & 0 & 0 & 0 & 0 \\
0 & 0 & 1 & 0 & 0 & 0 \\
0 & \frac{1}{l_{p}^{*}} & 0 & 0 & 0 & 0 \\
\frac{-1}{l_{p}^{*}} & 0 & 0 & 0 & 0 & 0 \\
0 & 0 & 0 & 0 & 0 & 1
\end{array}\right] \text {, } \\
& \tilde{C}=\left(\frac{\partial g}{\partial x}\right)_{x_{e}}=\left[\begin{array}{cccccccccccc}
1 & 0 & 0 & 0 & l_{p}^{*} & 0 & 0 & 0 & 0 & 0 & 0 & 0 \\
0 & 1 & 0 & -l_{p}^{*} & 0 & 0 & 0 & 0 & 0 & 0 & 0 & 0 \\
0 & 0 & 1 & 0 & 0 & 1 & 0 & 0 & 0 & 0 & 0 & 0
\end{array}\right] \\
& \tilde{D}=\left(\frac{\partial g}{\partial \tilde{u}}\right)_{x_{e}}=0,
\end{aligned}
$$

where dependence on the pendulum length is indicated by elements containing $l_{p}^{*}$.

In this section, a nonlinear multibody dynamic model of a variable length spherical pendulum and trolley system was developed and linearised about an operating point. The model is applied in the control algorithm as described in Chapter 6. In the following subsection, the dynamic models are confirmed with an existing software package. 


\subsubsection{Confirmation of Dynamic Models}

In this subsection, the multibody dynamic models of the variable length spherical pendulum are confirmed with existing software. The derived nonlinear model is summarized by Equations 4.134-4.137, whereas the linearised model is summarized by Equations 4.138-4.144. To confirm the derivations, each model is implemented in parallel with a Simulink/Simscape model, and the system is allowed to run for 60 seconds with data logging at $10 \mathrm{msec}$ intervals for each test. The Simscape modelling software is used to verify the derivations as it is a widely-accepted industrial tool for dynamic modelling. The software automatically derives and solves the equations of motion based on a block diagram model. The difference between the derived model and the Simulink/Simscape model is quantified as a root-mean-squared difference (RMSD) of the euclidean distance between the derived model output and the Simulink/Simscape output relative to an 'expected' euclidean distance of zero. The mathematical models used during the confirmation are provided in Appendix D. The main components of the Simscape model are shown in Figure 4.14 with a Cartesian Joint to control the trolley/tip position relative to the world frame, a universal joint to allow the pendulum to rotate with two degrees of freedom, a prismatic joint to control the length of the pendulum, and a mass to act as the load.

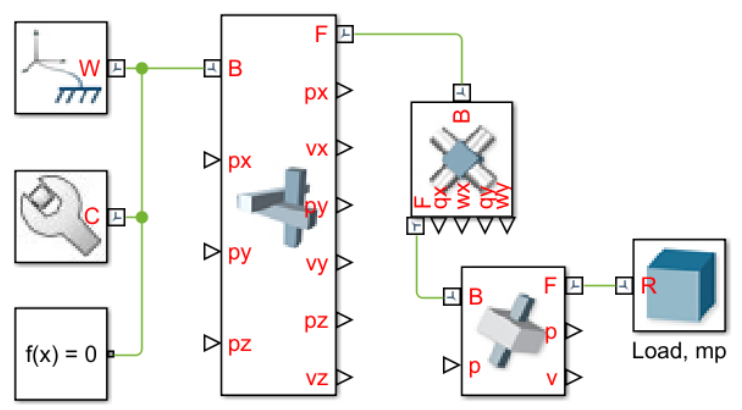

Figure 4.14: Simscape model of the variable length spherical pendulum used to confirm the dynamic analysis. 
The first test evaluates the free response of the pendulum for various initial conditions. For this test, the trolley is held at a position of $x_{t}=y_{t}=z_{t}=0$ and the length of the pendulum is held at its initial length of $l_{p}=250 \mathrm{~mm}$ by specifying zeros for the acceleration inputs. The initial pendulum angles, $\theta_{x}(0)$ and $\theta_{y}(0)$, are varied from $-20^{\circ}$ to $20^{\circ}$ and the model outputs for each 60 second test period are compared to the Simscape model. The results are summarized for the nonlinear model in Figure 4.15a and for the linearised model in Figure 4.15b where the $x$ - and $y$-axis indicate the initial angles, whereas the $z$-axis indicates the RMSD value. The nonlinear model differences may be attributed to numerical error and are considered negligible. The linear model differences, however, are mainly due to a small angle assumption implicitly applied through the linearisation process. The RMSD of the linear model is recorded as less than $5 \mathrm{~mm}$ for a deviation of $\pm 5^{\circ}$, whereas the differences increase substantially to approximately $120 \mathrm{~mm}$ as the initial angles approach $\pm 20^{\circ}$. In the current research, the assumption is made that the pendulum angles will remain small with respect to the world frame such that the resulting error does not greatly impact the motion compensation efforts. As the motion compensation algorithm is intended to reduce the undesired pendulum motion, the angles should remain small during normal operation. 


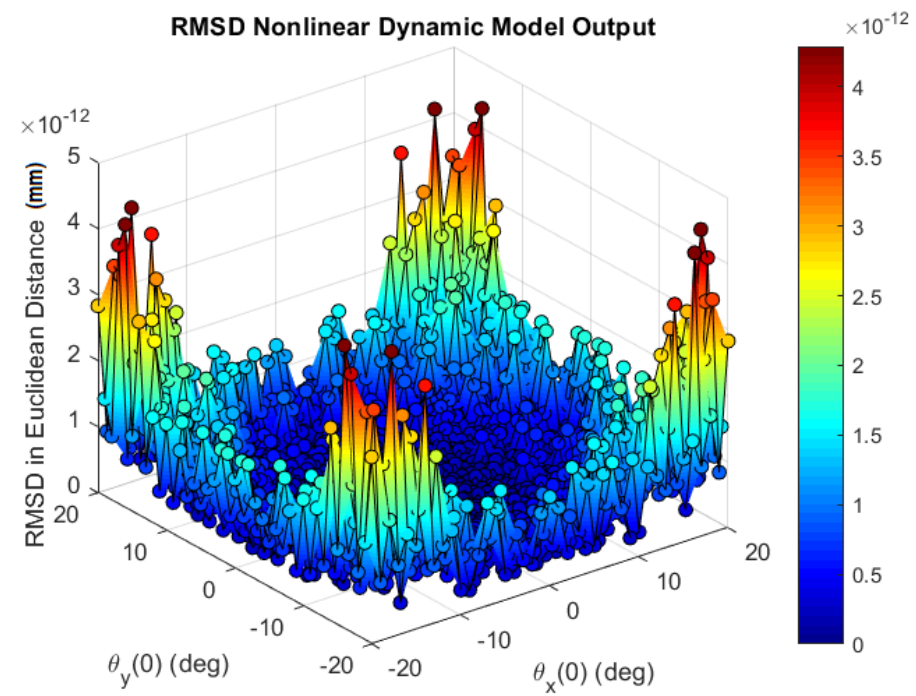

(a) Nonlinear RMSD

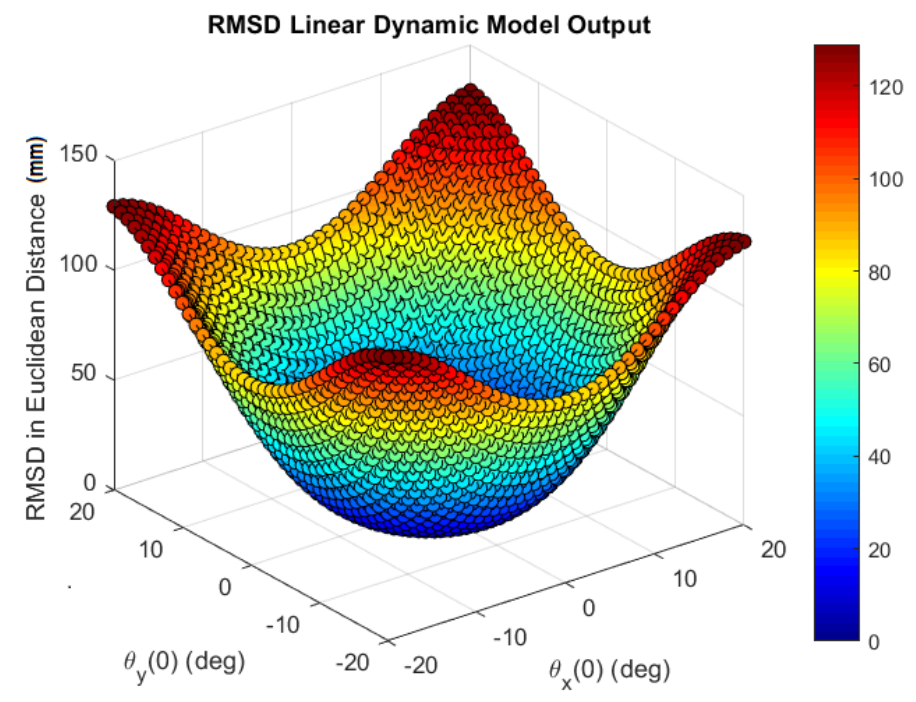

(b) Linear RMSD

Figure 4.15: Differences in the free response of each dynamic model caused by varying initial angles. 
The second test evaluates the effect of inaccurate linear system matrices due to changes in cable length that occur after linearisation. To analyse the effect of using an inaccurate length in the linearised model, the system matrices is initialized with a length of $l_{p}^{*}=250 \mathrm{~mm}$ and the initial condition of $l_{p}(0)=l_{p}^{*}+\Delta l_{p}$ is set for the actual length at the start of the simulation. For each trial, the trolley position is fixed at $x_{t}=y_{t}=z_{t}=0$, the initial condition for the secondary pendulum angle is $\theta_{y}(0)=0^{\circ}$, and zeros are specified for all acceleration inputs. The pendulum length is specified by varying $\Delta l_{p}$ from -200 to $200 \mathrm{~mm}$, and the initial condition for the primary pendulum angle $\theta_{x}(0)$ is varied from $-20^{\circ}$ to $20^{\circ}$. The model outputs for each 60 second test period are compared to the Simscape model. The results are summarized for the nonlinear model in Figure 4.16a and for the linearised model in Figure 4.16b where the $x$-axis indicates the initial angle, the $y$-axis indicates the variation in initial $\Delta l_{p}$ with respect to the linearised length $l_{p}^{*}=250 \mathrm{~mm}$, and the $z$-axis indicates the RMSD value. Again, the nonlinear model differences are considered negligible. The linear model differences, however, show that an inaccurate length may substantially impact the accuracy of the model for large angles. When the correct length is specified with $\Delta l_{p}=0$, the previously discussed small angle approximation causes the RMSD to reach $10 \mathrm{~mm}$ for an initial angle of $\theta_{x}(0)=-10^{\circ}$. However, for a slight variation in the cable length, this RMSD increases by a factor of approximately four. For smaller angles, the variations in cable length are less significant as the majority of cases show RMSD values under $15 \mathrm{~mm}$ for initial angles within the $\theta_{x}(0)= \pm 5^{\circ}$ range. To mitigate the effects of variations in length when performing model-based control with a linear model, it is recommended that the system matrices be updated periodically. Lifting operations could also be performed separately from the regular crane tip motion to reduce the probability of large angles occurring in combination with large variations of cable length. 


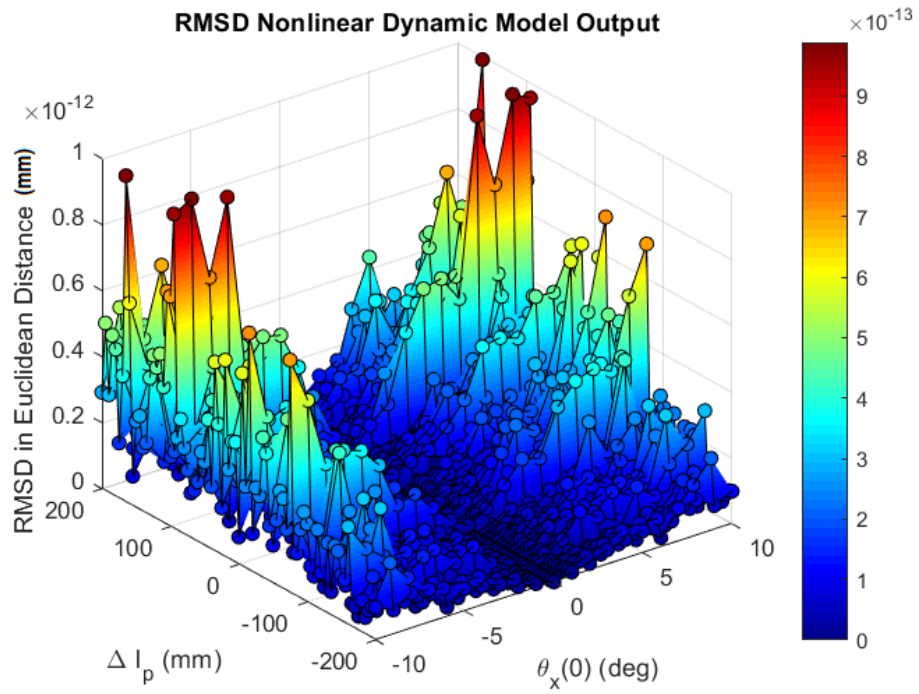

(a) Nonlinear RMSD

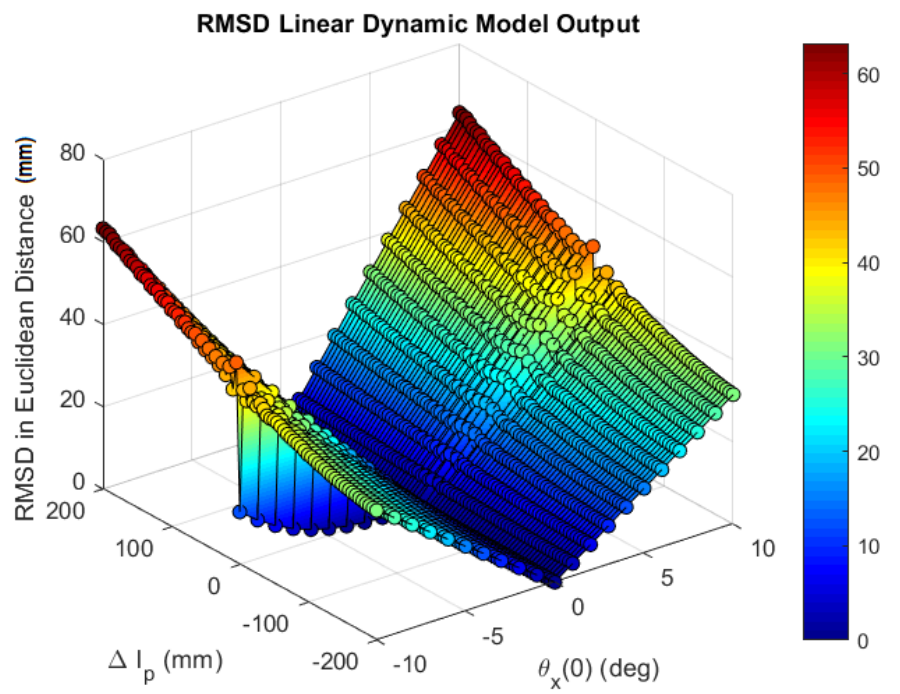

(b) Linear RMSD

Figure 4.16: Differences in the free response of each dynamic model for various initial angles and lengths. 
In this section, the linear and nonlinear models of the previous subsection were confirmed and briefly analysed by comparing the system outputs to those of an equivalent Simscape model. The linear model showed decreased performance that limits its applicability to small angles. When subject to variations in cable length, it is also noted that the linear model should be reinitialized periodically to maintain short-term accuracy when applying model-predictive techniques. The models developed within this section will be applied in the control algorithm of Chapter 6 . 


\subsection{Conclusion/Summary}

In this chapter, the kinematic and dynamic models that are applied throughout the current thesis work were described. In Section 4.1, a previously developed model of the motion platform was presented and compared to a newly developed Simscape model. Due to improved accuracy in several test cases, the Simscape model is used to perform the inverse kinematics and thereby generate set-points for the motion platform. The set-points correspond to realistic ship motion for the test-scale evaluation of the system, which is required to complete the first and second objectives of the current thesis. In Section 4.2, the forward and inverse kinematic models of the crane and related actuator mechanisms were developed mathematically and confirmed with a Simscape model. The forward and inverse kinematic models are required to complete the first and second objectives of the current thesis and are applied in the control algorithm of Chapter 6. Finally, in Section 4.3, a dynamic model of the load was developed as a variable length spherical pendulum suspended from a three-degreeof-freedom trolley that represents the crane tip. The model was confirmed with a Simscape model in both a nonlinear and linearised form. The dynamic model is required to complete the first objective of the current thesis, and it is applied/used in Chapter 6. In the next chapter, the state estimation and sensor fusion techniques used to determine the system states are discussed. 


\section{Chapter 5}

\section{State Estimation and Sensor}

\section{Fusion}

This chapter provides details of the state estimation and sensor fusion algorithms used to interpret the stochastic sensor data. These algorithms are required to determine and control the location of the load and to compensate for the periodic motion of the ship. In Section 5.1, the pose of the ship frame relative to the world frame is estimated with a sensor fusion algorithm that combines the raw data from the accelerometers, gyroscopes, and magnetometers of the IMU1. The surge/sway/heave estimation is not applied in the current work due to limitations of the test-scale experimental apparatus and poor signal-to-noise ratios for the accelerometer feedback; however, the algorithm is still discussed here for completeness and to allow for future investigation. In Section 5.2 , the pose estimation technique is adapted to estimate the pose of the load with the IMU2. Removal of estimation biases and low-pass filtering are briefly discussed in Section 5.3. 


\subsection{Estimating Ship Motion}

In this section, the motion of the ship is estimated by fusing the raw sensor signals from the gyroscopes, accelerometers, and magnetometers of the IMU1 located on the ship deck. The estimation problem is shown in Figure 5.1 with the same notation as used in Figures 4.6 and 4.7 and defined in Table 4.4. The body frame of the ship $B_{1}$ is considered to be moving with six degrees of freedom relative to the world frame $W_{0}$. This motion is considered a physical six-degree-of-freedom joint $e_{1}$ connecting frames $J_{0,2}$ to $J_{1,1}$. In parallel, a virtual six-degree-of-freedom joint $e_{10}$ is assigned to relate the IMU1 frame $S_{1,1}$ to the world frame $W_{0}$. The inverse kinematic problem considered in this section is to determine the pose of the ship body frame relative to the world frame ${ }^{W_{0}} \hat{\mathbf{Q}}_{B_{1}} \equiv{ }^{J_{0,2}} \hat{\mathbf{Q}}_{J_{1,1}}$ using an estimated pose of the IMU1 frame relative to the world frame ${ }^{W_{0}} \hat{\mathbf{Q}}_{S_{1,1}}$. The ship pose may then be decomposed into its corresponding joint variables $\left\{d_{1 x}^{*}, d_{1 y}^{*}, d_{1 z}^{*}, \theta_{1 z}^{*}, \theta_{1 y}^{*}, \theta_{1 x}^{*}\right\}$ to define the surge, sway, heave, yaw, pitch, and roll of the vessel.

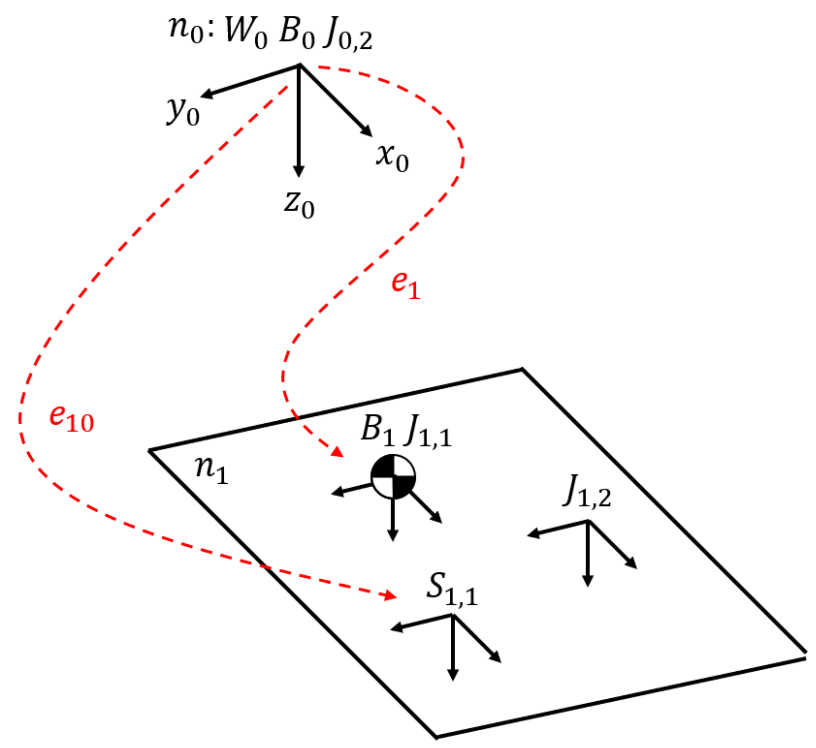

Figure 5.1: Diagram of the ship motion estimation problem. 
To form the pose loop for the kinematic analysis, three transformations are first defined and/or restated from Equation 4.7 as

$$
\begin{aligned}
{ }^{W_{0}} \hat{\mathbf{Q}}_{B_{1}} & ={ }^{J_{0,2}} \hat{\mathbf{Q}}_{J_{1,1}}=\mathbf{Q}_{1}\left(d_{1 x}^{*}, d_{1 y}^{*}, d_{1 z}^{*}, \theta_{1 z}^{*}, \theta_{1 y}^{*}, \theta_{1 x}^{*}\right) \\
& =\mathbf{Q}_{1,1}\left(d_{1 x}^{*}\right) \mathbf{Q}_{1,2}\left(d_{1 y}^{*}\right) \mathbf{Q}_{1,3}\left(d_{1 z}^{*}\right) \mathbf{Q}_{1,4}\left(\theta_{1 z}^{*}\right) \mathbf{Q}_{1,5}\left(\theta_{1 y}^{*}\right) \mathbf{Q}_{1,6}\left(\theta_{1 x}^{*}\right), \\
{ }^{B_{1}} \hat{\mathbf{Q}}_{S_{1,1}} & =1+0 \hat{\imath}+0 \hat{\jmath}+0 \hat{k}+\frac{\epsilon}{2}\left(0+s_{1 x} \hat{\imath}+s_{1 y} \hat{\jmath}+s_{1 z} \hat{k}\right) \\
{ }^{W_{0}} \hat{\mathbf{Q}}_{S_{1,1}} & =\mathbf{Q}_{10}\left({ }^{S_{1,1}} \vec{\omega},{ }^{S_{1,1}} \vec{a},{ }^{S_{1,1}} \vec{m}\right)
\end{aligned}
$$

where the transformation between the ship body frame and the world frame ${ }^{W_{0}} \hat{\mathbf{Q}}_{B_{1}}$ is stated as a function of the $e_{1}$ joint variables, and the transformation between the body frame and the sensor frame ${ }^{B_{1}} \hat{\mathbf{Q}}_{S_{1,1}}$ is stated in terms of constant offsets $\left\{s_{1 x}, s_{1 y}, s_{1 z}\right\}$ relative to the body frame. The transformation between the world frame and the sensor frame ${ }^{W_{0}} \hat{\mathbf{Q}}_{S_{1,1}}$ is expressed as a function of three vectors $\left({ }^{S_{1,1}} \vec{\omega}\right.$, $S_{1,1} \vec{a}$, and $\left.S^{S_{1,1}} \vec{m}\right)$ that represent the feedback from the IMU provided in the sensor frame. The local angular rates are defined within the vector $S_{1,1} \vec{\omega}$, the local linear accelerations are defined within the vector $S_{1,1} \vec{a}$, and the local magnetic field readings are defined within the vector ${ }^{S_{1,1}} \vec{m}$. The pose loop closure equation may be stated compactly by setting the product of Equations 5.1 and 5.2 equal to Equation 5.3 as

$$
{ }^{W_{0}} \hat{\mathbf{Q}}_{S_{1,1}}={ }^{W_{0}} \hat{\mathbf{Q}}_{B_{1}}^{B_{1}} \hat{\mathbf{Q}}_{S_{1,1}}
$$

where each side of the equation represents an equivalent dual quaternion expression for the pose of the sensor frame relative to the world frame. To solve the loop, the dependence of the pose on the sensor feedback is first defined with a sensor fusion algorithm in the following subsections. To simplify the notation, the frames are stated simply as $S \equiv S_{1,1}, B \equiv B_{1}$, and $W \equiv W_{0}$ for the remainder of this section. 
The IMU provides feedback signals in a local sensor frame for the angular rates ${ }^{S} \vec{\omega}=\left[\omega_{x}, \omega_{y}, \omega_{z}\right]^{T}$, the linear accelerations ${ }^{S} \vec{a}=\left[a_{x}, a_{y}, a_{z}\right]^{T}$, and the magnetic field readings ${ }^{S} \vec{m}=\left[m_{x}, m_{y}, m_{z}\right]^{T}$. It is assumed that the factory calibration has enforced orthogonality of the sensor axis through internal correction factors such that the components of each vector are decoupled. It is possible to gain information regarding the pose through numerical integration of the angular rates and linear accelerations. However, the resulting pose would be subject to a large amount of drift and would only provide an expression of the pose relative to some previous or initial pose. The result would be a 'dead-reckoning' estimate with unacceptable error, which motivates the application of a sensor fusion algorithm. Furthermore, as in the previous chapter, the dual quaternion ${ }^{W} \hat{\mathbf{Q}}_{S}$ may be represented as the concatenation of a translation and a rotation such that

$$
\begin{aligned}
{ }^{W} \hat{\mathbf{Q}}_{S} & ={ }^{W} \hat{\mathbf{Q}}_{D, S}{ }^{W} \hat{\mathbf{Q}}_{R, S}, \\
{ }^{W} \hat{\mathbf{Q}}_{D, S} & =\mathbf{Q}_{10,1}\left(d_{1 x}^{*}\right) \mathbf{Q}_{10,2}\left(d_{1 y}^{*}\right) \mathbf{Q}_{10,3}\left(d_{1 z}^{*}\right), \\
{ }^{W} \hat{\mathbf{Q}}_{R, S} & =\mathbf{Q}_{10,4}\left(\theta_{1 z}^{*}\right) \mathbf{Q}_{10,5}\left(\theta_{1 y}^{*}\right) \mathbf{Q}_{10,6}\left(\theta_{1 x}^{*}\right),
\end{aligned}
$$

where the rotation component is a real quaternion ${ }^{W} \hat{\mathbf{Q}}_{R, S}$, and the translation component is a dual quaternion ${ }^{W} \hat{\mathbf{Q}}_{D, S}$. The dual quaternions $\mathbf{Q}_{10, i}(\cdot)$ for $i=1 \ldots 6$ represent the six components of the transformation defined by edge $e_{10}$ in Figure 5.1 as functions of the joint variables (surge, sway, heave, roll, pitch, and yaw). By distinguishing between the translation and rotation components, the pose estimation problem is split into an orientation estimate and a position estimate.

To estimate the orientation, a sensor fusion algorithm is adapted and summarized from the work of Valenti et al. [34] in the following subsection. 


\subsubsection{Orientation Algorithm: Complementary Filter}

The algorithm presented herein is adapted from the work of Valenti et al. [34] with alterations to the notation, the world frame z-direction, the adaptation functions, and the integration technique. The first step in the algorithm is to estimate the relative orientation by numerical integration of a quaternion rate. The quaternion rate may be defined in terms of the local angular velocity as a system of differential equations,

$$
{ }^{W} \dot{\mathbf{q}}_{S}=\frac{1}{2}{ }^{W} \mathbf{q}_{S}^{S} \vec{\omega}
$$

where the lower-case $\mathbf{q}=q_{0}+q_{1} \hat{\imath}+q_{2} \hat{\jmath}+q_{3} \hat{k}$ is used to indicate a real quaternion and the vector ${ }^{S} \vec{\omega}$ is expressed in a pure quaternion form as ${ }^{S} \vec{\omega}=0+\omega_{x} \hat{\imath}+\omega_{y} \hat{\jmath}+\omega_{z} \hat{k}$. Expanding Equation 5.8 and equating components yields four differential equations,

$$
\begin{aligned}
& \dot{q_{0}}=-\frac{1}{2}\left(q_{1} \omega_{x}+q_{2} \omega_{y}+q_{3} \omega_{z}\right), \\
& \dot{q_{1}}=\frac{1}{2}\left(q_{0} \omega_{x}-q_{3} \omega_{y}+q_{2} \omega_{z}\right), \\
& \dot{q_{2}}=\frac{1}{2}\left(q_{3} \omega_{x}+q_{0} \omega_{y}-q_{1} \omega_{z}\right), \\
& \dot{q_{3}}=-\frac{1}{2}\left(q_{2} \omega_{x}-q_{1} \omega_{y}-q_{0} \omega_{z}\right),
\end{aligned}
$$

which are equivalent to those published by Küchler et al. [36]. In the complementary filter algorithm published by Valenti et al. [34], an approximate numerical integration method is implemented in which higher-order terms are omitted. In the current thesis, however, it is noted that the omission of higher-order terms may cause the resulting quaternion to drift from its unity norm. This drifting issue is discussed by Zhao and Wachem [64], and, although the quaternion may be renormalised, the rotation it represents may become skewed. In the current work, a more robust integration method is applied using a quaternion exponential. 
Assuming discrete times $t_{k}=k \Delta t$ for $k=\{0,1,2, \ldots\}$ with an initial condition of ${ }^{W} \mathbf{q}_{S, t_{0}}=\mathbf{q}_{\mathbf{I}}=1$ at $t_{0}=0$ and a time step of $\Delta t$, the numerical integration of Equations 5.9-5.12 may be performed with a quaternion exponential such that

$$
{ }^{W} \mathbf{q}_{S, t_{k+1}}={ }^{W} \mathbf{q}_{S, t_{k}} \exp \left(\frac{\Delta t^{S} \vec{\omega}}{2}\right)
$$

The quaternion exponential may be derived from an equivalent matrix form of Equations 5.9-5.12 by taking a matrix exponential of the skew symmetric matrix containing the angular velocity terms. The resulting matrix exponential may be converted back to a quaternion form as

$$
\exp \left(\frac{\Delta t^{S} \vec{\omega}}{2}\right)=\cos \left(\frac{\Delta t}{2}\left\|^{S} \vec{\omega}\right\|\right)+\frac{S \vec{\omega}}{\|S \vec{\omega}\|} \sin \left(\frac{\Delta t}{2}\left\|^{S} \vec{\omega}\right\|\right)
$$

which is similar to a technique discussed by Zhao and Wachem [64]. The result is analogous to both the matrix exponential for systems of differential equations and the Euler identity for complex numbers. In the current work, the angular velocity vector ${ }^{S} \vec{\omega}$ of Equations $5.13-5.14$ is assumed to be approximately constant for the duration of the time step and is substituted as an average of the previous and current rates such that ${ }^{S} \vec{\omega}=\frac{1}{2}\left({ }^{S} \vec{\omega}_{t_{k}}+{ }^{S} \vec{\omega}_{t_{k+1}}\right)$. By applying the quaternion exponential in the integration step, the unity norm is preserved in theory. In practice, continuous integration may introduce numerical error and the quaternion should be renormalised periodically. By integrating the quaternion rate, an estimated orientation is achieved relative to a previous estimate. However, due to uncertainty in both the previous orientation and the angular rate measurements, corrections must be applied to prevent drift and improve accuracy. 


\section{Accelerometer-Based Correction (Roll/Pitch)}

Returning to the algorithm of Valenti et al. [34], the next step is to correct the relative orientation estimate by considering the measured linear accelerations. The acceleration measurements ${ }^{S} \vec{a}=\left[a_{x}, a_{y}, a_{z}\right]^{T}$ are composed of two vectors,

$$
S_{\vec{a}}={ }^{S} \vec{a}_{S / W}+{ }^{S} \vec{g},
$$

where the first vector ${ }^{S} \vec{a}_{S / W}$ represents the acceleration of the sensor frame with respect to the world frame expressed in the sensor frame. The second vector ${ }^{S} \vec{g}$ represents a directed measurement of the gravitational acceleration expressed in the sensor frame. Assuming that the dominant acceleration is caused by gravity such that $S_{\vec{a}} \approx S_{\vec{g}}$ where the contribution of gravity is known in the world frame as ${ }^{W} \vec{g}=[0,0,-g]^{T}$ with $g \approx 9.80665 \mathrm{~m} / \mathrm{s}^{2}$, the previous estimate may be corrected through a secondary estimate that is based on the direction of gravity. It is noted that the contribution of gravity in the world frame is in the negative $z$-direction (up) due to the characteristics of the internal sensors. It is also noted that the gravitybased estimate is used for correcting roll and pitch angles as the yaw angle is about an axis parallel to the gravity vector and, therefore, allows for any number of solutions.

The method applied by Valenti et al. [34] to solve for the gravity correction factor involves predicting the direction of gravity with the previously estimated quaternion, and then solving for a delta quaternion that rotates the predicted gravity to the actual gravity in the world frame. The delta quaternion is used in the complementary filter to fuse the acceleration-based estimate with the angular rate-based estimate by performing a linear or spherical linear interpolation. 
In the procedure that follows, normalised direction vectors are indicated by a circumflex/hat. Consistent with the work of Valenti et al. [34], a prediction of the gravity vector ${ }^{W} \hat{g}_{P}$ is formed by rotating the normalised acceleration measurement vector ${ }^{S} \hat{a}$ into the world frame using the previously estimated transformation as

$$
{ }^{W} \hat{g}_{P}={ }^{W} \mathbf{q}_{S, t_{k}}\left({ }^{S} \hat{a}\right){ }^{W} \mathbf{q}_{S, t_{k}}^{*}
$$

where the direction vectors are treated as pure quaternions with $\hat{\imath}, \hat{\jmath}, \hat{k}$ components to perform the multiplication. The small deviation between the predicted gravity and the actual gravity may be found as a delta quaternion $\Delta \mathbf{q}_{a}$ such that

$$
\Delta \mathbf{q}_{a}\left({ }^{W} \hat{g}\right) \Delta \mathbf{q}_{a}^{*}={ }^{W} \hat{g}_{P}
$$

where ${ }^{W} \hat{g}=[0,0,-1]^{T}$ and ${ }^{W} \hat{g}_{P}=\left[g_{p x}, g_{p y}, g_{p z}\right]^{T}$. If either the $\hat{k}$ or $\hat{\jmath}$ components of $\Delta \mathbf{q}_{a}$ is selected as zero, the result is similar to that of Valenti et al. [34] with sign reversals caused by the assumed direction of gravity, such that

$$
\Delta \mathbf{q}_{a}=\left\{\begin{array}{l}
\frac{g_{y p}}{\sqrt{2\left(1+g_{z p}\right)}}+\sqrt{\frac{1+g_{z p}}{2}} \hat{\imath}+0 \hat{\jmath}+\frac{-g_{x p}}{\sqrt{2\left(1+g_{z p}\right)}} \hat{k}, \quad \text { if } g_{p z}>0 \\
\sqrt{\frac{1-g_{z p}}{2}+\frac{g_{y p}}{\sqrt{2\left(1-g_{z p}\right)}} \hat{\imath}+\frac{-g_{x p}}{\sqrt{2\left(1-g_{z p}\right)}} \hat{\jmath}+0 \hat{k},} \quad \text { if } g_{p z} \leq 0
\end{array},\right.
$$

where two cases are necessary to avoid division by zero. The delta quaternion $\Delta \mathbf{q}_{a}$ represents a small difference between the orientation that was estimated by integrating the angular rates and the orientation indicated by the direction of gravity. Neither estimate is ideal, so a confidence-based interpolation is performed prior to correcting the previous estimate [34]. 
The intended result of the interpolation is a new delta quaternion $\widehat{\Delta \mathbf{q}}_{a}$ that represents a compromise between the two estimates. If more trust is placed on the acceleration measurements due to low non-gravitational acceleration ${ }^{\vec{a}} \approx{ }^{S} \vec{g}$, the correction tends towards the previously discussed delta quaternion such that the majority of the correction is applied as ${\widehat{\Delta q_{a}}}_{\Delta} \approx \mathbf{q}_{a}$. However, if more trust is placed on the angular rate integrations due to high non-gravitational acceleration ${ }^{S} \vec{a} \neq S \vec{g}$, the correction tends towards an identity quaternion such that $\widehat{\Delta \mathbf{q}}_{a} \approx \mathbf{q}_{\mathbf{I}}$. The interpolation may be performed as either linear interpolation with re-normalisation when the delta quaternion is sufficiently close to the identity quaternion; or as spherical linear interpolation for larger differences. Consistent with the work of Valenti et al. [34], a threshold value $\beta$ of 0.9 is used to select the interpolation method by comparing it to the scalar component of the delta quaternion $\Delta \mathbf{q}_{g, 0}$. The linear interpolation (LERP) with normalisation and spherical linear interpolation (SLERP) may now be stated respectively as [34]

$$
\widehat{\Delta \mathbf{q}_{a}}= \begin{cases}\frac{\left(1-\alpha_{a}\right) \mathbf{q}_{\mathbf{I}}+\alpha_{a} \Delta \mathbf{q}_{a}}{\left\|\left(1-\alpha_{a}\right) \mathbf{q}_{\mathbf{I}}+\alpha_{a} \Delta \mathbf{q}_{a}\right\|}, & \text { if } \Delta \mathbf{q}_{g, 0}>\beta \\ \frac{\sin \left(\left|1-\alpha_{a}\right| \Omega\right)}{\sin (\Omega)} \mathbf{q}_{\mathbf{I}}+\frac{\sin \left(\alpha_{a} \Omega\right)}{\sin (\Omega)} \Delta \mathbf{q}_{a}, & \text { if } \Delta \mathbf{q}_{g, 0} \leq \beta\end{cases}
$$

where the subtended angle $\Omega$ is given as $\Omega=\operatorname{acos}\left(\Delta \mathbf{q}_{a} \cdot \mathbf{q}_{\mathbf{I}}\right)$ and the gain or weight is $\alpha_{a} \in[0,1]$. As was noted by Valenti et al. [34], the gain $\alpha_{a}$ defines the filter's cut-off frequency and it represents the level of trust placed on the acceleration measurements during each update. An adaptive gain is selected based on an estimated magnitude error caused by measurements of non-gravitational acceleration. 
The acceleration magnitude error $e_{a}$ is defined by Valenti et al. [34] as

$$
e_{a}=\frac{\left.||\right|^{S} \vec{a}||-g \mid}{g},
$$

and the corresponding adaptive gain is calculated as

$$
\alpha_{a}\left(e_{a}\right)= \begin{cases}\bar{\alpha}_{a} & \text { if } e_{a} \leq \beta_{a, l l} \\ \bar{\alpha}_{a}\left(\frac{\beta_{a, u l}-e_{a}}{\beta_{a, u l}-\beta_{a, l l}}\right) & \text { if } \beta_{a, l l}<e_{a}<\beta_{a, u l} \\ 0 & \text { if } e_{a} \geq \beta_{a, u l}\end{cases}
$$

where the constant gain $\bar{\alpha}_{a} \in[0,1]$ is selected to minimize errors in static testing and the saturation limits $\beta_{a, u l}>\beta_{a, l l}>0$ are selected to minimize errors during dynamic testing. The piecewise linear adaptation function of Equation 5.21 is a generalized form of the function described by Valenti et al. [34]. The adaptive gain reduces the contribution of acceleration measurements when non-gravitational components would result in a skewed estimate. Finally, the interpolated delta quaternion may be applied as a correction factor to the previous estimate such that

$$
{ }^{W} \mathbf{q}_{S, t_{k}}^{\prime}=\widehat{\Delta \mathbf{q}_{a}^{*} W} \mathbf{q}_{S, t_{k}}
$$

where ${ }^{W} \mathbf{q}_{S, t_{k}}^{\prime}$ is a new estimate of the sensor orientation that includes fused information from both the angular rate and linear acceleration measurements.

\section{Magnetometer-Based Correction (Yaw)}

As a final step in the algorithm, Valenti et al. [34] apply a similar process to derive a second correction factor based on the magnetic field readings. This correction factor is only applied to correct the yaw angle or heading such that the roll and pitch angles 
are not corrupted by magnetic disturbances.

Similar to the above process and following the work of Valenti et al. [34] with an adapted notation, a predicted magnetic field direction vector ${ }^{W} \hat{\eta}_{P}$ is formed by rotating the normalised measurement vector ${ }^{S} \hat{m}$ into the world frame with the corrected transformation as

$$
{ }^{W} \hat{\eta}_{P}={ }^{W} \mathbf{q}_{S, t_{k}}^{\prime}\left({ }^{S} \hat{m}\right)^{W} \mathbf{q}_{S, t_{k}}^{\prime *}
$$

Assuming that the global magnetic field is directed predominantly along the world $x$-axis (North), a delta quaternion is formed by rotating the world field vector as

$$
\Delta \mathbf{q}_{m}\left({ }^{W} \hat{\eta}\right) \Delta \mathbf{q}_{m}^{*}={ }^{W} \hat{\eta}_{P}
$$

where $\Delta \mathbf{q}_{m}=\Delta q_{\eta, 0}+0 \hat{\imath}+0 \hat{\jmath}+\Delta q_{\eta, 0} \hat{k}$ applies a rotation about the $z$-axis only such that ${ }^{W} \hat{\eta}=\left[\sqrt{\eta_{x}^{2}+\eta_{y}^{2}}, 0, \eta_{z}\right]^{T}$ lies in the $x z$-plane as a rotation of ${ }^{W} \hat{\eta}_{P}=\left[\eta_{x}, \eta_{y}, \eta_{z}\right]^{T}$. By applying only a z-axis rotation, the world magnetic field is allowed to have an arbitrary $z$-component $\eta_{z}$ and the correction is only applied to the heading or yaw angle. The delta quaternion may be found from Equation 5.24 as

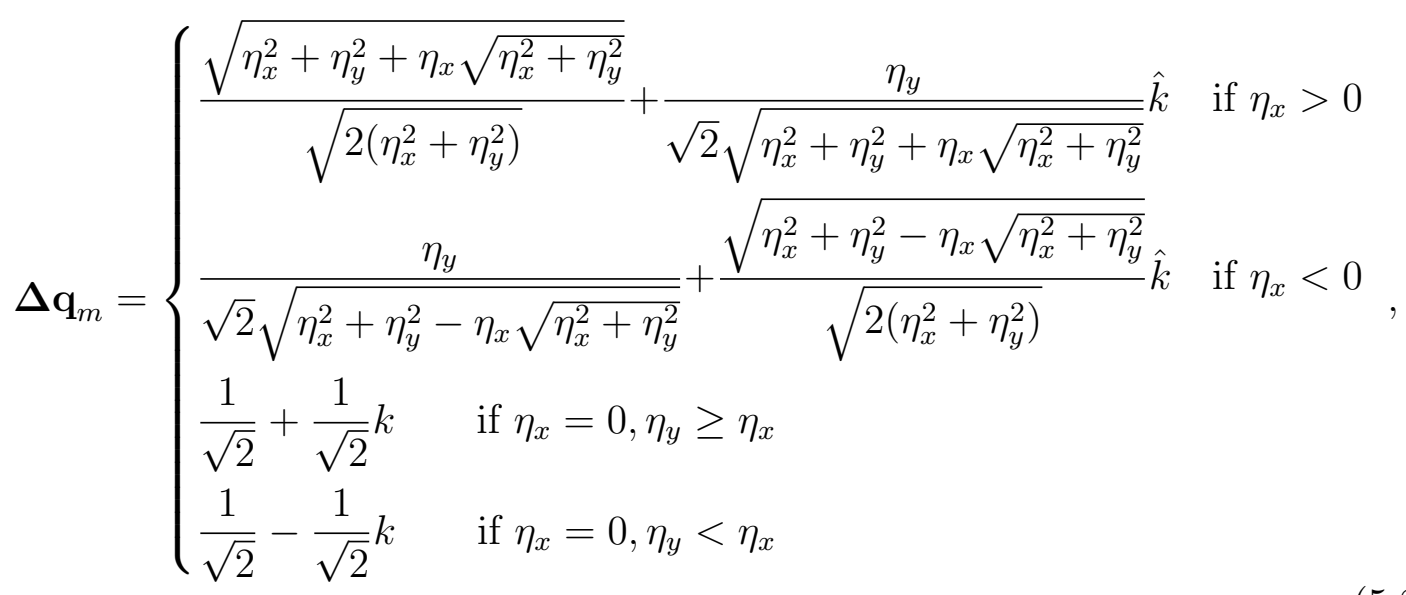

where the two additional cases are defined in the current thesis to avoid division by zero and simplify calculations when $\eta_{x}=0$. 
Linear or spherical linear interpolation between the delta quaternion and an identity quaternion may be performed again with the new delta quaternion as

$$
\widehat{\Delta \mathbf{q}_{m}}= \begin{cases}\frac{\left(1-\alpha_{m}\right) \mathbf{q}_{\mathbf{I}}+\alpha_{m} \boldsymbol{\Delta} \mathbf{q}_{m}}{\left\|\left(1-\alpha_{m}\right) \mathbf{q}_{\mathbf{I}}+\alpha_{m} \boldsymbol{\Delta} \mathbf{q}_{m}\right\|}, & \text { if } \boldsymbol{\Delta} \mathbf{q}_{m, 0}>\beta \\ \frac{\sin \left(\left|1-\alpha_{m}\right| \Omega\right)}{\sin (\Omega)} \mathbf{q}_{\mathbf{I}}+\frac{\sin \left(\alpha_{m} \Omega\right)}{\sin (\Omega)} \Delta \mathbf{q}_{m}, & \text { if } \boldsymbol{\Delta} \mathbf{q}_{m, 0} \leq \beta\end{cases}
$$

where the subtended angle is given as $\Omega=\operatorname{acos}\left(\Delta \mathbf{q}_{m} \cdot \mathbf{q}_{\mathbf{I}}\right)$ and the gain or weight is $\alpha_{m} \in[0,1]$. In the current thesis, an adaptive gain is defined to apply an exponential penalty to the correction such that large deviations are rejected. The adaptive gain is defined in terms of the product $\left(\Delta \mathbf{q}_{m} \cdot \mathbf{q}_{\mathbf{I}}\right) \in[-1,1]$ as

$$
\alpha_{m}=\bar{\alpha}_{m} \exp \left(-\beta_{m}\left|1-\Delta \mathbf{q}_{m} \cdot \mathbf{q}_{\mathbf{I}}\right|\right)
$$

where $\bar{\alpha}_{m} \in[0,1]$ and $\beta_{m}>0$ are tuning parameters from static and dynamic testing, respectively. The gain is intended to exponentially reduce the effect of the correction factor on the previous estimate when it deviates substantially due to magnetic disturbances. Finally, the interpolated delta quaternion may be applied as a correction factor to the previous estimate such that

$$
{ }^{W} \mathbf{q}_{S, t_{k}}^{\prime \prime}=\widehat{\Delta \mathbf{q}_{m}^{*}{ }^{W}} \mathbf{q}_{S, t_{k}}^{\prime},
$$

where ${ }^{W} \mathbf{q}_{S, t_{k}}^{\prime \prime}$ is a new estimate of the sensor orientation that includes information from the angular rate, linear acceleration, and magnetic field measurements. This estimate is propagated to the next iteration to continuously filter the data. This result concludes the algorithm discussed by Valenti et al. [34] with alterations to notation, world frame z-direction, adaptation functions, and integration technique. The parameters used to estimate the ship orientation are summarized in Table 5.1. 
In the next section, the sensor orientation is converted to the ship motion estimates.

Table 5.1: Summary of complementary filter parameters for the ship estimation.

\begin{tabular}{|c|c|c|c|c|c|c|}
\hline Parameter & $\beta$ & $\bar{\alpha}_{a}$ & $\beta_{a, l l}$ & $\beta_{a, u l}$ & $\bar{\alpha}_{m}$ & $\beta_{m}$ \\
\hline Value & 0.9 & 0.05 & 0.1 & 0.2 & 1 & 1 \\
\hline
\end{tabular}




\subsubsection{Estimating Yaw, Pitch, and Roll}

In this subsection, the estimated orientation quaternion is expressed as yaw, pitch, and roll angles of the ship with respect to the world frame. The dual quaternion pose loop of Equation 5.4 may be restated as

$$
{ }^{W} \hat{\mathbf{Q}}_{B}={ }^{W} \hat{\mathbf{Q}}_{S}^{S} \hat{\mathbf{Q}}_{B}
$$

where the rotational/real component of ${ }^{W} \hat{\mathbf{Q}}_{S}$ was estimated in the previous section; and the rotational/real component of the rigid transform ${ }^{S} \hat{\mathbf{Q}}_{B}={ }^{B} \hat{\mathbf{Q}}_{S}^{*}=\mathbf{Q}_{\mathbf{I}}$ is an identity quaternion as the sensor axes are aligned with the body frame of the ship. If the sensor axes are not aligned with the body frame, the rotational component of the rigid transform may be defined accordingly. The rotational/real quaternion part of Equation 5.29 represents a set of four equations. Applying the yaw-pitch-roll rotation sequence defined for ${ }^{W} \hat{\mathbf{Q}}_{B}$ in terms of the joint variables $\left\{\theta_{1 z}^{*}, \theta_{1 y}^{*}, \theta_{1 x}^{*}\right\}$ and equating components, the set of equations may be expressed as

$$
\begin{aligned}
& q_{0}=\cos \left(\frac{\theta_{1 x}^{*}}{2}\right) \cos \left(\frac{\theta_{1 y}^{*}}{2}\right) \cos \left(\frac{\theta_{1 z}^{*}}{2}\right)+\sin \left(\frac{\theta_{1 x}^{*}}{2}\right) \sin \left(\frac{\theta_{1 y}^{*}}{2}\right) \sin \left(\frac{\theta_{1 z}^{*}}{2}\right), \\
& q_{1}=\sin \left(\frac{\theta_{1 x}^{*}}{2}\right) \cos \left(\frac{\theta_{1 y}^{*}}{2}\right) \cos \left(\frac{\theta_{1 z}^{*}}{2}\right)-\cos \left(\frac{\theta_{1 x}^{*}}{2}\right) \sin \left(\frac{\theta_{1 y}^{*}}{2}\right) \sin \left(\frac{\theta_{1 z}^{*}}{2}\right), \\
& q_{2}=\cos \left(\frac{\theta_{1 x}^{*}}{2}\right) \sin \left(\frac{\theta_{1 y}^{*}}{2}\right) \cos \left(\frac{\theta_{1 z}^{*}}{2}\right)+\sin \left(\frac{\theta_{1 x}^{*}}{2}\right) \cos \left(\frac{\theta_{1 y}^{*}}{2}\right) \sin \left(\frac{\theta_{1 z}^{*}}{2}\right), \\
& q_{3}=\cos \left(\frac{\theta_{1 x}^{*}}{2}\right) \cos \left(\frac{\theta_{1 y}^{*}}{2}\right) \sin \left(\frac{\theta_{1 z}^{*}}{2}\right)-\sin \left(\frac{\theta_{1 x}^{*}}{2}\right) \sin \left(\frac{\theta_{1 y}^{*}}{2}\right) \cos \left(\frac{\theta_{1 z}^{*}}{2}\right),
\end{aligned}
$$

where $q_{0}, q_{1}, q_{2}, q_{3}$ are the ordered components of the estimated orientation quaternion from the previous subsection such that ${ }^{W} \mathbf{q}_{S}=q_{0}+q_{1} \hat{\imath}+q_{2} \hat{\jmath}+q_{3} \hat{k}$. 
Noting that the left- and right-hand sides of Equation 5.29 represent equivalent transformations, the solution for the joint variables is simplified by using each quaternion to rotate three unit vectors $\hat{\imath}, \hat{\jmath}, \hat{k}$ and then equating the resulting components. This method is equivalent to converting each quaternion to a rotation matrix and then equating elements. The result is a set of nine equations that correspond to rotation matrix elements such that

$$
\begin{aligned}
& {\left[\begin{array}{ccc}
q_{0}^{2}+q_{1}^{2}-q_{2}^{2}-q_{3}^{2} & 2\left(q_{1} q_{2}-q_{0} q_{3}\right) & 2\left(q_{0} q_{2}+q_{1} q_{3}\right) \\
2\left(q_{1} q_{2}+q_{0} q_{3}\right) & q_{0}^{2}-q_{1}^{2}+q_{2}^{2}-q_{3}^{2} & 2\left(q_{2} q_{3}-q_{0} q_{1}\right) \\
2\left(q_{1} q_{3}-q_{0} q_{2}\right) & 2\left(q_{0} q_{1}+q_{2} q_{3}\right) & q_{0}^{2}-q_{1}^{2}-q_{2}^{2}+q_{3}^{2}
\end{array}\right]} \\
& =\left[\begin{array}{ccc}
c_{z} c_{y} & c_{z} s_{x} s_{y}-c_{x} s_{z} & s_{x} s_{z}+c_{x} c_{z} s_{y} \\
s_{z} c_{y} & c_{x} c_{z}+s_{x} s_{y} s_{z} & c_{x} s_{y} s_{z}-c_{z} s_{x} \\
-s_{y} & c_{y} s_{x} & c_{x} c_{y}
\end{array}\right],
\end{aligned}
$$

where $c_{a}=\cos \left(\theta_{1 a}^{*}\right)$ and $s_{a}=\sin \left(\theta_{1 a}^{*}\right)$ for $a=\{x, y, z\}$ are substituted to simplify the expression. By selecting convenient equations from the set, the solution follows as

$$
\begin{aligned}
& \theta_{1 x}^{*}=\operatorname{atan}_{2}\left(\frac{2\left(q_{0} q_{1}+q_{2} q_{3}\right)}{q_{0}^{2}-q_{1}^{2}-q_{2}^{2}+q_{3}^{2}}\right), \\
& \theta_{1 y}^{*}=\operatorname{atan}_{2}\left(\frac{-2\left(q_{1} q_{3}-q_{0} q_{2}\right)}{\sqrt{1-4\left(q_{1} q_{3}-q_{0} q_{2}\right)^{2}}}\right), \\
& \theta_{1 z}^{*}=\operatorname{atan}_{2}\left(\frac{2\left(q_{1} q_{2}+q_{0} q_{3}\right)}{q_{0}^{2}+q_{1}^{2}-q_{2}^{2}-q_{3}^{2}}\right),
\end{aligned}
$$

where the $\operatorname{atan}_{2}$ function is used to return a quadrant-corrected result based on the signs of the numerator and denominator. The result is consistent with the relationships reported by Küchler et al. [36]; however, the pitch angle $\theta_{1 y}^{*}$ is written here using the arctangent function rather than the arcsine function as was used in the work of Henderson [65]. 
It is noted that the yaw angle, as defined within this section, contains both the heading and the yaw perturbations. In the current work, a Northern heading is assumed such that the yaw angle is directly representative of the yaw perturbations with a long-term mean value of zero.

Now that the yaw, pitch, and roll orientation estimates of the ship motion are established, the surge, sway, and heave position estimates of the ship may be found with an algorithm based on the Extended Kalman Filter. 


\subsubsection{Position Algorithm: Extended Kalman Filter}

The position of the ship may be estimated from the acceleration measurements with an Extended Kalman Filter (EKF). The work contained in this subsection is considered an extension of the heave estimation work of Küchler et al. [35] to include the surge and sway motions of the vessel. In the current thesis work, the test-scale acceleration measurements have poor signal-to-noise ratios, which prevent the application of the algorithm for the test-scale system. However, the algorithm is still summarized here for completeness as the work performed by Küchler et al. showed potential for fullscale systems, and the reader is referred to [35] for further information.

By noting that the ship motion is predominantly composed of periodic signals, a frequency based analysis is applied from the work of Küchler et al. [35]. It is assumed that the linear accelerations are sufficiently decoupled from the other degrees of freedom to allow for estimation of the surge, sway, and heave of the vessel. The Extended Kalman Filter is constructed as a state observer for a system of undamped oscillators such that the natural frequencies of the system correspond to the dominant frequencies of the ship motion. A Fast Fourier Transform (FFT) and peak-detection are applied to identify the dominant modes and initialize the filter.

To begin the process, the acceleration of the sensor is first expressed in the world frame using the orientation estimate of the previous subsection such that Equation 5.15 can be rearranged as

$$
S_{\vec{a}_{S / W}}={ }^{S} \vec{a}-{ }^{S} \vec{g}
$$

where the ${ }^{S} \vec{a}_{S / W}$ is expressed by removing the gravitational component ${ }^{S} \vec{g}$ from the sensor measurement vector ${ }^{S} \vec{a}$. By applying the quaternion rotation from the previous 
section, Equation 5.38 becomes

$$
\begin{aligned}
{ }^{W} \mathbf{q}_{S}\left({ }^{S} \vec{a}_{S / W}\right)^{W} \mathbf{q}_{S}^{*} & ={ }^{W} \mathbf{q}_{S}\left({ }^{S} \vec{a}-{ }^{S} \vec{g}\right){ }^{W} \mathbf{q}_{S}^{*} \\
{ }^{W} \vec{a}_{S / W} & ={ }^{W} \mathbf{q}_{S}\left({ }^{S} \vec{a}\right){ }^{W} \mathbf{q}_{S}^{*}-{ }^{W} \vec{g}
\end{aligned}
$$

where the vectors are expressed as pure quaternions with $\hat{\imath}, \hat{\jmath}, \hat{k}$ components, and the gravitational contribution in the world frame is ${ }^{W} \vec{g}=-g \hat{k}$. Noting that the sensor is rotating about the ship body frame $B \equiv B_{1}$ with an angular rate of ${ }^{S} \vec{\omega}$, its nongravitational acceleration may be expressed using general/relative motion equations such that

$$
{ }^{W} \vec{a}_{S / W}={ }^{W} \vec{a}_{B / W}+{ }^{W} \overrightarrow{\dot{\omega}} \times{ }^{W} \vec{r}_{S / B}+{ }^{W} \vec{\omega} \times\left({ }^{W} \vec{\omega} \times{ }^{W} \vec{r}_{S / B}\right)
$$

where the superscript $W$ indicates that all vectors are expressed with world frame components, and the subscript notation $S / W$ indicates a quantity regarding frame $S$ measured relative to frame $W$. The rigid arm ${ }^{W} \vec{r}_{S / B}$ contains the sensor offsets, which were previously defined within Equation 5.2 as body frame coordinates of the sensor such that ${ }^{B} \vec{r}_{S / B}=s_{1 x} \hat{\imath}+s_{1 y} \hat{\jmath}+s_{1 z} \hat{k}$.

Rearranging Equation 5.41 yields the acceleration of the body frame as

$$
{ }^{W} \vec{a}_{B / W}={ }^{W} \vec{a}_{S / W}-{ }^{W} \overrightarrow{\dot{\omega}} \times{ }^{W} \vec{r}_{S / B}-{ }^{W} \vec{\omega} \times\left({ }^{W} \vec{\omega} \times{ }^{W} \vec{r}_{S / B}\right),
$$

where, for convenience, the vectors of the right-hand side are restated here in terms 
of previously defined vectors and quaternion rotations as

$$
\begin{aligned}
{ }^{W} \vec{a}_{S / W} & ={ }^{W} \mathbf{q}_{S}\left({ }^{S} \vec{a}_{S / W}\right)^{W} \mathbf{q}_{S}^{*}, \\
{ }^{W} \overrightarrow{\dot{\omega}} & ={ }^{W} \mathbf{q}_{S}\left({ }^{S} \overrightarrow{\dot{\omega}}\right)^{W} \mathbf{q}_{S}^{*}, \\
{ }^{W} \vec{r}_{S / B} & =\left({ }^{W} \mathbf{q}_{S}^{S} \mathbf{q}_{B}\right)\left({ }^{B} \vec{r}_{S / B}\right)\left({ }^{W} \mathbf{q}_{S}^{S} \mathbf{q}_{B}\right)^{*},
\end{aligned}
$$

and vectors are again represented as pure quaternions by their $\hat{\imath}, \hat{\jmath}, \hat{k}$ components. Due to alignment of the sensor and body frames, the rotation quaternion ${ }^{S} \mathbf{q}_{B}=$ $\mathbf{q}_{\mathbf{I}}=1$ is an identity quaternion. The angular acceleration ${ }^{S} \overrightarrow{\dot{\omega}}$ may be estimated as a discrete time derivative at each iteration. For simplicity, the acceleration of the ship frame may be expressed with a modified notation as $\vec{a}_{B} \equiv{ }^{W} \vec{a}_{B / W}$ with components corresponding to the second derivative of the ship surge, sway, and heave coordinates as $\vec{a}_{B}=\ddot{d}_{1 x}^{*} \imath+\ddot{d}_{1 y}^{*} \imath+\ddot{d}_{1 z}^{*} k$.

In the current work, the algorithm applied by Küchler et al. [35] for heave motion is extended to include surge and sway by selecting a general coordinate $\zeta$ to represent any of the ship coordinates $\left\{d_{1 x}^{*}, d_{1 y}^{*}, d_{1 z}^{*}\right\}$. Any of the linear coordinates of the ship may be decomposed into a set of $N_{m}$ sine waves or modes as

$$
\zeta(t)=\sum_{i=1}^{N_{m}} A_{i} \cos \left(w_{i} t+\phi_{i}\right)+v(t),
$$

where $A_{i}$ is the amplitude, $w_{i}=2 \pi f_{i}$ is the eigenfrequency corresponding to a frequency of $f_{i}$, and $\phi_{i}$ is the phase for the $i^{t h}$ mode. 
The first and second derivatives of the position may be expressed as

$$
\begin{aligned}
& \dot{\zeta}(t)=\sum_{i=1}^{N_{m}} \dot{\zeta}_{i}+\dot{v}(t)=-\sum_{i=1}^{N} w_{i} A_{i} \sin \left(w_{i} t+\phi_{i}\right)+\dot{v}(t), \\
& \ddot{\zeta}(t)=\sum_{i=1}^{N_{m}} \ddot{\zeta}_{i}+\ddot{v}(t)=-\sum_{i=1}^{N} w_{i}^{2} A_{i} \cos \left(w_{i} t+\phi_{i}\right)+\ddot{v}(t),
\end{aligned}
$$

where the amplitude of each derivative is directly related to the amplitude of the corresponding position by the eigenfrequency. Therefore, by applying an FFT to the measured accelerations, the periodic motion of the ship may be identified. Consistent with Küchler et al. [35], the slow-varying offset $v(t)$ and its derivatives are neglected, and each mode is modelled as an undamped oscillator of the form $\ddot{\zeta}_{i}+w_{i}^{2} \zeta_{i}=0$ such that a state-space model for the $i^{\text {th }}$ mode may be defined as

$$
\begin{array}{ll}
\dot{x}_{i}=f\left(x_{i}\right)=\left[\begin{array}{c}
x_{i, 2} \\
-x_{i, 3}^{2} x_{i, 1} \\
0
\end{array}\right] \quad \text { where } \quad x_{i}=\left[\begin{array}{c}
x_{i, 1} \\
x_{i, 2} \\
x_{i, 3}
\end{array}\right]=\left[\begin{array}{c}
\zeta_{i} \\
\dot{\zeta}_{i} \\
w_{i}
\end{array}\right] \\
y_{i}=g\left(x_{i}\right)=-x_{i, 3}^{2} x_{i, 1} & \text { where } \quad y_{i}=\ddot{\zeta}_{i}
\end{array}
$$

where the eigenfrequency is modelled as a random walk parameter and the initial conditions are found through the output of the FFT. The system is discretized and solved in a real-time controller with a matrix exponential for each mode. Offset terms $x_{o f f}$ and $y_{o f f}$ are also introduced as random walk processes to account for any bias in the system/measurement. 
The final set of discrete equations to be solved by the controller are [35]

$$
\begin{aligned}
& x_{t_{k}}=\left[\begin{array}{c}
x_{1, t_{k}} \\
\vdots \\
x_{N_{m}, t_{k}} \\
x_{o f f, t_{k}}
\end{array}\right]=\left[\begin{array}{c}
\Phi_{1}\left(x_{1, t_{k-1}}, \Delta t\right) \\
\vdots \\
\Phi_{N_{m}}\left(x_{N_{m}, t_{k-1}}, \Delta t\right) \\
x_{o f f, t_{k-1}}
\end{array}\right]=\Phi\left(x_{\left.t_{k-1}, \Delta t\right)}\right. \\
& y_{t_{k}}=y_{1, t_{k}}+\ldots+y_{N_{m}, t_{k}}+y_{o f f, t_{k}}
\end{aligned}
$$

where the initial conditions $x_{t_{0}}$ are determined from the FFT data and $\Phi_{i}\left(x_{i, t_{k}}, \Delta t\right)$ is the discrete state transition matrix. An Extended Kalman Filter is used as an observer to propagate states. The system covariance matrix is used to place a penalty on higher frequency components, the measurement covariance is taken from the sensor noise, the system is periodically reinitialized as frequencies appear and/or disappear, and an FFT is performed and dominant peaks are selected repetitively [35].

In combination with the previous subsection, the surge, sway, heave, roll, pitch, and yaw of the vessel are estimated from a set of measurements such that the transformation between the world and ship frames ${ }^{W} \hat{\mathbf{Q}}_{B}$ is fully defined. In the following section, estimation of the load motion is discussed. 


\subsection{Estimating Load Motion}

In this section, the motion of the pendulum load is estimated using fused raw sensor signals from the gyroscopes, accelerometers, and magnetometers of the second inertial measurement unit (IMU2). The estimation problem is shown in Figure 5.2 with the same notation used in Figures 4.6 and 4.7 and defined in Table 4.4. Several additional elements are included in the diagram to assist with the estimation problem. The dashed edges shown in red represent joints that are consistent with Figure 4.6 and explicitly defined in Table 4.4 during the kinematic analysis of the crane. The additional dashed edges shown in black were implicitly defined during the dynamic analysis of the pendulum load. The edge $e_{11}$ represents a 3 -DOF Cartesian joint that defines the trolley/tip position relative to the world frame with a corresponding transformation of ${ }^{W_{0}} \hat{\mathbf{Q}}_{T}$; the edge $e_{12}$ represents a 3-DOF spherical joint that constrains the trolley/tip frame origin to be coincident with the origin of the joint frame $J_{4,2}$ at the crane tip; and the edge $e_{13}$ represents a compound physical joint consisting of a 3-DOF spherical joint at the tip followed by a 1-DOF prismatic joint corresponding to the cable/pendulum length.

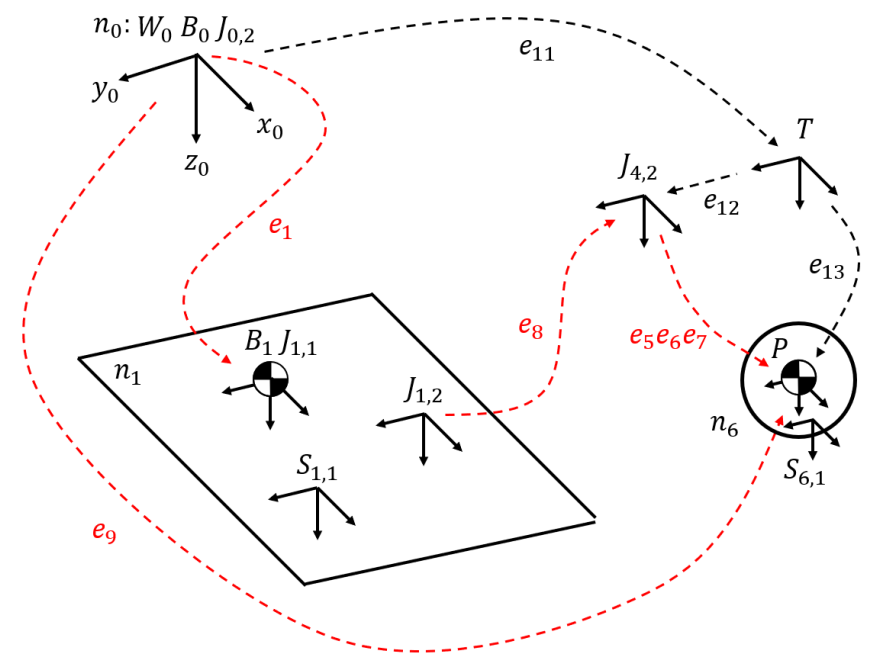

Figure 5.2: Diagram of the load motion estimation problem. 
The body frame of the load $P \equiv B_{6}$ is considered to be moving with three unconstrained degrees of freedom relative to the world frame such that its pose is fully defined by the estimation of three additional coordinates. The pose of the ship ${ }^{W_{0}} \hat{\mathbf{Q}}_{B_{1}}$ is considered to be known from the estimation algorithm of the previous section, and the relative pose of the crane's base frame ${ }^{B_{1}} \hat{\mathbf{Q}}_{J_{1,2}}$ is defined by rigid offsets. The location of the crane tip relative to its base was defined in the forward kinematic analysis of the previous chapter and by the dual translation quaternion ${ }^{J_{1,2}} \hat{\mathbf{Q}}_{D, J_{4,2}}$. Through the constraints implied by the 3-DOF spherical joint, the three aforementioned transformations may be used to define the pose of the trolley/tip frame relative to the world frame ${ }^{W_{0}} \hat{\mathbf{Q}}_{T}$. The length of the cable $l_{p}$ is directed along its local $z$-axis and defined within the transformation ${ }^{T} \hat{\mathbf{Q}}_{P}$, which was applied in the dynamic analysis of the previous chapter. Due to the assumed point mass load, the dynamic analysis considered only the $x$ and $y$ rotations. However, when considering the attached sensor frame, 3-DOF rotations are considered such that the transformation ${ }^{T} \hat{\mathbf{Q}}_{P}$ may be redefined by the sequence $\left\{\theta_{x}, \theta_{y}, \theta_{z}, l_{p}\right\}$. The sensor frame is assumed to be aligned with the body frame such that ${ }^{P} \hat{\mathbf{Q}}_{S}=\mathbf{Q}_{\mathbf{I}}$. The inverse kinematic problem of the current chapter is to estimate the unknown variables $\theta_{x}, \theta_{y}, \theta_{z}$ based on the feedback from the IMU and, using the estimated values, to determine the position of the load relative to the world frame. Given that the unknown variables represent three rotations in a sequence, they may be directly estimated from the orientation of the IMU and, therefore, the orientation algorithm of the previous section is implemented with the parameters shown in Table 5.2. The output of the complementary filter ${ }^{W} \hat{\mathbf{q}}_{S}$ is used to estimate the motion in the following subsection.

Table 5.2: Summary of complementary filter parameters for the load estimation.

\begin{tabular}{|c|c|c|c|c|c|c|}
\hline Parameter & $\beta$ & $\bar{\alpha}_{a}$ & $\beta_{a, l l}$ & $\beta_{a, u l}$ & $\bar{\alpha}_{m}$ & $\beta_{m}$ \\
\hline Value & 0.9 & 0.005 & 0.05 & 0.2 & 1 & 1 \\
\hline
\end{tabular}




\subsubsection{Estimating Pendulum Angles and Position}

In this subsection, the estimated orientation quaternion is expressed as pendulum angles $\theta_{x}, \theta_{y}, \theta_{z}$ of the load with respect to the world frame and/or translating world frame. These angles are illustrated in Figure 5.3 using a modified version of Figure 4.13. The $\theta_{x}$ and $\theta_{y}$ angles correspond to those used in the derivation of the dynamic model of Chapter 4 in Section 4.3. The $\theta_{z}$ angle is introduced here as a third degree of freedom that, when using a roll-pitch-yaw (x-y-z) rotation sequence, represents an angle of twist about the cable axis that may be present in the physical system. When assuming a point mass for the load, the angle of twist represented by $\theta_{z}$ is considered arbitrary for determining its position. However, a solution is still provided here for the benefit of future work when more complex loads are considered.

It is noted that this procedure is similar to that of Section 5.1.2; however, a yaw-pitch-roll (z-y-x) rotation sequence was considered in Section 5.1.2 for the ship, whereas a roll-pitch-yaw (x-y-z) sequence is considered here for the pendulum.

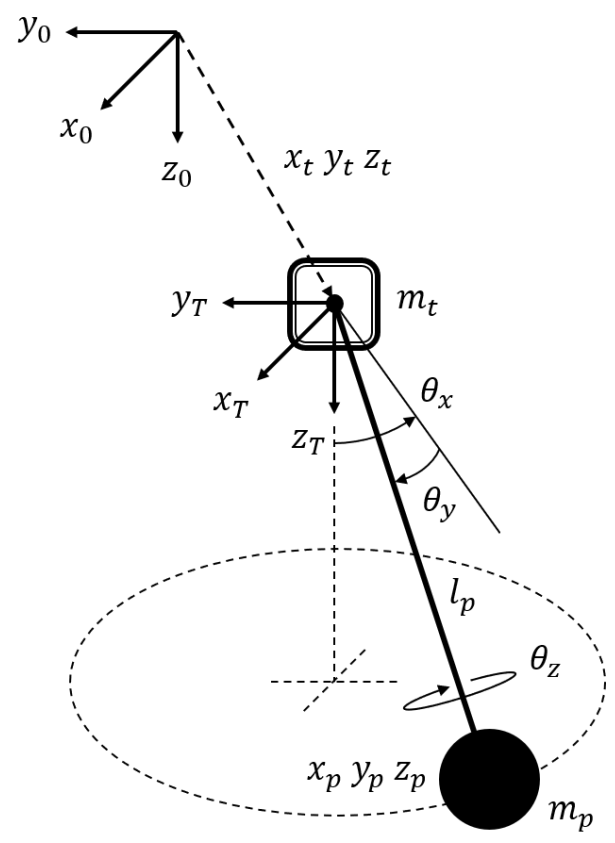

Figure 5.3: Diagram of the variable length pendulum with twist. 
The dual quaternion pose loop for the load may be stated equivalently as

$$
{ }^{W} \hat{\mathbf{Q}}_{P}={ }^{W} \hat{\mathbf{Q}}_{T}^{T} \hat{\mathbf{Q}}_{P}={ }^{W} \hat{\mathbf{Q}}_{S}^{S} \hat{\mathbf{Q}}_{P},
$$

where the rotational/real component of the world to sensor frame transformation ${ }^{W} \hat{\mathbf{Q}}_{S}$ is estimated with the previously defined complementary filter algorithm; and the rotational/real component of the rigid transform ${ }^{S} \hat{\mathbf{Q}}_{P}$ is an identity quaternion as the sensor axes are aligned with the body frame of the load. If the sensor axes are not aligned with the body frame, the rotational component of the rigid transform may be defined accordingly. The rotational part of Equation 5.53 represents a set of four equations. Applying the roll-pitch-yaw rotation sequence defined for the transformation ${ }^{W} \hat{\mathbf{Q}}_{P}$ in terms of the pendulum angles $\left\{\theta_{x}, \theta_{y}, \theta_{z}\right\}$ and equating components, the set of equations may be expressed as

$$
\begin{aligned}
& q_{0}=\cos \left(\frac{\theta_{x}}{2}\right) \cos \left(\frac{\theta_{y}}{2}\right) \cos \left(\frac{\theta_{z}}{2}\right)-\sin \left(\frac{\theta_{x}}{2}\right) \sin \left(\frac{\theta_{y}}{2}\right) \sin \left(\frac{\theta_{z}}{2}\right), \\
& q_{1}=\sin \left(\frac{\theta_{x}}{2}\right) \cos \left(\frac{\theta_{y}}{2}\right) \cos \left(\frac{\theta_{z}}{2}\right)+\cos \left(\frac{\theta_{x}}{2}\right) \sin \left(\frac{\theta_{y}}{2}\right) \sin \left(\frac{\theta_{z}}{2}\right), \\
& q_{2}=\cos \left(\frac{\theta_{x}}{2}\right) \sin \left(\frac{\theta_{y}}{2}\right) \cos \left(\frac{\theta_{z}}{2}\right)-\sin \left(\frac{\theta_{x}}{2}\right) \cos \left(\frac{\theta_{y}}{2}\right) \sin \left(\frac{\theta_{z}}{2}\right), \\
& q_{3}=\cos \left(\frac{\theta_{x}}{2}\right) \cos \left(\frac{\theta_{y}}{2}\right) \sin \left(\frac{\theta_{z}}{2}\right)+\sin \left(\frac{\theta_{x}}{2}\right) \sin \left(\frac{\theta_{y}}{2}\right) \cos \left(\frac{\theta_{z}}{2}\right),
\end{aligned}
$$

where $q_{0}, q_{1}, q_{2}, q_{3}$ are the ordered components of the estimated orientation quaternion from the previous subsection such that ${ }^{W} \mathbf{q}_{S}=q_{0}+q_{1} \hat{\imath}+q_{2} \hat{\jmath}+q_{3} \hat{k}$. 
Noting that the expressions of Equation 5.53 represent equivalent transformations, the solution for the joint variables is simplified by using each quaternion to rotate three unit vectors $\hat{\imath}, \hat{\jmath}, \hat{k}$ and then equating the resulting components. This method is equivalent to converting each quaternion to a rotation matrix and then equating elements. The result is a set of nine equations that correspond to rotation matrix elements such that

$$
\begin{aligned}
& {\left[\begin{array}{ccc}
q_{0}^{2}+q_{1}^{2}-q_{2}^{2}-q_{3}^{2} & 2\left(q_{1} q_{2}-q_{0} q_{3}\right) & 2\left(q_{0} q_{2}+q_{1} q_{3}\right) \\
2\left(q_{1} q_{2}+q_{0} q_{3}\right) & q_{0}^{2}-q_{1}^{2}+q_{2}^{2}-q_{3}^{2} & 2\left(q_{2} q_{3}-q_{0} q_{1}\right) \\
2\left(q_{1} q_{3}-q_{0} q_{2}\right) & 2\left(q_{0} q_{1}+q_{2} q_{3}\right) & q_{0}^{2}-q_{1}^{2}-q_{2}^{2}+q_{3}^{2}
\end{array}\right]} \\
& =\left[\begin{array}{ccc}
c_{y} c_{z} & -c_{y} s_{z} & s_{y} \\
c_{x} s_{z}+c_{z} s_{x} s_{y} & c_{x} c_{z}-s_{x} s_{y} s_{z} & -c_{y} s_{x} \\
s_{x} s_{z}-c_{x} c_{z} s_{y} & c_{z} s_{x}+c_{x} s_{y} s_{z} & c_{x} c_{y}
\end{array}\right],
\end{aligned}
$$

where $c_{a}=\cos \left(\theta_{a}\right)$ and $s_{a}=\sin \left(\theta_{a}\right)$ for $a=\{x, y, z\}$ are substituted to simplify the expression. By selecting convenient equations from the set, the solution follows as

$$
\begin{aligned}
& \theta_{x}=\operatorname{atan}_{2}\left(\frac{-2\left(q_{2} q_{3}-q_{0} q_{1}\right)}{q_{0}^{2}-q_{1}^{2}-q_{2}^{2}+q_{3}^{2}}\right), \\
& \theta_{y}=\operatorname{atan}_{2}\left(\frac{2\left(q_{0} q_{2}+q_{1} q_{3}\right)}{\sqrt{1-4\left(q_{0} q_{2}+q_{1} q_{3}\right)^{2}}}\right), \\
& \theta_{z}=\operatorname{atan}_{2}\left(\frac{-2\left(q_{1} q_{2}-q_{0} q_{3}\right)}{q_{0}^{2}+q_{1}^{2}-q_{2}^{2}-q_{3}^{2}}\right),
\end{aligned}
$$

where the $\operatorname{atan}_{2}$ function is used to return a quadrant-corrected result based on the signs of the numerator and denominator. The result is similar to the relationships reported by Henderson [65]. 
The pendulum position relative to the world frame is given by Equations 4.944.96, which are repeated here as

$$
\begin{aligned}
& x_{p}=x_{t}+l_{p} \sin \left(\theta_{y}\right), \\
& y_{p}=y_{t}-l_{p} \cos \left(\theta_{y}\right) \sin \left(\theta_{x}\right), \\
& z_{p}=z_{t}+l_{p} \cos \left(\theta_{y}\right) \cos \left(\theta_{x}\right),
\end{aligned}
$$

where the length $l_{p}$ is a known quantity, the angles $\theta_{x}$ and $\theta_{y}$ are determined from the IMU2 feedback, and the tip/trolley position $\left\{x_{t}, y_{t}, z_{t}\right\}$ is defined relative to the crane's base frame $J_{1,2}$ by Equations 4.73-4.75. To convert the position from the base frame coordinates to world frame coordinates, a transformation sequence may be applied such that

$$
\left[\begin{array}{c}
{ }^{W} x_{t} \\
{ }^{W} y_{t} \\
{ }^{W} z_{t}
\end{array}\right]=\left({ }^{W} \hat{\mathbf{Q}}_{B_{1}}^{B_{1}} \hat{\mathbf{Q}}_{J_{1,2}}\right)\left[\begin{array}{c}
{ }^{J_{1,2}} x_{t} \\
{ }^{J_{1,2}} y_{t} \\
J_{1,2} z_{t}
\end{array}\right]\left({ }^{W} \hat{\mathbf{Q}}_{B_{1}}^{B_{1}} \hat{\mathbf{Q}}_{J_{1,2}}\right)^{*}
$$

where the transformation between the world and ship frame ${ }^{W} \hat{\mathbf{Q}}_{B_{1}}$ was defined in the previous section, and the transformation between the ship frame and the base frame ${ }^{B_{1}} \hat{\mathbf{Q}}_{J_{1,2}}$ defines a rigid x-y-z offset. To perform the dual quaternion multiplication, the vectors shown are expressed by their dual quaternion equivalents. Substituting the resulting components of Equation 5.65 into Equations 5.62-5.64 yields the position of the load relative to the world frame, which will be applied in the control algorithm of Chapter 6.

Now that the estimates of the pendulum angles/position are defined, estimation biases will be removed and low-pass filtering will be applied to correct the angles in the following section. 


\subsection{Removal of Estimation Biases and Filtering}

In the current thesis work, the ship is assumed to have a Northern heading for simplicity. Although the magnetic field is defined in the Northern direction to enforce this assumption in the simulations, the experimental apparatus is subject to magnetic field variations and is not aligned for a Northern heading. To correct for these factors, the world frame is artificially rotated to remove the yaw angle detected by IMU1 on the ship. This correction results in both an artificial Northern heading and zero yaw for the ship, which are assumptions of the current thesis work. The nominal value of

the detected yaw angle is automatically determined by low-pass filtering $(0.005 \mathrm{~Hz})$ for several minutes prior to testing.

Similarly, corrections must be applied to remove biases in the pendulum angle estimates. These biases may result from time-varying sensor biases and/or imperfect mounting of the IMU. In particular, if the IMU is mounted such that a small angular offset exists between the pendulum/load frame and the IMU2 frame, the angles detected by the estimation algorithm will not accurately reflect the pendulum angles. Again, these nominal/steady-state offsets are determined and removed via low-pass filtering. Additional low-pass filters $(1 \mathrm{~Hz})$ are applied on the output angles of each of the estimation algorithms to further reduce the noise observed in the experimental estimates. It is noted that these low-pass filters result in a phase shift for smaller pendulum lengths (i.e. higher natural frequencies); however, preliminary testing suggests that the performance of the compensation system is not significantly impacted for pendulum lengths of $400 \mathrm{~mm}$ or greater. The effects of the filtering/estimation algorithm are investigated in Chapter 7 by comparing performance with sensor-based estimations to truth data feedback. 


\subsection{Conclusion/Summary}

In this chapter, the sensor fusion and state estimation algorithms used to determine the ship and load motion were described. In Section 5.1, a complementary filter from the work of Valenti et al. [34] was adapted to estimate the orientation of the ship. A frequency-based method with an Extended Kalman Filter was discussed from the work of Küchler et al. [35] to estimate the position of the ship relative to the world frame. Although the surge/sway/heave estimation is not applied in the current work due to limitations of the test-scale apparatus, it is included for completeness and for the purpose of future investigation. In the simulations and experiments, truth data is supplied in place of the surge/sway/heave estimates. The position and orientation of the ship are required for motion compensation performance and for determining the world frame position of the load, which is required for the first and second objectives of the current thesis. In Section 5.2, the complementary filter algorithm was reapplied to estimate the orientation of the load. In combination with the previously defined kinematic relationships, the orientation estimate was used to determine the position of the load relative to the world frame. The selected parameters for the ship and load complementary filters are summarized in Table 5.1 and Table 5.2, respectively; however, optimal tuning is recommended to improve the estimates in future work. The

position of the load relative to the world frame is required for the control algorithm can now be developed. 


\section{Chapter 6}

\section{Motion Compensation and Control}

This chapter develops independent joint control and world-frame motion compensation algorithms. In Section 6.1, four proportional-integral-derivative (PID) controllers with saturation, anti-windup, and deadband considerations are applied to control the displacement of each actuator. In Section 6.2, a motion compensation algorithm is developed from a linear-quadratic regulator (LQR) that is based on the linearised dynamic model of Chapter 4. The LQR-based motion compensation algorithm operates in a cascade control architecture by providing set-points to the aforementioned PID joint controllers. The controllers are applied to the test-scale physical system and hardware-in-the-loop/operator-in-the-loop simulation, and the performance of the proposed system is evaluated in Chapter 7 . 


\subsection{Independent Joint Control}

The displacement of each actuator is controlled with a proportional-integral-derivative (PID) controller. To implement the controller in a real-time digital system, the common continuous PID controller must be discretized. By considering a time step of $\Delta t$, the discrete form of the controller may be found with trapezoidal integration and backwards differentiation as

$$
\begin{aligned}
& u_{k}=K_{p} e_{k}+\sum_{j=0}^{k} K_{i} \Delta t\left(\frac{e_{j}+e_{j-1}}{2}\right)+K_{d}\left(\frac{e_{k}-e_{k-1}}{\Delta t}\right), \\
& e_{k}=r_{k}-y_{k}
\end{aligned}
$$

where $u(t)$ is the control action or system input; $e(t)$ is the error between a reference signal $r(t)$ and the system output $y(t)$; the set of gains $\left\{K_{p}, K_{i}, K_{d}\right\}$ are respectively

referred to as the proportional, integral, and derivative gains; $u_{k}$ is the control action; and $e_{k}$ is the error between the reference $r_{k}$ and the output $y_{k}$ at the discrete time $t_{k}=k \Delta t$ for $k \geq 0$. A past error of $e_{-1}=-e_{0}$ may be used to initialize the integration/summation term, and $e_{-1}=e_{0}$ may be used to initialize the differentiation term and thereby avoid undesired initial control actions for $k=0$. An abrupt change in the reference signal may result in an undesired derivative action or 'kick' as the error suddenly increases over a small time step. To avoid this undesired control action, the derivative term may be rewritten such that it acts only on the system output.

To remove dependence on the reference signal, Equation 6.1 can be expressed as

$$
u_{k}=K_{p} e_{k}+\sum_{j=0}^{k} K_{i} \Delta t\left(\frac{e_{j}+e_{j-1}}{2}\right)-K_{d}\left(\frac{y_{k}-y_{k-1}}{\Delta t}\right)
$$

where past values of $e_{-1}=-e_{0}$ and $y_{-1}=y_{0}$ may be assumed to initialize the integral 
and derivative terms respectively. Due to physical limitations, the control input $u_{k}$ is subject to upper and lower saturation limits such that

$$
\operatorname{sat}\left(u_{k}\right)=\left\{\begin{array}{lll}
u_{s a t, u l} & \text { if } & u_{k} \geq u_{\text {sat }, u l} \\
u_{k} & \text { if } & u_{\text {sat }, l l}<u_{k}<u_{\text {sat }, u l} \\
u_{\text {sat }, l l} & \text { if } & u_{k} \leq u_{\text {sat }, l l}
\end{array}\right.
$$

where the upper and lower limits of the control action are denoted as $u_{\text {sat,ul }}$ and $u_{\text {sat }, l l}$, respectively. If the control input becomes saturated with non-zero tracking error, the integral term will continuously grow until the error is reduced, which is known as integral 'windup'. To implement an anti-windup algorithm, the discrete PID controller of Equation 6.3 is first rewritten as

$$
\begin{aligned}
u_{k} & =u_{p, k}+u_{i, k}+u_{d, k}, \\
u_{p, k} & =K_{p} e_{k}, \\
u_{i, k} & =u_{a w, k-1}+K_{i} \Delta t\left(\frac{e_{k}+e_{k-1}}{2}\right), \\
u_{d, k} & =-K_{d}\left(\frac{y_{k}-y_{k-1}}{\Delta t}\right),
\end{aligned}
$$

where $u_{p, k}, u_{i, k}$, and $u_{d, k}$ are the proportional, integral, and derivative control actions, respectively. An anti-windup term $u_{a w, k-1}$ is included to reduce the integral action when the controller is saturated. The anti-windup term is defined as

$$
u_{a w, k-1}=\operatorname{sat}\left(u_{p, k-1}+u_{i, k-1}\right)-u_{p, k-1}
$$

where the saturation function $s a t(\cdot)$ is defined from Equation 6.4 by replacing $u_{k}$ with $u_{p, k-1}+u_{i, k-1}$. The anti-windup term reduces to $u_{a w, k-1}=u_{i, k-1}$ for normal 
integration if the PI control action is within the saturation limits. However, if the PI action is saturated, the anti-windup term reduces the previous PI action such that it does not exceed the saturation limits for the purpose of limiting the integral action. To initialize the controller at $k=0$, the integral and derivative control actions are set to zero such that $u_{i, 0}=0$ and $u_{d, 0}=0$. Therefore, on the first iteration of the control loop, only the proportional action is applied.

To finalize the PID control algorithm, several deadbands are considered. First, an error deadband is added to reduce excitation of the actuator by adjusting the feedback as

$$
y_{k}^{\prime}= \begin{cases}r_{k} & \text { if } \quad\left|r_{k}-y_{k}\right|<e_{d b} \\ y_{k} & \text { otherwise }\end{cases}
$$

such that the error is set to zero when it is sufficiently small. For the modified controller of Equations 6.5-6.8, this deadband is accomplished by substituting $y_{k}=$ $y_{k}^{\prime}$. This symmetric deadband reduces the control action that may result from noise in the feedback signal and defines an allowable tracking error to reduce oscillations about the set-point. The second deadband is a nonlinear characteristic of the actuator that results in negligible response for control actions or inputs that are within the limits of the deadband. To reduce the tracking errors caused by this nonlinearity, the deadband is 'jumped' such that the adjusted input $u_{k}^{\prime}$ becomes

$$
u_{k}^{\prime}=\left\{\begin{array}{lll}
\operatorname{sat}\left(u_{k}\right)+u_{d b, u l} & \text { if } \quad & \operatorname{sat}\left(u_{k}\right) \geq u_{d b} \\
0 & \text { if } \quad-u_{d b}<\operatorname{sat}\left(u_{k}\right)<u_{d b} \\
\operatorname{sat}\left(u_{k}\right)+u_{d b, l l} & \text { if } \quad \operatorname{sat}\left(u_{k}\right) \leq-u_{d b}
\end{array},\right.
$$

where the saturated input $\operatorname{sat}\left(u_{k}\right)$ is defined by Equations $6.4-6.8$ and the asymmet- 
rical upper and lower limits of the actuator deadband are defined as $u_{d b, u l}$ and $u_{d b, l l}$, respectively. To avoid undesired motion for small control actions, the deadband is only 'jumped' if the control action exceeds a minimum limit defined by $\pm u_{d b}$. The minimum jump limit $u_{d b}$ reduces the asymmetrical actuator deadband to a small symmetric control deadband. The control deadband allows for a smoother transition between forward/reverse and prevents motion for small inputs.

Figure 6.1 summarizes the algorithm in a block diagram where the inputs to the controller are the reference signal $r_{k}$ and feedback or measured system output $y_{k}$, and the output of the controller is the adjusted control action $u_{k}^{\prime}$. The z-transforms $z^{-1}$ are used to represent unit delays on the input signals such that a block input of $(\cdot)_{k}$ is transformed to a block output of $(\cdot)_{k-1}$. The PID control algorithm applied for each of the actuators is defined as

- apply the error deadband of Equation 6.10,

- calculate the unsaturated control action with Equations 6.4-6.9,

- apply saturation with Equation 6.4, and

- jump the deadband according to Equation 6.11.

In the current thesis work, a sampling frequency of $f_{s}=100 \mathrm{~Hz}$ is used for control, which corresponds to a sampling period or time step of $T_{s}=\Delta t=0.010 \mathrm{~s}$. Error deadbands are selected for the linear actuators as $e_{d b}=0.25 \mathrm{~mm}$ and for the winch motor as $e_{d b}=0.25^{\circ}$. The controller gains are chosen through a combination of manual and auto-tuning with estimated transfer functions in Simulink to achieve a desirable position tracking response. The tuned values are recorded in Table 6.1.

The tuned controllers are implemented in LabVIEW. The linear actuator setpoints are defined as extended lengths $(\mathrm{mm})$, whereas the winch motor set-point is 


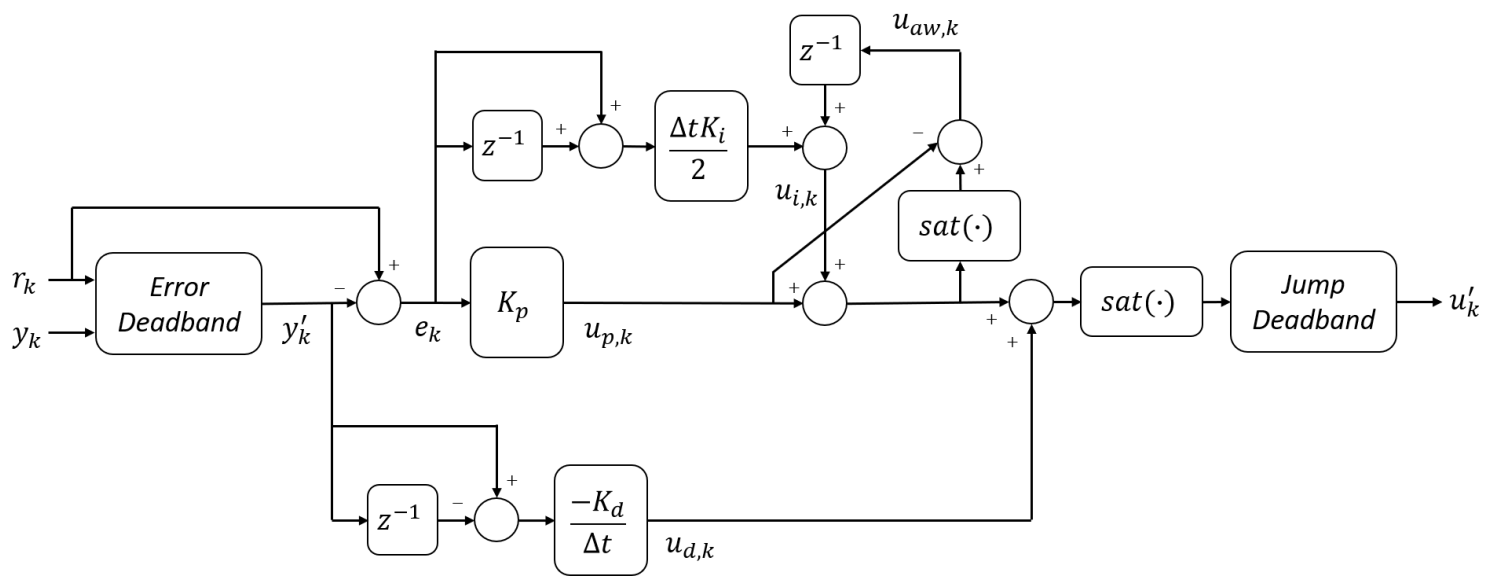

Figure 6.1: Block diagram of the PID algorithm.

Table 6.1: Independent joint control parameters.

\begin{tabular}{|c|c|c|c|c|}
\hline Parameter & $\begin{array}{c}\text { LA1 } \\
(\text { Base })\end{array}$ & $\begin{array}{c}\text { LA2 } \\
(\text { Boom })\end{array}$ & $\begin{array}{c}\text { LA3 } \\
(\mathrm{Jib})\end{array}$ & $\begin{array}{c}\text { Winch } \\
\text { Motor }\end{array}$ \\
\hline$e_{d b}$ & $0.25 \mathrm{~mm}$ & $0.25 \mathrm{~mm}$ & $0.25 \mathrm{~mm}$ & $0.25^{\circ}$ \\
$K_{p}$ & 0.6 & 0.45 & 0.59 & 0.015 \\
$K_{i}$ & 0 & 0 & 0 & 0.2 \\
$K_{d}$ & 0 & 0 & 0 & 0.00075 \\
$\Delta t$ & $0.010 \mathrm{~s}$ & $0.010 \mathrm{~s}$ & $0.010 \mathrm{~s}$ & $0.010 \mathrm{~s}$ \\
$u_{\text {sat }, l l}$ & $-1.505 \mathrm{~V}$ & $-1.505 \mathrm{~V}$ & $-1.505 \mathrm{~V}$ & -0.1535 \\
$u_{\text {sat }, u l}$ & $1.505 \mathrm{~V}$ & $1.125 \mathrm{~V}$ & $1.475 \mathrm{~V}$ & 0.17 \\
$u_{d b, l l}$ & $-0.145 \mathrm{~V}$ & $-0.145 \mathrm{~V}$ & $-0.145 \mathrm{~V}$ & -0.0165 \\
$u_{d b, u l}$ & $0.145 \mathrm{~V}$ & $0.525 \mathrm{~V}$ & $0.175 \mathrm{~V}$ & 0 \\
$u_{d b}$ & $0.005 \mathrm{~V}$ & $0.005 \mathrm{~V}$ & $0.005 \mathrm{~V}$ & 0.0035 \\
\hline
\end{tabular}

defined as an angular position (deg) relative to its zero position. The zero position corresponds to a pendulum length of $325 \mathrm{~mm}$ with the jib fully retracted. As the winch is used to control the pendulum length, the measured angle from the encoder is offset by an angle corresponding to both the initial length and the increase in length caused by extending the jib as shown in Figure 3.15 and described by Equations 3.1-3.4. These lengths and the desired length are converted to angular positions by equating them to an arc length with a nominal winch diameter of $41.32 \mathrm{~mm}$ and solving for the central angle. The error for the winch PID controller is then calculated as an 
angular error in degrees. Now that the joint control is established, a control method for motion compensation can be developed. 


\subsection{LQR-Based Motion Compensation}

A nonlinear variable length pendulum-trolley model was derived in Chapter 4 to represent the crane tip and the suspended load. The model was linearised about an operating point to produce a linear state-space model of the form given by Equations 4.138-4.139, which is repeated here for convenience as

$$
\begin{aligned}
& \dot{x}=\tilde{A} x+\tilde{B} \tilde{u}, \\
& y=\tilde{C} x+\tilde{D} \tilde{u},
\end{aligned}
$$

where the system matrices $\{\tilde{A}, \tilde{B}, \tilde{C}, \tilde{D}\}$ are defined by Equations 4.141-4.144. The system states are defined as

$$
x=\left[\begin{array}{llllllllllll}
x_{t} & y_{t} & z_{t} & \theta_{x} & \theta_{y} & l_{p} & \dot{x_{t}} & \dot{y}_{t} & \dot{z}_{t} & \dot{\theta_{x}} & \dot{\theta}_{y} & \dot{l}_{p}
\end{array}\right]^{T},
$$

where $\left\{x_{t}, y_{t}, z_{t}\right\}$ are the Cartesian coordinates of the crane tip in the world frame, $\left\{\theta_{x}, \theta_{y}\right\}$ are the pendulum angles about the $\mathrm{x}$ - and $\mathrm{y}$-axis of a translating world frame attached to the crane tip, and $l_{p}$ is the length of the pendulum from the crane tip to the centre of the load. For motion compensation, the system must be brought to a desired equilibrium point that is defined as

$$
x_{d}=\left[\begin{array}{llllllllllll}
x_{t d} & y_{t d} & z_{t d} & 0 & 0 & l_{p d} & 0 & 0 & 0 & 0 & 0 & 0
\end{array}\right]^{T},
$$

where the crane tip position is given as $\left\{x_{t d}, y_{t d}, z_{t d}\right\}$ with zero pendulum angles and a pendulum length of $l_{p d}$ to indirectly define the desired load position. To bring the system to the desired equilibrium, a control algorithm based on the linear-quadratic regulator (LQR) is developed. 
The state feedback control law of a linear-quadratic regulator, $\tilde{u}=-K x$, is chosen to minimize a quadratic cost function $J(\tilde{u})$ defined in MATLAB [66] as

$$
J(\tilde{u})=\int_{0}^{\infty}\left(x^{T} Q x+\tilde{u}^{T} R \tilde{u}+2 x^{T} N \tilde{u}\right) d t
$$

where $x$ is the vector of system states, $\tilde{u}$ is the vector of system inputs, and $Q, R$, and $N$ are cost function matrices used to penalize states/inputs. The optimal gain matrix $K$ that minimizes the above cost function is defined as

$$
K=R^{-1}\left(B^{T} S_{R}+N^{T}\right)
$$

where $S_{R}$ is the solution of

$$
\tilde{A}^{T} S_{R}+S_{R} \tilde{A}-\left(S_{R} \tilde{B}+N\right) R^{-1}\left(\tilde{B}^{T} S_{R}+N^{T}\right)+Q=0
$$

which is a Riccati equation [66]. Considering the feedback control law, $\tilde{u}=-K x$, the state-space model of Equation 6.12 becomes

$$
\dot{x}=(\tilde{A}-\tilde{B} K) x,
$$

which defines a linear autonomous system. The linear-quadratic regulator is used to adjust the eigenvalues/poles of the system such that $(\tilde{A}-\tilde{B} K)$ is a Hurwitz matrix and the system is stabilized about the origin of the state-space. From the stability of the linear system, the nonlinear system may be considered locally stable by the indirect method of Lyapunov. For motion compensation, the system must be brought to a general equilibrium point by moving the origin, which is equivalent to defining a controller to stabilise the error dynamics about the origin of the error state-space. 
The error state is defined as $e=x_{d}-x$ and the corresponding control law is selected as $\tilde{u}=K e$, and substituting into the model of Equation 6.12 yields

$$
\dot{x_{d}}-\dot{e}=\tilde{A} x_{d}-\tilde{A} e+\tilde{B} K e,
$$

where the desired equilibrium point $x_{d}$ is a solution of the original system such that substituting $x=x_{d}$ into Equation 6.12 yields $\dot{x_{d}}=\tilde{A} x_{d}+\tilde{B} e=\tilde{A} x_{d}+\tilde{B} K\left(x_{d}-x_{d}\right)=$ $\tilde{A} x_{d}$. Therefore, the error model may be rewritten as

$$
\dot{e}=\tilde{A} e-\tilde{B} K e=\tilde{A} e+\tilde{B} v
$$

where a modified control law $v=-K e$ is defined to match the standard model form. The controller may be found as a linear-quadratic regulator using the method described above. The LQR will modify the eigenvalues of the system such that the error states converge to the origin of the error state-space and, therefore, the system approaches the desired state. It is noted that the original control law $\tilde{u}=K e$ is defined by the same $K$ matrix as the modified control law.

The $\left[K, S \_R, e i g\right]=\operatorname{LQR}(A, B, Q, R, N)$ MATLAB command is used to return the gain matrix $K$, the solution of the Riccati equation $S_{R}$, and the modified eigenvalues eig. The $A$ and $B$ matrices are defined by the Jacobians presented in Equations 4.1414.142 with an initial pendulum length $l_{p}$. The cost function matrices of Equation 6.16 are defined with diagonal matrices such that

$$
\begin{aligned}
& Q=\operatorname{diag}\left(\left[\begin{array}{llll}
Q_{1,1} & Q_{2,2} & \ldots & Q_{12,12}
\end{array}\right]\right), \\
& R=\operatorname{diag}\left(\left[\begin{array}{llll}
R_{1,1} & R_{2,2} & \ldots & R_{6,6}
\end{array}\right]\right) \text {, } \\
& N=0 \text {, }
\end{aligned}
$$


where the numerical values for each element in $\mathrm{Q}$ and $\mathrm{R}$ are selected to penalize the corresponding states and inputs, respectively. In the current thesis work, $Q_{1,1}$ to $Q_{12,12}$ are selected as 10 , and $R_{1,1}$ to $R_{6,6}$ are selected as 1 . Given the cost function matrices, the optimal gain matrix $K$ is found in the form

$$
K=\left[\begin{array}{cccccccccccc}
K_{1} & 0 & 0 & 0 & -K_{5} & 0 & K_{7} & 0 & 0 & 0 & -K_{11} & 0 \\
0 & K_{2} & 0 & K_{4} & 0 & 0 & 0 & K_{8} & 0 & K_{10} & 0 & 0 \\
0 & 0 & K_{3} & 0 & 0 & 0 & 0 & 0 & K_{9} & 0 & 0 & 0 \\
0 & 0 & 0 & 0 & 0 & 0 & 0 & 0 & 0 & 0 & 0 & 0 \\
0 & 0 & 0 & 0 & 0 & 0 & 0 & 0 & 0 & 0 & 0 & 0 \\
0 & 0 & 0 & 0 & 0 & K_{6} & 0 & 0 & 0 & 0 & 0 & K_{12}
\end{array}\right]
$$

which reveals an equivalent proportional-derivative (PD) control structure with gains defined by $K_{1}-K_{12}$ that act on the error in world-frame tip coordinates, pendulum angles, and cable length. Linearising the system with various pendulum lengths in the range of $0.050-1.000 \mathrm{~m}$ does not have a significant effect on the final gain matrix, so the controller will likely be robust to changes in length. It is noted that the control action $\tilde{u}$ was defined in Chapter 4 from the kinematic constraints as

$$
\tilde{u}=\ddot{u}=\left[\begin{array}{llllll}
\ddot{u}_{x_{t}} & \ddot{u}_{y_{t}} & \ddot{u}_{z_{t}} & 0 & 0 & \ddot{u}_{l_{p}}
\end{array}\right]^{T}
$$

where $\ddot{u}$ defines the controllable kinematic inputs as the accelerations of the crane tip in the world frame and the acceleration of the pendulum length. However, the feedback control of the system is accomplished through position sensors and joint controllers that are designed to track position set-points. Therefore, it is both desirable and convenient to redefine the control action as a change in position such that the world-frame position set-points are offset from the current measured position by a 
delta control action before converting to the joint-space for independent joint control. Thus, the delta control action is defined as

$$
u_{\Delta}=\left[\begin{array}{llllll}
\Delta x_{t} & \Delta y_{t} & \Delta z_{t} & 0 & 0 & \Delta l_{p}
\end{array}\right]^{T}
$$

and position set-points are calculated relative to the measured states as

$$
\begin{aligned}
& x_{t, s p}=x_{t}+\Delta x_{t}, \\
& y_{t, s p}=y_{t}+\Delta y_{t}, \\
& z_{t, s p}=z_{t}+\Delta z_{t}, \\
& l_{p, s p}=l_{p}+\Delta l_{p},
\end{aligned}
$$

where the position set-points $\left\{x_{t, s p}, y_{t, s p}, z_{t, s p}, l_{p, s p}\right\}$ are converted to actuator setpoints using the inverse kinematic model for independent joint control. The delta control law is assumed to be similar to the acceleration control such that $u_{\Delta}=K_{\Delta} e$ and a gain matrix is assumed as

$$
\begin{aligned}
K_{\Delta}= & {\left[\begin{array}{cc}
K_{P \Delta} & K_{D \Delta}
\end{array}\right], } \\
K_{P \Delta}= & {\left[\begin{array}{cccccc}
K_{P \Delta x_{t}} & 0 & 0 & 0 & -K_{P \Delta \theta_{y}} & 0 \\
0 & K_{P \Delta y_{t}} & 0 & K_{P \Delta \theta_{x}} & 0 & 0 \\
0 & 0 & K_{P \Delta z_{t}} & 0 & 0 & 0 \\
0 & 0 & 0 & 0 & 0 & 0 \\
0 & 0 & 0 & 0 & 0 & 0 \\
0 & 0 & 0 & 0 & 0 & K_{P \Delta l_{p}}
\end{array}\right], }
\end{aligned}
$$




$$
K_{D \Delta}=\left[\begin{array}{cccccc}
K_{D \Delta x_{t}} & 0 & 0 & 0 & -K_{D \Delta \theta_{y}} & 0 \\
0 & K_{D \Delta y_{t}} & 0 & K_{D \Delta \theta_{x}} & 0 & 0 \\
0 & 0 & K_{D \Delta z_{t}} & 0 & 0 & 0 \\
0 & 0 & 0 & 0 & 0 & 0 \\
0 & 0 & 0 & 0 & 0 & 0 \\
0 & 0 & 0 & 0 & 0 & K_{D \Delta l_{p}}
\end{array}\right] \text {, }
$$

where the $K_{P \Delta}$ elements are proportional gains and the $K_{D \Delta}$ elements are derivative gains with structure and signs that are inherited from the acceleration control matrix in Equation 6.25.

Figure 6.2 shows the implementation of the LQR-based delta control algorithm. The delta control action is converted to actuator set-points by adding the current values of the states and then applying the inverse kinematic model of Chapter 4 . The states are estimated using the techniques discussed in Chapter 5. The structure of the controller is based on the LQR gain matrix. By applying delta control actions to determine the position set-points as described above, an increase in the delta control action will result in an increase in the actuator position error and a corresponding increase in the actuator velocity through the proportional controllers used for the individual actuators. This result is similar to the effect of applying the LQR control at the acceleration level; however, it not considered optimal control and tuning is necessary to ensure adequate performance. Through manual tuning, the gains are chosen as $K_{P \Delta x_{t}}=K_{P \Delta y_{t}}=K_{P \Delta z_{t}}=K_{P \Delta l_{p}}=1, K_{P \Delta \theta_{x}}=K_{P \Delta \theta_{y}}=0.1$, and all elements of $K_{D \Delta}$ are set to zero due to noise in the velocity estimates. These gains are selected to keep the set-point within the workspace and ensure stability for all the test cases considered in this thesis. 


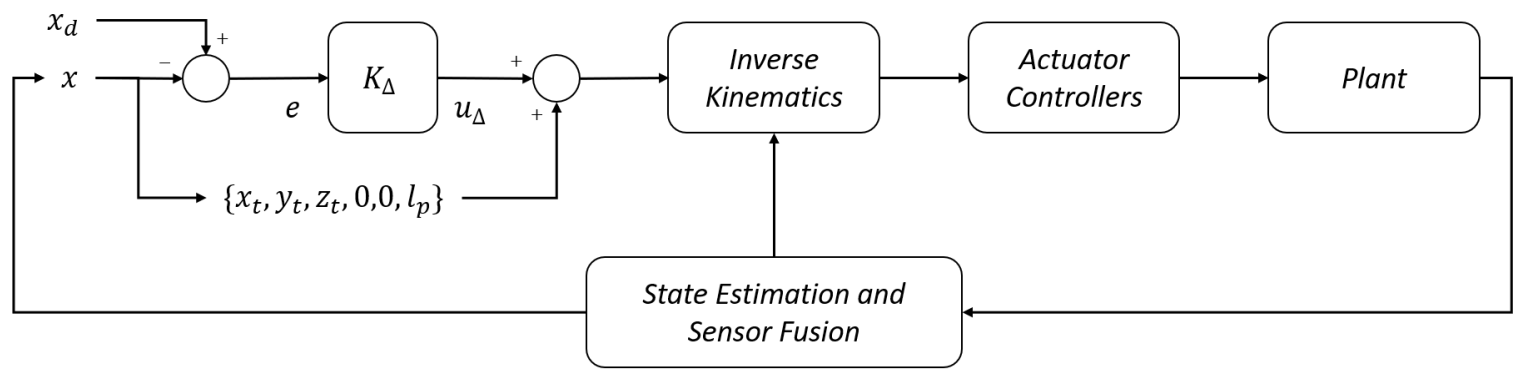

Figure 6.2: Block diagram of the LQR-based algorithm.

As noted, the form of the LQR control law is equivalent to a PD control architecture. Figure 6.3 shows the LQR-based controller replaced by an equivalent set of six PD controllers that may be initialised using the gains of the $K_{\Delta}$ matrix. The control architecture revealed by converting the gain matrix to PD controllers may also be used as a base for the application of advanced tuning algorithms in future work.

In practice, sensor noise and/or minor inaccuracies in the estimation algorithm result in inaccurate error states that create oscillations in the set-points determined by the controller and thereby reduce the effectiveness of the motion compensation efforts. To avoid these oscillations, error deadbands are implemented by setting the corresponding error to zero if its magnitude is below a deadband threshold. The threshold for the linear motion errors of $x_{t}, y_{t}, z_{t}$ and $l_{p}$ is $0.0005 \mathrm{~m}(0.5 \mathrm{~mm})$, and the threshold for the angular motion errors of $\theta_{x}$ and $\theta_{y}$ is $0.0175 \mathrm{rad}(1 \mathrm{deg})$. In addition, the control law is modified during implementation by offsetting the desired pendulum length $l_{p d}$ by the error in the z-position of the tip such that $l_{p d, n e w}=l_{p d, o l d}+\left(z_{t d}-z_{t}\right)$ is used as the new desired pendulum length. This modification allows the winch to further reduce tracking error of the world-frame load position when the crane tip is unable to track its desired position. For example, if substantial heave motion causes the z-position of the tip to saturate at the bound of the workspace, the pendulum length could be varied such that heave compensation is still performed. 


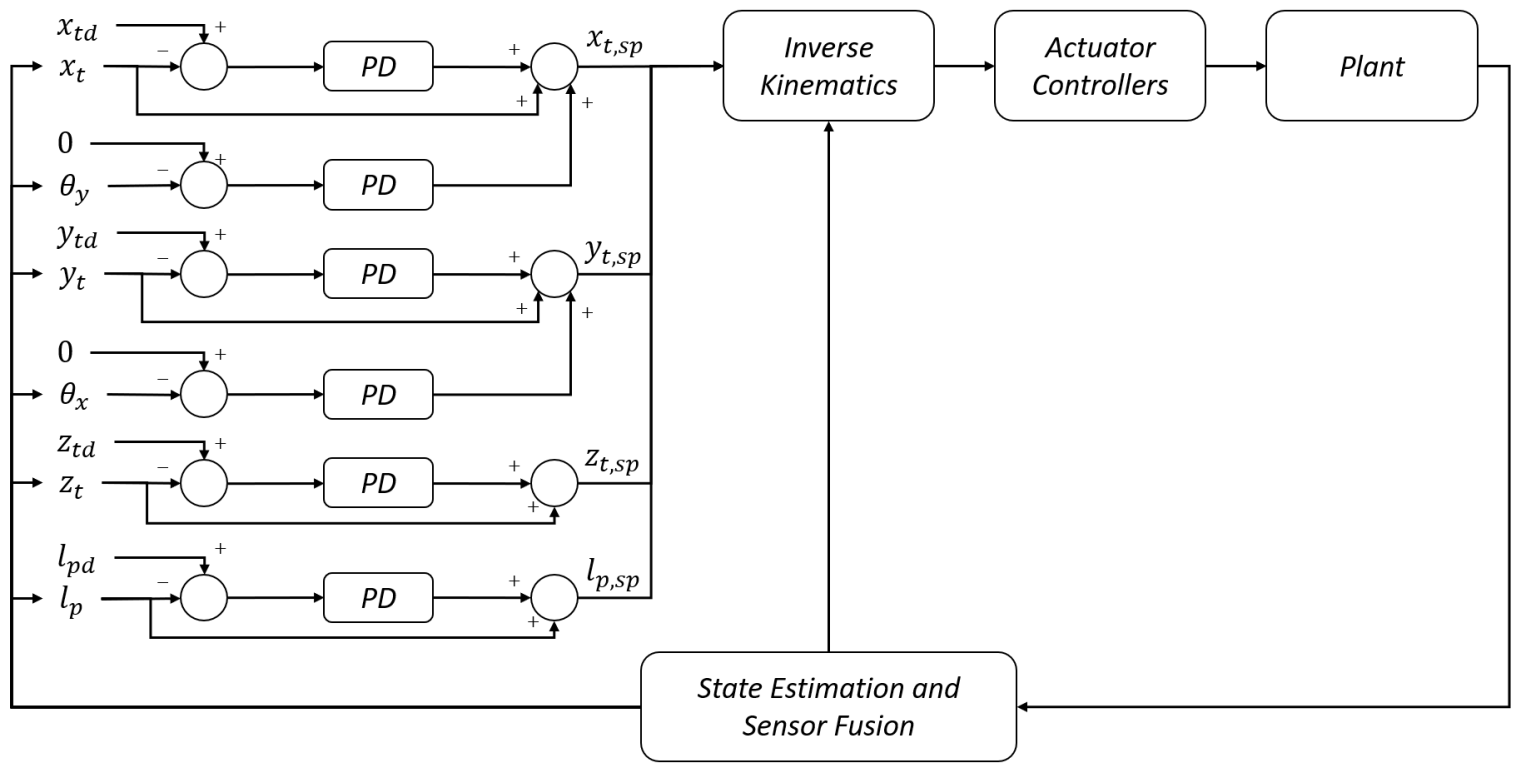

Figure 6.3: Block diagram of the LQR-based algorithm as equivalent PD controllers. 


\subsection{Conclusion/Summary}

In this chapter, a combined compensation system was presented with independent PID joint control and an LQR-based world-frame compensation algorithm. It is noted that the compensation system predominantly uses the linear actuators to compensate for the ship motion by moving the crane tip. The combined compensation system presented herein is intended for off-ship operations in which an operator must position a load accurately with respect to another body while the crane base experiences general ship motion. Ideally, the target body would be stationary with respect to the world frame, such as in offshore construction projects or in ship-to-platform transfers. If the body is not stationary, substantially more operator input may be required to accurately position the load. In particular, the vertical tip position should be maintained sufficiently above the other body and the operator must manually deposit the load. In future work, the accuracy of ship-to-ship transfer may be improved by compensating for the relative motion while simultaneously depositing the load during a period of minimal ship motion. The work included in this chapter is directly applicable to the first thesis objective. In the next chapter, the results of testing on the physical system and in simulation are presented and discussed. 


\section{Chapter 7}

\section{Results and Discussion}

This chapter presents and discusses the results of the 437 test-scale experiments and the corresponding simulations. In Section 7.1, the results of the experiments and simulations are briefly summarized. In Section 7.2 , the ability of the compensation system to dissipate energy is evaluated and compared to the free response of the pendulum. The first set of 40 tests and simulations in this section quantify the natural (uncompensated) response of the system for a range of eight initial angles with varying magnitude and direction for five pendulum lengths. These tests provide an estimate of the natural damping characteristics for use in simulation and create a baseline for evaluating the energy dissipation performance of the compensation system. The energy dissipation performance is evaluated through the $\theta_{x}, \theta_{y}$, and height time constants by fitting an exponential decay function to the measured response. In addition to the time constants, the cumulative distance travelled by the load while its velocity exceeds a noise threshold is examined, and the set-point tracking rootmean-squared error (RMSE) is calculated by considered the Euclidean distance from the set-point/equilibrium as a tracking error. The second set of 40 tests and simulations in this section quantify the forced (compensated) response of the system to 
the same initial angles and pendulum lengths. These tests are evaluated using the same performance metrics applied to the uncompensated cases, including the angular and height decay time constants, distance travelled, and set-point tracking RMSE. To improve the statistical significance of the conclusions, the energy dissipation tests are repeated between 3-12 times for a total of 345 experiments. The simulations are repeated with two variations, including truth data feedback (estimation algorithms are bypassed by inputting truth data to the controller) and simulated sensor feedback (noise is included on all sensors and the IMU feedback is emulated for both the load and ship), to analyse the performance impact of imperfect estimates of the ship and load motion.

In Section 7.3, the ability of the compensation system to track a fixed set-point while subject to base excitation is evaluated and compared to an uncompensated system. The uncompensated system uses only the inverse kinematics to position the crane tip and extend/retract cable without generating anti-pendulum control actions. Three sets of scaled ship motion profiles are applied for a fixed set-point, and an additional five sets of sinusoidal surge/sway motion profiles are applied to excite the natural frequencies of the pendulum load for five additional set-points. The tests are repeated 3-6 times for a total of 61 experiments, and the corresponding simulations are repeated with the truth/sensor variations described above to analyse performance impacts of imperfect estimates of the ship and load motion. The performance of the compensation system is evaluated by examining the ellipsoid volume, distance travelled, and set-point tracking RMSE. The ellipsoid volume is calculated from a fitted ellipsoid that contains the centroid of the load for $95 \%$ of the test period.

In Section 7.4, the ability of the compensation system to track a time-varying set-point while subject to base excitation is evaluated and compared to an uncompensated algorithm. The same three sets of scaled ship motion profiles are applied 
with a variable set-point profile for the crane motion that is intended to represent an off-ship pick-and-place operation. A case without ship motion is included to form a baseline for the tracking performance. The pendulum length varies to alter the natural frequency of the system throughout the test period, and undesired motion of load is introduced through both the base excitation and the motion of the crane tip relative to its base. The tests are repeated 3-5 times for a total of 31 experiments, and the corresponding simulations are repeated with the truth/sensor variations described above to analyse performance impacts of imperfect estimates of the ship and load motion. Similar to the fixed set-point tracking tests, the performance of the compensation system is evaluated by examining the ellipsoid volume, distance travelled, and set-point tracking RMSE. For these variable set-point tests, the ellipsoid volume is calculated by considering the position of the load relative to its set-point throughout the test period rather than its absolute position. 


\subsection{Summary of Results}

The detailed results of the experiments and simulations are described throughout the chapter using bar graphs, and percentage reductions or changes are used to describe the improvements of the compensated metrics compared to the corresponding uncompensated metrics. For the purpose of this work, a percentage reduction, change, or difference is defined as

$$
\% \text { Reduction, Change, or Difference }=\left(\frac{\text { Value }- \text { Reference }}{\text { Reference }}\right) \times 100,
$$

where "a reduction of 50\%" is considered equivalent to a percentage change or difference of $-50 \%$ as the negative change is implied by the term "reduction." The "Value" in Equation 7.1 corresponds to the value of the compensated metric, whereas the "Reference" corresponds to the uncompensated metric. In this section, the results of the chapter are briefly summarized by mean percentage reductions for the experiments, the simulations (1) with truth data feedback, and the simulations (2) with simulated sensor feedback. The truth data simulations indicate the performance of the compensation system with ideal/perfect knowledge of the load and ship motion, whereas the sensor-based simulations demonstrate the effects of sensor noise and the imperfect estimation algorithm on the overall performance of the system.

The results of the Energy Dissipation experiments and simulations of Section 7.2 are summarized in Table 7.1 as mean percentage reductions for each of the performance metrics. In experiments, the angular time constants exhibit mean reductions of $88.7-90.9 \%$ and the height time constant shows a mean reduction of $88.2 \%$. Similar results are observed in the simulations with mean reductions of $89.5-95.0 \%$ for the angular time constants and $93.5-97.2 \%$ for the height time constant. The reductions in these time constants correspond directly to reduced settling times and increased 
rates of energy dissipation. The energy dissipation tests also show mean reductions in distance travelled of $77.8 \%$ in experiments and $92.7-95.2 \%$ in simulations. Similarly, the energy dissipation tests show mean reductions in set-point tracking RMSE of $54.8 \%$ in experiments and $72.2-75.7 \%$ in simulations. The reductions in distance travelled and RMSE indicate significantly less undesired motion of the load; however, the measurement noise associated with the stereo vision system results in larger deviations between the simulated and experimental results. The large percentage reductions for all metrics in the energy dissipation tests demonstrate the ability of the compensation system to dissipate energy at a rate that is considerably higher than the natural damping of the system while reducing tracking error and undesired motion.

Differences between the simulations and experiments are attributed to the unmodelled imperfections of the real-world system, including cable twist and variations in the magnetic field experienced by the two IMUs. The sensor-based simulations (2) are more representative of the complete experimental system as the IMUs are emulated, sensor noise is applied, and the estimation algorithm is used. It is noted that imperfections in the estimation algorithm result in performance improvements over the truth data simulations (1) in many cases. These performance improvements are a result of increased control actions due to overshoot and/or estimation errors that result in motion compensation control actions after the pendulum has entered the angular deadband of the controller. However, these improvements are specific to the simulations as the negative impact of rotation about the cable axis and variations in the magnetic fields experienced by the IMUs would outweigh the potential benefits of the estimation error, which is confirmed through the decreased performance of the experimental system.

The results of the Fixed Set-Point Tracking experiments and simulations of Sec- 
tion 7.3 are summarized in Table 7.2 as mean percentage reductions for each of the performance metrics. The upper half of the table represents the mean values for cases with scaled ship motion profiles, whereas the lower half of the table represents mean values for cases where sinusoidal base excitations were applied to excite the natural frequency of the pendulum.

In the upper half of Table 7.2, the mean change in the experimental ellipsoid volume represents an increase for the test cases with scaled ship motion, which is indicated by the bracketed value with an up-arrow. The increase in volume, which is discussed in Section 7.3, is likely a result of the ellipsoid fitting procedure coupled with minor deviations in the shape of the point cloud under compensation. The simulation results, however, show mean reductions of $49.9-66.8 \%$ in ellipsoid volume that are not affected by measurement error or other imperfections of the experimental system. Due to negligible motion above the velocity noise threshold, the distance travelled metric is excluded and considered inappropriate for these cases. The setpoint tracking RMSE shows mean reductions of $8.6 \%$ in experiments and $7.1-25.5 \%$ in simulations. The RMSE results are not affected by the shape of the point cloud, which is a limitation of the ellipsoid volume metric. Similarly, the RMSE results do not include a noise threshold, which is a limitation of the distance travelled metric in cases where the load motion occurs predominantly below the threshold. Therefore, the RMSE results are considered the most appropriate for the fixed set-point tracking performance when scaled ship motion is applied to excite the base of the crane. The mean reductions in the RMSE values indicate improved set-point tracking performance when the compensation system is applied for these cases.

In the lower half of Table 7.2, significant improvements in all performance metrics are shown for the test cases where sinusoidal base excitations were applied to excite the natural frequency of the pendulum. The ellipsoid volume shows mean reductions 
of $99.4 \%$ for experiments and $100 \%$ for simulations, the distance travelled shows mean reductions of $85.4 \%$ for experiments and $89.6-93.5 \%$ for simulations, and the set-point tracking RMSE shows mean reductions of $80.1 \%$ for experiments and $87.6-89.6 \%$ for simulations. These significant reductions demonstrate the ability of the compensation system to decouple the motion of the ship from that of the load and thereby reduce undesired motion.

The results of the Variable Set-Point Tracking experiments and simulations of Section 7.4 are summarized in Table 7.3 as mean percentage reductions for each of the performance metrics. The ellipsoid volume shows mean reductions of $34.5 \%$ in experiments and $52.9-77.0 \%$ in simulations, which indicates that the load remains in a smaller volume with the compensation system. The distance travelled also shows a mean reduction of $78.8 \%$ for the experimental results; however, the majority of motion occurs at or below the noise threshold and comparative simulation values can not be computed. The set-point tracking RMSE shows mean reductions of $36.4 \%$ in experiments and 7.8-29.9\% in simulation. Again, due to low levels of excitation caused by the scaled ship motion, the RMSE results are considered the most appropriate for evaluating the variable set-point tracking performance. The reductions in RMSE indicate improved tracking performance for the compensation system compared to uncompensated cases.

In the following section, the detailed results of the Energy Dissipation experiments and simulations are provided and discussed. 
Table 7.1: Summary of mean energy dissipation results.

\begin{tabular}{|c|c|c|c|}
\hline Performance Metric & $\begin{array}{c}\text { \% Reduction } \\
\text { Experimental }\end{array}$ & $\begin{array}{c}\text { \% Reduction } \\
\text { Simulation (1) }\end{array}$ & $\begin{array}{c}\text { \% Reduction } \\
\text { Simulation (2) }\end{array}$ \\
\hline$\theta_{x}$ Time Constant & $88.7 \%$ & $89.7 \%$ & $95.0 \%$ \\
$\theta_{y}$ Time Constant & $90.9 \%$ & $89.5 \%$ & $94.7 \%$ \\
Height Time Constant & $88.2 \%$ & $93.5 \%$ & $97.2 \%$ \\
Distance Travelled & $77.8 \%$ & $92.7 \%$ & $95.2 \%$ \\
Set-Point Tracking RMSE & $54.8 \%$ & $72.2 \%$ & $75.7 \%$ \\
\hline
\end{tabular}

Table 7.2: Summary of mean fixed set-point tracking results.

\begin{tabular}{|c|c|c|c|}
\hline & $\begin{array}{c}\% \text { Reduction } \\
\text { Experimental }\end{array}$ & $\begin{array}{c}\text { Reduction } \\
\text { Simulation (1) }\end{array}$ & $\begin{array}{c}\% \text { Reduction } \\
\text { Simulation (2) }\end{array}$ \\
\hline Performance Metric & & & \\
Ship Motion: & $(74.7 \% \uparrow)$ & $66.8 \%$ & $49.9 \%$ \\
Set-Point Tracking RMSE & $8.6 \%$ & $7.1 \%$ & $25.5 \%$ \\
\hline \hline Natural Frequency Excitation: & & & \\
Ellipsoid Volume & $99.4 \%$ & $100 \%$ & $100 \%$ \\
Distance Travelled & $85.4 \%$ & $93.5 \%$ & $89.6 \%$ \\
Set-Point Tracking RMSE & $80.1 \%$ & $89.6 \%$ & $87.6 \%$ \\
\hline
\end{tabular}

Table 7.3: Summary of variable set-point tracking results.

\begin{tabular}{|c|c|c|c|}
\hline Performance Metric & $\begin{array}{c}\text { \% Reduction } \\
\text { Experimental }\end{array}$ & $\begin{array}{c}\text { \% Reduction } \\
\text { Simulation (1) }\end{array}$ & $\begin{array}{c}\text { \% Reduction } \\
\text { Simulation (2) }\end{array}$ \\
\hline Ellipsoid Volume & $34.5 \%$ & $77.0 \%$ & $52.9 \%$ \\
Distance Travelled & $78.8 \%$ & - & - \\
Set-Point Tracking RMSE & $36.4 \%$ & $7.8 \%$ & $29.9 \%$ \\
\hline
\end{tabular}




\subsection{Energy Dissipation}

In this section, the ability of the compensation system to dissipate energy is evaluated and compared to the free response of the pendulum load. The first set of 40 tests are used to quantify the natural (uncompensated) response of the pendulum to a range of eight initial angles with varying magnitude and direction for five pendulum lengths. The parameters for these tests are summarized in Table 7.4. The crane and motion platform are off for all tests, no base excitation is applied, and five pendulum lengths 400, 550, 700, 850, and $1000 \mathrm{~mm}$ are examined. The lengths represent a set of natural frequencies $4.95,4.22,3.74,3.40$, and $3.13 \mathrm{rad} / \mathrm{s}$, which provide some insight into the frequency response of the system. The initial conditions are reported as mean initial angles in the $\theta_{x}$ and $\theta_{y}$ directions to further subdivide the results into eight groups ranging from $-8.5^{\circ}$ to $-23^{\circ}$ in the $\theta_{x}$ direction and $7.6^{\circ}$ to $24^{\circ}$ in the $\theta_{y}$ direction. The tests provide an estimate of the natural damping characteristics for use in the simulations and create a baseline for evaluating the energy dissipation performance of the compensated system. Summarized in Table 7.5, the second set of 40 tests quantify the compensated response of the system with the same pendulum lengths and initial conditions as the free response tests of Table 7.4. The compensation system is on for all tests, no base excitation is applied, and all settings are kept constant throughout the testing. It is noted that tests with angles greater than $20^{\circ}$ could not be performed for $850 \mathrm{~mm}$ and $1000 \mathrm{~mm}$ pendulum lengths due to physical constraints in the operating environment. The tests that could not be performed are indicated by a value of 0 in the 'Repetitions' column of Table 7.4 and Table 7.5. 
Table 7.4: Energy dissipation experiments/simulations (001-040).

\begin{tabular}{|c|c|c|c|c|c|}
\hline Exp/Sim No. & Compensation & $l_{p, s p}(\mathrm{~mm})$ & $\theta_{x, 0}(\mathrm{deg})$ & $\theta_{y, 0}(\operatorname{deg})$ & Repetitions \\
\hline 001 & No & 400 & -8.5 & 0 & 5 \\
\hline 002 & $\mathrm{No}$ & 400 & -13 & 0 & 5 \\
\hline 003 & No & 400 & -18 & 0 & 6 \\
\hline 004 & No & 400 & -23 & 0 & 7 \\
\hline 005 & $\mathrm{No}$ & 400 & 0 & 7.6 & 5 \\
\hline 006 & No & 400 & 0 & 13 & 5 \\
\hline 007 & $\mathrm{No}$ & 400 & 0 & 18 & 7 \\
\hline 008 & No & 400 & 0 & 24 & 7 \\
\hline 009 & No & 550 & -8.5 & 0 & 3 \\
\hline 010 & No & 550 & -13 & 0 & 3 \\
\hline 011 & No & 550 & -18 & 0 & 4 \\
\hline 012 & No & 550 & -23 & 0 & 4 \\
\hline 013 & $\mathrm{No}$ & 550 & 0 & 7.6 & 3 \\
\hline 014 & No & 550 & 0 & 13 & 3 \\
\hline 015 & No & 550 & 0 & 18 & 3 \\
\hline 016 & No & 550 & 0 & 24 & 4 \\
\hline 017 & No & 700 & -8.5 & 0 & 6 \\
\hline 018 & No & 700 & -13 & 0 & 6 \\
\hline 019 & No & 700 & -18 & 0 & 7 \\
\hline 020 & $\mathrm{No}$ & 700 & -23 & 0 & 8 \\
\hline 021 & No & 700 & 0 & 7.6 & 5 \\
\hline 022 & No & 700 & 0 & 13 & 5 \\
\hline 023 & No & 700 & 0 & 18 & 5 \\
\hline 024 & No & 700 & 0 & 24 & 5 \\
\hline 025 & $\mathrm{No}$ & 850 & -8.5 & 0 & 3 \\
\hline 026 & $\mathrm{No}$ & 850 & -13 & 0 & 3 \\
\hline 027 & No & 850 & -18 & 0 & 4 \\
\hline 028 & $\mathrm{No}$ & 850 & -23 & 0 & 0 \\
\hline 029 & No & 850 & 0 & 7.6 & 3 \\
\hline 030 & No & 850 & 0 & 13 & 3 \\
\hline 031 & No & 850 & 0 & 18 & 5 \\
\hline 032 & No & 850 & 0 & 24 & 0 \\
\hline 033 & No & 1000 & -8.5 & 0 & 10 \\
\hline 034 & No & 1000 & -13 & 0 & 5 \\
\hline 035 & No & 1000 & -18 & 0 & 12 \\
\hline 036 & No & 1000 & -23 & 0 & 0 \\
\hline 037 & No & 1000 & 0 & 7.6 & 5 \\
\hline 038 & No & 1000 & 0 & 13 & 7 \\
\hline 039 & No & 1000 & 0 & 18 & 5 \\
\hline 040 & No & 1000 & 0 & 24 & 0 \\
\hline
\end{tabular}


Table 7.5: Energy dissipation experiments/simulations (041-080).

\begin{tabular}{|c|c|c|c|c|c|}
\hline Exp/Sim No. & Compensation & $l_{p, s p}(\mathrm{~mm})$ & $\theta_{x, 0}(\mathrm{deg})$ & $\theta_{y, 0}(\mathrm{deg})$ & Repetitions \\
\hline 041 & Yes & 400 & -8.5 & 0 & 5 \\
\hline 042 & Yes & 400 & -13 & 0 & 6 \\
\hline 043 & Yes & 400 & -18 & 0 & 5 \\
\hline 044 & Yes & 400 & -23 & 0 & 5 \\
\hline 045 & Yes & 400 & 0 & 7.6 & 5 \\
\hline 046 & Yes & 400 & 0 & 13 & 5 \\
\hline 047 & Yes & 400 & 0 & 18 & 5 \\
\hline 048 & Yes & 400 & 0 & 24 & 5 \\
\hline 049 & Yes & 550 & -8.5 & 0 & 3 \\
\hline 050 & Yes & 550 & -13 & 0 & 4 \\
\hline 051 & Yes & 550 & -18 & 0 & 3 \\
\hline 052 & Yes & 550 & -23 & 0 & 5 \\
\hline 053 & Yes & 550 & 0 & 7.6 & 3 \\
\hline 054 & Yes & 550 & 0 & 13 & 3 \\
\hline 055 & Yes & 550 & 0 & 18 & 3 \\
\hline 056 & Yes & 550 & 0 & 24 & 4 \\
\hline 057 & Yes & 700 & -8.5 & 0 & 5 \\
\hline 058 & Yes & 700 & -13 & 0 & 6 \\
\hline 059 & Yes & 700 & -18 & 0 & 5 \\
\hline 060 & Yes & 700 & -23 & 0 & 5 \\
\hline 061 & Yes & 700 & 0 & 7.6 & 5 \\
\hline 062 & Yes & 700 & 0 & 13 & 5 \\
\hline 063 & Yes & 700 & 0 & 18 & 5 \\
\hline 064 & Yes & 700 & 0 & 24 & 6 \\
\hline 065 & Yes & 850 & -8.5 & 0 & 3 \\
\hline 066 & Yes & 850 & -13 & 0 & 3 \\
\hline 067 & Yes & 850 & -18 & 0 & 3 \\
\hline 068 & Yes & 850 & -23 & 0 & 0 \\
\hline 069 & Yes & 850 & 0 & 7.6 & 3 \\
\hline 070 & Yes & 850 & 0 & 13 & 3 \\
\hline 071 & Yes & 850 & 0 & 18 & 3 \\
\hline 072 & Yes & 850 & 0 & 24 & 0 \\
\hline 073 & Yes & 1000 & -8.5 & 0 & 5 \\
\hline 074 & Yes & 1000 & -13 & 0 & 5 \\
\hline 075 & Yes & 1000 & -18 & 0 & 5 \\
\hline 076 & Yes & 1000 & -23 & 0 & 0 \\
\hline 077 & Yes & 1000 & 0 & 7.6 & 5 \\
\hline 078 & Yes & 1000 & 0 & 13 & 5 \\
\hline 079 & Yes & 1000 & 0 & 18 & 5 \\
\hline 080 & Yes & 1000 & 0 & 24 & 0 \\
\hline
\end{tabular}


In addition to the 80 tests listed in Table 7.4 and Table 7.5, 80 simulations are performed with the same parameters as the corresponding experiment. Two variations are run for each simulation, which are denoted by ' 1 ' for simulations that use truth data of the ship and load positions as feedback to the compensation controller, and '2' for simulations that provide noisy simulated sensor feedback to emulate the real-world conditions. During the 'truth' simulations, the estimation algorithms are bypassed in the control software and the truth data is directly used as an ideal 'estimate.' Both simulations allow for confirmation of the performance trends observed in the experiments, and the simulations with simulated sensor feedback provide a preliminary analysis of how imperfect estimates of the load and ship motion impact performance.

The results of the marker tracking procedure of Chapter 3 in Section 3.6 are illustrated by the world data points in Figure 7.1. Figure 7.1a represents the world data points for the first repetition of Test 020, which is an uncompensated test with an initial angle in the $\theta_{x}$ direction. Figure $7.1 \mathrm{~b}$ represents the world data points for the first repetition of Test 060, which is a compensated test with the same parameters as Test 020 . The five points in the top left of each plot represent the five markers drawn on the bottom of the deck to identify the ship frame. Below these deck points are four levels of red points that represent each of the uniformly spaced markers along the cable of the pendulum. By fitting a line to these marker points and using the known offset between the markers and the centroid of the pendulum load, the layer of black points are found as an indication of the pendulum centroid at each point in time. Finally, the world frame marker points are shown as they are drawn/detected on the floor underneath the platform. 


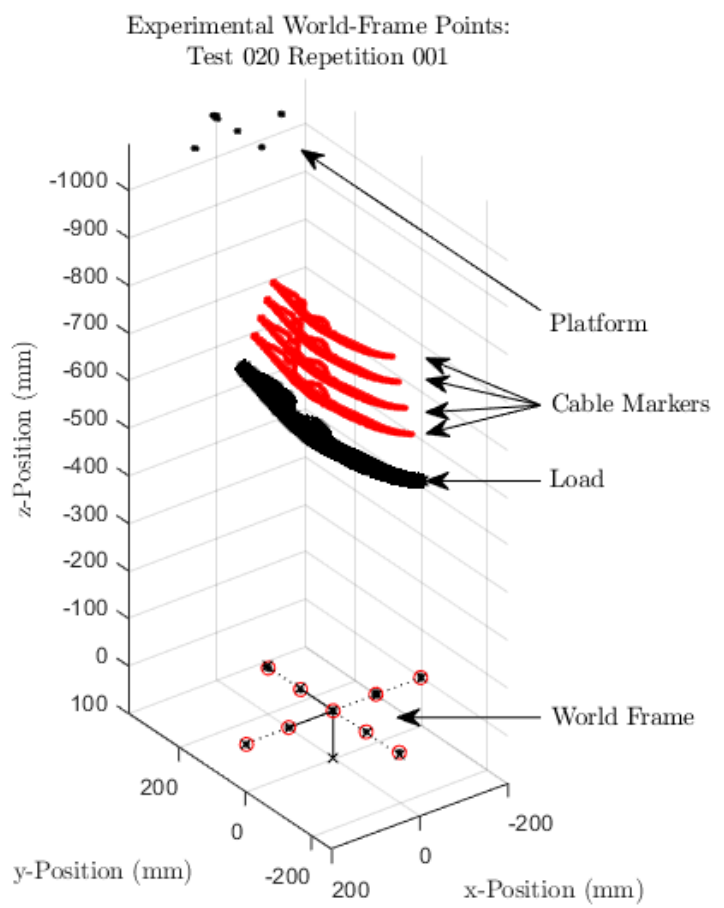

(a) Test 020 Repetition 001

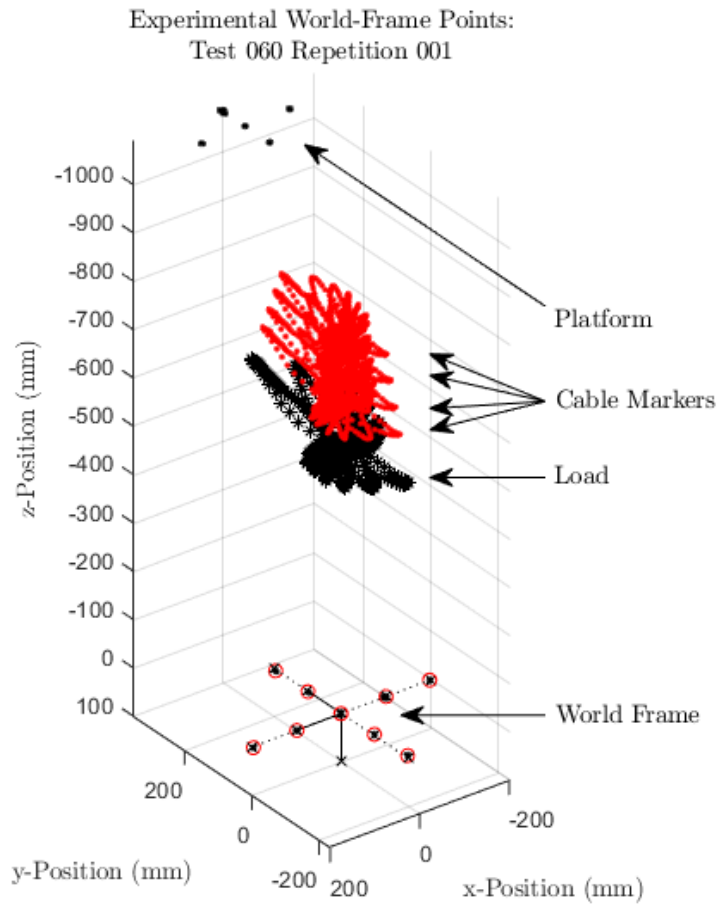

(b) Test 060 Repetition 001

Figure 7.1: World position tracking data for energy dissipation tests. 
To analyse the response of the pendulum, the world data points discussed above are used to generate several key performance indicators, including the angular time constants, the height time constant, the $95 \%$ ellipsoid volume, the distance travelled, and the set-point tracking RMSE. In the upper plots of Figure 7.2 and Figure 7.3, the height of the pendulum relative to its set-point (equilibrium point) is calculated throughout the test duration. These sample results correspond to Test 020 Repetition 001 and Test 060 Repetition 001, and are derived from the world data points of Figure 7.1. The peaks in height are an indication of the mechanical energy stored in the system, and the exponential decay of the height is an indication of the dissipation of energy through damping. A peak detection and least-squares exponential fitting procedure is applied to the detected height, and the time constant of the exponential decay function is calculated as the inverse of the fitted decay constant. The initial height also provides an indicator of the potential energy associated with the initial conditions. The height responses, peak detection results, and exponential fitting results (i.e. the time constant and fitted line) are shown on the plot.

The cable marker points are also used to estimate the pendulum angles $\theta_{x}$ and $\theta_{y}$ by fitting a line to the points and solving for the two angles. In the second and third plots of Figure 7.2 and Figure 7.3, the pendulum angles $\theta_{x}$ and $\theta_{y}$ are calculated throughout the test duration. Similar to the height response, the angular response of the pendulum and the exponential decay of the peak values is an indication of the dissipation of mechanical energy. A similar peak detection and exponential fitting procedure is applied to determine the corresponding time constant; however, as the angular response is sinusoidal with decaying amplitude, the peak detection procedure is applied to a single side of the response that represents motion in the opposite direction of the initial angle. The positive peaks are detected for negative initial $\theta_{x}$ angles, and the negative peaks are detected for positive initial $\theta_{y}$ angles. Therefore, in 
general, the magnitude of the maximum peak in the opposite direction of the initial angle represents the decay over a single half-cycle swing of the pendulum and does not include manual errors that occur while positioning the pendulum at its starting position. The $\theta_{y}$ response for Test 020 Repetition 001 (Figure 7.2) does not include an exponential fit as no significant peak angles were detected and exponential fitting was deemed inappropriate. It is also noted here that Figure $7.1 \mathrm{a}$ contains a set of points that deviate from the natural path of the pendulum as a result of the variations in the $\theta_{y}$ angle that occurred while manually moving the pendulum to its starting position, which is shown in the second plot of Figure 7.1 prior to the start time at 0 seconds.

Each of the aforementioned metrics provide significant insight into the response of the system; however, they are not necessarily sufficient for evaluating the performance of the compensation system due to several limitations. In particular, intentional changes in pendulum length may affect the transient information contained within the height response and thereby influence the exponential fitting procedure. Similarly, for the two angular responses, the damping of one pendulum angle could be a result of the angular motion transferring to the other pendulum angle. The transference of motion from one angular direction to the other is referred to as off-axis motion in the current work. This off-axis motion is more prevalent in compensated test cases, and it is discussed in the following subsection. 
Pendulum Response: Test 020 Repetition 001
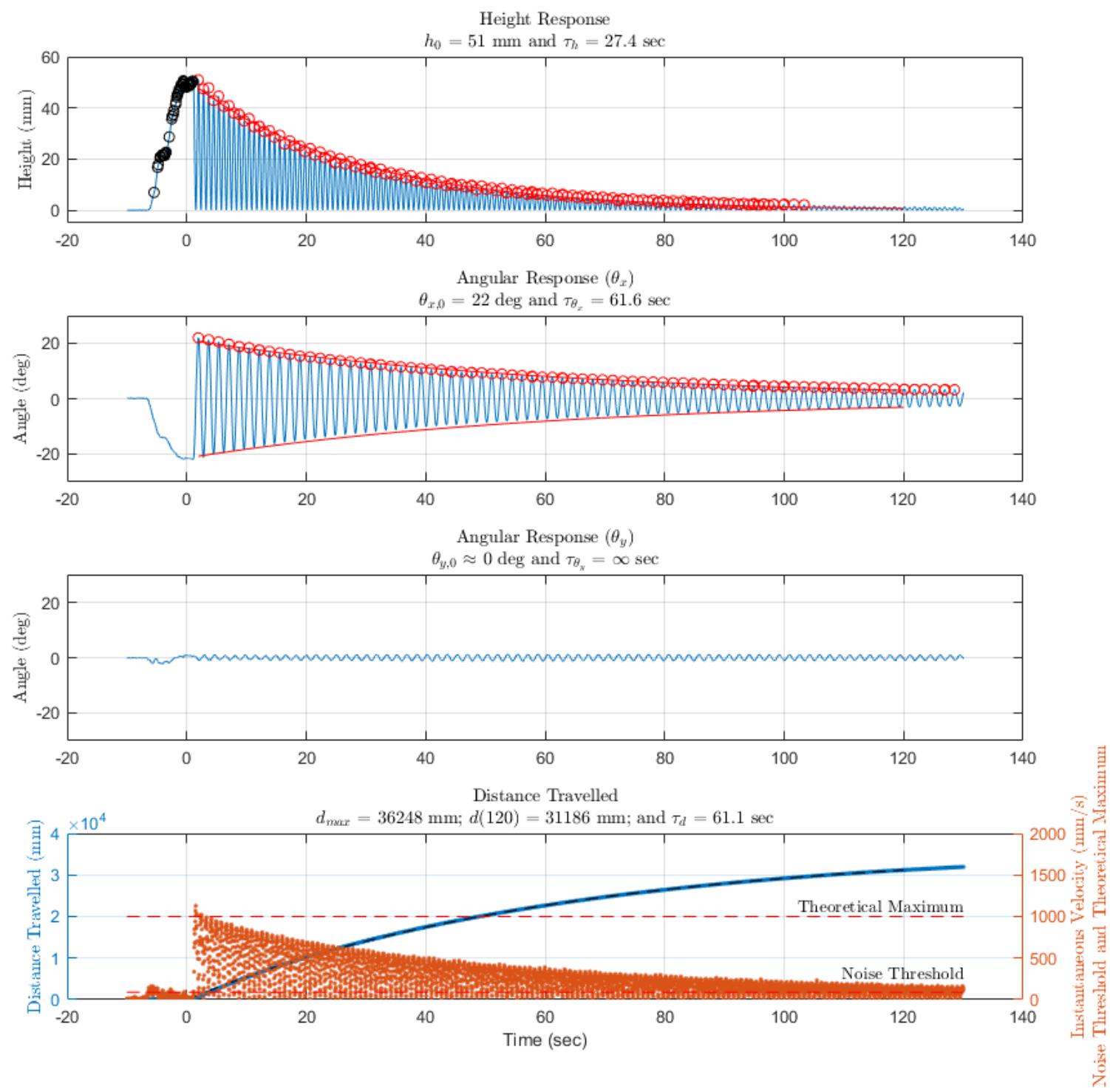

Figure 7.2: Pendulum response with exponential fitting for Test 020 Repetition 001 (uncompensated/free response of a $700 \mathrm{~mm}$ pendulum). 
Pendulum Response: Test 060 Repetition 001
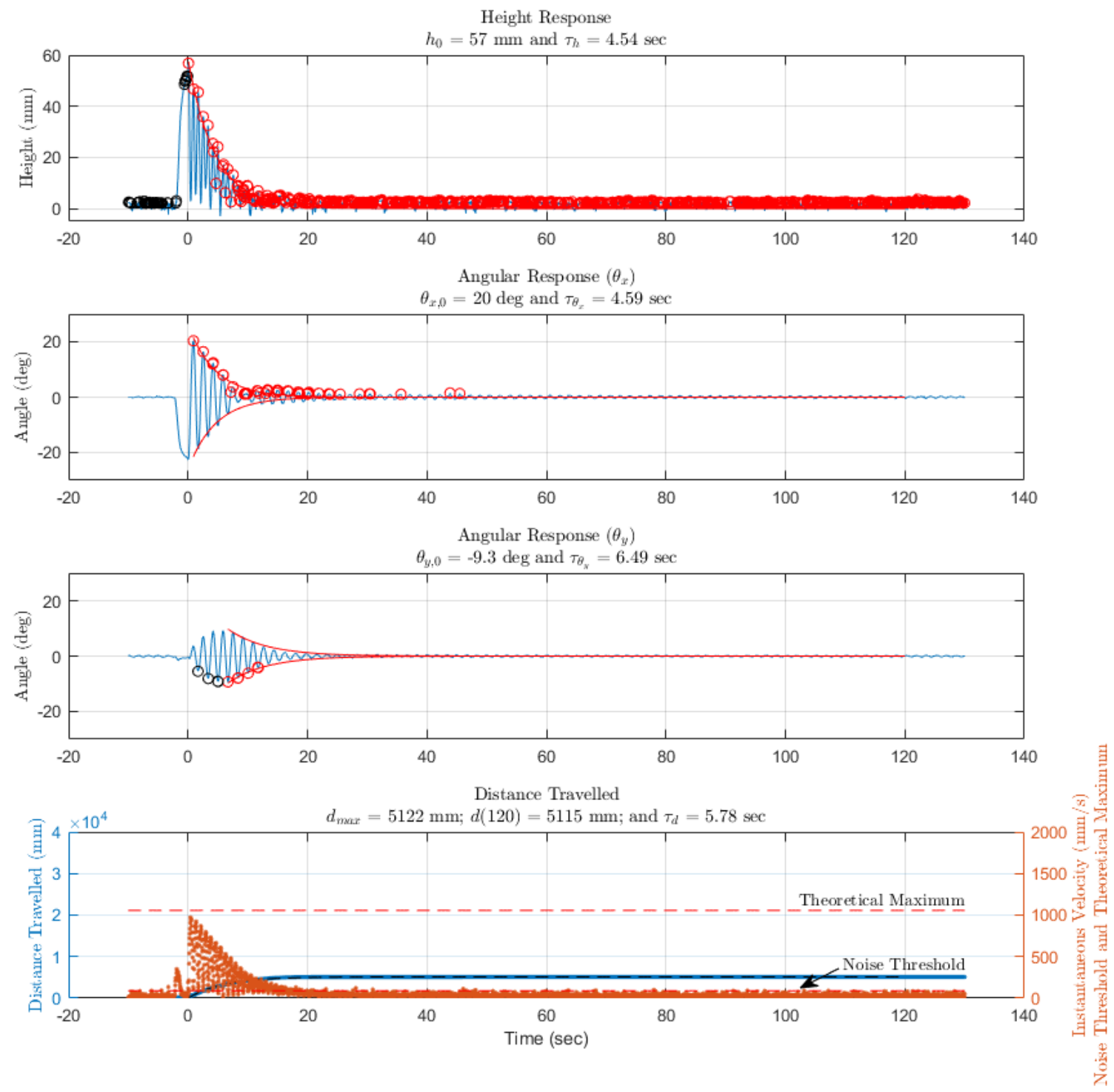

Figure 7.3: Pendulum response with exponential fitting for Test 060 Repetition 001 (compensated response of a $700 \mathrm{~mm}$ pendulum). 


\subsubsection{Off-Axis Motion}

The magnitude of the maximum peak in the opposite direction of the initial angle generally represents the decay over a single half-cycle swing of the pendulum. However, an exception to this observation is for off-axis compensated motion where imperfections in the system lead to a transference of motion from one angular direction to the other as demonstrated in the second and third plots of Figure 7.3 for Test 060 Repetition 001. Observing the second plot of Figure 7.3, the initial angle is applied in the negative $\theta_{x}$ direction and is quickly damped out. However, examining the third plot, the motion is partially transferred to the $\theta_{y}$ direction as demonstrated by the exponential growth and decay during the first 20 seconds of the test. The peaks prior to the decay are illustrated by black circles and are not used in the exponential fitting procedure, whereas the peaks following the maximum peak are indicated with red circles and are fitted with the exponential decay function. This transference of motion is likely due to errors in estimation caused by the load rotating about the cable axis during the test, as well as differences in the magnetic fields and/or magnetometer sensor biases for each of the two IMUs. Due to imperfections in the estimation algorithm, rotations about the cable axis could cause a small portion of the correctly sensed angle to be briefly split between the two directions. Similarly, any differences in the magnetic field experienced by the two IMUs and/or biases in the magnetometer sensor data would result in discrepancies between the Northern direction identified by IMU1 on the deck of the ship and IMU2 on the load.

During the experiments, the assumption of zero-yaw and a Northern heading was applied by rotating the world frame to remove the mean yaw angle sensed by IMU1. The mean yaw angle was identified with a low-pass filter over an initialization period of several minutes prior to testing, and the rotation was applied for both the estimation of the ship and the load motion under the assumption that each IMU 
sensed the same global magnetic field and thereby identified the 'correct' direction for magnetic North. If IMU2 on the load sensed a different magnetic field than that of IMU1 on the ship, the estimation algorithm would resolve the angles into a frame that does not match the assumed world frame and partial transference would occur. This conclusion was confirmed in simulation by applying differently biased magnetic fields to each of the IMUs with a $5.95^{\circ}$ discrepancy in field directions, which resulted in an erroneous estimation of an off-axis angle during the initialization period. It is noted that the current implementation allows for world-frame positioning of the load and steady-state or low frequency twists of the load about the cable axis; however, it relies on agreement between the magnetic field readings to avoid off-axis motion. Although energy dissipation will still occur in the majority of cases, the current implementation of the system is not recommended where the IMUs may experience significantly different magnetic fields with above $5-10^{\circ}$ differences in the sensed field directions, as off-axis motion will result and the stability of the system could be compromised for severe cases.

Regardless of its cause, the issue with off-axis motion in the compensated response motivates the investigation of three additional metrics, namely the distance travelled, the $95 \%$ ellipsoid volume, and the set-point tracking RMSE, to avoid the potential limitations of those discussed in the previous subsection. 


\subsubsection{Additional Performance Metrics}

The issue with off-axis motion and the limitations of the previously discussed performance metrics motivate the use of three additional metrics to further quantify the response of the compensated system. These metrics are the distance travelled by the load during the test period, the volume of an ellipsoid that encompasses $95 \%$ of the world-frame points identified for the load during the test period, and the set-point tracking root-mean-squared error.

\section{Distance Travelled}

The first additional metric, the distance travelled during the test period, is calculated by the cumulative sum of the Euclidean distance between pendulum points in adjacent/subsequent video frames. This Euclidean distance represents the distance travelled between the two video frames or in $1 / 30$ seconds, which is based on the video frame rate of 30 frames-per-second. The magnitude of the pendulum velocity may also be estimated by dividing the Euclidean distance between frames by the period. Assuming negligible decay over the initial quarter-swing, the theoretical maximum velocity $v_{\max }$ may be estimated from the initial height $h_{0}$ such that

$$
v_{\text {max }} \approx \sqrt{2 g h_{0}},
$$

where $g$ is the gravitational constant. From the pendulum lengths and initial angles of Table 7.4, the maximum velocity is expected to be within the range of $260-1300 \mathrm{~mm} / \mathrm{s}$. This brief theoretical calculation serves as a logical check regarding the magnitude of the calculated values.

In addition to the maximum velocity, a noise threshold must be considered to avoid unbounded summations of noise when the pendulum is at rest. This thresh- 
old is determined to be approximately $3 \mathrm{~mm}$ of movement between adjacent frames, which corresponds to a velocity of approximately $90 \mathrm{~mm} / \mathrm{s}$. Motion below this noise threshold is not included in the summation of the total distance travelled. An exponential fit of the cumulative distance travelled reveals a time constant similar in magnitude to the angular response in many cases and the velocity decay resembles the height decay.

The lower plots of Figure 7.2 and Figure 7.3 show the distance travelled by the pendulum, its instantaneous velocity, and exponential fitting results (the time constant and fitted line) that are derived from the world data points of Figure 7.1. The solid blue line is plotted against the left axis and represents the cumulative distance travelled throughout the test period, and the distance travelled at the end of the test period (120 seconds) is indicated in the title. An exponential fit yields a dashed black line that resembles the cumulative distance travelled with a time constant and theoretical maximum distance that are also listed in the title of the plot. The instantaneous velocity between pendulum points identified in subsequent frames is plotted as a dotted orange/red line against the right axis. An upper dashed line is used to indicate the maximum theoretical velocity found using Equation 7.2, and the velocity noise threshold is indicated by a lower red dashed line to indicate data that was not included in the summation due to low frame-to-frame velocities.

\section{Ellipsoid Volume}

The second additional metric investigated is the volume of an ellipsoid that encompasses $95 \%$ of the pendulum points. That is, the pendulum would reside within this ellipsoid for $95 \%$ of the test period. Figure 7.4 shows the ellipsoid fitting result for Test 020 Repetition 001, and Figure 7.5 shows the ellipsoid fitting result for Test 060 Repetition 001. The ellipsoid was used in previous compensation system development 
efforts [21], and the fitting procedure is modified in the current work to facilitate automatic fitting with minimal user input. The ellipsoid dimensions are based on the standard deviation of the data points such that the ratios of the principal ellipsoid dimensions are defined by the ratios of the standard deviations of the point cloud in the principal directions and, therefore, a single scale factor defines the size of the ellipsoid.

For the energy dissipation tests, the percentage change in ellipsoid volume caused by the compensation system ranges from $-97 \%$ to $186 \%$ (mean: $-33 \%$ ) for the experimental cases and the simulated results are separated from the experimental results by up to 10 orders of magnitude. The simulated results exhibit substantial percentage increases in the ellipsoid volume caused by the compensation system. These substantial increases are a result of the negligible volume of the ideal free response, which generates a point cloud with all points on an arc with negligible standard deviation in one of its principal directions. It is concluded that the current ellipsoid fitting procedure does not produce a strong, appropriate, or consistent metric for analysing the energy dissipation results. The data is not well represented by an ellipsoid, and the ellipsoid volume results are omitted for the energy dissipation tests; however, this metric is used in the set-point tracking tests. 


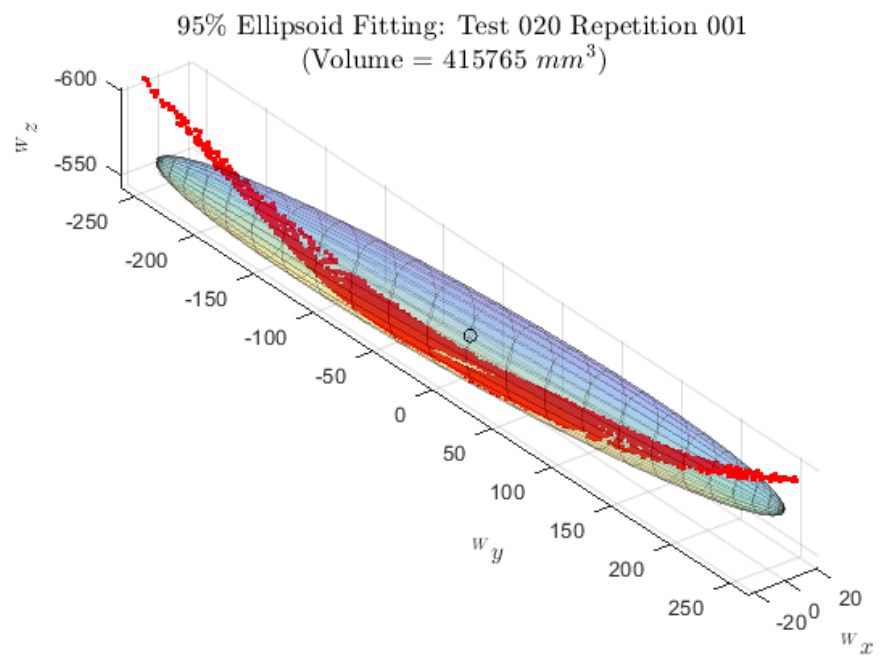

Figure 7.4: Ellipsoid fitting result for Test 020 Repetition 001 (uncompensated/free response of a $700 \mathrm{~mm}$ pendulum).

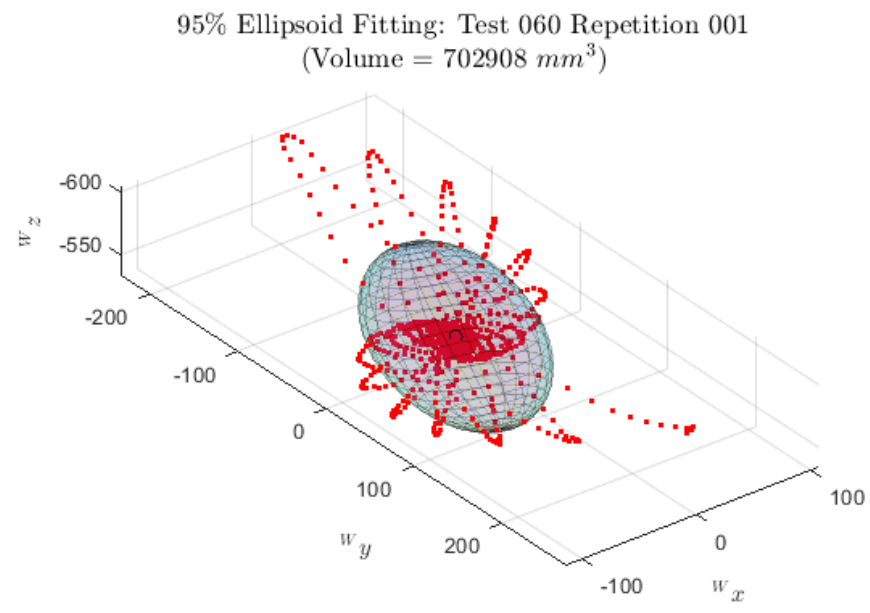

Figure 7.5: Ellipsoid fitting result for Test 060 Repetition 001 (compensated response of a $700 \mathrm{~mm}$ pendulum). 


\section{Set-Point Tracking RMSE}

The third additional metric considered in the current work is the set-point tracking root-mean-squared error (RMSE), which is calculated by taking the Euclidean distance between each pendulum point relative to the set-point as the error compared to a desired distance of zero. This metric represents the tracking performance based on the distance to the set-point for the entire test duration. Each of the aforementioned performance metrics are calculated the same way for the simulation results. Although the noise-free world data points are directly taken from the simulation, the noise threshold is still applied when calculating the cumulative distance travelled to improve consistency between the simulation and experimental results.

Prior to performing the simulations and comparing the uncompensated and compensated results, the natural damping of the system must be quantified for use in the simulation. 


\subsubsection{Natural Damping of the Pendulum}

Prior to performing the simulations, the appropriate damping torque on the pendulum is determined from a brief analysis of the experimental results. The pendulum damping is assumed to be predominantly caused by drag forces on the body of the pendulum load and, therefore, the damping constants are largely dependent on the shape of the load. For this research, the load is disk-shaped as shown in Figure 3.20 and has a significantly different projected area in each direction. By considering the minor rotations of the load about the cable to have a negligible effect on the damping, and considering independent damping in the $\theta_{x}$ and $\theta_{y}$ directions, the damping characteristics may be determined from the exponential decay of the oscillation amplitudes. Following the notation of [67], the exponential decay of the peak angles is represented by the exponential decay function

$$
\theta=\theta_{0} e^{-\kappa t / 2}
$$

where $\theta$ is the pendulum angle, $\theta_{0}$ is the initial angle, $\kappa$ is a damping constant, and $t$ is the time. The division of the damping constant in the exponential term is used for consistency/convenience in [67], and the damping constant is defined with units of $\sec ^{-1}$ as

$$
\kappa=\frac{k L D}{3 m}+\frac{c}{m}
$$

where $k$ is a constant associated with the cable drag, $L$ is the cable length, $D$ is the cable diameter, $m$ is the mass, and $c$ is the damping constant for the pendulum body under the assumption that the drag force is directly proportional to the velocity of the body. In the current work, the first term of Equation 7.4, which represents the drag on the pendulum cable, is considered negligible as the experimental results do not show 
increases in drag with increases in cable length. Therefore, the damping constant $\kappa$ in Equation 7.4 is dependent only on the pendulum mass and the damping constant for the pendulum body. Updating the notation to be consistent with the current work and considering each angular direction of the pendulum motion independently, the decaying peak heights may be represented as

$$
\begin{aligned}
& \theta_{x}=\theta_{x, 0} e^{-\kappa_{\theta_{x}} t / 2}=\theta_{x, 0} e^{-\lambda_{\theta_{x}} t}=\theta_{x, 0} e^{-t / \tau_{\theta_{x}}}, \\
& \theta_{y}=\theta_{y, 0} e^{-\kappa_{\theta_{y}} t / 2}=\theta_{y, 0} e^{-\lambda_{\theta_{y}} t}=\theta_{y, 0} e^{-t / \tau_{\theta_{y}}},
\end{aligned}
$$

where $\kappa_{\theta_{x}}$ and $\kappa_{\theta_{y}}$ are the damping constants, $\lambda_{\theta_{x}}$ and $\lambda_{\theta_{y}}$ are the decay constants, $\tau_{\theta_{x}}$ and $\tau_{\theta_{y}}$ are the time constants for each angular direction, and $\theta_{x, 0}$ and $\theta_{y, 0}$ are the initial angles for the $\theta_{x}$ and $\theta_{y}$ directions. Separate damping parameters must be determined for each angular direction due to the lack of symmetry demonstrated in Figure 3.20, which results in different drag coefficients for each angular direction. The damping constant to mass ratio for the pendulum body in each direction may be determined from the free response angular time constants as

$$
\begin{aligned}
& \frac{c_{\theta_{x}}}{m_{p}}=\kappa_{\theta_{x}}=2 \lambda_{\theta_{x}}=2 / \tau_{\theta_{x}}, \\
& \frac{c_{\theta_{y}}}{m_{p}}=\kappa_{\theta_{y}}=2 \lambda_{\theta_{y}}=2 / \tau_{\theta_{y}},
\end{aligned}
$$

where the mass of the pendulum $m_{p}$ is 301.8 grams. The torques caused by the drag forces on the body may then be applied in the simulation as

$$
\begin{aligned}
& T_{\theta_{x}}=m_{p}\left(\frac{c_{\theta_{x}}}{m_{p}}\right) l_{p} v_{p y}, \\
& T_{\theta_{y}}=-m_{p}\left(\frac{c_{\theta_{y}}}{m_{p}}\right) l_{p} v_{p x},
\end{aligned}
$$


where $T_{\theta_{x}}$ and $T_{\theta_{y}}$ are the damping torques, $v_{p x}$ and $v_{p y}$ are components of the worldframe velocities of the pendulum load directed along its local $\mathrm{x}$ - and $\mathrm{y}$-axes, and the ratios $\frac{c_{\theta_{x}}}{m_{p}}$ and $\frac{c_{\theta_{y}}}{m_{p}}$ may be approximated from the mean experimental results in each direction. For each of the free response test cases (Test 001-040), the angular time constants are determined through the previously described exponential fitting procedure.

In Figure 7.6, the time constants from each repetition of all of the free response test cases are inverted to show the corresponding decay constants. The $\theta_{x}$ decay constants are shown in the upper plot and the $\theta_{y}$ decay constants are shown in the lower plot. The mean decay constants are indicated by the dashed lines and the corresponding ratios are determined from these mean values as $\frac{c_{\theta_{x}}}{m_{p}}=2 \lambda_{\theta_{x}} \approx 0.0272 \mathrm{sec}^{-1}$ and $\frac{c_{\theta_{y}}}{m_{p}}=2 \lambda_{\theta_{y}} \approx 0.018 \mathrm{sec}^{-1}$. Consistent with the shape of the pendulum, the larger area results in a higher drag for the $\theta_{x}$ direction. The damping torques are calculated and applied in simulation. Given the application of the damping torque, preliminary simulations are performed in the following section to demonstrate the similarities between the analysis of the simulated results and the experimental results. 
Energy Dissipation Results:

Angular Decay Constants for Free Response

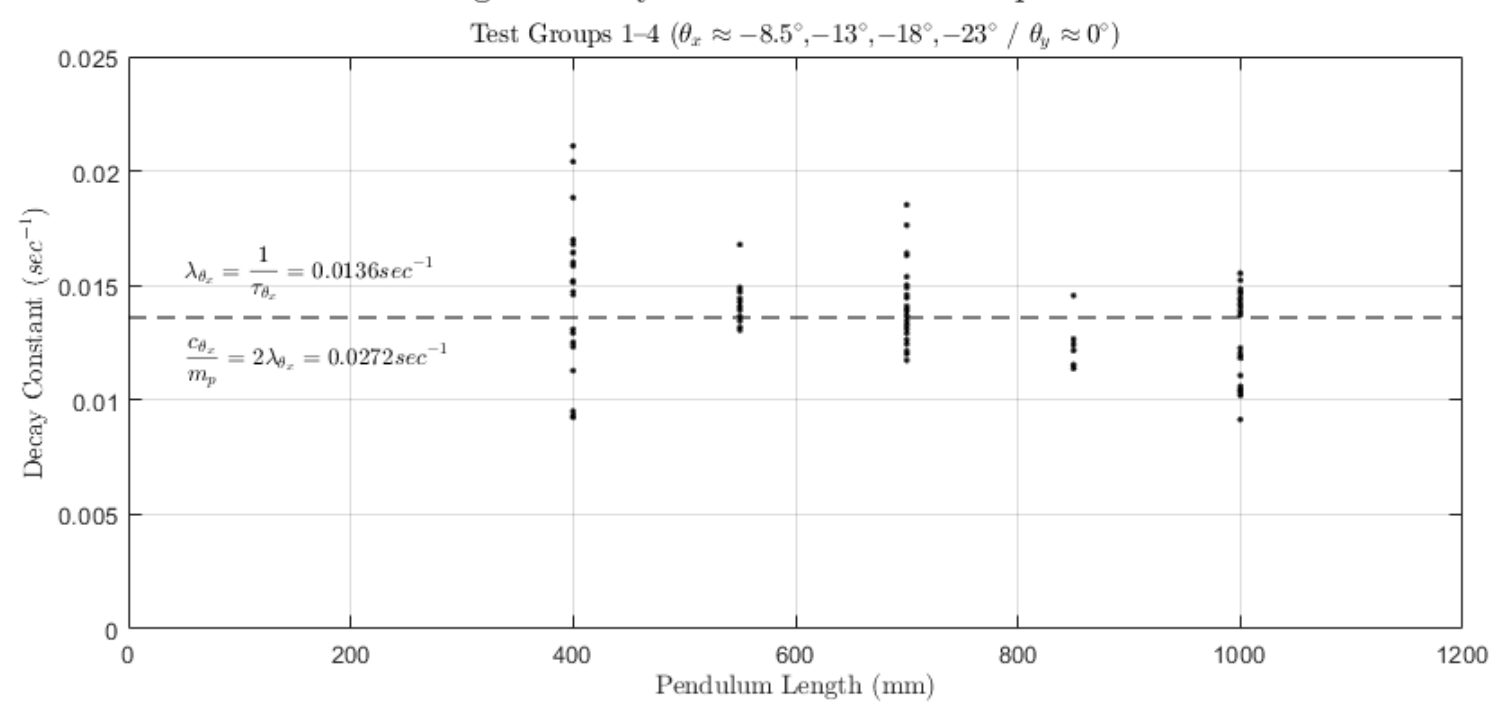

Test Groups $5-8\left(\theta_{x} \approx 0^{\circ} / \theta_{y} \approx 7.6^{\circ}, 13^{\circ}, 18^{\circ}, 24^{\circ}\right)$

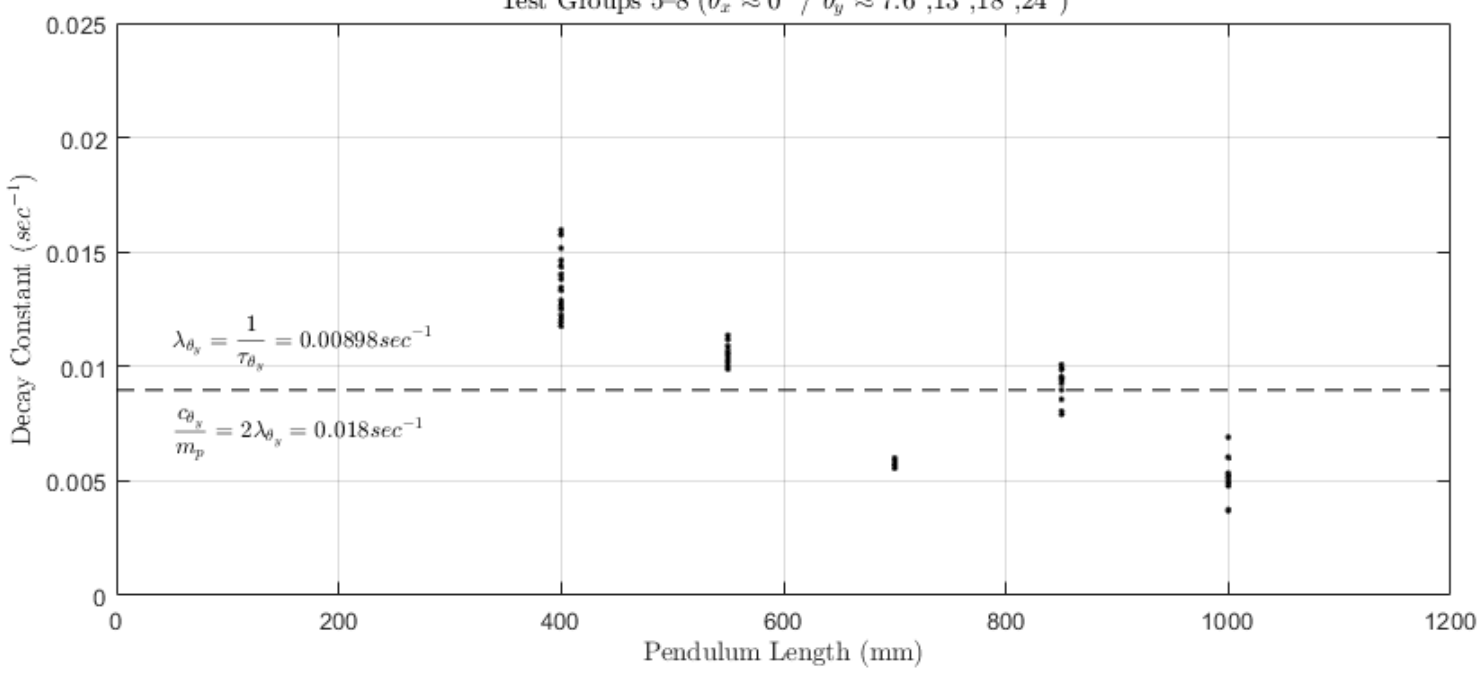

Figure 7.6: Decay constants found from fitting exponential decay functions to the angular response of each uncompensated test case (Test 001-040). The mean value is indicated by a dashed line. 


\subsubsection{Preliminary Simulation Results}

By applying the two damping torques in simulation, simulated results may be found that correspond to the experimental results. Complementary to the experimental results in Figure 7.2 for Test 020 Repetition 001, Figure 7.7 shows the corresponding simulation results for Simulation 020-1. Comparing these simulated results to the experimental results yields $0.15-1.36 \%$ difference in the initial/peak values, $15.41-$ $33.76 \%$ difference in the fitted time constants, and $22.32 \%$ difference in the distance travelled. These differences are expected due to the use of the mean decay constant when determining the damping torque in simulation. Similarly, the simulation results for Simulation 060-1 and 060-2 are shown in Figure 7.8 and Figure 7.9, respectively. These simulated results are comparable to the experimental results shown for Test 060 Repetition 001 in Figure 7.3 with $2.00-5.42 \%$ difference in the initial/peak values, $11.18-53.12 \%$ difference in the fitted time constants, and $38.51-48.84 \%$ difference in the distance travelled. Although some differences are expected as a result of the mean decay constant in simulation, the larger differences for the compensated cases may be largely attributed to the off-axis motion. The simulated off-axis motion is considered negligible when compared to the off-axis motion observed in the experimental results. This observation is attributed to the simulated constraint that prevents rotation of the load about the cable axis, which limits cross-over of the angular response through the estimation algorithm, as well as the assumption that both IMUs experience the same unbiased magnetic field.

It is interesting to note that, in Figure 7.8, the simulation that bypasses the estimation algorithm and supplies 'truth' data for the ship and load motion to the controller exhibits slightly poorer performance compared to Figure 7.9 where the simulation applies noise to all sensor measurements and emulates the inertial measurement unit (IMU) feedback for use in the estimation algorithms. The reason for 
this result is demonstrated in Figure 7.10, which shows the tip velocity and pendulum angle over the test duration. In the upper plot, the truth data is supplied to the controller, so the set-points are calculated without overshoot and the control action (i.e. the tip motion) stops as soon as the pendulum angle enters the deadband. In the lower plot, however, the simulated sensor data is supplied to the controller and the estimation algorithm is used to estimate the pendulum angle. The noise, overshoot, and low-pass filtering in the estimated angle result in larger control efforts prior to entering the deadband, as well as some control effort due to noise/overshoot after the actual angle enters the deadband. The result is slightly improved performance, which is demonstrated by the faster exponential decay of the pendulum angle. This phenomenon is specific to the simulations, however, as it is likely that the negative impact of estimation errors caused by rotation of the load about the cable axis or magnetic field bias in the real-world system would outweigh the potential benefits of overshooting/lagging at key points in the motion.

In the following subsection, the results of the energy dissipation simulations and experiments are discussed to draw conclusions about the performance of the compensation system. 
Pendulum Response: Simulation 020-1
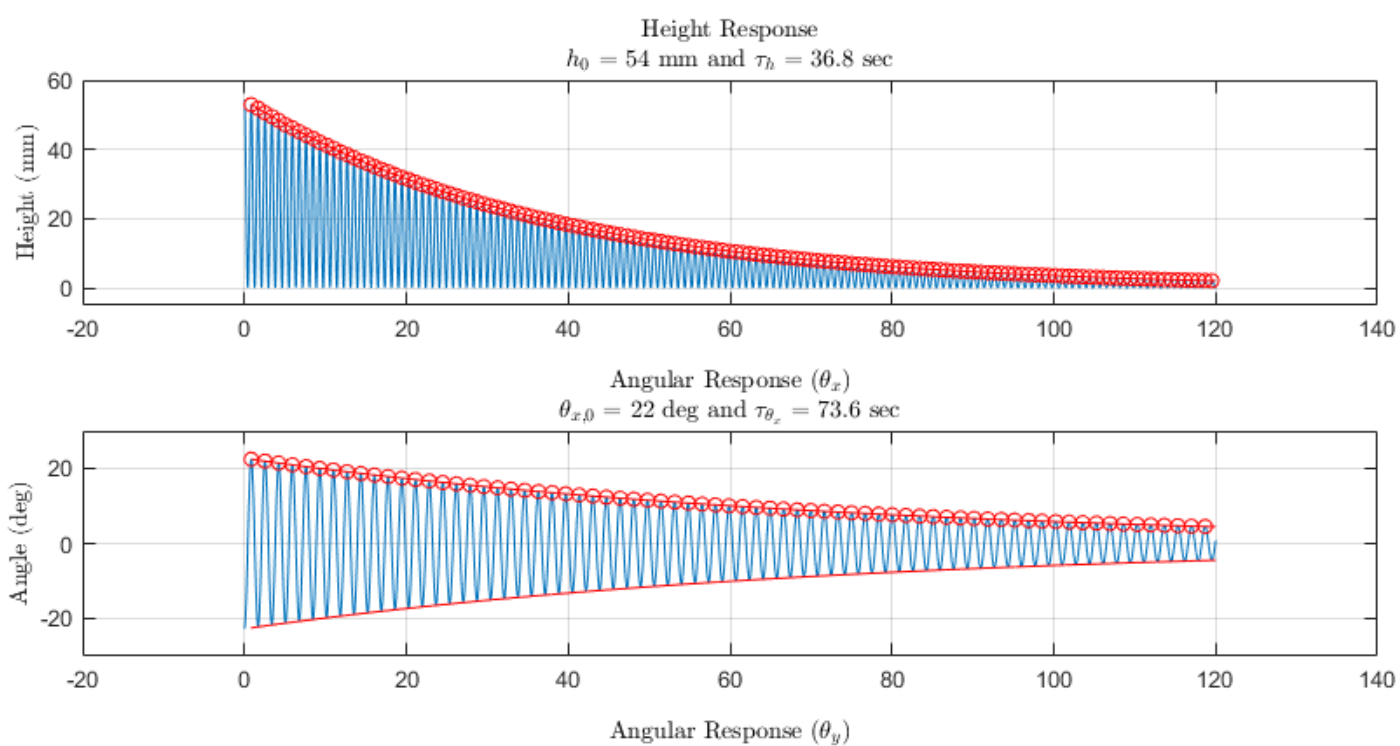

$\theta_{y, 0} \approx 0$ deg and $\tau_{\theta_{3}}=\infty$ sec
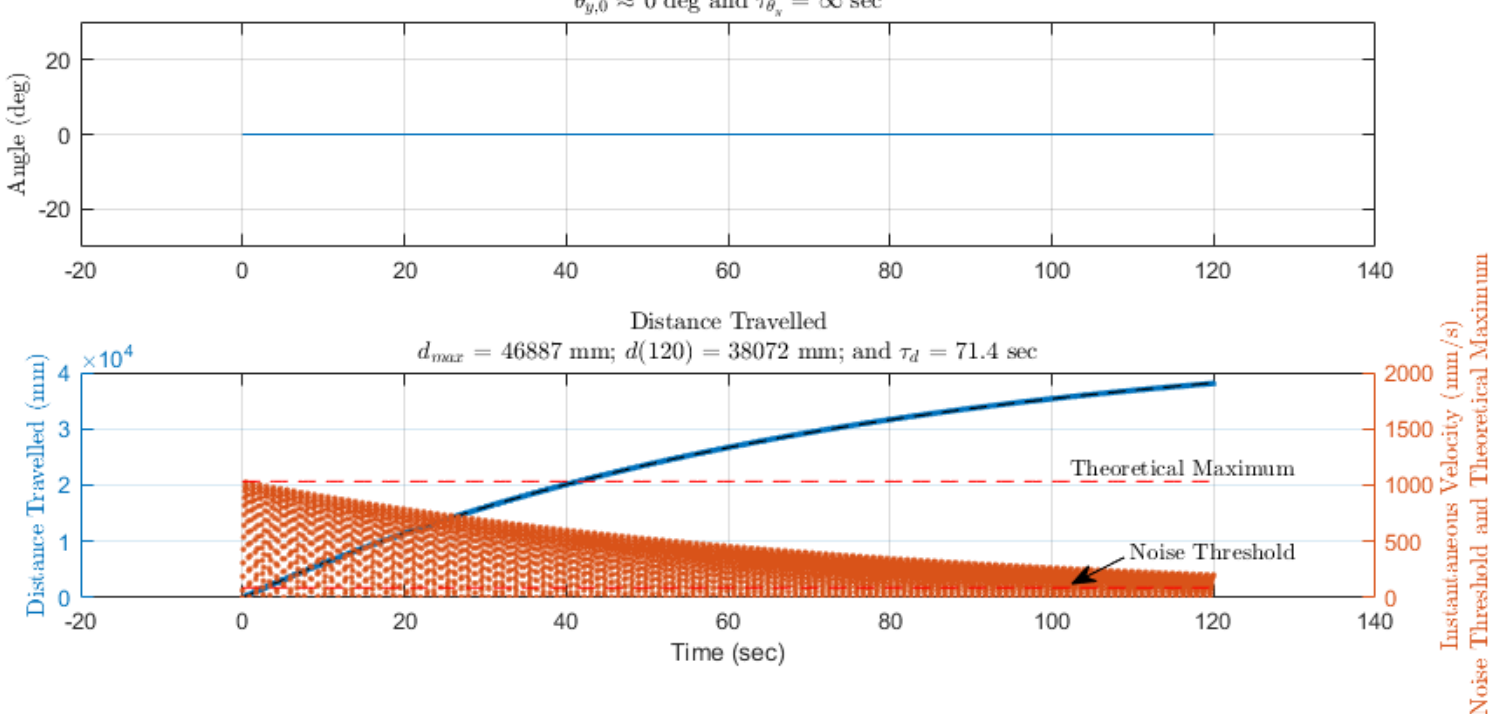

Figure 7.7: Pendulum response with exponential fitting for Simulation 020-1 (uncompensated/free response of a $700 \mathrm{~mm}$ pendulum). 
Pendulum Response: Simulation 060-1
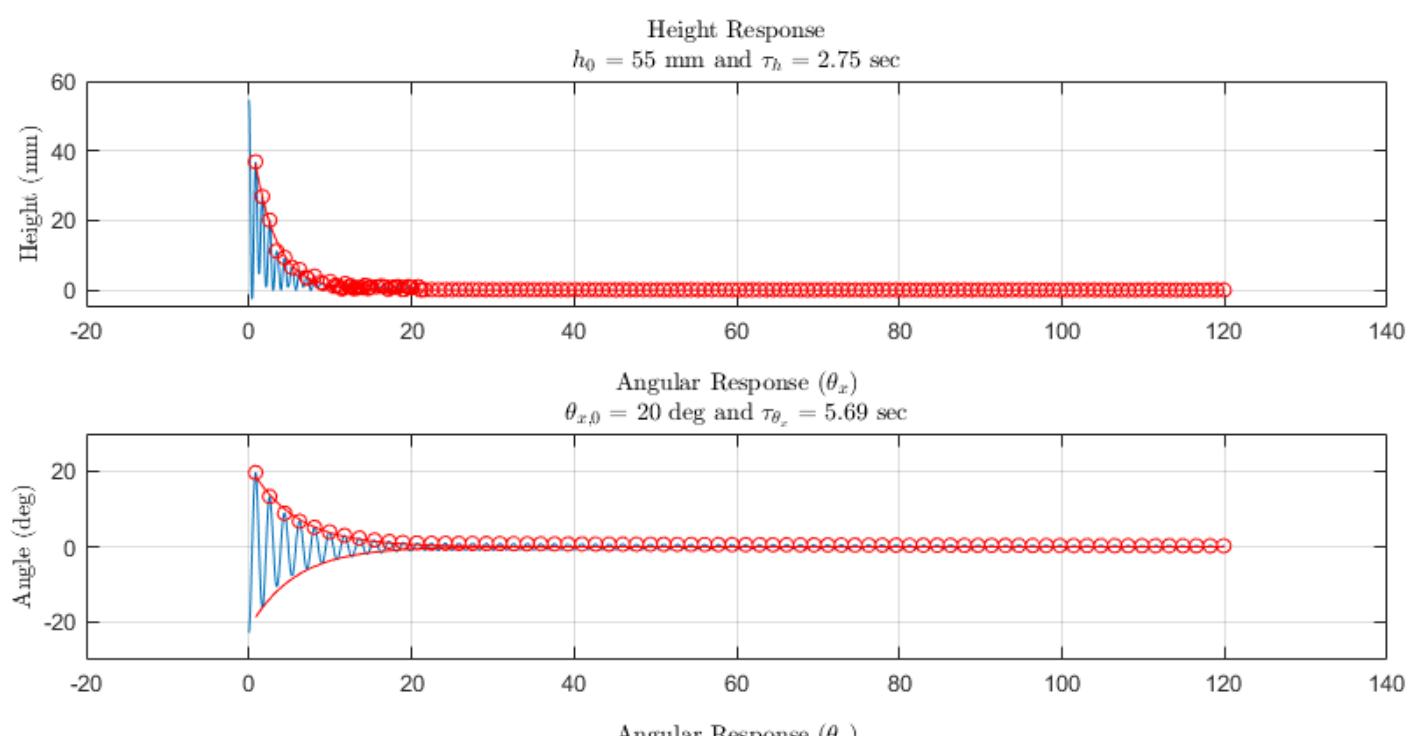

$\theta_{y, 0} \approx 0$ deg and $\tau_{\theta_{y}}=\infty \sec$
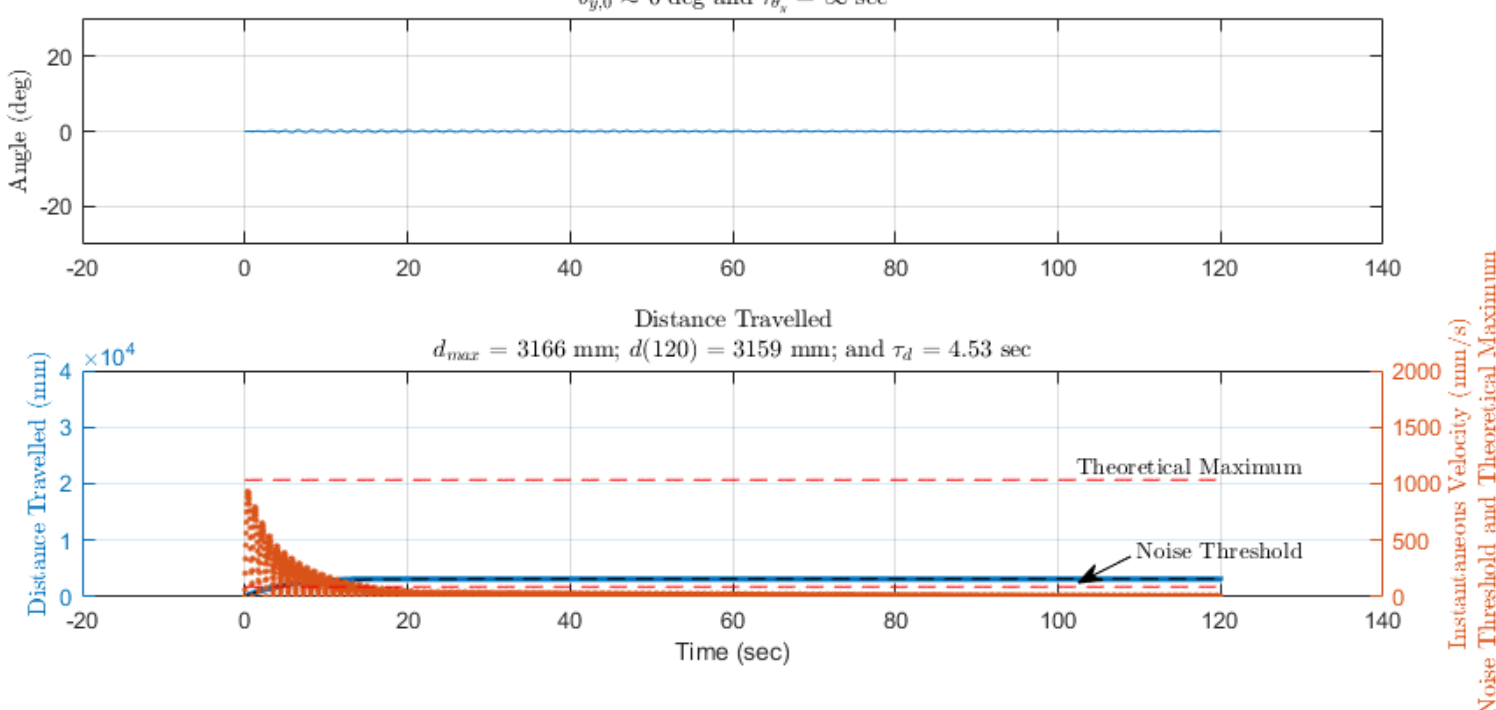

Figure 7.8: Pendulum response with exponential fitting for Simulation 060-1 (compensated response of a $700 \mathrm{~mm}$ pendulum with truth data feedback). 
Pendulum Response: Simulation 060-2
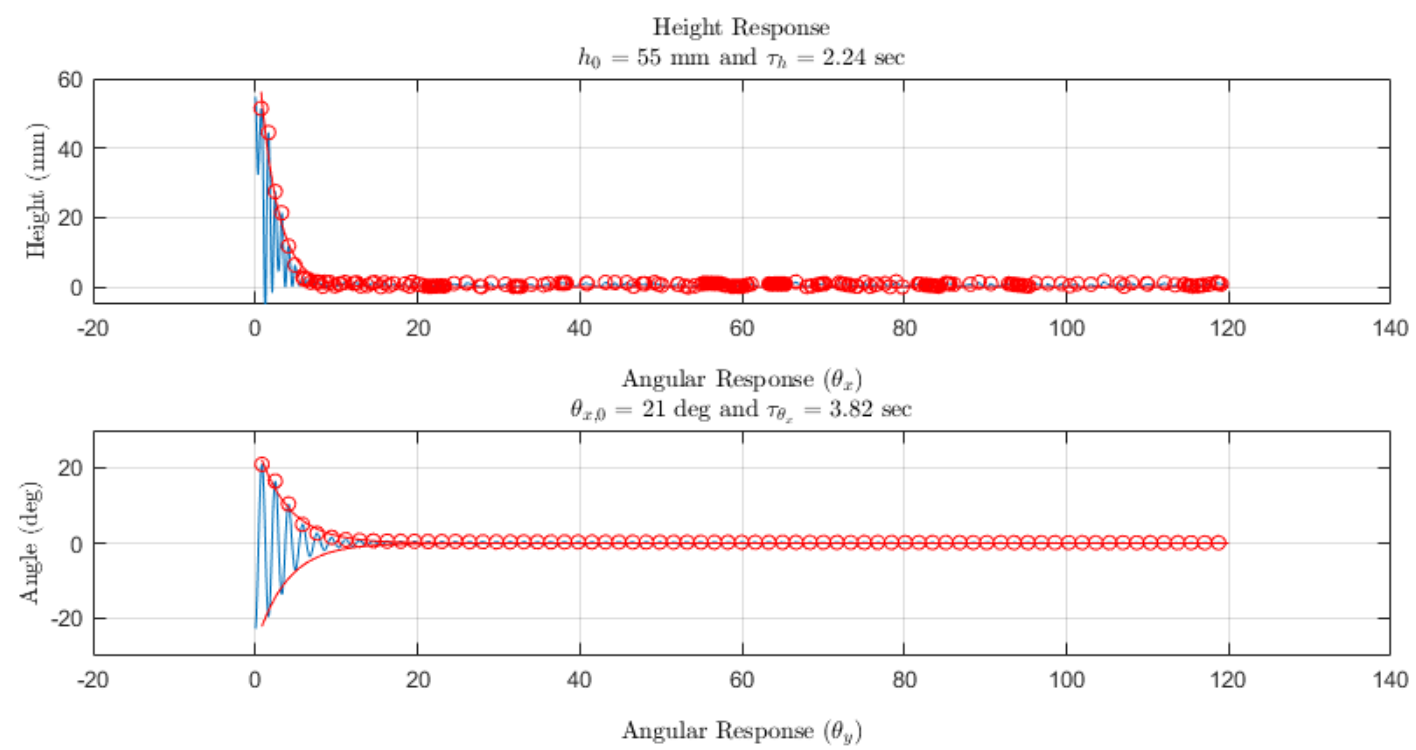

$\theta_{y, 0} \approx 0 \operatorname{deg}$ and $\tau_{\theta_{y}}=\infty$ sec
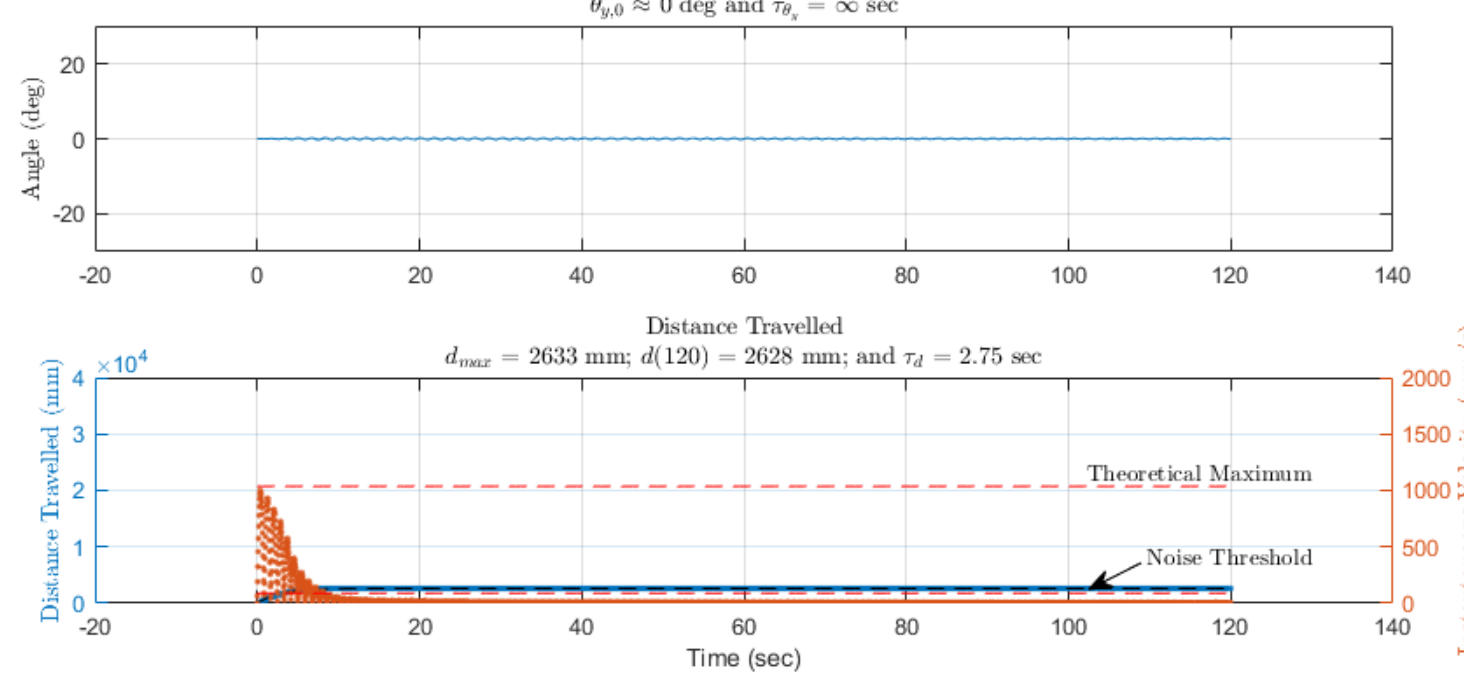

Figure 7.9: Pendulum response with exponential fitting for Simulation 060-2 (compensated response of a $700 \mathrm{~mm}$ pendulum with simulated sensor feedback). 
Tip Velocity and Pendulum Angle

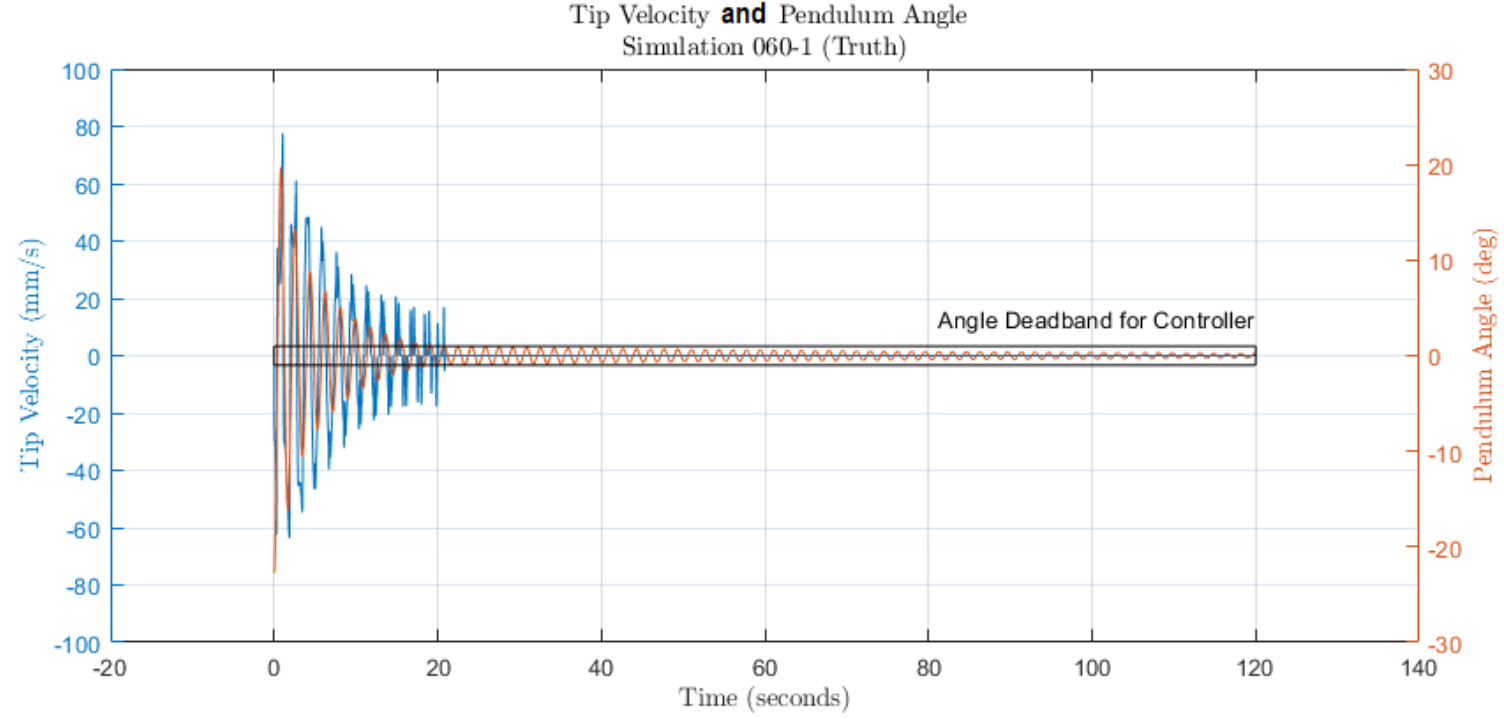

Tip Velocity and Pendulum Angle

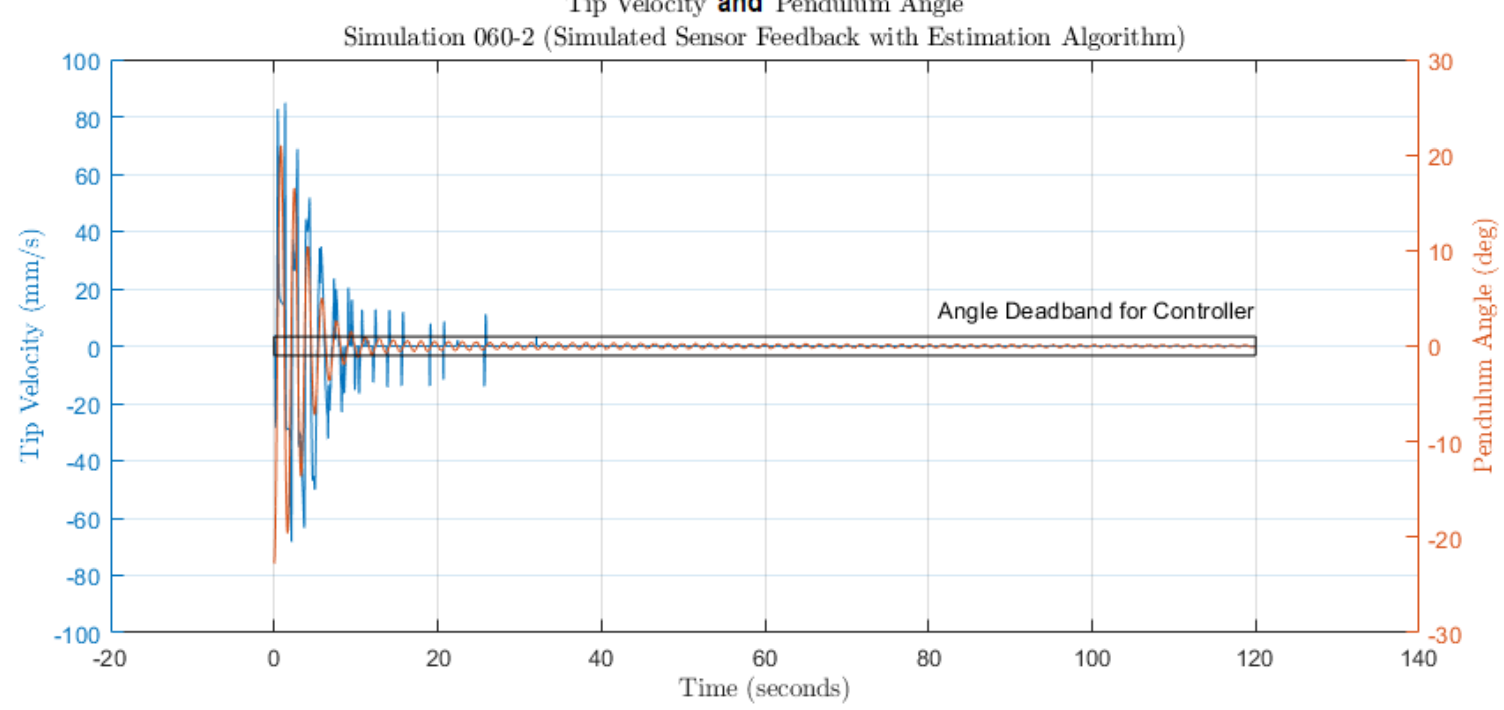

Figure 7.10: The crane tip velocity and pendulum angle for truth and simulated sensor simulations, Simulation 060-1 and Simulation 060-2, respectively. 


\subsubsection{Energy Dissipation Results}

The results of the energy dissipation experiments and simulations are generated and summarized in Figures 7.11-7.13 and 7.16-7.20. The results represent all repetitions of Tests 001-080 (total of 345 experiments) as listed in Tables 7.4-7.5, as well as both variations of the corresponding Simulations 001-080 (total of 160 simulations). The experimental results are summarized by the mean value with an error bar to indicate the standard error of each of the performance metrics. The key performance metrics discussed in this subsection include the peak $\theta_{x}$ angles in Figure 7.11, the peak $\theta_{y}$ angles in Figure 7.12, the maximum height above the set-point in Figure 7.13, the time constants for exponential decay in Figures 7.16-7.18, the $95 \%$ ellipsoid volume, the distance travelled above the noise threshold in Figure 7.19, and the set-point tracking root-mean-squared error (RMSE) in Figure 7.20.

The results of Figures 7.11-7.13 and 7.16-7.20 are presented as a series of bar graphs that are divided into eight Test Groups based on the initial conditions/angles. Experiments that could not be performed are indicated by an ' $\mathrm{x}$ ' in place of the bar. An asterisk $\left(^{*}\right)$ is used to indicate bars that may be difficult to see due to values that are below $2 \%$ of the maximum listed on the vertical axis. The graph for each Test Group summarizes the data across all tested lengths with five bars per length. The bars represent, from left to right:

\section{Exp: No Compensation}

The uncompensated experimental results to demonstrate the free response of the pendulum (Test 001-040).

\section{Exp: Compensation}

The compensated experimental results to demonstrate the compensated response of the pendulum (Test 041-080). 


\section{Sim: No Compensation $(1 / 2)$}

Both variations of the uncompensated simulation results (Simulation 001-040). The variations are combined as the free response is independent of the compensation system.

\section{Sim: Compensation (1)}

The first variation of the compensated simulation results to demonstrate the compensated response of the pendulum when truth data is supplied as feedback to the controller (Simulation 041-1 to 080-1).

\section{Sim: Compensation (2)}

The second variation of the compensated simulation results to demonstrate the compensated response of the pendulum when noisy sensor data is supplied to the controller and the estimation algorithm is used to determine the pendulum angles (Simulation 041-2 to 080-2).

To indicate off-axis motion, a note is placed on the $\theta_{x}$ or $\theta_{y}$ angular data plots when the intended/ideal angle is zero and the initial angle was applied only in the orthogonal direction. 


\section{Energy Dissipation Results: Peak Angles}

In Figure 7.11 and Figure 7.12, the maximum peak $\theta_{x}$ and $\theta_{y}$ angles that occurred in the opposite direction of the initial angles are shown. These values represent the peak angle reached during the first swing-up of the pendulum as it moves away from its initial position and thereby exclude the applied disturbance (initial angle) as well as the human error that occurs while manually bringing the pendulum to its initial position. The value of the mean initial angle is negated and displayed as a dashed line across all lengths of each test group to show both the consistency of the experiments and act as a benchmark for evaluating the initial decay in the first half-swing. In all uncompensated cases, the pendulum approaches the negative of its initial angle as very little energy is dissipated through natural damping during the first halfswing. In the compensated cases, however, there is a visible reduction in the angle caused by the rapid decay of the compensated response. When an initial $\theta_{x}$ angle is applied, the experimental results indicate a reduction of 4.8-47.1\% (mean: 18.6\%), the truth simulation results (1) indicate a reduction of $10.3-35.2 \%$ (mean: $19.2 \%$ ), and the results of simulations with simulated sensors (2) indicate a reduction of 2.7$23.8 \%$ (mean: 13.1\%) for the compensated cases when compared to the corresponding uncompensated cases. Similarly, when an initial $\theta_{y}$ angle is applied, the experimental results indicate a reduction of 5.6-28.6\% (mean: $14.2 \%$ ), the truth simulation results (1) indicate a reduction of 18.5-43.7\% (mean: $27.8 \%$ ), and the results of simulations with simulated sensors (2) indicate a reduction of 10.6-15.3\% (mean: 12.3\%) for the compensated cases when compared to the corresponding uncompensated cases. These observations demonstrate the potential for rapid reduction of the pendulum angles through the compensation system during the first half-swing of the pendulum.

It is noted that, in the above discussion, the reductions for the experimental results are listed as 4.8-47.1\% (mean: $18.6 \%$ ) for the $\theta_{x}$ direction and 5.6-28.6\% (mean: 
$14.2 \%)$ for the $\theta_{y}$ direction. The apparent differences in the two ranges of observed reductions are a result of a $47.1 \%$ reduction for Test 001 with a $400 \mathrm{~mm}$ pendulum and $-8.5^{\circ}$ initial angle in the $\theta_{x}$ direction, which may be partially attributed to an increase in human error for the lower pendulum length and small initial angle. Excluding Test 001 from the comparison yields 4.8-33.1\% (mean: 16.9\%) for the $\theta_{x}$ direction and 5.6-28.6\% (mean: $14.2 \%$ ) for the $\theta_{y}$ direction, which demonstrates similar performance for both directions in the majority of test cases.

Despite the potential for rapid reduction of the initial angle, imperfections in the estimation algorithm and the physical system result in off-axis motion in nearly all compensated cases that rely on the estimation algorithm. The off-axis motion for Test Groups 5-8 are shown in Figure 7.11 as the peak $\theta_{x}$ angles that were generated as a result of the initial angle in the $\theta_{y}$ direction, whereas the off-axis motion for Test Groups 1-4 are shown in Figure 7.12 as the peak $\theta_{y}$ angles that were generated as a result of the initial angle in the $\theta_{x}$ direction. The off-axis motion is likely generated due to the combination of sensor noise, imperfect actuator motion resulting in offaxis/nonlinear motion of the crane tip, twisting of the load about the cable axis, and variations in the magnetic field sensed by the two IMUs.

Although the twisting motion may be detected in the experimental system, the estimation is dependent on noisy measurements and low-pass filtering. As the cable twists, the lag introduced by the low-pass filter may result in a portion of the motion being resolved into the incorrect axis. As the inaccurate off-axis angle is 'detected,' the system attempts to compensate for it and thereby generates an actual angle in the off-axis direction. Similarly, differences in the magnetic fields experienced by the two IMUs would also generate off-axis motion as discussed in Section 7.2.1. It is noted here that several uncompensated $400 \mathrm{~mm}$ test cases also experience significant off-axis motion, which is a result of the inaccuracies and magnification of human error 
while manually applying the initial angle to such a short length.

The percentage differences of the simulated results relative to experimental results for cases with an initial $\theta_{x}$ angle range from $-26.6 \%$ to $14.4 \%$ (mean: $-0.2 \%$ ) for uncompensated motion, $-19.6 \%$ to $14.2 \%$ (mean: $-1.6 \%$ ) for compensated motion with truth simulations, and $-7.4 \%$ to $15.3 \%$ (mean: $5.9 \%$ ) for compensated motion with simulated sensor data. Similarly, the percentage differences of the simulated results relative to experimental results for cases with an initial $\theta_{y}$ angle range from $-12.3 \%$ to $14.8 \%$ (mean: $-0.4 \%$ ) for uncompensated motion, $-0.1 \%$ to $-31.5 \%$ (mean: $-16.5 \%$ ) for compensated motion with truth simulations, and $-5.1 \%$ to $13.5 \%$ (mean: $2.0 \%$ ) for compensated motion with simulated sensor data. Percentage differences that approach $0 \%$ indicate good agreement between the experimental and simulated results; however, the simulations produce far less off-axis motion due to an ideal initial angle, a simulated constraint that prevents twisting about the cable of the pendulum, and the assumption that both IMUs experience the same global magnetic field. The off-axis motion in simulation is attributed predominantly to tracking errors for the crane tip that result from using the three linear actuators to rotate the crane joints while attempting to generate a linear motion of the crane tip. This effect is more evident when a $\theta_{y}$ angle is applied as the three linear actuators must all work together to generate the anti-pendulum motion; whereas motion in the $\theta_{x}$ direction may be dissipated predominantly through the boom and jib actuators when the crane is extended directly off the port side of the vessel. It is noted that additional off-axis motion is produced in the sensor-based simulations due to the combination of noise in the feedback signals and imperfections in the estimation algorithm. 
Energy Dissipation Results:

Significant Peak Angles in Positive $\theta_{x}$ Direction (Excluding Initial Disturbance)
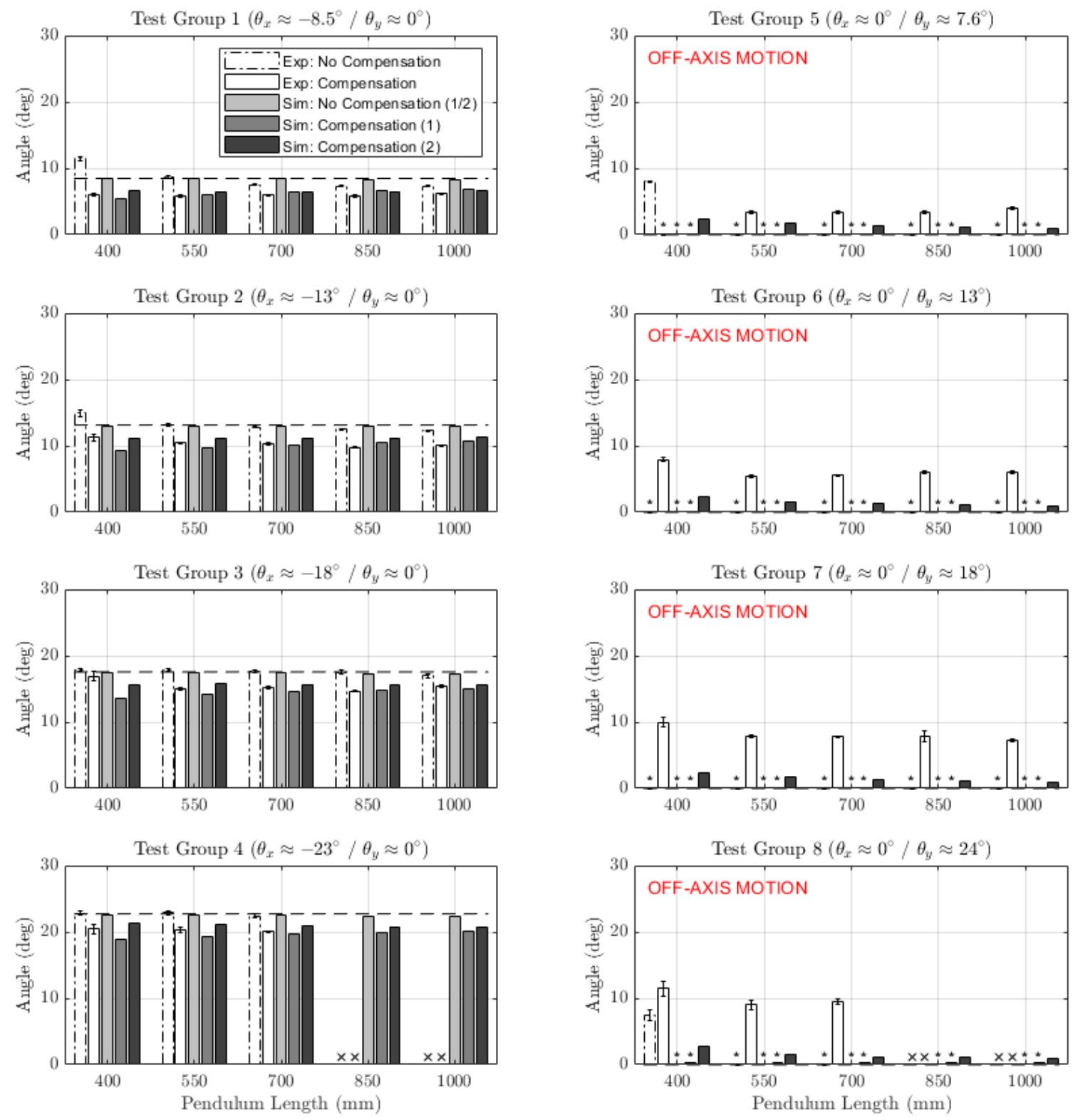

Figure 7.11: Significant peak angles in the positive $\theta_{x}$ direction for all energy dissipation tests (Test 001-080) and simulations (Simulation 001-1/2-080-1/2). The experimental results are summarized by the mean of all repetitions with error bars to indicate the standard errors. The results are grouped by the mean initial angle, the negative of which is indicated by a dashed line. 
Energy Dissipation Results:

Significant Peak Angles in Negative $\theta_{y}$ Direction (Excluding Initial Disturbance)
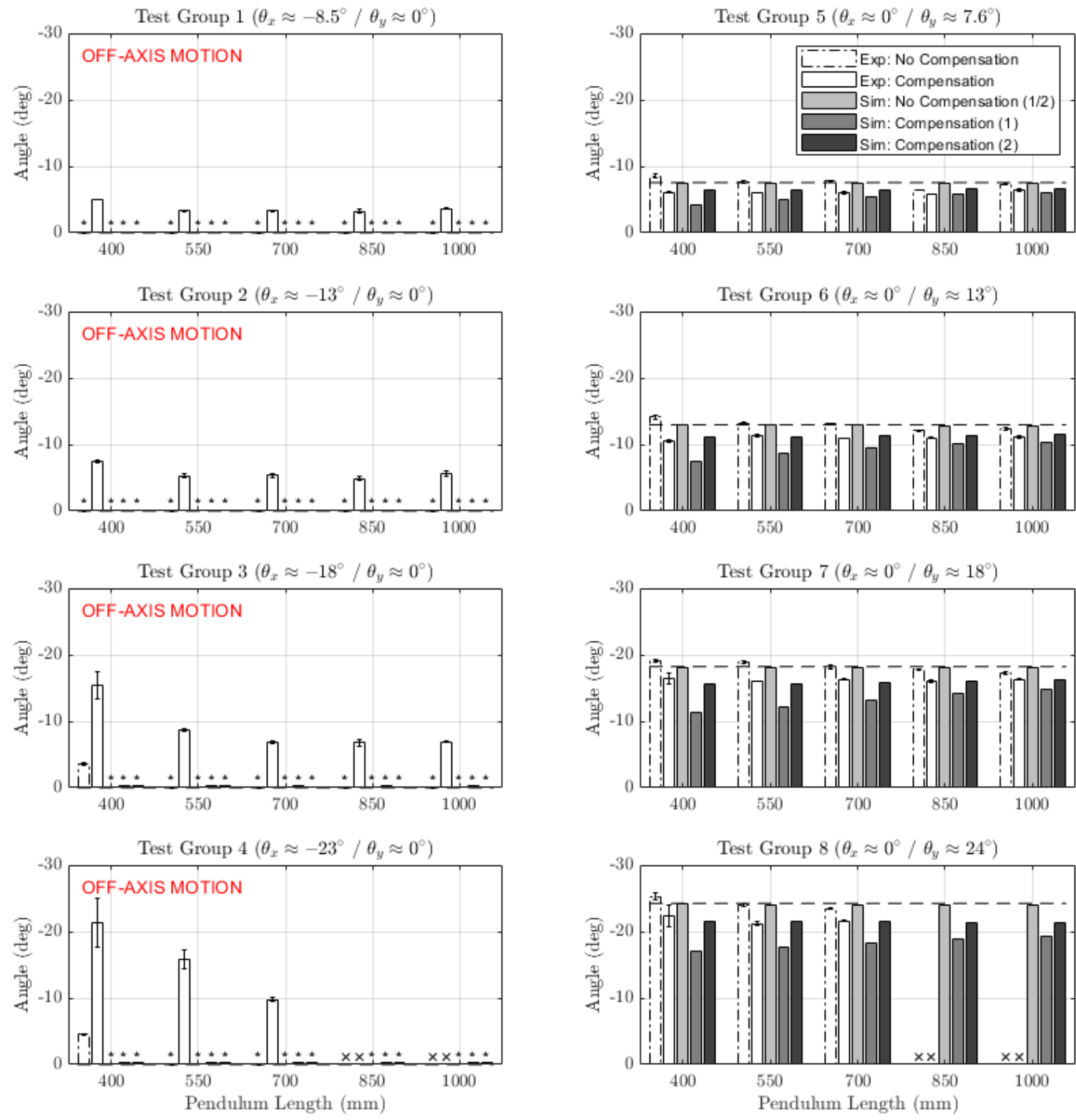

Figure 7.12: Significant peak angles in the negative $\theta_{y}$ direction for all energy dissipation tests (Test 001-080) and simulations (Simulation 001-1/2-080-1/2). The experimental results are summarized by the mean of all repetitions with error bars to indicate the standard errors. The results are grouped by the mean initial angle, the negative of which is indicated by a dashed line. 


\section{Energy Dissipation Results: Maximum Height}

In Figure 7.13, the maximum height relative to the set-point is shown for each case. The maximum height is an indication of the initial potential energy of the system. The initial potential energy of the pendulum may be expressed in terms of either the initial angles or the initial height as

$$
E_{P}=m_{p} g h_{0}=m_{p} g l_{p}\left(1-\cos \left(\theta_{x, 0}\right) \cos \left(\theta_{y, 0}\right)\right)
$$

where $E_{P}$ is the potential energy associated with the initial conditions, $m_{p}$ is the mass of the pendulum, $g$ is the gravitational constant, $h_{0}$ is the initial height relative to the set-point/equilibrium, $l_{p}$ is the length of the pendulum, and $\theta_{x, 0}$ and $\theta_{y, 0}$ are the initial angles. Due to the trigonometric/nonlinear relationship between the initial height and the initial angles, the changes in potential energy caused by increasing pendulum lengths become more pronounced as larger initial angles are applied.

Due to the use of a mean angle in the simulations, some deviation is expected between the maximum heights experienced in simulation compared to the experiments. For the uncompensated responses, the percentage difference of the maximum simulation height relative to the experimental height ranges from $-72.3 \%$ to $34.5 \%$ (mean: $-5.3 \%)$. It is noted that the previously discussed human error for the experimental test cases with a $400 \mathrm{~mm}$ pendulum length result in percentage differences from $-25.3 \%$ to $-72.3 \%$ when comparing the uncompensated simulated values to the experimental values. These high percentage differences account for a large portion of the deviation between the simulation and experimental values reported above. Similar percentage

differences are also found when comparing the results for compensated simulations with truth data (Sim 1) to the compensated experimental results. In these cases, the percentage difference ranges from $-29.9 \%$ to $37.3 \%$ (mean: $5.8 \%$ ). 
In the sensor-based simulation, however, a significant deviation from the other results is observed in many test cases. These deviations result in percentage differences ranging from $1.6 \%$ to $845 \%$ (mean: $210 \%$ ) when comparing the results of compensated simulations with sensor feedback (Sim 2) to the compensated experimental results. This deviation represents a 'kick' that is introduced as a result of the initial estimation error. In the sensor-based simulation, the initial pendulum angles are assumed to be zero (i.e. the filter is not initialized), so the actual initial angle is excluded from the initial estimation. As the pendulum begins to move from its initial position, the estimation updates based on the angular velocity measurement and moves away from the equilibrium point in the direction of the rotation. The gravity measurement acts as a low-pass filter and causes the estimation to converge towards the true value; however, the initial error results in incorrect control actions that produce a deviation in height prior to convergence.

To demonstrate the estimation error caused by the uninitialized algorithm, Figure 7.14 shows the $\mathrm{x} / \mathrm{y} / \mathrm{z}$ load position estimates compared to the true values for the first 10 seconds of a compensated simulation, Simulation 060-2. The actual position of the load is indicated by a solid blue line, the estimated position is indicated by a dotted red/orange line, and the set-point/equilibrium is indicated by a dashed black line. Examining the values at the initial time of 0 seconds on each plot, the initial estimate assumes that the load is at the set-point/equilibrium, whereas the actual position is up to $200 \mathrm{~mm}$ away due to the application of the initial angle. As the pendulum oscillates, the estimated amplitude and frequency begin to converge to those of the actual motion with a phase lag; however, the large errors in the z-position estimate prior to convergence may cause an abrupt change in length or 'kick' as described above. For the example case in Figure 7.14, the effect of the error may be observed in the third plot by examining the actual z-position of the load. In this case, the error 
results in control actions that contribute to erratic behaviour and overshoot during the first 2 seconds of the simulation.

For comparison, Figure 7.15 shows the load position estimate with the true values for the first 10 seconds of the uncompensated version of the above simulation, Simulation 020-2. In this case, the same initial estimation error is observed for the $\mathrm{x} / \mathrm{y} / \mathrm{z}$ positions in each plot; however, no compensation effort or control action is applied based on these erroneous values. As the simulation progresses, the actual position of the pendulum varies periodically to demonstrate the free response. During the first 10 seconds of the simulation, the estimated position converges to the actual amplitude and frequency with a phase lag, which may be seen by comparing the actual and estimated values on each plot for simulation times in the 8-10 second range. These results suggest that a minimum initialization period is necessary prior to activating the compensation system to avoid errors in the applied control actions. 
Energy Dissipation Results:

Maximum Height Relative to Set-Point
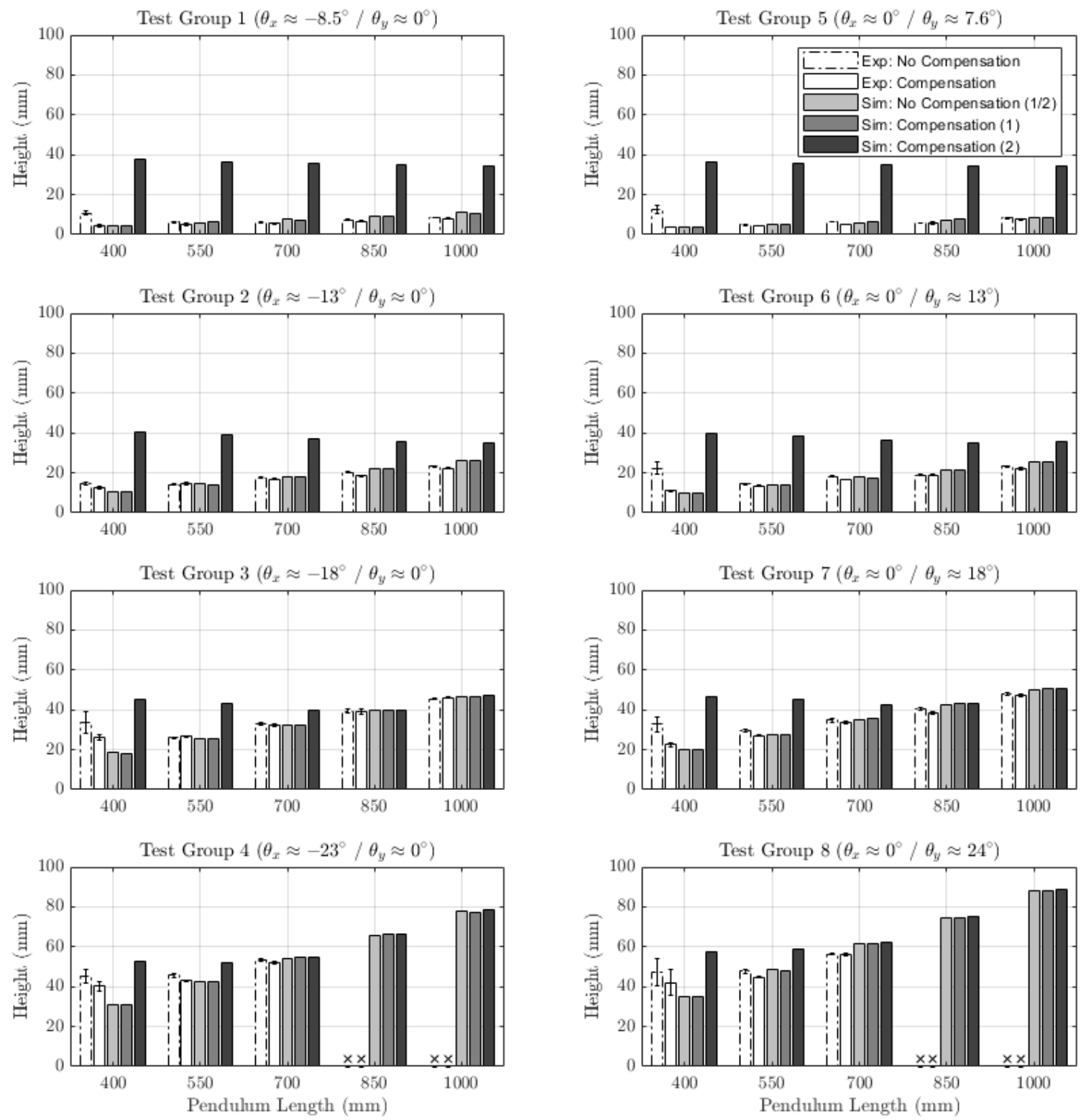

Figure 7.13: Maximum height relative to the set-point as an indication of the potential energy introduced to the system for all energy dissipation tests (Test 001-080) and simulations (Simulation 001-1/2-080-1/2). The experimental results are summarized by the mean of all repetitions with error bars to indicate the standard errors, and the results are grouped by the mean initial angle. 
Load Position Estimate: Simulation 060-2
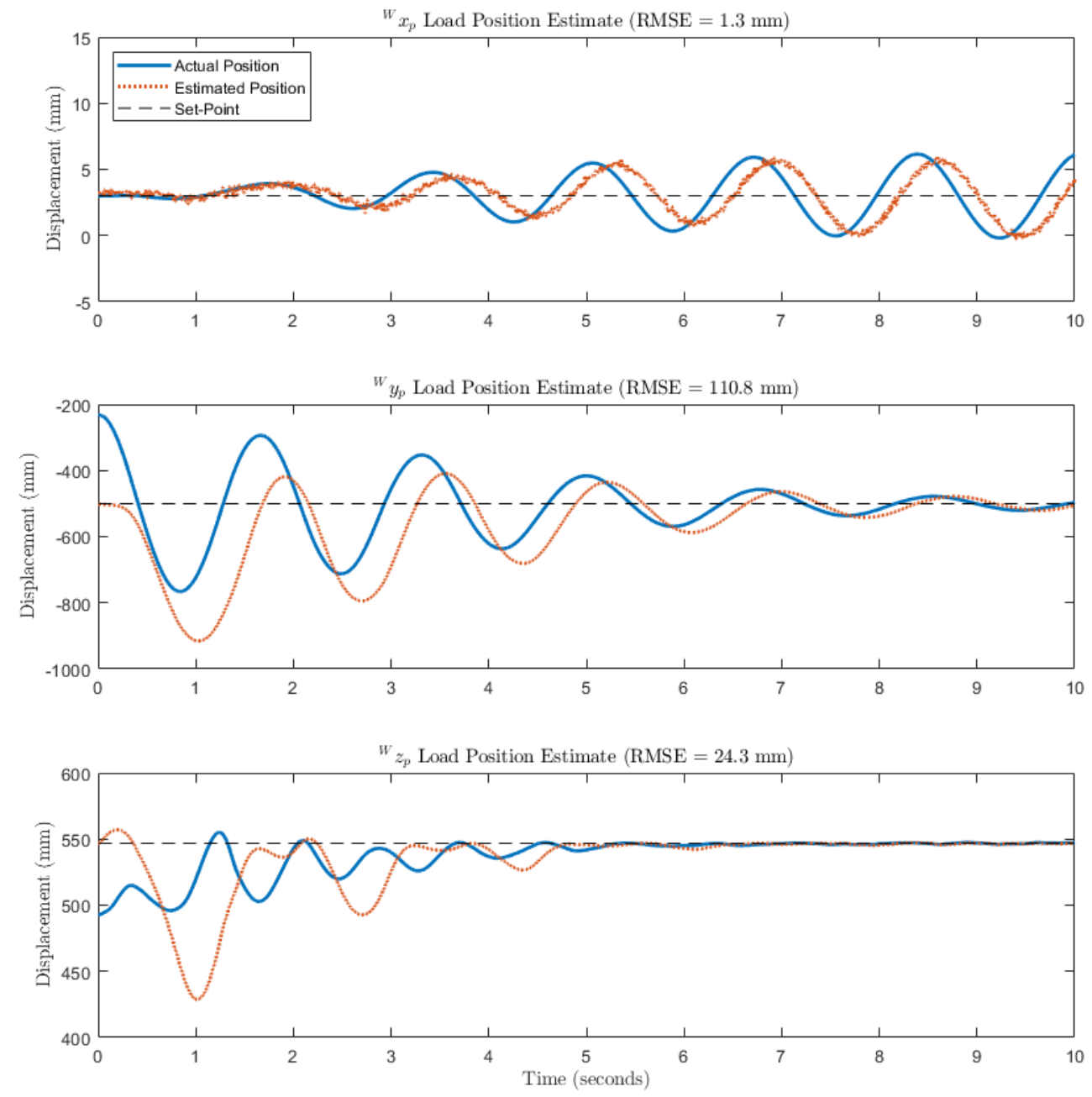

Figure 7.14: Load position estimate for the first 10 seconds of the compensated sensor-based simulation, Simulation 060-2. The large errors in the z-direction over the first 2 seconds of the initialization period result in an abrupt change in pendulum length/height in some cases. 
Load Position Estimate: Simulation 020-2
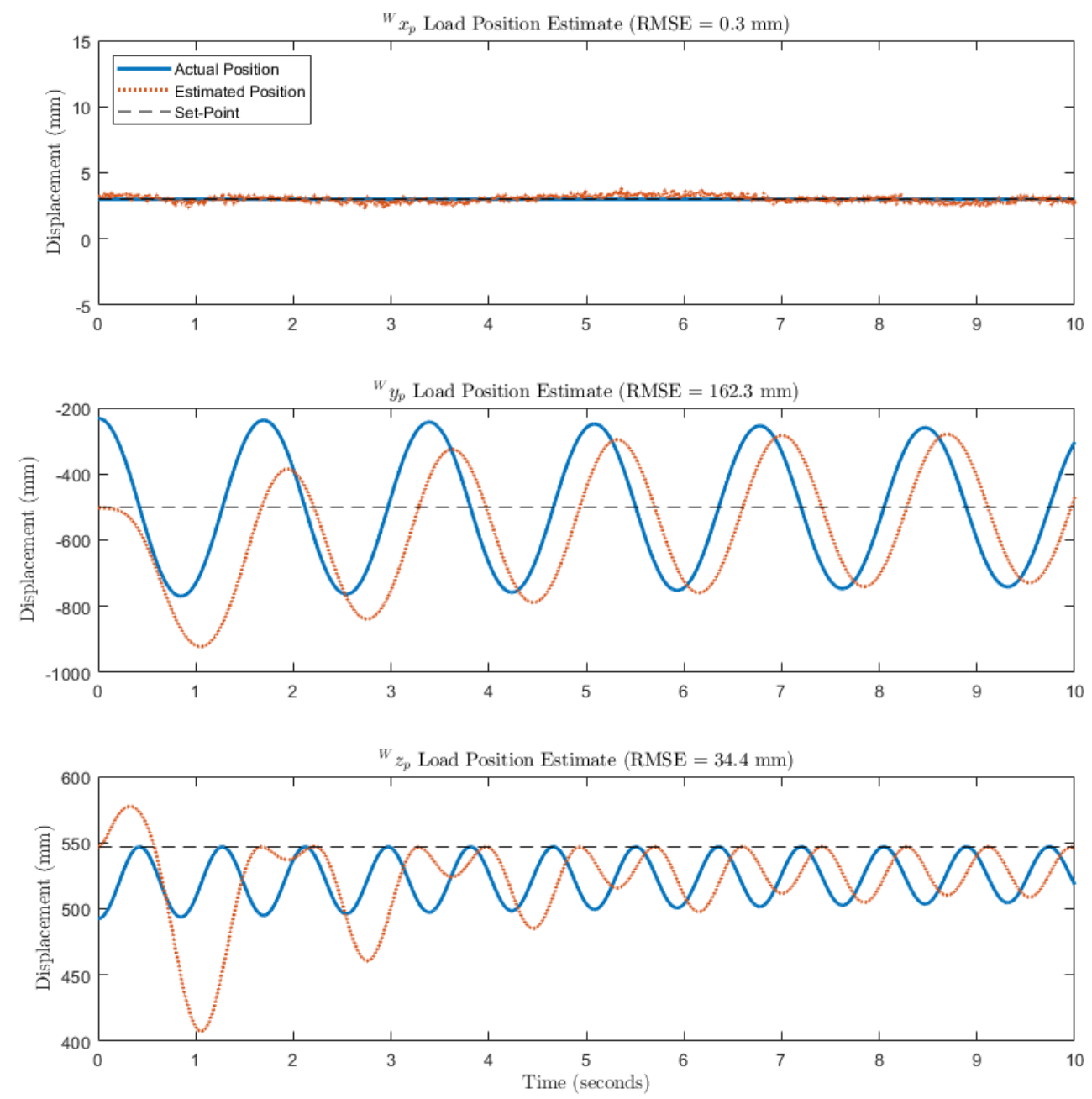

Figure 7.15: Load position estimate for the first 10 seconds of the uncompensated sensor-based simulation, Simulation 020-2. The estimation is shown to converge to the actual value with a phase lag within this time, which suggests a minimum initialization period for the estimation algorithm. 


\section{Energy Dissipation Results: Time Constants for Exponential Decay}

In Figure 7.16 and Figure 7.17, respectively, the time constants associated with the exponential decay of the $\theta_{x}$ and $\theta_{y}$ peak angles are shown. These time constants are an indication of the time required for the angular response to decay after the maximum peak angle is reached. The uncompensated cases were used to identify the natural decay/damping constants in Section 7.2.3, which are equivalent to mean time constants of 73.5 seconds for the $\theta_{x}$ decay and 111.4 seconds for the $\theta_{y}$ decay across all repetitions of the experiments. These time constants are observed for the uncompensated simulated responses with less than $1 \%$ error resulting from the exponential fitting procedure. For the experimental results, however, the time constants range from 53.6-97.0 seconds for the $\theta_{x}$ decay, and from $67.3-214.3$ seconds for the $\theta_{y}$ decay. These variations result in percentage differences in the range of $-24.2 \%$ to $37.6 \%$ (mean: $-0.8 \%$ ) for $\theta_{x}$ and $-48.1 \%$ to $65.5 \%$ (mean: $-0.4 \%$ ) for $\theta_{y}$ time constants when comparing the uncompensated simulation values to the experimental values. It is noted that the uncompensated $\theta_{y}$ time constants show more significant variations during the experiments, which are attributed to changes in drag caused by twisting motion about the cable.

When comparing the time constants identified from the truth data simulated results (1) to the experimental results for the compensated cases, percentage differences ranging from $-76.6 \%$ to $83.9 \%$ (mean: $-5.8 \%$ ) are found for the $\theta_{x}$ results, and percentage differences of $-83.8 \%$ to $886.7 \%$ (mean: $73.1 \%$ ) are found for the $\theta_{y}$ results. The most significant differences between the simulation and the experimental results for the $\theta_{y}$ time constants occur for the 400-700 mm pendulum lengths with the smallest initial angle $\theta_{y} \approx 7.6^{\circ}$. If these values are excluded, the range of percentage differences is reduced to $-83.8 \%$ to $69.7 \%$ (mean: $-5.6 \%$ ) for the $\theta_{y}$ time constant. Similarly, the

percentage differences found from comparing the time constants identified from the 
simulations with simulated sensor feedback (2) to the experimental results for the compensated cases range from $-15.3 \%$ to $-84.6 \%$ (mean: $-55.2 \%$ ) for $\theta_{x}$ and from 92.1\% to $422.0 \%$ (mean: $-36.3 \%$ ) for $\theta_{y}$. By excluding experimental outliers as above, the range of percentage differences is reduced to $-30.6 \%$ to $-92.1 \%$ (mean: $-63.3 \%$ ) for $\theta_{y}$ time constants. The differences between the compensated experiments and simulations are attributed to unmodelled imperfections such as twisting about the cable, magnetic field variations that lead to estimation error, backlash in the joints of the crane, and other factors that may affect the energy dissipation of the compensated system.

In all cases, substantial improvements are observed for compensated motion compared to the uncompensated motion for both angular directions. The percentage reduction in the $\theta_{x}$ time constant realised by the compensation system ranges from $82.5 \%$ to $95.1 \%$ (mean: $88.7 \%$ ) in the experimental cases, $80.6 \%$ to $96.7 \%$ (mean: $89.7 \%$ ) in the truth simulations, and $90.1 \%$ to $98.5 \%$ (mean: $95.0 \%$ ) in the sensorbased simulations. Similarly, the percentage reduction in the $\theta_{y}$ time constant realised by the compensation system ranges from $79.7 \%$ to $97.9 \%$ (mean: $90.9 \%$ ) in the experimental cases, $52.0 \%$ to $98.0 \%$ (mean: $89.5 \%$ ) in the truth simulations, and $49.2 \%$ to $99.5 \%$ (mean: $94.7 \%$ ) in the sensor-based simulations.

Although the compensation system yields substantial improvements for the time constants, imperfections in the estimation algorithm and unsynchronised actuator motion result in substantial off-axis motion. After this off-axis motion is generated and reaches the peak values, the compensated system damps out the motion. The dissipation of off-axis motion is quantified by the non-zero time constants in the righthand side of Figure 7.16 (off-axis motion in $\theta_{x}$ for an initial $\theta_{y}$ ) and the left-hand side of Figure 7.17 (off-axis motion in $\theta_{y}$ for an initial $\theta_{x}$ ). For the simulated results in Figure 7.16 and Figure 7.17, the small magnitude of the off-axis motion is near the deadband 
of the compensation system and therefore the dissipation occurs predominantly due to natural damping with time constants that are similar to those shown for the free response. In the experimental results, the off-axis motion is more substantial and the compensation system dissipates the energy much more rapidly after the peak value is reached. The sensor-based simulations generally show improvements over the truth simulations with an average percentage difference of $-46.3 \%$ for the $\theta_{x}$ time constant and $-60.3 \%$ for the $\theta_{y}$ time constant when comparing the sensor-based simulation to the truth simulation. A potential reason for this phenomenon was discussed in Section 7.2.4 and demonstrated in Figure 7.10 where imperfections in the estimation algorithm caused increased control actions.

Figure 7.18 shows the time constants for the height decay of the experimental and simulated results for the same test cases as above. In all cases, substantial improvements are realised with compensation regardless of potential off-axis motion. The percentage reduction in the height time constant realised by the compensation system ranges from $70.3 \%$ to $96.1 \%$ (mean: $88.2 \%$ ) in the experimental cases, $85.2 \%$ to $98.5 \%$ (mean: $93.5 \%$ ) in the truth simulations, and $91.6 \%$ to $99.5 \%$ (mean: $97.2 \%$ ) in the sensor-based simulations. These results directly represent the improved dissipation of potential energy through the compensation system regardless of off-axis motion.

Through the above analysis, the decay time constants exhibit reductions of up to $99.5 \%$ when the compensation system is applied. These reductions correspond to reduced settling times and indicate the improved ability of the compensation system to dissipate the initial energy when compared to the natural damping. It is noted that imperfections in the physical system result in off-axis motion and deviations between the simulated and experimental results; however, the trends are consistent and similar improvements are observed in all cases. 
Energy Dissipation Results:

Time Constants for $\theta_{x}$ Decay
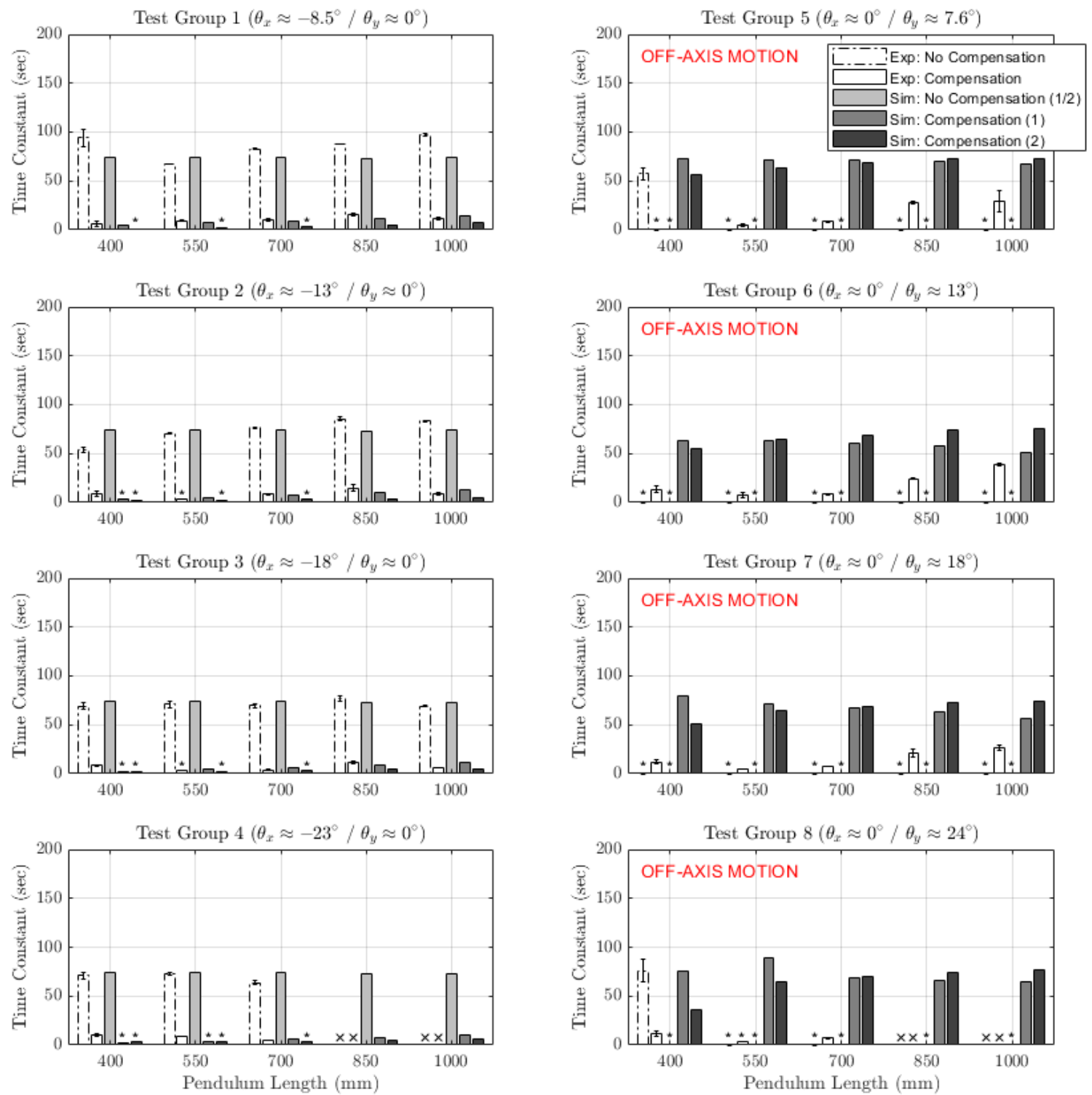

Figure 7.16: Time constants for the decay of peak $\theta_{x}$ angles for all energy dissipation tests (Test 001-080) and simulations (Simulation 001-1/2-080-1/2). The experimental results are summarized by the mean of all repetitions with error bars to indicate the standard errors, and the results are grouped by the mean initial angle. 
Energy Dissipation Results:

Time Constants for $\theta_{y}$ Decay
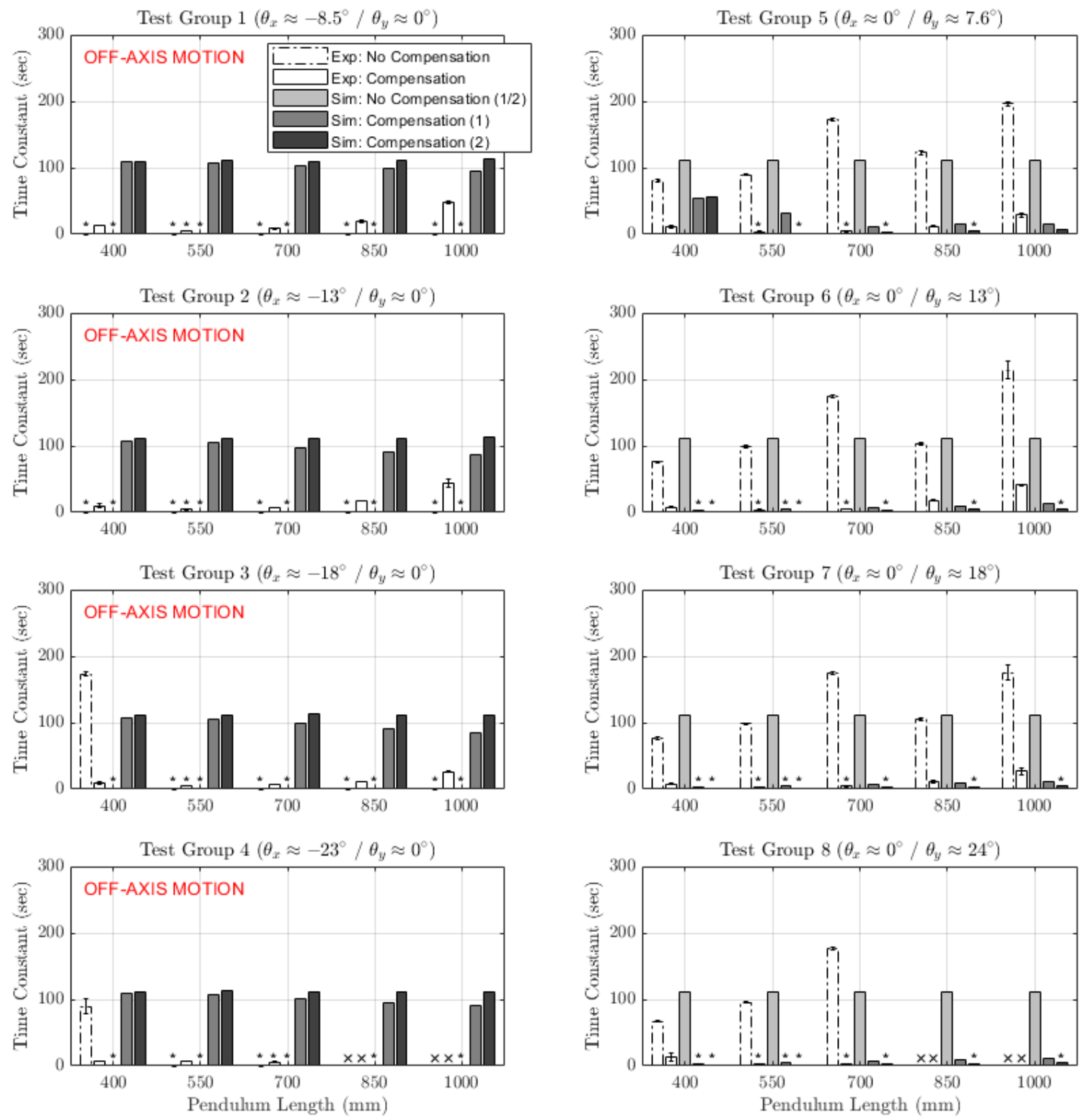

Figure 7.17: Time constants for the decay of peak $\theta_{y}$ angles for all energy dissipation tests (Test 001-080) and simulations (Simulation 001-1/2-080-1/2). The experimental results are summarized by the mean of all repetitions with error bars to indicate the standard errors, and the results are grouped by the mean initial angle. 
Energy Dissipation Results:

Time Constants for Height Decay
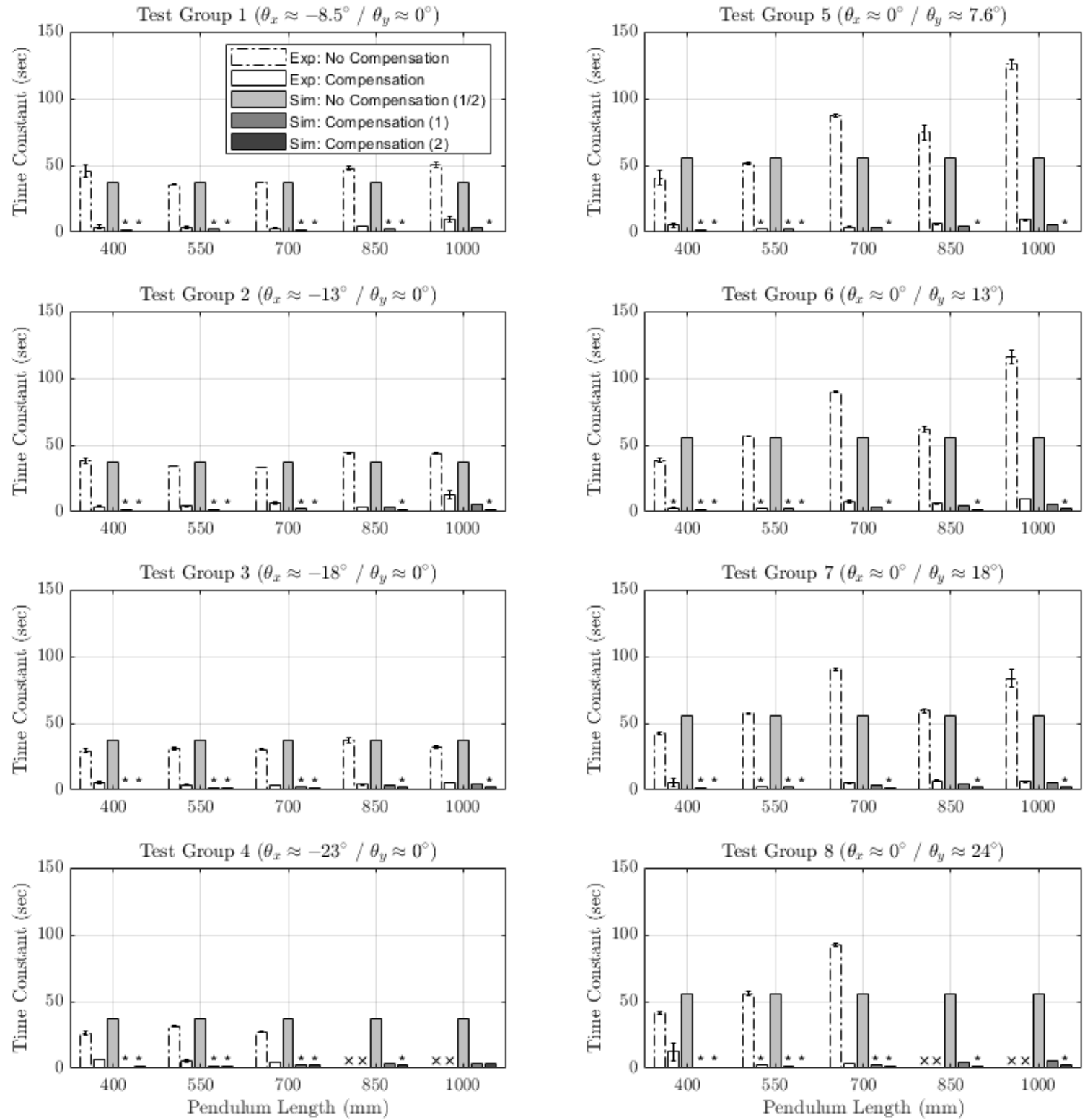

Figure 7.18: Time constants for the decay of peak heights for all energy dissipation tests (Test 001-080) and simulations (Simulation 001-1/2-080-1/2). The experimental results are summarized by the mean of all repetitions with error bars to indicate the standard errors, and the results are grouped by the mean initial angle. 


\section{Energy Dissipation Results: Distance Travelled}

Figure 7.19 shows the distance travelled while the velocity was above the noise threshold for the experimental and simulated test cases. The average percentage difference of the simulated to the experimental distance travelled is $-2.4 \%$ for the uncompensated cases, which indicates good agreement between the simulated and experimental responses. The average percentage difference of the truth simulations is $-56.2 \%$ and the average percentage difference of the sensor-based simulations is $-71.5 \%$ when taken relative to the experimental values for the compensated cases. These large percentage differences may be attributed to unmodelled imperfections in the real-world system that negatively impact its performance, including twisting about the cable or variations in the magnetic fields sensed by the two IMUs. The percentage reduction in distance travelled realised by the compensation system ranges from $41.1 \%$ to $94.7 \%$ (mean: $77.8 \%$ ) in the experimental cases, $84.9 \%$ to $98.4 \%$ (mean: $92.7 \%$ ) in the truth simulations, and $90.2 \%$ to $98.3 \%$ (mean: $95.2 \%$ ) in the sensor-based simulations.

The distance travelled metric is more appropriate for quantifying the dissipation of the initial energy than the ellipsoid volume, and it reinforces the conclusions drawn from the height time constants. The experimental distance travelled deviates from the simulated distance travelled in many cases due to the combination of noise in the detected position and imperfections in the real-world system. The simulation results also include mean damping terms, assume no rotation of the pendulum about the cable axis, and assume that each IMU senses the same global magnetic field. Each of these assumptions may result in further deviations between the simulated and experimental results for the compensated cases in which the twist about the cable axis or magnetic field variations may interfere with the estimation algorithm. 
Energy Dissipation Results:

Distance Travelled with Noise Threshold
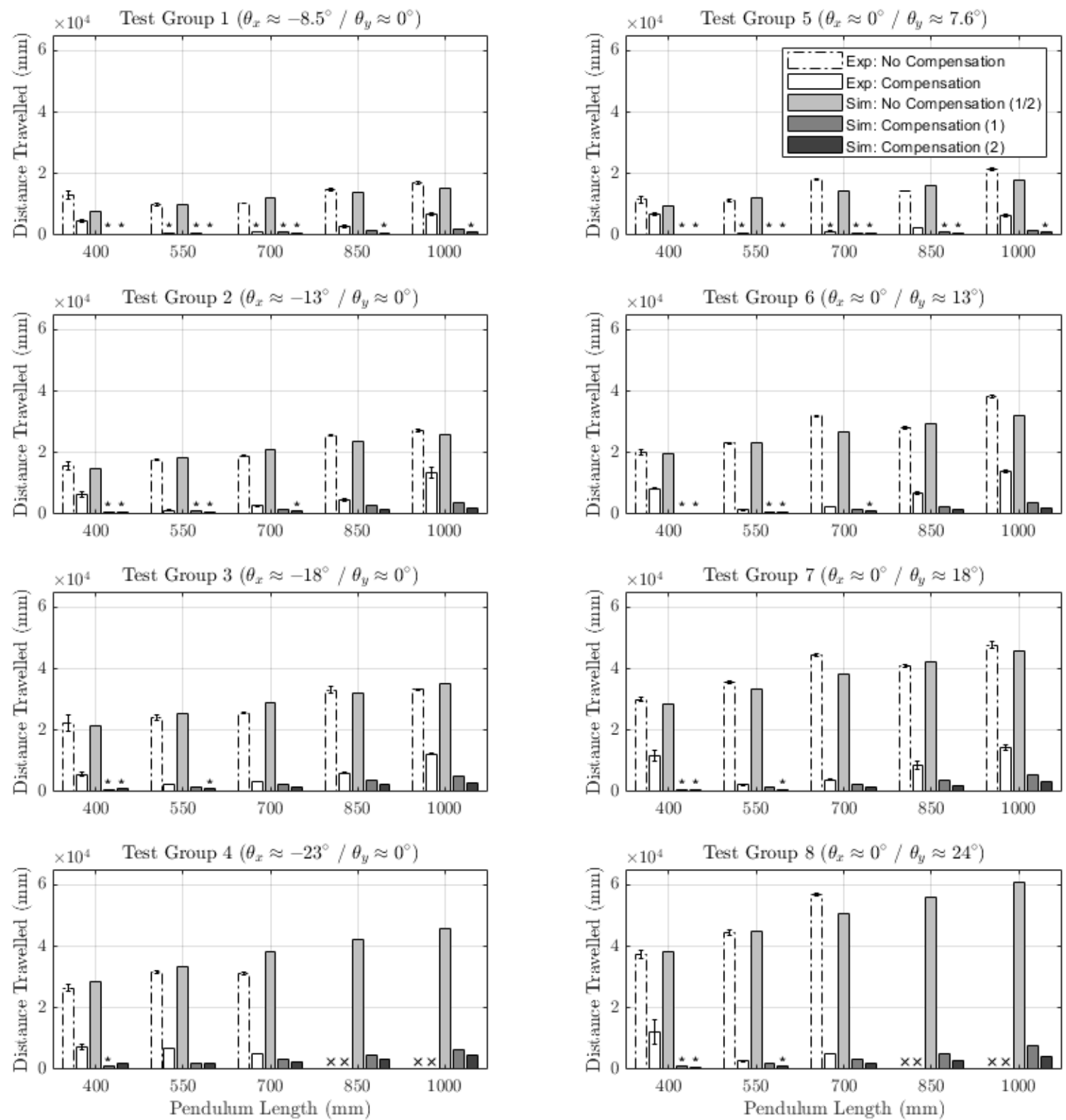

Figure 7.19: Distance travelled above a noise threshold during the test period for all energy dissipation tests (Test 001-080) and simulations (Simulation 001-1/2-080$1 / 2$ ). The experimental results are summarized by the mean of all repetitions with error bars to indicate the standard errors, and the results are grouped by the mean initial angle. 


\section{Energy Dissipation Results: Set-Point Tracking RMSE}

In Figure 7.20, the root-mean-squared error (RMSE) is shown as the final metric for evaluating the energy dissipation performance for each of the test cases. The RMSE is calculated by taking the Euclidean distance from the detected position of the load to its desired position, the equilibrium/set-point, and comparing this value to a reference value of zero for all data points throughout the duration of the test. The average percentage difference of the simulated RMSE relative to the experimental RMSE is $0.3 \%$ for the uncompensated cases, which indicates good agreement between the simulated and experimental responses. The average percentage difference of the truth simulations is $-38.6 \%$, and the average difference of the sensor-based simulations is $-46.1 \%$ when taken relative to the experimental values for the compensated cases. It is believed that the large differences between the simulation and the experiment may be attributed to unmodelled effects.

The percentage reduction in RMSE realised by the compensation system ranges from $34.6 \%$ to $68.0 \%$ (mean: $54.8 \%$ ) in the experimental cases, $58.9 \%$ to $84.0 \%$ (mean: $72.2 \%$ ) in the truth simulations, and $67.4 \%$ to $83.2 \%$ (mean: $75.7 \%$ ) in the sensorbased simulations. These reductions in RMSE demonstrate the improved ability of the compensation system to dissipate energy while simultaneously improving the tracking error in cases where a disturbance or initial angle is applied.

Through the analysis presented in this section, the energy dissipation performance was shown to improve substantially when the compensation system was applied. The time constants yielded the most direct insight into the energy dissipation performance as they represented the exponential decay of the initial energy. The distance travelled and RMSE indicated similar performance improvements over the uncompensated cases; however, the ellipsoid volume metric was deemed inappropriate for the energy dissipation tests due to non-elliptical pendulum motion. 
The truth data simulations demonstrated the performance of the compensation system with ideal knowledge of the load motion, whereas the sensor-based simulations included sensor noise and used the imperfect estimation algorithm. The sensor-based simulations showed improvement over the truth data simulations in many cases due to differences in overshoot and altered behaviour near the angular deadband of the motion compensation controller. Although the sensor-based simulations are more representative of the real-world system due to the emulated IMUs and sensor noise, unmodelled phenomena impact the experimental performance and result in several differences between the simulation and experimental results. However, the trends and potential for performance improvements are still confirmed in all simulations via comparison to the uncompensated cases.

In the following section, the ability of the compensation system to track a fixed set-point while subject to base excitation is investigated. 
Energy Dissipation Results:

Set-Point Tracking RMSE
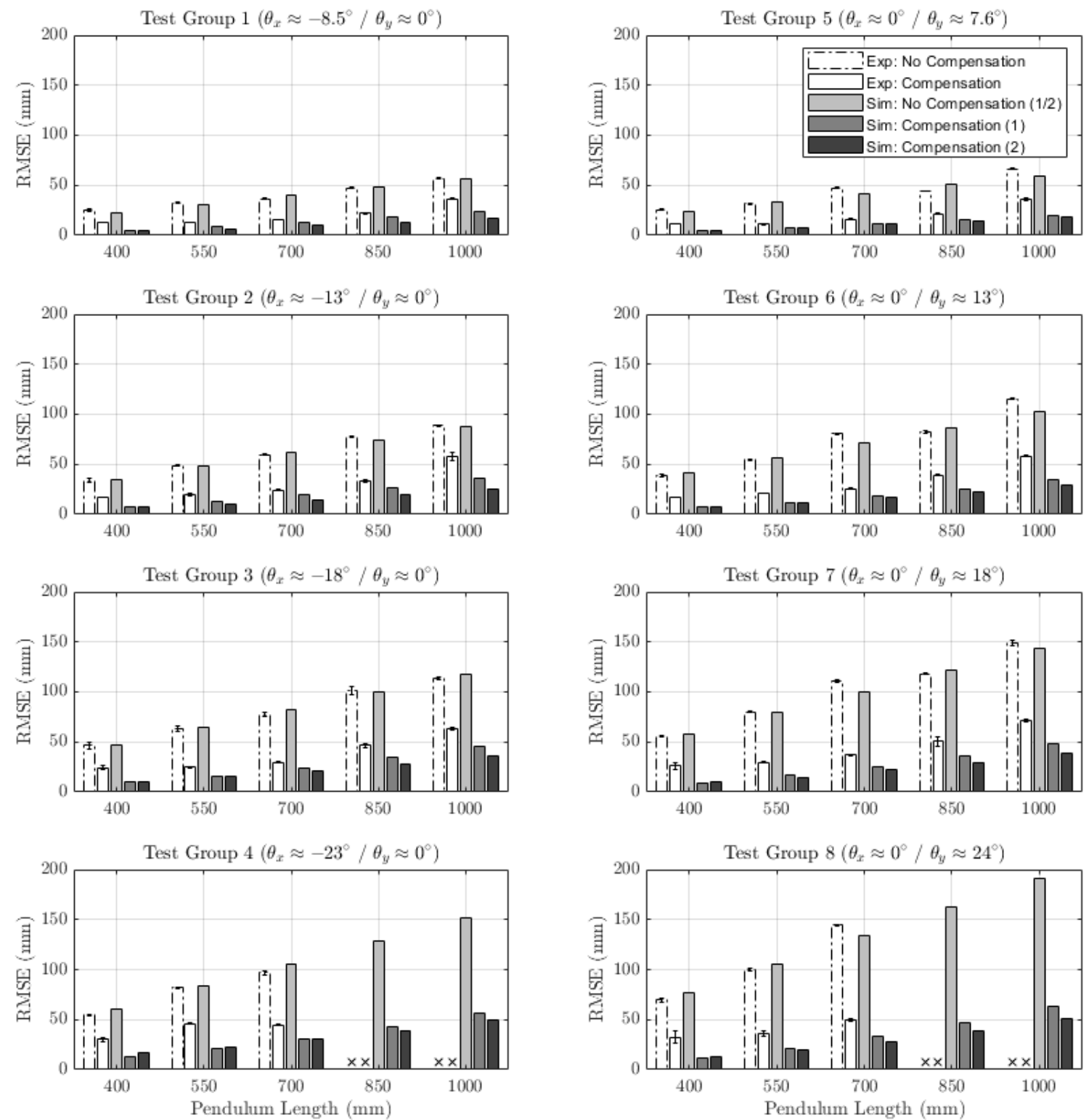

Figure 7.20: Root-mean-squared error (RMSE) of the distance between the load position and the set-point during the test period for all energy dissipation tests (Test 001-080) and simulations (Simulation 001-1/2-080-1/2). The experimental results are summarized by the mean of all repetitions with error bars to indicate the standard errors, and the results are grouped by the mean initial angle. 


\subsection{Fixed Set-Point Tracking}

In this section, the ability of the compensation system to track a fixed set-point while subject to base excitation is evaluated and compared to the performance of an uncompensated algorithm that uses only the inverse kinematics to position the crane tip and extend/retract cable without motion compensation control actions. The three sets of scaled ship motion profiles 1-3 from Appendix A are applied for a fixed setpoint with $700 \mathrm{~mm}$ length, and an additional set of five sinusoidal surge/sway motion profiles are applied to excite the natural frequencies of the pendulum load for five additional set-points with 400, 550, 700, 850, and $1000 \mathrm{~mm}$ lengths. Each test is repeated between 3-6 times, and the test parameters are summarized in Table 7.6. The corresponding simulations are also described in Table 7.6, and the simulations are again performed with two variations: truth data feedback (1) and simulated sensor feedback (2).

For the test-scale system, the high signal-to-noise ratio and imperfect tracking of the motion platform prevents the accurate estimation of surge, sway, and heave in the current work. Therefore, these linear ship motion set-points are supplied directly to the controller for motion profiles 1-3 to be used in the inverse kinematics for both compensated and uncompensated control. It is noted that estimating the linear heave motion of the ship showed promising results for full-scale ship motion in previous work [35]; however, a more in-depth analysis is recommended as future work.

To perform the experiments in this section, the system was brought to rest with the initial ship position, the load was positioned at the set-point, the compensation system was turned off, and the cameras were activated. A trigger was pressed to simultaneously start the ship motion, turn the compensation system on for compensated test cases, and activate an LED to indicate the start time in each camera. The 
tests were performed for a duration of 120 seconds with an additional 10 seconds of recording before and after the test to allow for initialization of the estimation algorithms, and to allow for initialization of the machine vision software. As previously discussed, markers were placed on the cable, ship deck, and floor to simplify the vision-based analysis.

Table 7.6: Fixed set-point tracking experiments/simulations (081-096).

\begin{tabular}{|c|c|c|c|}
\hline Exp/Sim No. & Control & Ship Motion & Repetitions \\
\hline 081 & Inverse Kinematic & Profile 1 & 3 \\
082 & Inverse Kinematic & Profile 2 & 5 \\
083 & Inverse Kinematic & Profile 3 & 5 \\
084 & Inverse Kinematic & $w_{n}\left(l_{p}=0.70 m\right)$ & 5 \\
085 & Inverse Kinematic & $w_{n}\left(l_{p}=0.40 m\right)$ & 3 \\
086 & Inverse Kinematic & $w_{n}\left(l_{p}=0.55 m\right)$ & 3 \\
087 & Inverse Kinematic & $w_{n}\left(l_{p}=0.85 m\right)$ & 3 \\
088 & Inverse Kinematic & $w_{n}\left(l_{p}=1.00 m\right)$ & 3 \\
\hline 089 & World-Frame Compensated & Profile 1 & 3 \\
090 & World-Frame Compensated & Profile 2 & 5 \\
091 & World-Frame Compensated & Profile 3 & 5 \\
092 & World-Frame Compensated & $w_{n}\left(l_{p}=0.70 m\right)$ & 6 \\
093 & World-Frame Compensated & $w_{n}\left(l_{p}=0.40 m\right)$ & 3 \\
094 & World-Frame Compensated & $w_{n}\left(l_{p}=0.55 m\right)$ & 3 \\
095 & World-Frame Compensated & $w_{n}\left(l_{p}=0.85 m\right)$ & 3 \\
096 & World-Frame Compensated & $w_{n}\left(l_{p}=1.00 m\right)$ & 3 \\
\hline
\end{tabular}

Similar to the previous section, the marker tracking procedure is applied and world data points are generated such as those shown in Figure 7.21a for Profile 1 without compensation (Test 081 Repetition 001), Figure 7.21b for Profile 1 with compensation (Test 089 Repetition 001), Figure 7.21c for $w_{n}\left(l_{p}=0.70 \mathrm{~m}\right)$ without compensation (Test 084 Repetition 001), and Figure 7.21d for $w_{n}\left(l_{p}=0.70 m\right.$ ) with compensation (Test 092 Repetition 001). To analyse the response of the system, the world data points are used to generate several key performance indicators. First, the 95\% ellipsoid volume is calculated as previously described as the point cloud data is expected to more closely resemble an ellipsoid for these test cases. The cumulative 
distance travelled and the set-point tracking root-mean-squared error (RMSE) are also calculated as in the previous section.

It is noted that, due to the scale of the experimental apparatus and limitations in the motion platform, the base excitation frequencies applied through the ship motion profiles are relatively far removed from the natural frequencies of the pendulum. The difference in frequencies avoids resonance and results in less significant motion of the load as illustrated in Figure 7.21a and Figure 7.21b for the first ship motion profile. However, this separation in frequencies would be less pronounced in realworld operations as increased cable lengths result in lower natural frequencies that may be excited by the ship motion. This observation motivates the additional test cases in which the set-point is selected at lengths ranging evenly from 400-1000 mm, and surge/sway motion is generated using sine waves with frequencies that matches the theoretical natural frequency of the system for each set-point as $w_{n}=\sqrt{g / l_{p}}$. In these test cases, resonance occurs and substantial motion is generated for the uncompensated case in Figure 7.21c. The substantial motion is then avoided using the compensation system as shown in Figure 7.21d, which demonstrates the ability of the compensation system to decouple the motion of the load from that of the ship. 


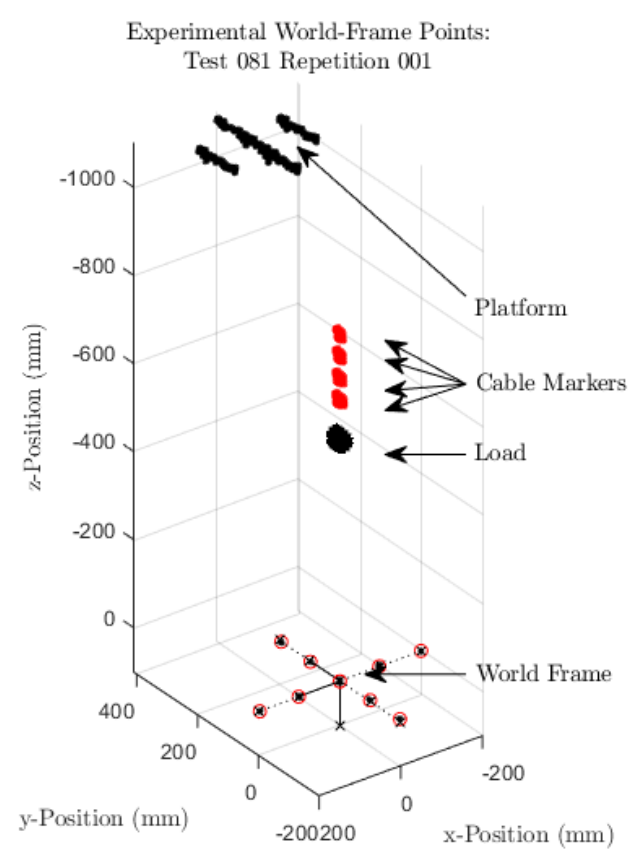

(a) Uncompensated; Profile 1

Experimental World-Frame Points:

Test 084 Repetition 001

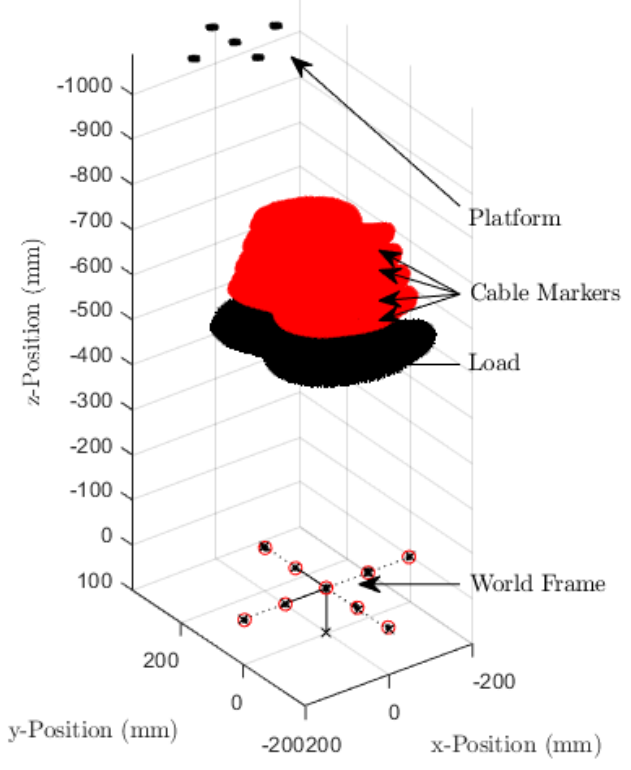

(c) Uncompensated; $w_{n}\left(l_{p}=0.70 m\right)$

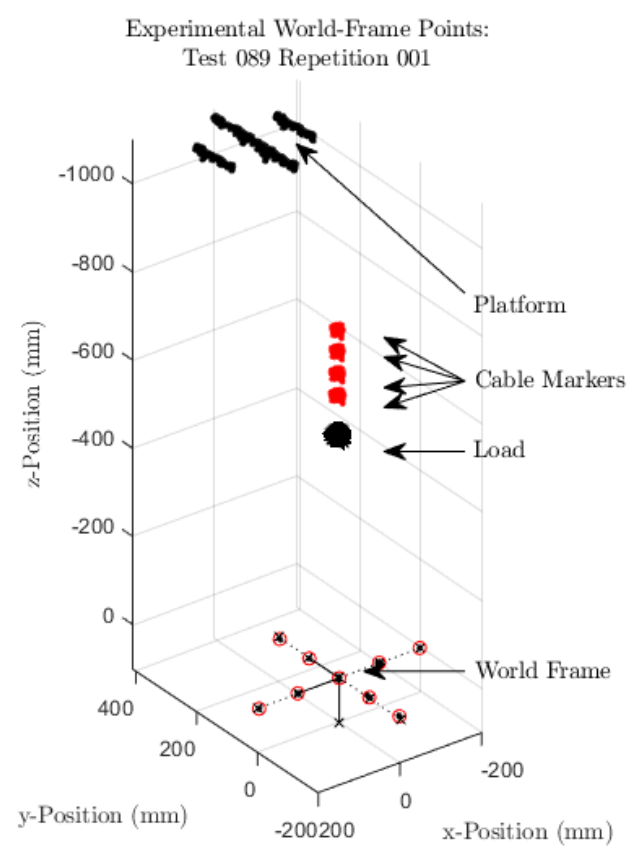

(b) Compensated; Profile 1

Experimental World-Frame Points: Test 092 Repetition 001

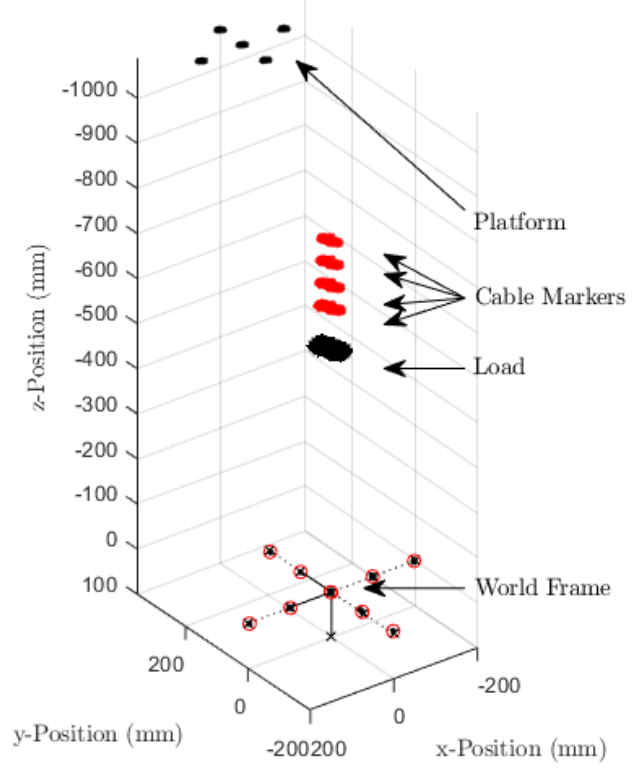

(d) Compensated; $w_{n}\left(l_{p}=0.70 m\right)$

Figure 7.21: World position tracking data for fixed set-point tests. 


\subsubsection{Ship Motion Results}

The results of this section are presented as a series of bar graphs that are grouped by the input motion profile. An asterisk $\left(^{*}\right)$ is used to indicate bars that may be difficult to see due to values that are below $2 \%$ of the maximum listed on the vertical axis. From left-to-right, the bars in each group represent:

\section{Exp: No Compensation}

The uncompensated experimental results to demonstrate the system response when only the inverse kinematics are used to position the crane tip without compensation (Test 081-088).

\section{Exp: Compensation}

The compensated experimental results to demonstrate the system response when the world-frame compensation system is used (Test 089096).

\section{Sim: No Compensation (1)}

The first variation (truth data feedback) of the uncompensated simulation results to demonstrate the system response when the inverse kinematics are used to position the crane tip without compensation (Simulation 081-1 to 088-1).

\section{Sim: No Compensation (2)}

The second variation (simulated sensor feedback) of the uncompensated simulation results to demonstrate the system response when the inverse kinematics are used to position the crane tip without compensation (Simulation 081-2 to 088-2). 


\section{Sim: Compensation (1)}

The first variation (truth data feedback) of the compensated simulation results to demonstrate the system response when the world-frame compensation system is used (Simulation 089-1 to 096-1).

\section{Sim: Compensation (2)}

The second variation (simulated sensor feedback) of the compensated simulation results to demonstrate the system response when the worldframe compensation system is used (Simulation 089-2 to 096-2).

Figure 7.22 summarizes the results of the fixed set-point tracking experiments and simulations for the three ship motion profiles. The experimental results are summarized as mean values with error bars to indicate the standard error. The first plot shows the ellipsoid volume, the second plot shows the distance travelled by the load, and the third plot shows the set-point tracking RMSE.

The ellipsoid volume exhibited a reduction of $9.2 \%$ for the third motion profile, whereas the first and second profiles showed a growth of $18.1 \%$ and $215 \%$ for the experimental test cases, respectively. The frequencies of the applied ship motion are far removed from the natural frequency of the pendulum, so very little excitation occurs as a result of the ship motion. Given the small uncompensated motion, it is likely that inaccuracies in the experimental compensation efforts and the stereo vision analysis could alter the ellipsoid dimensions enough to produce a relatively large increase in volume that does not accurately reflect the performance of the system. In the truth simulations, the compensation system reduces the ellipsoid volume by $52.1 \%, 84.4 \%$, and $63.8 \%$ for the first, second, and third motion profiles, respectively. Similarly, in the sensor-based simulations, the compensation system reduces the ellipsoid volume by $39.6 \%, 55.0 \%$, and $54.9 \%$ for the first, second, and third motion 
profiles, respectively. These simulation results are not affected by measurement error or imperfections in the stereo vision system as the simulated position of the pendulum is known.

It is noted that the majority of the motion occurs at low velocities with only small changes in position between video frames. The resulting velocities are near the noise threshold, so the distance travelled is very small with relatively large standard error. In the compensated cases, the compensation control actions are based on inaccurate estimates of the load motion and contribute to slightly higher load velocities at various points throughout the test. The higher load velocities result in more accumulation of distance travelled during the test period, which is indicated by the higher mean distances travelled when the compensation system and load estimation algorithm are used. However, due to the low velocity motion that was observed in both simulations and experiments, the distance travelled is not an ideal indicator of performance for fixed set-point tracking when the ship motion profiles are applied.

The set-point tracking RMSE is a representation of the tracking error throughout the test duration and is based on the calculated distance between the load position and its set-point. For the experimental test cases, the compensation system results in percentage reductions in RMSE of $6.7 \%$, and $22.8 \%$ for the second and third motion profiles, respectively. The first motion profile experiences an insignificant increase of $0.35 \mathrm{~mm}$ in RMSE. In the truth simulations, the compensation system results in $1.85 \%$ reduction, $20.0 \%$ reduction, and $0.46 \%$ increase in RMSE for the first, second, and third motion profiles, respectively. The small changes in RMSE for the first and third motion profiles are considered negligible and correspond to less than 0.08 $\mathrm{mm}$ differences. In the sensor-based simulations, the compensation system results in $20.6 \%, 21.2 \%$, and $34.7 \%$ reductions in RMSE for the first, second, and third motion profiles, respectively. The increased error for the uncompensated motion is likely due 
to the poor initial estimate leading to inverse kinematic control actions that excite the pendulum. The compensation system, however, is able to recover and apply anti-pendulum control actions to reduce the error as the estimations converge.

Through the above analysis, the fixed set-point tracking RMSE shows reductions of up to $34.7 \%$ when the compensation system is applied. Limited excitation occurs as the frequencies of the ship motion are removed from the natural frequencies of the pendulum, so the ellipsoid volume and distance travelled metrics provide limited insight into the experimental performance. The simulated results, however, are not affected by measurement noise and show potential reductions of up to $84.4 \%$ in ellipsoid volume. 
Fixed Set-Point Tracking Results

(with Ship Motion)
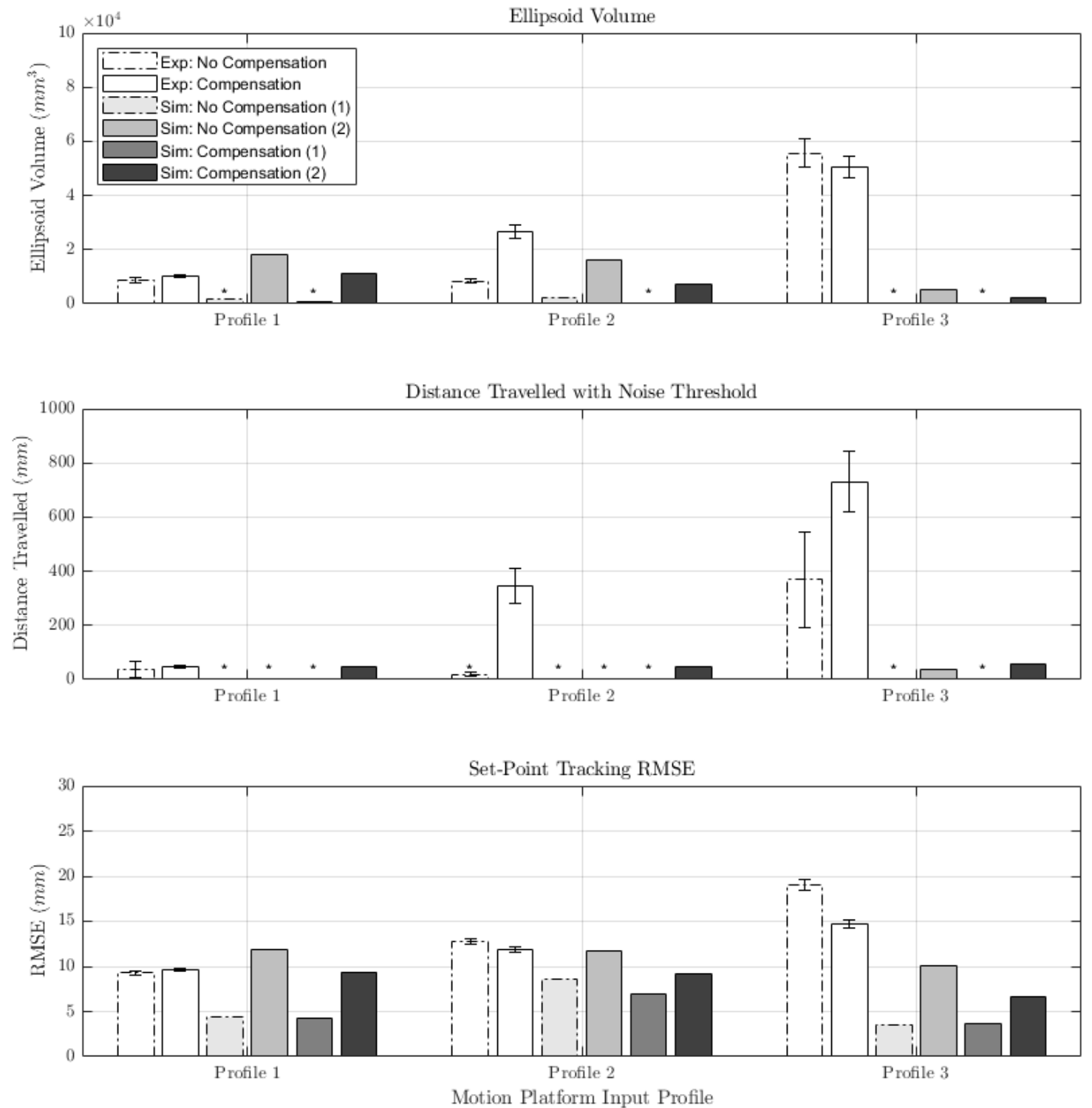

Figure 7.22: Ellipsoid volume, distance travelled, and set-point tracking RMSE for fixed set-point tracking tests and simulations with base excitation derived from scaled ship motion (Test 081-083 and 089-091; Simulation 081-1/2-083-1/2 and 089-1/2091-1/2). The experimental results are summarized by the mean of all repetitions with error bars to indicate the standard errors, and the results are grouped by the corresponding ship motion profile. 


\subsubsection{Natural Frequency Excitation Results}

Figure 7.23 shows the results of the fixed set-point tracking simulations and experiments for base excitations that correspond to the natural frequency of the pendulum. In these cases, substantial improvements are realised through the compensation system for all performance metrics in all cases. The experimental results show $98.7 \%$ to $99.8 \%$ reductions in ellipsoid volume, $73.2 \%$ to $96.5 \%$ reductions in distance travelled above the noise threshold, and $75.7 \%$ to $83.7 \%$ reductions in RMSE. In the truth simulations, the compensation system results in $100 \%$ reductions in ellipsoid volume, $89.0 \%$ to $99.8 \%$ reductions in distance travelled, and $86.9 \%$ to $91.9 \%$ reductions in RMSE. Similarly, the sensor-based simulations result in $100 \%$ reductions in ellipsoid volume, $87.0 \%$ to $91.9 \%$ reductions in distance travelled, and $85.7 \%$ to $91.9 \%$ reductions in RMSE. These significant improvements demonstrate the ability of the compensation system to effectively decouple the motion of the load from that of the ship and thereby avoid unwanted motion.

In the following section, the ability of the compensation system to improve trajectory tracking is investigated by varying the set-point over time to mimic a maritime pick-and-place operation with ship motion. 
Fixed Set-Point Tracking Results (with Natural Frequency Excitation)
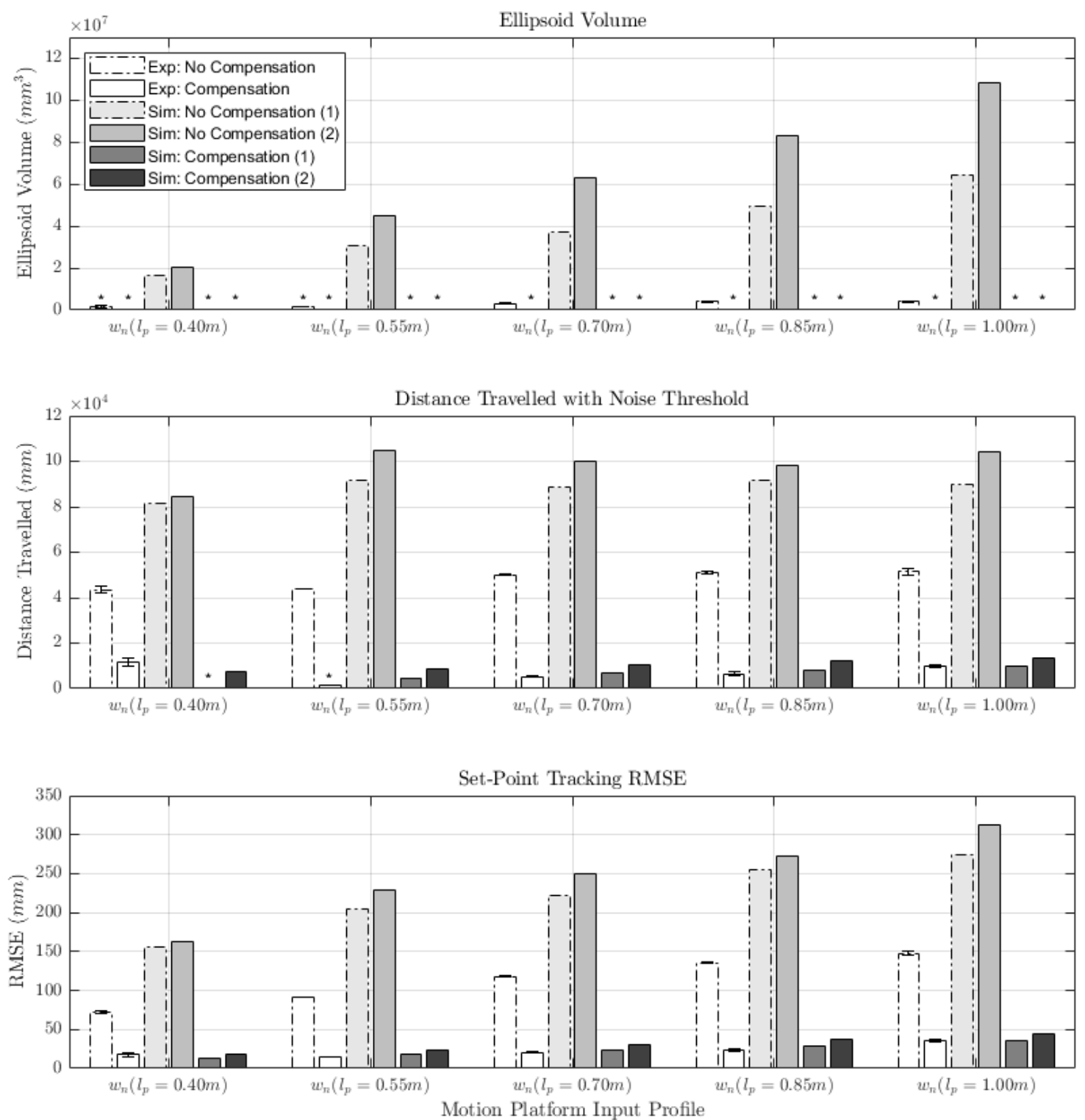

Figure 7.23: Ellipsoid volume, distance travelled, and set-point tracking RMSE for fixed set-point tracking tests and simulations with sinusoidal base excitation derived from the natural frequency of the pendulum $w_{n}=\sqrt{g / l_{p}}$ (Test 084-088 and 092096; Simulation 084-1/2-088-1/2 and 092-1/2-096-1/2). The experimental results are summarized by the mean of all repetitions with error bars to indicate the standard errors, and the results are grouped by the corresponding motion profile. 


\subsection{Variable Set-Point Tracking}

In this section, the ability of the compensation system to track a variable set-point or trajectory is investigated and compared to the performance of an uncompensated algorithm that uses only the inverse kinematics to position the crane tip and extend/retract cable without anti-pendulum control actions. The same three sets of scaled ship motion profiles 1-3 are applied, and a desired set-point profile is applied to mimic a pick-and-place maritime crane operation. The set-point profile is also applied for a case with no ship motion, and each experiment is performed $3-5$ times. The test parameters are summarized in Table 7.7 for the experiments and the corresponding simulations.

Table 7.7: Variable set-point tracking experiments/simulations (097-104).

\begin{tabular}{|c|c|c|c|}
\hline Exp/Sim No. & Control & Ship Motion & Repetitions \\
\hline 097 & Inverse Kinematic & No Motion & 5 \\
098 & Inverse Kinematic & Profile 1 & 3 \\
099 & Inverse Kinematic & Profile 2 & 4 \\
100 & Inverse Kinematic & Profile 3 & 4 \\
\hline 101 & World-Frame Compensated & No Motion & 4 \\
102 & World-Frame Compensated & Profile 1 & 3 \\
103 & World-Frame Compensated & Profile 2 & 4 \\
104 & World-Frame Compensated & Profile 3 & 4 \\
\hline
\end{tabular}


The procedure for running the experiments is identical to that of the previous section; however, the set-point varies as the operation is performed. Similar to the previous analysis, the world data is generated by tracking a series of markers through each recorded video frame.

Figure 7.24a shows the world data for the no motion case without compensation (Test 097 Repetition 001), which exhibits small deviations from the trajectory. In contrast, Figure $7.24 \mathrm{~b}$ shows the world data for the no motion case with compensation (Test 101 Repetition 001), which exhibits slight oscillations about the trajectory.

When ship motion is applied in Figure 7.24c, the oscillations about the trajectory become substantial for the uncompensated case with the first ship motion profile (Test 098 Repetition 001). However, in Figure 7.24d, the compensation system is able to significantly reduce these tracking errors for the compensated case with the first ship motion profile (Test 102 Repetition 001).

To evaluate the results, the distance travelled and set-point tracking RMSE are applied as in the previous sections. The ellipsoid volume is calculated by subtracting the set-point from the detected world data point prior to the ellipsoid fitting procedure. This subtraction results in a point cloud that represents the variation of the load about its time-varying set-point. The results are summarized in bar graphs in the same format as described/used in the previous section. 

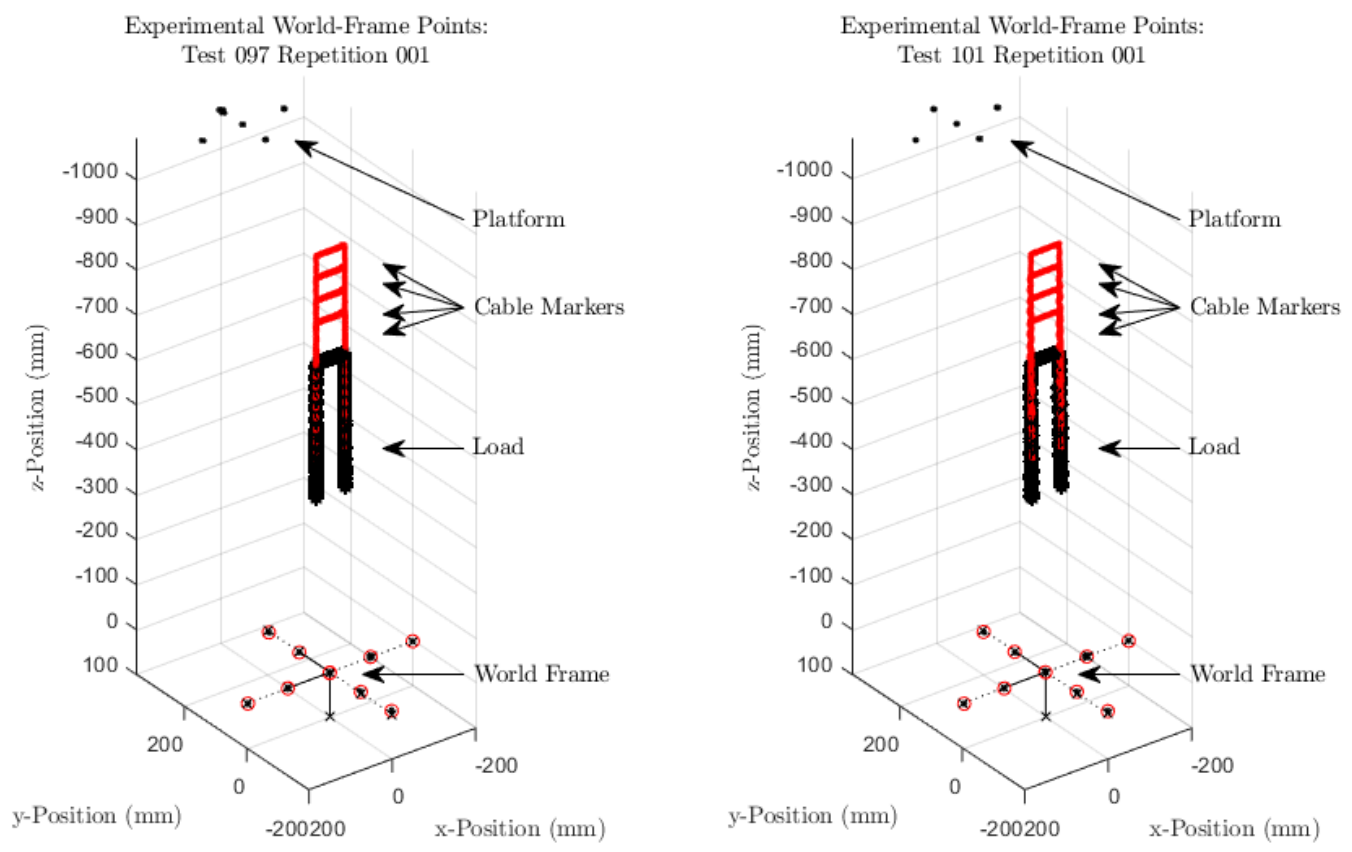

(a) Uncompensated; No Motion Experimental World-Frame Points: Test 098 Repetition 001

(b) Compensated; No Motion Experimental World-Frame Points: Test 102 Repetition 001
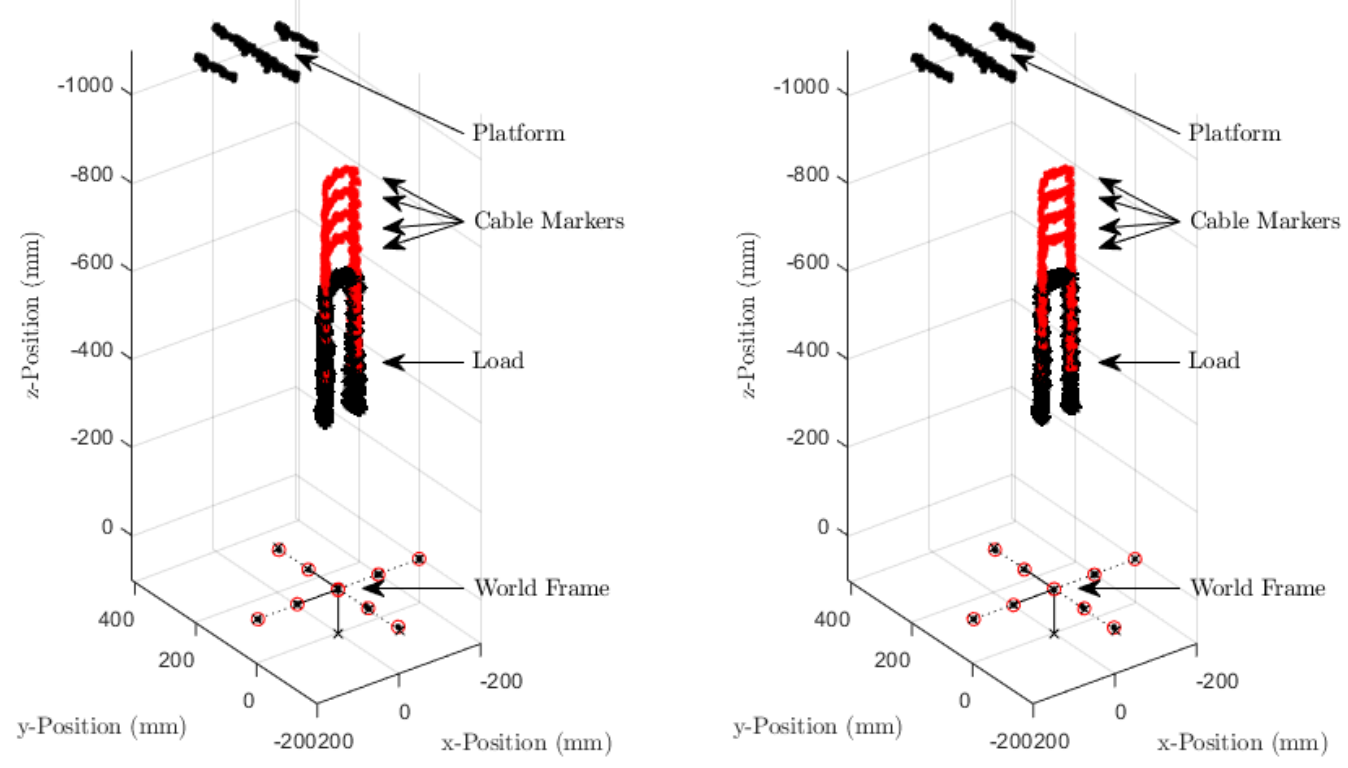

(c) Uncompensated; Profile 1

(d) Compensated; Profile 1

Figure 7.24: World position tracking data for variable set-point tests. 
Figure 7.25 summarizes the set-point tracking results for the 95\% Ellipsoid Volume, Distance Travelled, and RMSE metrics. The experimental results show 63.8\%, $14.0 \%$, and $25.8 \%$ reduction in the relative ellipsoid volume; $73.7 \%, 86.8 \%$, and $75.9 \%$ reduction in the distance travelled; and $24.3 \%, 36.5 \%$, and $48.5 \%$ reductions in the RMSE when comparing the compensated values to the uncompensated values for the first, second, and third ship motion profiles, respectively. The truth simulation results yield $81.3 \%, 82.1 \%$, and $67.6 \%$ reduction in the relative ellipsoid volume; and $24.4 \%$ reduction, $5.0 \%$ reduction, $6.1 \%$ increase in the RMSE for the first, second, and third ship motion profiles, respectively. The RMSE increase in the third profile results from a $0.33 \mathrm{~mm}$ change in RMSE and may be caused by anti-pendulum control actions deviating from the desired set-point trajectory while attempting to damp out the pendulum angles. The sensor-based simulation results yield $33.9 \%, 57.1 \%$, and $67.7 \%$ reduction in the relative ellipsoid volume; and $18.0 \%, 36.6 \%$, and $35.1 \%$ reduction in the RMSE. In all simulations, the distance travelled is considered negligible as the majority of the motion occurs below the velocity threshold.

The variable set-point tracking performance exhibits 14.0-86.8\% improvements across all experimental metrics, and the motion of the load about the desired trajectory is visibly reduced when the compensation system is applied. Similar performance improvements are noted for the ellipsoid volume and RMSE in the sensor-based simulations, whereas the truth data simulations indicate only minor improvements over the uncompensated approach. 
Variable Set-Point Tracking Results

(with Ship Motion)
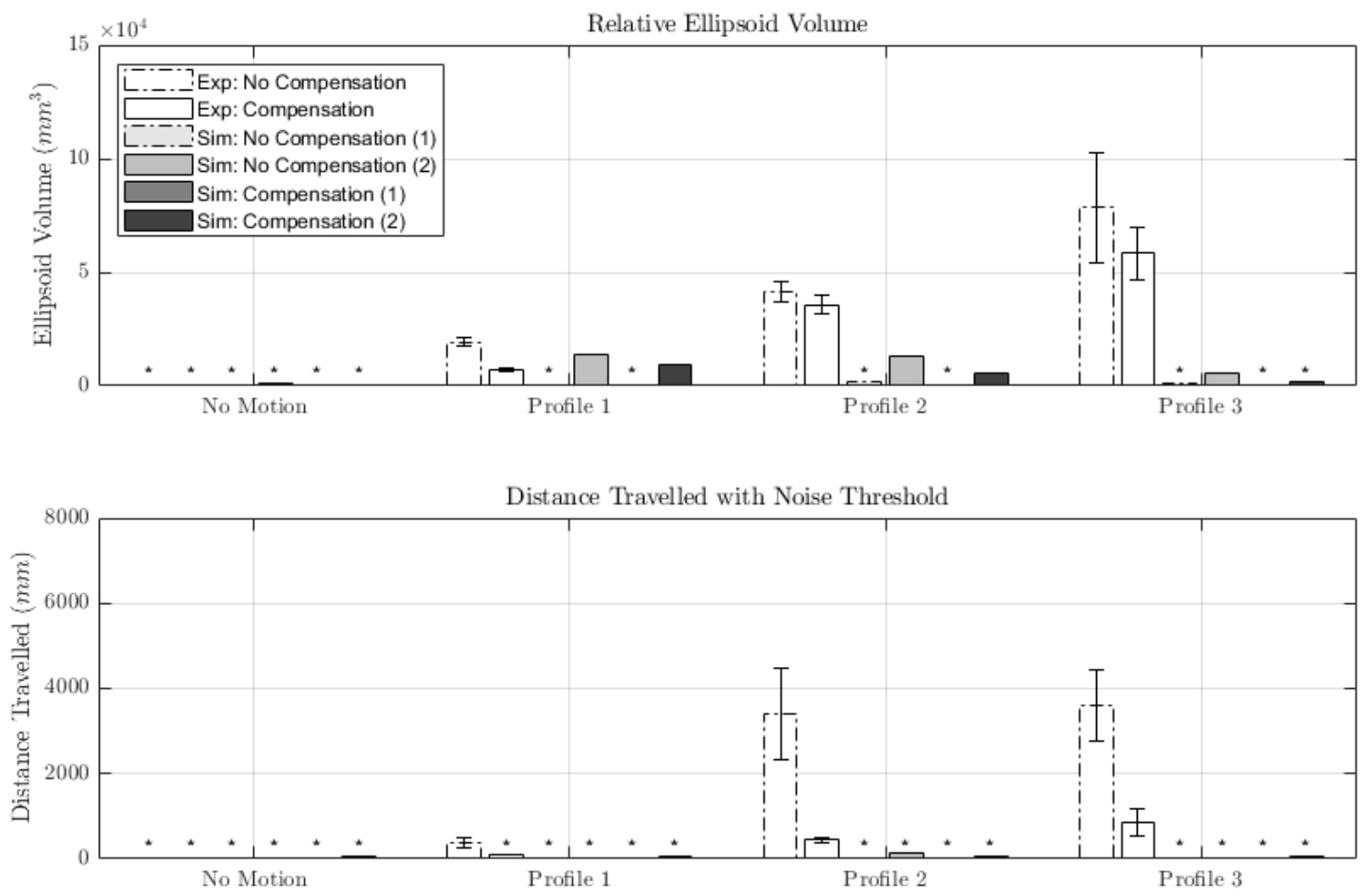

Set-Point Tracking RMSE

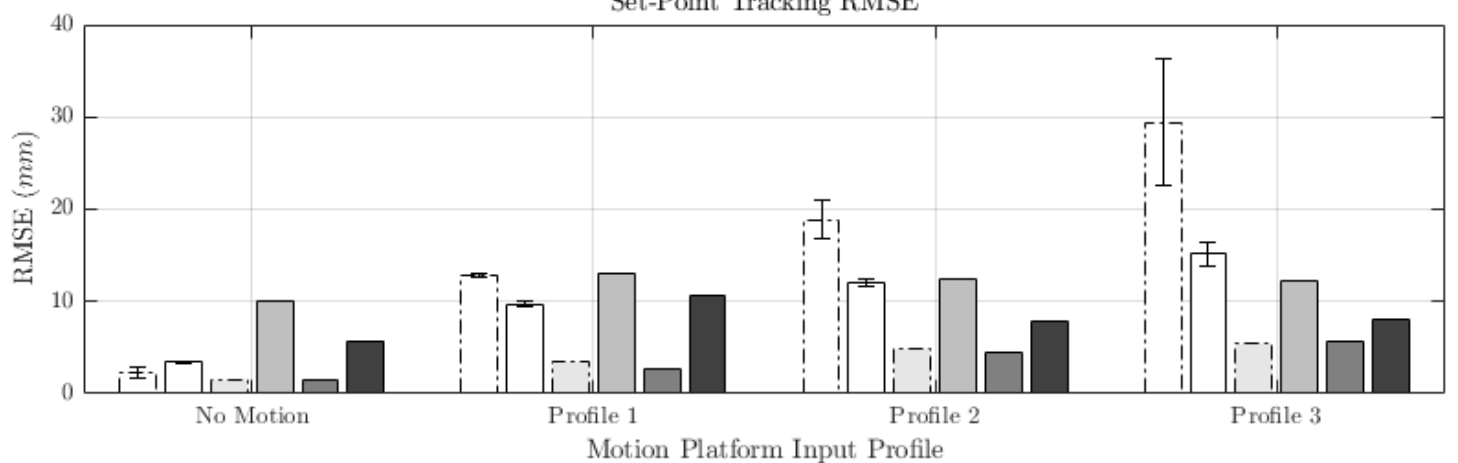

Figure 7.25: Relative ellipsoid volume, distance travelled, and set-point tracking RMSE for variable set-point tracking tests and simulations with base excitation derived from scaled ship motion (Test 097-104; Simulation 097-1/2-104-1/2). The experimental results are summarized by the mean of all repetitions with error bars to indicate the standard errors, and the results are grouped by the corresponding ship motion profile. 


\subsection{Conclusion/Summary}

In this chapter, the performance of the compensation system was analysed for three different scenarios. The results were summarized in Section 7.1 as mean reductions for the experiments, truth simulations, and sensor-based simulations. In Section 7.2, substantial improvements in energy dissipation were shown for time constants associated with the exponential decay of the initial energy through $88.2-97.2 \%$ mean reductions, as well as the distance travelled by the pendulum through $77.8-95.2 \%$ mean reductions and the set-point tracking RMSE through 54.8-75.7\% mean reductions when the compensated response was compared to the uncompensated/free response. In Section 7.3, improvements were noted for the RMSE values through 7.1-25.5\% mean reductions for tracking a fixed set-point with ship motion. Although the majority of simulations and experiments showed a $9.2-84.4 \%$ reduction in ellipsoid volume, an increase of $18.1-215 \%$ was noted for the first and second ship motion profiles. These increases are likely a result of the ellipsoid fitting procedure coupled with minor deviations in the shape of the point cloud under compensation. The frequency range of the ship motion profiles is relatively far removed from the natural frequency of the pendulum. Therefore, to further investigate the capabilities of the system, sinusoidal base excitation was applied in several additional test cases to excite the natural frequency of the pendulum. Substantial improvements in the fixed set-point tracking performance were realised for ellipsoid volume through $99.4-100 \%$ mean reductions, distance travelled through 85.4-93.5\% mean reductions, and set-point tracking RMSE through $80.1-89.6 \%$ mean reductions. Finally, in Section 7.4 , the ability of the system to track a trajectory or variable set-point was analysed. Again, the compensation system resulted in substantial improvements in relative ellipsoid volume through 34.5-77.0\% mean reductions and set-point tracking RMSE through 7.8-36.4\% mean 
reductions relative to the uncompensated test cases. Although the simulated distance travelled could not be meaningfully compared due to velocities that were below the noise threshold, the experimental results also yielded a mean reduction of $78.8 \%$ in distance travelled for variable set-point tracking.

The time constants, distance travelled, and RMSE showed similar performance improvements for the energy dissipation tests; however, the ellipsoid volume metric was deemed inappropriate for these tests due to the non-elliptical pendulum motion. For the set-point tracking tests, the RMSE metric provided the most consistent comparison across all test cases, whereas the distance travelled and ellipsoid volume did not produce meaningful results in cases where the load motion was below the velocity noise threshold and/or little excitation occurred. For substantial load motion, such as the motion observed for the natural frequency base excitation, all of the performance metrics showed similar performance improvements.

The truth data simulations demonstrated the performance of the compensation system with ideal/perfect knowledge of the position and orientation of the load and ship, whereas the sensor-based simulations demonstrated the effects of the imperfect estimation algorithm on the overall performance of the system. The effect of a poor initial estimate was also observed through erroneous behaviour and changes in pendulum length during the first 10 seconds of the energy dissipation simulations, and it was suggested that the algorithm should be initialized for a period prior to activating the compensation system to avoid these erroneous control actions.

The work included in this chapter represents the completion of the first and second objectives of this thesis. The key contribution of this chapter is the test-scale experimental validation and simulation of the compensation and control techniques presented in this thesis for a variety of cases, including energy dissipation, fixed setpoint tracking, and variable set-point tracking test cases. 


\section{Chapter 8}

\section{Conclusions and Recommendations}

\subsection{Objectives}

The main objectives of this thesis were:

1. To develop a method for combining active-heave compensation and anti-pendulum control for a combined world-frame compensation system.

2. To integrate the robotic control technique with the combined compensation system for hardware-in-the-loop testing, and to evaluate the effectiveness of the control and compensation techniques through test-scale experiments and simulations.

The first objective of this thesis was accomplished through Chapter 4, Chapter 5, and Chapter 6. In Chapter 4, the forward and inverse kinematic models of the crane were developed for use in the compensation algorithm. In particular, the inverse kinematic models were necessary to convert from world-frame set-points to set-points for the linear actuators. A constrained kinematic/dynamic model of the pendulum load was also derived for use in the development of the compensation system. In Chap- 
ter 5, an estimation algorithm was implemented to relate the ship and load motion to the world frame. When combined with the inverse kinematic models, the results of the estimation algorithm allowed for set-point transformations between the world frame, the tip/load positions, and the required joint positions. Finally, Chapter 6 presented the individual joint controllers and the world-frame compensation system. The compensation system was developed in the world frame and used the aforementioned transformations to provide set-points to the joint controllers. To perform heave compensation, the crane tip position is maintained at a specified world-frame z-position and the cable length is adjusted to reduce the tracking error. To perform anti-pendulum control, the crane tip is used to dissipate energy by moving towards the load.

The second objective of this thesis was accomplished predominantly through Chapter 3 and Chapter 7. In Chapter 3, the test-scale experimental apparatus and simulations were developed. The hardware-in-the-loop simulations were developed in Simulink/Simscape based on the components used in the experimental apparatus. The robotic control and world-frame compensation algorithms from the first objective were implemented on a National Instruments myRIO controller, which was used to control both the simulations and the experimental apparatus with identical control software. In Chapter 7, the results of the test-scale experiments and simulations were presented and the performance of the compensation system was evaluated.

\subsection{Contributions}

The key contributions of this thesis were:

1. Further development of a test-scale experimental apparatus for evaluation of motion compensation systems. 
2. Development of parametric hardware-in-the-loop simulations to facilitate future development and testing. Although operator studies and human factors analysis were beyond the scope of the current work, the simulations allow for operatorin-the-loop testing in future work.

3. Development of a combined compensation system with potential for extended application to a variety of crane types in future work.

4. Application of a robotic control method to reduce the potential for operator error during compensated crane operations, as well as to facilitate task automation in future work.

5. Application of sensor fusion techniques to estimate both the ship and load motion through low-cost inertial sensor feedback.

6. Test-scale experimental validation and simulations of the compensation and control techniques for a variety of test conditions.

\subsection{Future Work}

Potential avenues for future work that stem from the work of this thesis include:

1. To improve the accuracy of the simulations, sensor biases and variations in the magnetic fields experienced by the two IMUs could be quantified and simulated. In addition, an investigation could be performed to determine if the lab conditions are representative of industrial conditions by gathering data from IMUs during real-world crane operations.

2. To improve the estimation algorithm, alternative sensor fusion algorithms could be investigated and applied in both test-scale and full-scale simulations to deter- 
mine the accuracy of the estimation and its effects on the system performance. The linear motion estimation algorithm and its effects on system performance could also be confirmed for surge, sway, and heave.

3. To improve the applicability of the research, the double-pendulum effects that typically occur between the hook and load could be modelled and investigated. If an IMU is to be used in an industrial case, the placement (i.e. on the hook or on the load) should be considered.

4. Full-scale simulations could be performed with hydraulic actuator models that are representative of those currently used within the industry. To confirm the simulations, full-scale testing could be performed in a controlled environment with a mid-sized crane.

5. An in-depth human factors analysis and operator investigation should be performed to evaluate how the operator interacts with the system and to determine which control structure is most intuitive. A variety of control structures and compensation systems should be compared to the standard joint-space control to determine the most appropriate avenues for future work.

6. Advanced tuning algorithms could be applied to determine optimal values for the parameters used in both the estimation algorithm and the compensation system.

7. Advanced control techniques could be considered, including adaptive and/or intelligent control. It is anticipated that adaptive/intelligent control techniques may further improve performance while allowing the system to automatically adjust parameters as operating conditions change. 


\section{References}

[1] A. Valverde and P. Tsiotras, "Dual quaternion framework for modeling of spacecraft-mounted multibody robotic systems," Frontiers in Robotics and AI, vol. 5, 11 2018. [Online]. Available: http://dx.doi.org/10.3389/frobt.2018.00128

[2] E. Özgür and Y. Mezouar, "Kinematic modeling and control of a robot arm using unit dual quaternions," Robotics and Autonomous Systems, vol. 77, 12 2015. [Online]. Available: http://dx.doi.org/10.1016/j.robot.2015.12.005

[3] "Knuckle boom crane: GCC - 25 ton SWL," [Accessed December 2019]. [Online]. Available: https://www.vlmaritime.com/product/j0013-knuckle-boom-crane/

[4] J. Babicz, Encyclopedia of Ship Technology, 2nd ed. WÄRTSILÄ Corporation, 2015.

[5] M. Santoso, S.-F. Su, and A. Aisjah, "Nonlinear rudder roll stabilization using fuzzy gain scheduling - pid controller for naval vessel," 12 2013, pp. 94-99. [Online]. Available: http://dx.doi.org/10.1109/iFuzzy.2013.6825416

[6] A. M. Arney, "FFG-7 ship motion and airwake trial. part II: removal of ship motion effects from measured airwake data." Air Operations Division Aeronautical and Maritime Research Laboratory, Tech. Rep. DSTO-TR-0093, 
October 1994. [Online]. Available: https://apps.dtic.mil/dtic/tr/fulltext/u2/ a291132.pdf

[7] MEOPAR: Marine Environmental Observation Prediction and Response Network, "Strategic plan 2017-2022," 2017. [Online]. Available: https://meopar.ca/ wp-content/uploads/2019/06/MEOPAR_Strategic_Plan_2017_ENG_WEB.pdf

[8] S. Galić, L. Zvonimir, and I. Skoko, "The role and importance of safety in maritime transportation," in 6th International Maritime Science Conference, 2014, pp. 186-200. [Online]. Available: http://www.pfst.unist.hr/imsc/archive/ 2014/IMSC2014_proceedings.pdf

[9] Canada Newfoundland and Labrador Offshore Petroleum Board, "Annual report 2006/07," C-NLOPB, 2007. [Online]. Available: https://www.cnlopb.ca/ wp-content/uploads/ar2007e.pdf

[10] M. Laird, "A structured approach to offshore crane maintenance," Topsides UK 2017 Conf. and Exhibition, 2017. [Online]. Available: https://www.spe-aberdeen.org/wp-content/uploads/2017/07/ Topsides-UK-2017-EnerMech-Presentation-final.pdf

[11] J. Thomas, C. C. Baker, T. B. Malone, J. Malone, C. L. Hard, I. C. L. Rezende, S. Caruana, and M. Witten, "Application of human factors in reducing human error in existing offshore facilities," Proceedings of the 2nd International Workshop On Human Factors In Offshore Operations '02 (HFW2002), 2002. [Online]. Available: https://digitalcommons.unl.edu/usdot/34?utm_source=digitalcommons. unl.edu\%2Fusdot\%2F34\&utm_medium=PDF\&utm_campaign=PDFCoverPages

[12] L. Ramli, Z. Mohamed, A. M. Abdullahi, H. Jaafar, and I. M. Lazim, "Control strategies for crane systems: A comprehensive review," Mechanical 
Systems and Signal Processing, vol. 95, pp. 1 - 23, 2017. [Online]. Available: http://dx.doi.org/10.1016/j.ymssp.2017.03.015

[13] J. Woodacre, "Model-predictive control of a hydraulic active heave compensation system with heave prediction," MASc, Dalhousie University, Halifax, Nova Scotia, August 2015. [Online]. Available: https://carleton.ca/mdl/wp-content/ uploads/Woodacre-Jeffrey-MASc-MECH-Aug-2015.pdf

[14] S. Albada, G. Dick van Albada, H. Hildre, and H. Zhang, "A novel approach to anti-sway control for marine shipboard cranes," in Proceedings - 27th European Conference on Modelling and Simulation, ECMS 2013, 05 2013, pp. 249-256. [Online]. Available: http://dx.doi.org/10.7148/2013-0249

[15] J. Suthakorn and G. G. Parker, "Anti-swing control of suspended loads on shipboard robotic cranes," Systematics, Cybernetics and Informatics, vol. 3, no. 1, pp. 35-40, 2005. [Online]. Available: http://www.iiisci.org/Journal/SCI/ FullText.asp?var $=\& \mathrm{id}=\mathrm{P} 195107$

[16] PALFINGER MARINE, "Palfinger marine product catalogue," 2017.

[17] Y. Chu, F. Sanfilippo, V. Æsøy, and H. Zhang, "An effective heave compensation and anti-sway control approach for offshore hydraulic crane operations," in 2014 IEEE International Conference on Mechatronics and Automation, August 2014, pp. 1282-1287. [Online]. Available: http: //dx.doi.org/10.1109/ICMA.2014.6885884

[18] J. J. Craig, Introduction to Robotics: Mechanics and Control, 3rd ed. Pearson Prentice Hall, 2005.

[19] M. Pedersen, M. Hansen, and B. Morten, "Developing a tool point control scheme for a hydraulic crane using interactive real-time dynamic simulation," 
Modeling, Identification and Control, vol. 31, 10 2010. [Online]. Available: http://dx.doi.org/10.4173/mic.2010.4.2

[20] J. McPhee, "Control, simulation, and testbed development for improving maritime launch and recovery operations," MASc, Carleton University, Ottawa, Ontario, 2019. [Online]. Available: https://carleton.ca/mdl/wp-content/ uploads/mcphee-controlsimulationandtestbeddevelopmentfor.pdf

[21] C. Calnan, "Set-point algorithms for active heave compensation of towed bodies," MASc, Dalhousie University, 2016. [Online]. Available: https://carleton.ca/ mdl/wp-content/uploads/Calnan-Clark-MASc-ENG-December-2016.pdf

[22] J. Woodacre, R. Bauer, and R. Irani, "A review of vertical motion heave compensation systems," Ocean Engineering, vol. 104, pp. 140 - 154, 2015. [Online]. Available: http://dx.doi.org/10.1016/j.oceaneng.2015.05.004

[23] E. Abdel-Rahman, A. Nayfeh, and Z. Masoud, "Dynamics and control of cranes: A review," Journal of Vibration and Control, vol. 9, pp. 863-908, 07 2003. [Online]. Available: http://dx.doi.org/10.1177/1077546303009007007

[24] J. Hatleskog and M. Dunnigan, "Heave compensation simulation for non-contact operations in deep water," vol. 2006, 10 2006, pp. 1 - 6. [Online]. Available: https://doi.org/10.1109/OCEANS.2006.307096

[25] C. Calnan, R. J. Bauer, and R. A. Irani, "Controller design and motion compensation for marine towed bodies," in OCEANS 2016 MTS/IEEE Monterey, September 2016, pp. 1-9. [Online]. Available: https://doi.org/10. 1109/OCEANS.2016.7761290

[26] C. Calnan, R. J. Bauer and R. A. Irani, "Reference-point algorithms for active motion compensation of towed bodies," IEEE Journal of Oceanic 
Engineering, vol. 44, no. 4, pp. 1024-1040, October 2019. [Online]. Available: https://doi.org/10.1109/JOE.2018.2866317

[27] C. Westin, "Modelling and simulation of marine cables with dynamic winch and sheave contact," MASc, Carleton University, Ottawa, Ontario, 2018. [Online]. Available: https://carleton.ca/mdl/wp-content/uploads/ Westin-Cassidy-MASc-ENG-August-2018.pdf

[28] J. Woodacre, R. Bauer, and R. Irani, "Hydraulic valve-based active-heave compensation using a model-predictive controller with non-linear valve compensations," Ocean Engineering, vol. 152, pp. 47 - 56, 2018. [Online]. Available: https://doi.org/10.1016/j.oceaneng.2018.01.030

[29] P. G. Syvertsen, "Modeling and control of crane on offshore vessel," Master Thesis, NTNU: Norwegian University of Science and Technology, 2011. [Online]. Available: http://hdl.handle.net/11250/237968

[30] I. A. Martin and R. A. Irani, "Evaluation of both linear and non-linear control strategies for a shipboard marine gantry crane," in OCEANS 2019 Seattle, October 2019.

[31] Q. H. Ngo, N. Nguyen, C.-N. Nguyen, T. Tran, and Q. Ha, "Fuzzy sliding mode control of an offshore container crane," Ocean Engineering, vol. 140, pp. 125-134, 08 2017. [Online]. Available: https://www.researchgate.net/deref/ http\%3A\%2F\%2Fdx.doi.org\%2F10.1016\%2Fj.oceaneng.2017.05.019

[32] B. Kimiaghalam, A. Homaifar, and B. Sayarrodsari, "An application of model predictive control for a shipboard crane," in Proceedings of the 2001 American Control Conference. (Cat. No.01CH37148), vol. 2, June 2001, pp. 929-934 vol.2. [Online]. Available: https://doi.org/10.1109/ACC.2001.945838 
[33] Y. Chu, "Virtual prototyping for marine crane design and operations," PhD, Norwegian University of Science and Technology, 2018. [Online]. Available: https://ntnuopen.ntnu.no/ntnu-xmlui/bitstream/handle/11250/2488322/ Yingguang\%20Chu.pdf?sequence $=3$

[34] J. X. Roberto G. Valenti, Ivan Dryanovski, "Keeping a good attitude: A quaternion-based orientation filter for IMUs and MARGs," 2015. [Online]. Available: https://doi.org/10.3390/s150819302

[35] S. Küchler, J. Eberharter, K. Langer, K. Schneider, and O. Sawodny, "Heave motion estimation of a vessel using acceleration measurements," IFAC Proceedings Volumes, vol. 44, no. 1, pp. 14742 - 14 747, 2011, 18th IFAC World Congress. [Online]. Available: https://doi.org/10.3182/20110828-6-IT-1002. 01935

[36] S. Küchler, C. Pregizer, J. K. Eberharter, K. Schneider, and O. Sawodny, "Real-time estimation of a ship's attitude," in Proceedings of the 2011 American Control Conference, June 2011, pp. 2411-2416. [Online]. Available: https://doi.org/10.1109/ACC.2011.5990612

[37] B. Fan, Q. Li, C. Wang, and T. Liu, "An adaptive orientation estimation method for magnetic and inertial sensors in the presence of magnetic disturbances," Sensors, vol. 17, p. 1161, 05 2017. [Online]. Available: https://doi.org/10.3390/s17051161

[38] N. Filipe, M. Kontitsis, and P. Tsiotras, "Extended kalman filter for spacecraft pose estimation using dual quaternions," Journal of Guidance, Control, and Dynamics, vol. 2015, pp. 1-17, 05 2015. [Online]. Available: https://doi.org/10.2514/1.G000977 
[39] "Motion reference unit - mru - kongsberg maritime," [Accessed December 2019]. [Online]. Available: https://www.kongsberg.com/maritime/products/ vessel-reference-systems/motion-and-heading-sensors/motion-reference-unit/

[40] F. Sanfilippo, L. I. Hatledal, H. G. Schaathun, K. Y. Pettersen, and H. Zhang, "A universal control architecture for maritime cranes and robots using genetic algorithms as a possible mapping approach," in 2013 IEEE International Conference on Robotics and Biomimetics (ROBIO), Dec 2013, pp. 322-327. [Online]. Available: http://dx.doi.org/10.1109/ROBIO.2013.6739479

[41] J. Vaughan, A. Karajgikar, and W. Singhose, "A study of crane operator performance comparing pd-control and input shaping," in Proceedings of the 2011 American Control Conference, June 2011, pp. 545-550. [Online]. Available: http://dx.doi.org/10.1109/ACC.2011.5991506

[42] M. Spong, S. Hutchinson, and M. Vidyasagar, Robot Modeling and Control. Wiley, 2005.

[43] A. V. Salazar, "Dynamic modeling and control of spacecraft robotic systems using dual quaternions," PhD, Georgia Institute of Technology, 2018. [Online]. Available: https://smartech.gatech.edu/bitstream/handle/1853/ 59928/VALVERDE-DISSERTATION-2018.pdf

[44] J. Diebel, "Representing attitude: Euler angles, unit quaternions, and rotation vectors," 2006. [Online]. Available: https://www.astro.rug.nl/software/ kapteyn-beta/_downloads/attitude.pdf

[45] J. B. Kuipers, "Quaternions and rotation sequences," in Proceedings of the International Conference on Geometry, Integrability and Quantization. Sofia, 
Bulgaria: Coral Press Scientific Publishing, 2000, pp. 127-143. [Online]. Available: https://doi.org/10.7546/giq-1-2000-127-143

[46] J. Solà, "Quaternion kinematics for the error-state kalman filter," November 2017. [Online]. Available: https://arxiv.org/pdf/1711.02508.pdf

[47] K. Daniilidis, "Hand-eye calibration using dual quaternions," The International Journal of Robotics Research, vol. 18, pp. 286 - 298, 1999.

[48] A. Cibicik and O. Egeland, "Dynamic modelling and force analysis of a knuckle boom crane using screw theory," Mechanism and Machine Theory, vol. 133, pp. 179 - 194, 2019. [Online]. Available: http: //www.sciencedirect.com/science/article/pii/S0094114X18313119

[49] C. Westin and R. A. Irani, "Cable-pulley interation with dynamic wrap angle using the absolute nodal coordinate formulation," in Proceedings of the 4th International Conference of Control, Dynamic Systems, and Robotics (CDSR'17), 2017. [Online]. Available: https://avestia.com/CDSR2017_Proceedings/files/ paper/CDSR_133.pdf

[50] C. Westin and R. A. Irani, "Vortex-induced vibrations of a low-tension cable-sheave system modeled using nonlinear finite elements," in Proceedings of The Canadian Society for Mechanical Engineering International Congress 2018, 2018. [Online]. Available: https://carleton.ca/mdl/wp-content/uploads/ Westin-etal-CSME-Preprint.pdf

[51] Y. Chu, V. Æsøy, S. Ehlers, and H. Zhang, "Integrated multi-domain system modelling and simulation for offshore crane operations," Ship Technology Research, vol. 62, no. 1, pp. 36-46, 2015. [Online]. Available: https://doi.org/10.1179/0937725515Z.0000000004 
[52] A Multi-Body Dynamic Model Based on Bond Graph for Maritime Hydraulic Crane Operations, ser. International Conference on Offshore Mechanics and Arctic Engineering, vol. Volume 1: Offshore Technology; Offshore Geotechnics, 05 2015, v001T01A010. [Online]. Available: https: //doi.org/10.1115/OMAE2015-41616

[53] Y. Chu, V. Æsøy, H. Zhang, and O. Bunes, "Modelling and simulation of an offshore hydraulic crane," in ECMS 2014 Proceedings, 05 2014, pp. 87-93. [Online]. Available: https://doi.org/10.7148/2014-0087

[54] DSA, "ShipMo3D," [Accessed December 2019]. [Online]. Available: https: //dsaocean.com/shipmo3d/overview/

[55] Actuonix Motion Devices, "Miniature linear motion series p16 datasheet," [Accessed December 2019]. [Online]. Available: https://s3.amazonaws.com/ actuonix/Actuonix +P16+Datasheet.pdf

[56] Pololu Corporation, "Pololu simple motor controller user's guide," [Accessed December 2019]. [Online]. Available: https://www.pololu.com/docs/pdf/0J44/ simple_motor_controllers.pdf

[57] Yost Labs, "Yost labs 3-space sensor wireless 2.4ghz dsss technical brief," [Accessed December 2019]. [Online]. Available: https://yostlabs.com/wp/ wp-content/uploads/pdf/TSS_Wireless_Tech_Brief_v2.2.1.pdf

[58] GoPro, "GoPro HERO7 Black," [Accessed December 2019]. [Online]. Available: https://gopro.com/en/ca/shop/cameras/hero7-black/CHDHX-701-master.html

[59] MathWorks, "Stereo camera calibrator app," [Accessed December 2019]. [Online]. Available: https://www.mathworks.com/help/vision/ug/ stereo-camera-calibrator-app.html 
[60] R. L. Norton, Design of Machinery, 5th ed. McGraw-Hill, 2012.

[61] J. H. Yang and S. H. Shen, "Novel approach for adaptive tracking control of a 3-d overhead crane system," J. Intell. Robotics Syst., vol. 62, no. 1, pp. 59-80, April 2011. [Online]. Available: http://dx.doi.org/10.1007/s10846-010-9440-9

[62] R. M. Murray, Z. Li, and S. S. Sastry, A Mathematical Introduction to Robotic Manipulation. CRC Press, 1994.

[63] J.-J. E. Slotine and W. Li, Applied Nonlinear Control, 1991.

[64] F. Zhao and B. G. M. van Wachem, "A novel quaternion integration approach for describing the behaviour of non-spherical particles," 2013. [Online]. Available: http://dx.doi.org/10.1007/s00707-013-0914-2

[65] D. M. Henderson, "Euler angles, quaternions, and transformation matrices for space shuttle analysis," NASA National Aeronautics and Space Administration, Design Note 1.4-8-020, 1977. [Online]. Available: https://ntrs.nasa.gov/archive/ nasa/casi.ntrs.nasa.gov/19770019231.pdf

[66] MathWorks. (2019) Linear-quadratic regulator design. [Online]. Available: https://www.mathworks.com/help/control/ref/lqr.html

[67] P. Mohazzabi and S. P. Shankar, "Damping of a simple pendulum due to drag on its string," vol. 5, pp. 120-130, 2017. [Online]. Available: http://dx.doi.org/10.4236/jamp.2017.51013

[68] S. Gull, A. Lasenby, and C. Doran, "Imaginary numbers are not real-the geometric algebra of spacetime," Foundations of Physics, vol. 23, no. 9, pp. 1175-1201, Sep 1993. [Online]. Available: https://doi.org/10.1007/BF01883676 


\section{Appendix A}

\section{Ship Motion Profiles}

This appendix provides the test-scale and full-scale ship motion profiles, as well as the recorded feedback of the test-scale actuators used throughout the current thesis work. When converting from the full-scale to the test-scale, the full-scale displacements are scaled such that the test-scale displacements and velocities are within the physical limits of the linear actuators. The linear actuator set-points are found using the Simscape model of Chapter 4 to perform the inverse kinematic analysis. The set-points are supplied to the system and the actuator feedback is recorded in LabVIEW. To determine simulation set-points that account for tracking error, the stochastic actuator feedback signals are fitted with a smoothing spline in MATLAB and derivatives are taken to fully define the set-points required for the Simscape model. The Simscape model of Chapter 4 is used to perform a forward kinematic analysis and convert the actuator feedback signals back to surge, sway, heave, roll, pitch, and yaw to define reference data for evaluating the experimental ship motion estimates. 


\section{A.1 Ship Motion Profile 1}
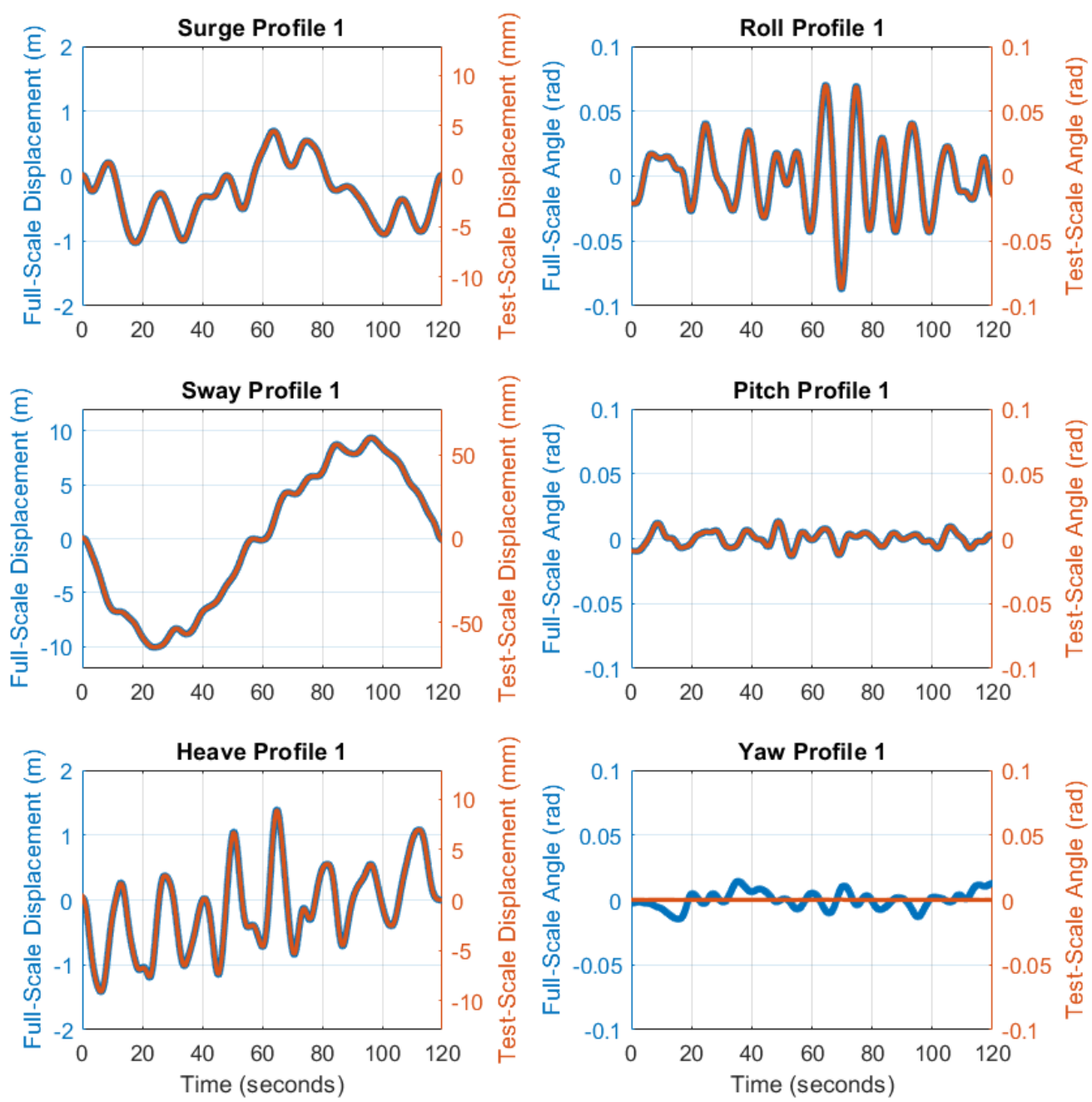

Figure A.1: Ship Motion Profile 1: Full-scale and test-scale displacements [6]. 

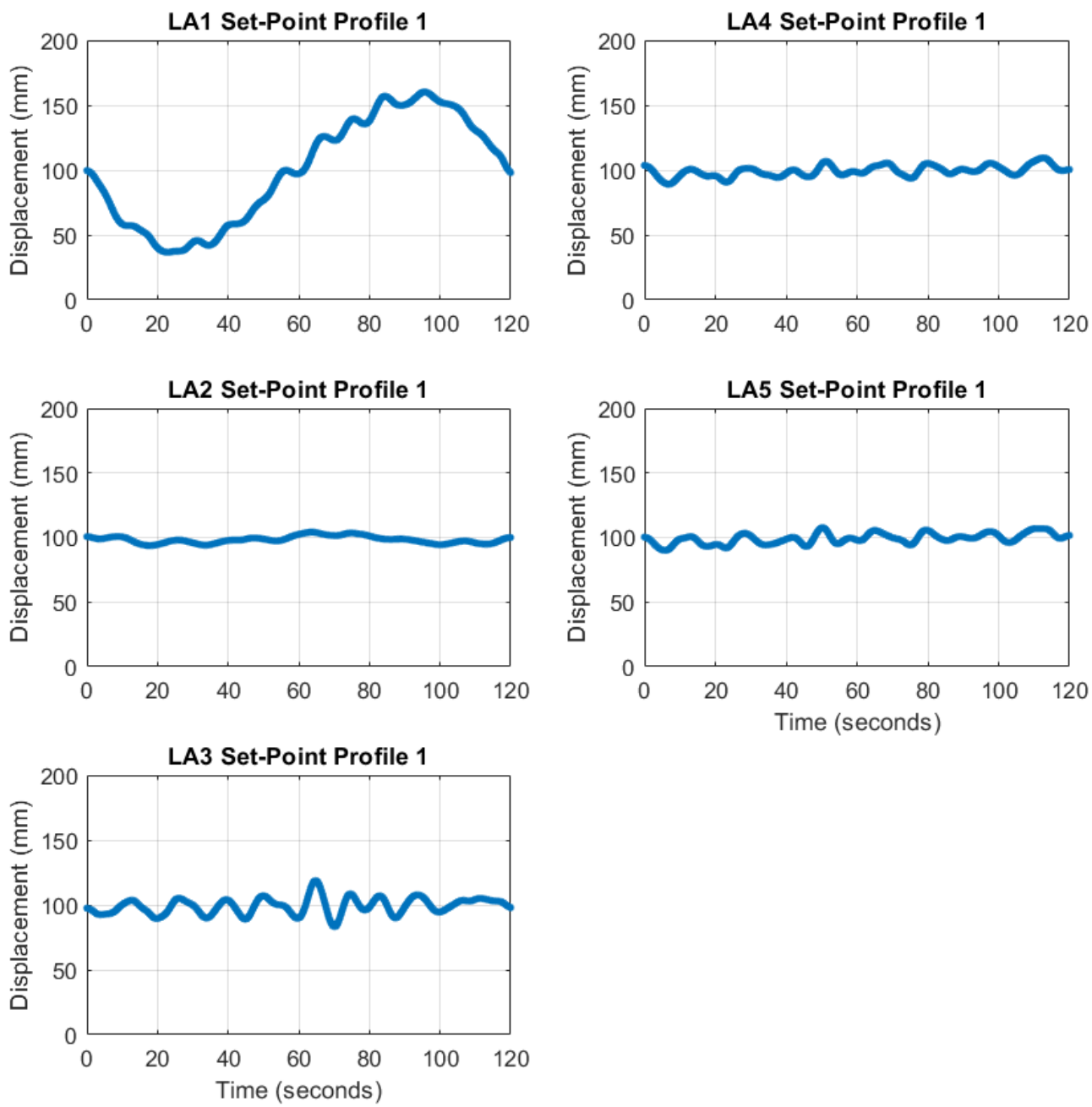

Figure A.2: Ship Motion Profile 1: Linear actuator set-points. 

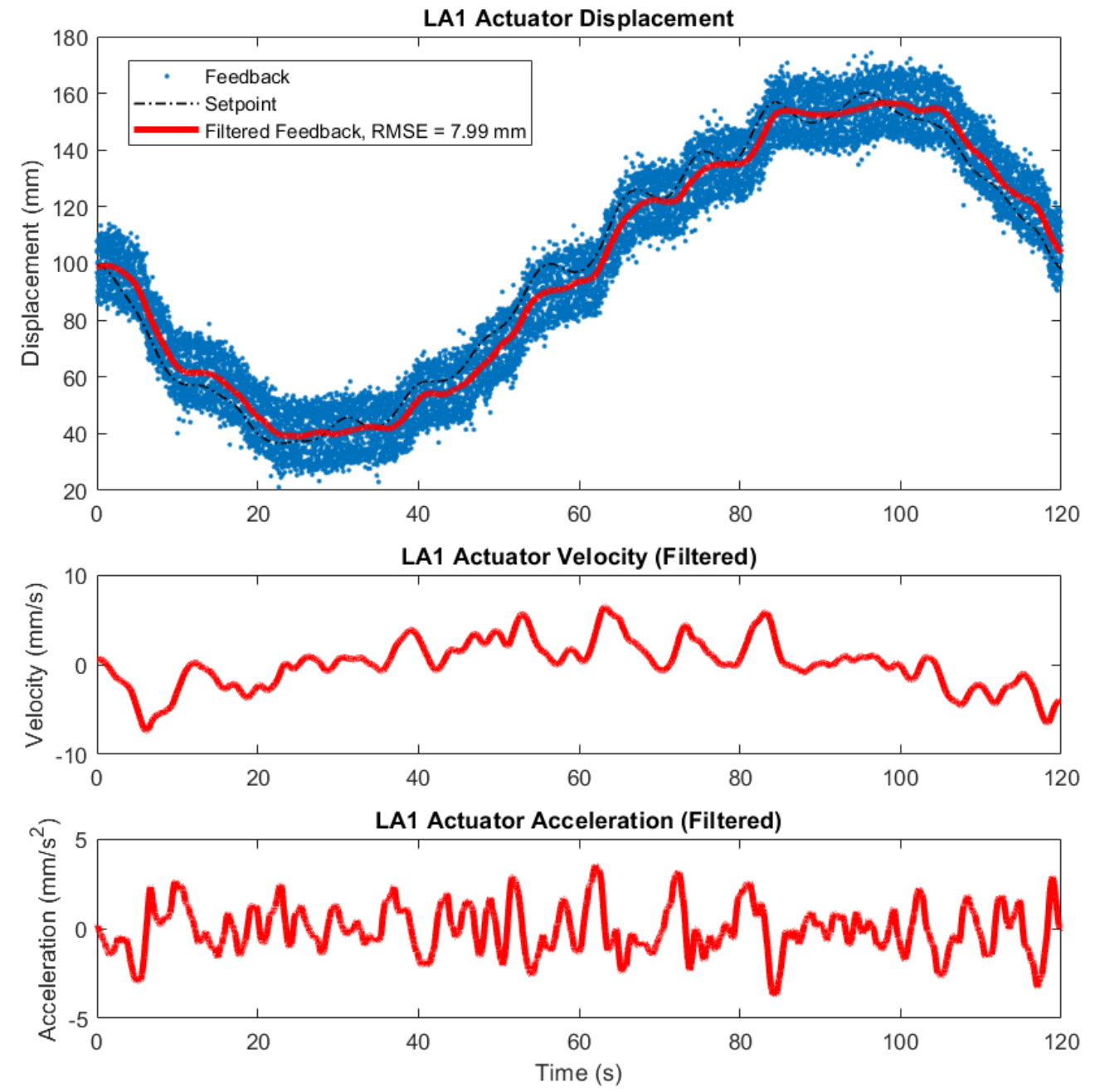

Figure A.3: Ship Motion Profile 1: LA1 set-points, feedback, and filtered feedback. 

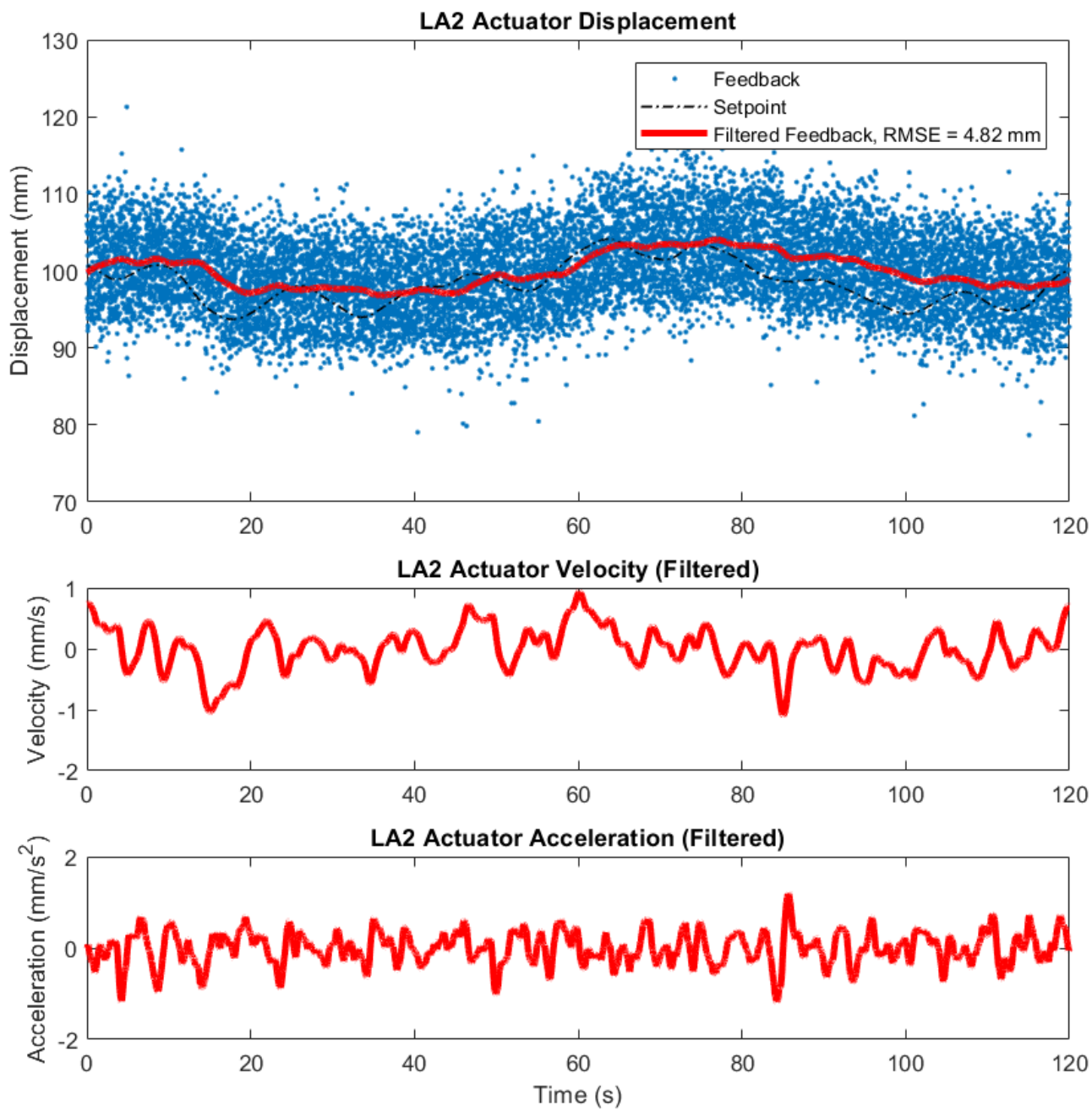

Figure A.4: Ship Motion Profile 1: LA2 set-points, feedback, and filtered feedback. 

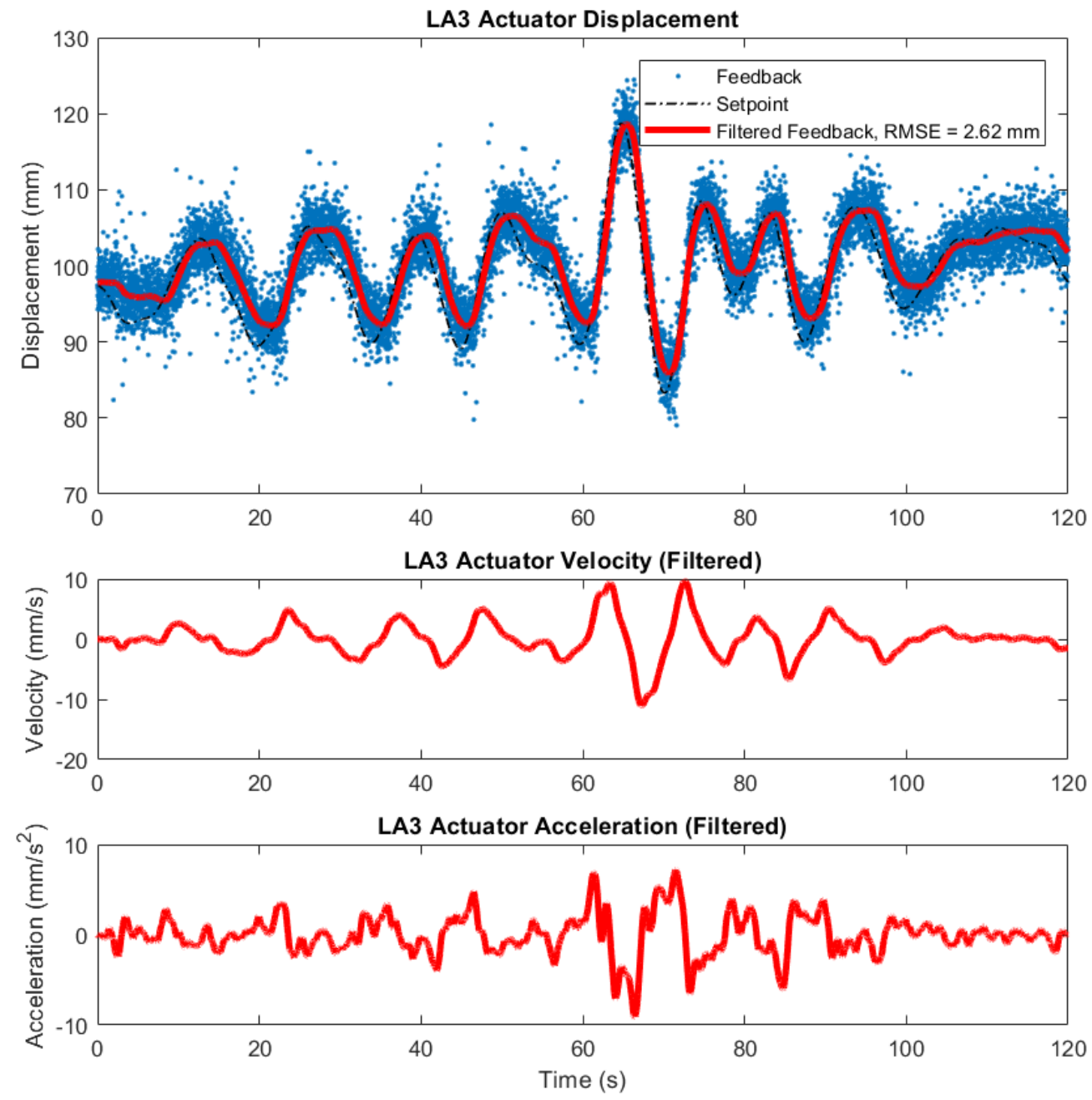

Figure A.5: Ship Motion Profile 1: LA3 set-points, feedback, and filtered feedback. 

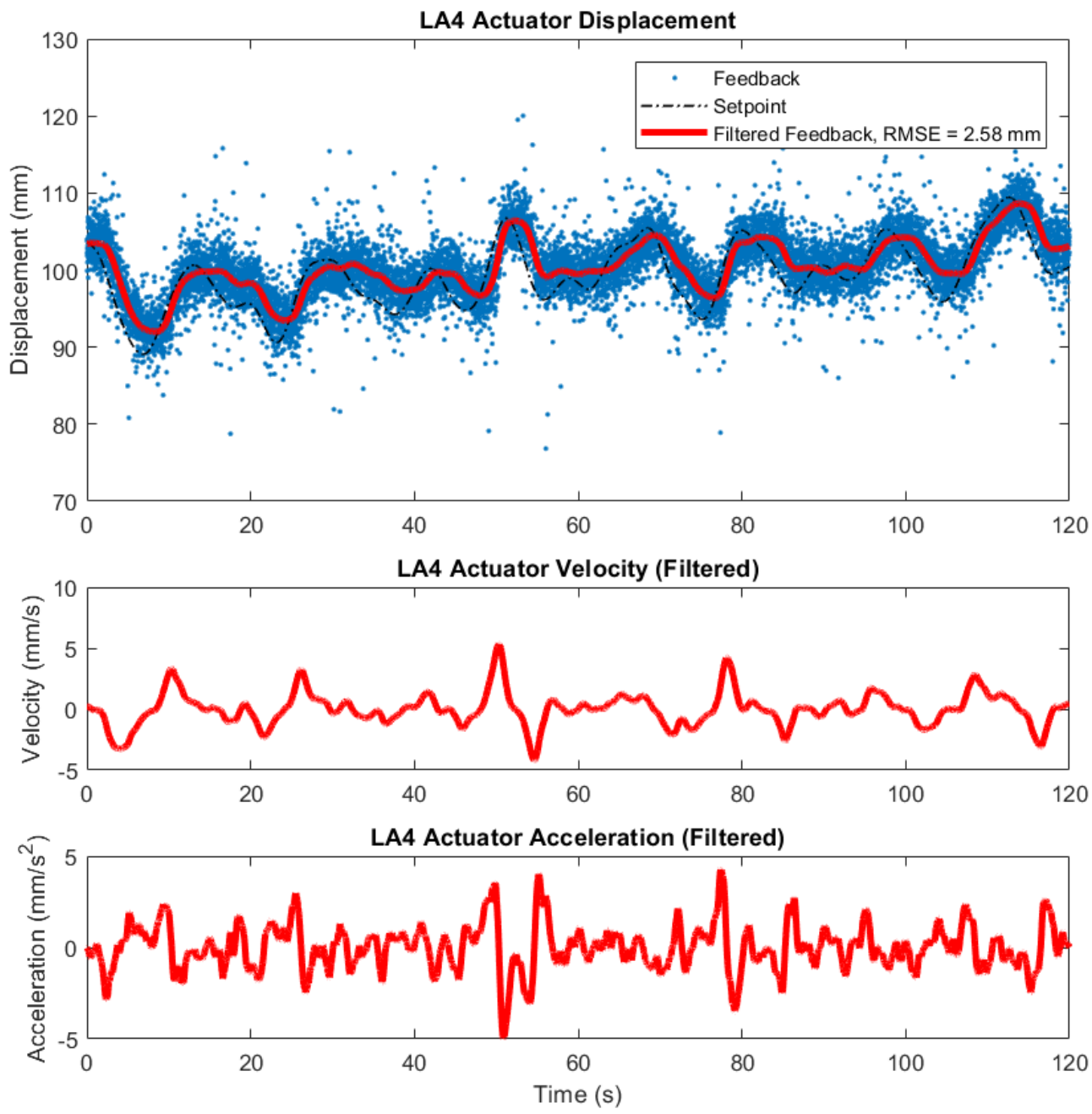

Figure A.6: Ship Motion Profile 1: LA4 set-points, feedback, and filtered feedback. 

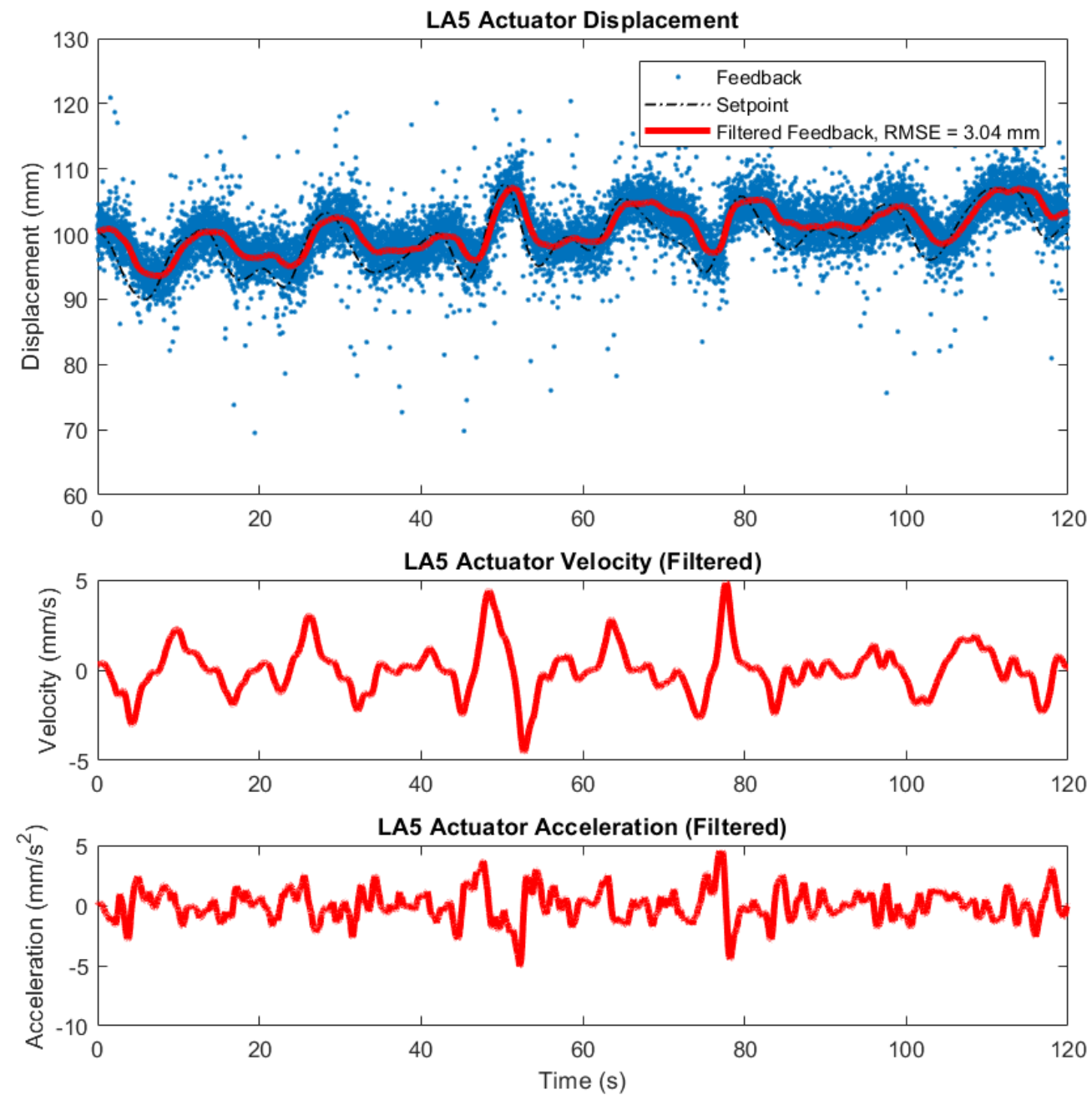

Figure A.7: Ship Motion Profile 1: LA5 set-points, feedback, and filtered feedback. 

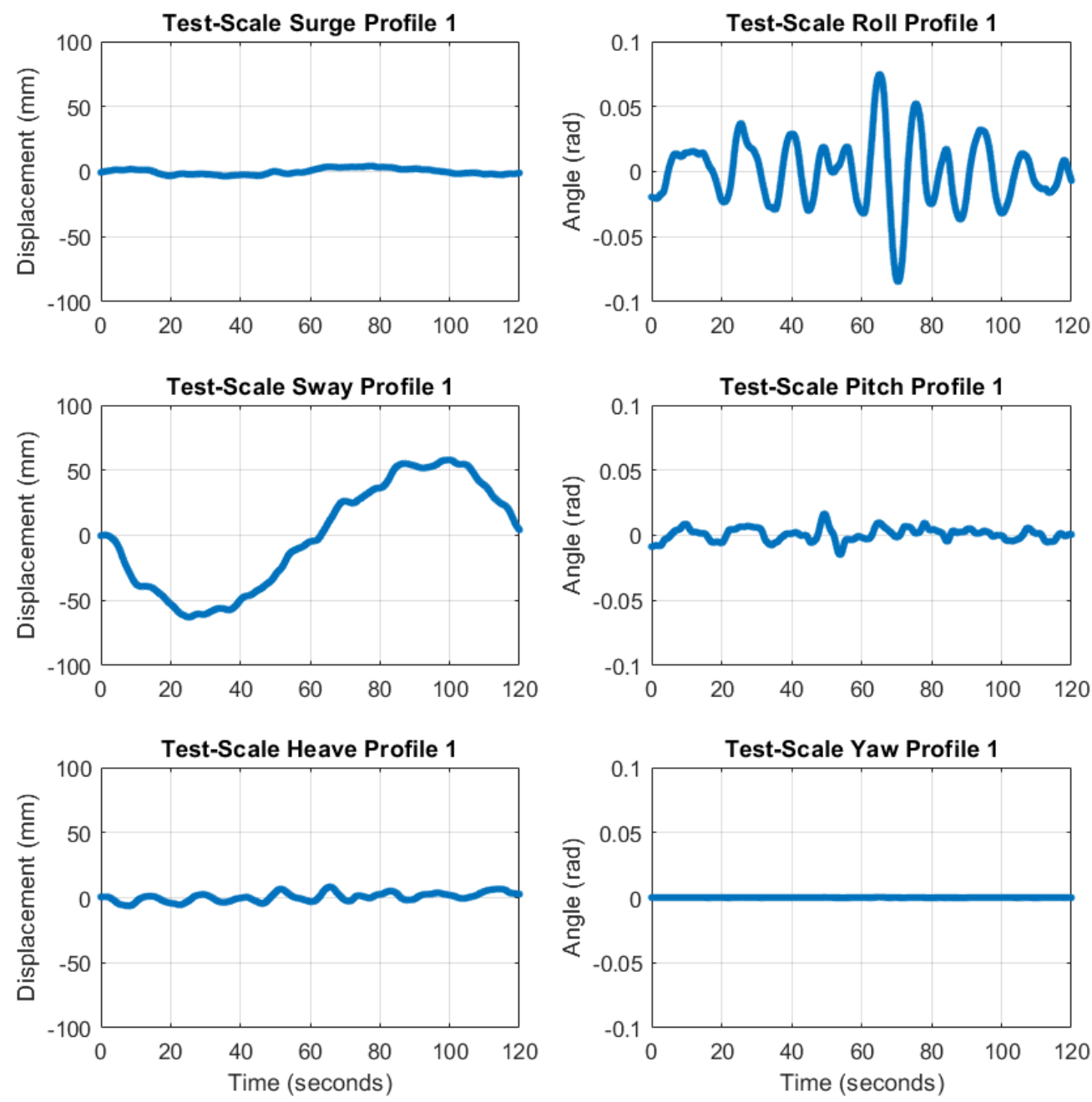

Figure A.8: Ship Motion Profile 1: Test-scale ship motion resulting from the filtered linear actuator feedback. 


\section{A.2 Ship Motion Profile 2}
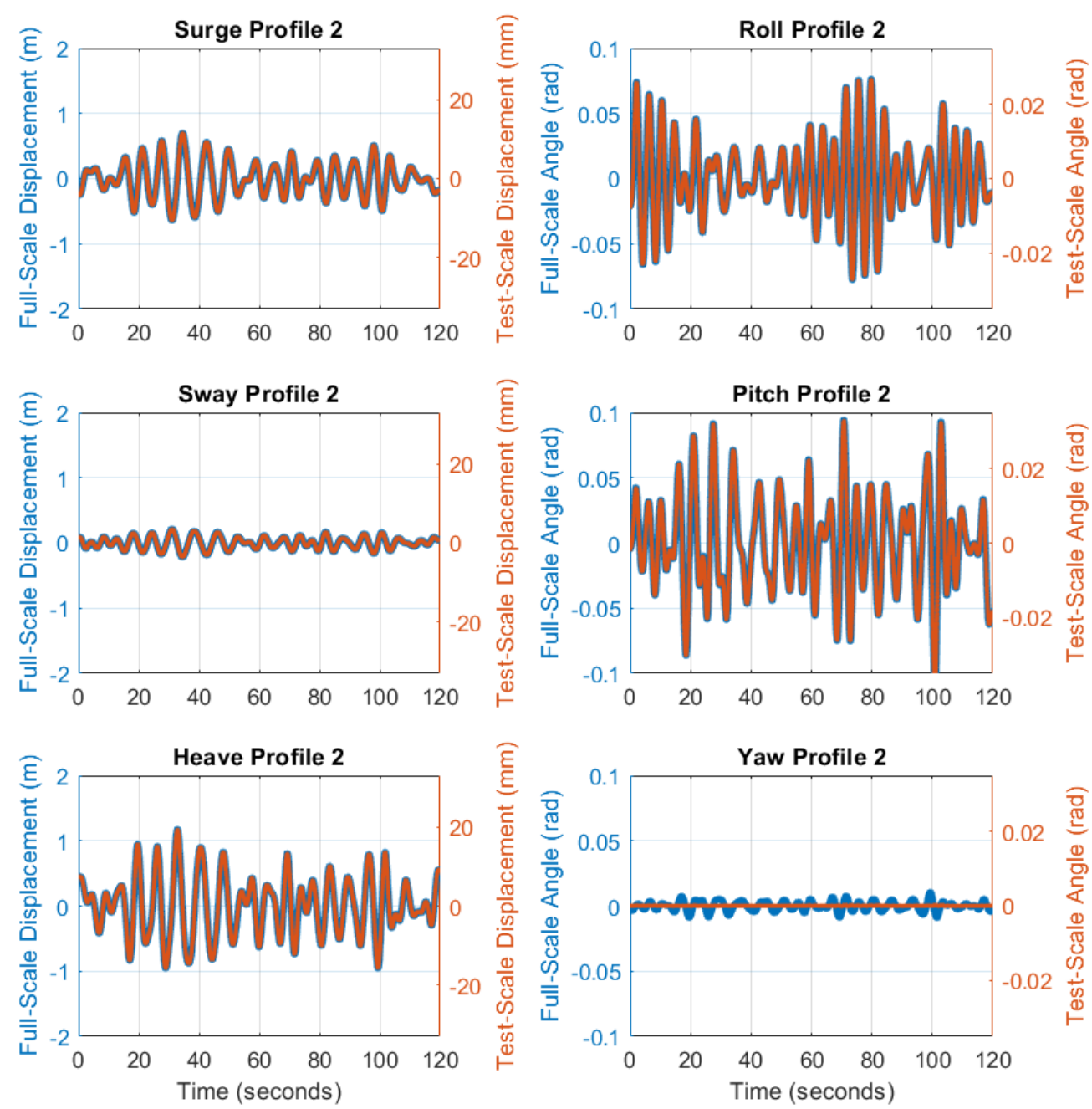

Figure A.9: Ship Motion Profile 2: Full-scale and test-scale displacements. 

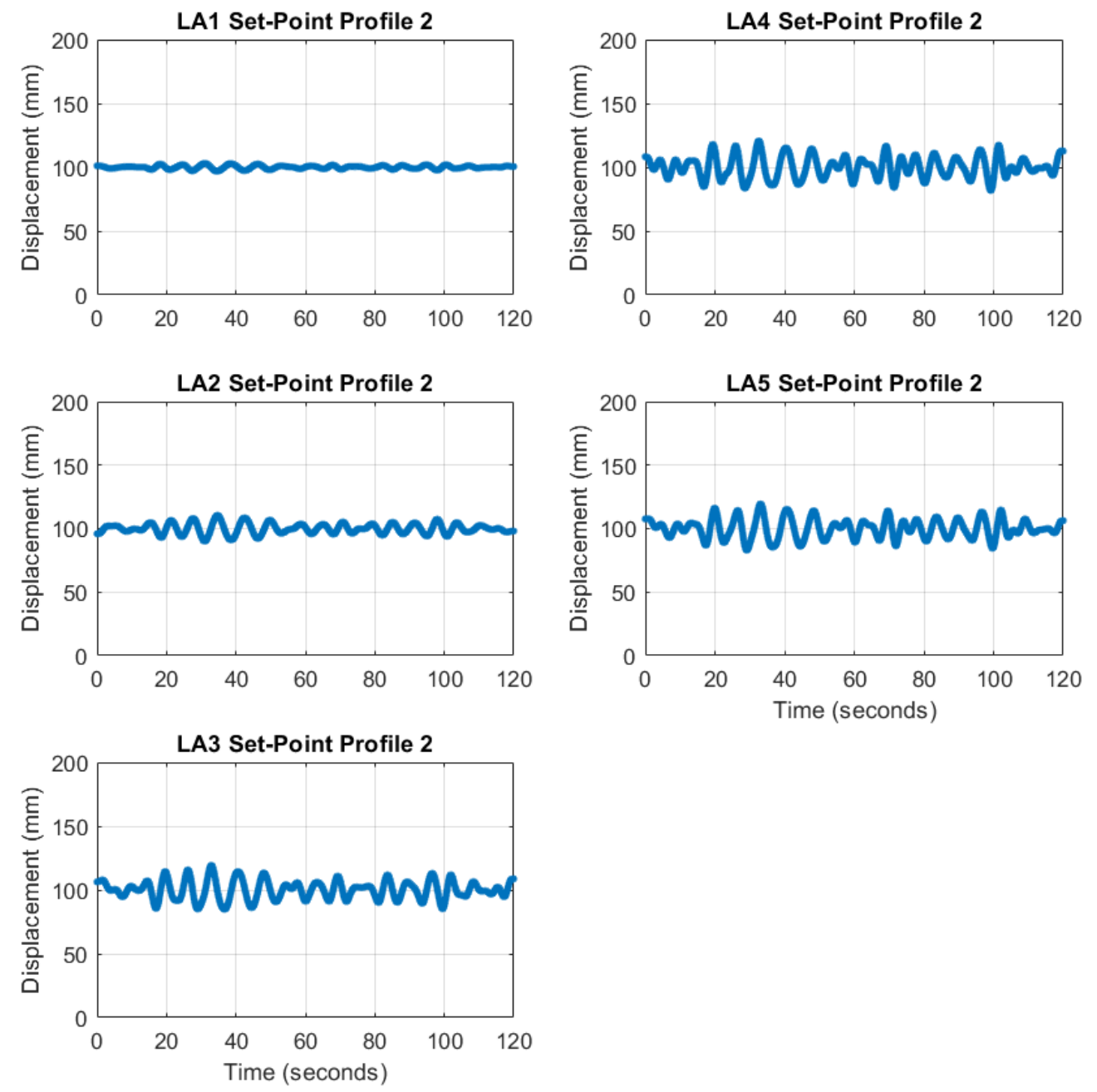

Figure A.10: Ship Motion Profile 2: Linear actuator set-points. 

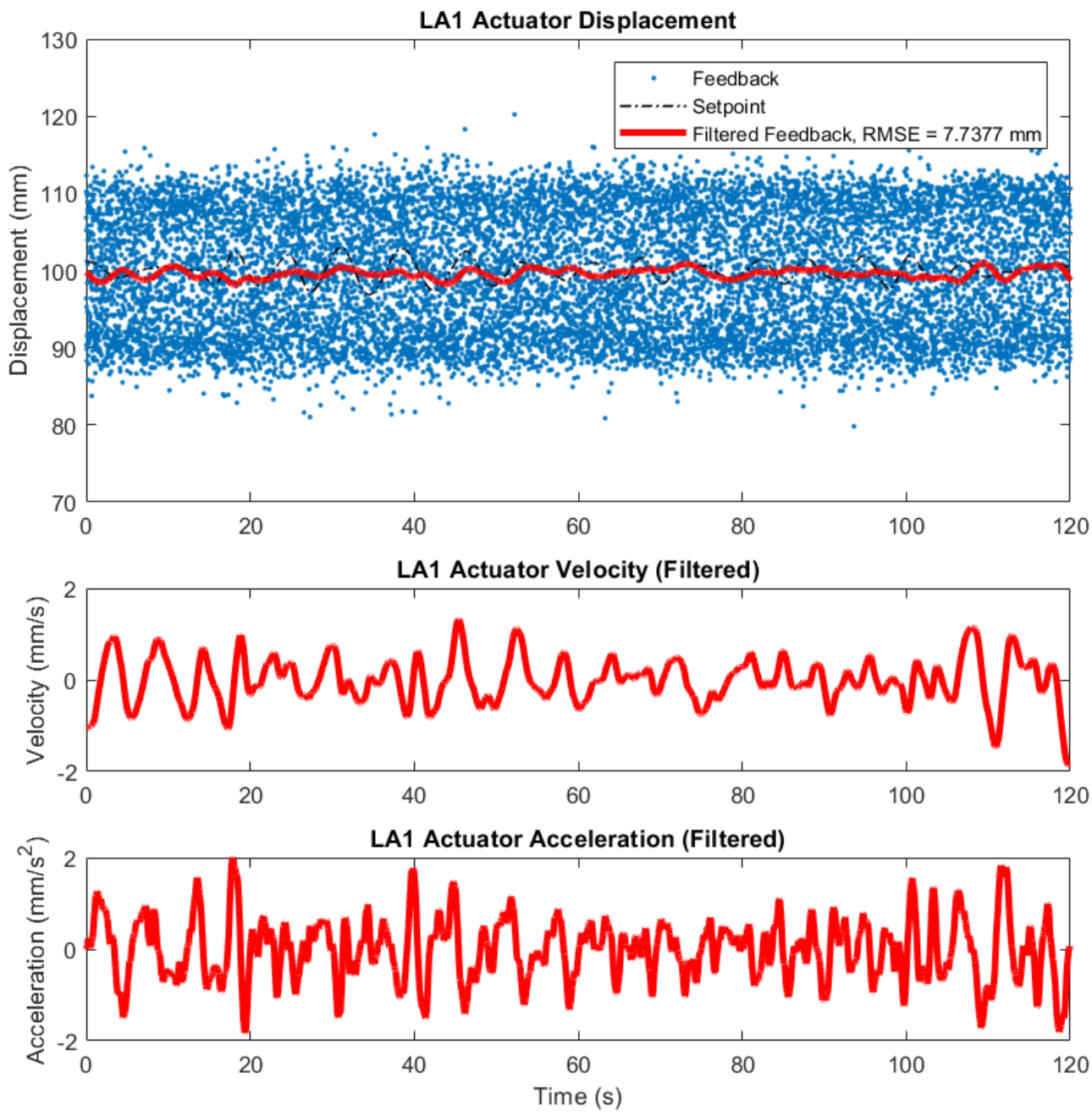

Figure A.11: Ship Motion Profile 2: LA1 set-points, feedback, and filtered feedback. 

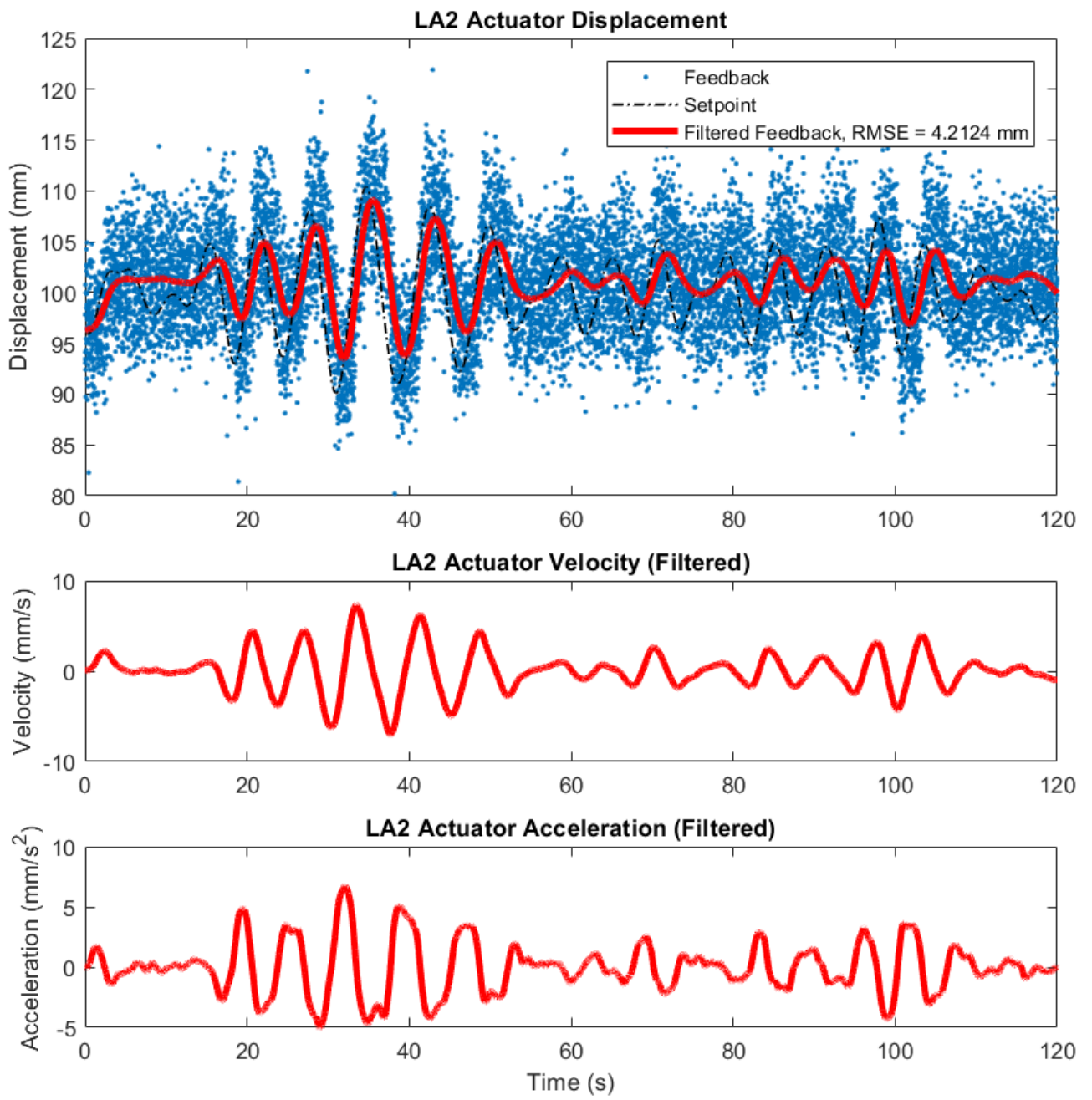

Figure A.12: Ship Motion Profile 2: LA2 set-points, feedback, and filtered feedback. 

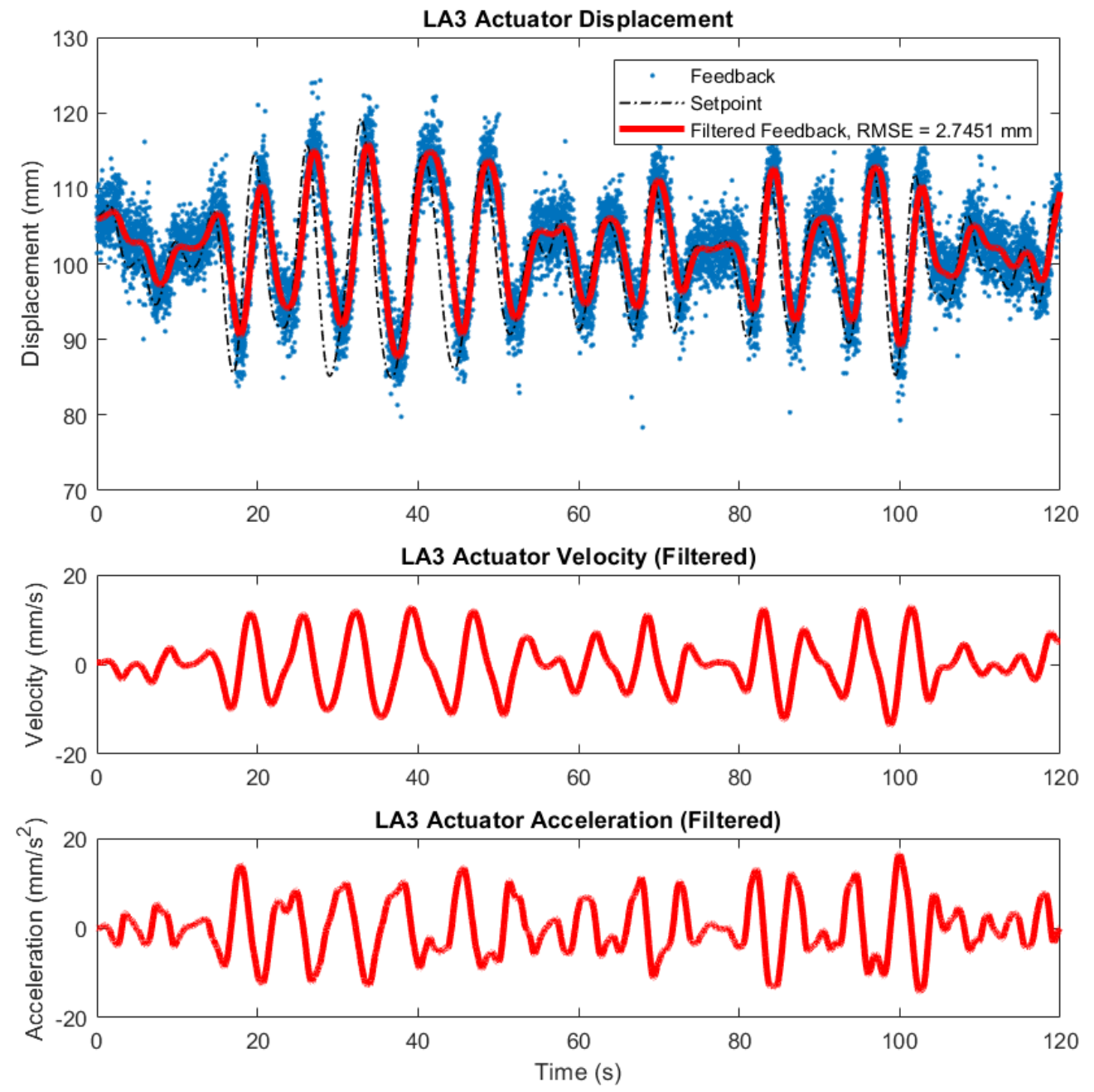

Figure A.13: Ship Motion Profile 2: LA3 set-points, feedback, and filtered feedback. 

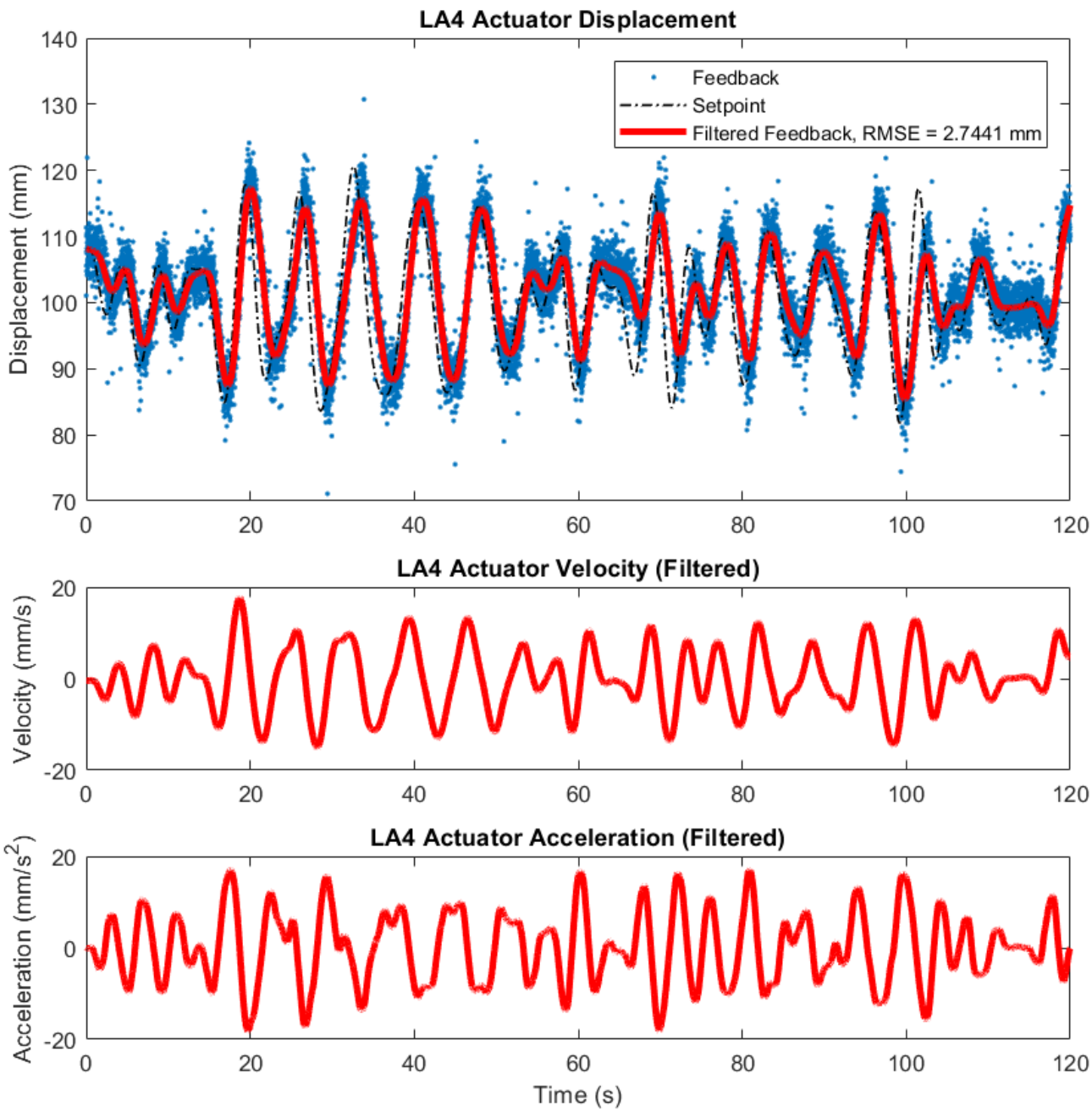

Figure A.14: Ship Motion Profile 2: LA4 set-points, feedback, and filtered feedback. 

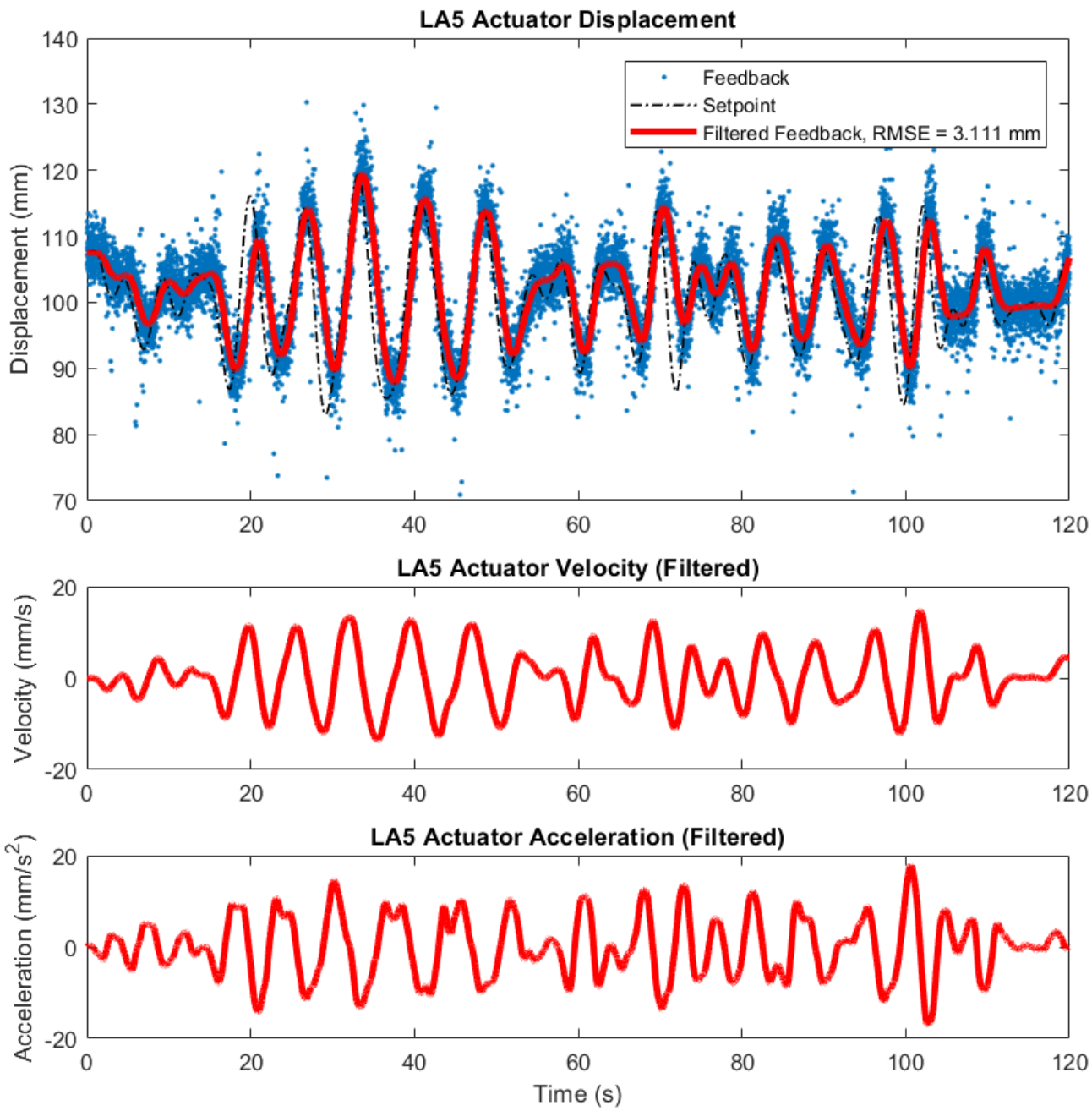

Figure A.15: Ship Motion Profile 2: LA5 set-points, feedback, and filtered feedback. 

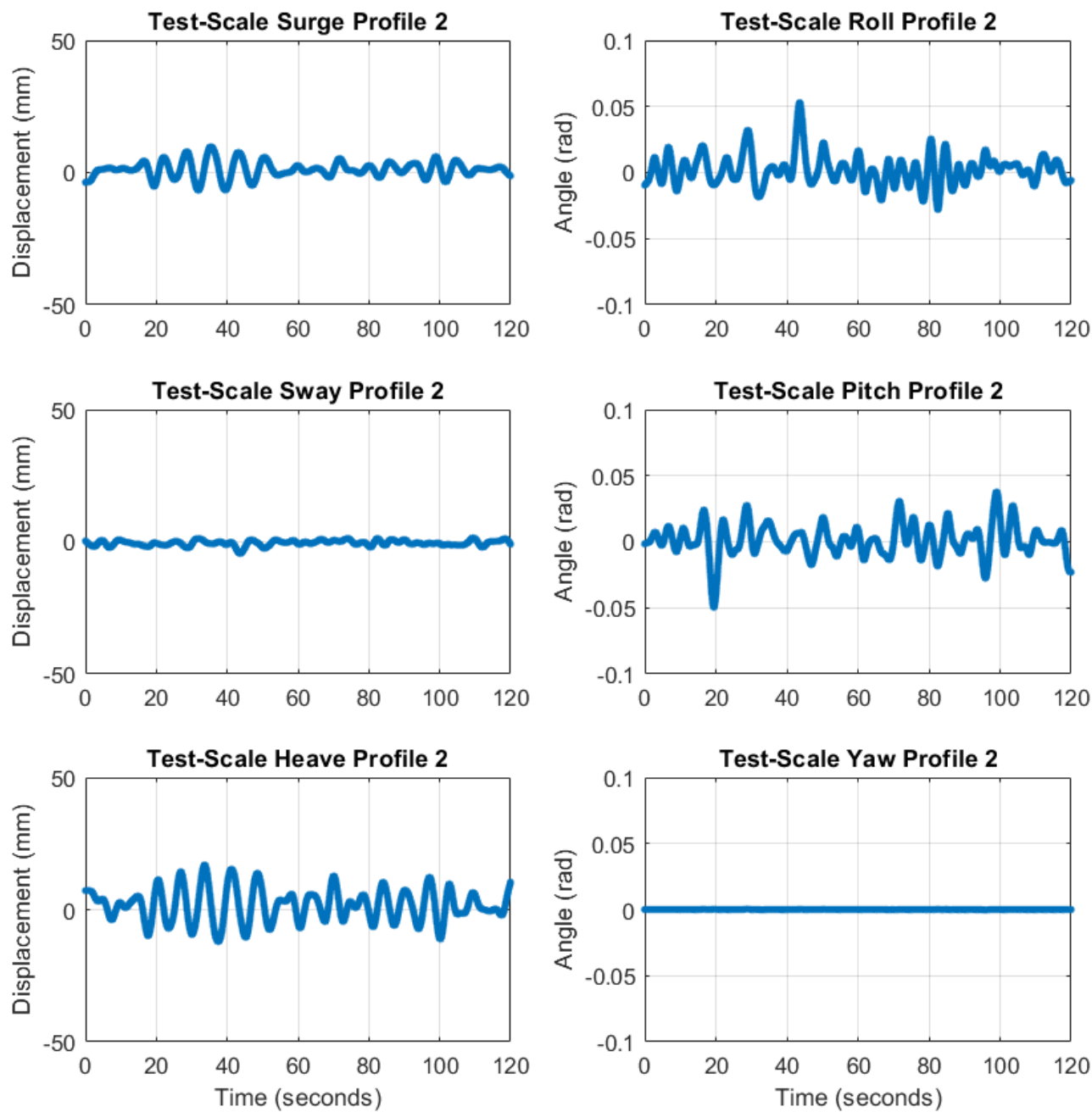

Figure A.16: Ship Motion Profile 2: Test-scale ship motion resulting from the filtered linear actuator feedback. 


\section{A.3 Ship Motion Profile 3}
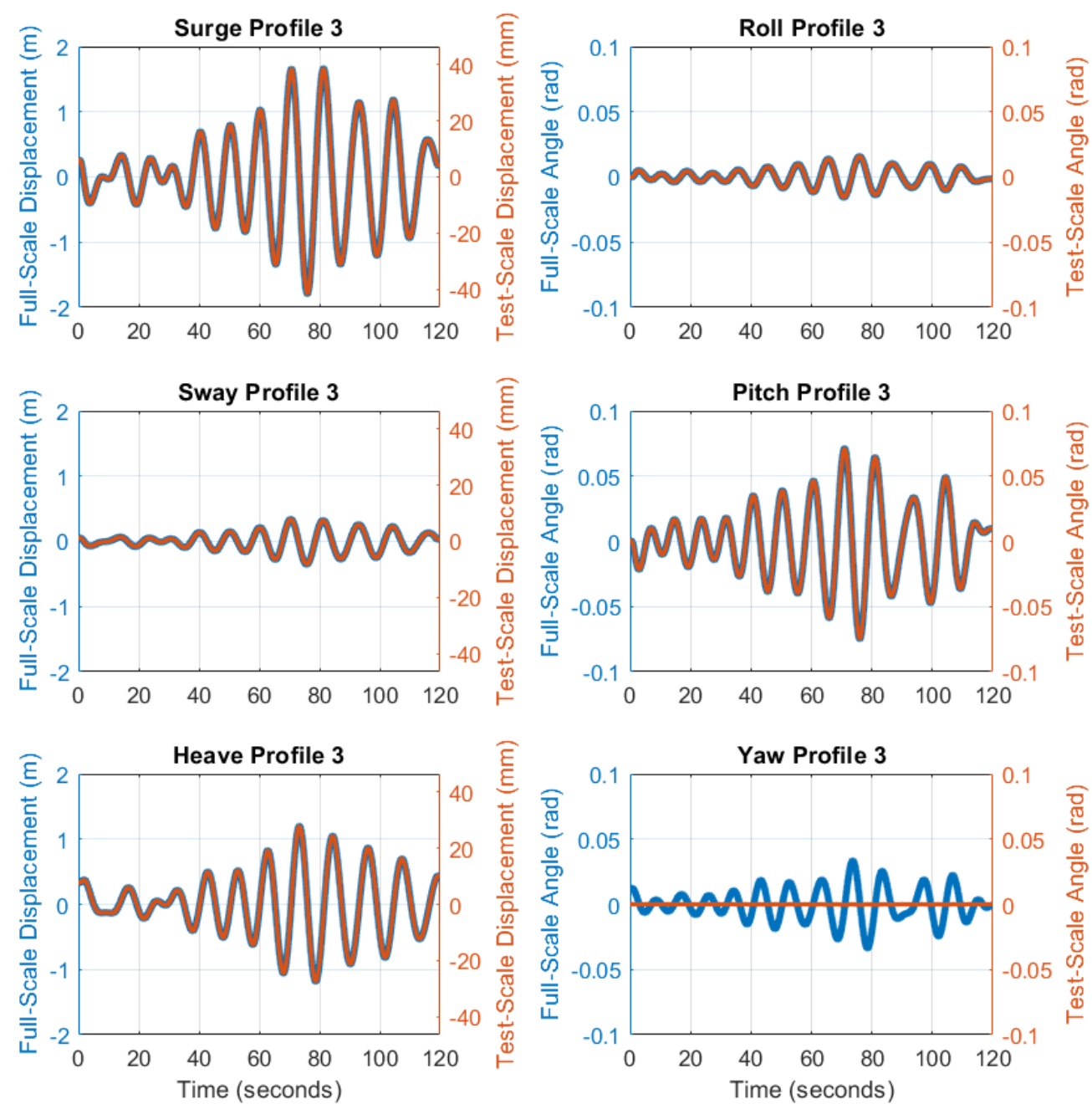

Figure A.17: Ship Motion Profile 3: Full-scale and test-scale displacements. 

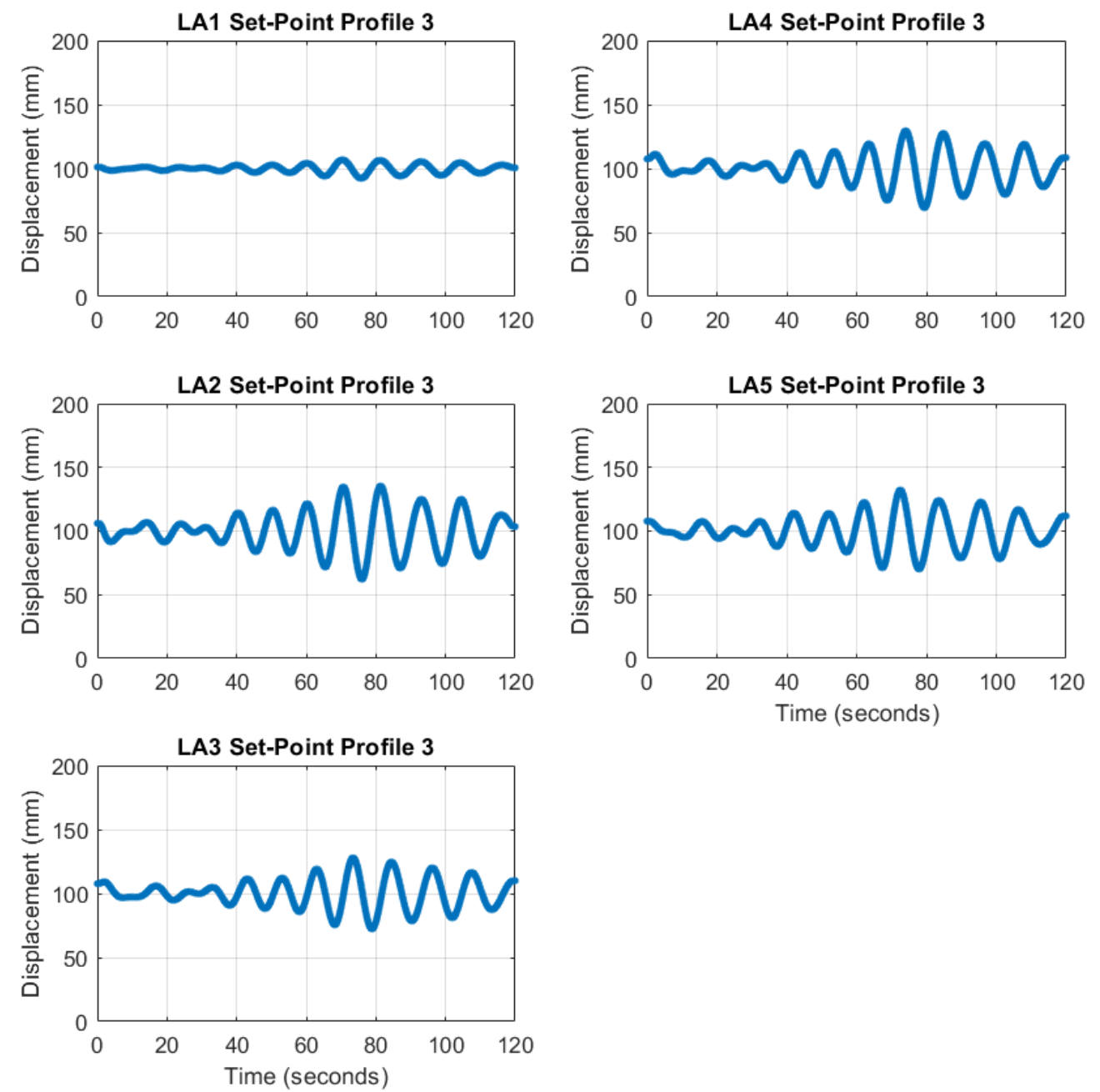

Figure A.18: Ship Motion Profile 3: Linear actuator set-points. 

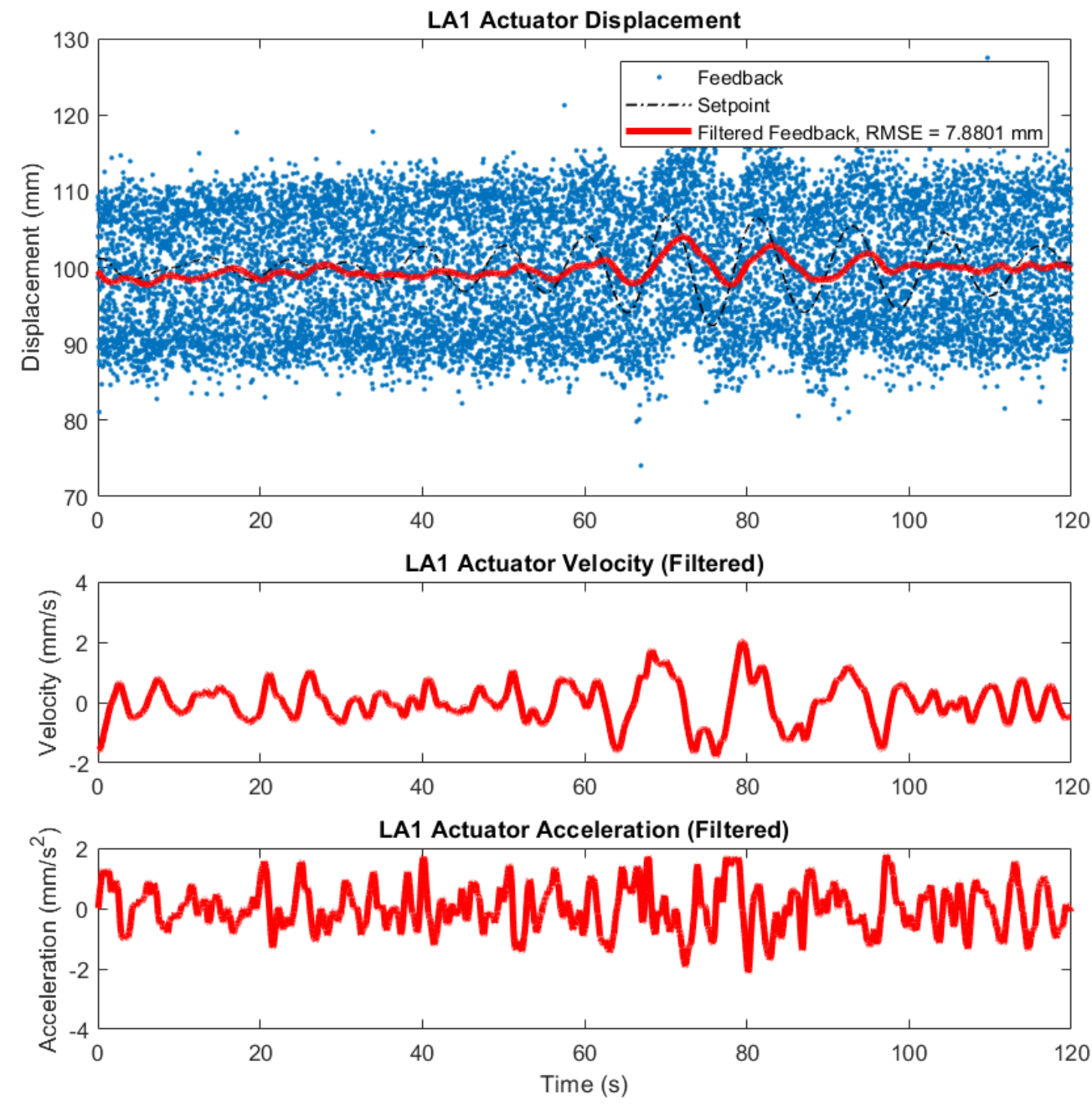

Figure A.19: Ship Motion Profile 3: LA1 set-points, feedback, and filtered feedback. 

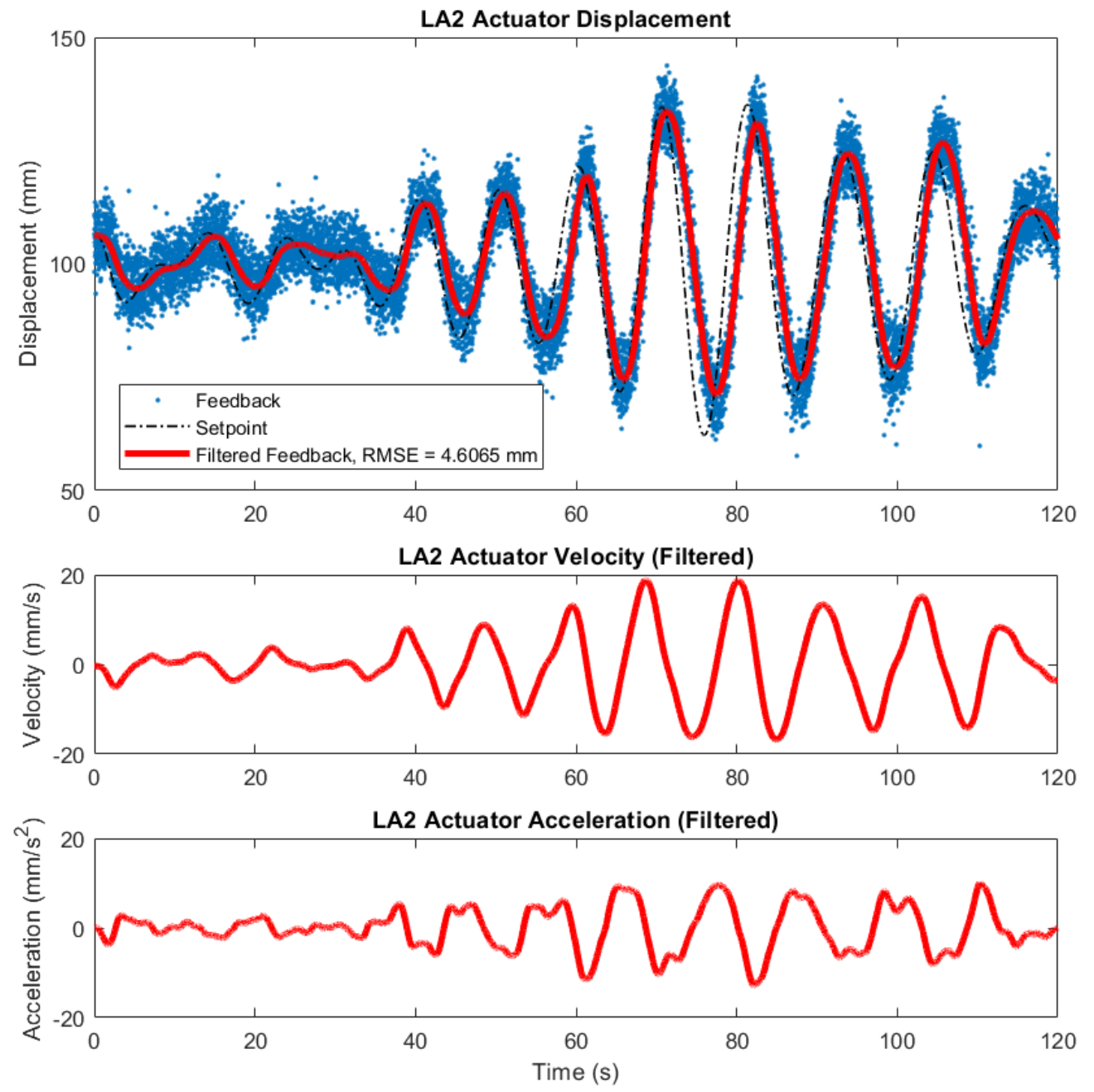

Figure A.20: Ship Motion Profile 3: LA2 set-points, feedback, and filtered feedback. 

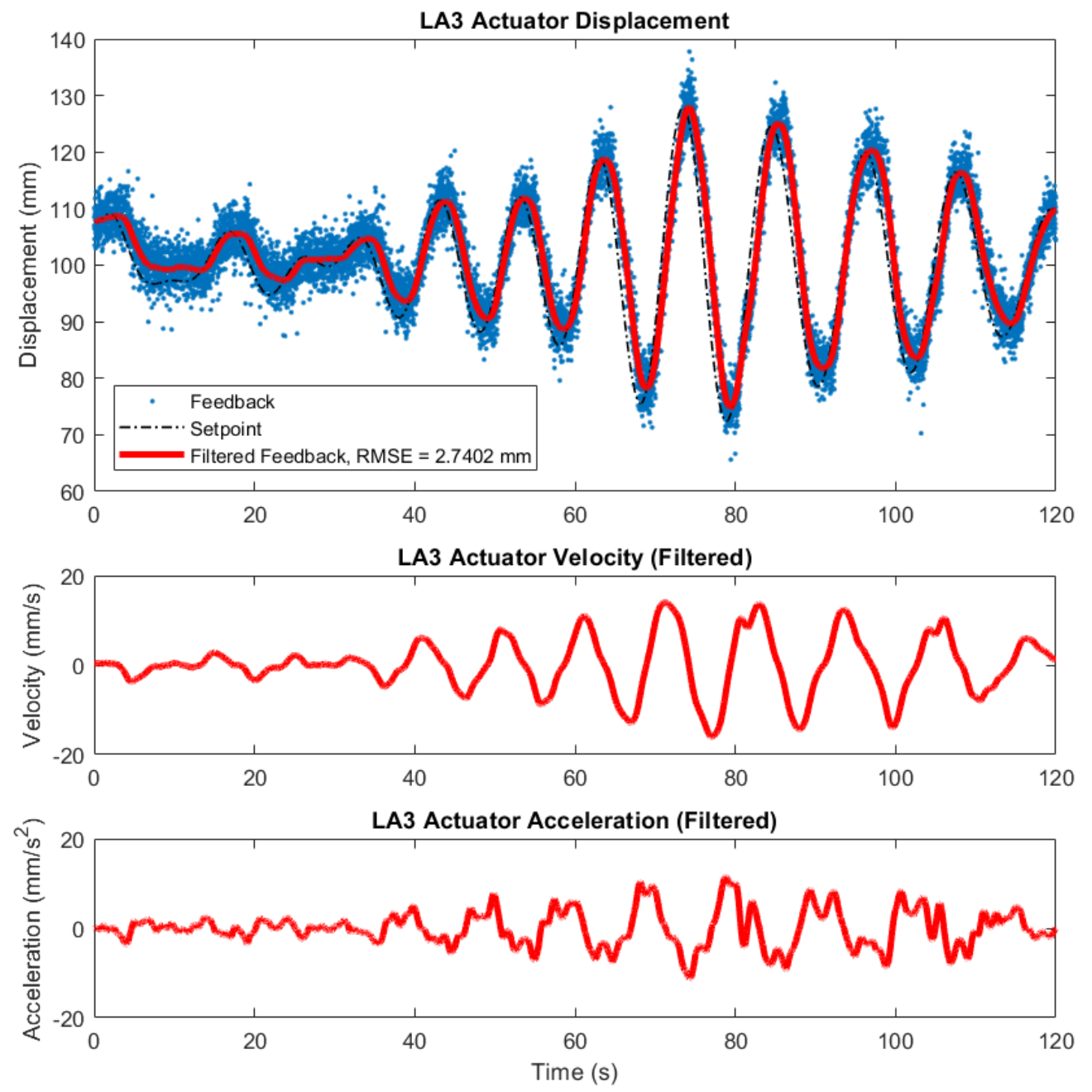

Figure A.21: Ship Motion Profile 3: LA3 set-points, feedback, and filtered feedback. 

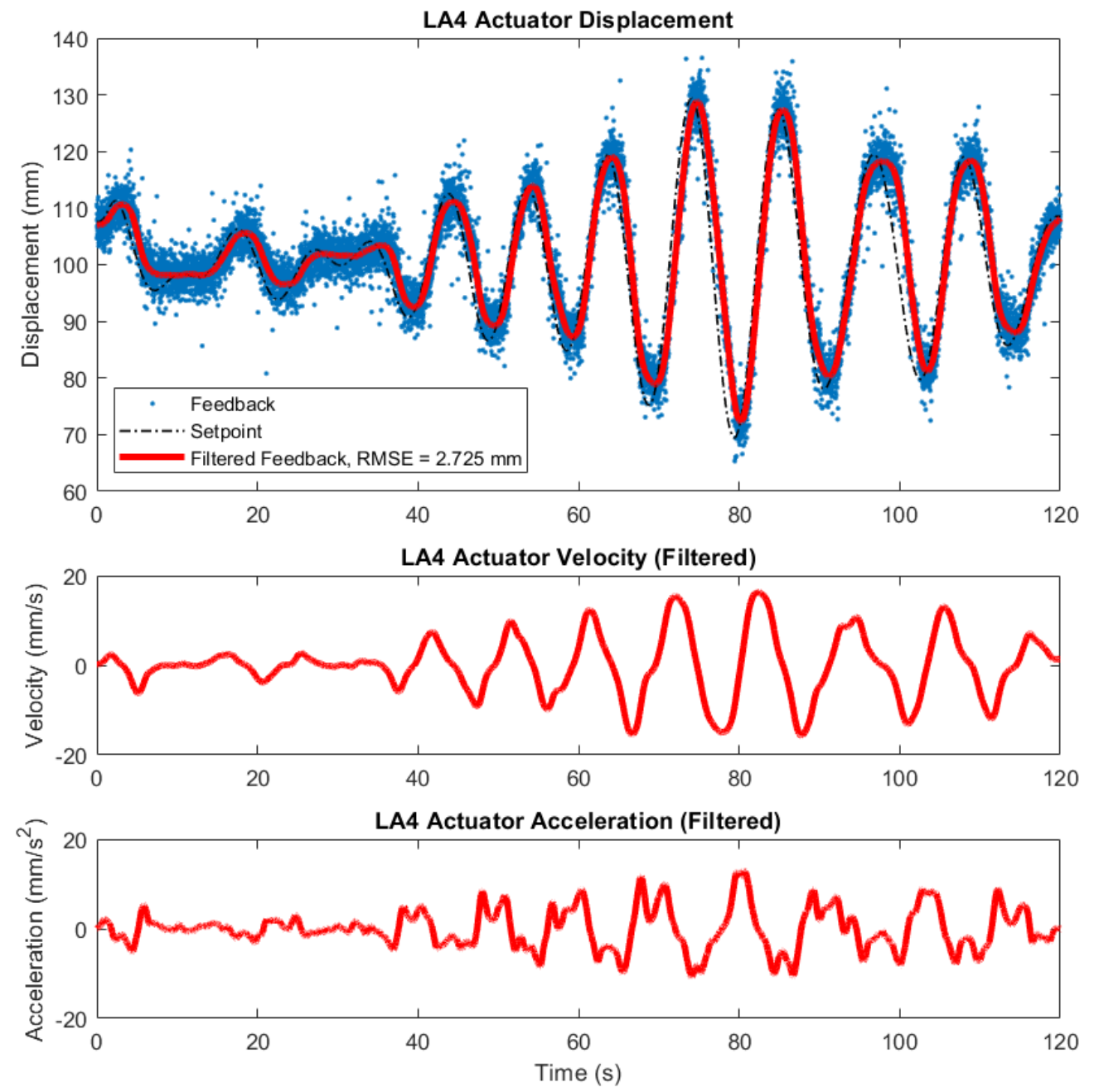

Figure A.22: Ship Motion Profile 3: LA4 set-points, feedback, and filtered feedback. 

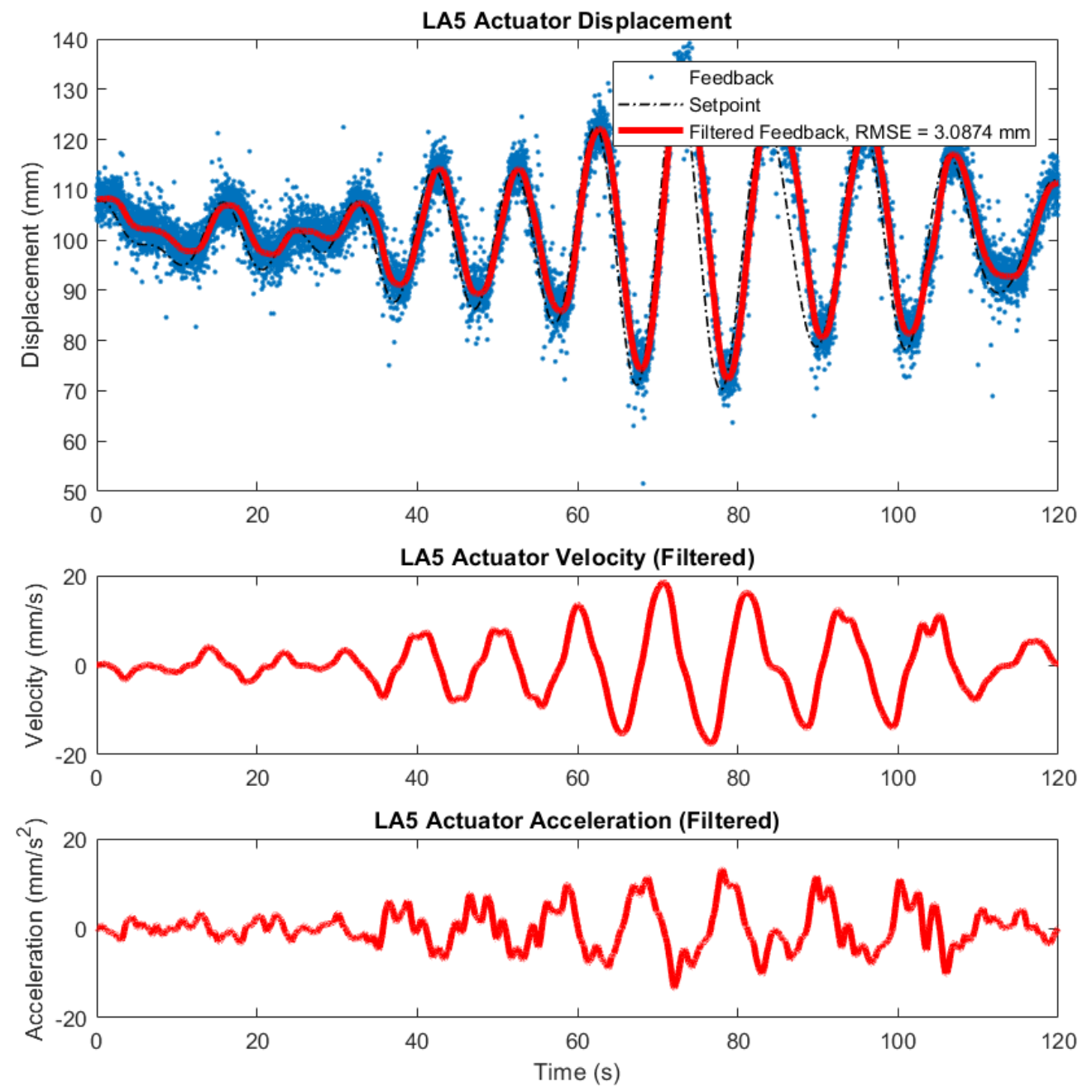

Figure A.23: Ship Motion Profile 3: LA5 set-points, feedback, and filtered feedback. 

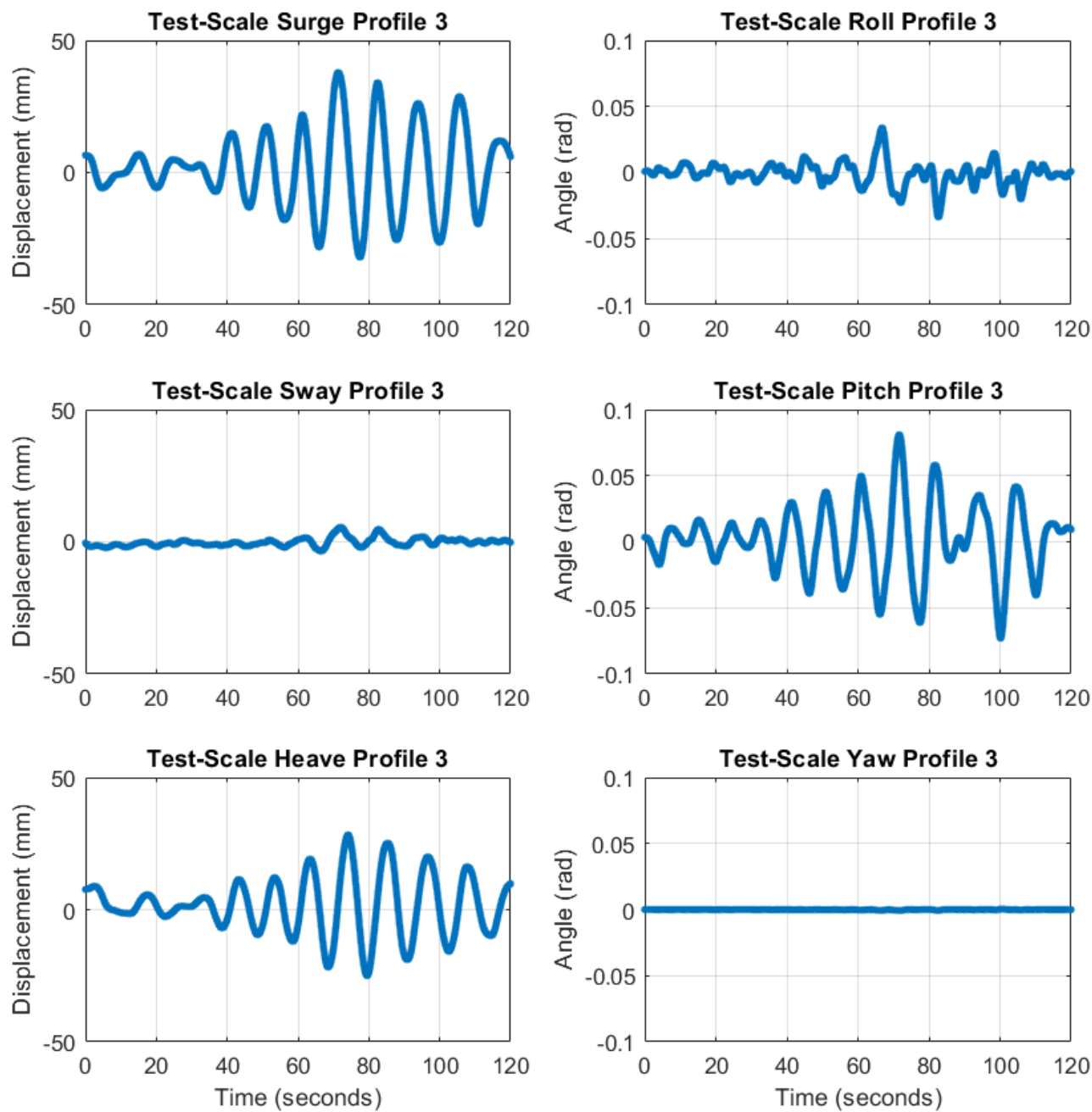

Figure A.24: Ship Motion Profile 3: Test-scale ship motion resulting from the filtered linear actuator feedback. 


\section{Appendix B}

\section{Mechanical Drawings}

Please note that the drawings provided within this appendix are not to scale as the margins were adjusted and the drawing sizes were reduced to conform to thesis formatting requirements. 


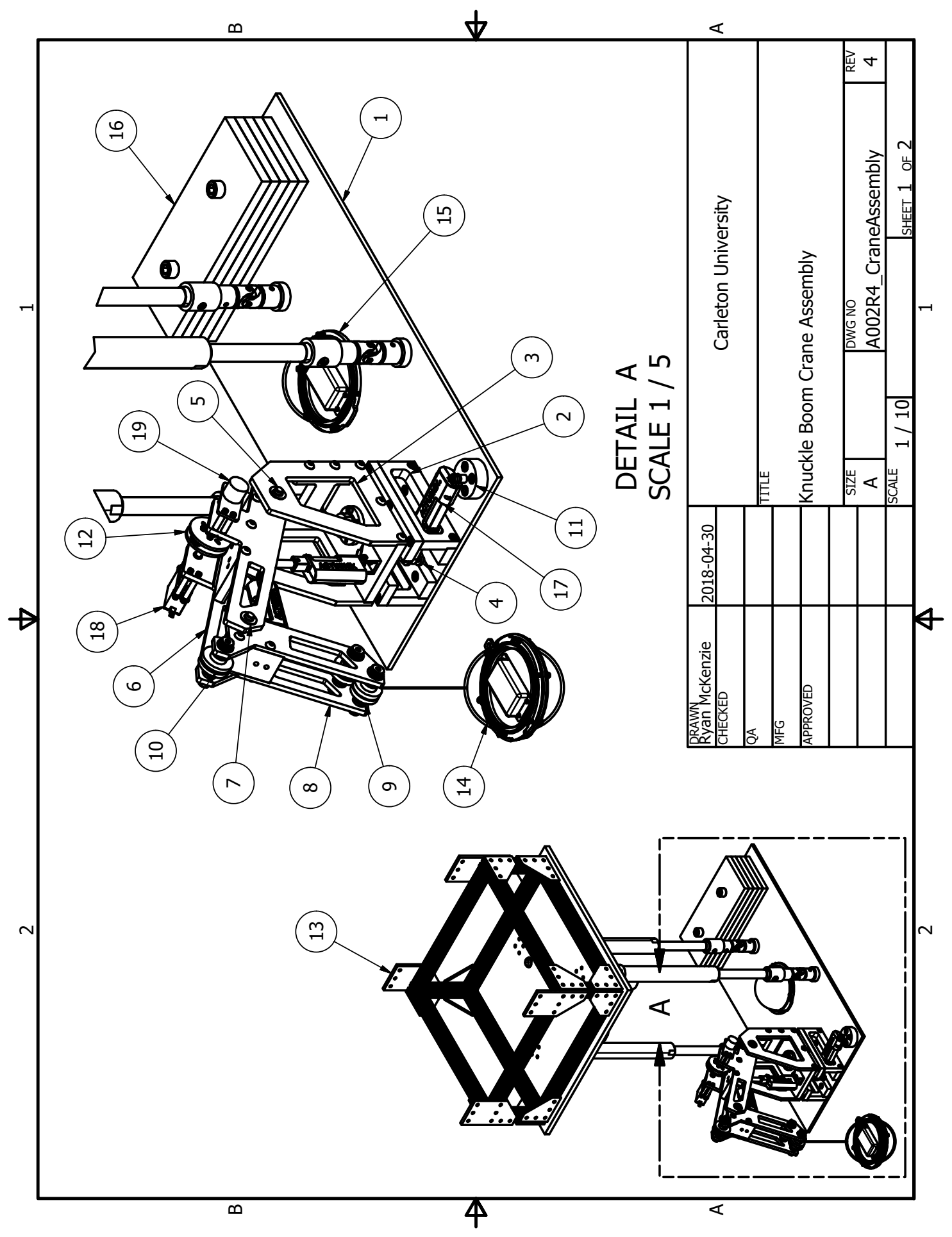




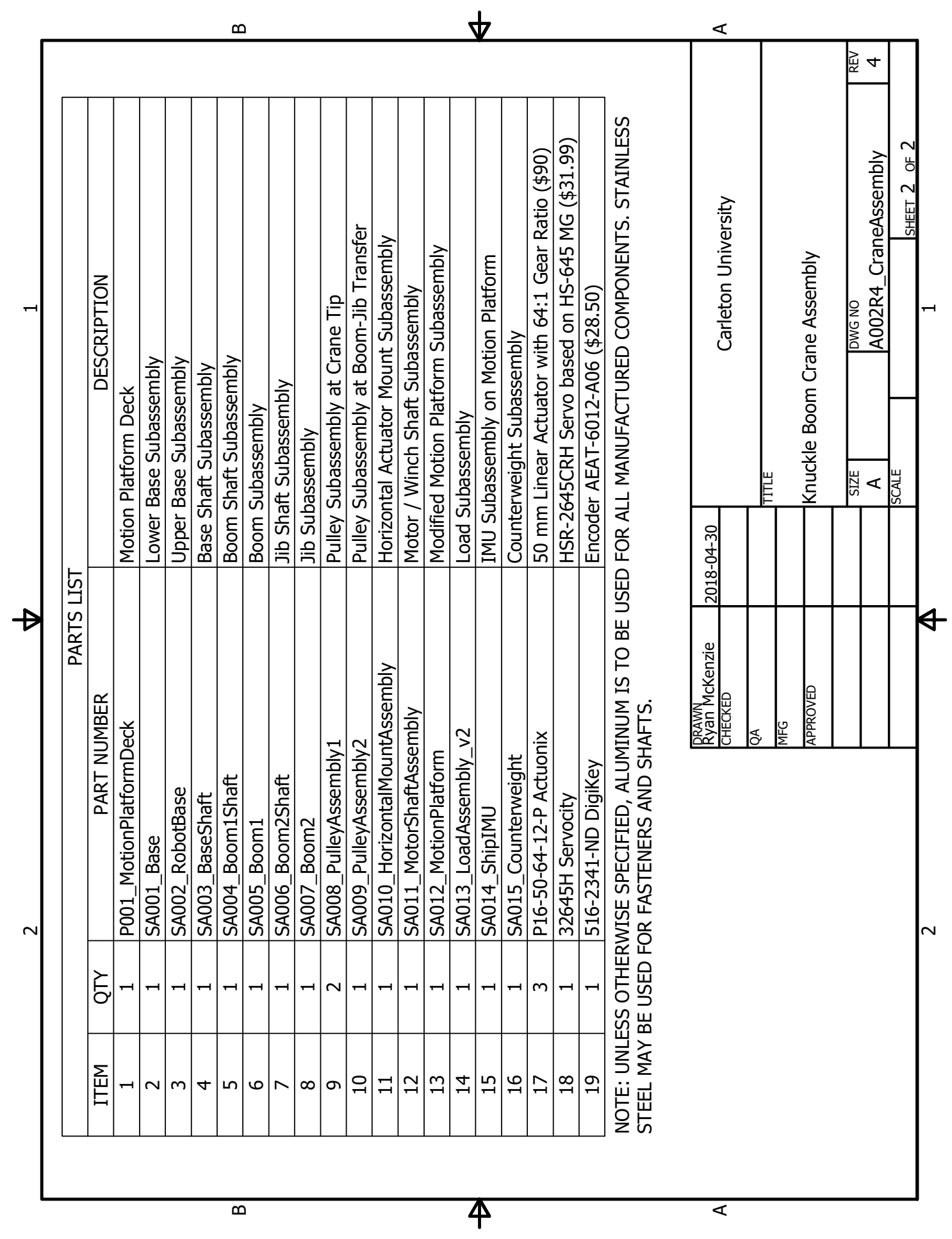




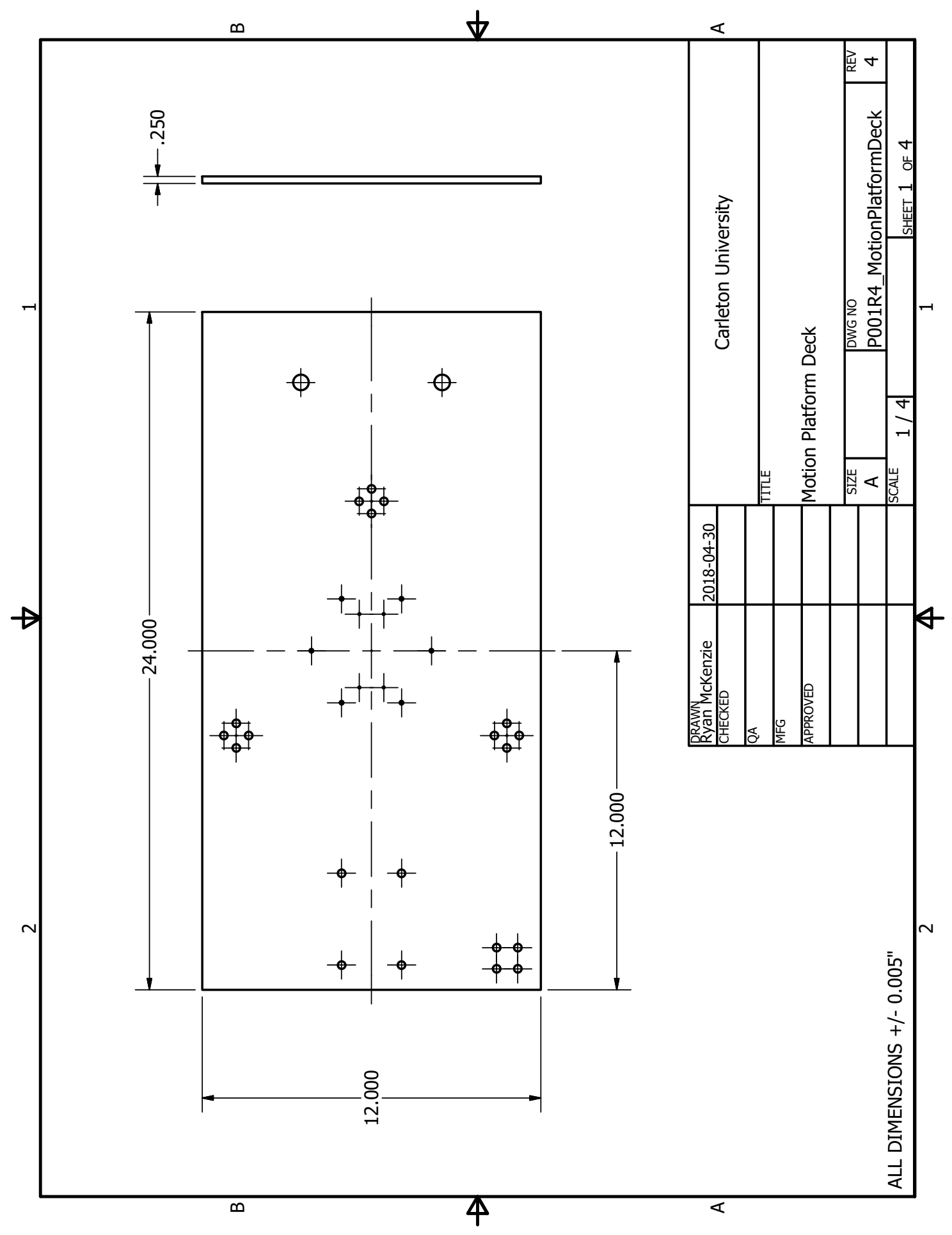




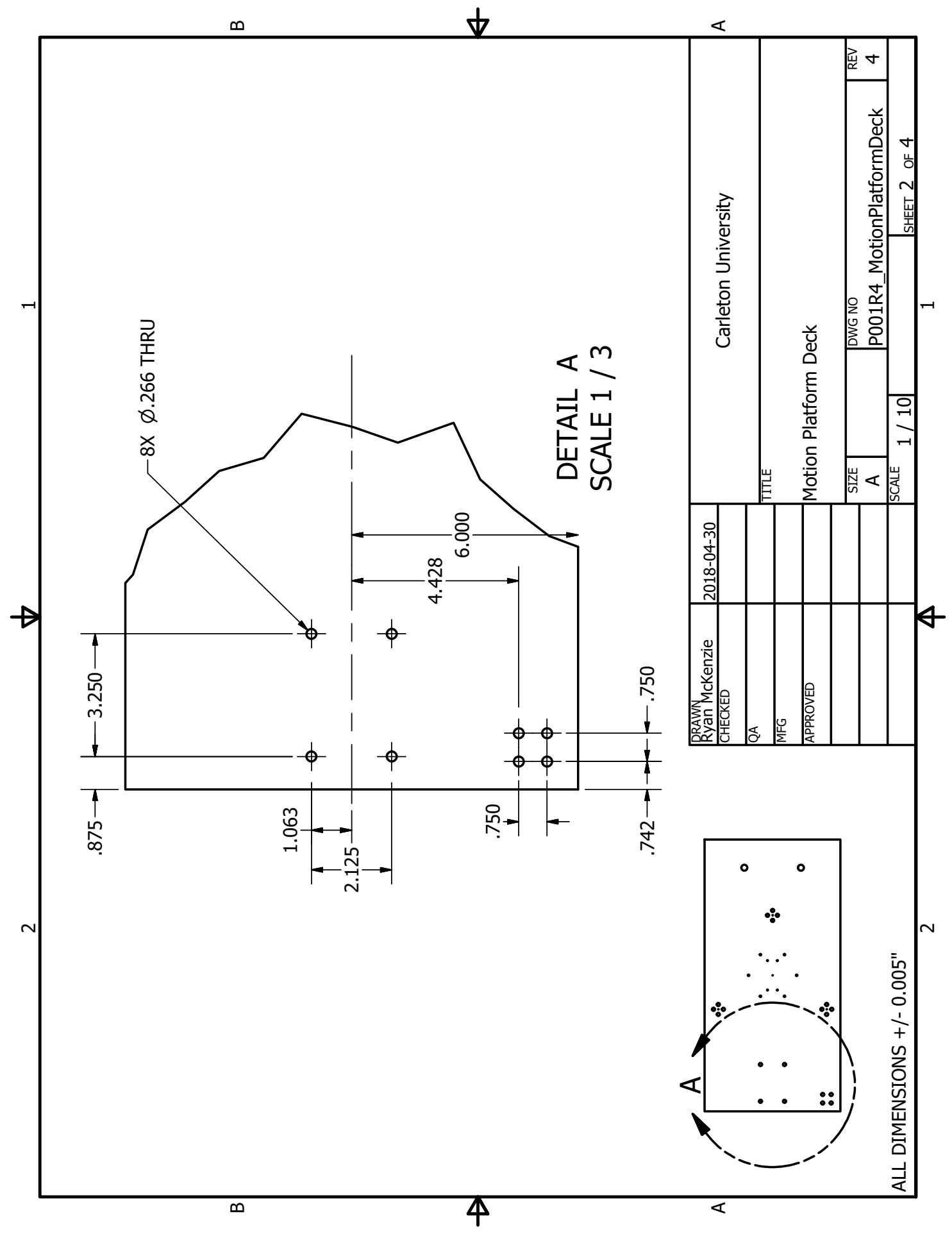




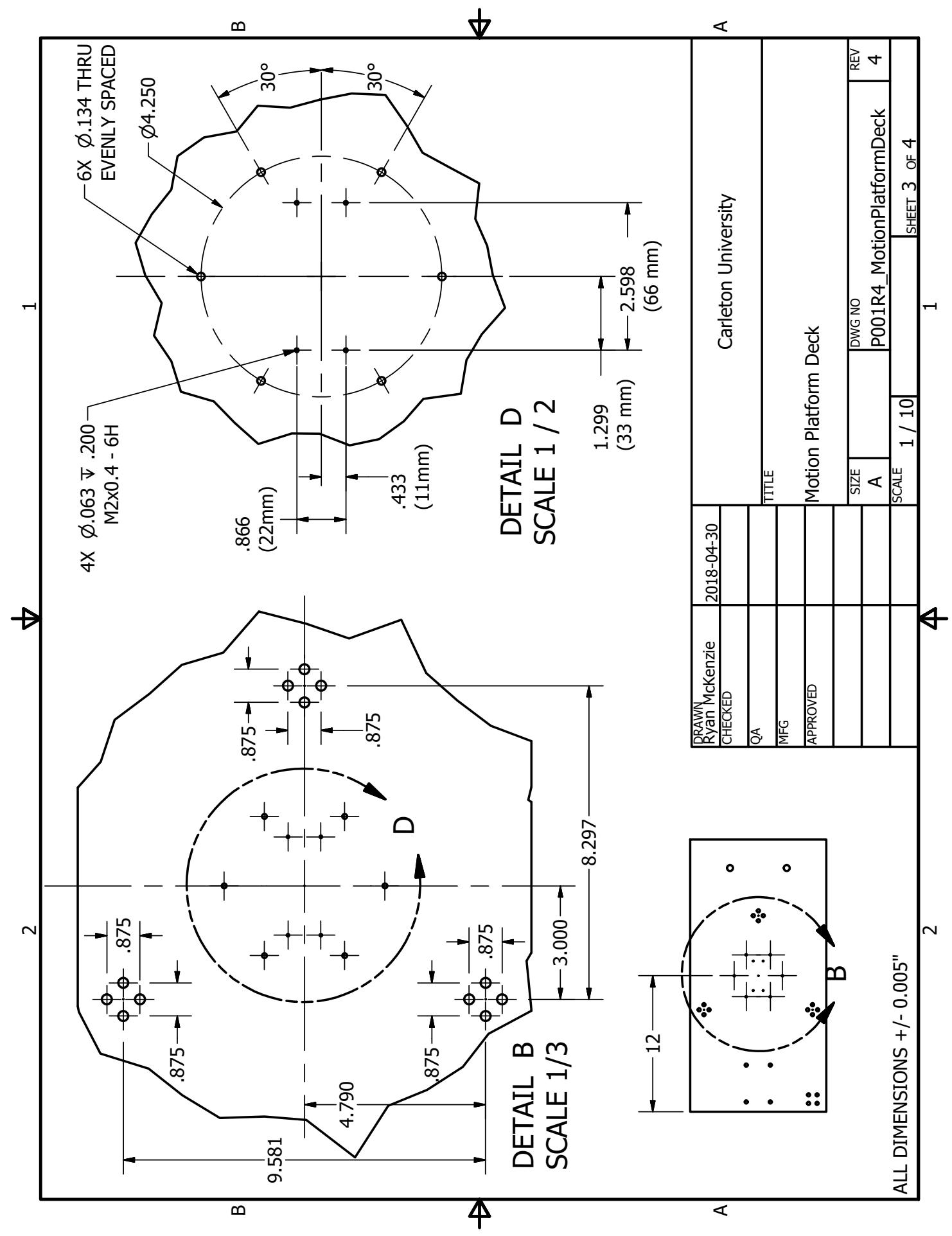




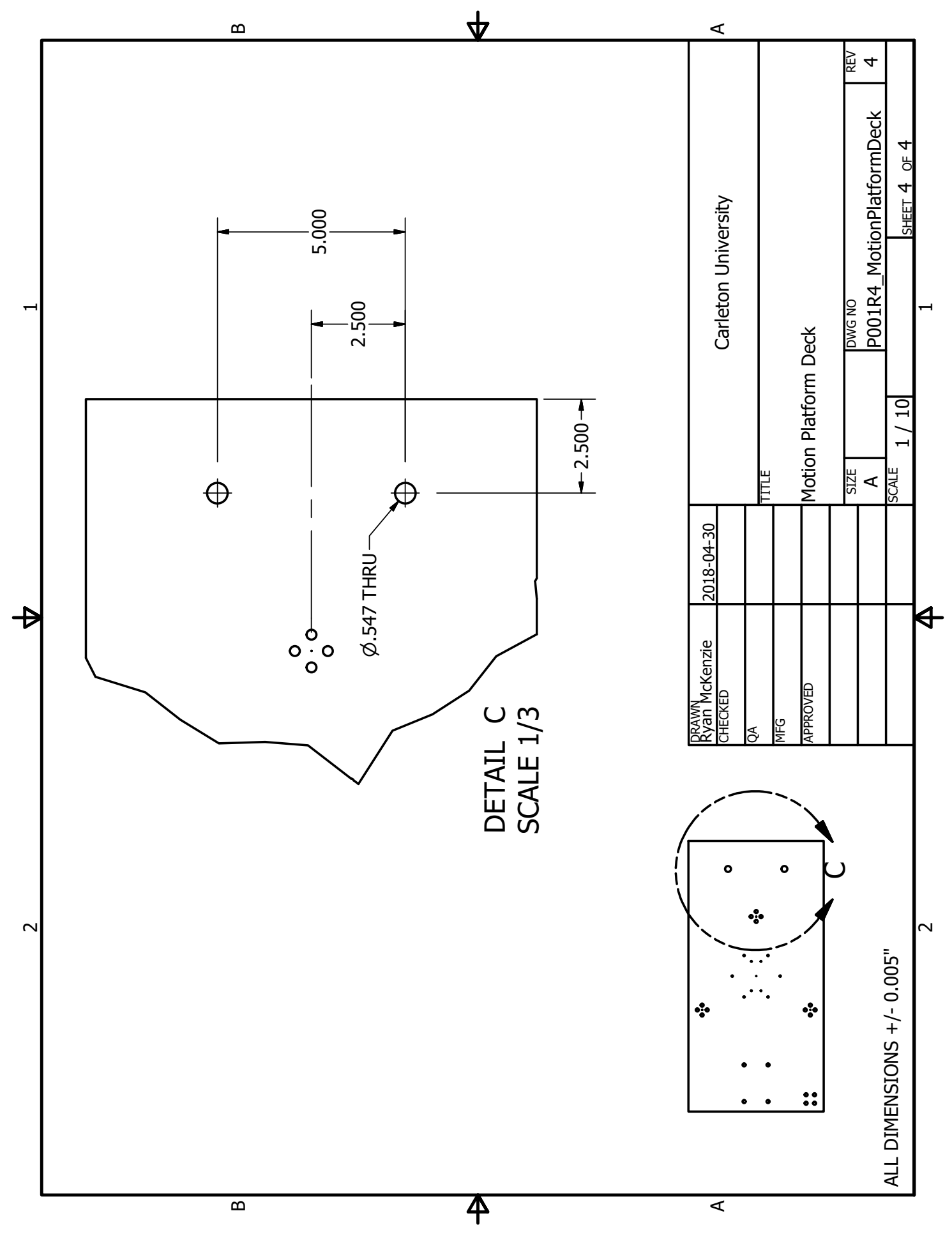




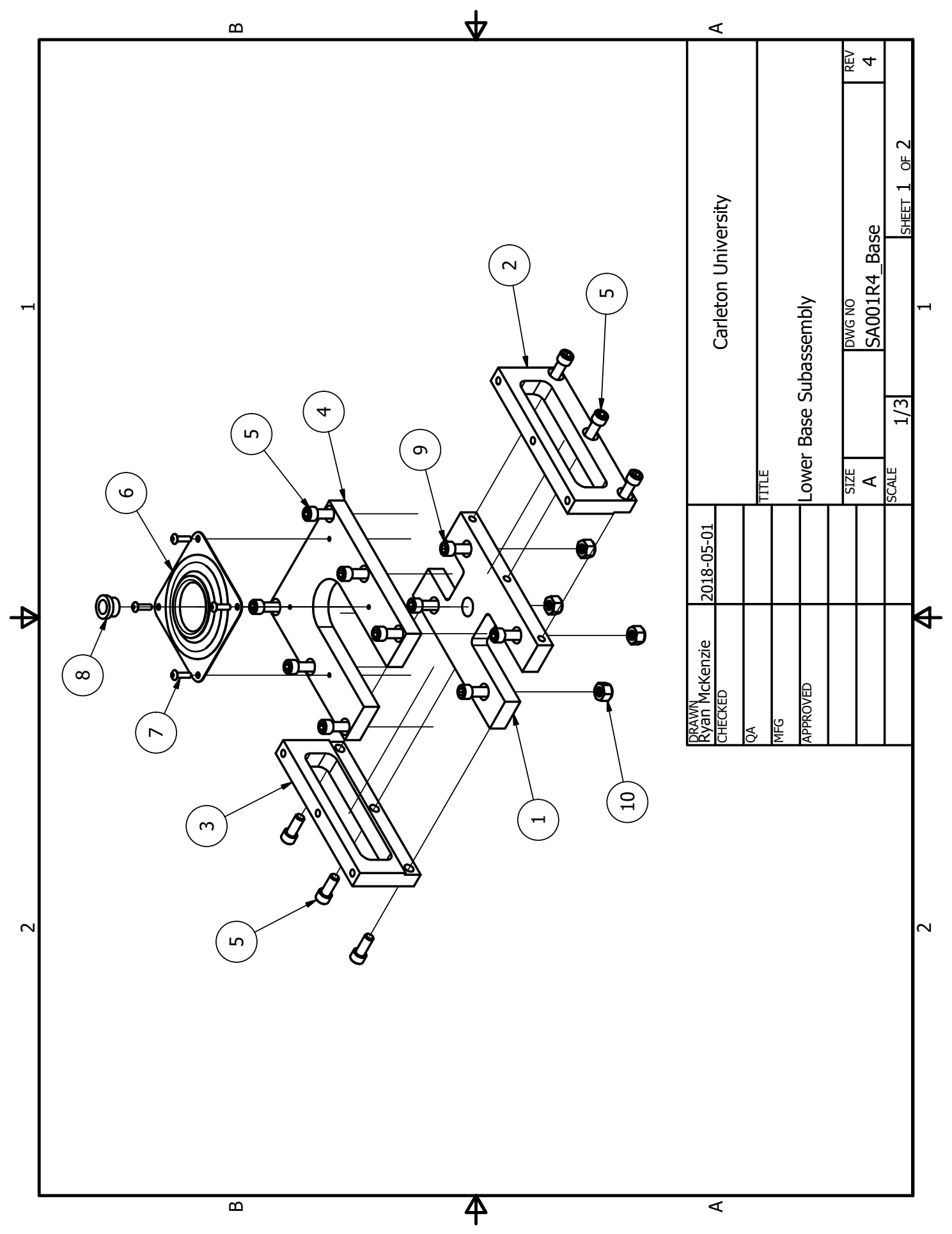




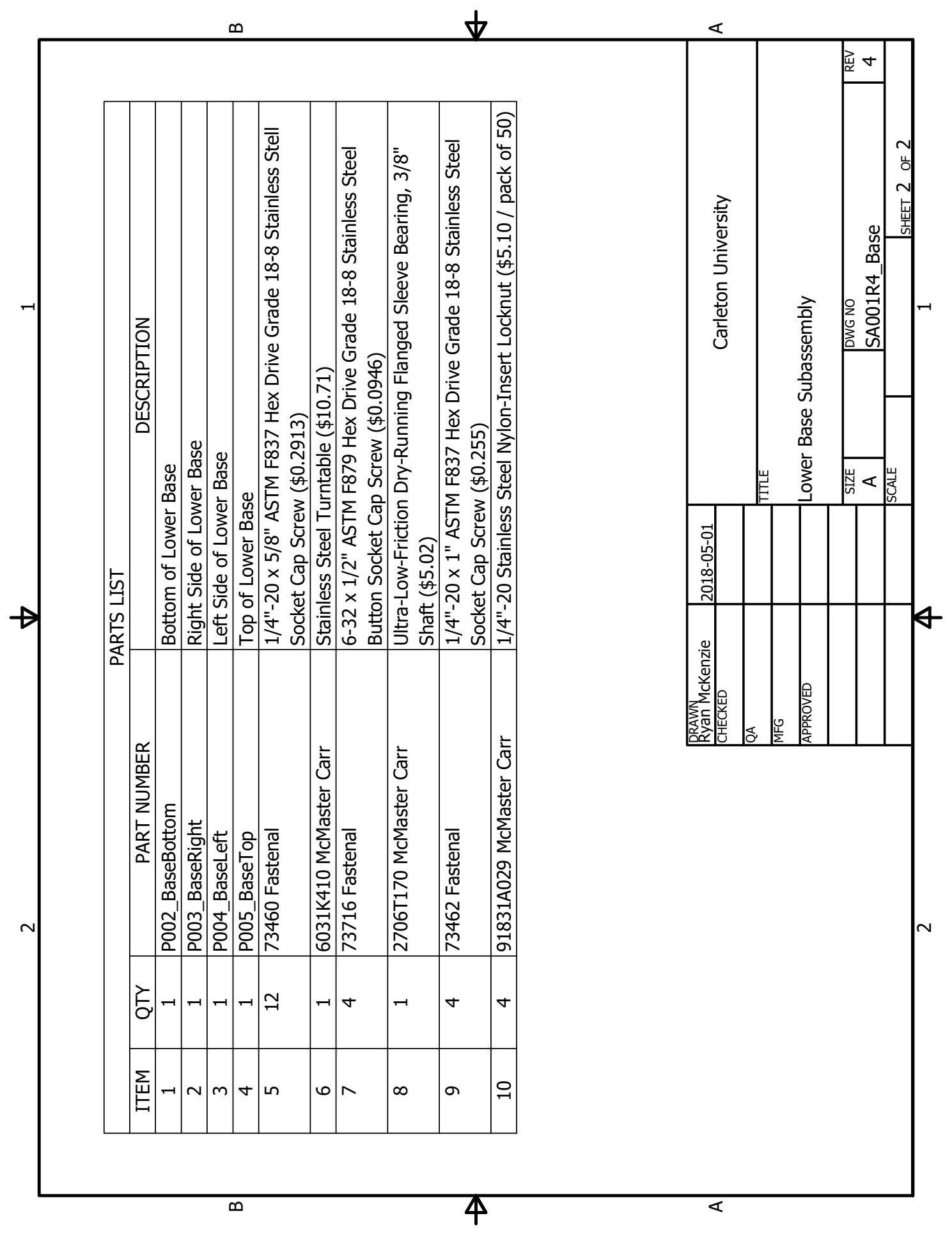




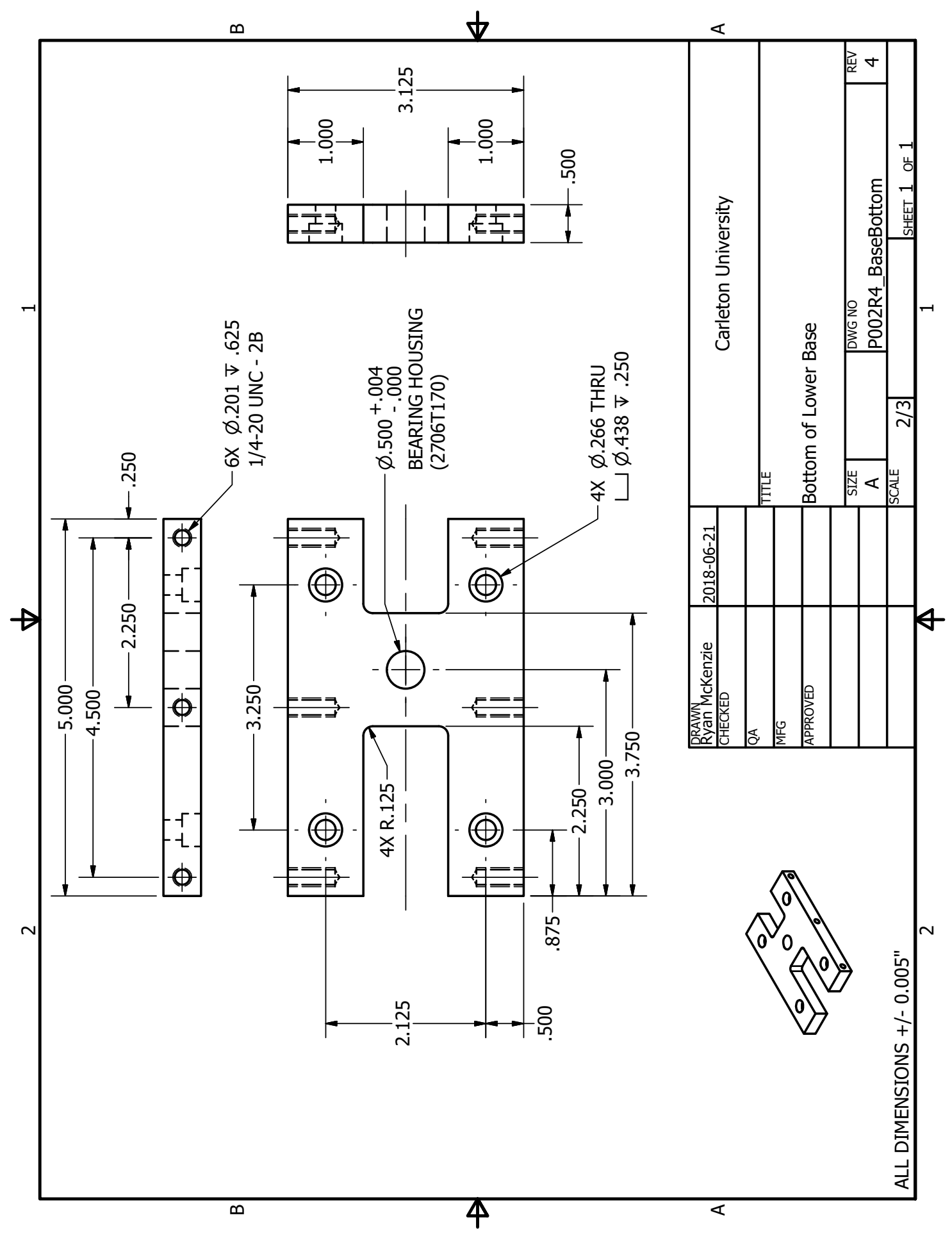




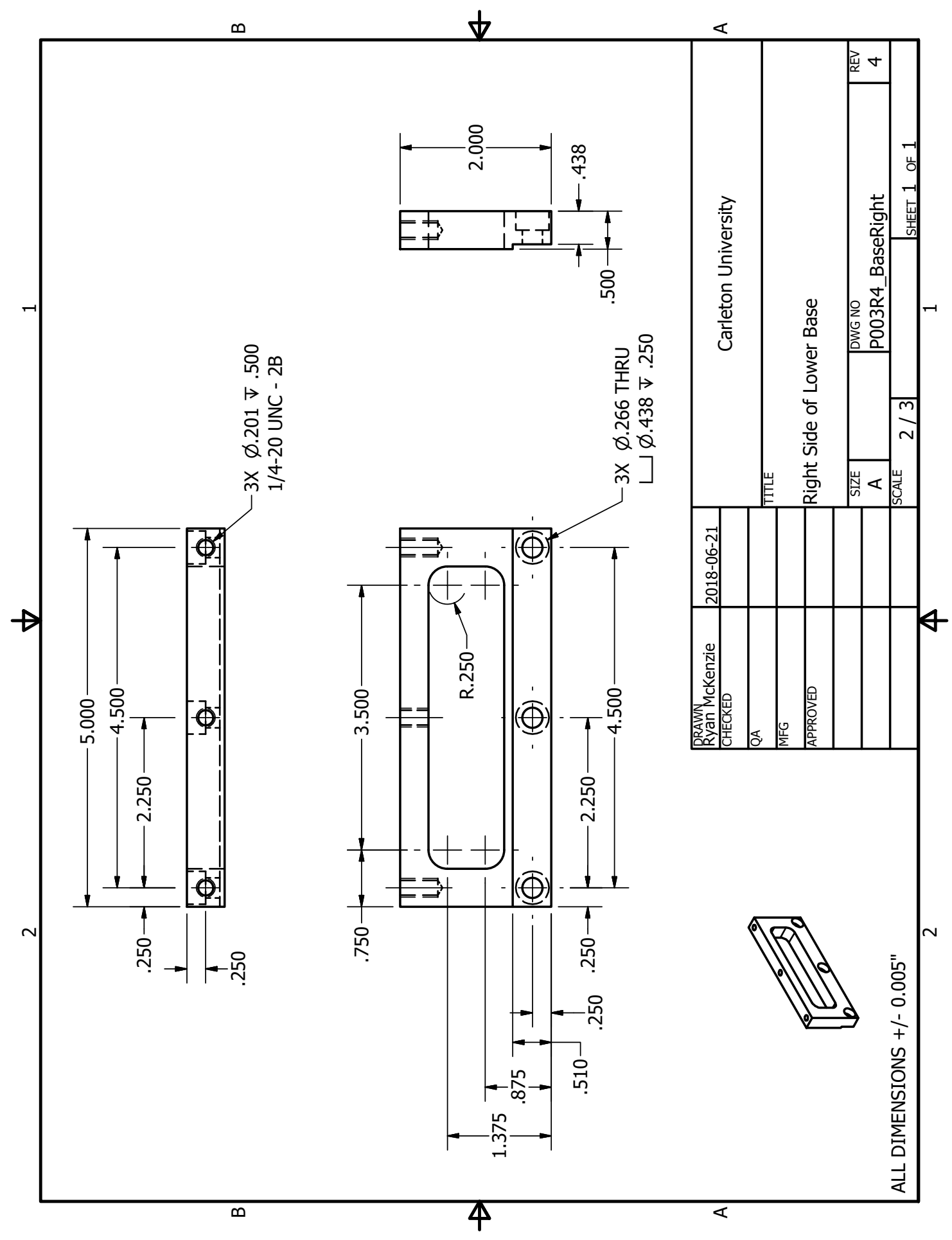




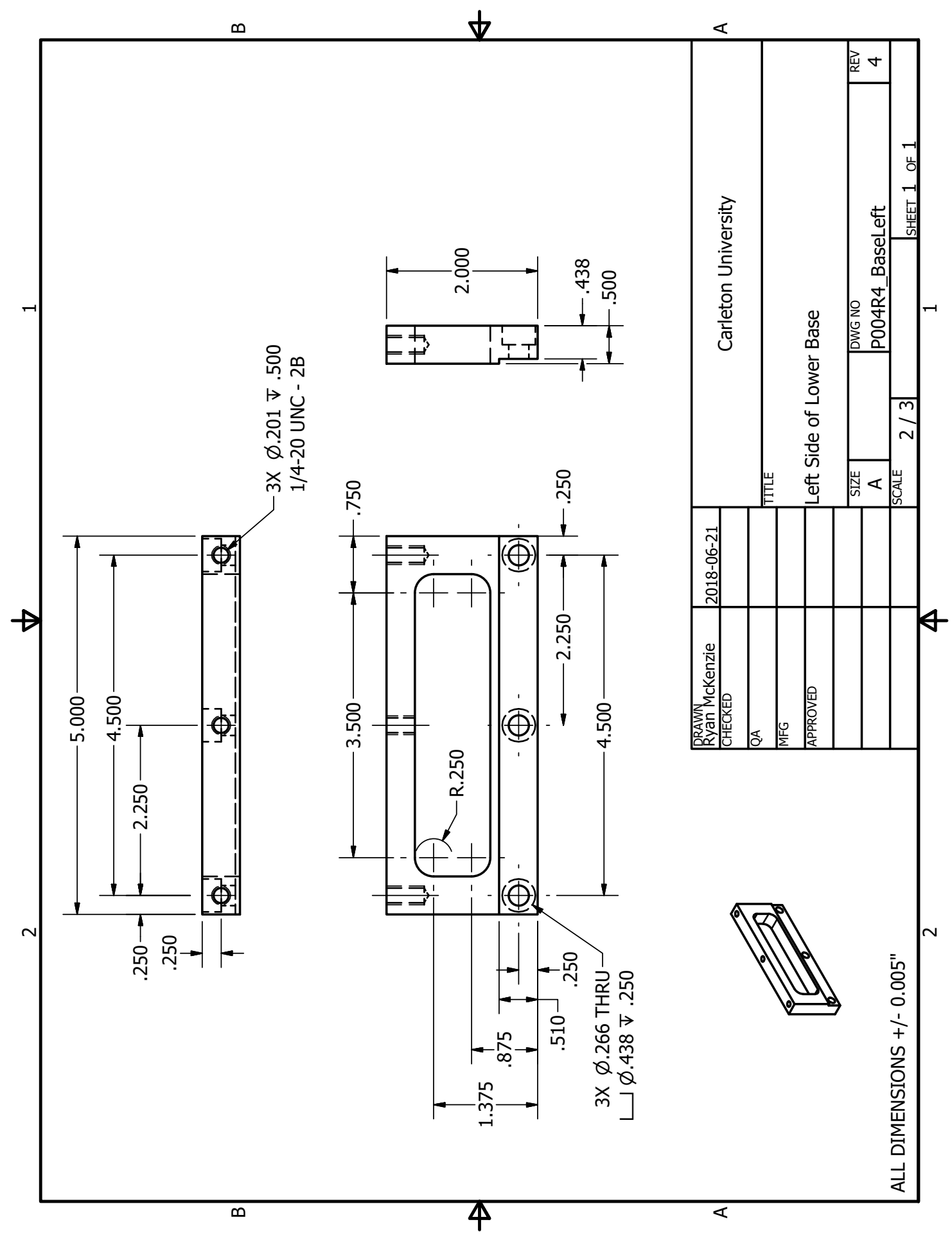




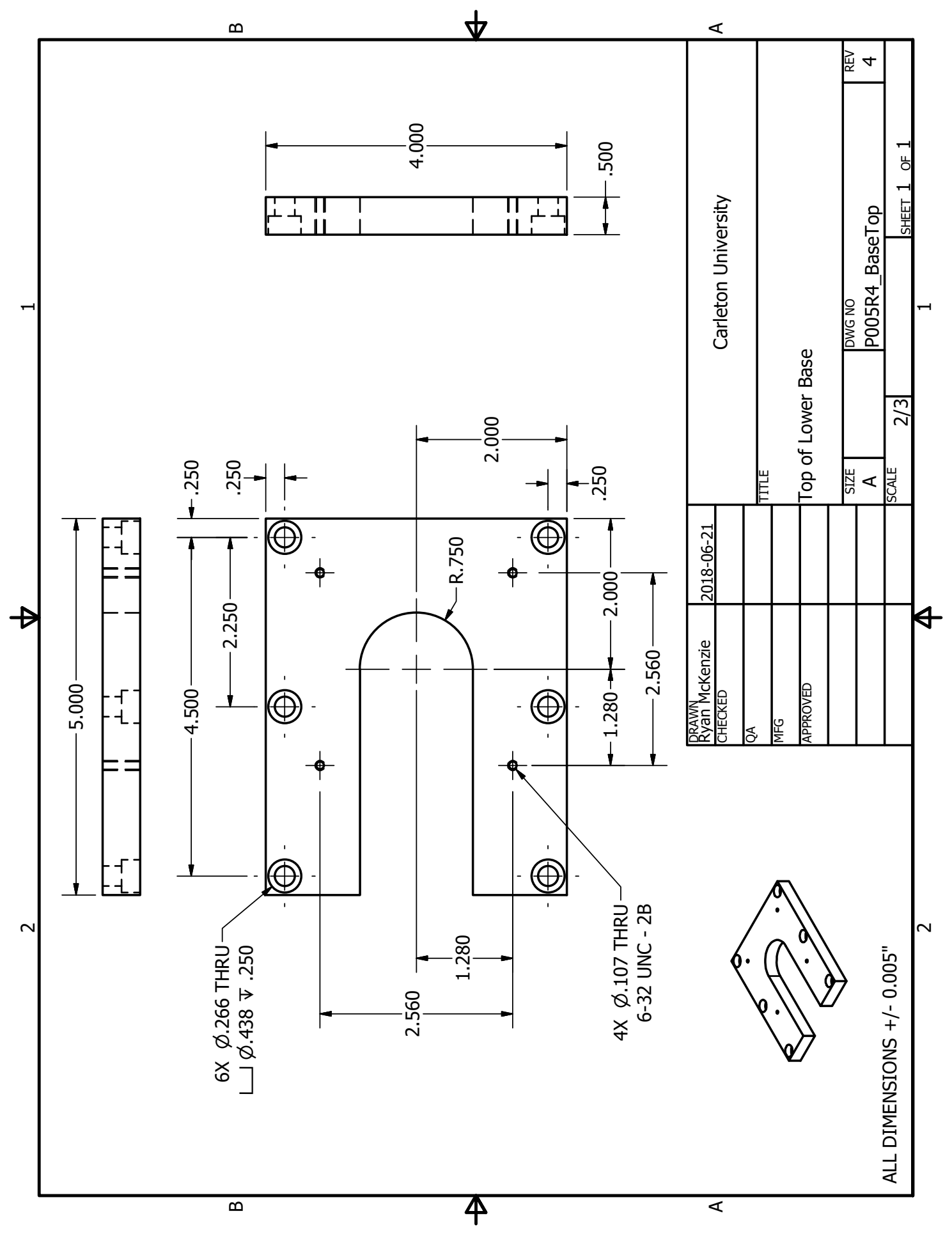




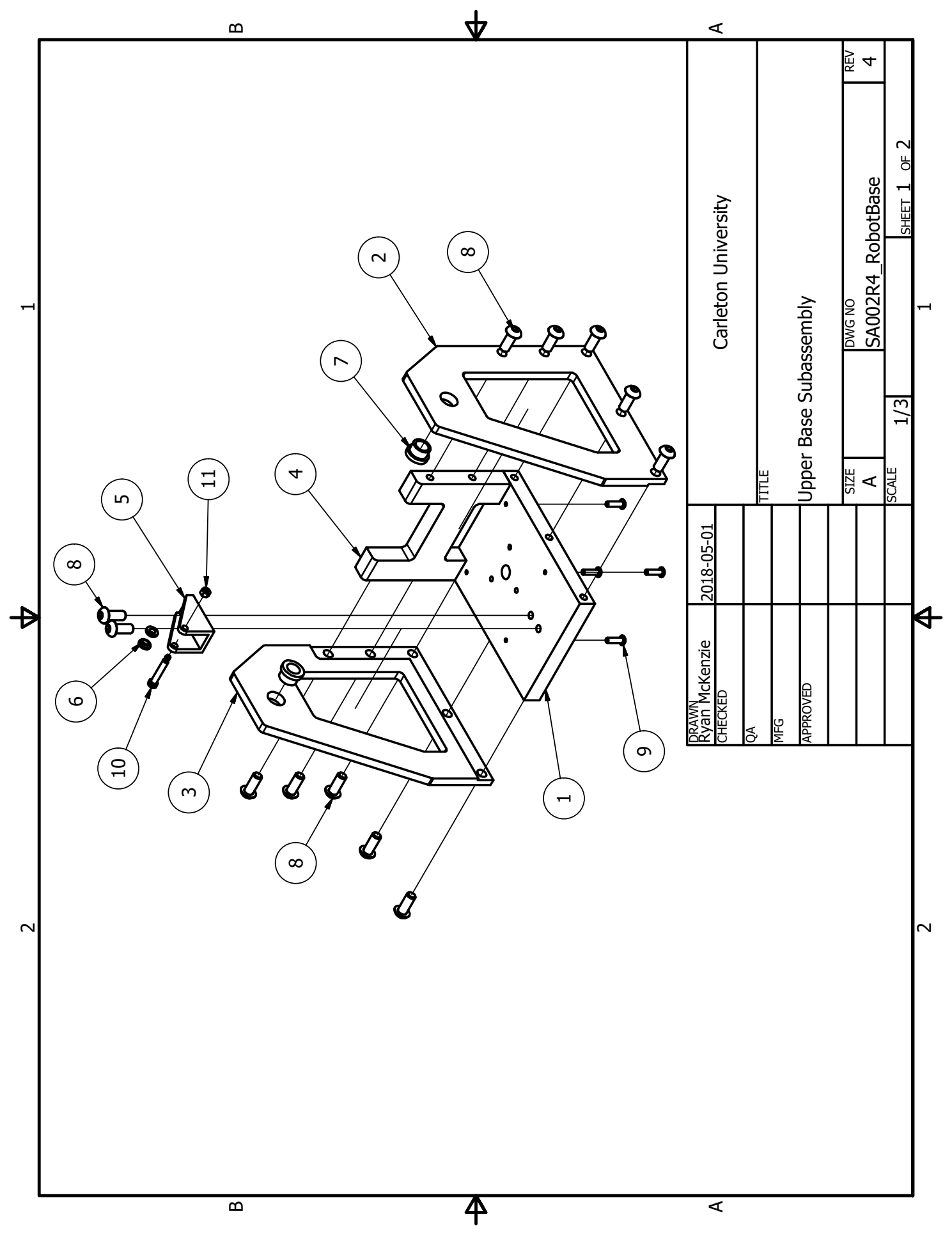




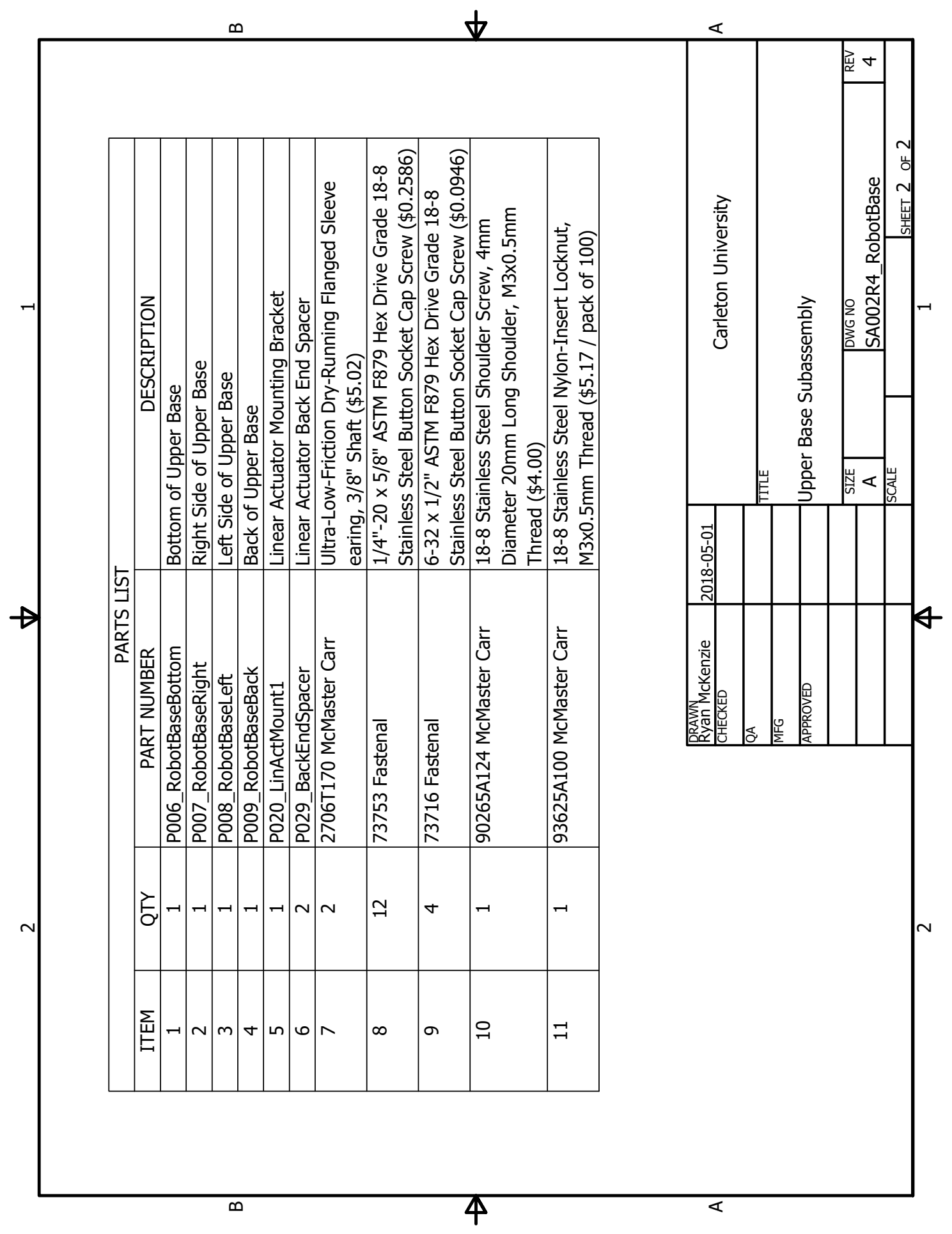




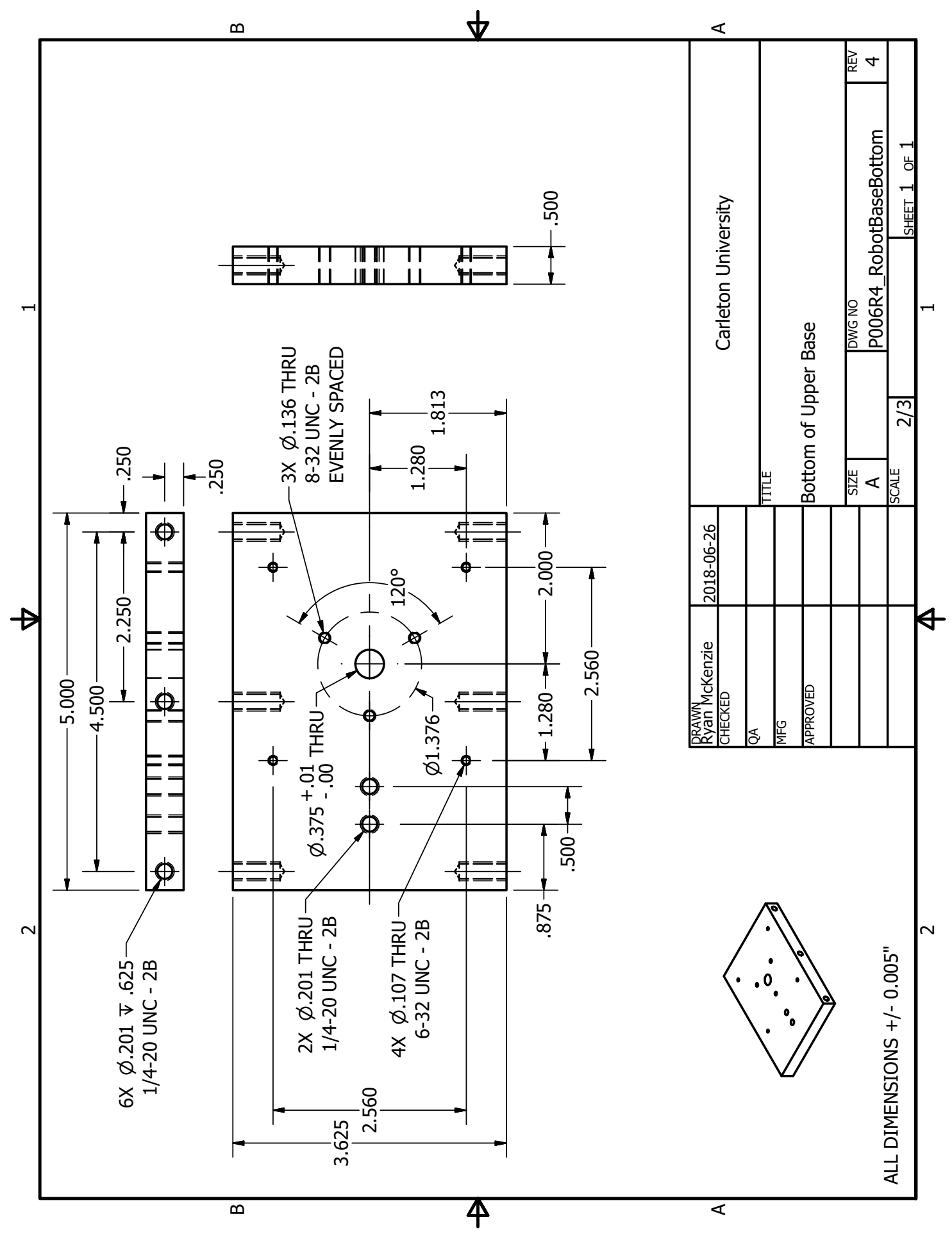




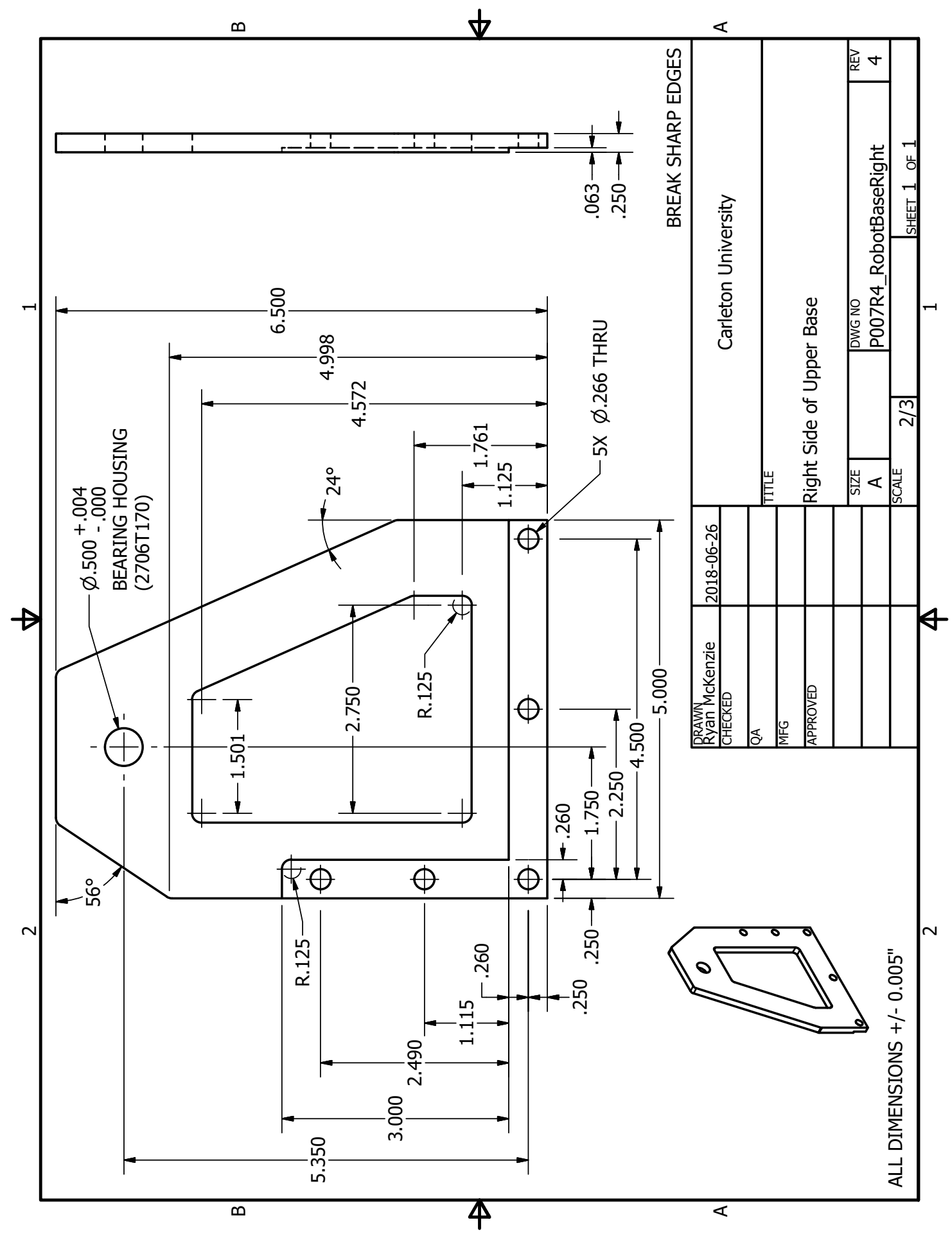




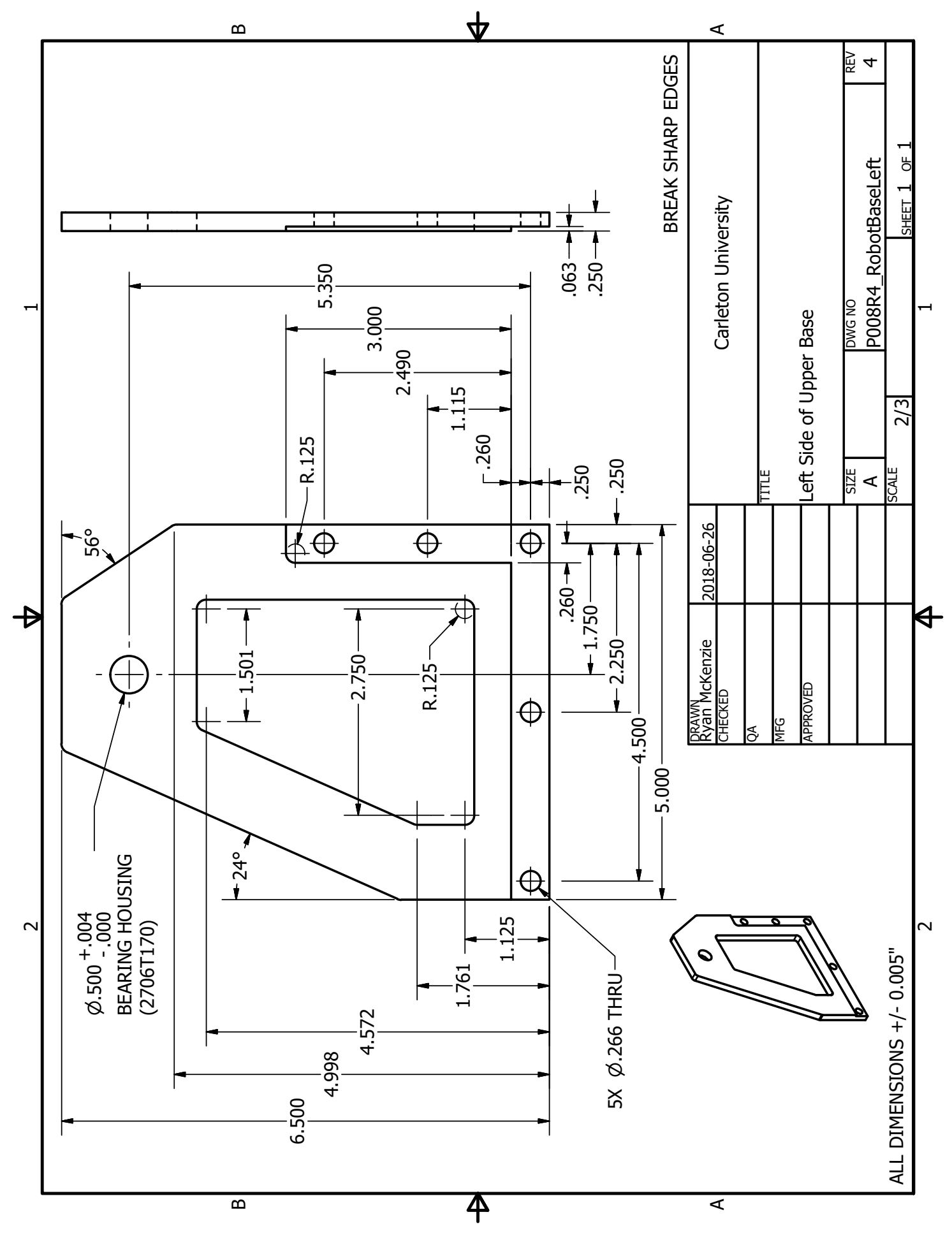




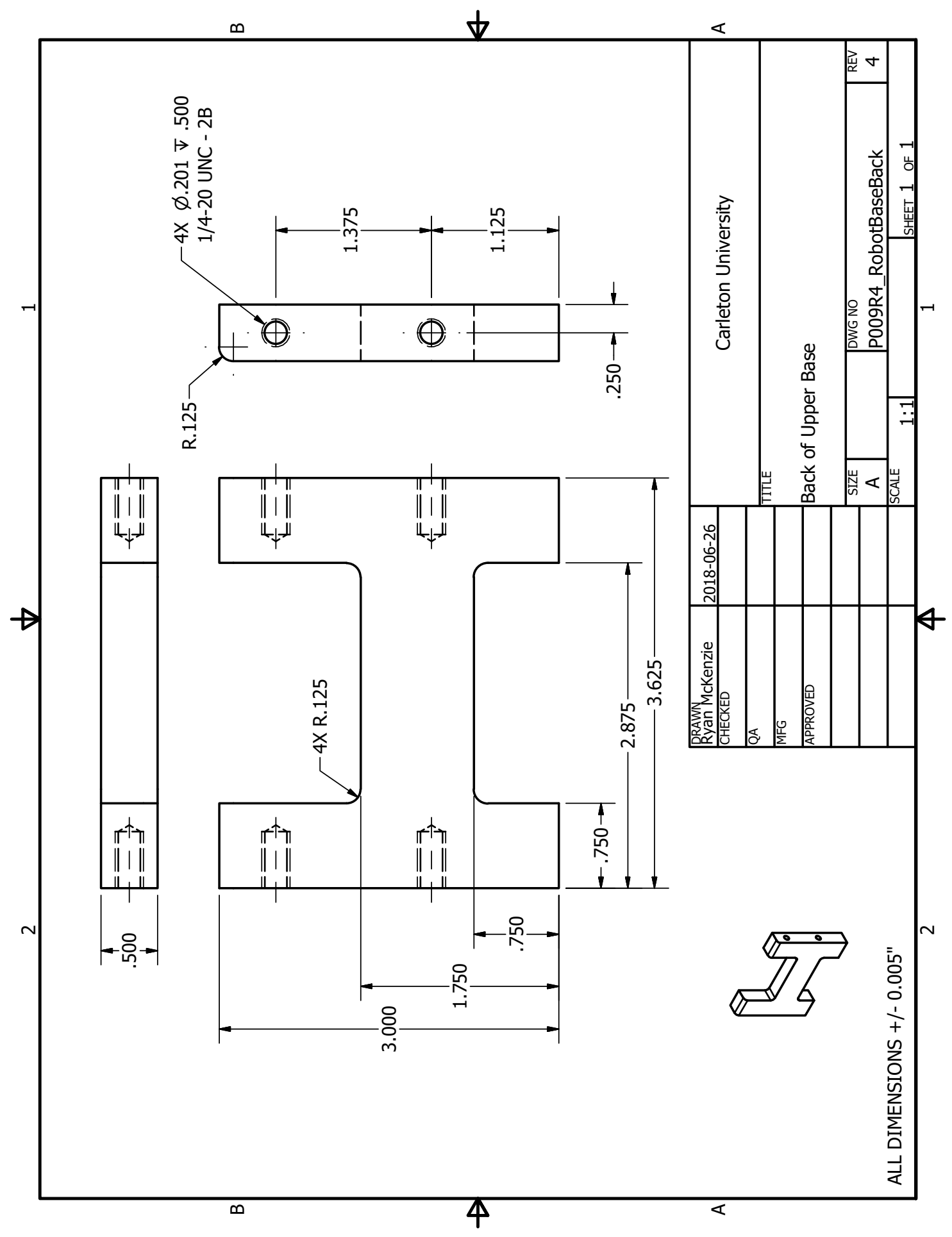




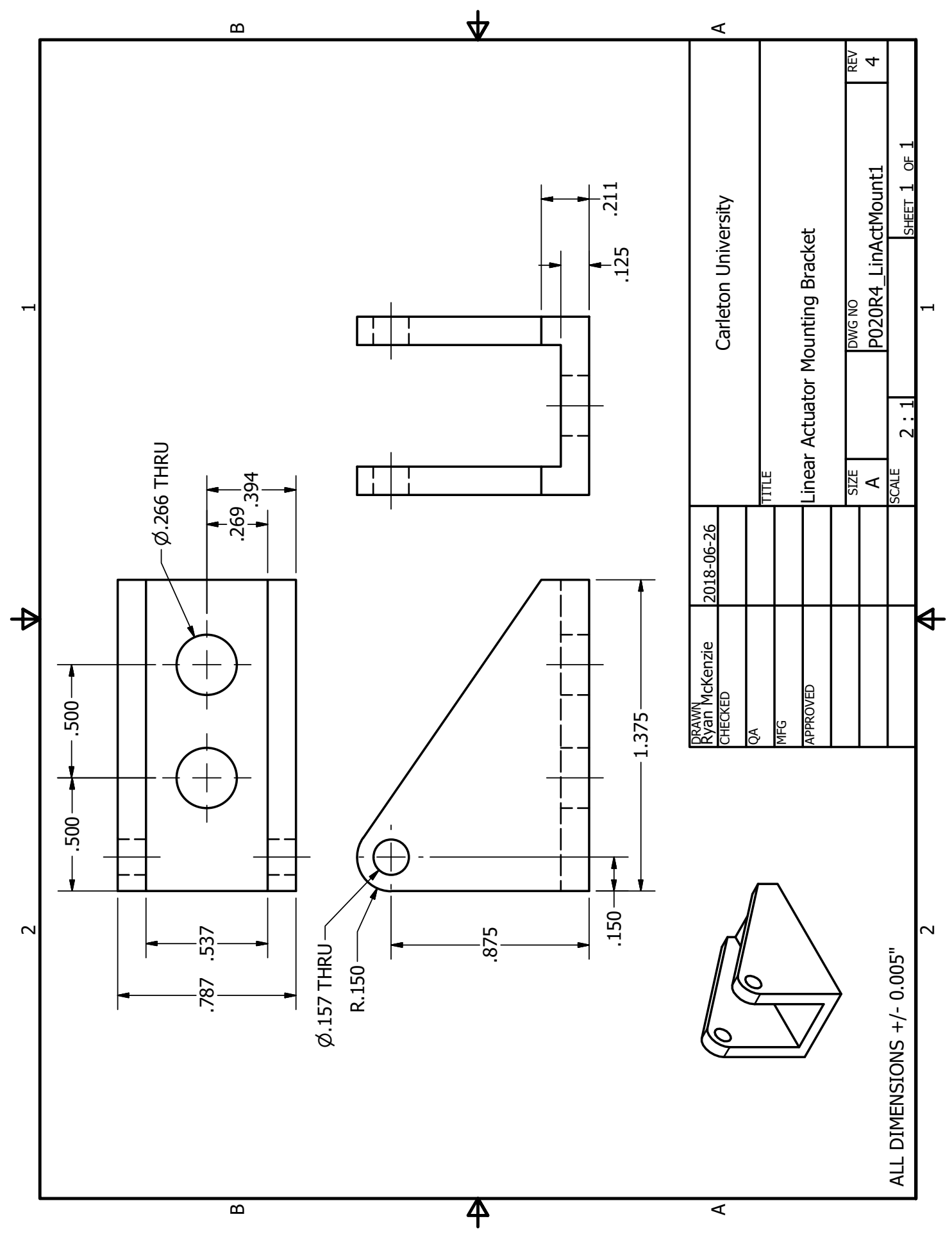




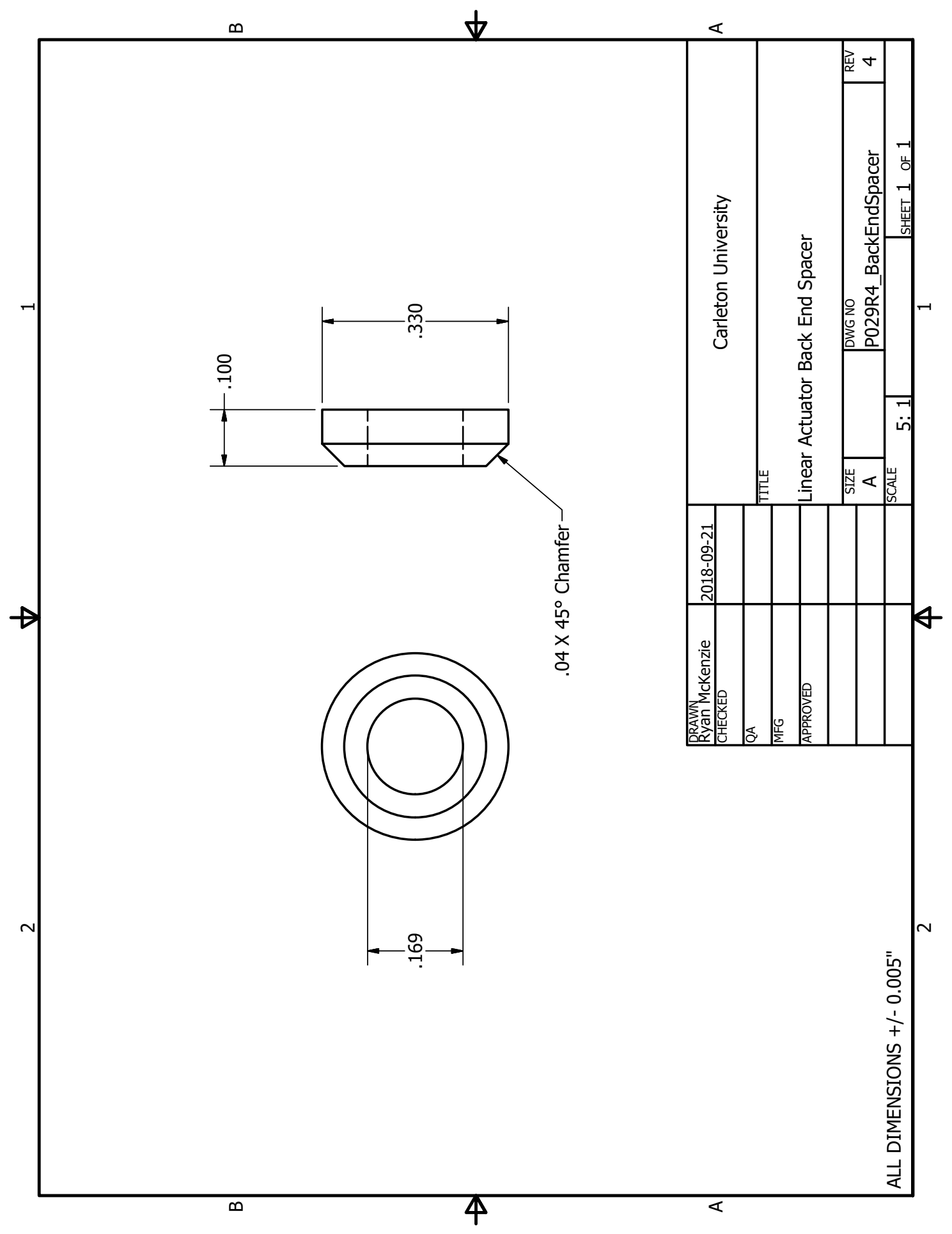




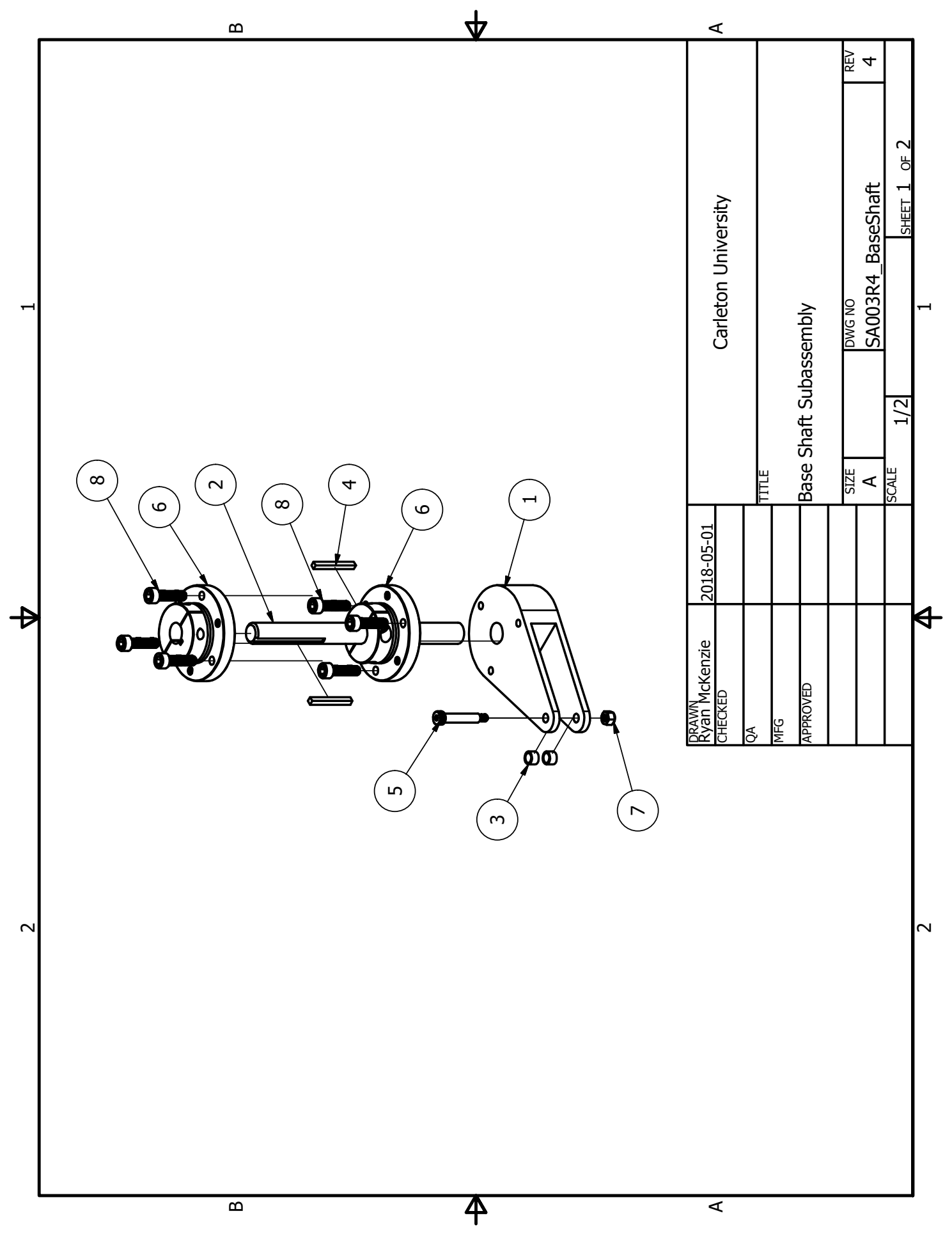




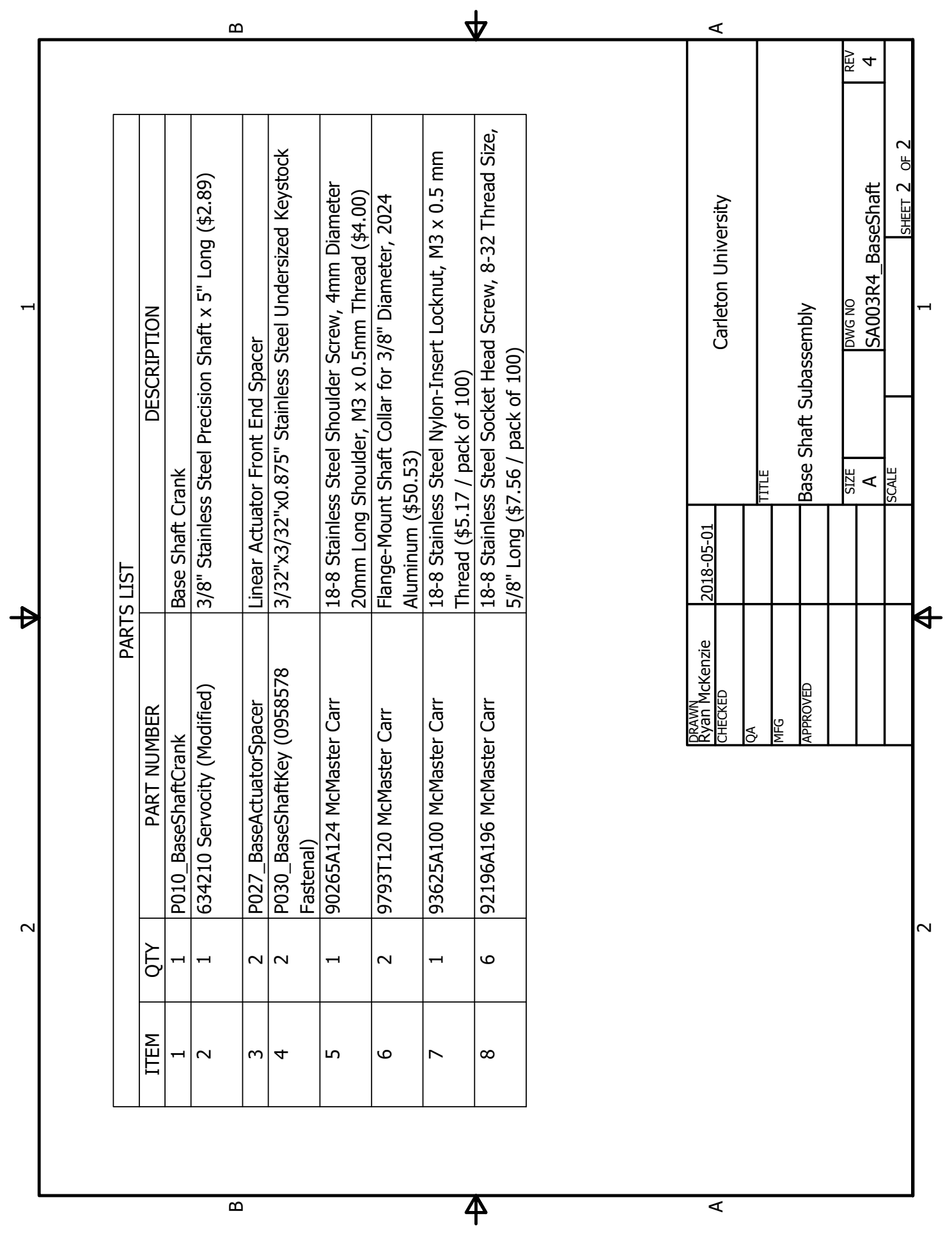




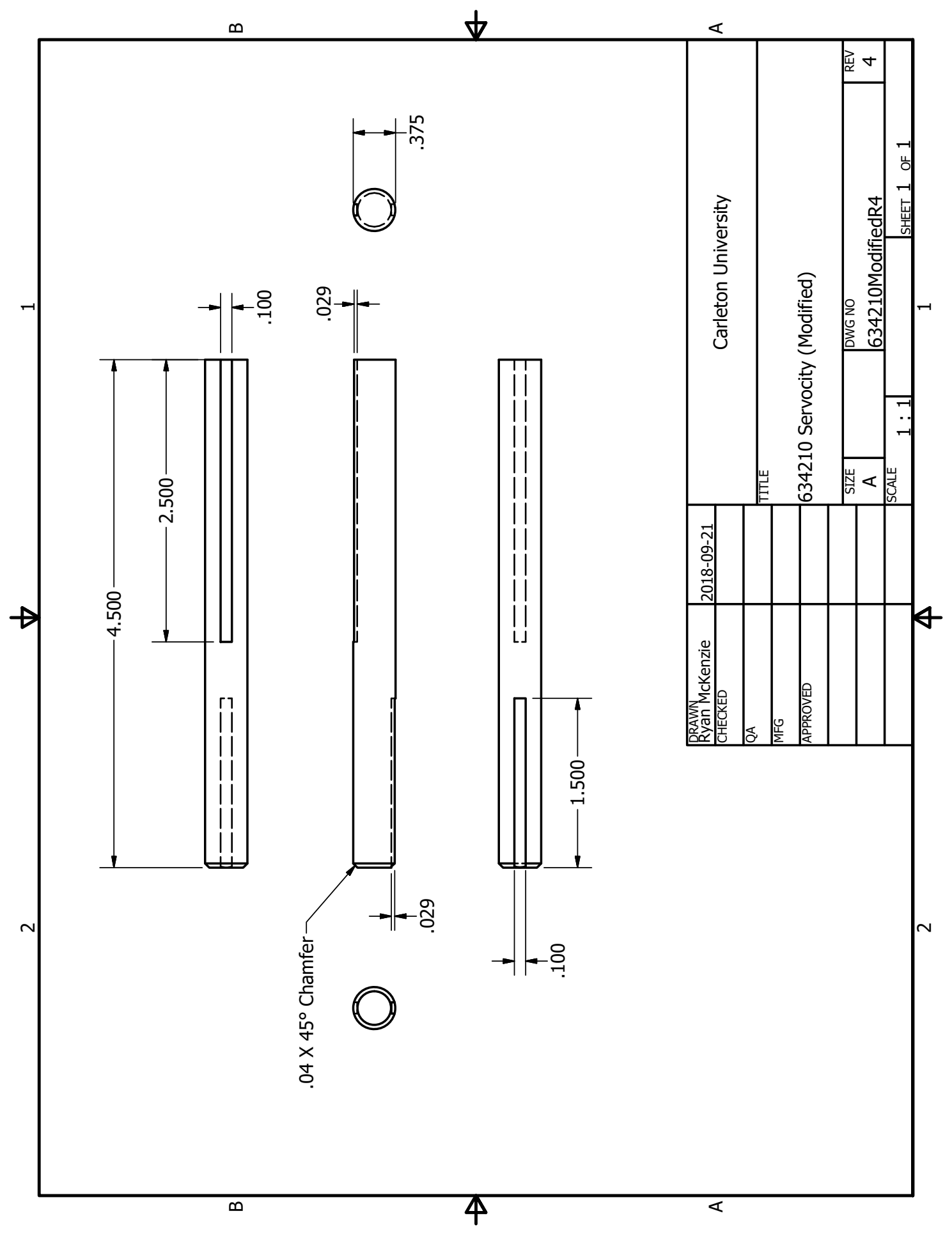




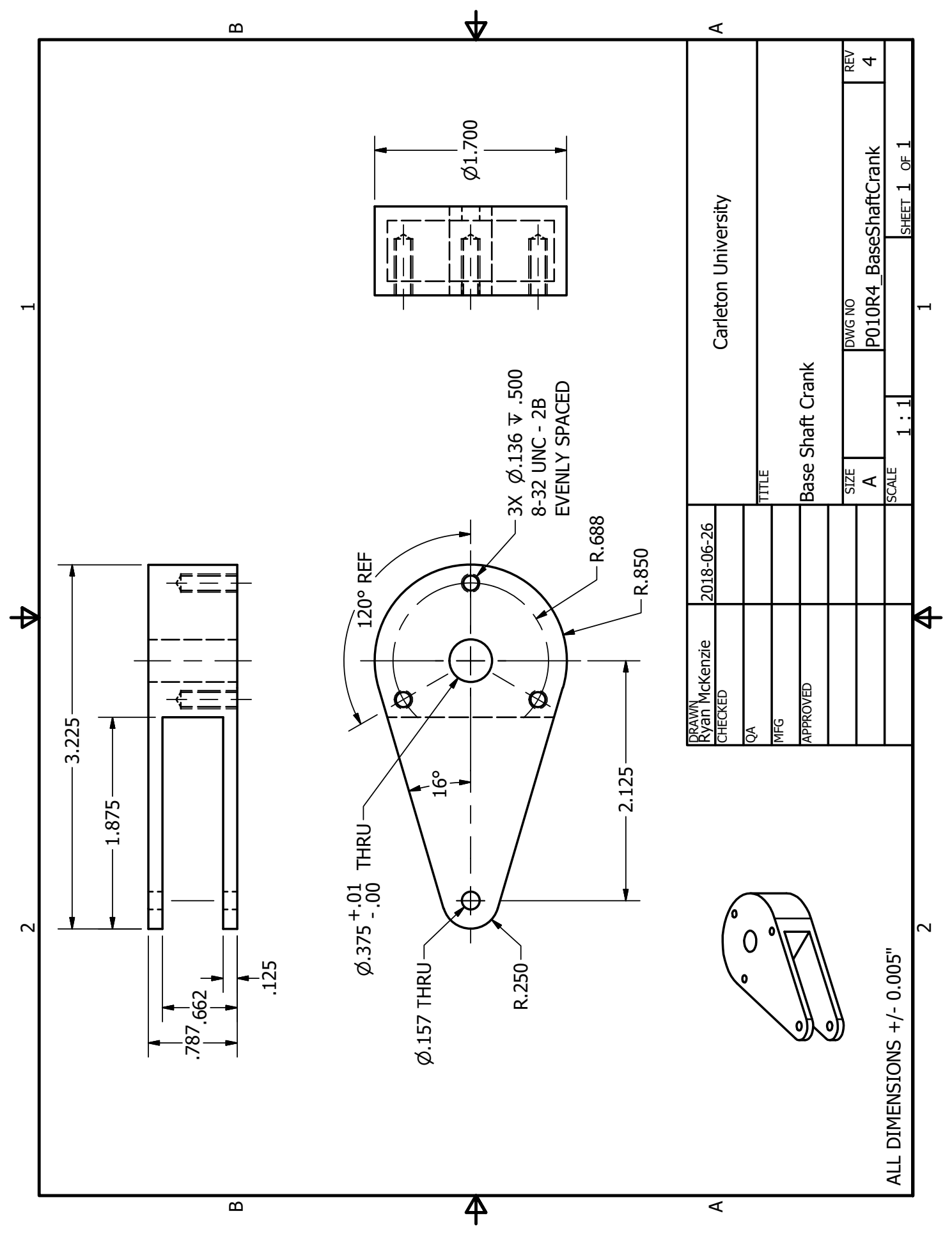




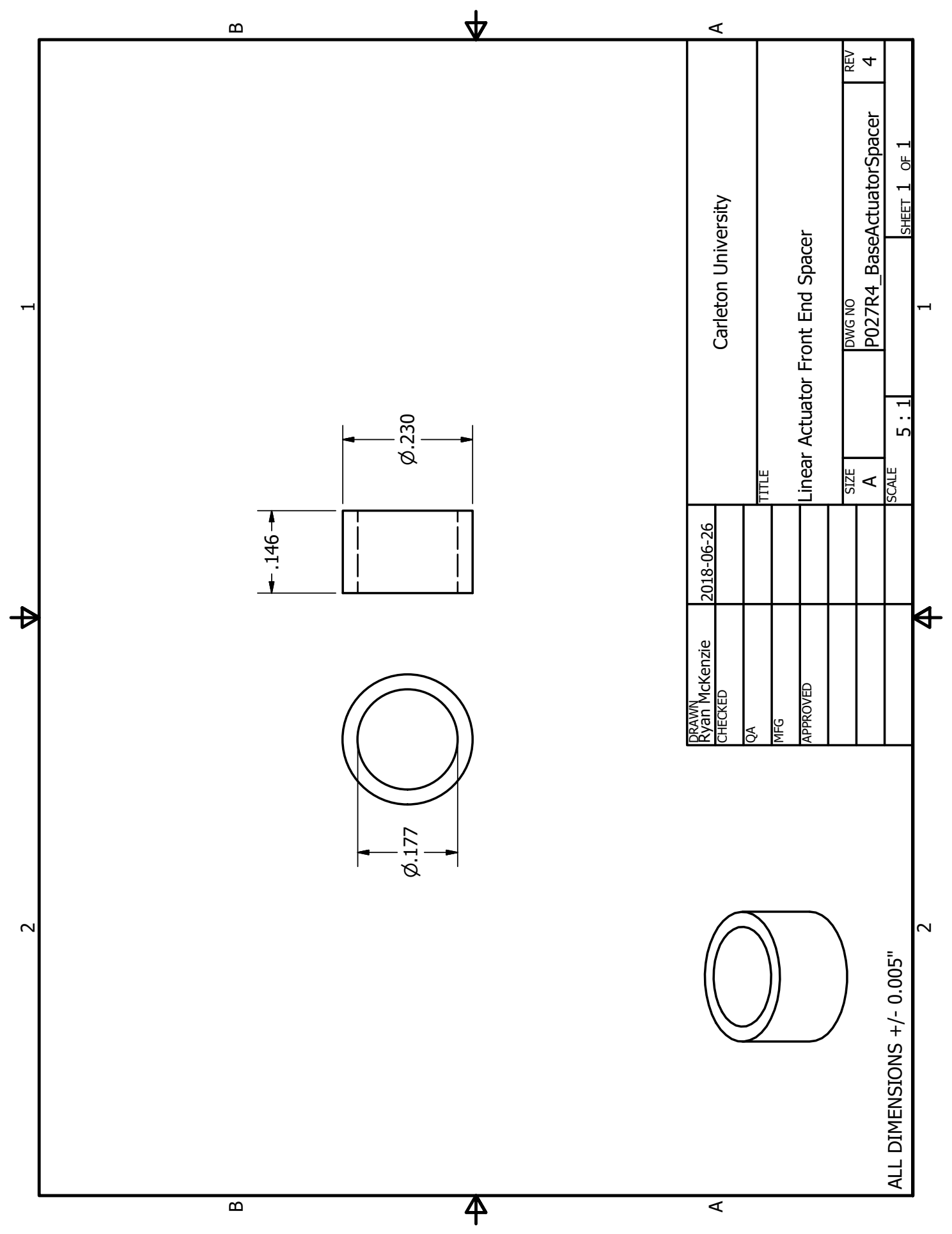




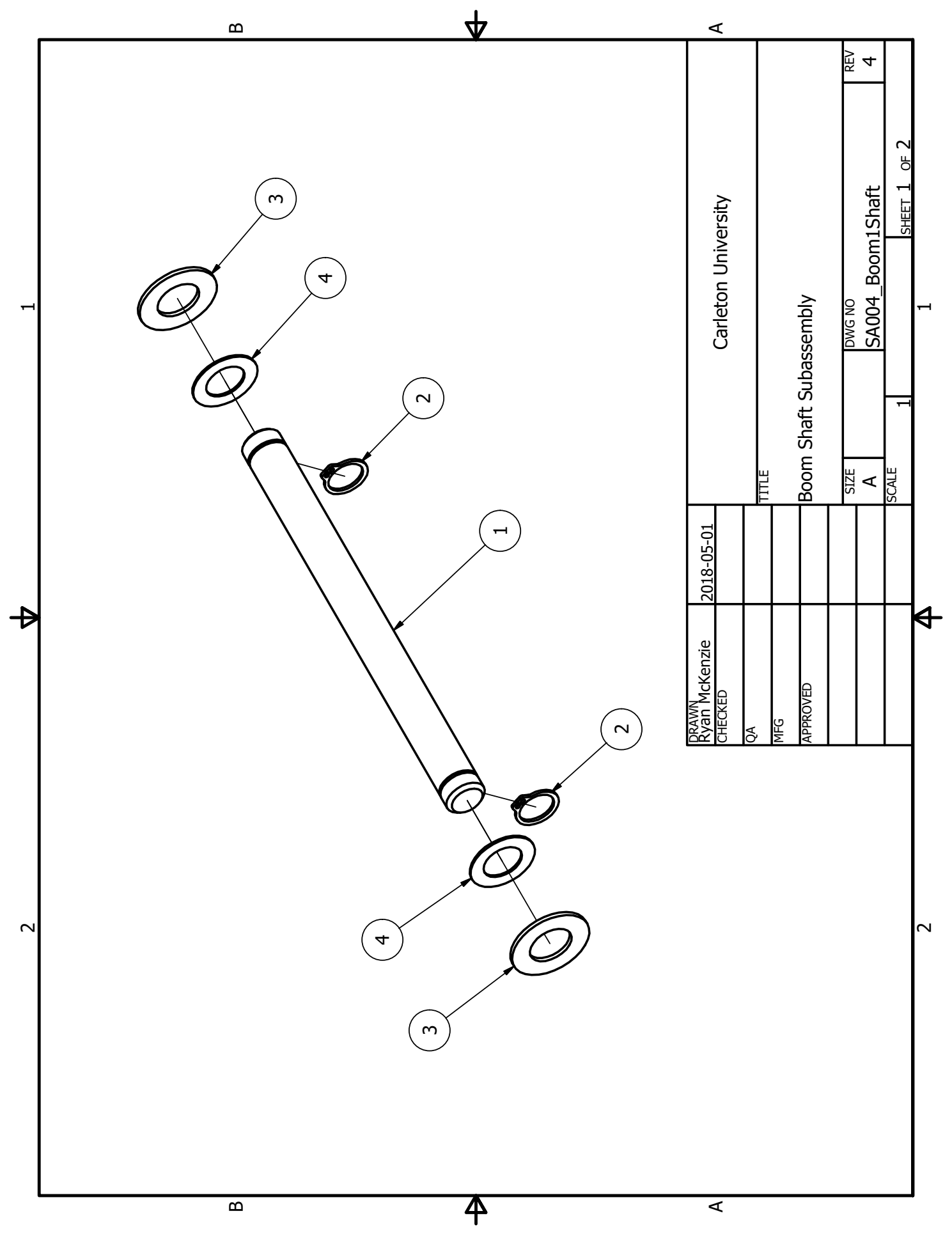




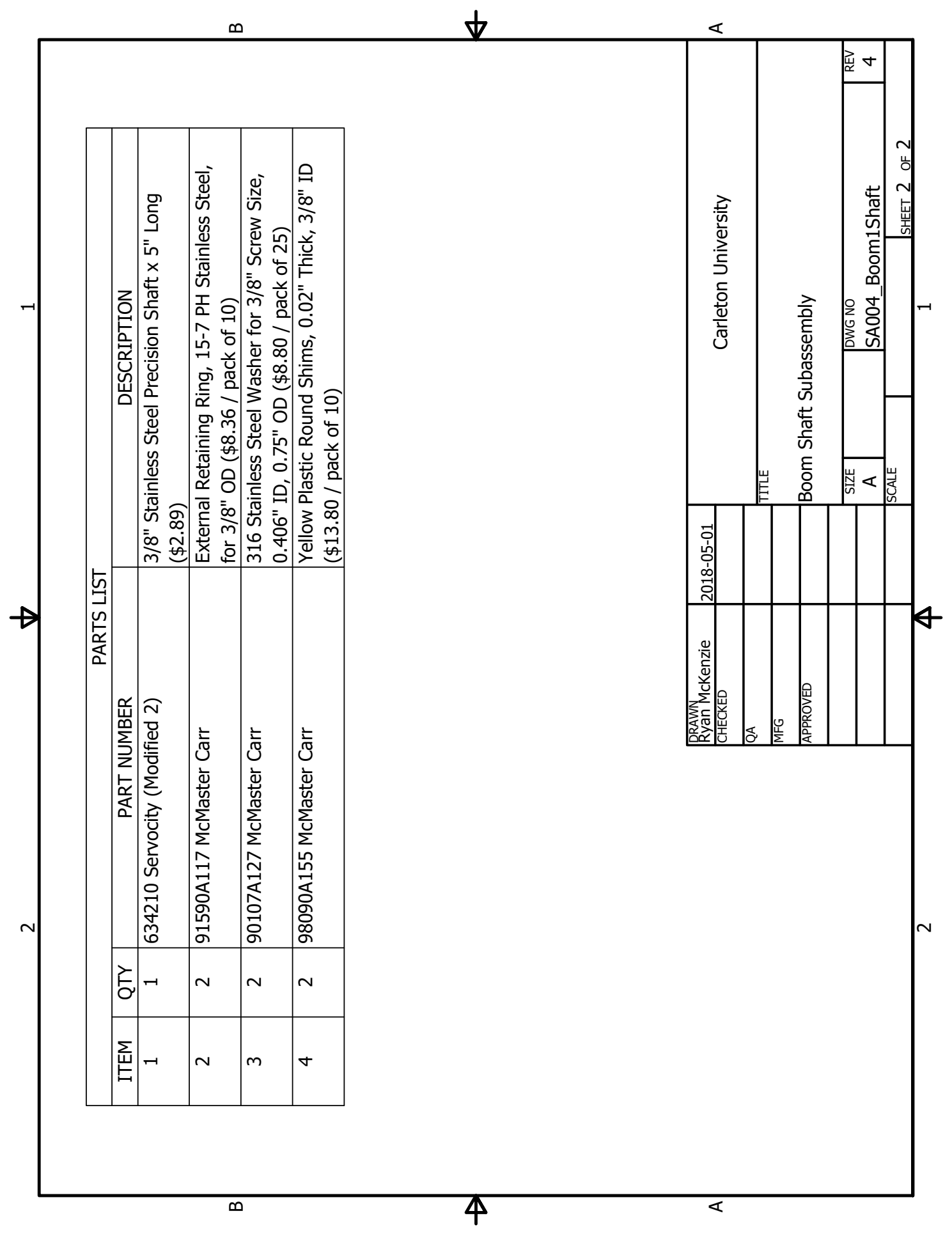




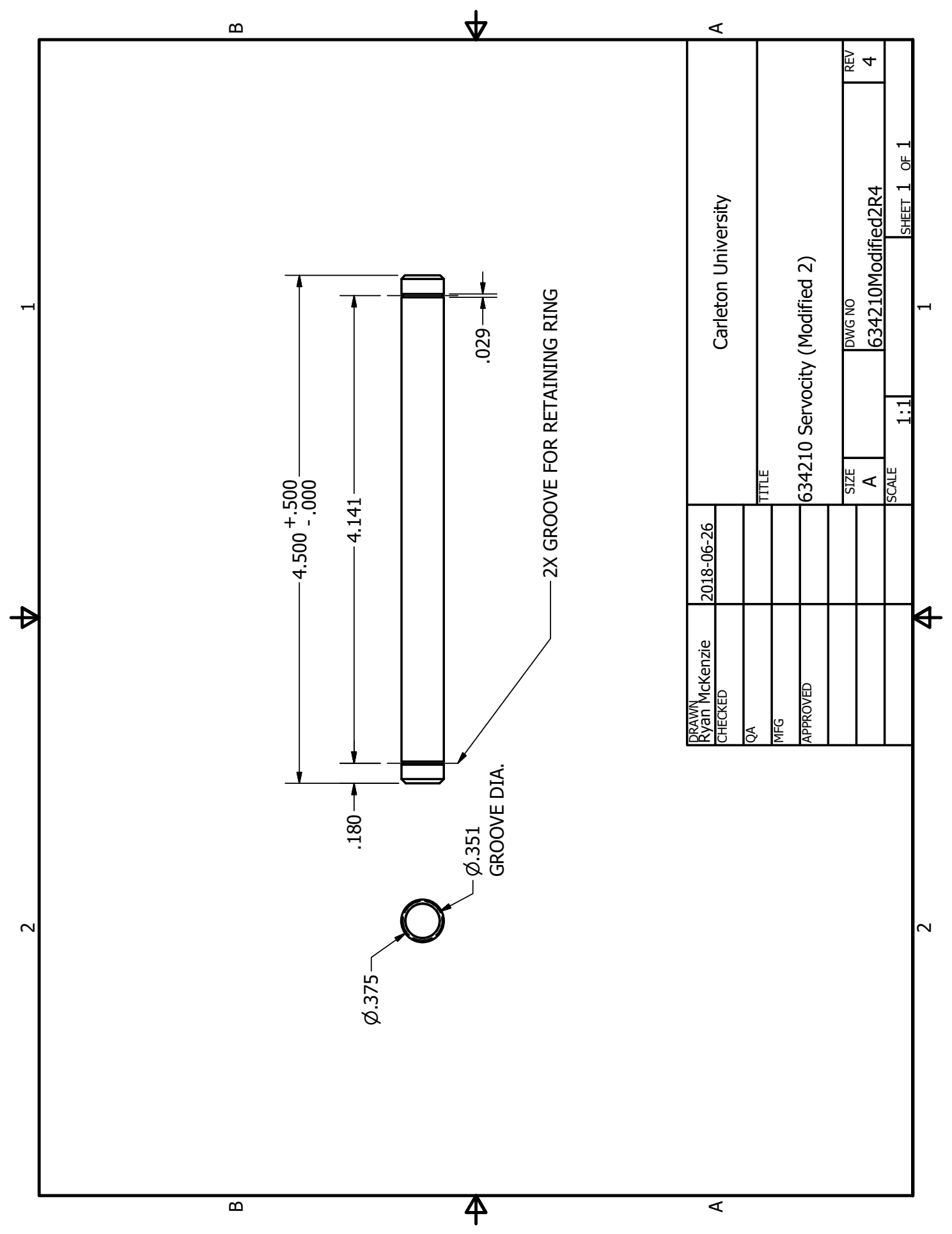




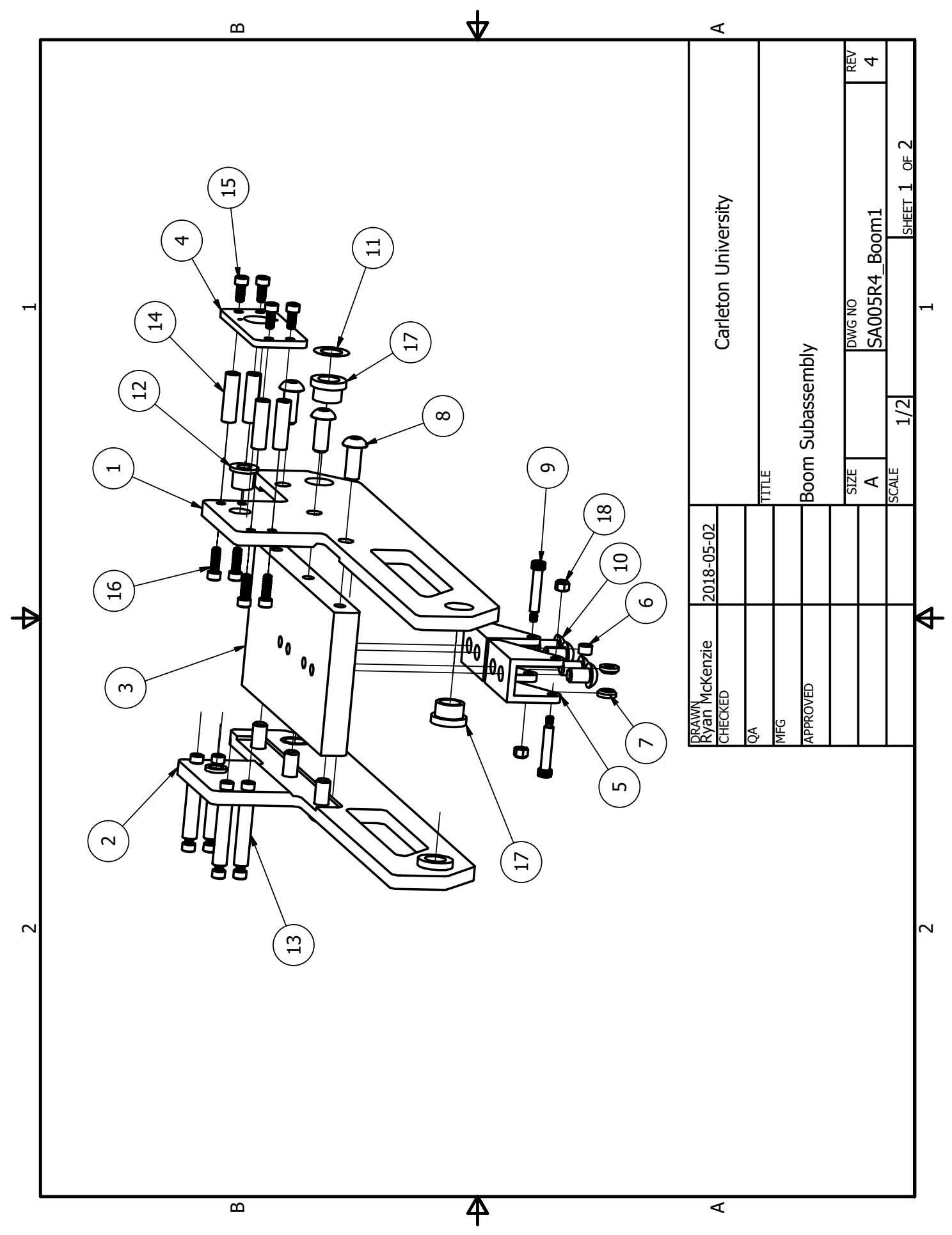




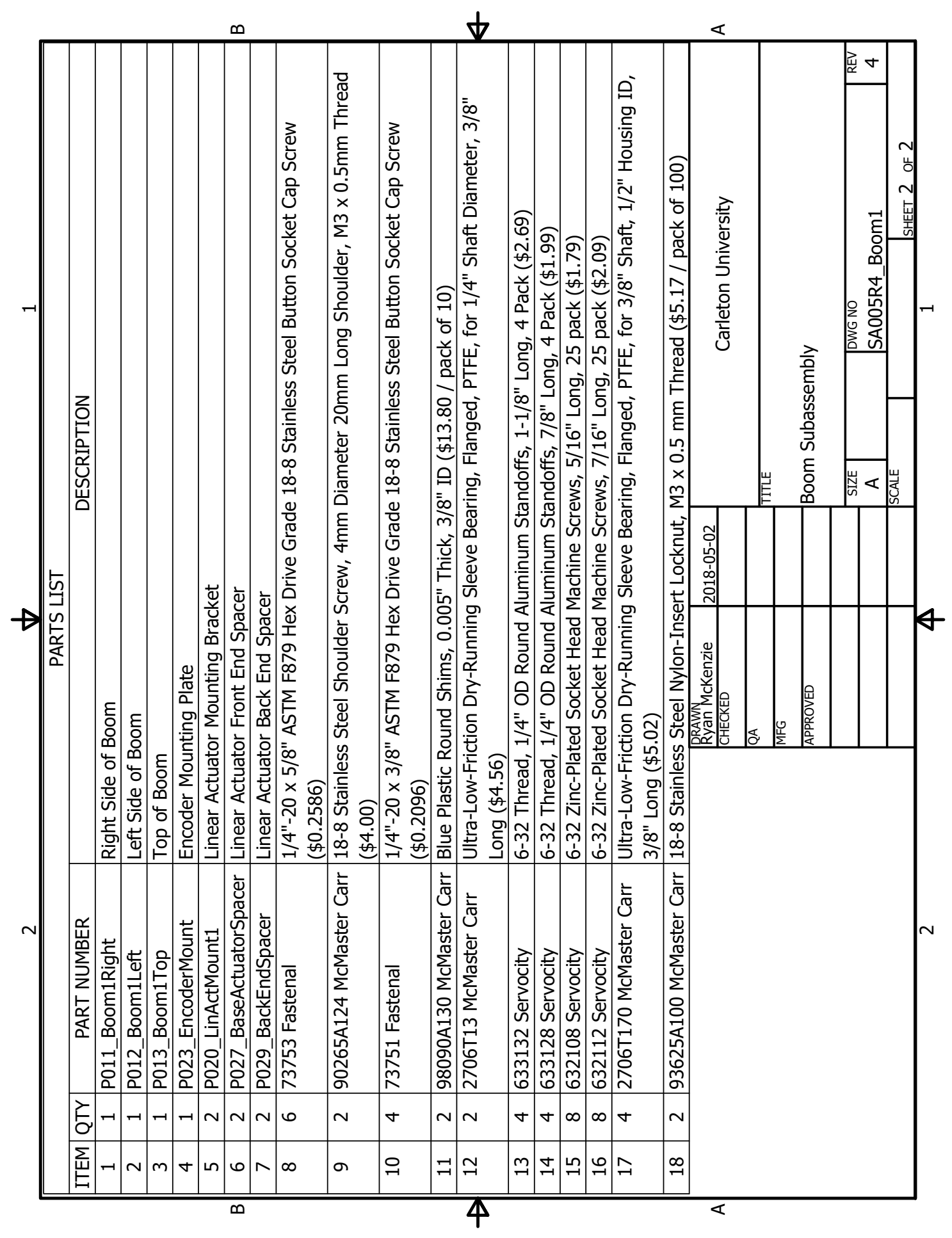




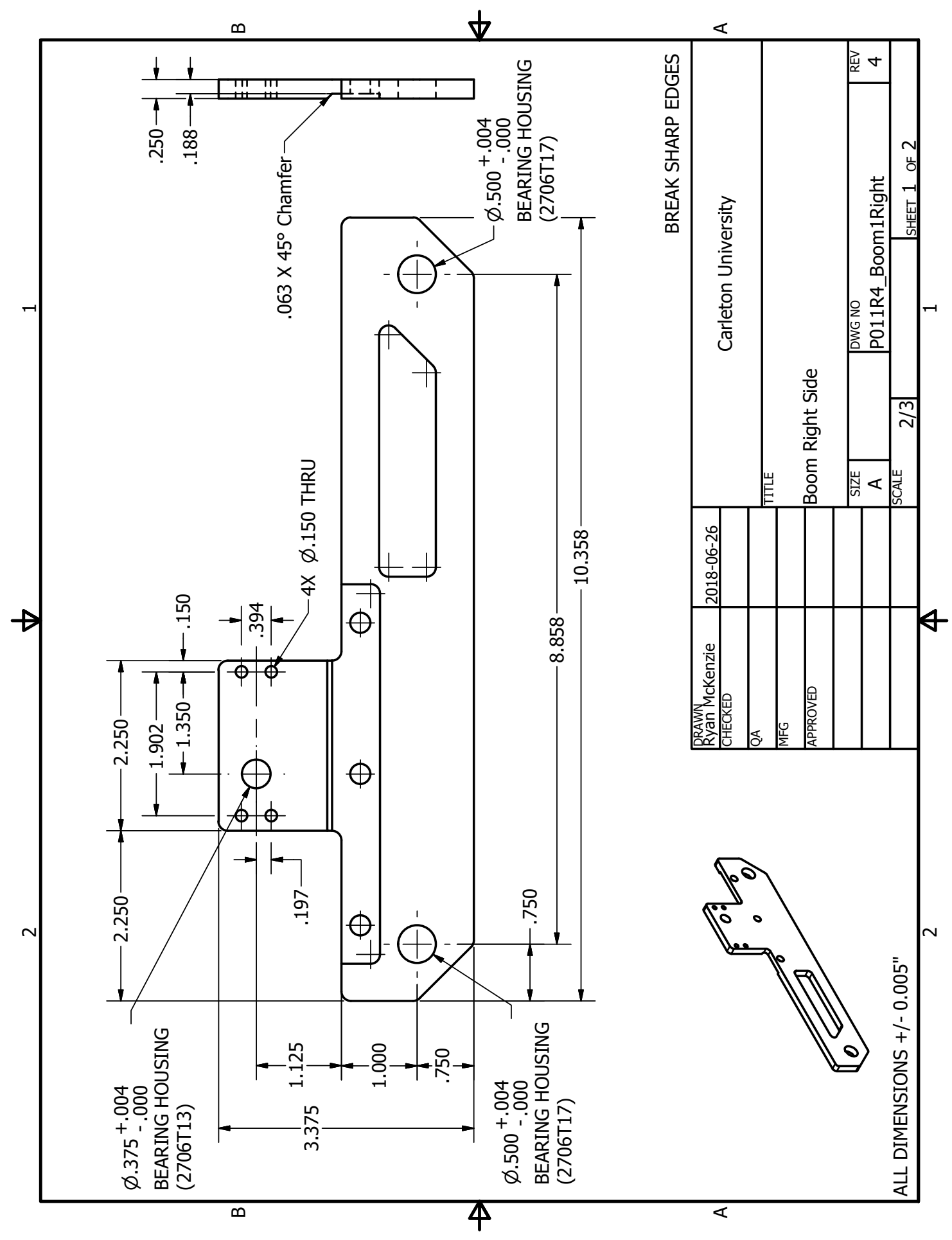




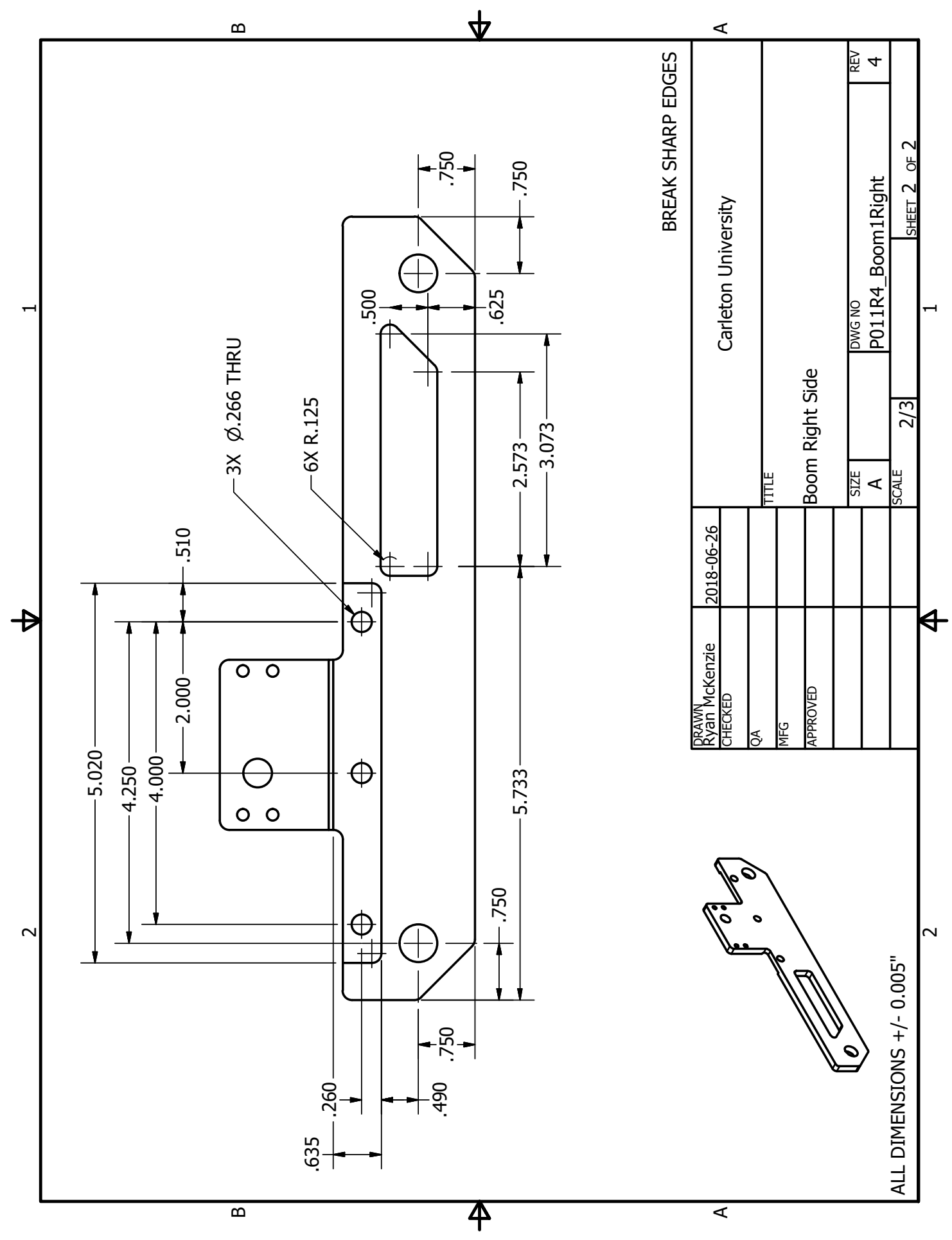




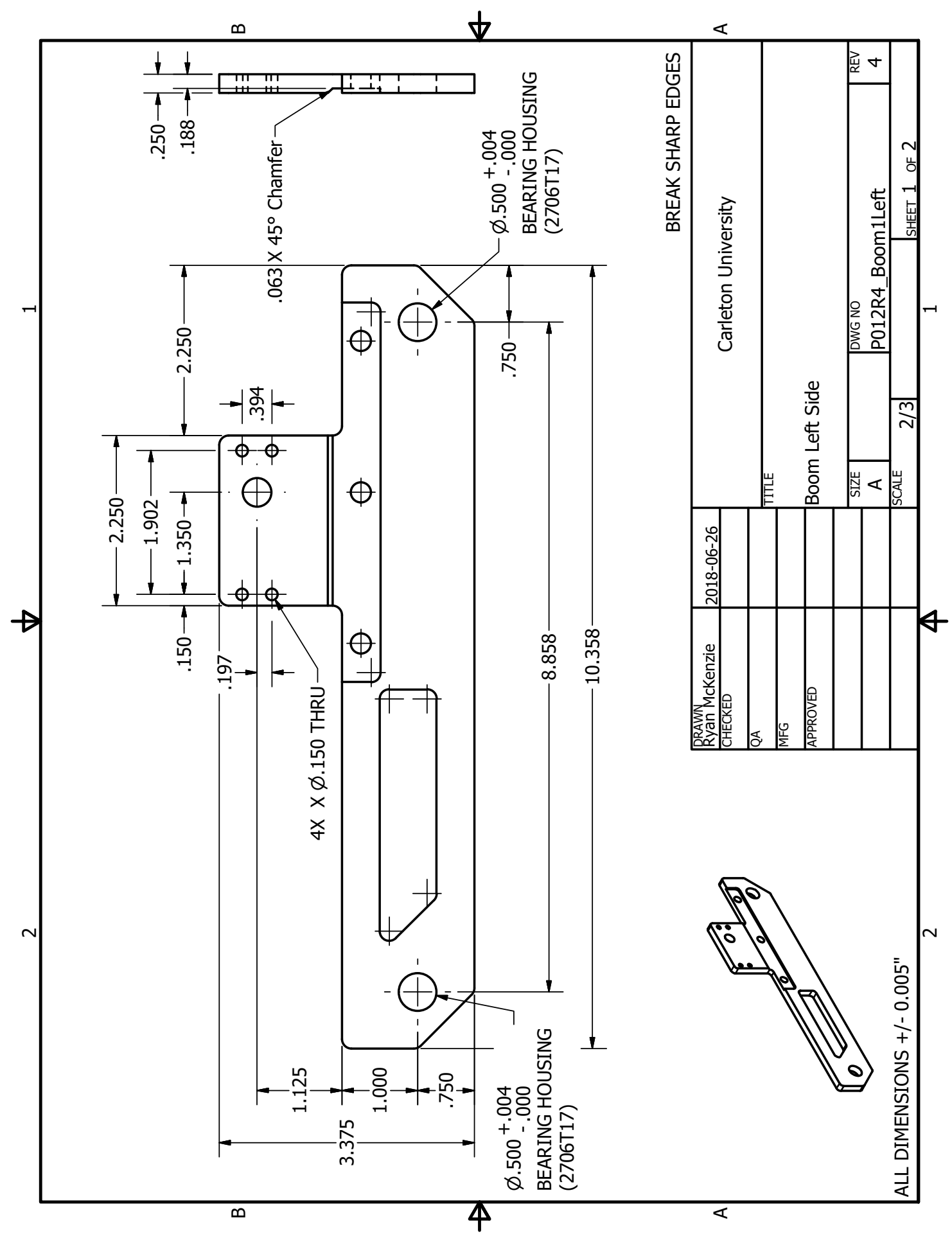




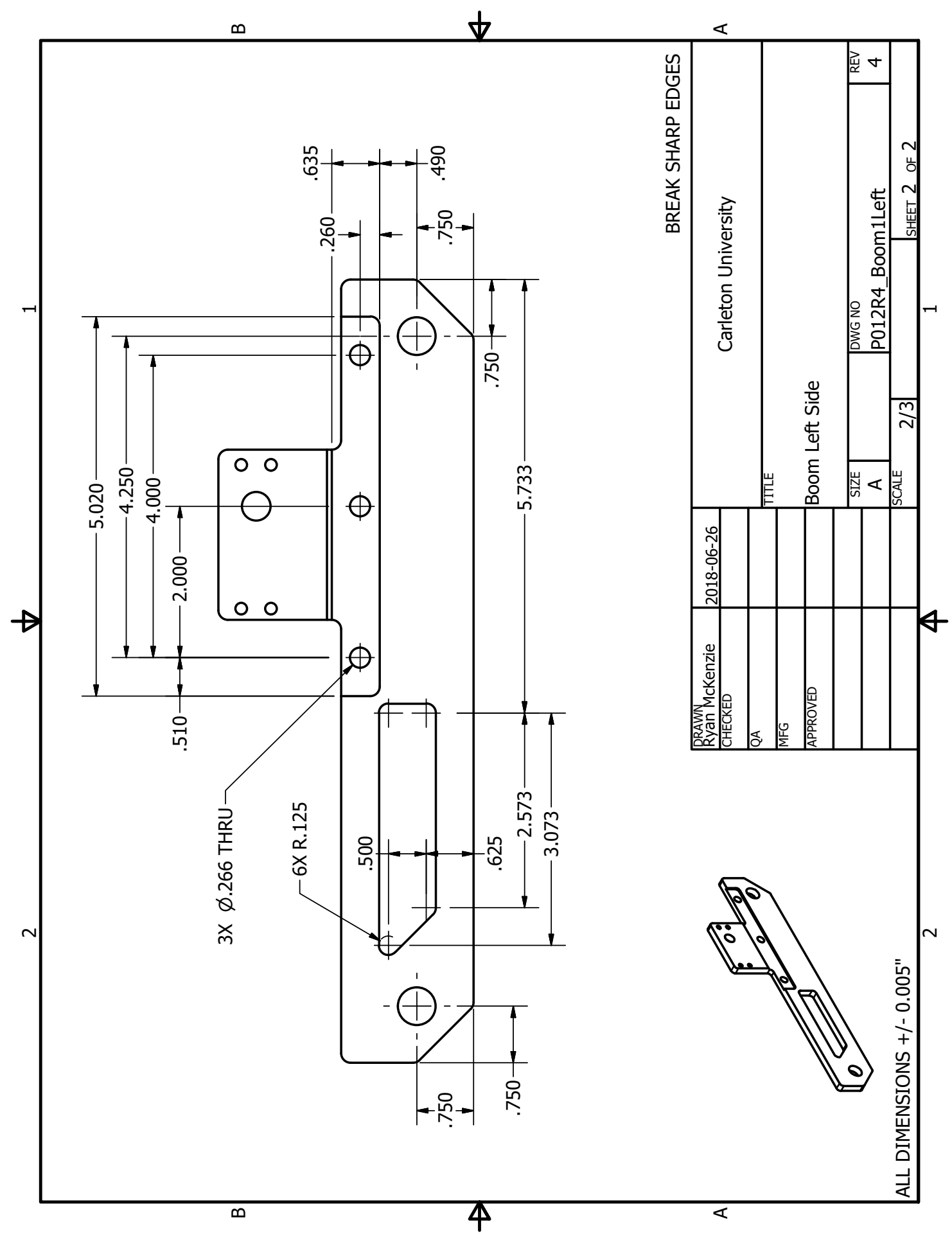




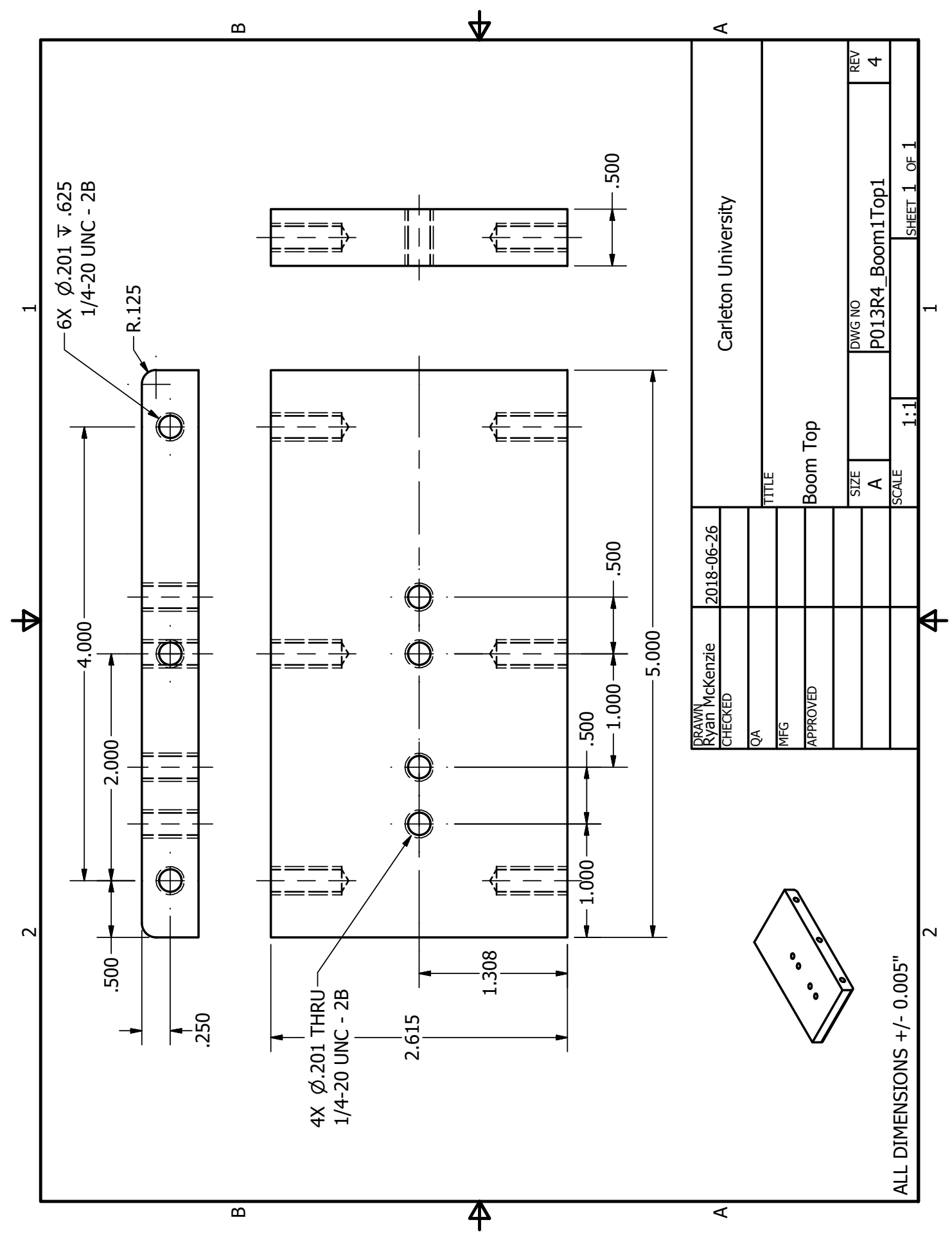




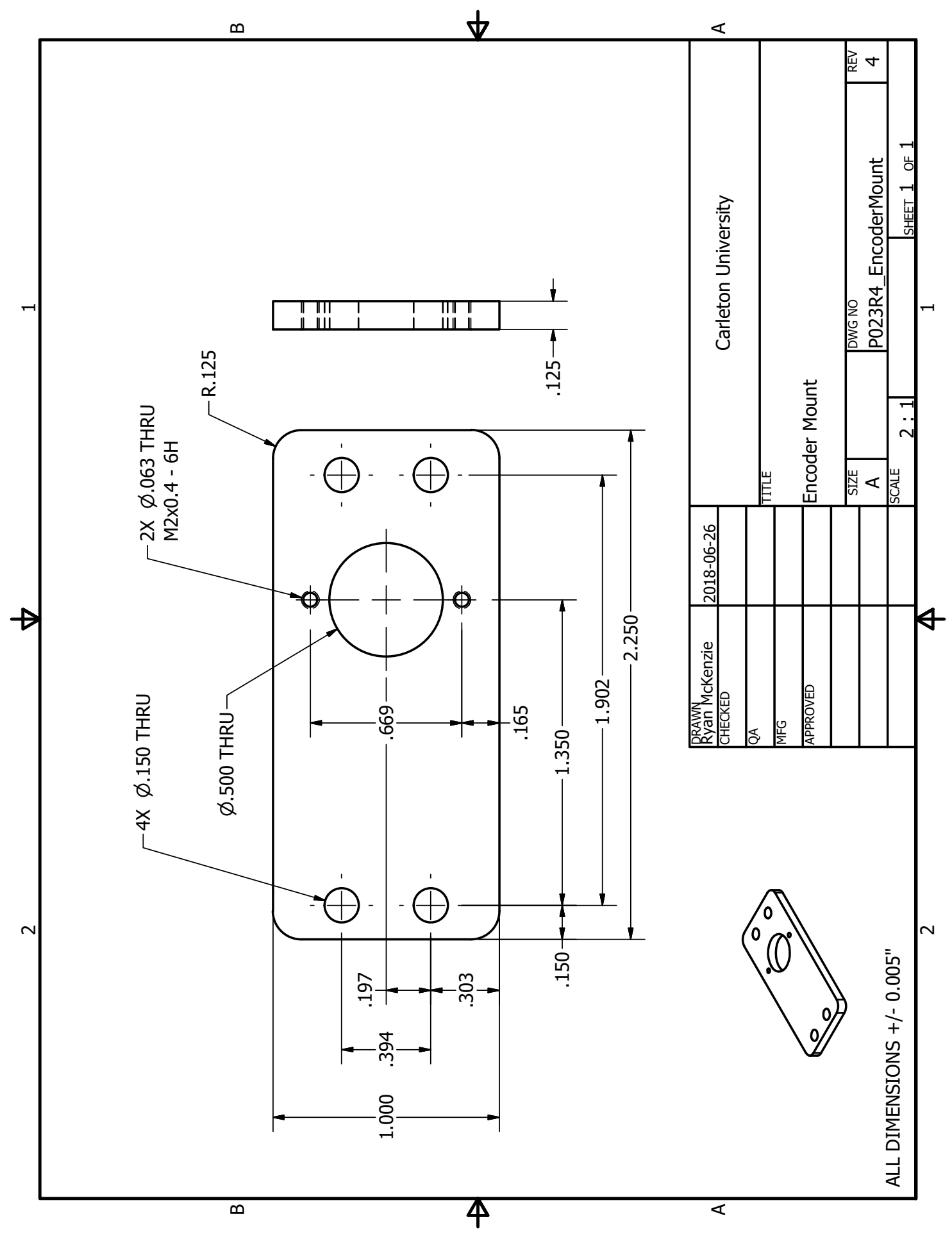




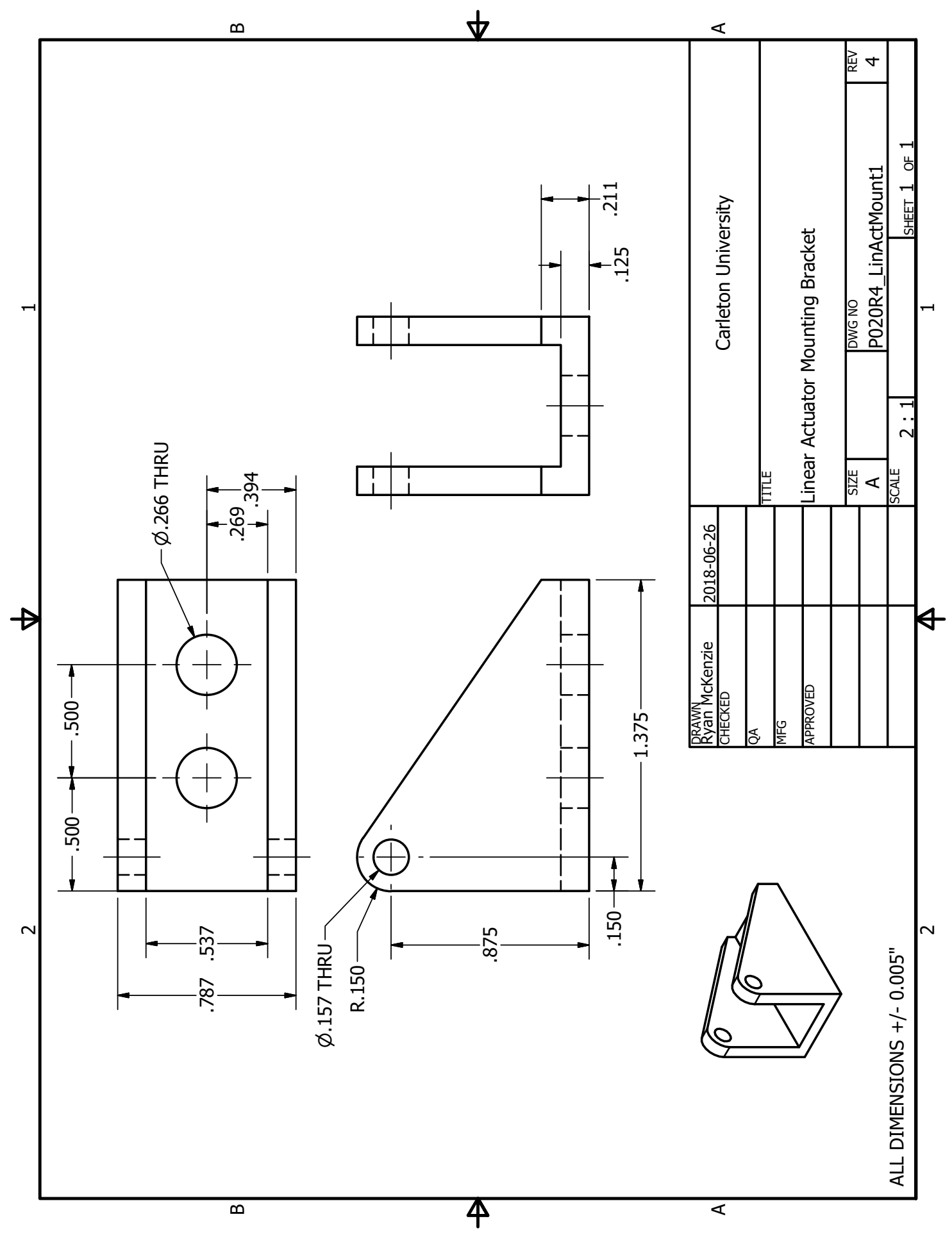




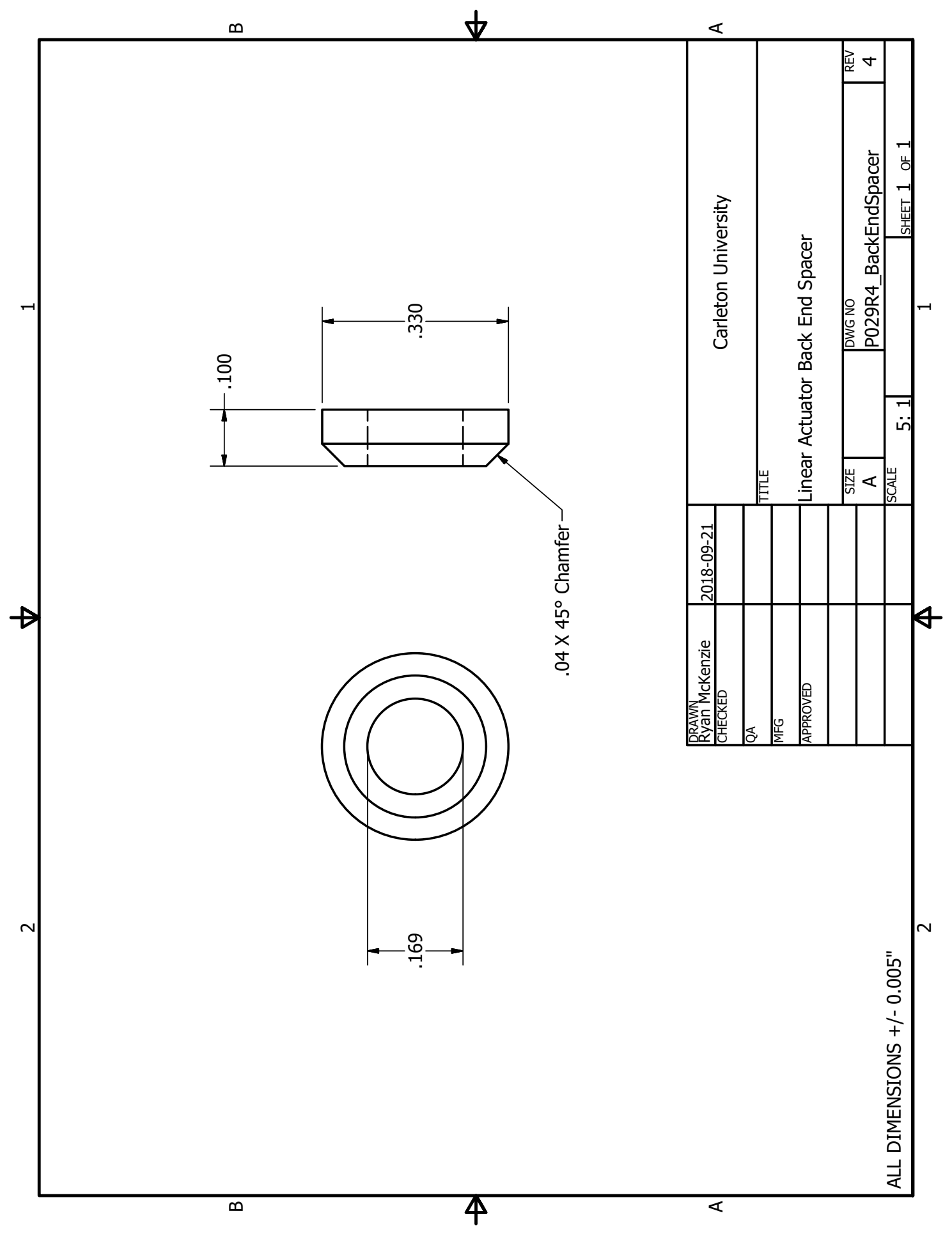




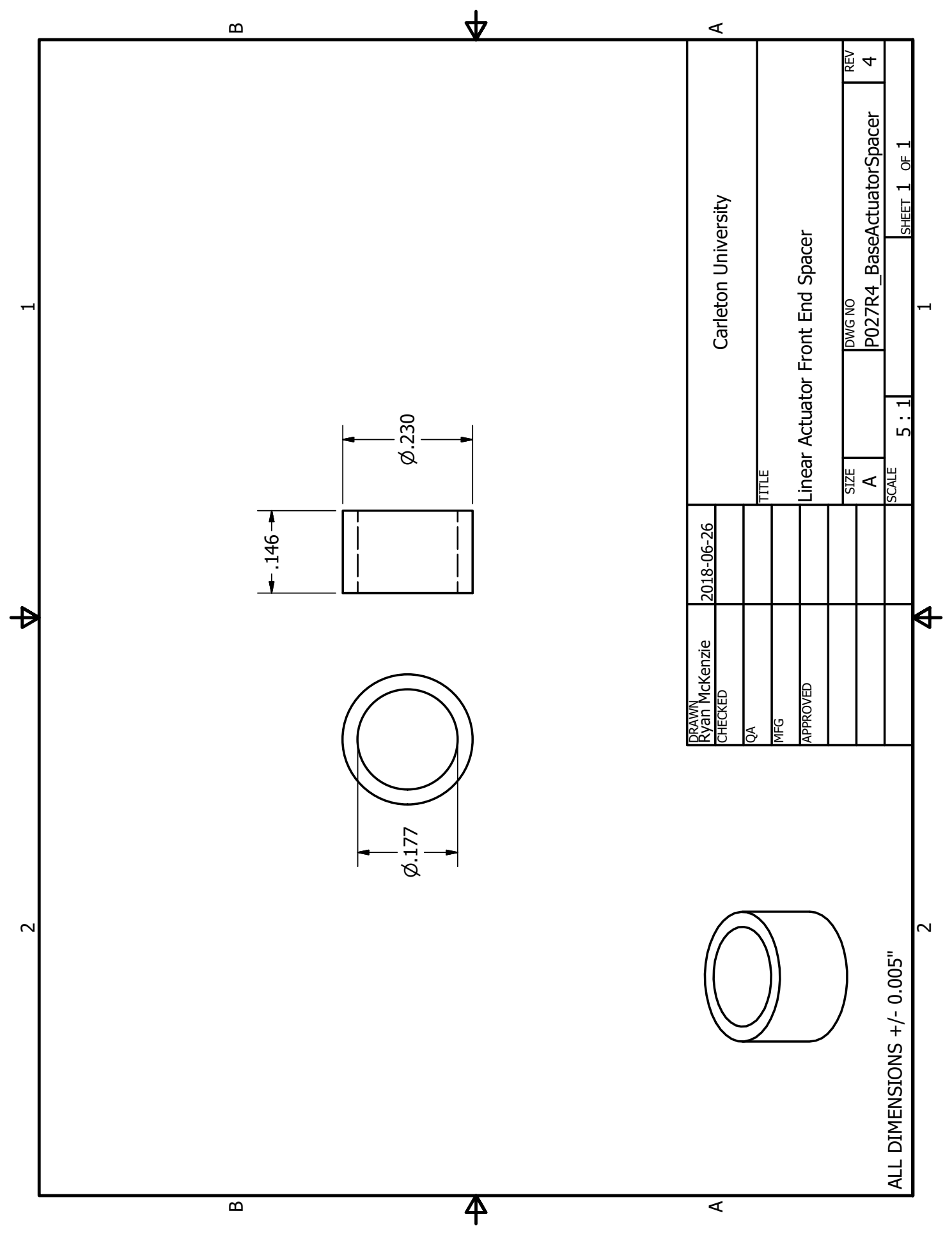




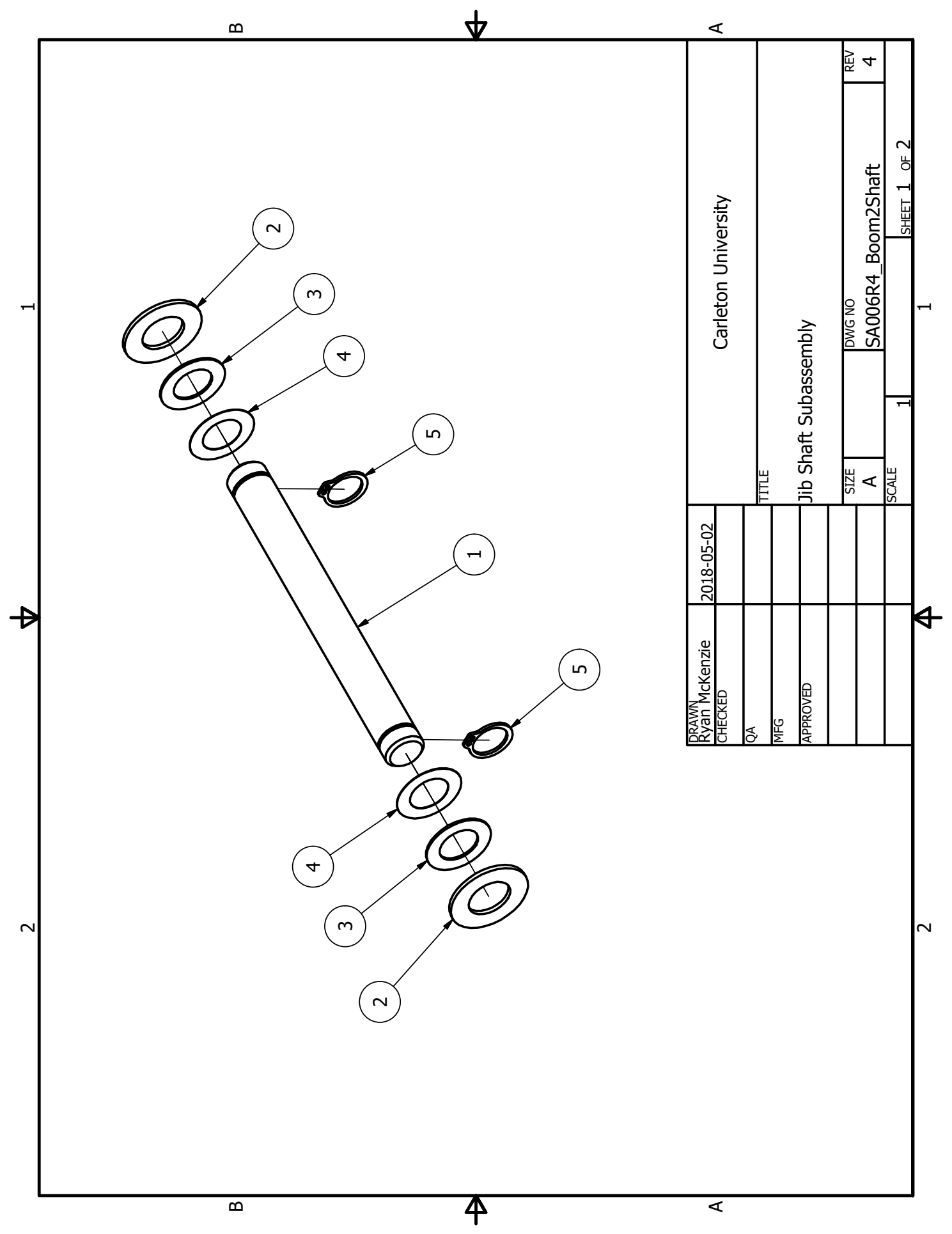




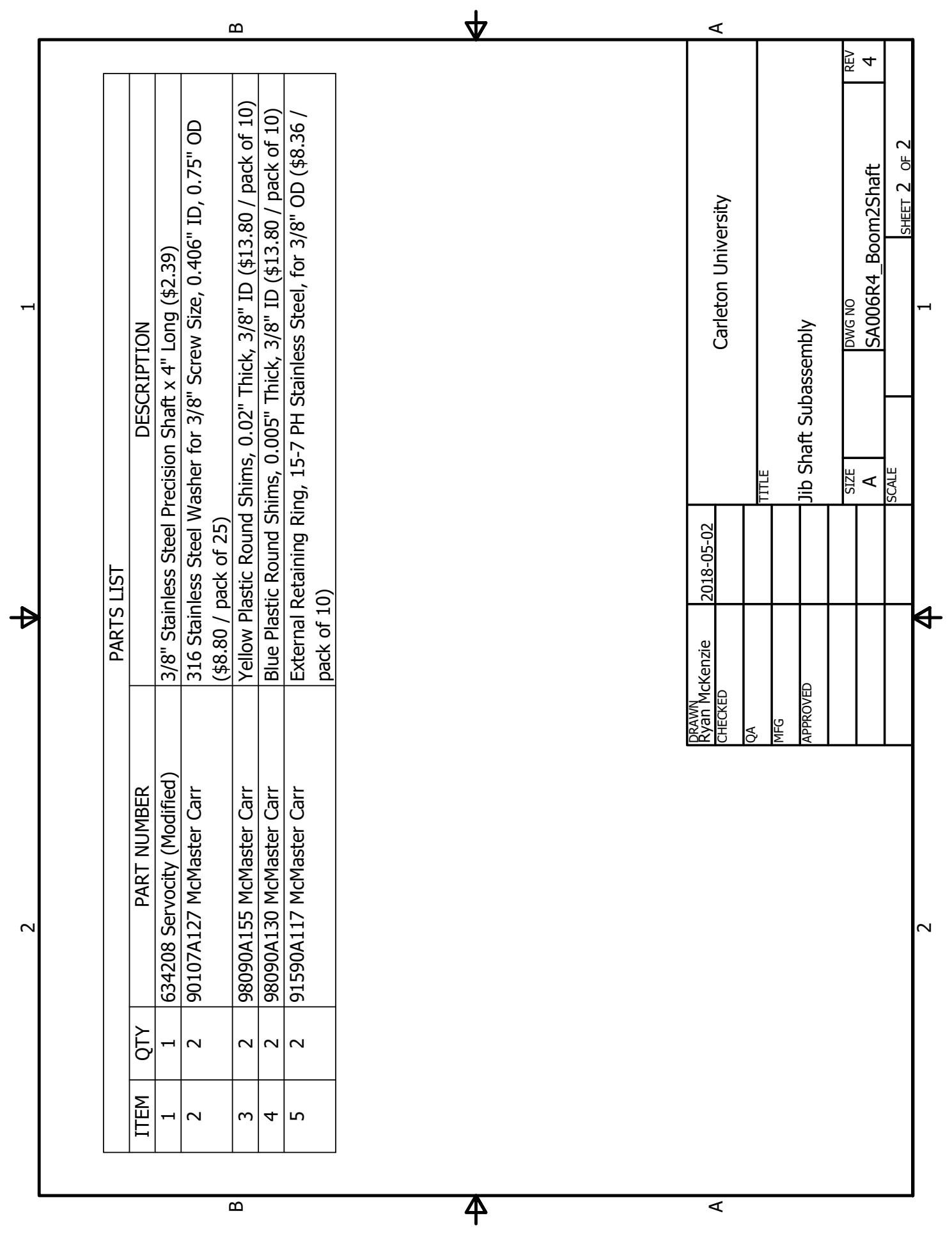




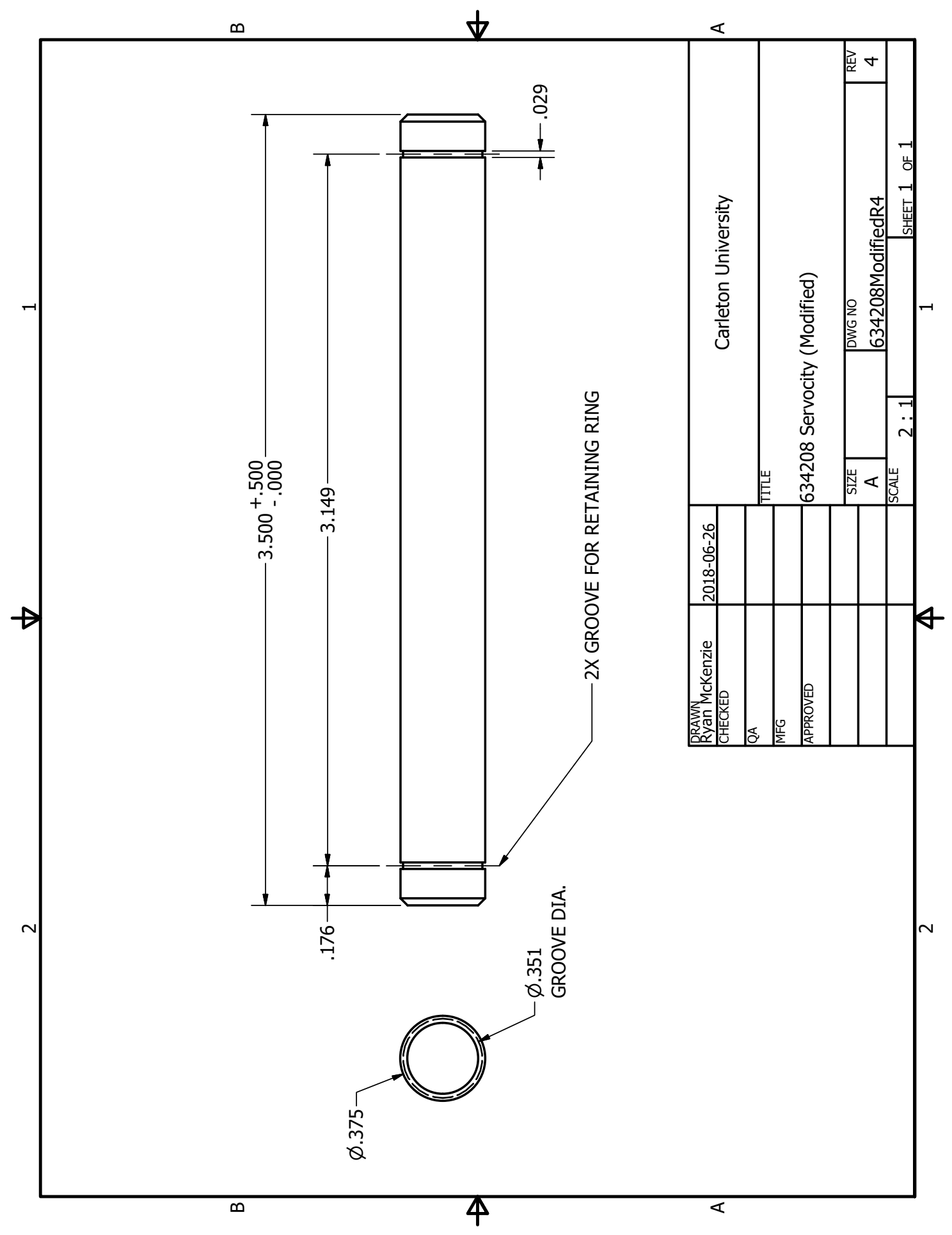




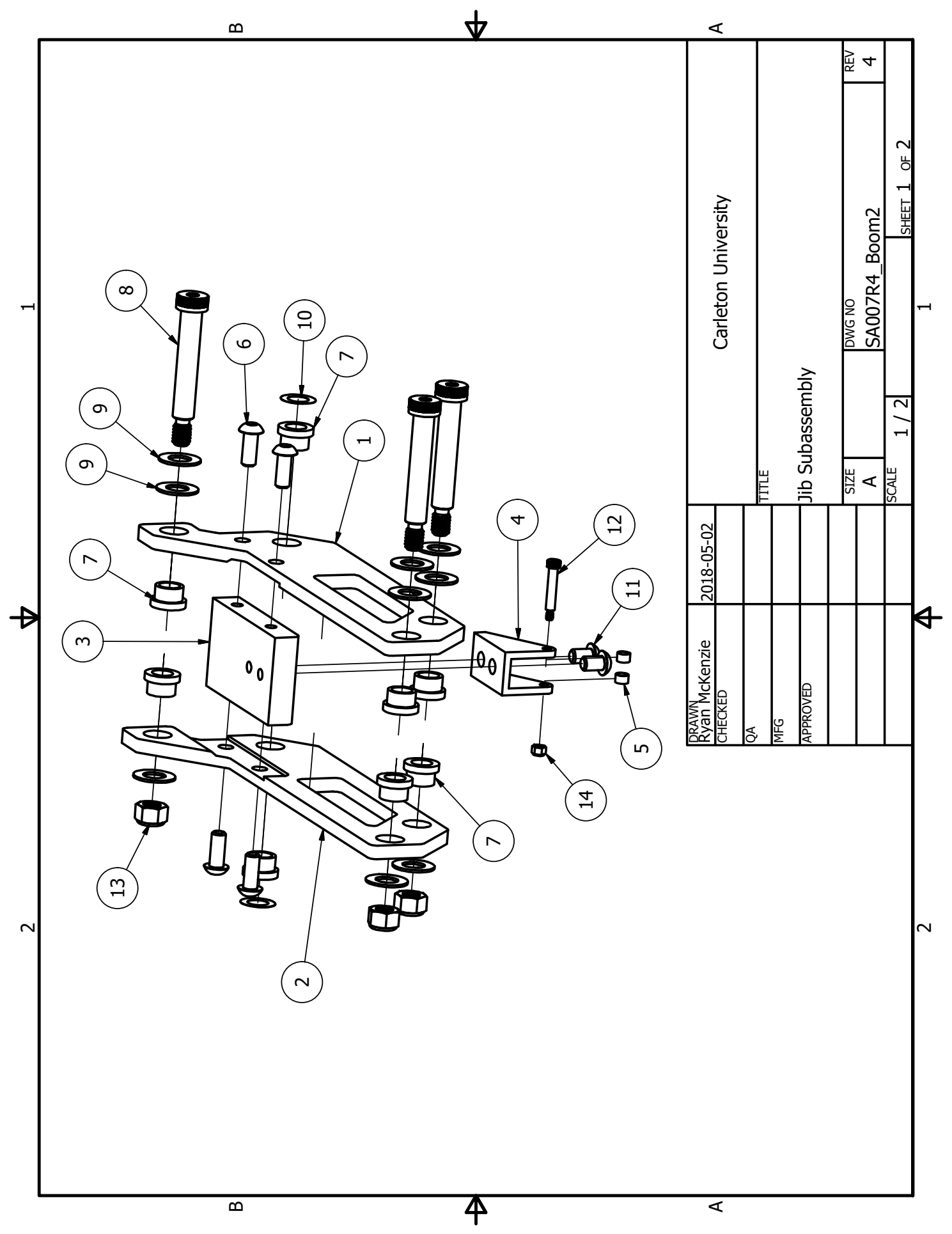




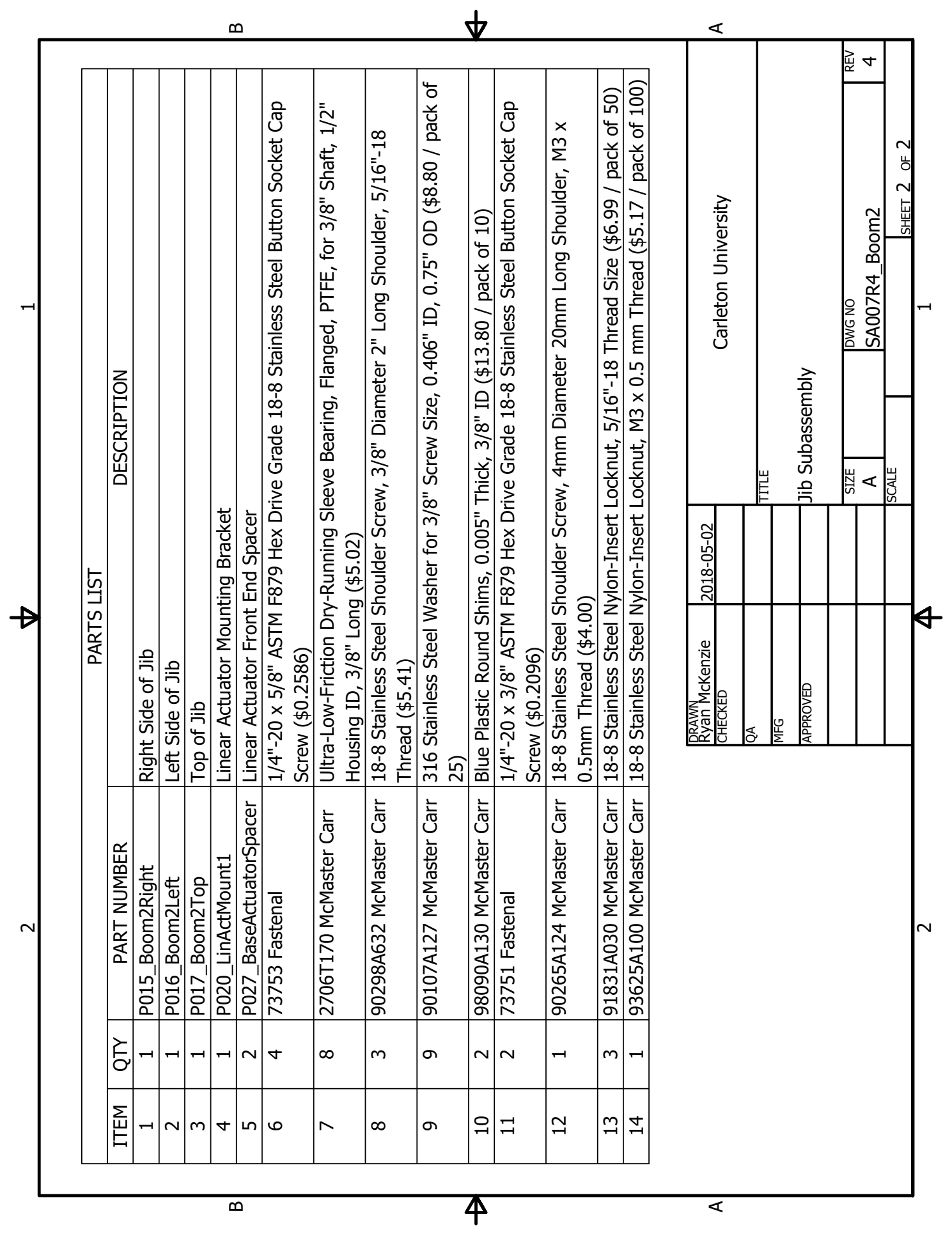




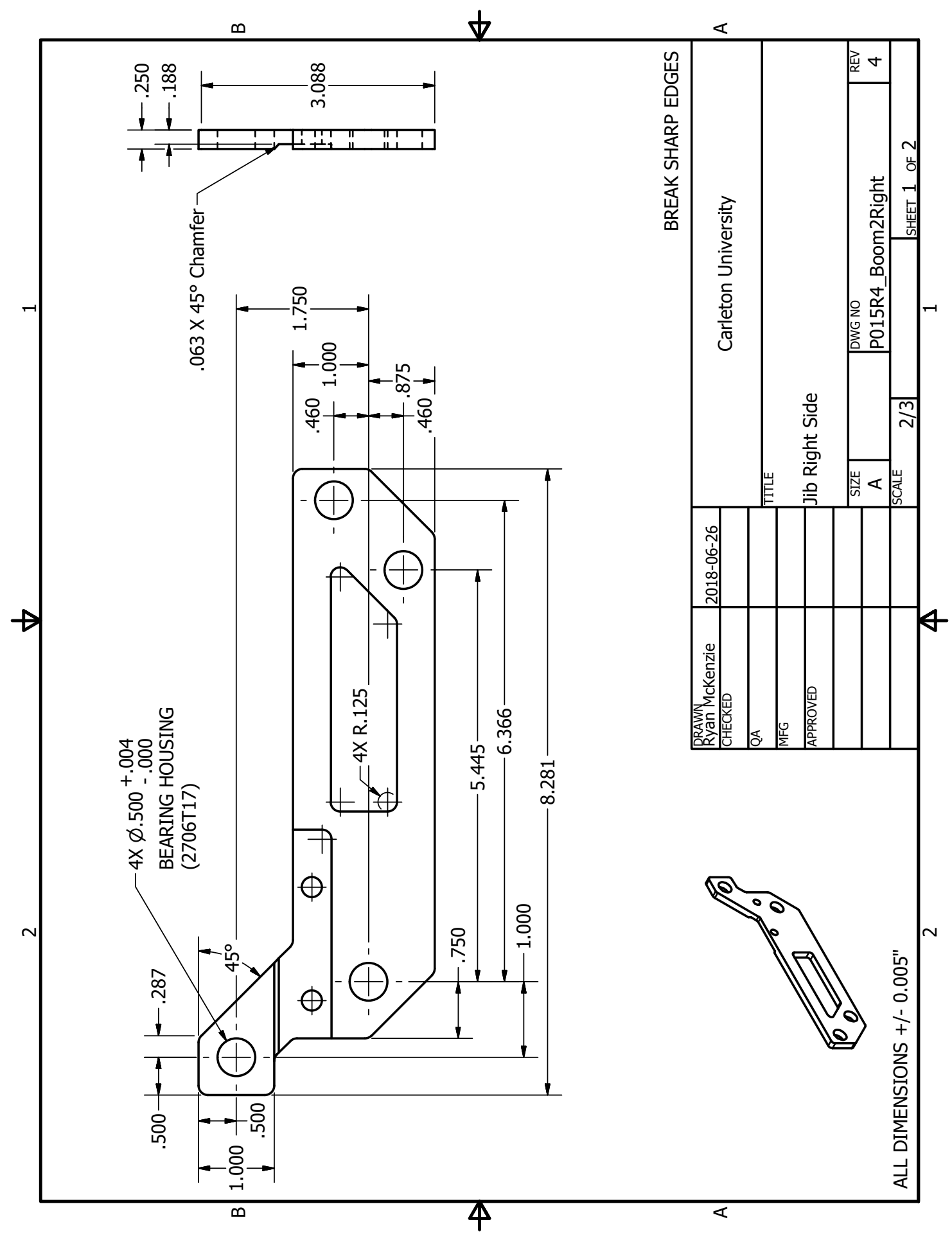




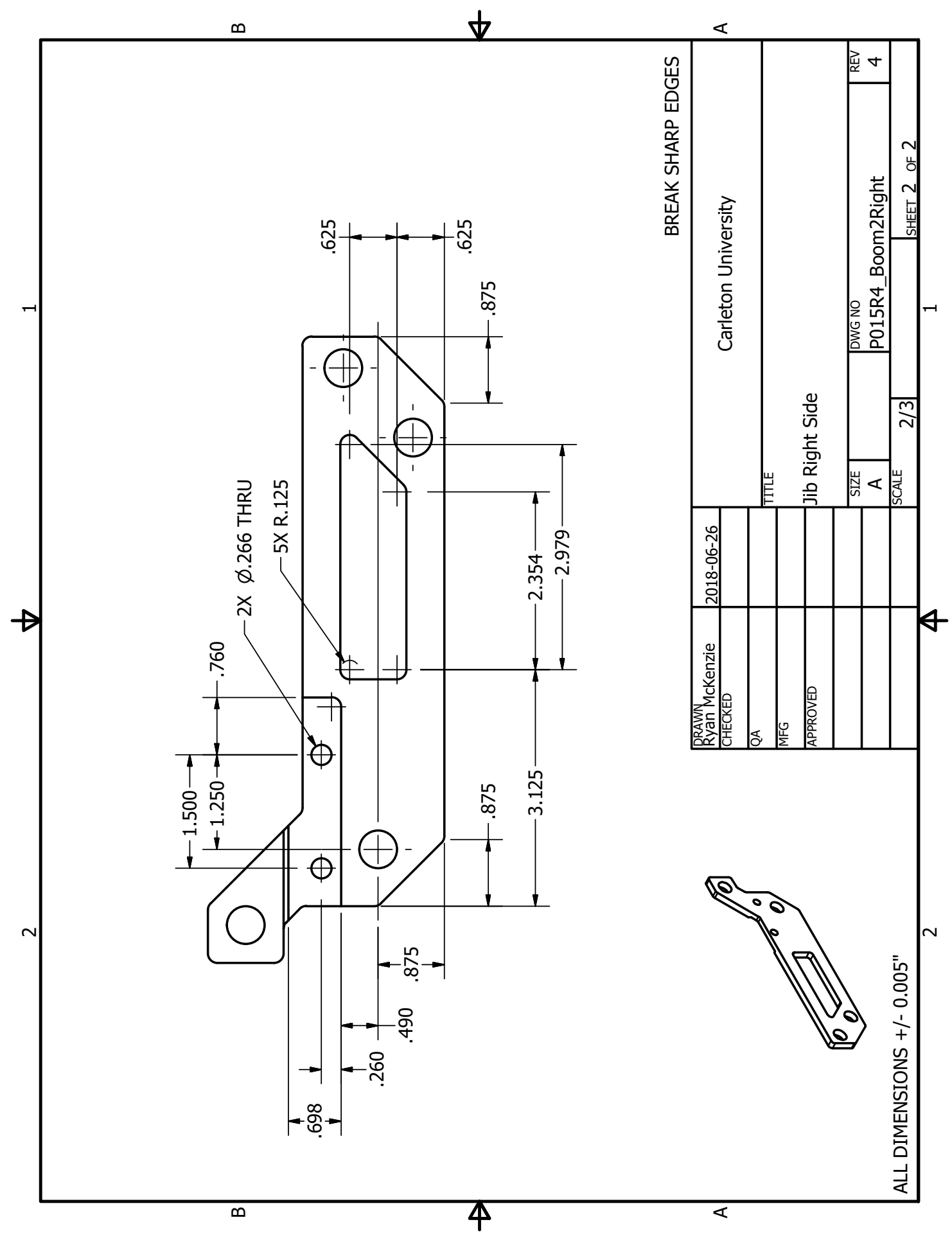




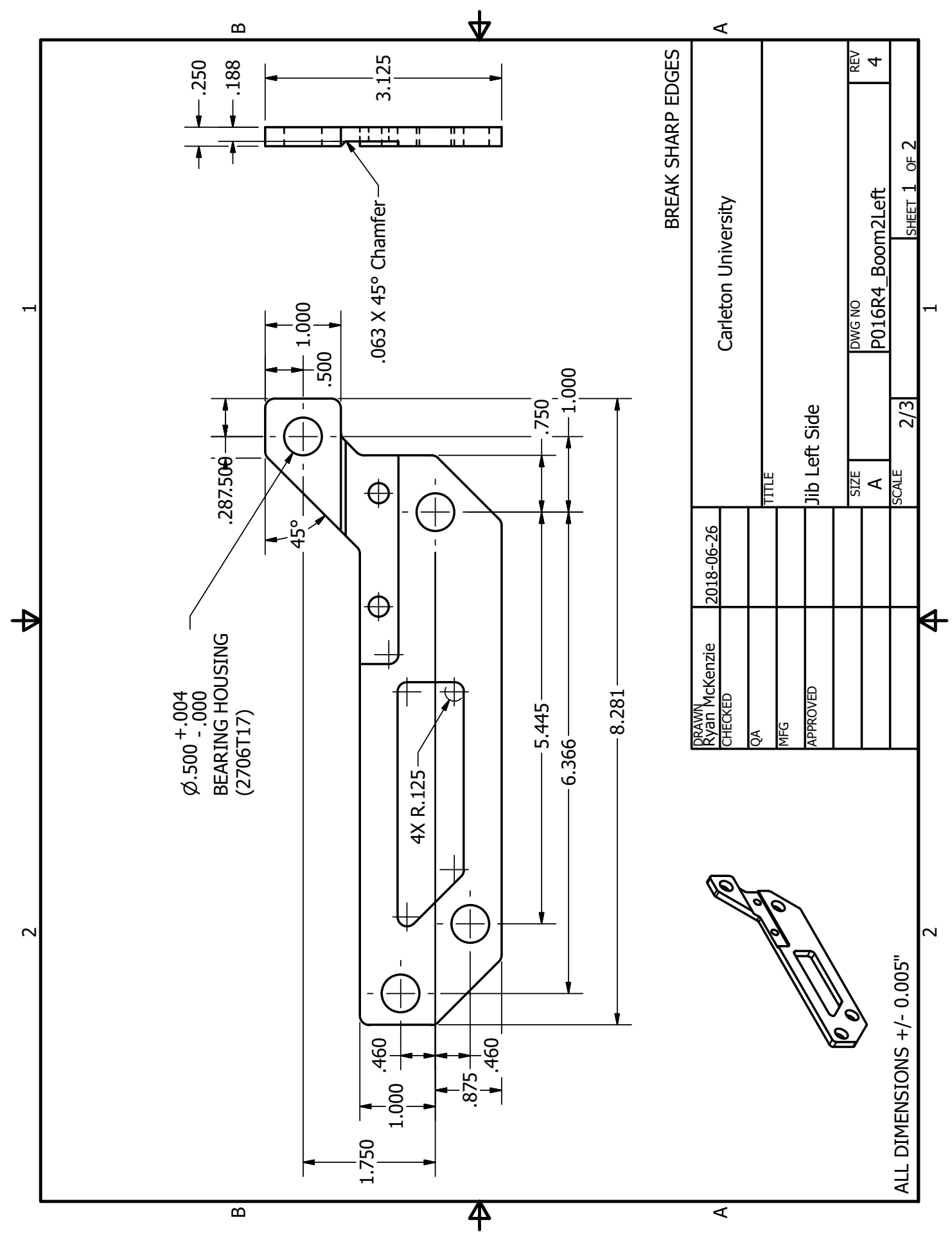




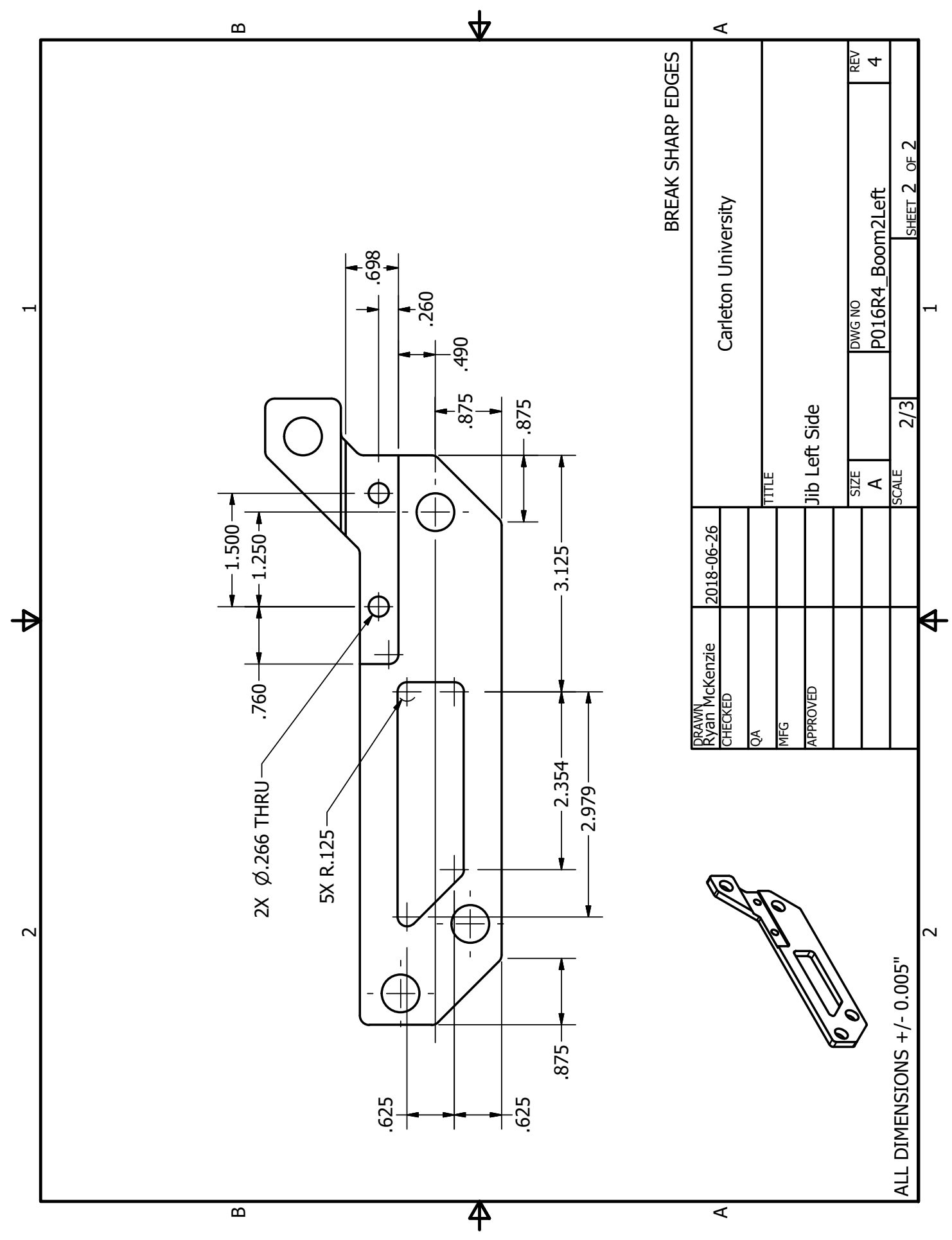




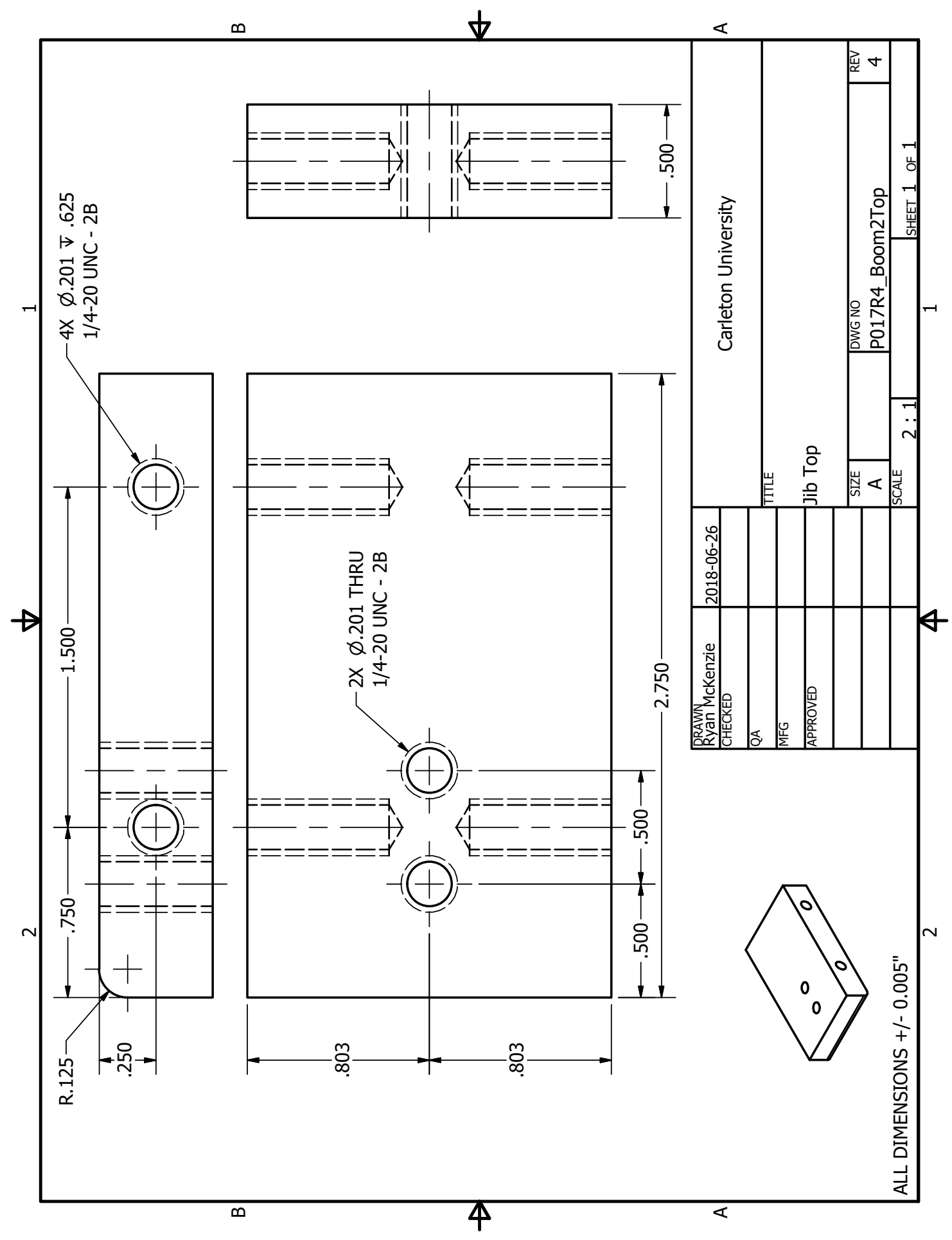




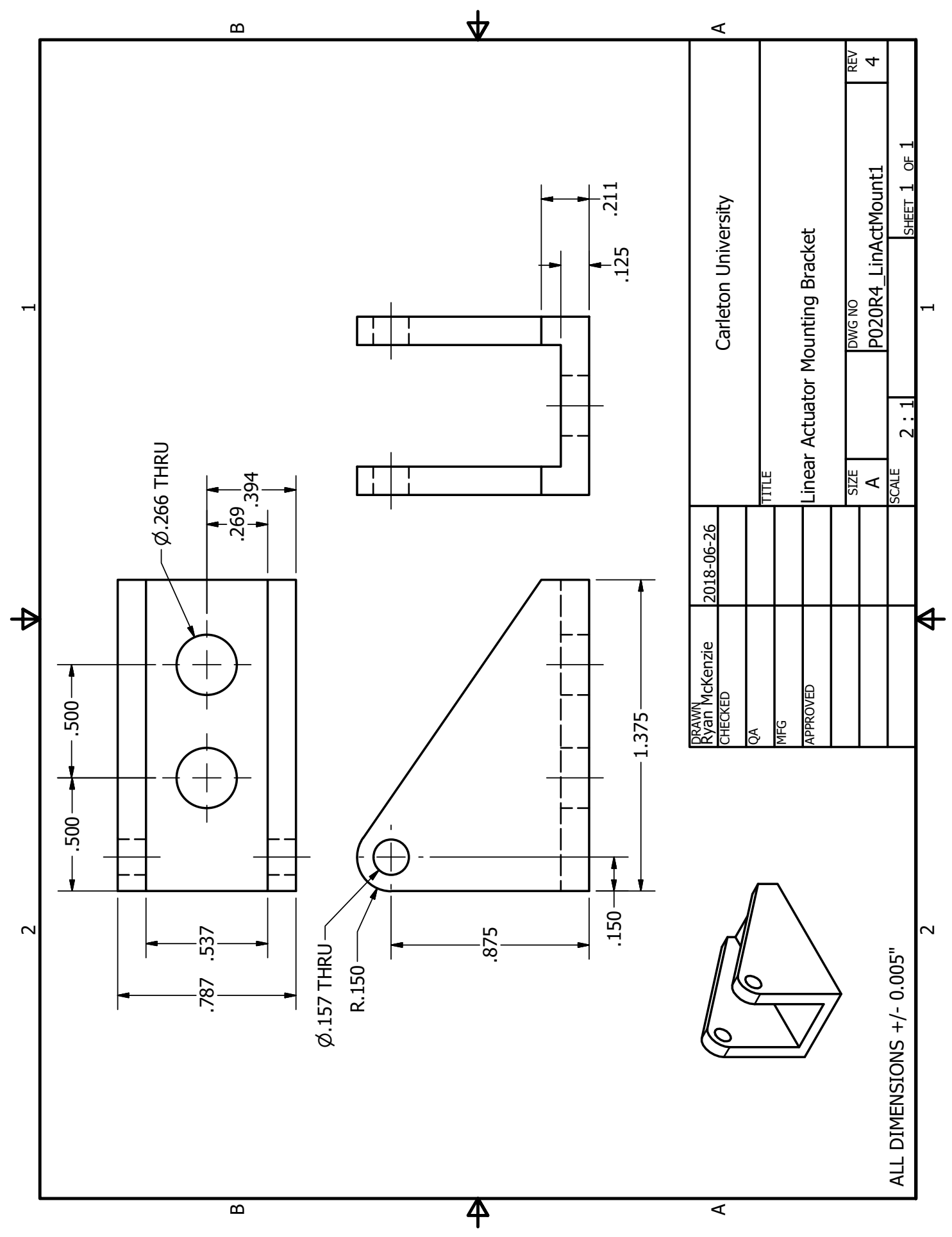




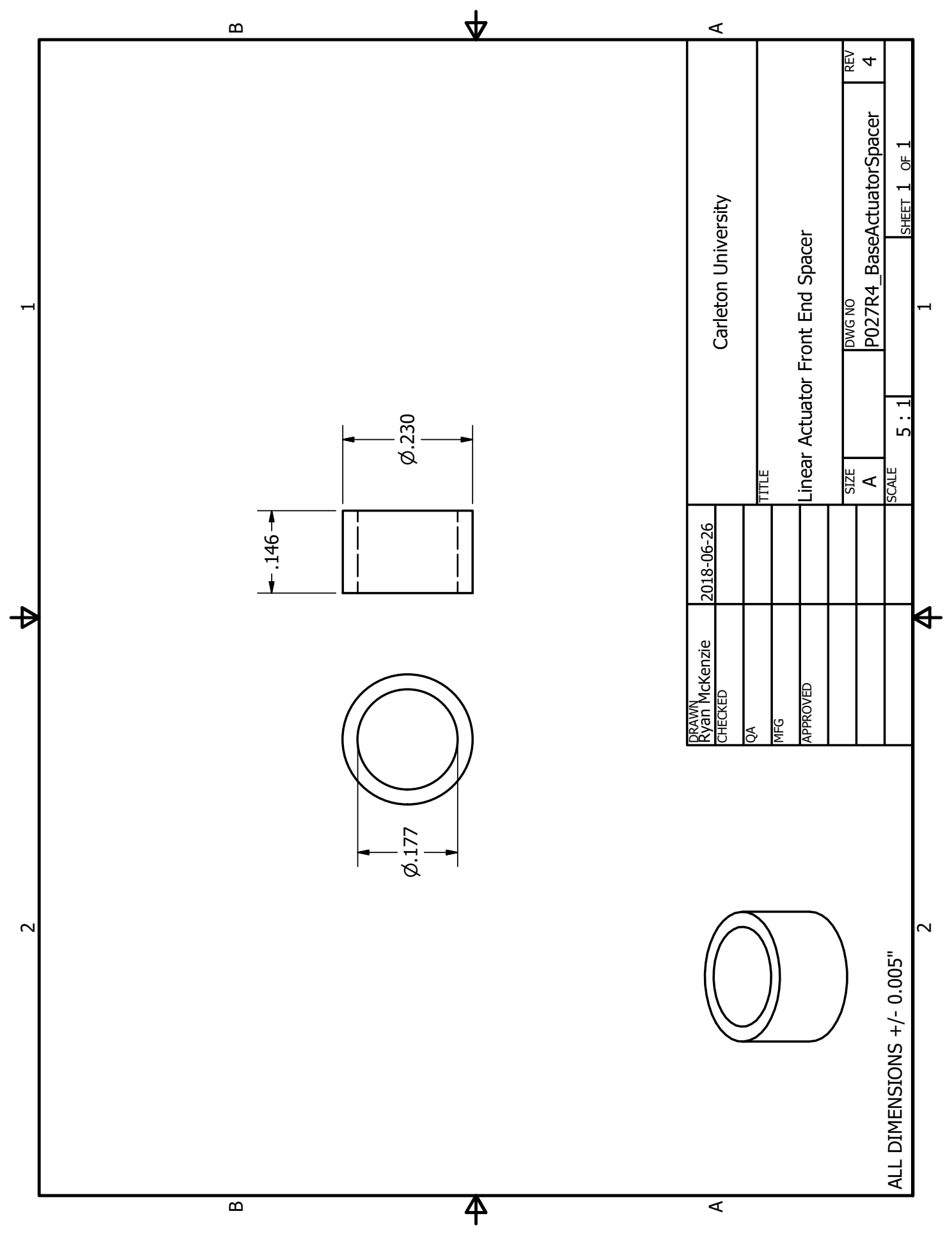




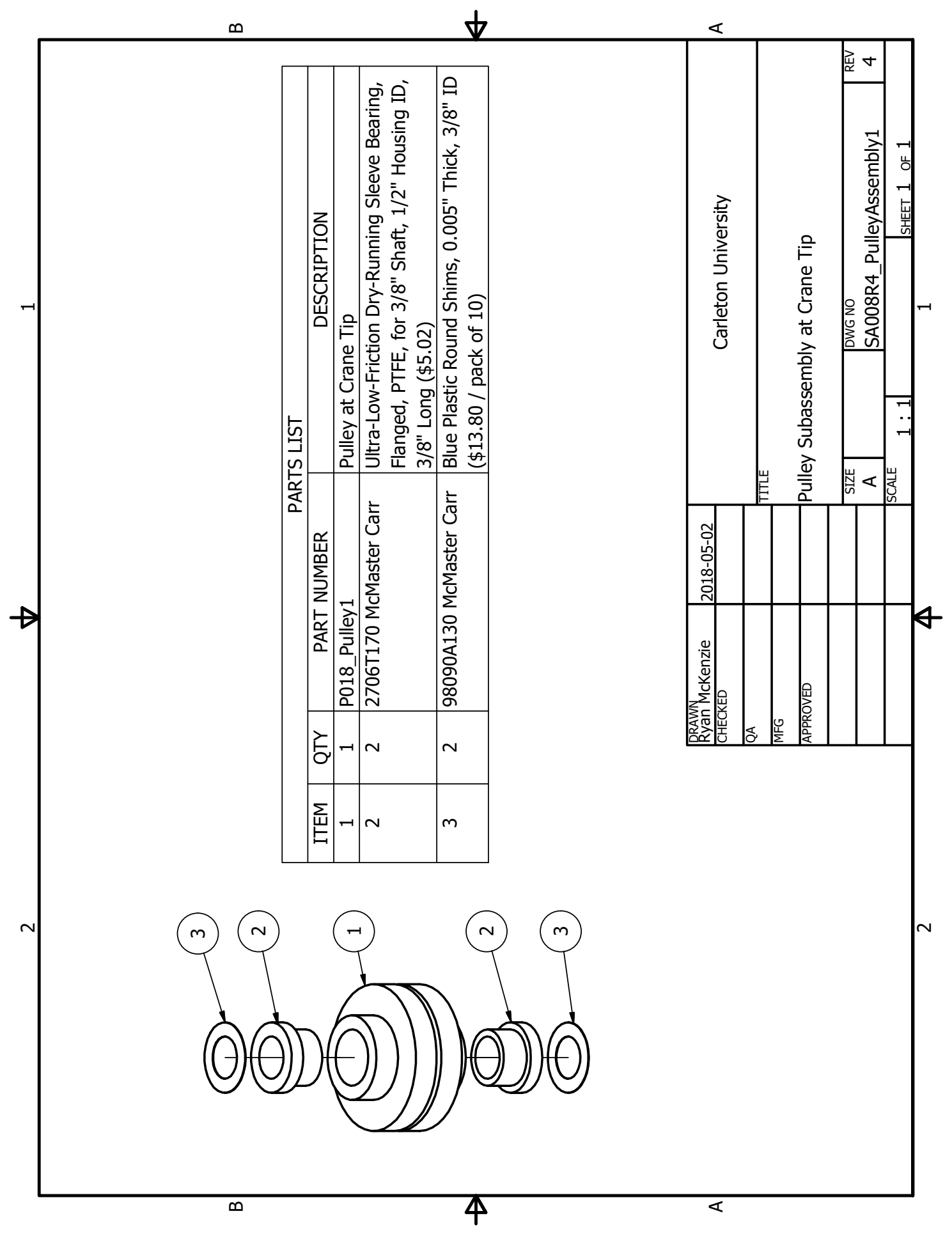




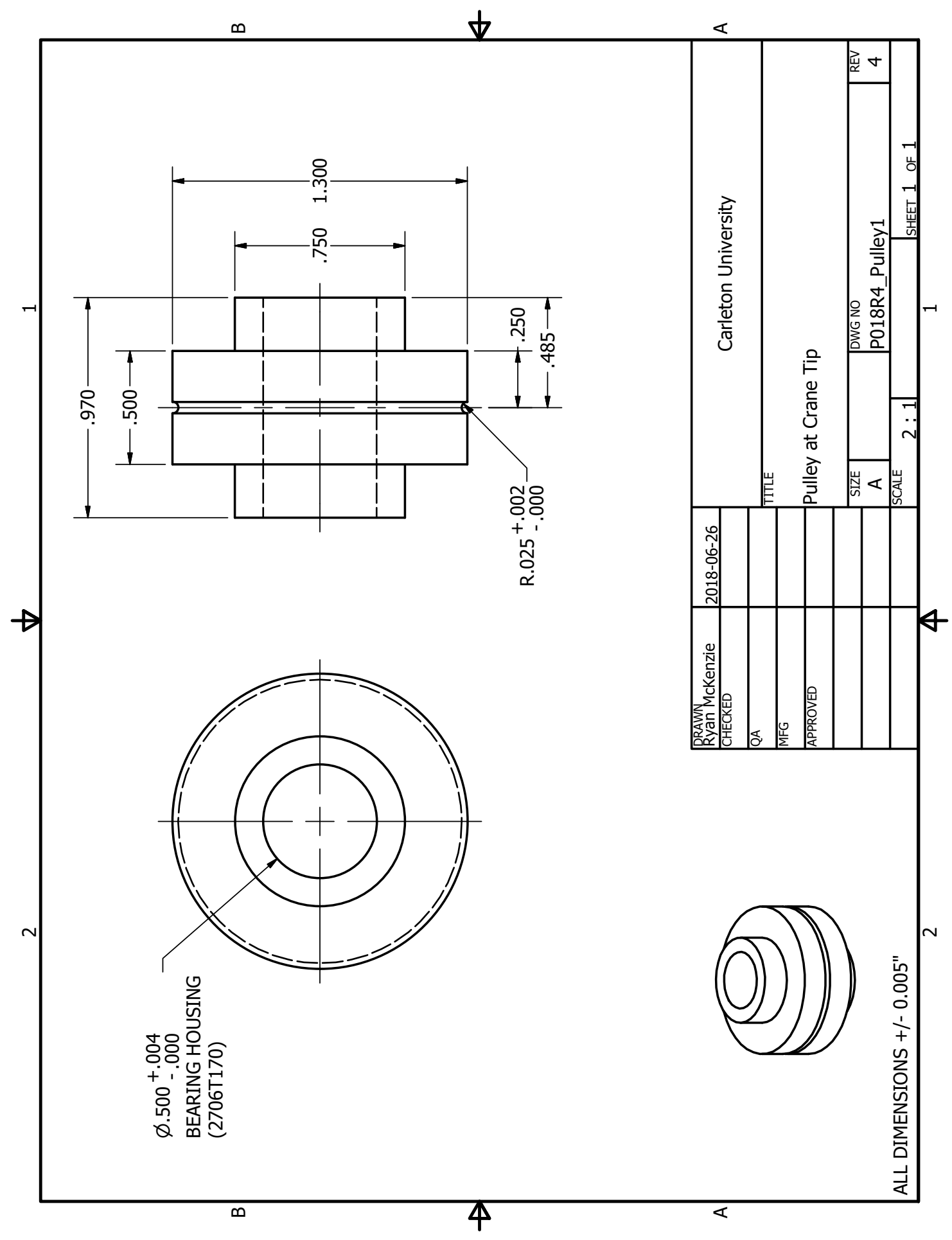




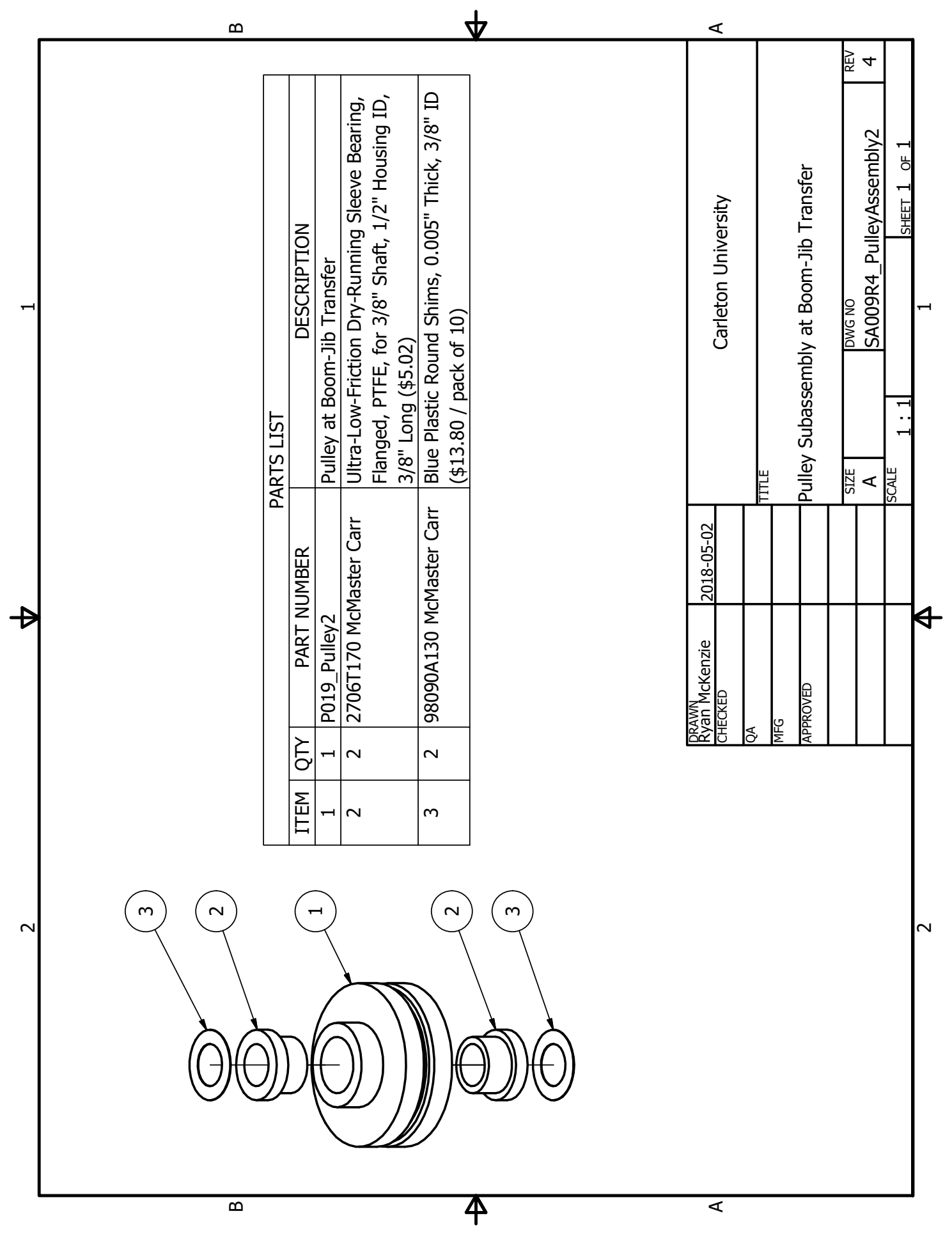




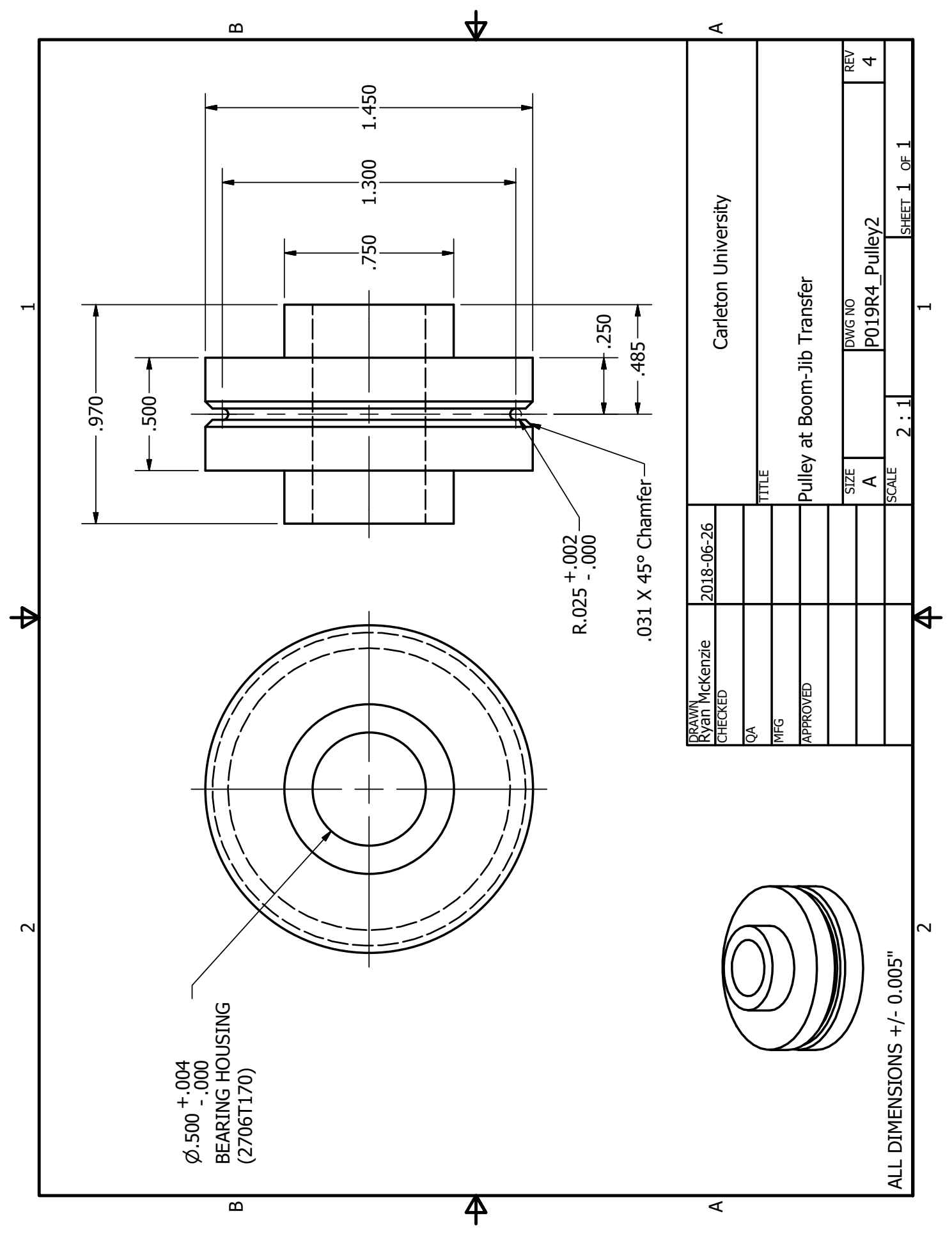




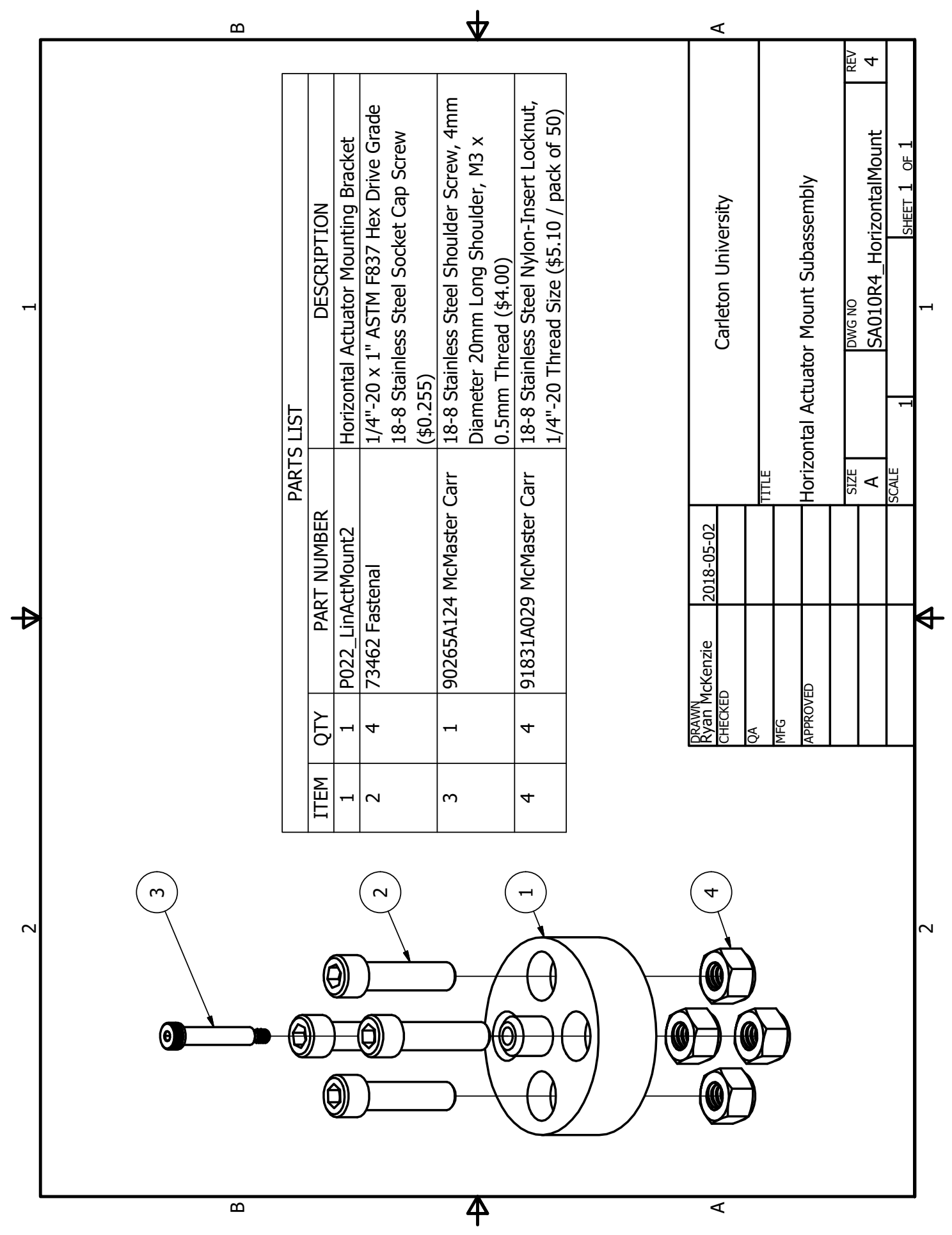




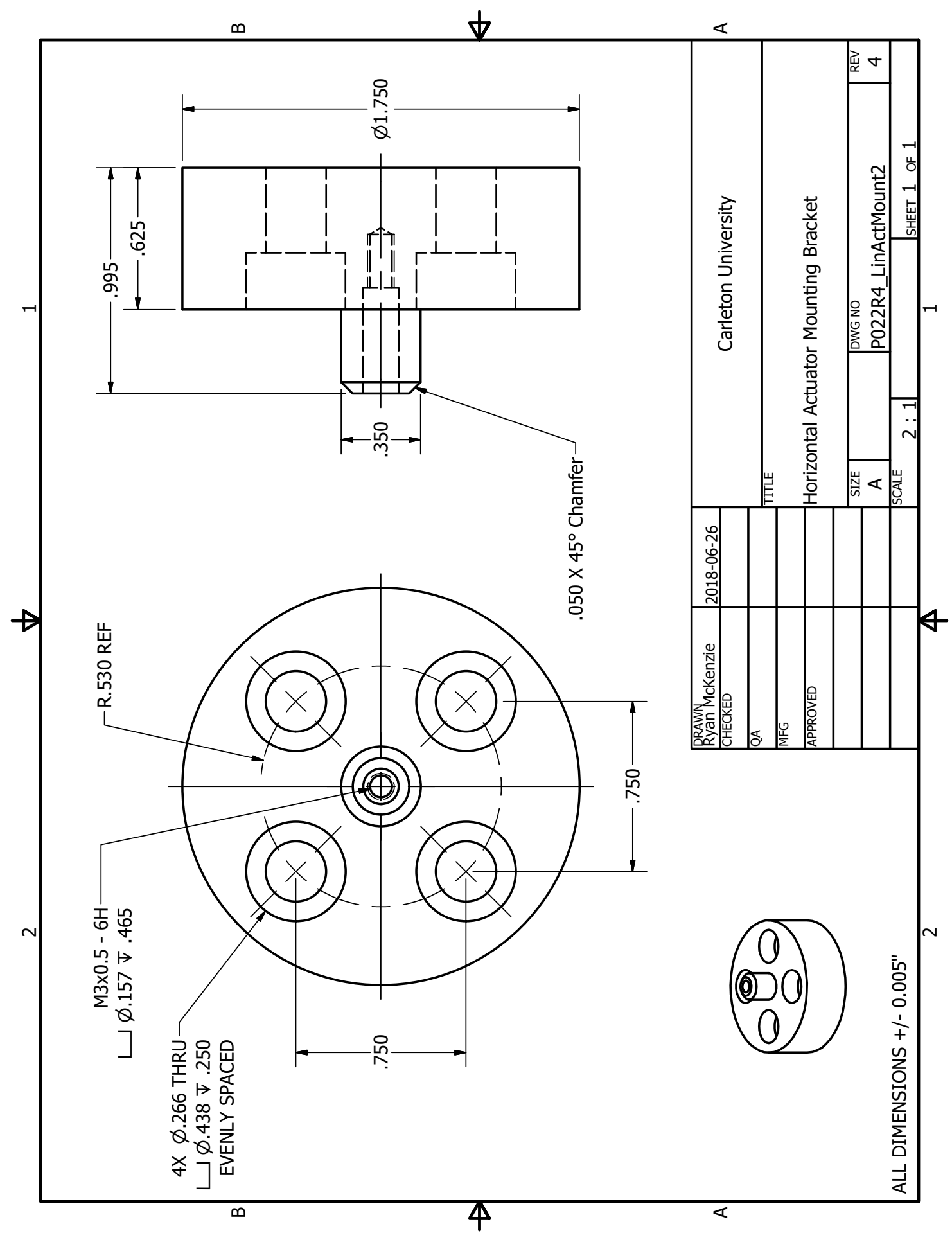




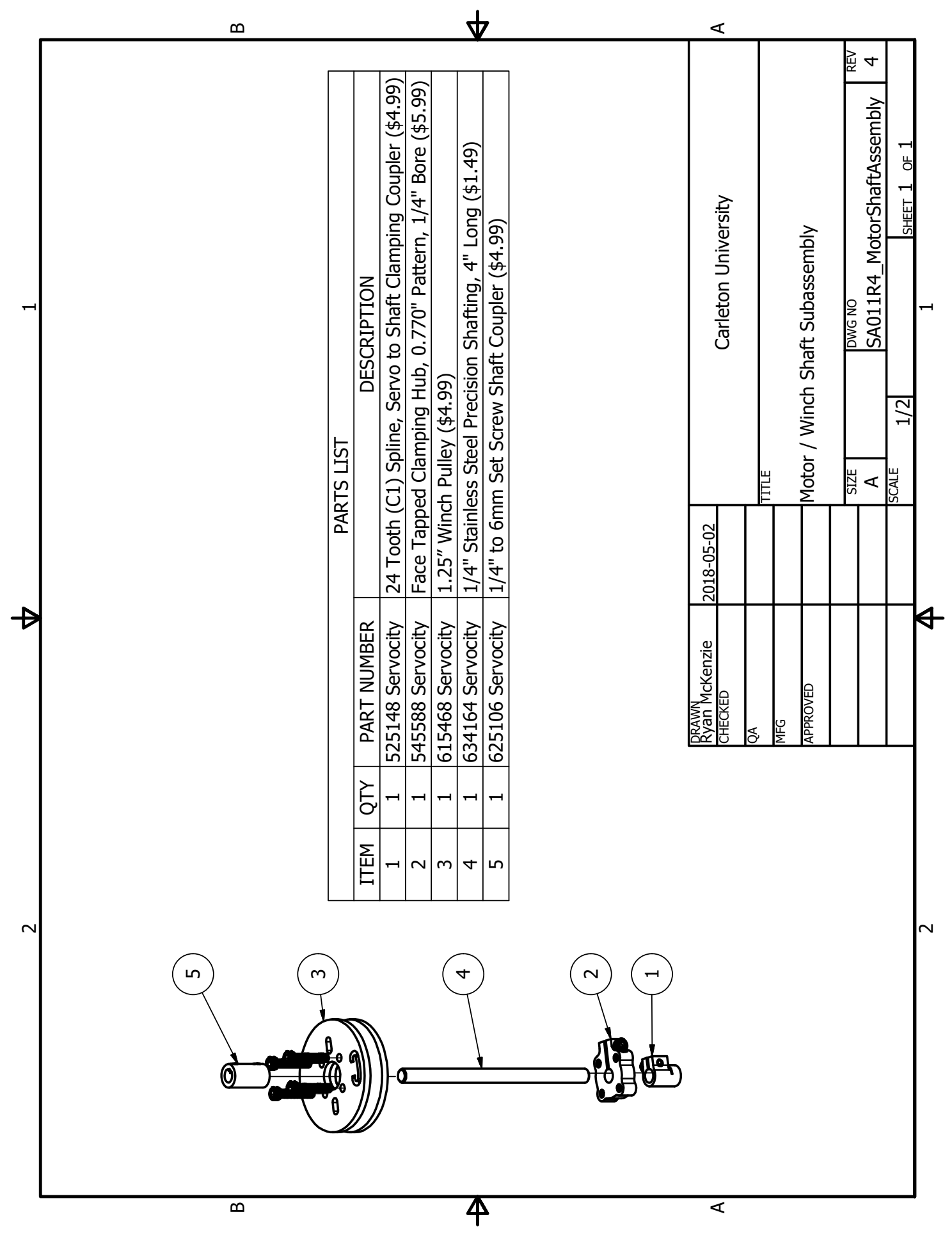




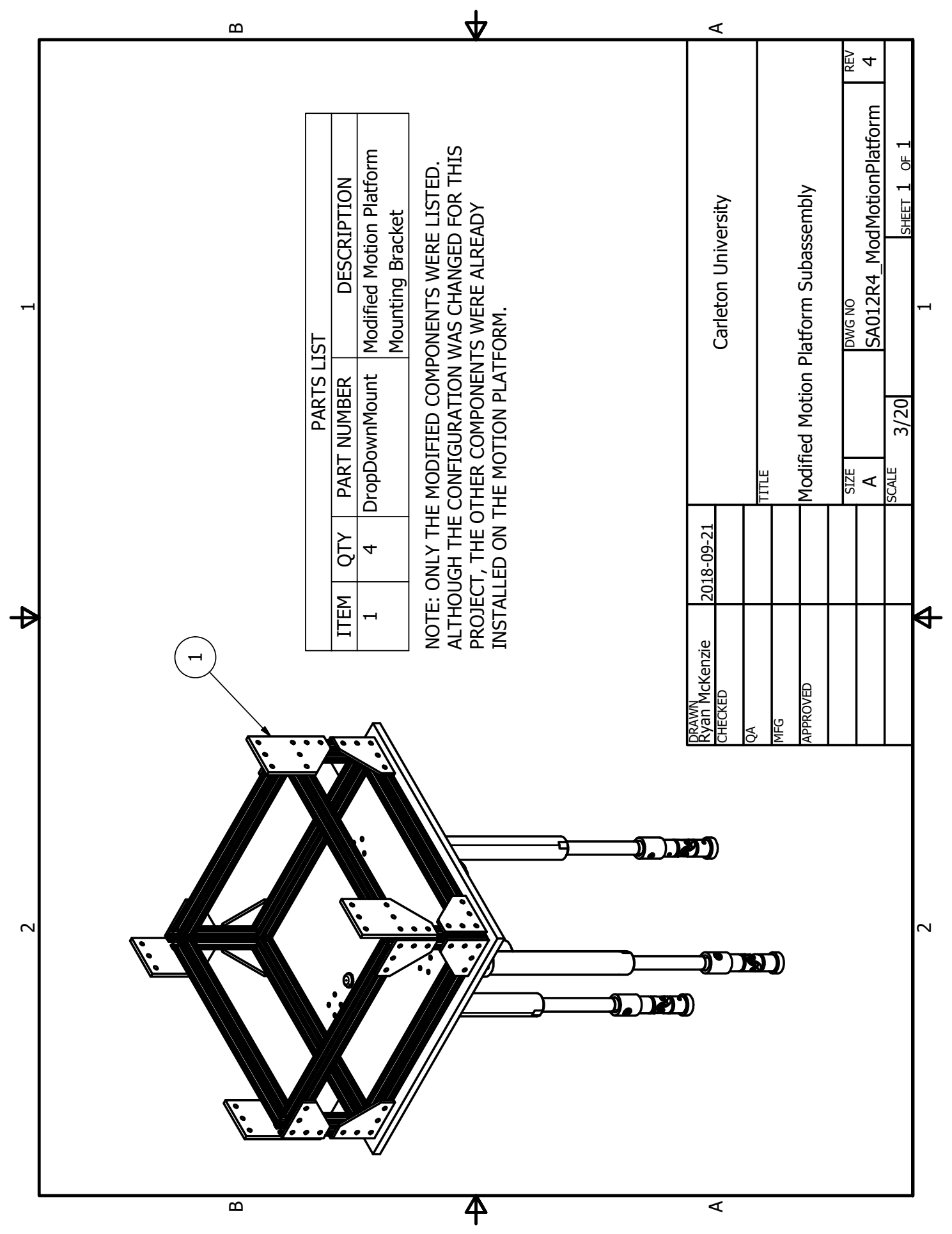




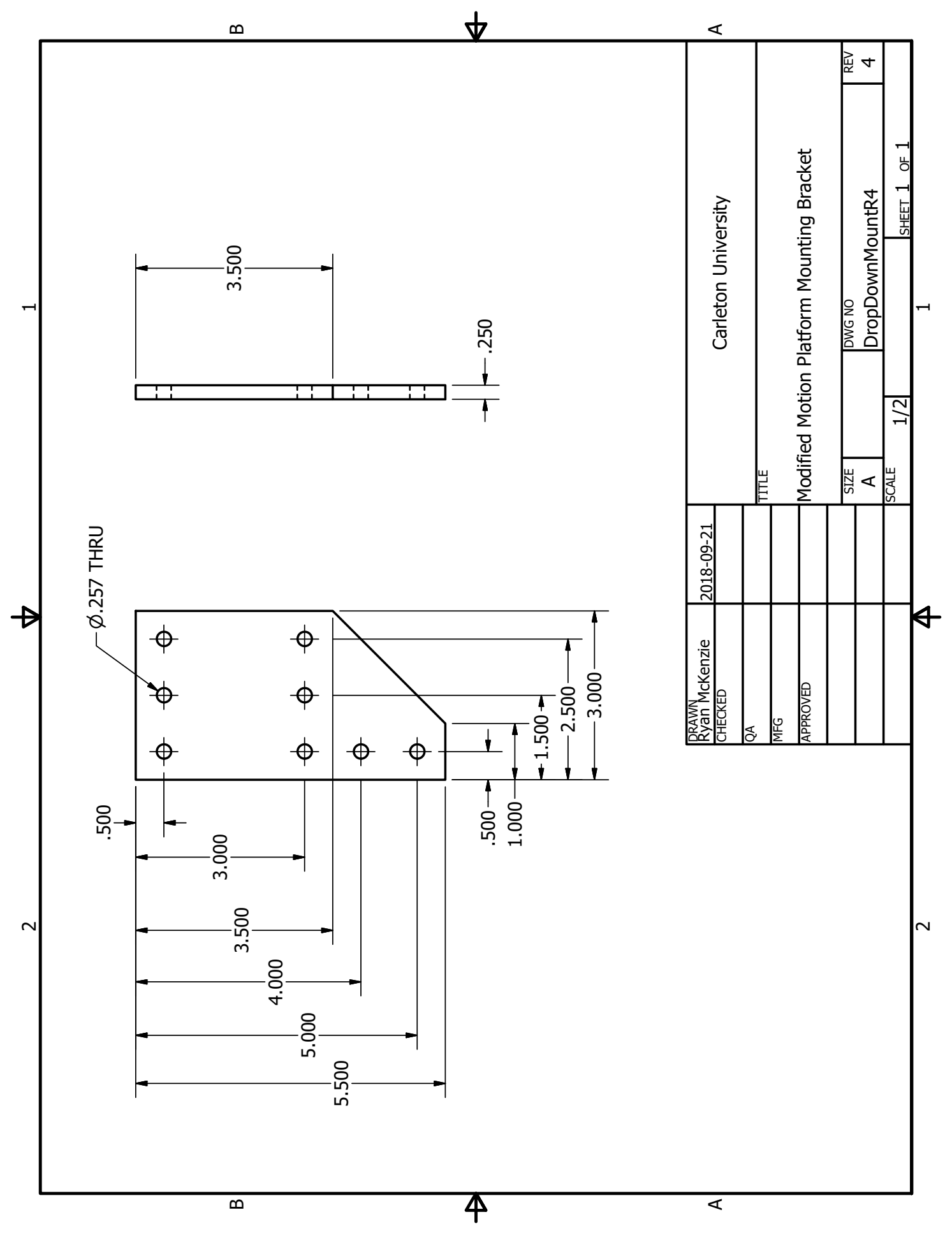




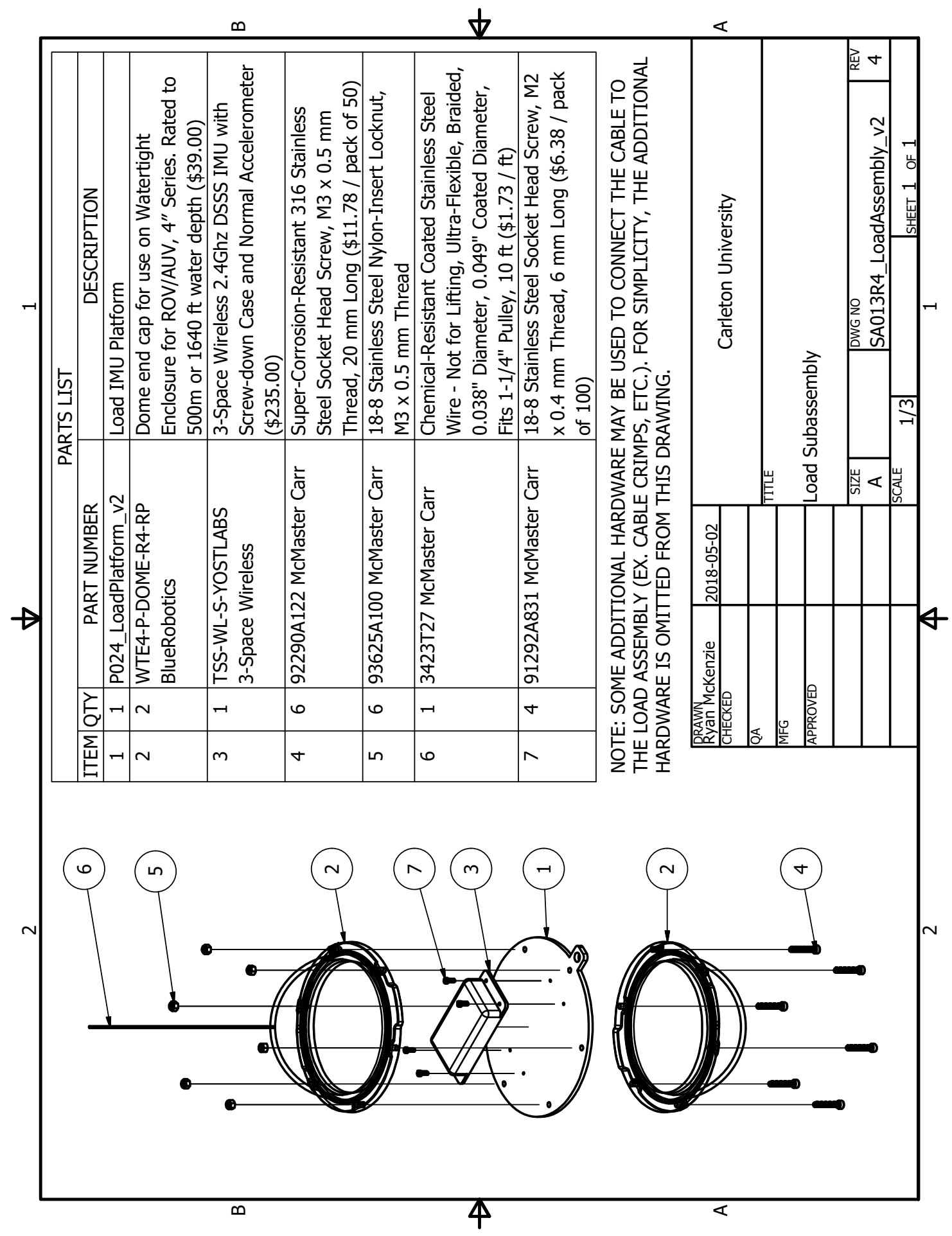




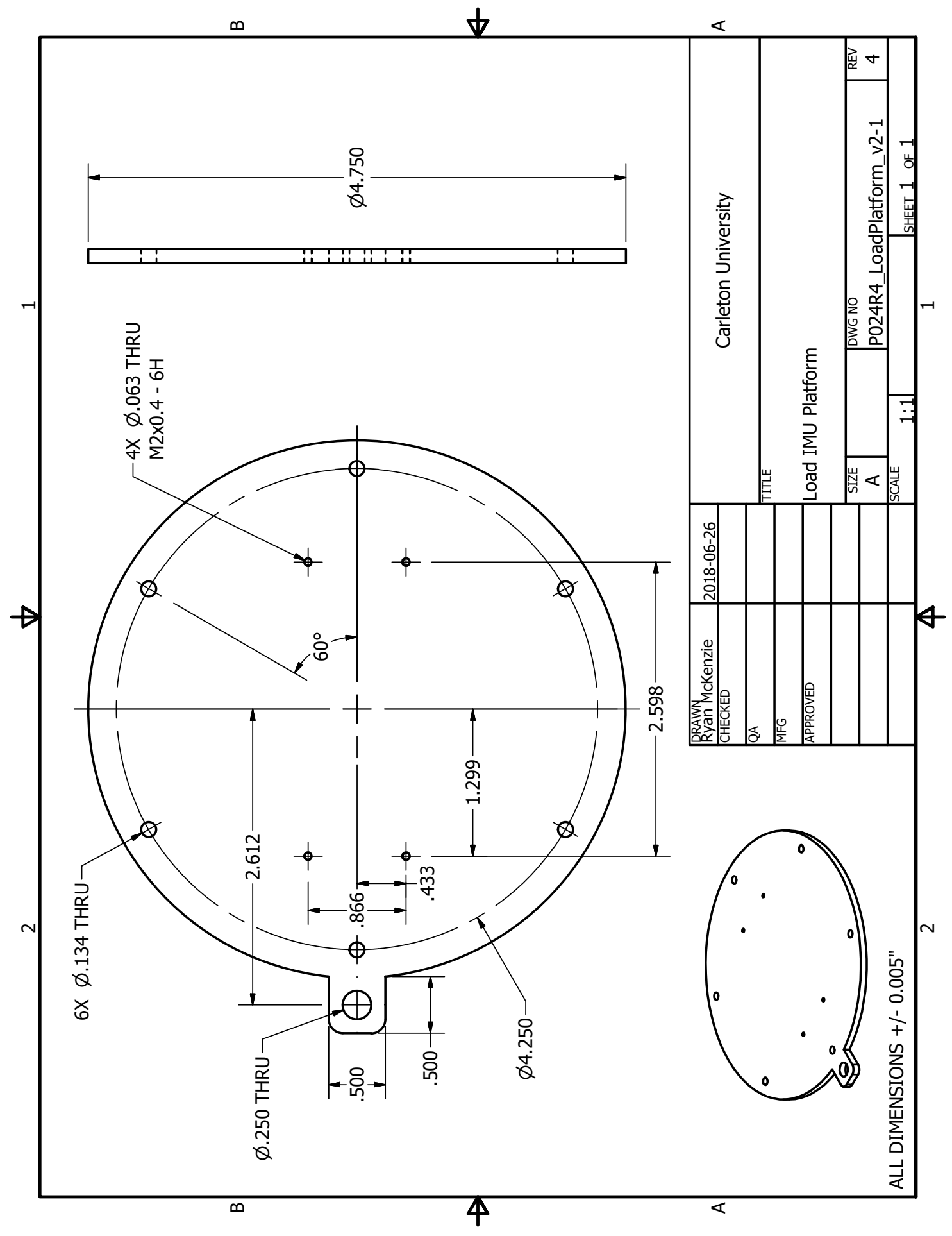




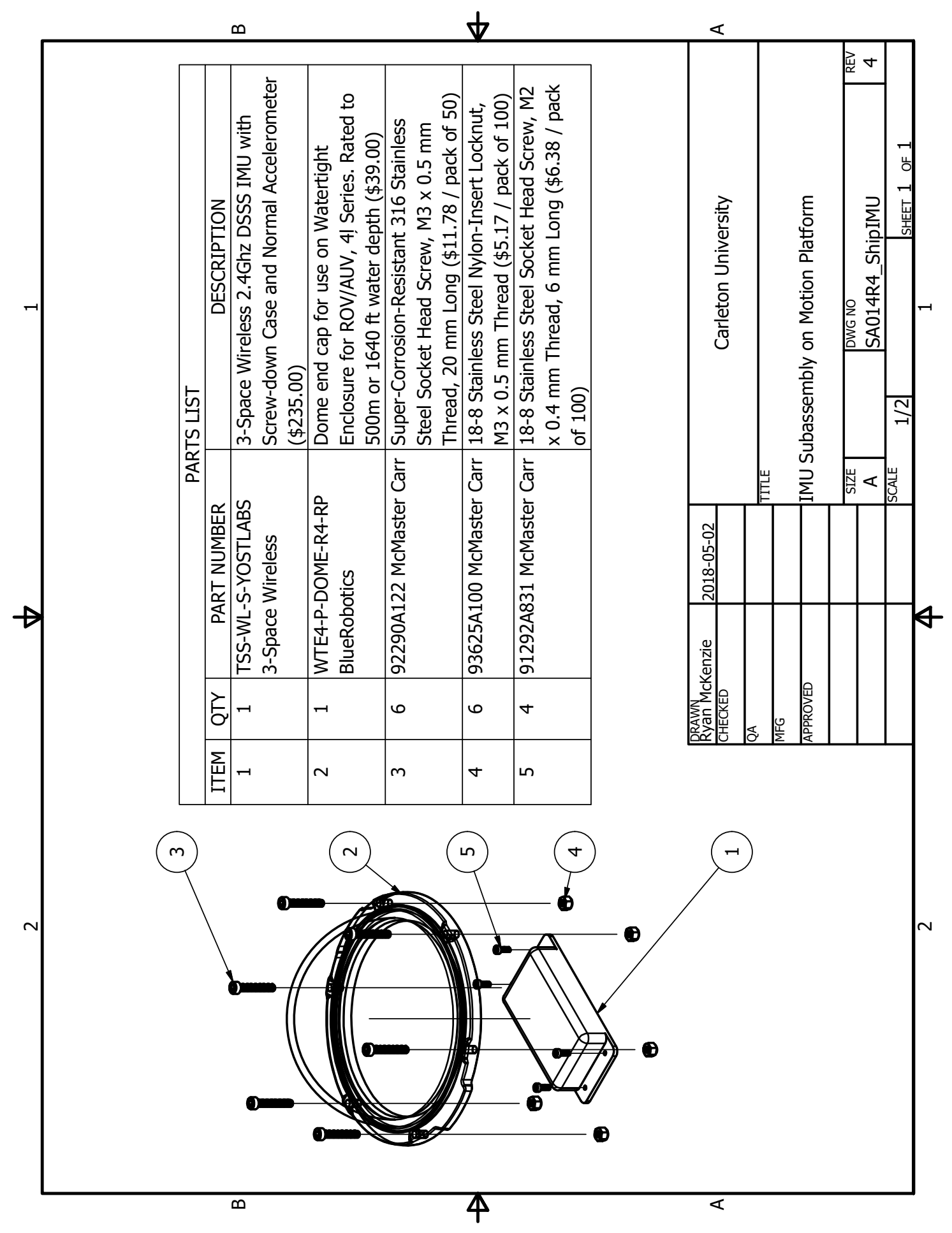




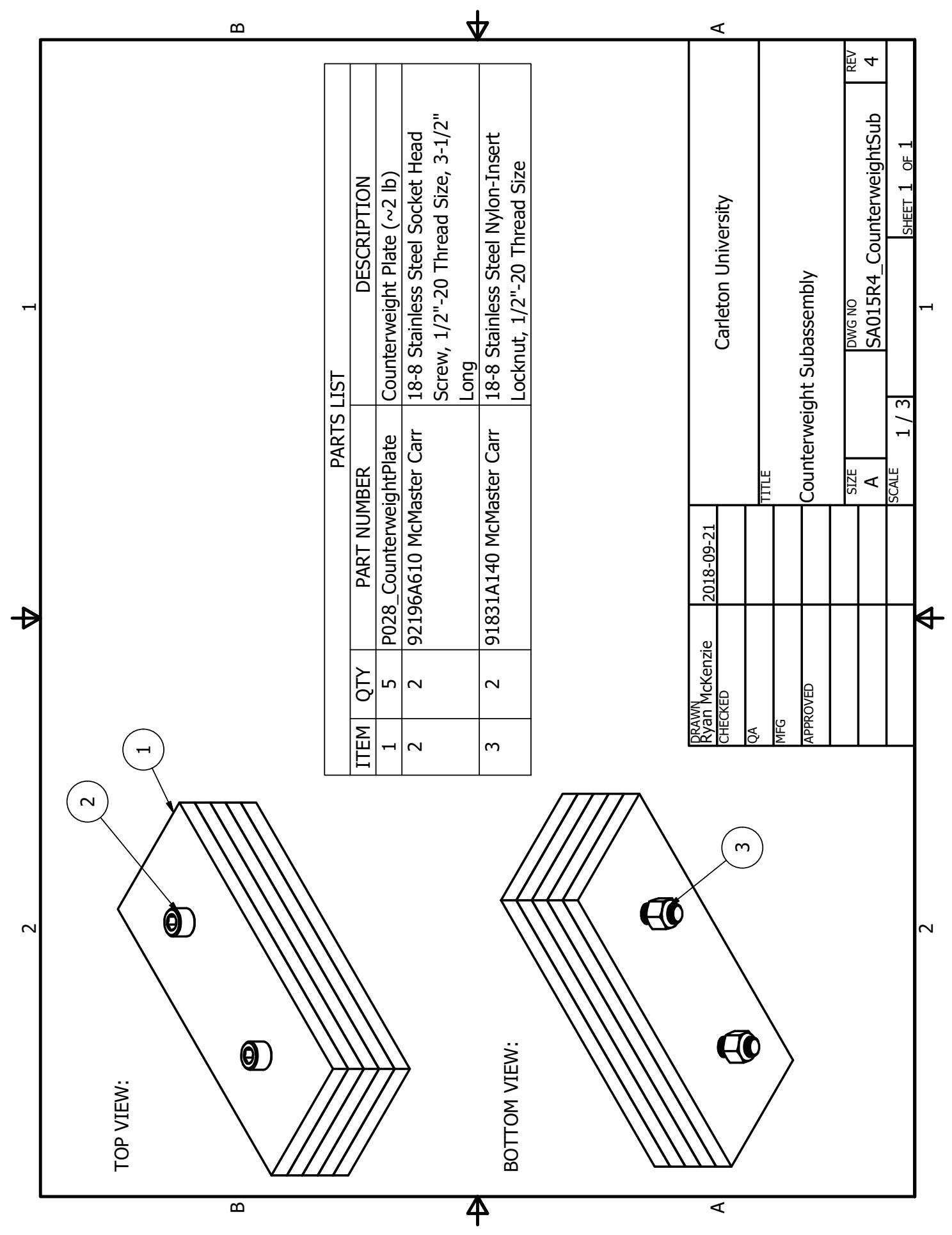




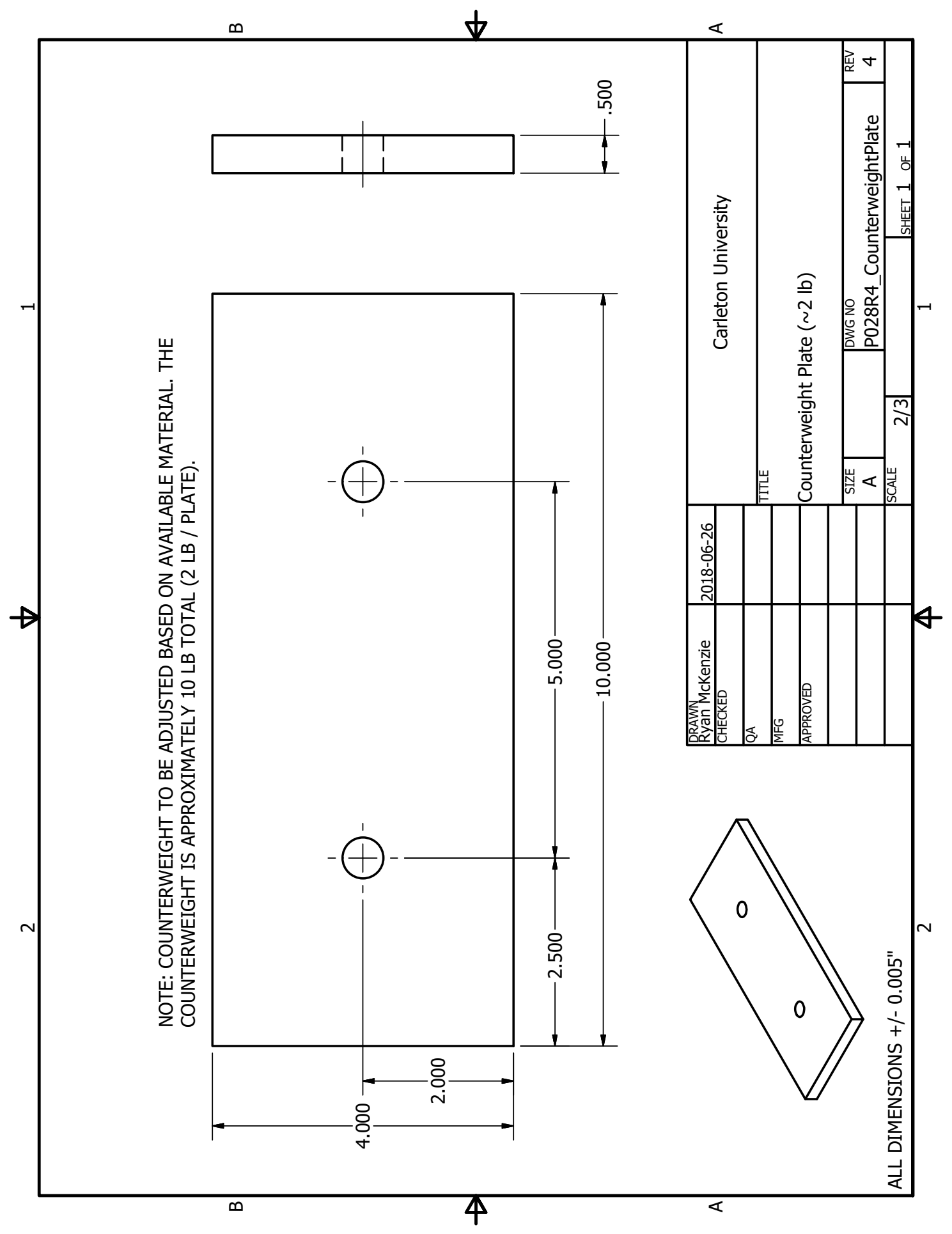




\section{Appendix C}

\section{Mathematical Preliminaries}

This appendix provides a brief summary of quaternion and dual quaternion algebra as it applies to the current thesis work. In the current work, quaternions are used to define rotations, and dual quaternions are used to define transformations (rotation and/or translation). These mathematical objects are useful for defining the orientation or pose (position and orientation) of one frame relative to another, and for transforming points or vectors between frames. Although less common than transformation matrices, the benefits of quaternion-based methods include

- reduced memory/storage requirements;

- improved computational efficiency for key operations such as

- concatenation of transformations,

- interpolation between orientations, and

- enforcement of type invariance/numerical stability; and

- improved singularity avoidance.

These and many other benefits motivate the use of quaternion-based methods in robotic applications, which is a commonly discussed topic in current research. 
In this appendix, the following topics will be briefly discussed:

- Rotation Sequences

- Rotation Matrices

- Quaternions

- Transformation Sequences

- Homogeneous Transformation Matrices

- Dual Quaternions 


\section{C.1 Rotation Sequences}

In the current work, rotation sequences are used to define the orientation of one frame relative to another. For example, given two frames $A$ and $B$, the orientation of frame $B$ relative to frame $A$ may be expressed as ${ }^{A} \mathcal{O}_{B}$ where $\mathcal{O}$ is a generalized orientation that may be represented by either a quaternion $\mathcal{O} \equiv q$ or rotation matrix $\mathcal{O} \equiv R$. The orientation may also be expressed as a set of Euler angles $\mathcal{O} \equiv\left\{\theta_{1}, \theta_{2}, \theta_{3}\right\}$ or an axis-angle combination $\mathcal{O} \equiv\{\hat{n}, \theta\}$; however, these expressions are less intuitive when describing large sequences and are typically converted to either a quaternion or a rotation matrix in practice. A rotation sequence is defined by a series of rotations between intermediate frames that relate the first frame to the final frame in the sequence. Each successive rotation, which is typically defined about a common principal axis, defines the relative orientation of two adjacent intermediate frames. For example, the yaw-pitch-roll (ZYX) sequence may be used to define the orientation of a frame $B$ relative to another frame $A$ by rotating frame $A$ into $A^{\prime}$ by an angle $\theta_{z}$ about a common $z$-axis, then rotating the new frame $A^{\prime}$ into $A^{\prime \prime}$ by an angle $\theta_{y}$ about a common $y$-axis, and finally rotating the new frame $A^{\prime \prime}$ into the final frame $B$ by an angle of $\theta_{x}$ about a common $x$-axis. The orientations of the intermediate frames may be expressed as functions of the rotation angles such that

$$
\begin{aligned}
{ }^{A} \mathcal{O}_{A^{\prime}} & =f\left(\theta_{z}\right) \\
A^{\prime} \mathcal{O}_{A^{\prime \prime}} & =f\left(\theta_{y}\right) \\
A^{\prime \prime} \mathcal{O}_{B} & =f\left(\theta_{x}\right)
\end{aligned}
$$

where the rotation angles are defined using the right-hand-rule about the axis of rotation as illustrated in the three steps of Figure C.1. 

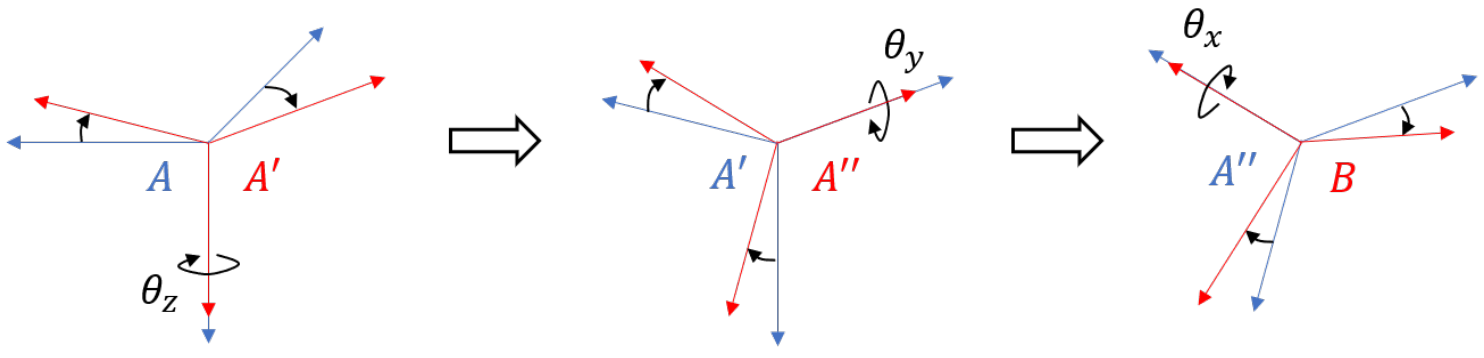

Figure C.1: Yaw-pitch-roll rotation sequence.

For both rotation matrices and quaternions, the intermediate orientations may be concatenated to define the orientation of the final frame $B$ relative to the first frame $A$ through multiplication such that

$$
{ }^{A} \mathcal{O}_{B}={ }^{A} \mathcal{O}_{A^{\prime}}{ }^{\prime} \mathcal{O}_{A^{\prime \prime}}{ }^{A^{\prime \prime}} \mathcal{O}_{B}={ }^{A} \mathcal{O}_{A^{\prime}}^{A^{\prime}} \mathcal{O}_{A^{\prime \prime}}^{A^{\prime \prime}} \mathcal{O}_{B}
$$

where the compressed form ${ }^{A} \mathcal{O}_{B}={ }^{A} \mathcal{O}_{A^{\prime}}^{A^{\prime}} \mathcal{O}_{A^{\prime \prime}}^{A^{\prime \prime}} \mathcal{O}_{B}$ is used to reduce the size of the expression. It is noted that, by equating two representations of the same orientation, an 'orientation loop' is formed that may be useful for solving rotational kinematic chains. The orientation ${ }^{A} \mathcal{O}_{B}$ may also be used as a rotation operator to convert a vector with components expressed in the second frame ${ }^{B} \vec{v}$ into a vector with components expressed in the first frame ${ }^{A} \vec{v}$ such that

$$
{ }^{A} \vec{v}={ }^{A} \mathcal{O}_{B}\left({ }^{B} \vec{v}\right)
$$

where the rotation operator ${ }^{A} \mathcal{O}_{B}(\cdot)$ defines either matrix-vector multiplication or pre-multiplication with post-multiplication by a conjugate depending on whether the orientation is expressed as a rotation matrix or a quaternion. The details of mathematical operations used to rotate vectors are discussed in the following two sections. 
If the origins of both frames $A$ and $B$ are coincident, then point coordinates may be represented as vectors and transformed in an identical manner. An inverse may be defined that expresses the orientation of the first frame $A$ relative to the final frame $B$ such that the inverse rotation becomes

$$
{ }^{B} \vec{v}={ }^{A} \mathcal{O}_{B}^{-1}\left({ }^{A} \vec{v}\right)={ }^{B} \mathcal{O}_{A}\left({ }^{A} \vec{v}\right)
$$

where ${ }^{B} \mathcal{O}_{A}={ }^{A} \mathcal{O}_{B}^{-1}$ is the orientation of the first frame $A$ relative to the final frame $B$. In the next section, rotation matrices are briefly discussed. 


\section{C.2 Rotation Matrices}

In this section, rotation matrices are briefly used to represent frame orientations and/or rotation operators such that ${ }^{A} \mathcal{O}_{B}$ with $\mathcal{O} \equiv R$. Returning to the yaw-pitchroll example of the previous section, the orientation of the final frame $B$ relative to the first frame $A$ may be expressed as a rotation matrix such that

$$
{ }^{A} R_{B}={ }^{A} R_{A^{\prime}}^{A^{\prime}} R_{A^{\prime \prime}}^{A^{\prime \prime}} R_{B}=\left[\begin{array}{ccc}
c_{z} c_{y} & c_{z} s_{x} s_{y}-c_{x} s_{z} & s_{x} s_{z}+c_{x} c_{z} s_{y} \\
s_{z} c_{y} & c_{x} c_{z}+s_{x} s_{y} s_{z} & c_{x} s_{y} s_{z}-c_{z} s_{x} \\
-s_{y} & c_{y} s_{x} & c_{x} c_{y}
\end{array}\right]
$$

where $c_{a}=\cos \left(\theta_{a}\right)$ and $s_{a}=\sin \left(\theta_{a}\right)$ are substituted to simplify the expression. The elemental/intermediate rotation matrices are defined as

$$
\begin{gathered}
{ }^{A} R_{A^{\prime}}=R_{z}=\left[\begin{array}{ccc}
\cos \left(\theta_{z}\right) & -\sin \left(\theta_{z}\right) & 0 \\
\sin \left(\theta_{z}\right) & \cos \left(\theta_{z}\right) & 0 \\
0 & 0 & 1
\end{array}\right] \\
{ }^{A^{\prime}} R_{A^{\prime \prime}}=R_{y}=\left[\begin{array}{ccc}
\cos \left(\theta_{y}\right) & 0 & \sin \left(\theta_{y}\right) \\
0 & 1 & 0 \\
-\sin \left(\theta_{y}\right) & 0 & \cos \left(\theta_{y}\right)
\end{array}\right] \\
A^{A^{\prime \prime}} R_{B}=R_{x}=\left[\begin{array}{lll}
1 & 0 & 0 \\
0 & \cos \left(\theta_{x}\right) & -\sin \left(\theta_{x}\right) \\
0 & \sin \left(\theta_{x}\right) & \cos \left(\theta_{x}\right)
\end{array}\right]
\end{gathered}
$$

and a column vector ${ }^{B} \vec{v}$ with components in the $B$ frame may be expressed with components in the $A$ frame by multiplication as ${ }^{A} \vec{v}={ }^{A} R_{B}^{B} \vec{v}$. Rotation matrices must be orthogonal with determinants of 1 , and the inverse orientation may be expressed as the transpose ${ }^{B} R_{A}={ }^{A} R_{B}^{-1}={ }^{A} R_{B}^{T}$. In the next section, quaternions are discussed. 


\section{C.3 Quaternions}

In this section, quaternions are briefly introduced as an alternative method of representing relative orientation/rotation. A quaternion may be defined as the linear combination of a real number and three imaginary numbers as

$$
\begin{aligned}
& \mathbf{q}=q_{0}+q_{1} \hat{\imath}+q_{2} \hat{\jmath}+q_{3} \hat{k} \\
& \hat{\imath}^{2}=\hat{\jmath}^{2}=\hat{k}^{2}=\hat{\imath} \hat{\jmath} \hat{k}=-1
\end{aligned}
$$

where $\left\{q_{0}, q_{1}, q_{2}, q_{3}\right\}$ are the scalar coefficients of the real and imaginary components. Several special quaternions exist, including the identity quaternion $q_{I}=1$, the zero quaternion $q_{0}=0$, and the pure quaternion which has a zero real component. A pure quaternion may be used to represent a vector such that

$$
\mathbf{v}=\vec{v}=0+v_{1} \hat{\imath}+v_{2} \hat{\jmath}+v_{3} \hat{k}=(0, \vec{v})
$$

where $(0, \vec{v})$ is an equivalent scalar-vector pair that may be used to represent a quaternion. Quaternion mathematics apply the operations shown in Table C.1 and may be derived intuitively using Clifford Algebra or Geometric Algebra [68]. A vector ${ }^{B} \vec{v}$ with components in the $B$ frame may be expressed with components in the $A$ frame by pre-multiplication and post-multiplication by the conjugate such that

$$
{ }^{A} \mathbf{v}={ }^{A} \hat{\mathbf{q}}_{B}\left({ }^{B} \mathbf{v}\right)^{A} \hat{\mathbf{q}}_{B}^{*}
$$

where a unit quaternion ${ }^{A} \hat{\mathbf{q}}_{B}$ is used to represent the orientation of the $B$ frame relative to the $A$ frame. Orientation/rotation quaternions must have a unity norm to ensure pure rotation. 
A common formulation for a quaternion-based orientation/rotation is based on an axis-angle representation with half-angles such that

$$
{ }^{A} \hat{\mathbf{q}}_{B}=\cos \left(\frac{\theta}{2}\right)+\hat{\vec{n}} \sin \left(\frac{\theta}{2}\right)
$$

where $\theta$ defines the angle of rotation about an axis defined by the unit vector $\hat{\vec{n}}$. The inverse of a unit quaternion is defined as its conjugate and may be used to express the inverse orientation as

$$
{ }^{B} \hat{\mathbf{q}}_{A}=\left({ }^{A} \hat{\mathbf{q}}_{B}\right)^{-1}={ }^{A} \hat{\mathbf{q}}_{B}^{*}
$$

where the conjugate negates the imaginary coefficients such that the inverse may be considered a rotation by the same angle about an opposite axis.

By noting that the columns of a rotation matrix may be found by simply expressing $B$ frame unit vectors $\hat{\imath}, \hat{\jmath}, \hat{k}$ in the $A$ frame, the orientation quaternion ${ }^{A} \hat{\mathbf{q}}_{B}$ may be converted to a rotation matrix as

$$
{ }^{A} R_{B}=\left[\begin{array}{ccc}
q_{0}^{2}+q_{1}^{2}-q_{2}^{2}-q_{3}^{2} & 2\left(q_{1} q_{2}-q_{0} q_{3}\right) & 2\left(q_{0} q_{2}+q_{1} q_{3}\right) \\
2\left(q_{1} q_{2}+q_{0} q_{3}\right) & q_{0}^{2}-q_{1}^{2}+q_{2}^{2}-q_{3}^{2} & 2\left(q_{2} q_{3}-q_{0} q_{1}\right) \\
2\left(q_{1} q_{3}-q_{0} q_{2}\right) & 2\left(q_{0} q_{1}+q_{2} q_{3}\right) & q_{0}^{2}-q_{1}^{2}-q_{2}^{2}+q_{3}^{2}
\end{array}\right]
$$

where ${ }^{A} R_{B}$ represents the same orientation as the quaternion ${ }^{A} \hat{\mathbf{q}}_{B}=q_{0}+q_{1} \hat{\imath}+q_{2} \hat{\jmath}+q_{3} \hat{k}$.

Table C.1: Quaternion operations [1].

\begin{tabular}{|l|l|}
\hline Addition & $\mathbf{a}+\mathbf{b}=\left(a_{0}+b_{0}, \vec{a}+\vec{b}\right)$ \\
\hline Scalar Multiplication & $\lambda \mathbf{a}=\left(\lambda a_{0}, \lambda \vec{a}\right)$ \\
\hline Multiplication & $\mathbf{a b}=\left(a_{0} b_{0}-\vec{a} \cdot \vec{b}, a_{0} \vec{b}+b_{0} \vec{a}+\vec{a} \times \vec{b}\right)$ \\
\hline Conjugate & $\mathbf{a}^{*}=\left(a_{0},-\vec{a}\right)$ \\
\hline Dot Product & $\mathbf{a} \cdot \mathbf{b}=\left(a_{0} b_{0}+\vec{a} \cdot \vec{b}, \overrightarrow{0_{3 \times 1}}\right)=\frac{1}{2}\left(\mathbf{a}^{*} \mathbf{b}+\mathbf{b}^{*} \mathbf{a}\right)$ \\
\hline Cross Product & $\mathbf{a} \times \mathbf{b}=\left(0, a_{0} \vec{b}+b_{0} \vec{a}+\vec{a} \times \vec{b}\right)=\frac{1}{2}\left(\mathbf{a b}-\mathbf{b}^{*} \mathbf{a}^{*}\right)$ \\
\hline Norm & $\|\mathbf{a}\|=\sqrt{\mathbf{a} \cdot \mathbf{a}}$ \\
\hline
\end{tabular}


Returning to the yaw-pitch-roll example of the previous sections, the orientation of the final frame $B$ relative to the first frame $A$ may be expressed as a quaternion such that

$$
\begin{aligned}
{ }^{A} \hat{\mathbf{q}}_{B} & ={ }^{A} \hat{\mathbf{q}}_{A^{\prime}}^{A^{\prime}} \hat{\mathbf{q}}_{A^{\prime \prime}}^{A^{\prime \prime}} \hat{\mathbf{q}}_{B}=q_{0}+q_{1} \hat{\imath}+q_{2} \hat{\jmath}+q_{3} \hat{k} \\
q_{0} & =\cos \left(\frac{\theta_{x}}{2}\right) \cos \left(\frac{\theta_{y}}{2}\right) \cos \left(\frac{\theta_{z}}{2}\right)+\sin \left(\frac{\theta_{x}}{2}\right) \sin \left(\frac{\theta_{y}}{2}\right) \sin \left(\frac{\theta_{z}}{2}\right) \\
q_{1} & =\sin \left(\frac{\theta_{x}}{2}\right) \cos \left(\frac{\theta_{y}}{2}\right) \cos \left(\frac{\theta_{z}}{2}\right)-\cos \left(\frac{\theta_{x}}{2}\right) \sin \left(\frac{\theta_{y}}{2}\right) \sin \left(\frac{\theta_{z}}{2}\right) \\
q_{2} & =\cos \left(\frac{\theta_{x}}{2}\right) \sin \left(\frac{\theta_{y}}{2}\right) \cos \left(\frac{\theta_{z}}{2}\right)+\sin \left(\frac{\theta_{x}}{2}\right) \cos \left(\frac{\theta_{y}}{2}\right) \sin \left(\frac{\theta_{z}}{2}\right) \\
q_{3} & =\cos \left(\frac{\theta_{x}}{2}\right) \cos \left(\frac{\theta_{y}}{2}\right) \sin \left(\frac{\theta_{z}}{2}\right)-\sin \left(\frac{\theta_{x}}{2}\right) \sin \left(\frac{\theta_{y}}{2}\right) \cos \left(\frac{\theta_{z}}{2}\right)
\end{aligned}
$$

where the elemental/intermediate orientation quaternions are defined as

$$
\begin{gathered}
{ }^{A} \hat{\mathbf{q}}_{A^{\prime}}=q_{z}=\cos \left(\frac{\theta_{z}}{2}\right)+0 \hat{\imath}+0 \hat{\jmath}+\sin \left(\frac{\theta_{z}}{2}\right) \hat{k} \\
A^{A^{\prime}} \hat{\mathbf{q}}_{A^{\prime \prime}}=q_{y}=\cos \left(\frac{\theta_{y}}{2}\right)+0 \hat{\imath}+\sin \left(\frac{\theta_{y}}{2}\right) \hat{\jmath}+0 \hat{k} \\
A^{A^{\prime \prime}} \hat{\mathbf{q}}_{B}=q_{x}=\cos \left(\frac{\theta_{x}}{2}\right)+\sin \left(\frac{\theta_{x}}{2}\right) \hat{\imath}+0 \hat{\jmath}+0 \hat{k}
\end{gathered}
$$

and concatenation is performed by quaternion multiplication as defined in Table C.1. If the intermediate yaw-pitch-roll angles are to be extracted from the final orientation quaternion, a direct solution may be found by considering the elements of the equivalent rotation matrix as demonstrated in Chapter 5. 


\section{C.4 Transformation Sequences}

In the current work, transformation sequences are used to define the pose (position and orientation) of one frame relative to another. For example, given two frames $A$ and $B$, the pose of frame $B$ relative to frame $A$ may be expressed as ${ }^{A} \mathcal{P}_{B}$ where $\mathcal{P}$ is a generalized pose that may be represented by either a dual quaternion $\mathcal{P} \equiv Q$ or a homogeneous transformation matrix $\mathcal{P} \equiv H$. A transformation sequence is defined by a series of translations and rotations between intermediate frames that relate the first frame to the final frame in the sequence. Each successive translation or rotation, which is typically defined along or about a common principal axis, defines the relative orientation of two adjacent intermediate frames. For example, the surge-sway-heaveyaw-pitch-roll sequence for defining ship motion may be used to define the orientation of a frame $B$ relative to another frame $A$ by translating frame $A$ into frame $A_{t}$ along the length of a displacement vector ${ }^{A} \vec{d}_{A_{t} / A}=d_{x y z}$, rotating frame $A_{t}$ into $A^{\prime}$ by an angle $\theta_{z}$ about a common $z$-axis, then rotating the new frame $A^{\prime}$ into $A^{\prime \prime}$ by an angle $\theta_{y}$ about a common $y$-axis, and finally rotating the new frame $A^{\prime \prime}$ into the final frame $B$ by an angle of $\theta_{x}$ about a common $x$-axis. The orientations of the intermediate frames may be expressed as functions of the displacements/angles such that

$$
\begin{aligned}
{ }^{A} \mathcal{P}_{A_{t}} & =f\left(d_{x y z}\right) \\
{ }^{{ }^{A}} \mathcal{P}_{A^{\prime}} & =f\left(\theta_{z}\right) \\
{ }^{A^{\prime}} \mathcal{P}_{A^{\prime \prime}} & =f\left(\theta_{y}\right) \\
{ }^{A^{\prime \prime}} \mathcal{P}_{B} & =f\left(\theta_{x}\right)
\end{aligned}
$$

where the rotation angles are defined using the right-hand-rule about the axis of rotation, and displacements are defined along a vector as illustrated in Figure C.2. 


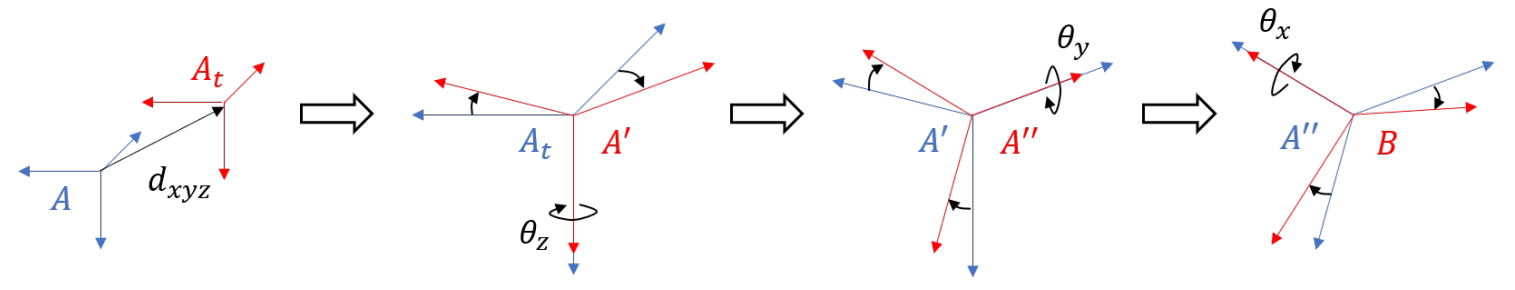

Figure C.2: Surge-sway-heave-yaw-pitch-roll transformation sequence.

For both transformation matrices and dual quaternions, the intermediate poses may be concatenated to define the pose of the final frame $B$ relative to the first frame $A$ through multiplication such that

$$
{ }^{A} \mathcal{P}_{B}={ }^{A} \mathcal{P}_{A^{\prime}}{ }^{A^{\prime}} \mathcal{P}_{A^{\prime \prime}}{ }^{A^{\prime \prime}} \mathcal{P}_{B}={ }^{A} \mathcal{P}_{A^{\prime}}^{A^{\prime}} \mathcal{P}_{A^{\prime \prime}}^{A^{\prime \prime}} \mathcal{P}_{B}
$$

where the compressed form ${ }^{A} \mathcal{P}_{B}={ }^{A} \mathcal{P}_{A^{\prime}}^{A^{\prime}} \mathcal{P}_{A^{\prime \prime}}^{A^{\prime \prime}} \mathcal{P}_{B}$ is used to reduce the size of the expression. It is noted that, by equating two representations of the same pose, a 'pose loop' is formed that may be useful for solving general kinematic chains. The pose ${ }^{A} \mathcal{P}_{B}$ may also be used as a transformation to convert a vector with components expressed in the second frame ${ }^{B} \vec{v}$ into a vector with components expressed in the first frame ${ }^{A} \vec{v}$ such that

$$
{ }^{A} \vec{v}={ }^{A} \mathcal{P}_{B}\left({ }^{B} \vec{v}\right)
$$

where the transformation operator ${ }^{A} \mathcal{O}_{B}(\cdot)$ defines either matrix-vector multiplication or pre-multiplication with post-multiplication by a conjugate depending on whether the transformation is expressed as a transformation matrix or a dual quaternion. The details of the mathematical operations used to transform vectors are discussed in the following two sections. 
As the origins of the frames $A$ and $B$ are not necessarily coincident, point coordinates must be transformed in such a way that the relative displacement is included in the transformation whereas vectors only require the rotational component. An inverse may be defined that expresses the pose of the first frame $A$ relative to the final frame $B$ such that the inverse transformation becomes

$$
{ }^{B} \vec{v}={ }^{A} \mathcal{P}_{B}^{-1}\left({ }^{A} \vec{v}\right)={ }^{B} \mathcal{P}_{A}\left({ }^{A} \vec{v}\right)
$$

where ${ }^{B} \mathcal{P}_{A}={ }^{A} \mathcal{P}_{B}^{-1}$ is the pose of the first frame $A$ relative to the final frame $B$. In the next section, homogeneous transformation matrices are briefly discussed. 


\section{C.5 Homogeneous Transformation Matrices}

In this section, homogeneous transformation matrices are briefly used to represent pose and/or transformation operators such that ${ }^{A} \mathcal{P}_{B}$ with $\mathcal{P} \equiv H$. Returning to the surge-sway-heave-yaw-pitch-roll example of the previous section, the pose of frame $B$ relative to frame $A$ may be expressed as

$$
{ }^{A} H_{B}={ }^{A} H_{A_{t}}^{A_{t}} H_{A^{\prime}}^{A^{\prime}} H_{A^{\prime \prime}}^{A^{\prime \prime}} H_{B}=\left[\begin{array}{cc}
{ }^{A} R_{B} & { }^{A} \vec{d}_{B / A} \\
\overrightarrow{0}^{T} & 1
\end{array}\right]
$$

with elemental/intermediate transformation matrices defined as

$$
\begin{aligned}
& { }^{A} H_{A_{t}}=H_{d x y z}=\left[\begin{array}{cc}
I & \vec{d}_{x y z} \\
\overrightarrow{0}^{T} & 1
\end{array}\right] \\
& { }^{A_{t}} H_{A^{\prime}}=H_{R z}=\left[\begin{array}{ll}
R_{z} & \overrightarrow{0} \\
\overrightarrow{0}^{T} & 1
\end{array}\right] \\
& { }^{A^{\prime}} H_{A^{\prime \prime}}=H_{R y}=\left[\begin{array}{ll}
R_{y} & \overrightarrow{0} \\
\overrightarrow{0}^{T} & 1
\end{array}\right] \\
& A^{\prime \prime} H_{B}=H_{R x}=\left[\begin{array}{ll}
R_{x} & \overrightarrow{0} \\
\overrightarrow{0}^{T} & 1
\end{array}\right]
\end{aligned}
$$

where ${ }^{A} \vec{d}_{B / A}=\vec{d}_{x y z}=\left[d_{x}, d_{y}, d_{z}\right]^{T}$ are the surge-sway-heave displacements of frame $B$ relative to frame $A$ with coordinates expressed in frame $A$. Point transformations ${ }^{A} p={ }^{A} H_{B}^{B} p$ and vector transformations ${ }^{A} v={ }^{A} H_{B}^{B} v$ may be defined using homogeneous coordinates such that ${ }^{B} p=\left[p_{x}, p_{y}, p_{z}, 1\right]^{T}$ and ${ }^{B} v=\left[v_{x}, v_{y}, v_{z}, 0\right]^{T}$. In the following section, dual quaternion pose representations are discussed. 


\section{C.6 Dual Quaternions}

Dual quaternions are an extension of quaternions to transformations that involve both rotation and translation. A dual quaternion is defined as the combination of a real component and a dual component such that

$$
\begin{aligned}
\mathbf{Q} & =\mathbf{q}_{R}+\epsilon \mathbf{q}_{D} \\
& =\left(q_{0}, \vec{q}_{R}\right)+\epsilon\left(q_{4}, \vec{q}_{D}\right) \\
& =q_{0}+q_{1} \hat{\imath}+q_{2} \hat{\jmath}+q_{3} \hat{k}+q_{4} \epsilon+q_{5} \epsilon \hat{\imath}+q_{6} \epsilon \hat{\jmath}+q_{7} \epsilon \hat{k} \\
\mathbf{q}_{R} & =q_{0}+q_{1} \hat{\imath}+q_{2} \hat{\jmath}+q_{3} \hat{k} \\
\mathbf{q}_{D} & =q_{4}+q_{5} \hat{\imath}+q_{6} \hat{\jmath}+q_{7} \hat{k} \\
\hat{\imath}^{2} & =\hat{\jmath}^{2}=\hat{k}^{2}=\hat{\imath} \hat{\jmath} \hat{k}=-1 \\
\epsilon^{2} & =0
\end{aligned}
$$

where $\mathbf{q}_{R}$ and $\mathbf{q}_{D}$ are the real and dual components, respectively, and a dual number is introduced in combination with the three imaginary numbers. Dual quaternion mathematics apply the operations shown in Table C.2, which build on the quaternion operations shown in Table C.1.

Similar to quaternions, the identity dual quaternion is defined as $\mathbf{Q}_{I}=1$, the zero dual quaternion is defined as $\mathbf{Q}_{0}=0$, and a dual representation of a vector is

$$
\mathbf{V}=(1, \overrightarrow{0})+\epsilon(0, \vec{v})
$$

where the real and dual quaternion components are shown as scalar-vector pairs. Points may also be represented as vectors; however, a combined conjugate is required when transforming point coordinates to account for the linear translation. 
Table C.2: Dual quaternion operations [1,2].

\begin{tabular}{|l|l|}
\hline Addition & $\mathbf{A}+\mathbf{B}=\left(\mathbf{a}_{R}+\mathbf{b}_{R}\right)+\epsilon\left(\mathbf{a}_{D}+\mathbf{b}_{D}\right)$ \\
\hline Scalar Multiplication & $\lambda \mathbf{A}=\left(\lambda \mathbf{a}_{R}\right)+\epsilon\left(\lambda \mathbf{a}_{D}\right)$ \\
\hline Multiplication & $\mathbf{A B}=\left(\mathbf{a}_{R} \mathbf{b}_{R}\right)+\epsilon\left(\mathbf{a}_{D} \mathbf{b}_{R}+\mathbf{a}_{R} \mathbf{b}_{D}\right)$ \\
\hline Conjugate 1 (Classic) & $\mathbf{A}^{*}=\left(\mathbf{a}_{R}^{*}\right)+\epsilon\left(\mathbf{a}_{D}^{*}\right)$ \\
\hline Conjugate 2 (Dual) & $\mathbf{A}^{* *}=\left(\mathbf{a}_{R}\right)-\epsilon\left(\mathbf{a}_{D}\right)$ \\
\hline Conjugate 3 (Combined) & $\mathbf{A}^{* * *}=\left(\mathbf{a}_{R}^{*}\right)-\epsilon\left(\mathbf{a}_{D}^{*}\right)$ \\
\hline Dot Product & $\mathbf{A} \cdot \mathbf{B}=\left(\mathbf{a}_{R} \cdot \mathbf{b}_{R}\right)+\epsilon\left(\mathbf{a}_{D} \cdot \mathbf{b}_{R}+\mathbf{a}_{R} \cdot \mathbf{b}_{D}\right)$ \\
\hline Cross Product & $\mathbf{A} \times \mathbf{B}=\left(\mathbf{a}_{R} \times \mathbf{b}_{R}\right)+\epsilon\left(\mathbf{a}_{D} \times \mathbf{b}_{R}+\mathbf{a}_{R} \times \mathbf{b}_{D}\right)$ \\
\hline Circle Product & $\mathbf{A} \circ \mathbf{B}=\left(\mathbf{a}_{R} \cdot \mathbf{b}_{R}+\mathbf{a}_{D} \cdot \mathbf{b}_{D}\right)+\epsilon \mathbf{0}$ \\
\hline Norm & $\|\mathbf{A}\|=\sqrt{\mathbf{A} \circ \mathbf{A}}$ \\
\hline
\end{tabular}

The dual quaternion pose of a $B$ frame relative to an $A$ frame may be defined by the concatenation of a pure translation ${ }^{A} \hat{\mathbf{Q}}_{\text {trans }, B}=(1, \overrightarrow{0})+\epsilon\left(0,{ }^{A} \vec{d}_{B / A}\right)$ and a pure rotation ${ }^{A} \hat{\mathbf{Q}}_{r o t, B}={ }^{A} \hat{\mathbf{q}}_{r, B}+\epsilon(0, \overrightarrow{0})$ such that

$$
\begin{aligned}
{ }^{A} \hat{\mathbf{Q}}_{B} & ={ }^{A} \hat{\mathbf{Q}}_{\text {trans }, B}{ }^{A} \hat{\mathbf{Q}}_{r o t, B} \\
& ={ }^{A} \hat{\mathbf{q}}_{r, B}+\epsilon\left(0, \frac{{ }^{A} \vec{d}_{B / A}}{2}\right){ }^{A} \hat{\mathbf{q}}_{r, B}
\end{aligned}
$$

where ${ }^{A} \hat{\mathbf{q}}_{r, B}$ is a quaternion that defines the orientation of frame $B$ relative to frame $A$ and ${ }^{A} \vec{d}_{B / A}$ is a displacement vector that defines the position of frame $B$ relative to frame $A$ with components expressed in frame $A$. By converting the orientation quaternion to a rotation matrix and extracting the displacement, a dual quaternion pose may be converted to a homogeneous transformation matrix of the form

$$
{ }^{A} H_{B}=\left[\begin{array}{cc}
{ }^{A} R_{B} & { }^{A} \vec{d}_{B / A} \\
\overrightarrow{0}^{T} & 1
\end{array}\right]
$$

where both ${ }^{A} H_{B}$ and ${ }^{A} \hat{\mathbf{Q}}_{B}$ represent the pose of frame $B$ relative to frame $A$. 
Dual quaternions may be constructed by forming a transformation sequence between intermediate frames and concatenating elemental/intermediate pose dual quaternions via dual quaternion multiplication. For example, the surge/sway/heave/yaw/pitch/roll transformation sequence that defines a ship body frame $B$ relative to a world frame $A \equiv W$ may be stated as

$$
{ }^{A} \hat{\mathbf{Q}}_{B}={ }^{A} \hat{\mathbf{Q}}_{A_{t}}^{A_{t}} \hat{\mathbf{Q}}_{A^{\prime}}^{A^{\prime}} \hat{\mathbf{Q}}_{A^{\prime \prime}}^{A^{\prime \prime}} \hat{\mathbf{Q}}_{B}
$$

where $A_{t}$ represents a translating world frame and the $\left\{A^{\prime}, A^{\prime \prime}, B\right\}$ frames are equivalent to those applied in the yaw-pitch-roll rotation sequence of Figure C.1. The elemental/intermediate pose dual quaternions are defined as

$$
\begin{aligned}
{ }^{A} \hat{\mathbf{Q}}_{A_{t}} & =Q_{d x y z}=1+0 \hat{\imath}+0 \hat{\jmath}+0 \hat{k}+\frac{\epsilon}{2}\left(0+d_{x} \hat{\imath}+d_{y} \hat{\jmath}+d_{z} \hat{k}\right) \\
{ }^{A_{t}} \hat{\mathbf{Q}}_{A^{\prime}} & =Q_{q z}=\cos \left(\frac{\theta_{z}}{2}\right)+0 \hat{\imath}+0 \hat{\jmath}+\sin \left(\frac{\theta_{z}}{2}\right) \hat{k}+\frac{\epsilon}{2}(0+0 \hat{\imath}+0 \hat{\jmath}+0 \hat{k}) \\
{ }^{A^{\prime}} \hat{\mathbf{Q}}_{A^{\prime \prime}} & =Q_{q y}=\cos \left(\frac{\theta_{y}}{2}\right)+0 \hat{\imath}+\sin \left(\frac{\theta_{y}}{2}\right) \hat{\jmath}+0 \hat{k}+\frac{\epsilon}{2}(0+0 \hat{\imath}+0 \hat{\jmath}+0 \hat{k}) \\
{ }^{A^{\prime \prime}} \hat{\mathbf{Q}}_{B} & =Q_{q x}=\cos \left(\frac{\theta_{x}}{2}\right)+\sin \left(\frac{\theta_{x}}{2}\right) \hat{\imath}+0 \hat{\jmath}+0 \hat{k}+\frac{\epsilon}{2}(0+0 \hat{\imath}+0 \hat{\jmath}+0 \hat{k})
\end{aligned}
$$

where the $x y z$-translations are grouped into a single dual quaternion for convenience. The components of a vector may be converted from the $B$ frame to the $A$ frame by pre-multiplying by the dual quaternion transformation and post-multiplying by its classic conjugate ${ }^{A} \mathbf{V}={ }^{A} \hat{\mathbf{Q}}_{B}\left({ }^{B} \mathbf{V}\right){ }^{A} \hat{\mathbf{Q}}_{B}^{*}$, and the inverse pose/transformation is also defined with the classic conjugate as ${ }^{B} \hat{\mathbf{Q}}_{A}={ }^{A} \hat{\mathbf{Q}}_{B}^{-1}={ }^{A} \hat{\mathbf{Q}}_{B}^{*}$. Point transformations are expressed using the combined conjugate to account for the translation between the reference frames as ${ }^{A} \mathbf{P}={ }^{A} \hat{\mathbf{Q}}_{B}\left({ }^{B} \mathbf{P}\right){ }^{A} \hat{\mathbf{Q}}_{B}^{* * *}$ where ${ }^{B} \mathbf{P}$ and ${ }^{A} \mathbf{P}$ are dual quaternion representations of a vector $\vec{p}$ containing the point coordinates expressed relative to frame $B$ and $A$, respectively. 


\section{Appendix D}

\section{Expanded Derivation of System}

\section{Models}

This appendix is an extension of Chapter 4. The MATLAB functions used to confirm the derived models are presented herein. The matrix form of the equations of motion for the variable length spherical pendulum with a three-degree-of-freedom trolley are also presented here for convenience.

\section{D.1 Confirmation of Kinematic Models}

The construction of the Simscape Multibody model, which is shown in Figure D.1 with its corresponding block diagram in Figures D.2 and D.3, is labelled to match the graph theoretic representation of Figure 4.6. In Figure D.1, a simplified skeleton model of the crane is developed with the same actuator mechanism dimensions that were reported in the previous subsections, as well as link lengths for the tower, boom, and jib defined by dimensions of the CAD model as $l_{2 z}=184.6 \mathrm{~mm}, l_{3 y}=225 \mathrm{~mm}$, and $l_{4 y}=150 \mathrm{~mm}$, respectively. In Figure D.1, the tower, boom, and jib $\left(n_{2}-n_{4}\right)$ are 
shown as grey prisms, and the actuator mechanisms are indicated by blue components to represent the joint locations $e_{j}^{\prime}-e_{j}^{\prime \prime \prime}$. The base frame on the platform $n_{2}$ and the tip frame on the jib $n_{4}$ are also indicated.

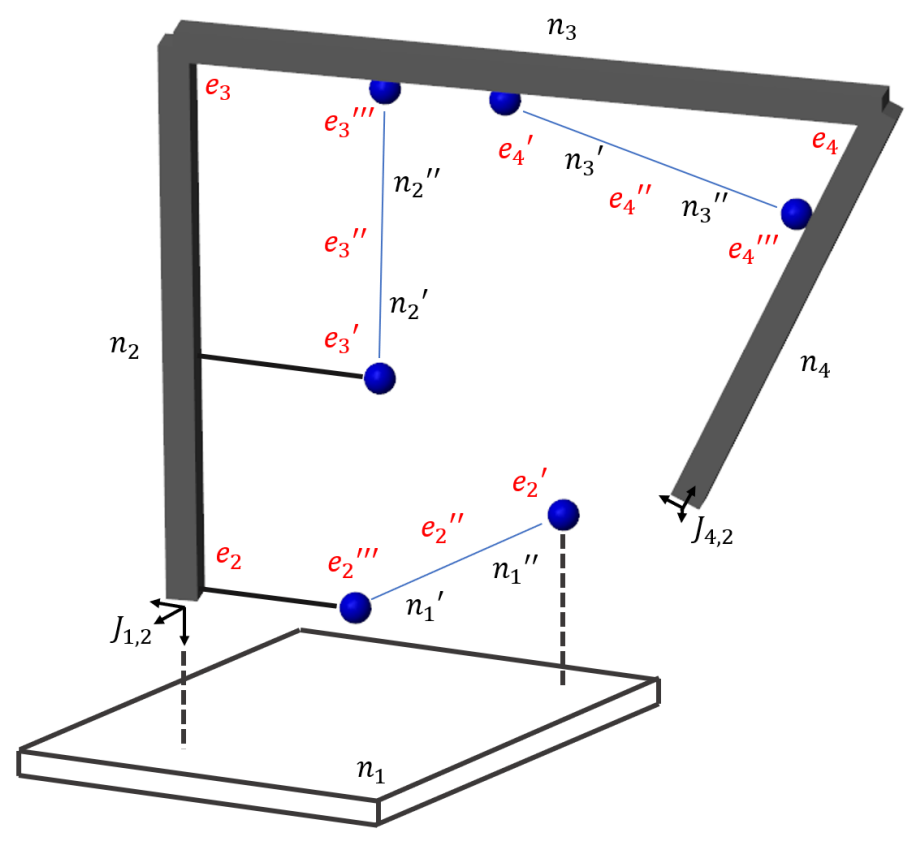

Figure D.1: Simscape model of the knuckle boom crane used to confirm the kinematic analysis.

In Figure D.2, the main serial structure of the crane is shown with the actuator mechanisms contained in the sub-diagrams for the revolute joints $e_{2}-e_{4}$. The 6-DOF virtual joint $e_{8}$ is constructed in Simscape using a 3-DOF Cartesian Joint for the $x-y-z$ displacement of the crane tip relative to its base and a 3-DOF Spherical Joint to allow unconstrained rotation. This construction defines a translating reference frame that is aligned with the base frame and has an origin that is coincident with the crane tip frame.

In Figure D.3, the base actuator sub-diagram is shown as the main revolute joint $e_{2}$ in parallel with the three actuator mechanism joints $e_{2}^{\prime} e_{2}^{\prime \prime} e_{2}^{\prime \prime \prime}$. The prismatic joint $e_{2}^{\prime \prime}$ accepts the extended length of the base actuator $d_{2 x}$ as an input for the forward 


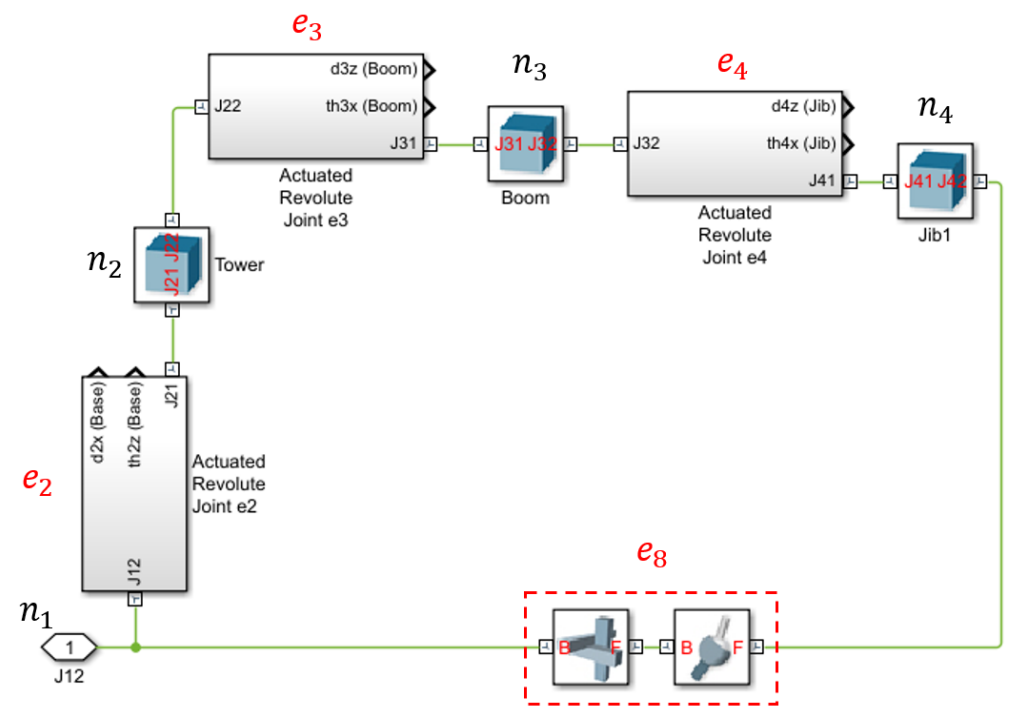

Figure D.2: Simscape block diagram of the knuckle boom crane used to confirm the kinematic analysis.

kinematic problem. Due to restrictions in the software, additional rigid transforms are included to align the coordinate frames so that the local joint axis aligns with a local $z$-axis. Both the boom actuator mechanism for joint $e_{3}$ and the jib actuator mechanism for joint $e_{4}$ are constructed in a similar manner.

To confirm the forward kinematic models, function blocks containing the mathematical models from the previous subsections are implemented in parallel with the Simscape model. The contents of the function blocks are provided below. 


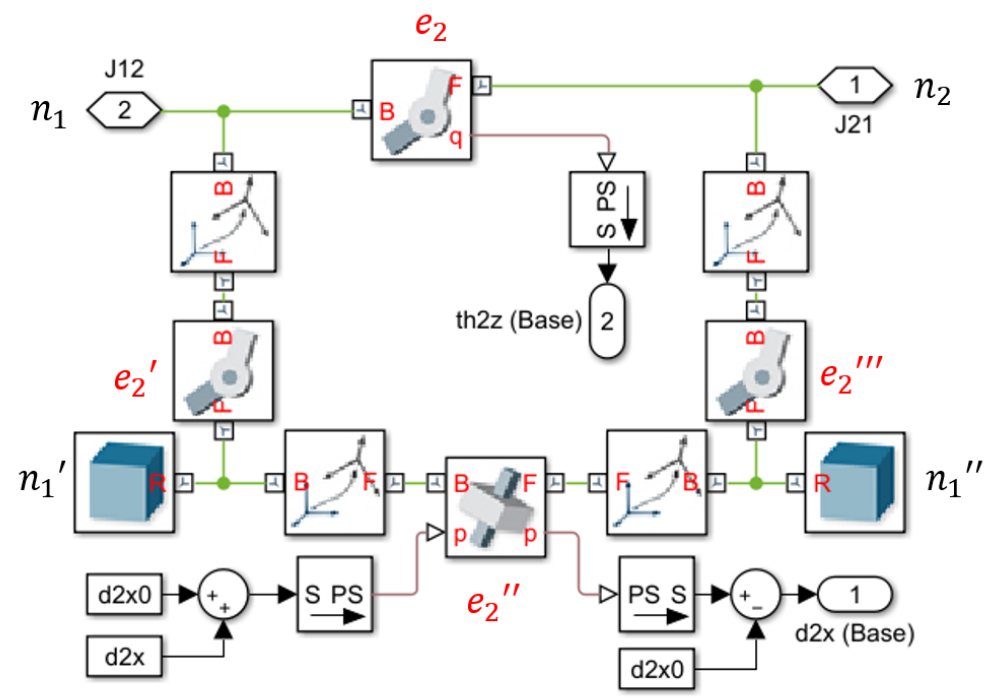

Figure D.3: Simscape block diagram for the base actuator mechanism. 


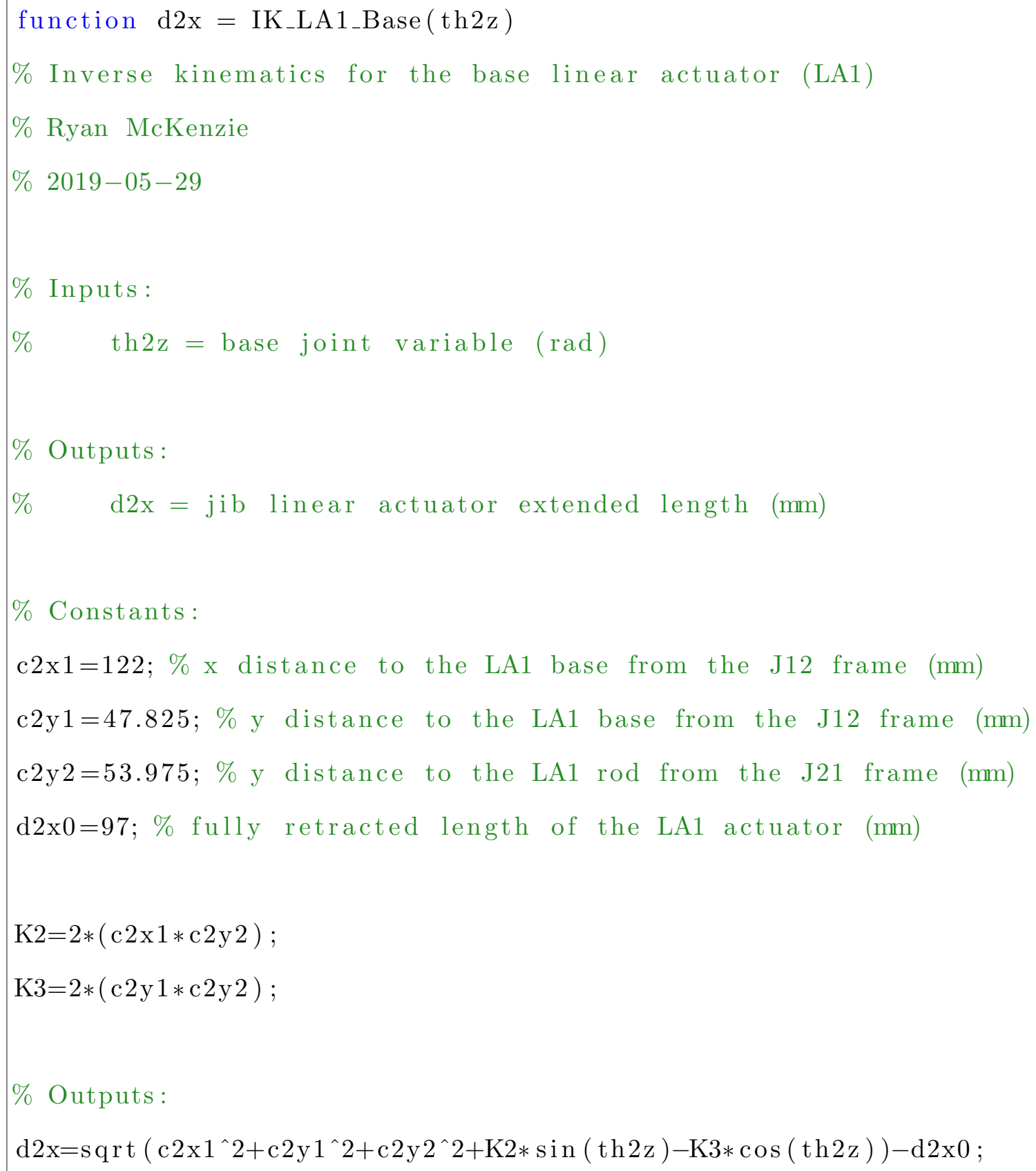


$\%$ Inputs :

$\% \quad$ th $3 x=$ boom joint variable $(\operatorname{rad})$

$\%$ Outputs:

$\% \quad \mathrm{~d} 3 \mathrm{z}=$ boom linear actuator extended length (mm)

$\%$ Constants:

$\mathrm{c} 3 \mathrm{y} 1=62.865 ; \% \mathrm{y}$ distance to the LA2 base from the J22 frame (mm) $\mathrm{c} 3 \mathrm{z} 1=107.315 ; \% \mathrm{z}$ distance to the LA2 base from the J22 frame (mm

c3y $2=66.04 ; \%$ y distance to the LA2 rod from the J31 frame (mm) $\mathrm{c} 3 \mathrm{z} 2=9.525 ; \% \mathrm{z}$ distance to the LA2 rod from the J31 frame (mm) $\mathrm{d} 3 \mathrm{z} 0=97 ; \%$ fully retracted length of the LA2 actuator (mm)

$\mathrm{K} 2=2 *(\mathrm{c} 3 \mathrm{y} 2 * \mathrm{c} 3 \mathrm{z} 1-\mathrm{c} 3 \mathrm{y} 1 * \mathrm{c} 3 \mathrm{z} 2)$

$\mathrm{K} 3=2 *(\mathrm{c} 3 \mathrm{y} 1 * \mathrm{c} 3 \mathrm{y} 2+\mathrm{c} 3 \mathrm{z} 1 * \mathrm{c} 3 \mathrm{z} 2) ;$

$\%$ Outputs:

$\mathrm{d} 3 \mathrm{z}=\operatorname{sqrt}\left(\mathrm{c} 3 \mathrm{y} 1^{\wedge} 2+\mathrm{c} 3 \mathrm{y} 2^{\wedge} 2+\mathrm{c} 3 \mathrm{z} 1^{\wedge} 2+\mathrm{c} 3 \mathrm{z} 2^{\wedge} 2+\mathrm{K} 2 * \sin (\operatorname{th} 3 \mathrm{x})-\mathrm{K} 3 * \cos (\operatorname{th} 3 \mathrm{x})\right)-$ $\mathrm{d} 3 \mathrm{z} 0$

function $\mathrm{d} 4 \mathrm{z}=$ IK_LA $3 \_J i b(\operatorname{th} 4 \mathrm{x})$

\% Inverse kinematics for the jib linear actuator (LA3)

\% Ryan McKenzie

$\% 2019-05-29$

$\%$ Inputs: 


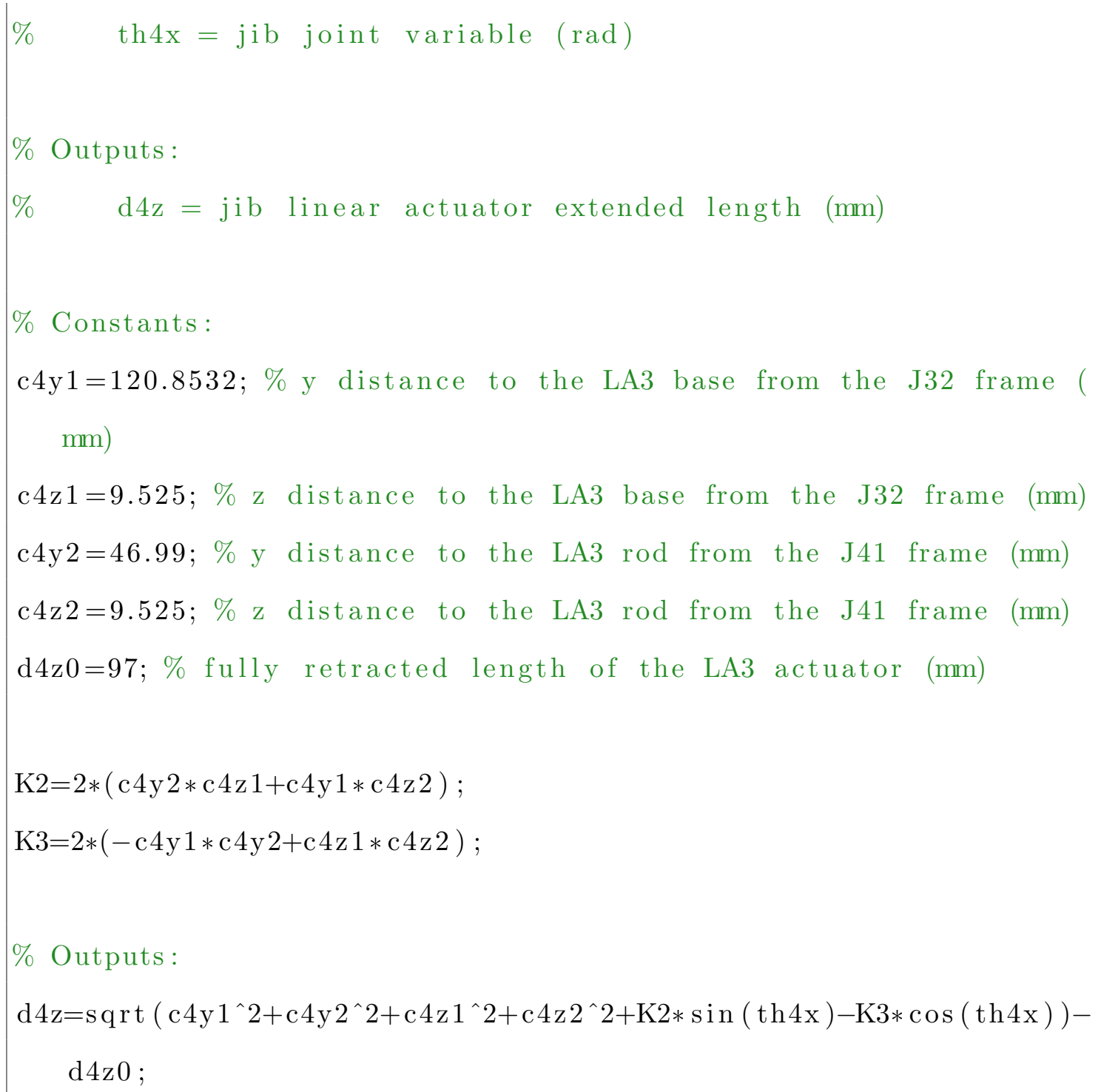




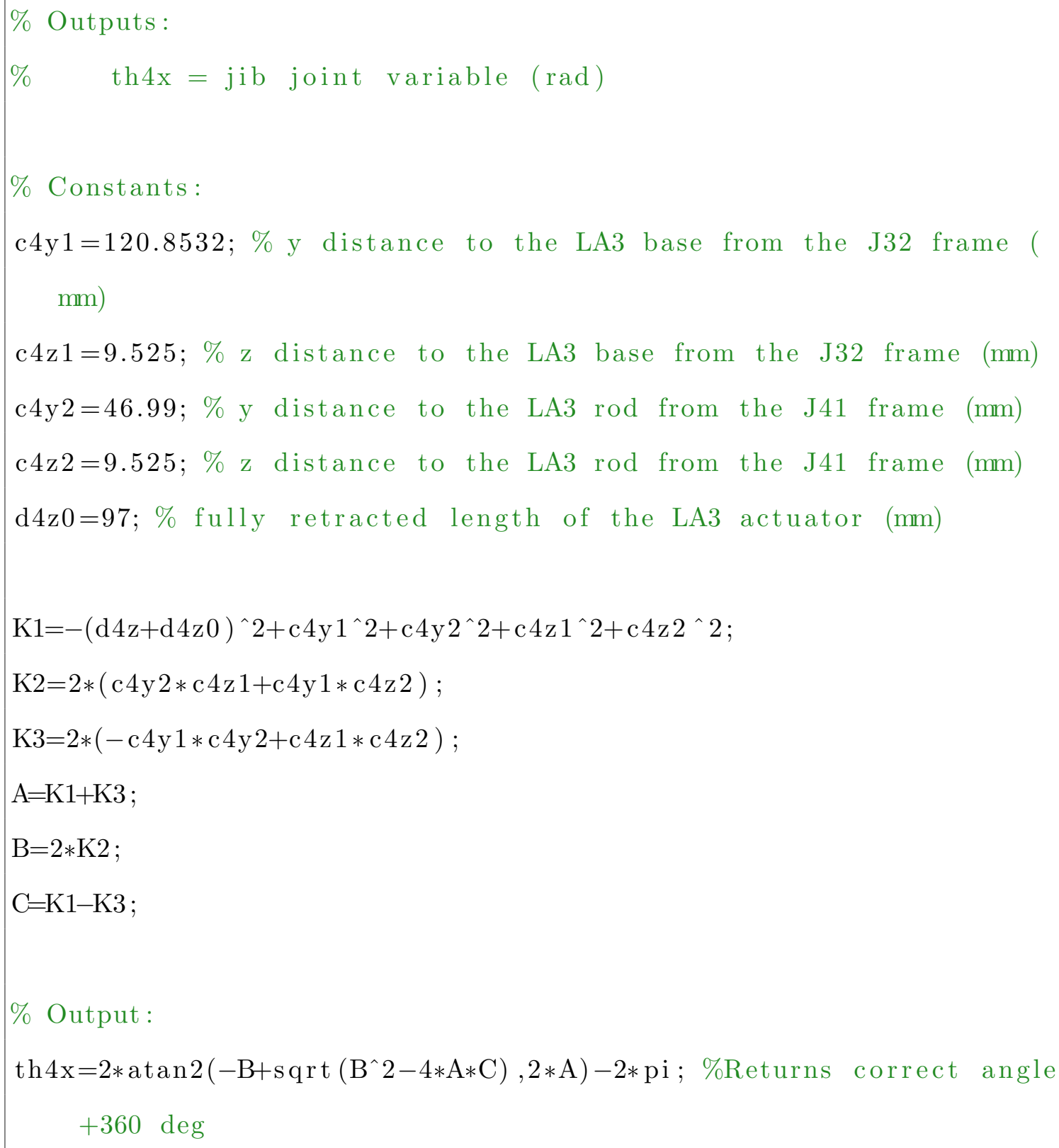




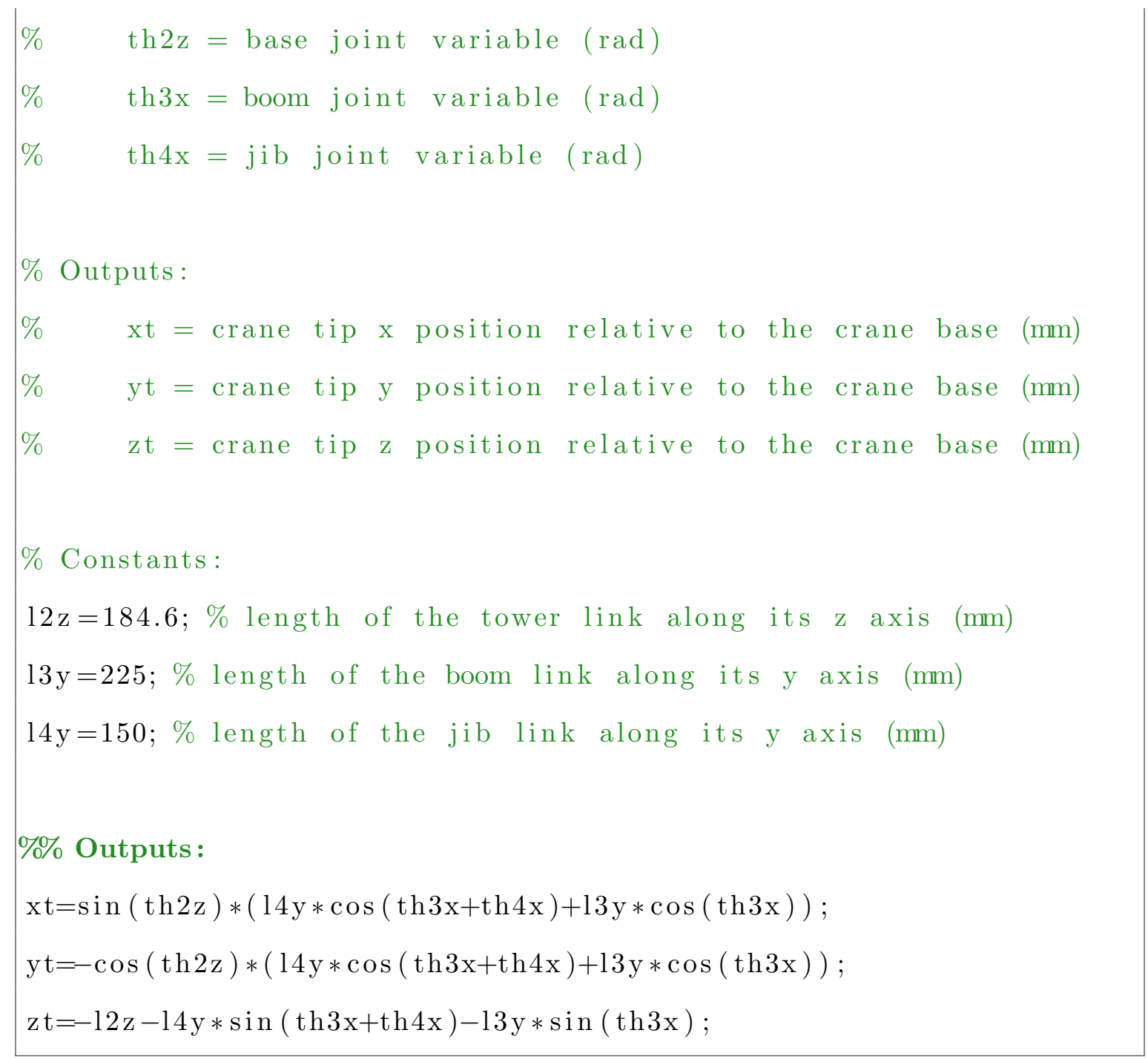

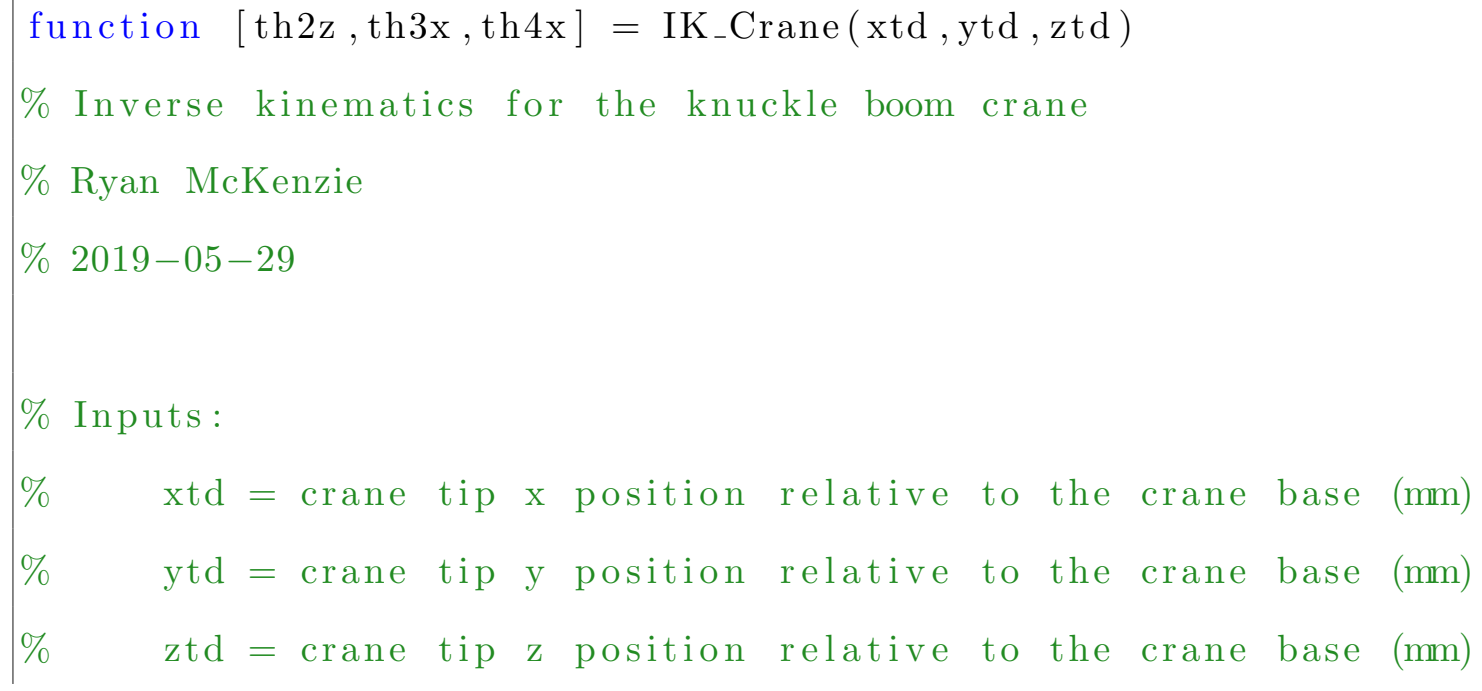


$\%$ Outputs :

$\% \quad$ th $2 \mathrm{z}=$ base joint variable $(\mathrm{rad})$

$\% \quad$ th $3 \mathrm{x}=$ boom joint variable $(\mathrm{rad})$

$\% \quad \operatorname{th} 4 \mathrm{x}=\mathrm{jib}$ joint variable $(\mathrm{rad})$

$\%$ Constants:

$12 \mathrm{z}=184.6$; \% length of the tower link along its $\mathrm{z}$ axis (mm)

$13 \mathrm{y}=225 ; \%$ length of the boom link along its y axis (mm)

$14 \mathrm{y}=150 ; \%$ length of the jib link along its y axis (mm)

$\% \%$ Output 1 - Base Joint Variable:

$\operatorname{th} 2 \mathrm{z}=\operatorname{atan} 2(\mathrm{xtd},-\mathrm{ytd}) ;$

$\% \%$ Output 2 - Boom Joint Variable:

$\mathrm{K} 1=\mathrm{xtd}^{\wedge} 2+\mathrm{ytd}^{\wedge} 2+2 * 12 \mathrm{z} * \mathrm{ztd}+13 \mathrm{y}^{\wedge} 2+12 \mathrm{z}^{\wedge} 2+\mathrm{ztd}{ }^{\wedge} 2-14 \mathrm{y}^{\wedge} 2 ;$

$\mathrm{K} 2=2 *(13 \mathrm{y} * 12 \mathrm{z}+13 \mathrm{y} * \mathrm{ztd}) ;$

$\mathrm{K} 3=2 * 13 \mathrm{y} * \operatorname{sqrt}\left(\operatorname{xtd}^{\wedge} 2+\mathrm{ytd}^{\wedge} 2\right) ;$

$\mathrm{A}=\mathrm{K} 1+\mathrm{K} 3$;

$\mathrm{B}=2 * \mathrm{~K} 2$

$\mathrm{C}=\mathrm{K} 1-\mathrm{K} 3$;

$\operatorname{th} 3 \mathrm{x}=2 * \operatorname{atan} 2\left(-\mathrm{B}+\operatorname{sqrt}\left(\mathrm{B}^{\wedge} 2-4 * \mathrm{~A} * \mathrm{C}\right), 2 * \mathrm{~A}\right) ;$

$\% \%$ Output 3 - Jib Joint Variable:

$\operatorname{th} 4 x=\operatorname{atan} 2\left(-z \operatorname{td}-12 z-13 y * \sin (\operatorname{th} 3 x), \operatorname{sqrt}\left(x \operatorname{td}^{\wedge} 2+y \operatorname{td}^{\wedge} 2\right)-13 y * \cos (\operatorname{th} 3 x)\right.$ )$-\operatorname{th} 3 x$ 


\section{D.2 Matrix Form of Equations of Motion}

The multibody dynamic equations for the variable length spherical pendulum with a three-degree-of-freedom trolley were derived in Chapter 4. In this section, the matrix form of the equations of motion are provided for convenience as they were used in the derivation and may be useful in future work. Equation 4.109 is restated here as

$$
\begin{aligned}
& M(\xi) \ddot{\xi}+B(\xi, \dot{\xi}) \dot{\xi}+G(\xi)=\tau \\
& {\left[\xi_{1}, \xi_{2}, \xi_{3}, \xi_{4}, \xi_{5}, \xi_{6}\right]^{T}=\left[x_{t}, y_{t}, z_{t}, \theta_{x}, \theta_{y}, l_{p}\right]^{T}} \\
& {\left[\tau_{1}, \tau_{2}, \tau_{3}, \tau_{4}, \tau_{5}, \tau_{6}\right]^{T}=\left[F_{x_{t}}, F_{y_{t}}, F_{z_{t}}, T_{\theta_{x}}, T_{\theta_{y}}, F_{l_{p}}\right]^{T}}
\end{aligned}
$$

where $\xi$ and $\tau$ are the $6 \times 1$ vectors of generalized coordinates and forces, $M(\xi)$ is the $6 \times 6$ positive definite and symmetric inertia matrix, $B(\xi, \dot{\xi}) \dot{\xi}$ represents the Coriolis terms $\left(\dot{\xi}_{i} \dot{\xi}_{j}, i \neq j\right)$ and centrifugal terms $\left(\dot{\xi}_{i}^{2}\right)$ as the product of a $6 \times 6$ matrix and the generalized velocities, and $G(\xi)$ is a $6 \times 1$ vector of the gravitational forces and torques. The definitions of these terms are provided here as 


$$
\begin{aligned}
M(\xi) & =\left[\begin{array}{cccccc}
M_{11} & 0 & 0 & 0 & M_{15} & M_{16} \\
0 & M_{22} & 0 & M_{24} & M_{25} & M_{26} \\
0 & 0 & M_{33} & M_{34} & M_{35} & M_{36} \\
0 & M_{42} & M_{43} & M_{44} & 0 & 0 \\
M_{51} & M_{52} & M_{53} & 0 & M_{55} & 0 \\
M_{61} & M_{62} & M_{63} & 0 & 0 & M_{66}
\end{array}\right] \\
M_{11} & =M_{22}=M_{33}=m_{t}+m_{p} \\
M_{44} & =m_{p} l_{p}^{2} c_{y}^{2} \\
M_{55} & =m_{p} l_{p}^{2} \\
M_{66} & =m_{p} \\
M_{15} & =M_{51}=m_{p} l_{p} c_{y} \\
M_{16} & =M_{61}=m_{p} s_{y} \\
M_{24} & =M_{42}=-m_{p} l_{p} c_{x} c_{y} \\
M_{25} & =M_{52}=m_{p} l_{p} s_{x} s_{y} \\
M_{26} & =M_{62}=-m_{p} c_{y} s_{x} \\
M_{34} & =M_{43}=-m_{p} l_{p} c_{y} s_{x} \\
M_{35} & =M_{53}=-m_{p} l_{p} c_{x} s_{y} \\
M_{36} & =M_{63}=m_{p} c_{x} c_{y}
\end{aligned}
$$




$$
\begin{aligned}
& B(\xi, \dot{\xi})=\left[\begin{array}{cccccc}
0 & 0 & 0 & 0 & B_{15} & B_{16} \\
0 & 0 & 0 & B_{24} & B_{25} & B_{26} \\
0 & 0 & 0 & B_{34} & B_{35} & B_{36} \\
0 & 0 & 0 & B_{44} & B_{45} & B_{46} \\
0 & 0 & 0 & B_{54} & B_{55} & B_{56} \\
0 & 0 & 0 & B_{64} & B_{65} & 0
\end{array}\right] \\
& B_{15}=-m_{p} l_{p} s_{y} \dot{\theta}_{y}+m_{p} c_{y} \dot{l}_{p} \\
& B_{16}=m_{p} c_{y} \dot{\theta_{y}} \\
& B_{24}=m_{p} l_{p} c_{y} s_{x} \dot{\theta_{x}}+m_{p} l_{p} c_{x} s_{y} \dot{\theta_{y}}-m_{p} c_{x} c_{y} \dot{l_{p}} \\
& B_{25}=m_{p} l_{p} c_{y} s_{x} \dot{\theta_{y}}+m_{p} l_{p} c_{x} s_{y} \dot{\theta_{x}}+m_{p} s_{x} s_{y} \dot{l}_{p} \\
& B_{26}=-m_{p} c_{x} c_{y} \dot{\theta_{x}}+m_{p} s_{x} s_{y} \dot{\theta_{y}} \\
& B_{34}=-m_{p} l_{p} c_{x} c_{y} \dot{\theta_{x}}+m_{p} l_{p} s_{x} s_{y} \dot{\theta_{y}}-m_{p} c_{y} s_{x} \dot{l_{p}} \\
& B_{35}=-m_{p} l_{p} c_{x} c_{y} \dot{\theta_{y}}+m_{p} l_{p} s_{x} s_{y} \dot{\theta_{x}}-m_{p} c_{x} s_{y} \dot{l_{p}} \\
& B_{36}=-m_{p} c_{y} s_{x} \dot{\theta_{x}}-m_{p} c_{x} s_{y} \dot{\theta_{y}} \\
& B_{44}=-m_{p} l_{p}^{2} c_{y} s_{y} \dot{\theta}_{y}+m_{p} l_{p} c_{y}^{2} \dot{l}_{p} \\
& B_{45}=-m_{p} l_{p}^{2} c_{y} s_{y} \dot{\theta_{x}} \\
& B_{46}=m_{p} l_{p} c_{y}^{2} \dot{\theta_{x}} \\
& B_{54}=m_{p} l_{p}^{2} c_{y} s_{y} \dot{\theta_{x}} \\
& B_{55}=m_{p} l_{p} \dot{l}_{p} \\
& B_{56}=m_{p} l_{p} \dot{\theta}_{y} \\
& B_{64}=-m_{p} l_{p} c_{y}^{2} \dot{\theta_{x}} \\
& B_{65}=-m_{p} l_{p} \dot{\theta}_{y}
\end{aligned}
$$




$$
G(\xi)=\left[\begin{array}{c}
0 \\
0 \\
-m_{p} g \\
m_{p} l_{p} c_{y} s_{x} g \\
m_{p} l_{p} c_{x} s_{y} g \\
-m_{p} c_{x} c_{y} g
\end{array}\right]
$$




\section{D.3 Confirmation of Dynamic Models}

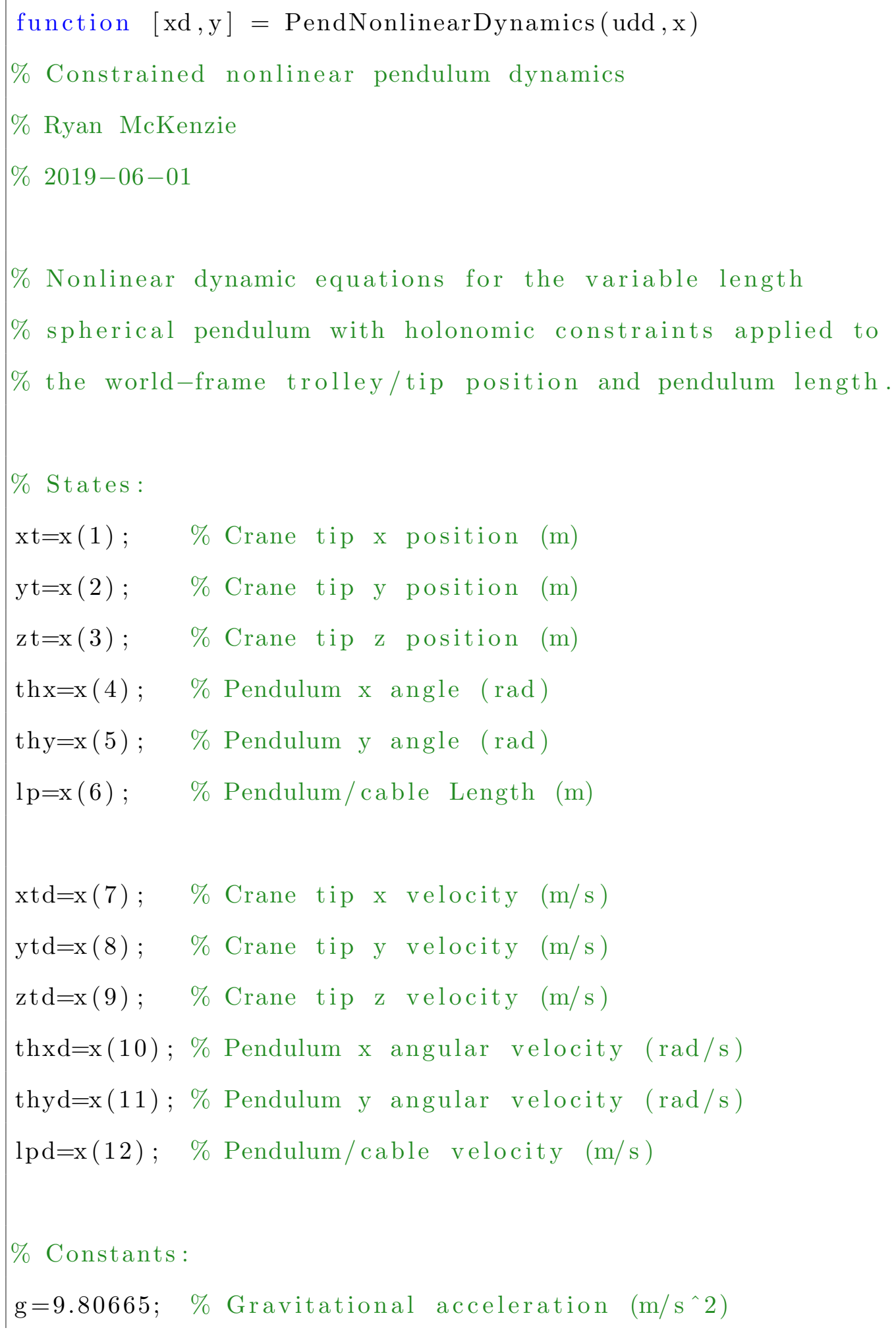


\% Calculate time derivative of state vector:

$c x=\cos (\operatorname{th} x)$

$\operatorname{sx}=\sin (\operatorname{th} x)$;

$\mathrm{cy}=\cos ($ thy $)$;

$\operatorname{sy}=\sin ($ thy $)$;

PhiBG $=[0 ; 0 ; 0 ;$

$(-\mathrm{g} * \mathrm{sx}-2 * \mathrm{cy} * \operatorname{lpd} * \operatorname{thx} d+2 * \operatorname{lp} * \operatorname{sy} * \operatorname{thx} d * \operatorname{thyd}) /(\operatorname{cy} * \operatorname{lp}) ;$

$\left(-\mathrm{cy} * \mathrm{lp} * \mathrm{sy} * \operatorname{thxd}{ }^{\wedge} 2-2 * \operatorname{lpd} * \operatorname{thyd}-\mathrm{cx} * \mathrm{sy} * \mathrm{~g}\right) / \mathrm{lp} ;$

$0]$;

Phiudd $=[$ eye $(3), \quad$ zeros $(3,3)$;

$[0, \quad \mathrm{cx}, \quad \mathrm{sx}, \quad 0, \quad 0,0] \cdot /(\mathrm{cy} * \mathrm{lp}) ;$

$\left[\begin{array}{llllll}-\mathrm{cy}, & -\mathrm{sx} * \mathrm{sy}, \quad \mathrm{cx} * \mathrm{sy}, \quad 0, \quad 0,0\end{array}\right] / \mathrm{lp} ;$

$[0,0,0,0,0,1]]$;

$\mathrm{xd}=[\mathrm{x}(7: 12) ;$ PhiBG $]+[$ zeros $(6,6) ;$ Phiudd $] *$ udd $;$

\% Calculate system output vector:

$\mathrm{y}=[\mathrm{xt}+\mathrm{lp} * \mathrm{sy} ; \quad \%$ Pendulum $\mathrm{x}$ position $(\mathrm{m})$

$\mathrm{yt}-\mathrm{lp} * \mathrm{cy} * \mathrm{sx} ; \quad \%$ Pendulum y position $(\mathrm{m})$

$\mathrm{zt}+\mathrm{lp} * \mathrm{cx} * \mathrm{cy}] ; \quad \%$ Pendulum z position $(\mathrm{m})$

function $[\mathrm{xd}, \mathrm{y}]=$ PendLinearDynamics $(\mathrm{u}, \mathrm{x})$

\% Constrained linear pendulum dynamics

\% Ryan McKenzie

$\%$ 2019-06-01 
$\%$ Linear dynamic equations for the variable length

$\%$ spherical pendulum with holonomic constraints applied to

$\%$ the world-frame trolley/tip position and pendulum length.

$\%$ Presented in state-space form with linearisation about an

$\%$ operating point due to dependence of the system matrices

$\%$ on the length of the pendulum.

$\mathrm{g}=9.80665 ; \quad \%$ Gravitational acceleration $\left(\mathrm{m} / \mathrm{s}^{\wedge} 2\right)$

$\mathrm{lp}=0.250 ; \quad \%$ Pendulum length $(\mathrm{m})$ at current operating point

$\%$ Define system matrices:

$\mathrm{A}=[\operatorname{zeros}(6,6)$, eye $(6) ;$

$\left[\begin{array}{llllll}0 & 0 & 0 & 0 & 0 & 0\end{array}\right.$

$\begin{array}{llllll}0 & 0 & 0 & 0 & 0 & 0\end{array}$

$\begin{array}{llllll}0 & 0 & 0 & 0 & 0 & 0\end{array}$

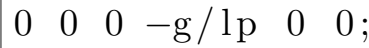

$\begin{array}{lllllll}0 & 0 & 0 & 0 & -\mathrm{g} / \mathrm{lp} & 0\end{array}$

$\left.\left.0 \begin{array}{llllll}0 & 0 & 0 & 0 & 0 & 0\end{array}\right], \operatorname{zeros}(6,6)\right]$;

$\mathrm{B}=[\operatorname{zeros}(6,6) ;$

eye $(3)$, zeros $(3,3)$;

$0,1 / \mathrm{lp}, 0,0,0,0$;

-1/lp , $0,0,0,0,0$;

$0,0,0,0,0,1]$; 


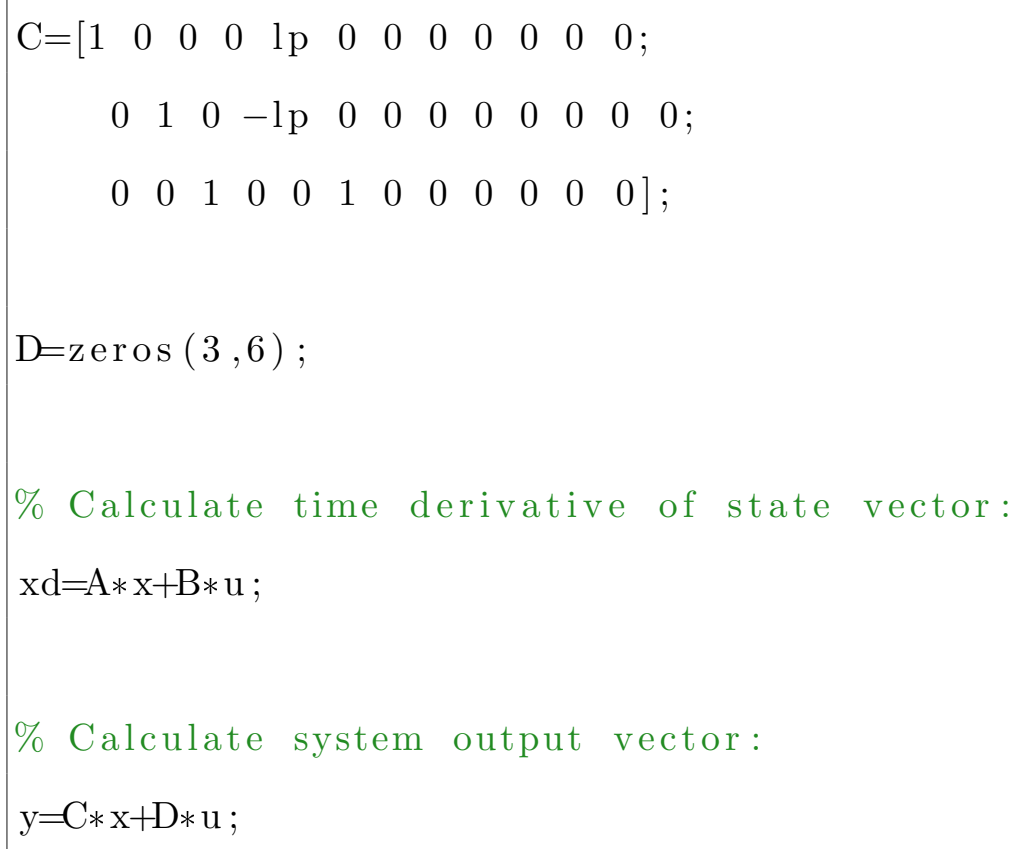

